\title{
CRITICAL SUCCESS FACTORS FOR OUTSOURCED SOFTWARE DEVELOPMENT PROJECTS FROM A VENDOR'S PERSPECTIVE: A STRUCTURAL EQUATION MODELLING ANALYSIS OF TRADITIONAL PLAN-BASED AND AGILE METHODOLOGIES
}

By

Arthur Ahimbisibwe

A thesis submitted to Victoria University of Wellington in fulfilment of the requirements for the degree of Doctor of Philosophy in Management

Victoria University of Wellington 


\begin{abstract}
There are many factors proposed as to why software projects fail, one of them is the inappropriate choice of a project management methodology. Although there is an increased range of available management choices, project managers do not frequently consider their alternatives. They tend to narrowly tailor project categorisation systems and use categorisation criteria that are not logically linked with objectives. To address this, this study develops and tests an integrative contingency fit model for contrasting perspectives of traditional plan-based and agile methodologies specifically for outsourced software development projects. In addition, it takes a vendor's perspective, rather than the client perspective that is mostly used. Overall, the research seeks to answer these questions: (RQ1) what are the Critical Success Factors (CSFs) for outsourced software development projects from a vendor's perspective? (RQ2) What are the differences in these CSFs for traditional plan-driven and agile methodologies towards project success from a vendor's perspective?
\end{abstract}

The IT literature reveals two major distinct categories of methodologies: traditional plan-based and agile. Previous research has identified CSFs with respect to project success with mixed findings. The recent increase in popularity of methodologies has shifted the debate, interest and controversy to CSFs that are the factors which are most important to make a methodology successful. While there is an increasing diversity of project types, project contexts and methodologies, the frameworks or theories connecting these are limited. To date software development projects studies have addressed generally one methodology per study and perceived candidate CSFs as a form of reasons of success amidst a wide range of project success criteria. Although contingency theory has been previously argued for outsourced software development projects, empirical models have frequently not fully incorporated contingency as fit or fit as moderation (i.e. traditional vs. agile). This study sought to fill this research gap.

Cross-sectional data from 984 senior vendor project managers and team leaders was collected by a global web-based survey. Structural Equation Modelling (SEM) (a multivariate statistical technique, in which parameters are estimated by minimizing the discrepancy between the model-implied covariance matrix and the observed covariance matrix) was used for data analysis. SEM results provide support for several contingency hypotheses theorizing relationships between candidate CSFs and project success. Project management methodology was found to moderate the effects of various CSFs on project success, and in different ways for various success measures. Similarly, the results show the level of project uncertainty moderates the impact of various CSFs on project success, and in different ways for various success measures. Together these findings provide empirical support for contingency as fit and more fully incorporate fit as moderation.

The study contributes towards understanding the differences between traditional plan-based and agile project management based on the perceptions of vendor respondents with regard to their client organizations, and also to understanding what are the most significant antecedents of success (the CSFs) in different project contexts. The study also examines the indirect and interaction effects, and the findings contribute towards understanding of the contingency perspective as a framework to be used by project managers and organizations. Practical implications of these results suggest that project managers should tailor project management methodologies according to various project types, which is likely to improve current project success rates. 


\section{ACKNOWLEDGEMENTS}

I would like to express my appreciation to all those who helped me to complete this thesis.

To Associate Professor Bob Cavana, my primary supervisor, thank you for patiently taking me through the theoretical and methodological challenges, imparting to me some knowledge and taking me through hard times. Without your input my $\mathrm{PhD}$ would have remained a nightmare.

To Associate Professor Urs Daellenbach, my secondary supervisor, I appreciate all your input in all forms, including your prompt feedbacks, agility and directions on SEM analyses. I also thank you for your moral support, good research skills and enormous encouragement to keep me going.

To my examiners, Dr. Mary Tate, Professor Nevan Wright, and Associate Professor Ofer Zwikael, thank you for the insightful feedback and your suggestions to further improve this work.

To all academic as well administrative staff of School of Management who provided me with important input during this project, I pray that the almighty God rewards you abundantly. My special thanks go to Megan Key, Sophia Lum and Laura Dimock as well as my senior colleagues Tibor Zsirmik and Debbie Maree Forsyth, am very humbled by your continuous support and I highly acknowledge your endless efforts towards completing this thesis.

To Victoria University of Wellington and the School of Management, I owe you a lot for this great opportunity and the financial support that you provided to me.

To my late parents, family, friends, and all research participants, I acknowledge your contribution towards my completion of this study. 


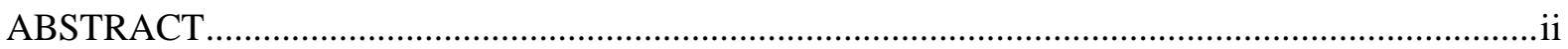

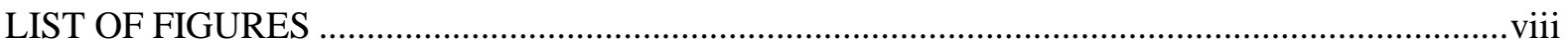

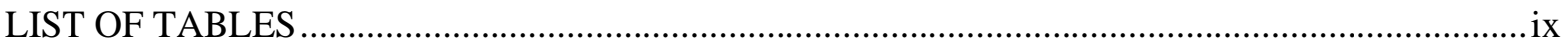

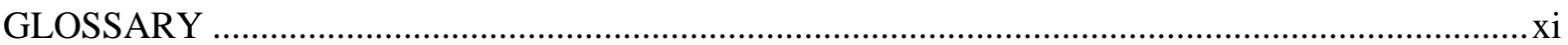

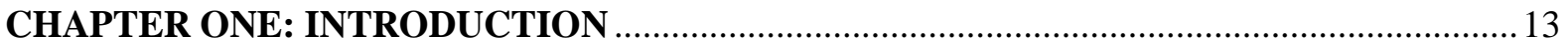

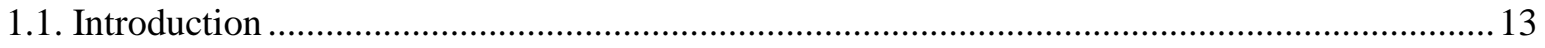

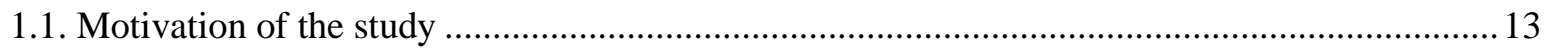

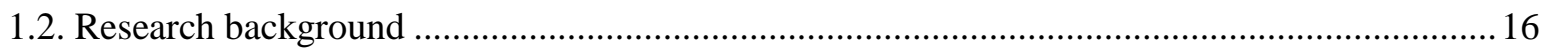

1.2.1. Communities of project management methodology practices ............................................ 19

1.2.2. Contrasting two major vendor communities of methodology practice................................24

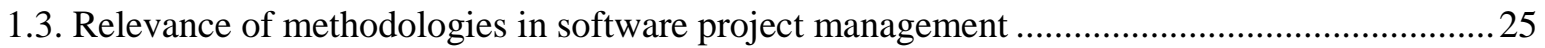

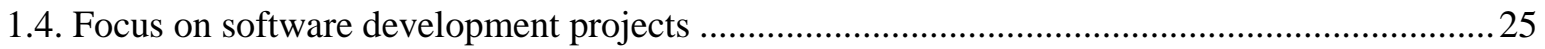

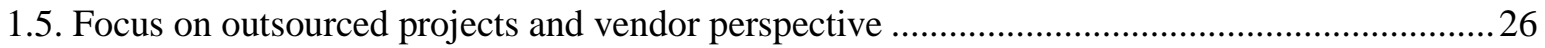

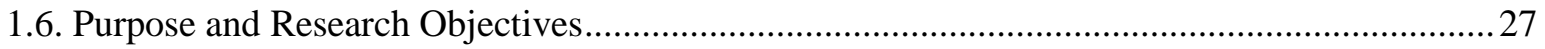

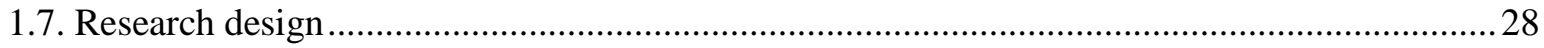

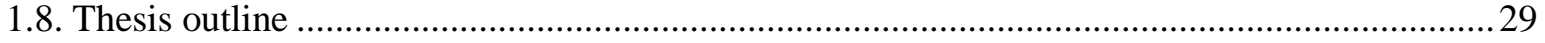

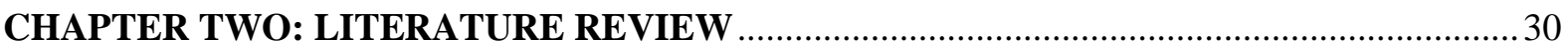

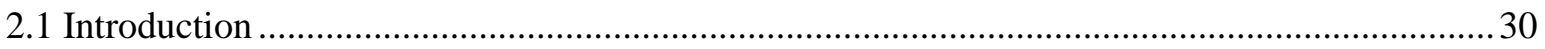

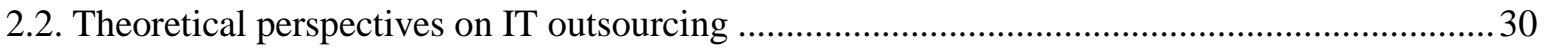

2.2.1. Approaches, types and quality of IT outsourcing relationships.......................................... 31

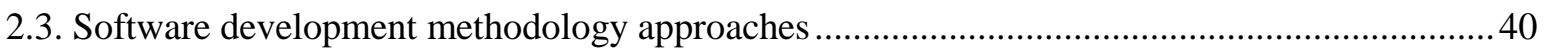

2.3.1. Comparison of software development methodologies........................................................ 42

2.4. The concept of project success in software project management research ................................. 48

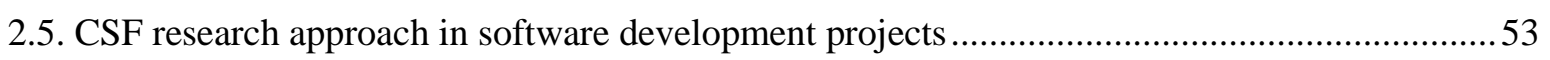

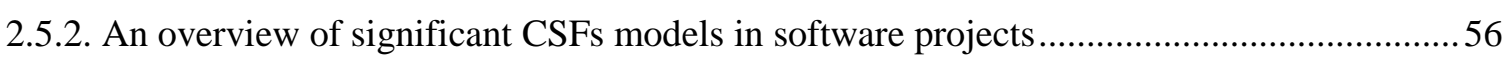

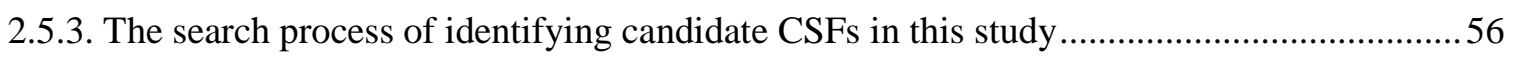

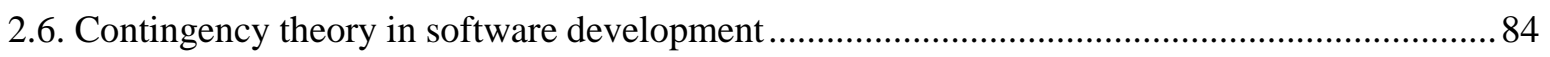

2.6.1. Empirical studies of contingency theory in software development .................................... 86

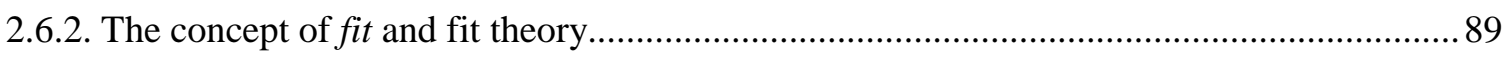

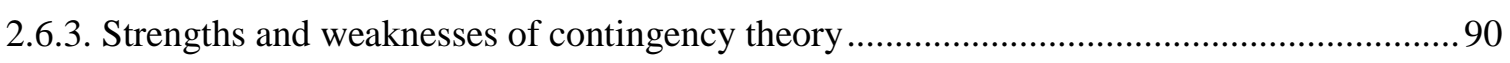

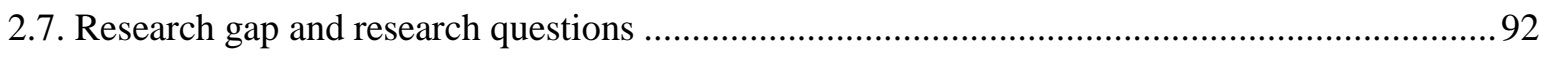

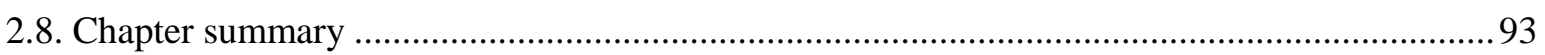




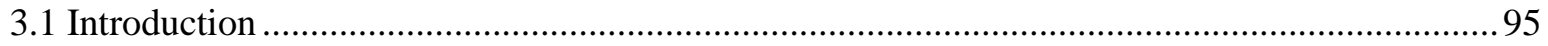

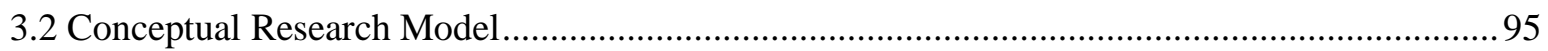

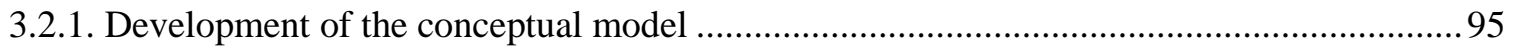

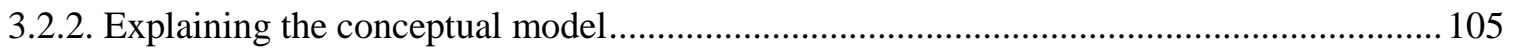

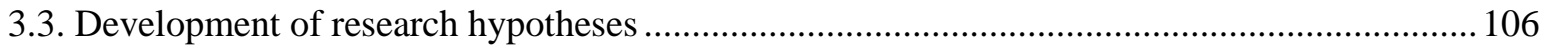

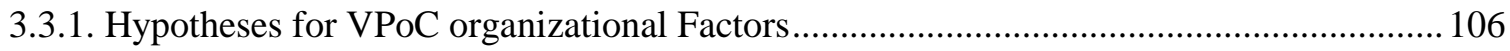

3.3.2 Hypotheses for vendor perception of team factors .......................................................... 112

3.3.3. Hypotheses for vendor perception of customer factors .................................................... 117

3.3.4. Hypotheses for vendor perception of project (uncertainty) factors ................................... 120

3.3.5. Hypotheses on other interrelationships between candidate CSFs and moderation............. 126

3.3.6. The conceptual research model including the associated hypotheses................................ 128

3.3.7. Preliminary relevant checks with software development project practitioners .................. 133

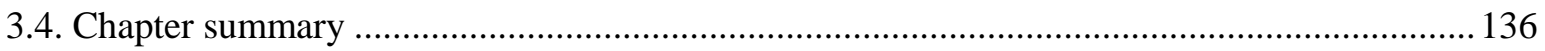

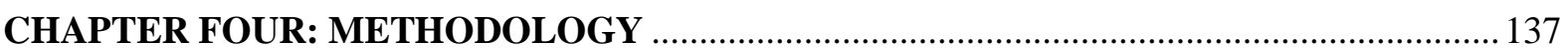

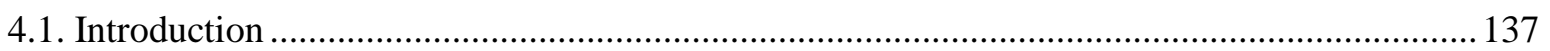

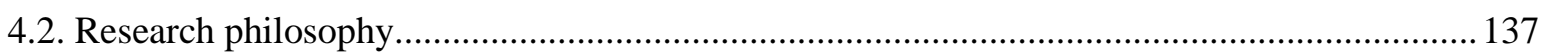

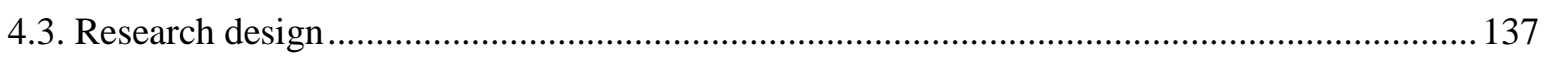

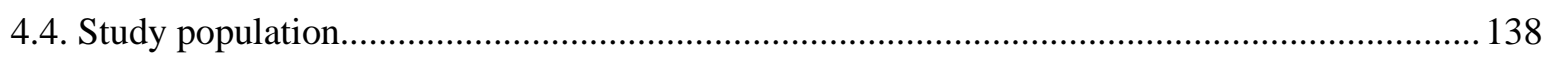

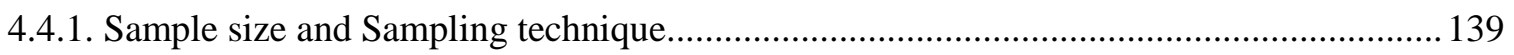

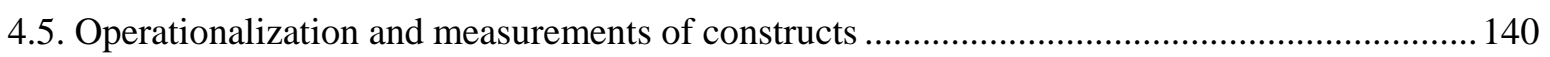

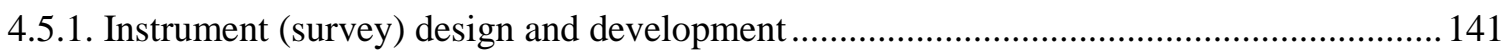

4.5.2. Measurement items identification and collection from literature ....................................... 141

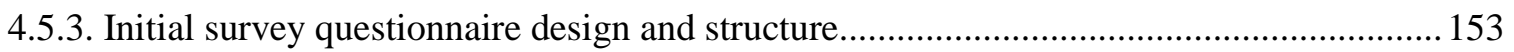

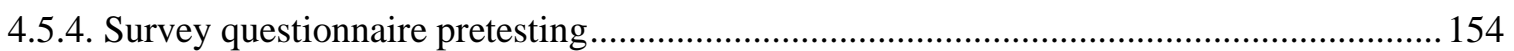

4.5.5. Item reduction in section B of the initial online survey draft ........................................... 163

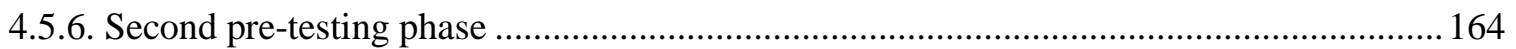

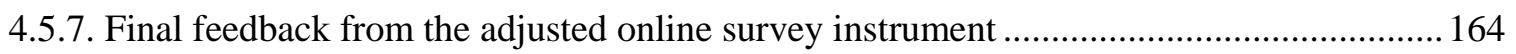

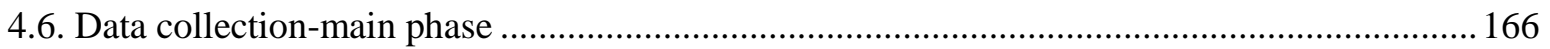

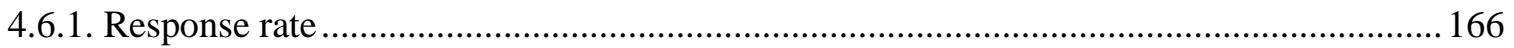

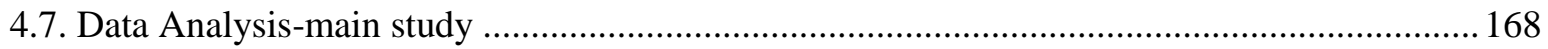

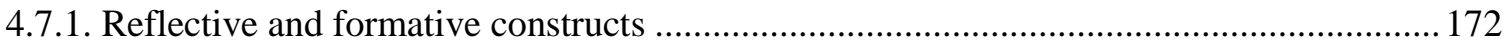

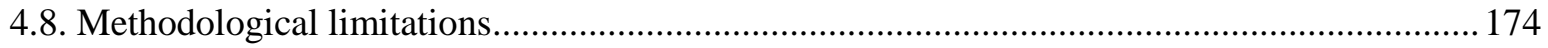

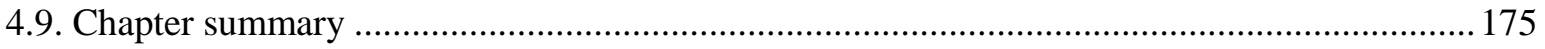




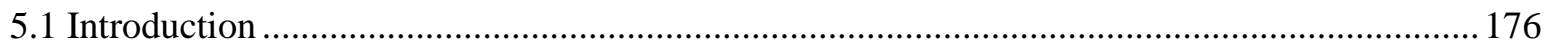

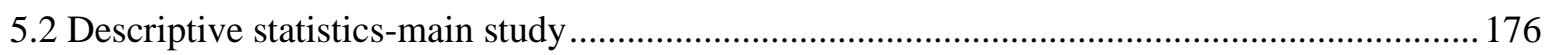

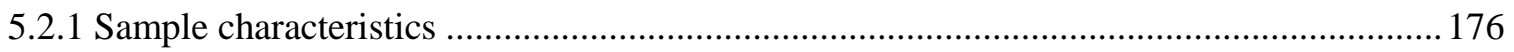

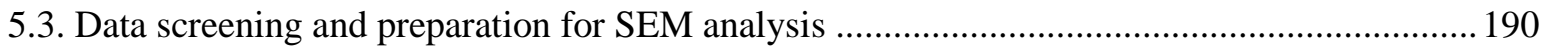

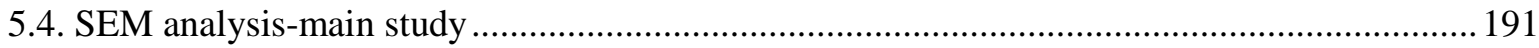

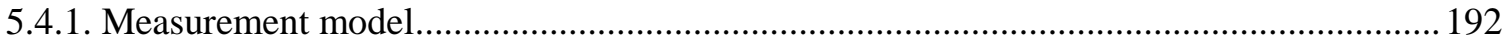

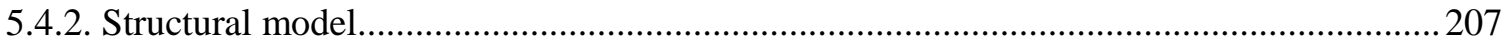

5.4.3. Total variance explained by the final structural models .....................................................2216

5.5. Group invariance testing (group comparisons) using SEM..................................................... 217

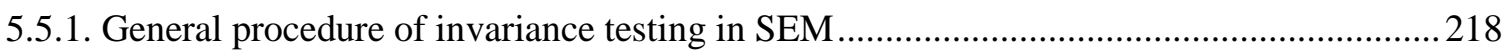

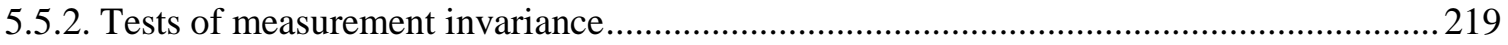

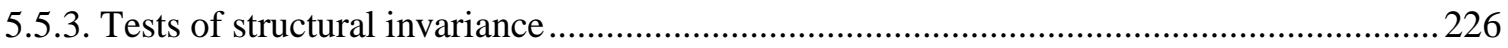

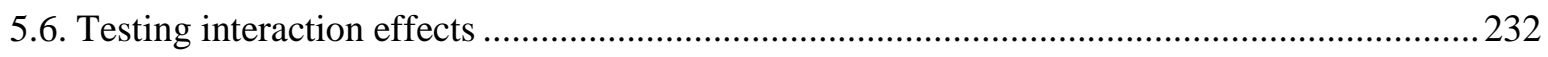

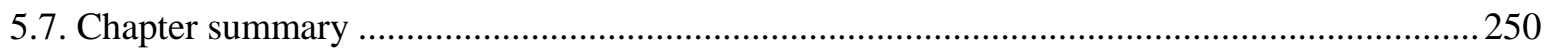

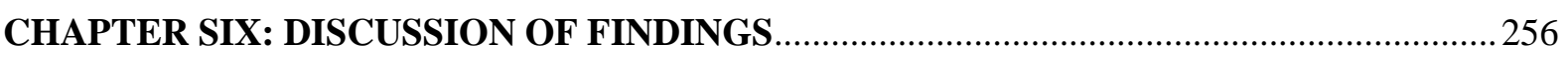

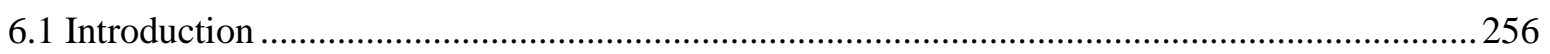

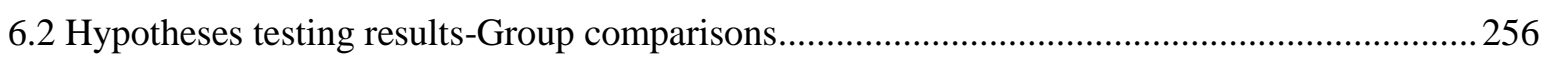

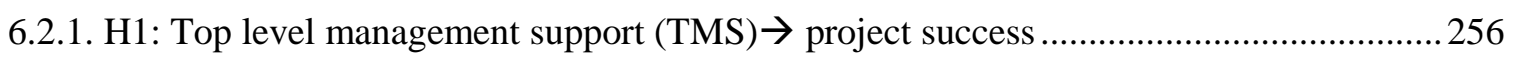

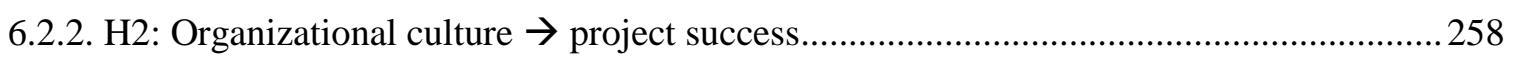

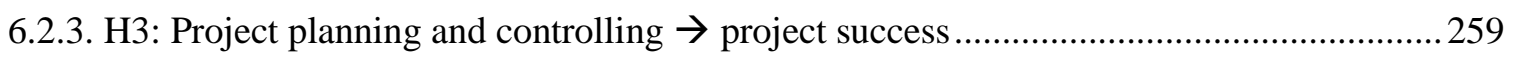

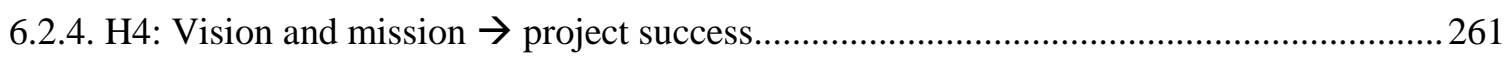

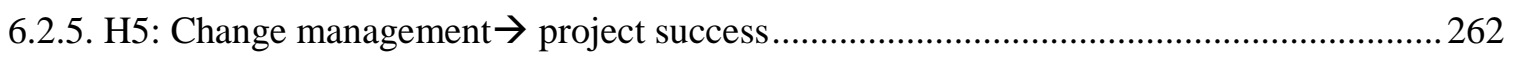

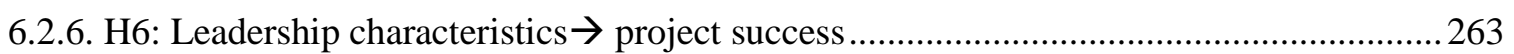

6.2.7. H7: Internal project communication $\rightarrow$ project success .................................................. 264

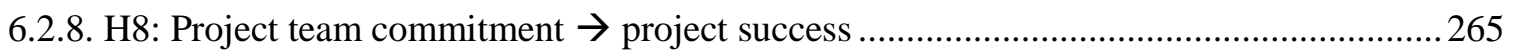

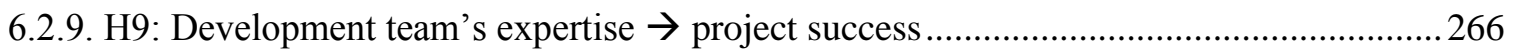

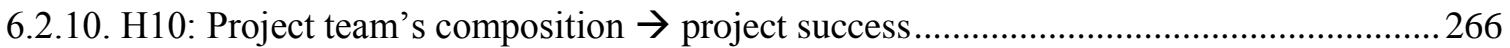

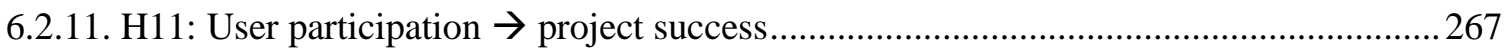

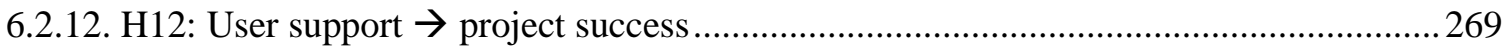

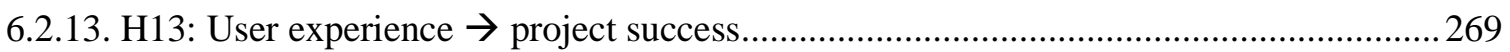

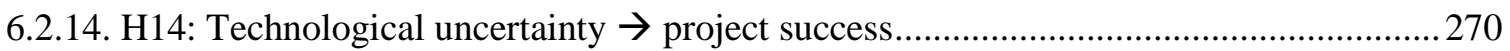

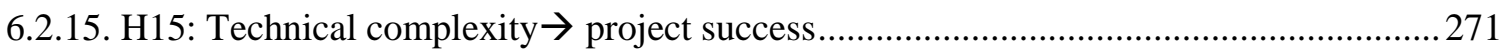

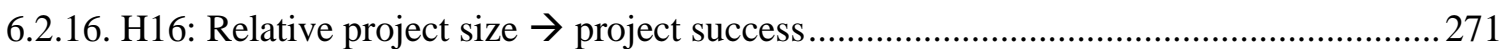


6.2.17. H17: Specification changes $\rightarrow$ project success

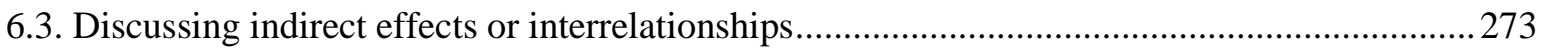

6.4. H19: Moderating effects of project management methodology ……….................................. 274

6.5. H20-24: Moderating effects of vendor perception of project (uncertainty) factors ................. 275

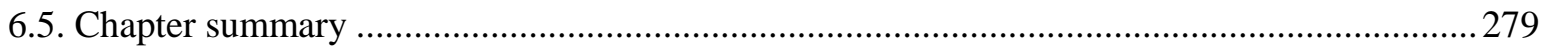

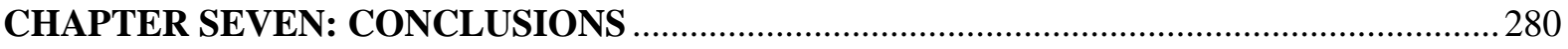

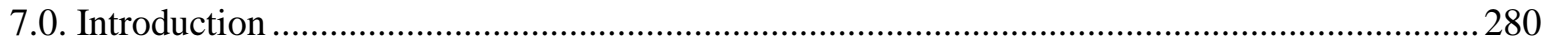

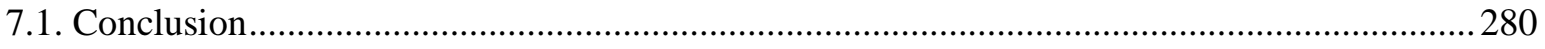

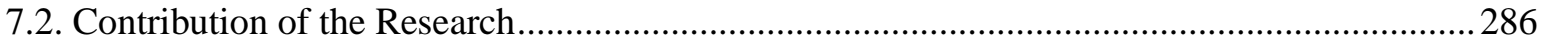

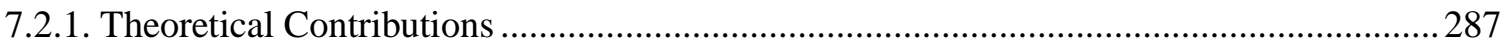

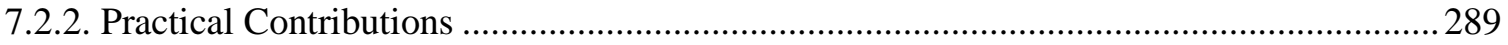

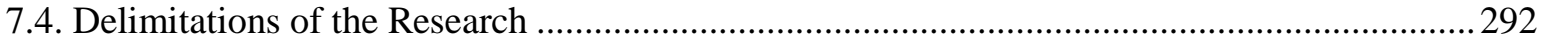

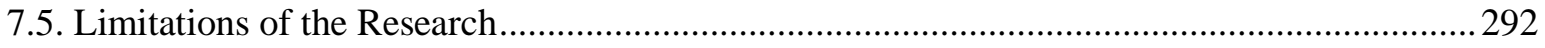

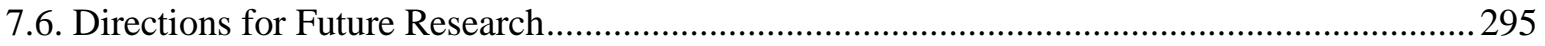

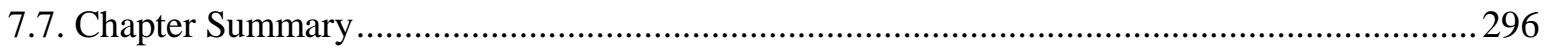

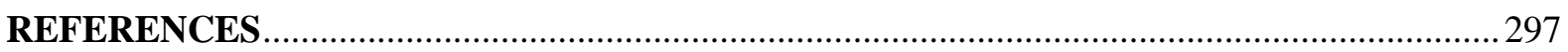

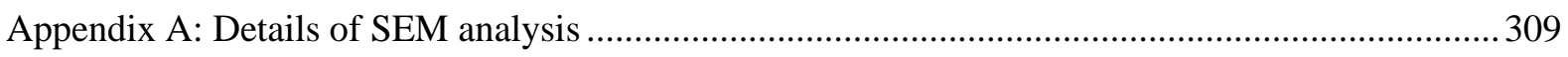

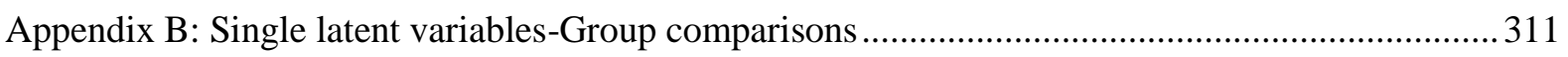

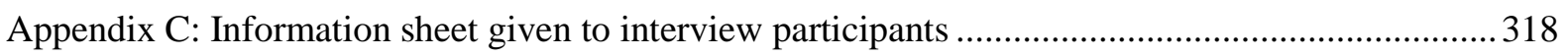

Appendix D: Research agreement signed by interviewees ............................................................ 319

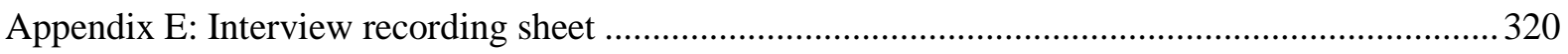

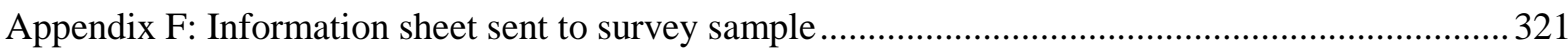

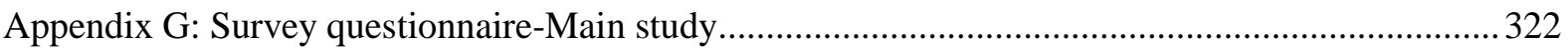




\section{LIST OF FIGURES}

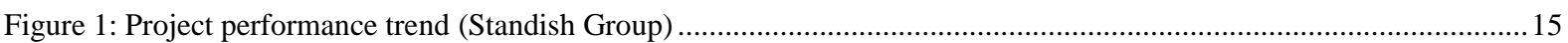

Figure 2: project management hierarchy and associated methodologies ..................................................................... 18

Figure 3:PRINCE2 model (OGC, 2009)

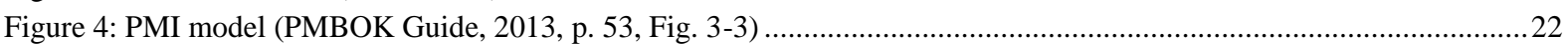

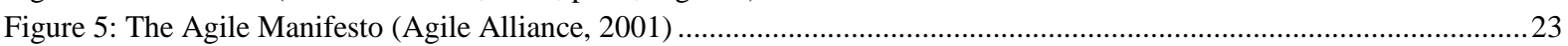

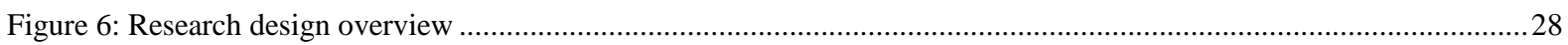

Figure 7: Four types of outsourcing relationships (source: Nam et al, 1996, p.38).......................................................32

Figure 8: Types of outsourcing relationships (Kern et al. 2002, Figure 4, p.66) .............................................................. 34

Figure 9: Evolution of outsourcing relationships (Kishore et al., 2003, Figure 2, p.91) .................................................35

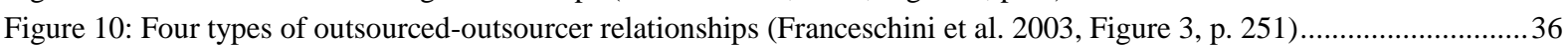

Figure 11: Types of relationship in IT outsourcing (Lacity \& Willcocks, 2000, Figure 18.1, p. 365) ................................37

Figure 12: Three stage-maturity model of outsourcing relationships (Gottschalk \& Solli-Saether, 2006, Figure 1, p.205) ....37

Figure 13: Success rates of Agile and waterfall or traditional projects .....................................................................42

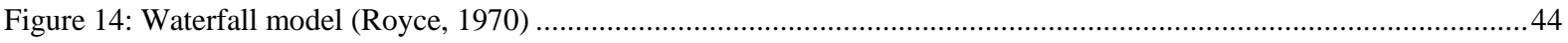

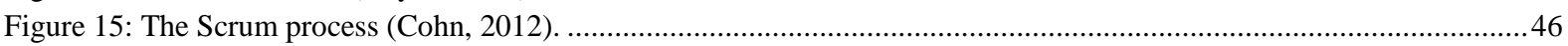

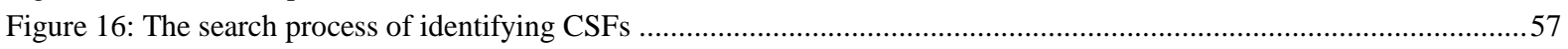

Figure 17: Application system success (Ratbe et al., 2000, Exhibit 2, p.28) ..................................................................5

Figure 18: CSFs for project performance (Yetton et al., 2000, Figure 1, p. 269) .............................................................59

Figure 19: Agile and plan-driven methodologies (Boehm \& Turner, 2003, Figure 2, p.6) ..............................................61

Figure 20: Success factors for agile software projects (Chow \& Cao, 2008, figure 1, p.964)............................................62

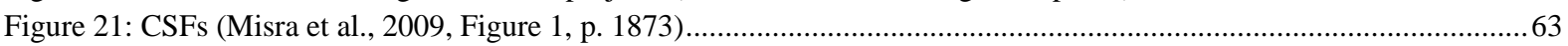

Figure 22: Software development performance (Lee \& Xia, 2010, Figure 1, p. 92) .........................................................65

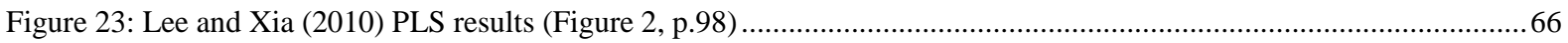

Figure 24: CSFs model for agile process improvement (Wan \& Wang, 2010, Fig 1, p.1134) .........................................66

Figure 25: Theoretical Framework for Successful Agile projects (Mohammad \& Al-Shargabi, 2011, Figure 4.1, p.1204) ...68

Figure 26: An integrative model for project performance (Jun et al., 2011, Fig. 1, p.926) ..................................................69

Figure 27: A proposed model of CSFs for software projects (Sudhakar, 2012, Figure 4, p.552)........................................70

Figure 28: Regression on software development agility (Sheffield \& Lemetayer, 2013, p.468, Fig. 3) .............................. 71

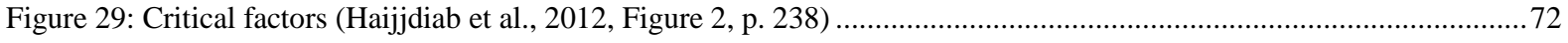

Figure 30: Comparing CSFs frequencies of traditional plan-based and agile methodologies ............................................... 77

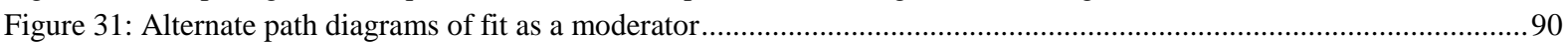

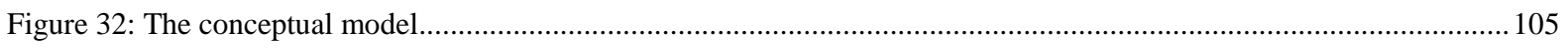

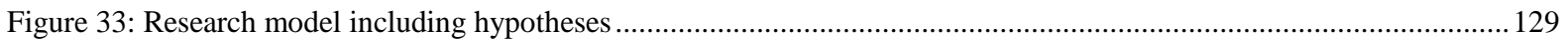

Figure 34: Respondents in traditional/agile methodology vs. vendor and client groups ...............................................168

Figure 35: Alternative measurement models (Diamantopoulos et al., 2008, Figure 1, pg. 1205) ...................................172

Figure 36: Significant moderating effects of project management methodology ............................................................232

Figure 37: A schematic representation of a SEM used to test for moderation for latent variables.....................................233

Figure 38 : Interaction effects of technological uncertainty and candidate CSFs on project success ...................................239

Figure 39: Interaction effects of technical complexity and candidate CSFs on project success ......................................243

Figure 40: Interaction effects of relative project size and candidate CSFs on project success .......................................246

Figure 41: Interaction effects of specification changes and candidate CSFs on project success ........................................250

Figure 42: Summary of CSFs for project success (standardised path coefficients) ..........................................................283

Figure 43: SEM model (main effects model) tested (without covariances for readability and clarity) ................................333 


\section{LIST OF TABLES}

Table 1: Two major communities of project management methodology practice: Traditional and Agile...........24

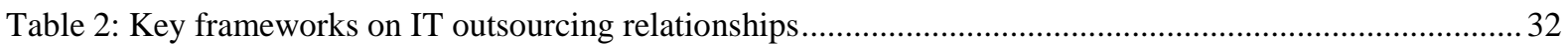

Table 3 Traditional plan based vs. Agile software development methodology ............................................47

Table 4: Summary of the criteria on project success used in previous studies .............................................50

Table 5: Mapping of some previous measures of software development project success ...................................51

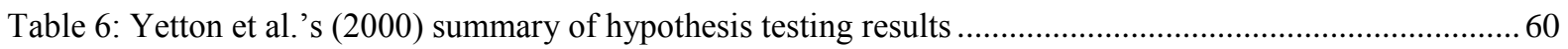

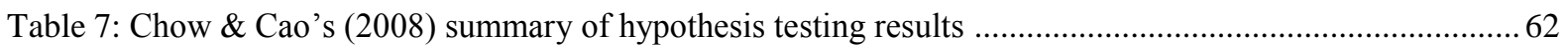

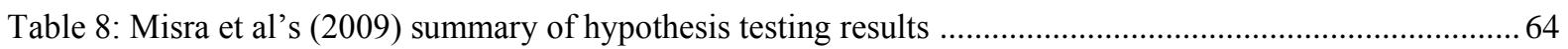

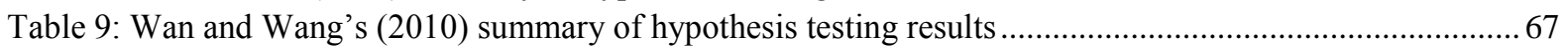

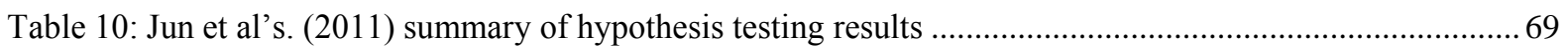

Table 11: Mapping of candidate CSFs from previous models to the current study ...........................................73

Table 12: Empirical studies related to only candidate CSFs for software development methodologies ..............74

Table 13: Candidate CSFs identified across 148 publications ...................................................................... 76

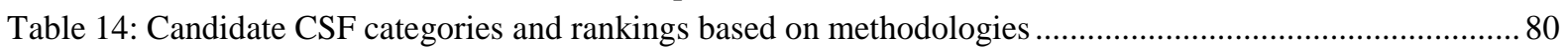

Table 15: Summary of research hypotheses and supporting references ........................................................ 131

Table 16: Research participants who contributed to the conceptual model discussion ...................................... 134

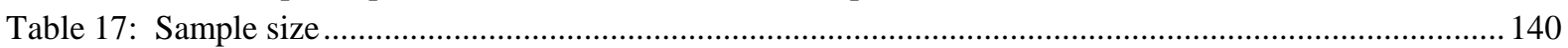

Table 18: Scales used previously to measure top level management support (Nah \& Delgado, 2006) ..............142

Table 19: Scales previously used to measure organizational culture (Strode et al. 2009) ................................ 143

Table 20: Scales previously used to measure project planning and controlling (Jun et al., 2011).................... 143

Table 21: Scales previously used to measure change management (Nah \& Delgado, 2006) ............................ 144

Table 22: Scales previously used to measure Vision and mission (Wan \& Wang, 2006) ................................ 144

Table 23: Scales previously used to measure vision and mission (Nah \& Delgado, 2006) .............................. 144

Table 24: Scales previously used to measure leadership characteristics ................................................... 145

Table 25: Scales previously used to measure internal project communication (Jun et al., 2011)..................... 146

Table 26: Scales previously used to measure team commitment (Meyer \& Allen (1991)................................ 146

Table 27: Scales previously used to measure project team commitment (Ahimbisibwe \& Nangoli, 2012) ....... 147

Table 28: Scales previously used to measure team's expertise and experience (Jun et al., 2011).................... 147

Table 29: Scales previously used to measure team's composition (Nah \& Delgado, 2006)............................. 148

Table 30: Scales previously used to measure user participation (Jun et al., 2011) ......................................... 148

Table 31: Scales previously used to measure user support (Jiang \& Klein, 2000) .......................................... 149

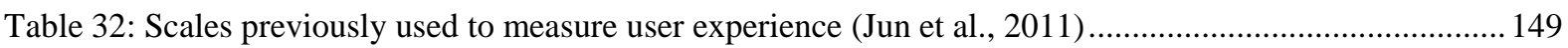

Table 33: Scales previously used to measure technological uncertainty (Nidumolu, 1995)............................. 150

Table 34: Scales previously used to measure technical complexity (Jun et al., 2011) ................................... 150

Table 35: Scales previously used to measure relative project size (Jun et al., 2011).................................... 151

Table 36: Scales previously used to measure project size (Jiang \& Klein, 2000) ........................................ 151

Table 37: Scales previously used to measure specification changes (Nidumolu, 1995).................................. 151

Table 38: Scales previously used to measure project criticality (Sheffield \& Lemetayer (2013)..................... 152

Table 39: Scales previously used to measure project success (Chow \& Cao, 2008) ...................................... 152

Table 40: Scales previously used to measure project success (Misra et al., 2009) .......................................... 152

Table 41: Scales previously used to measure project success (Jun et al., 2011)......................................... 153

Table 42: Scales previously used to measure project success (Sheffield \& Lemetayer, 2013) ......................... 153

Table 43: Profile of research participants who contributed in the semi-structured interviews .......................... 159

Table 44: Summary of questions that were discarded from the initial online survey draft............................... 164

Table 45: summary of initially sent out e-mail invitations, the invalid e-mails and final responses ................ 167

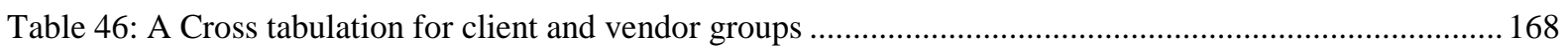

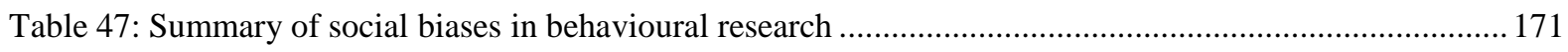

Table 48: Differences between reflective and formative constructs ......................................................... 173

Table 49: Experience in software development projects ................................................................... 177

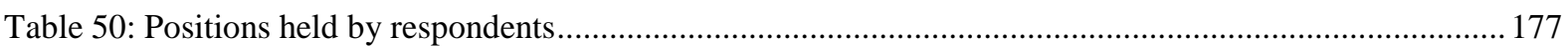




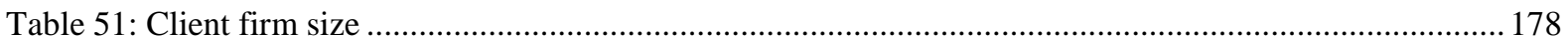

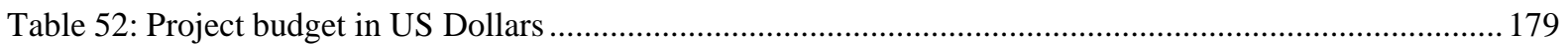

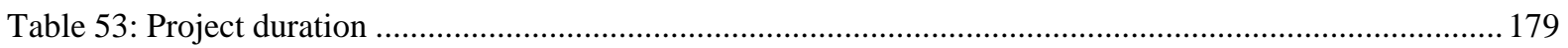

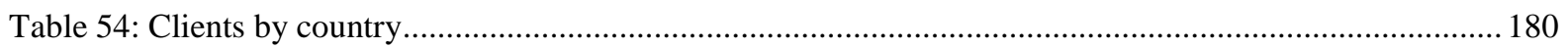

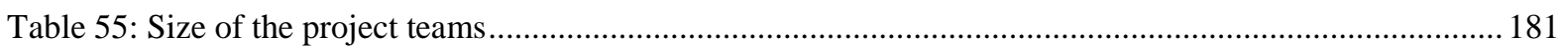

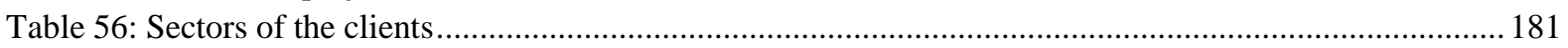

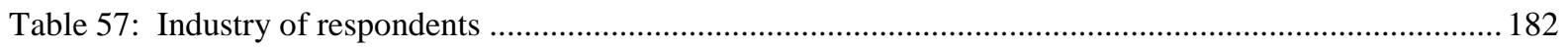

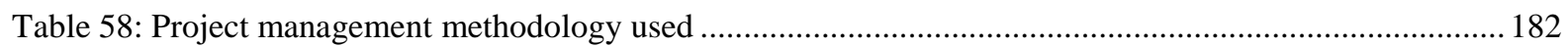

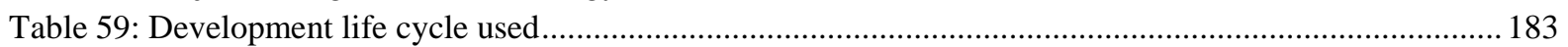

Table 60: Made a decision in the client organization of choosing the project management methodology ..........183

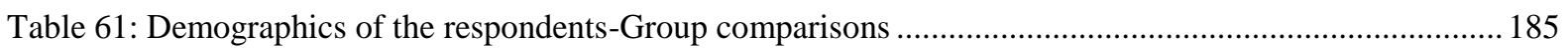

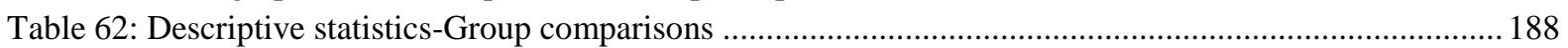

Table 63: Measurement models measures of Goodness-of-fit -Group comparisons ........................................... 196

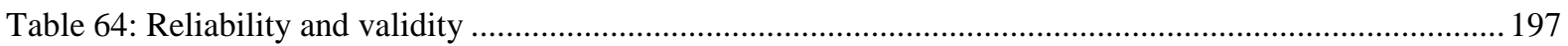

Table 65: Standardised regression weights from the measurement models-Group comparisons ....................... 199

Table 66: Correlates for projects that used the traditional plan-based methodology, $n=513 \ldots \ldots \ldots \ldots \ldots \ldots \ldots \ldots \ldots \ldots . . . . . . . . . . . . .202$

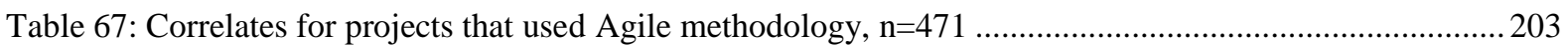

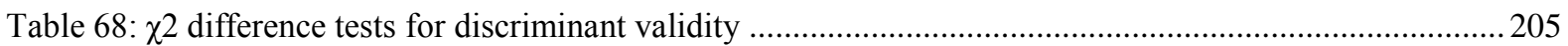

Table 69: Structural models measures of Goodness-of-fit-Group comparisons ................................................ 208

Table 70: Hypothesis testing results (only with direct link to project success)-Group comparisons...................209

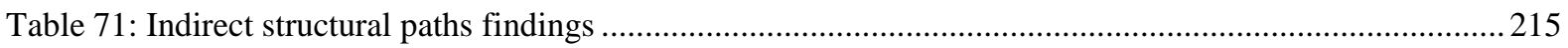

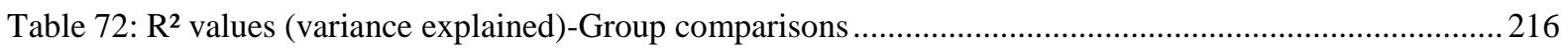

Table 73: Hierarchy and descriptions of the six stage process of measurement invariance testing ....................220

Table 74: A summary of Goodness-of-fit statistics from measurement invariance tests.....................................225

Table 75: Nested group model comparisons testing for structural invariance ..................................................226

Table 76: $\chi 2$ difference tests for moderating effects of project management methodology ...............................228

Table 77: Hierarchical SEM analysis of interaction terms of technological uncertainty ....................................238

Table 78: Hierarchical SEM analysis of interaction terms of technical complexity ...........................................241

Table 79: Hierarchical SEM analysis of interaction terms of relative project size ............................................245

Table 80: Hierarchical SEM analysis of interaction terms of specification changes ...........................................248

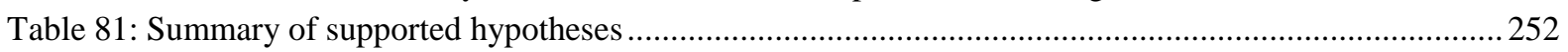

Table 82: Summary of path coefficients that differ across the two methodology groups ....................................253

Table 83: Summarized significant moderating effects and supported moderation hypotheses ........................... 255

Table 84: Hypothesis testing results (only with direct link to project success)-Group comparisons...................2282

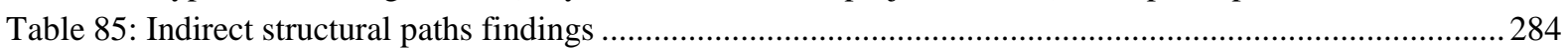

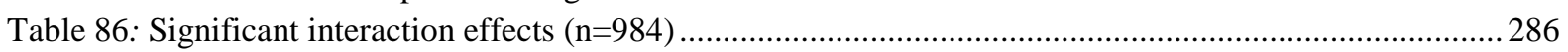

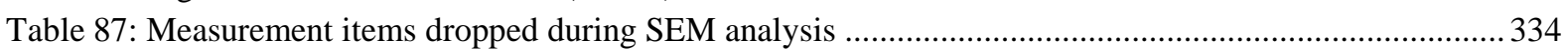




\section{GLOSSARY}

\section{Concept}

Agile software development

\section{Budget}

Change management

\section{Client/user experience \\ Cost}

Critical Success Factor

(CSF)/critical factor

Customer commitment

Development skill

Experience level of the team Fit

\section{Functionality \\ In-house \\ Interdependency \\ Internal communication}

Leadership characteristics

Market uncertainty

Mission

Organisational culture

Planning and control

Process performance/success

Product performance/success

Product Scope

Project inherent uncertainty

Project champion

Project criticality

Project factors

Project Life Cycle (PLC)

Project management (PM)

Project Management

Methodology

Project management processes

\section{Project Scope}

Project success

Project team

Project team commitment

Quality

Relative project size

SDM

Software development project

\section{Description}

A group of highly flexible methodologies that seek to embrace the changes and uncertainty involved in software development projects.

The project was completed within planned costs.

An approach to transitioning individuals, teams, projects and organizations from a current state to a desired future state.

Experience of the user.

Total budget of the project.

An issue that if addressed appropriately, will substantially increase the likelihood of chances of project success.

The level of engagement the customer is willing to put in the project.

Competence and expertise of the project development team.

Experience of the project team member that reduces need for supervision and guidance.

The choice of a methodology that delivers project success in the context of relevant CSFs/project characteristics and project environment.

The project met the customer's functional requirements.

Developing a software internally rather than using outsourced vendors

Many parts or projects to rely on during project life cycle

Management practices that increase information sharing and cohesion among project team members.

Traits and styles of top level management

The external environment is stable vs. unstable.

Fundamental purpose for organizational existence.

The influence of shared values on the process of software development.

The extent to which planning and controlling practices are used in a project.

The extent to which a project is delivered on schedule, scope and within budget.

Description of the performance of the system actually delivered to the users

The features and functions that characterize a product, service, or result.

Project-specific risk characteristics that initially exist in a project rather than emerge during the course of its implementation.

Not a project manager or team leader but a person who provides moral, psychological and physical support to the team and provides them with needed resources and advocates for the project's benefits and advantages to its stakeholders.

The level to which a defect on the product/software would impact the client.

Project characteristics e.g. inherent risks that cause variances in expected project results

A collection of generally sequential project phases whose name and number are determined by the control needs of the organization involved in the project

The art and science of managing projects to a specific schedule, at or below a predetermined budget, to the customer's performance requirements and within the resources available.

Approaches to managing project activities including lean, iterative, incremental, and phased approaches categorized as traditional linear project management methodology vs. agile iterative project management methodology

Set of interrelated actions and activities performed to achieve a specified project result. Processes interact for the purpose of initiating, planning, executing, monitoring and controlling, and closing projects.

The amount of work that needs to be accomplished to deliver a product, service, or result with the specified features and functions.

Meeting all the set project objectives for all stakeholders.

A team whose members usually are assigned to activities for the same project.

Willingness of a team to devote energy and royalty to a project as expressed in three forms: affective, continuance and normative.

The software developed is reliable as required

The duration of the project in months or years or cost.

A framework that is used to structure, plan, and control the process of developing software.

A complex undertaking within the boundaries of time; budget and staff resources intended to generate a new or enhanced computer code that adds significant business value to a new or existing business process. 
Team co-location Team empowerment Team size

Technical complexity

Technological uncertainty

Time

Top level management support (TMS)

Traditional software

development

Outsourced projects

\section{Urgency}

User

User participation

Vendor

Vision
Team members working in the same office vs. working in different places.

Feeling most comfortable being free to make decisions and empowered.

Number of team members working on the project.

Inability to predict the relationship between input and output during the project lifecycle

The technology used is well known by the project team vs. it is totally new.

The project was completed within schedule

Executives willingness to participate, involve and provide resources, authority and influence on the project.

A set of traditional, contract driven methodologies that seek adherence to a pre-established plan, presumed certainty and existing processes.

An individual or collaborative enterprise that is carried out by third parties or vendors carefully planned to achieve a particular aim within constraints of time, budget and quality.

The extent to which time constraints are a factor in project activities and decision making.

The client or beneficiary of the software development project including the general public.

The behaviours and activities of the user in relation to contributing to product development.

Supplier or an individual or an organization outsourced to make software for another company.

Statement outlining what the organization wants to be or how it wants the world in which it operates to be (big picture). 


\section{CHAPTER ONE: INTRODUCTION}

\subsection{Introduction}

This chapter describes the motivation of the study, research problem and why it is a topic of interest. A research background on project management methodology practices is explained and the relevance of methodologies in managing software projects to success is described. The focus on outsourced software development projects and vendor perspective are discussed. Finally, the purpose, research objectives, research design and the structure of the thesis are outlined.

\subsection{Motivation of the study}

Projects are a frequent activity in various organizations which invest substantial resources to drive innovation and change (Sauser, Reilly \& Shenhar, 2009; Shenhar \& Dvir, 2007). However, numerous software development (SD) projects fail to deliver on time or budget and do not give value (PMI's Pulse report, 2013; KPMG report, 2013). Shenhar (2008, p.15) reported that nearly two thirds of SD projects do not meet their time and budget goals, and many do not meet their business objectives. Although there are many reasons proposed for why SD fail in different contexts, numerous studies argue that SD projects fail due to inappropriate choice of a project management approach (Sauser et al., 2009; Murad \& Cavana, 2012). Indeed, the existence of several alternative project management methodologies often makes it difficult to select the most appropriate methodology (Sheffield \& Lemetayer, 2013). People tend to be emotionally attached and potentially biased towards one approach (Bohm \& Turner, 2003). Definitively describing project characteristics and then matching them to an appropriate project management approach appears problematic (Sauser et al., 2009). It is possible that users and/or software developers will tend to stick to what they are good at and will favour the project management methods with which they have had most experience (Boehm \& Turner, 2004). As a result, despite the increasing range of available management choices, project managers are seen to frequently fail to seriously consider their alternatives (Howell et al., 2010). When project categorisation systems and categorisation criteria are not logically matched with project objectives and environment, this may provide a key reason for why many SD projects are reported to fail to deliver on time, budget or do not give value to the organisation.

SD projects continue to fail even with the existence of communities of methodology practice such as PRINCE2, PMI and Agile that promote good practices (Standish Group, 2012). 
Whilst the traditional plan-driven development approaches are often regarded as too rigid to fit some environments, some project managers still try to force them to fit projects or fail to consider alternatives (Howell et al., 2010). According to Wysocki's (2009) testimonial data gathered from 10,000 project managers, no more than $20 \%$ of all projects have the characteristics of traditional projects, but research shows project managers continue to apply these traditional methods to projects for which they may not be suited. In contrast, emergent agile methodologies promise increased user satisfaction with lower defect rates, faster development times for solutions to rapidly changing requirements but are less understood (Sheffield \& Lemetayer, 2013, p.462). As another complication, Iivari and Huisman's (2007) study found that hierarchical organizations were not suitable for the deployment of agile methodologies. Thus, despite exhortation to move away from old practices, it has also been cautioned that the new methodologies are not silver bullets that guarantee success every time (Boehm \& Turner, 2004).

Sauser et al.'s (2009) case study of NASA's Mars Climate Orbiter (MCO), that was lost in space after completing its nine-month journey to Mars, demonstrates that when important SD projects fail, the investigation is often focused on the engineering and technical reasons for the failure. Yet, in many cases the root cause of the failure is not technical, but managerial (Sauser et al., 2009). Their conclusions revealed that the problem was rooted in management's failure to select the right methodology for the SD project (Sauser et al., 2009, p.665). Tiwana and Keil's (2004) study of 720 software projects similarly found that the use of an inappropriate methodology is actually the most critical risk driver for SD project failure (p.74). Therefore, there is a view that objective guidance in matching the project type and the software development methodology would be expected to increase the chances of SD project success. Howell et al. (2010) further suggest that the lack of a decision support tool and theory connecting project types and project management methodology discourages SD project managers from considering alternative methodologies (p.256). Overall, the best fit methodology on project success has been argued as crucial but the actual impact is not well understood.

The Chaos report (Standish Group, 2012) reveals that $86 \%$ of all traditional software development projects were unsuccessful. With a shocking $14 \%$ project success rate, $57 \%$ were challenged and $29 \%$ as completely failed software development projects. In fact, from 
over 15 years of their research, their findings support the low rate of success and high rate of failure for SD projects (Standish Group, 1994-2014).

The project performances trends in Figure 1 are based on Chaos reports that are regularly published by the Standish Group exemplify these concerns.

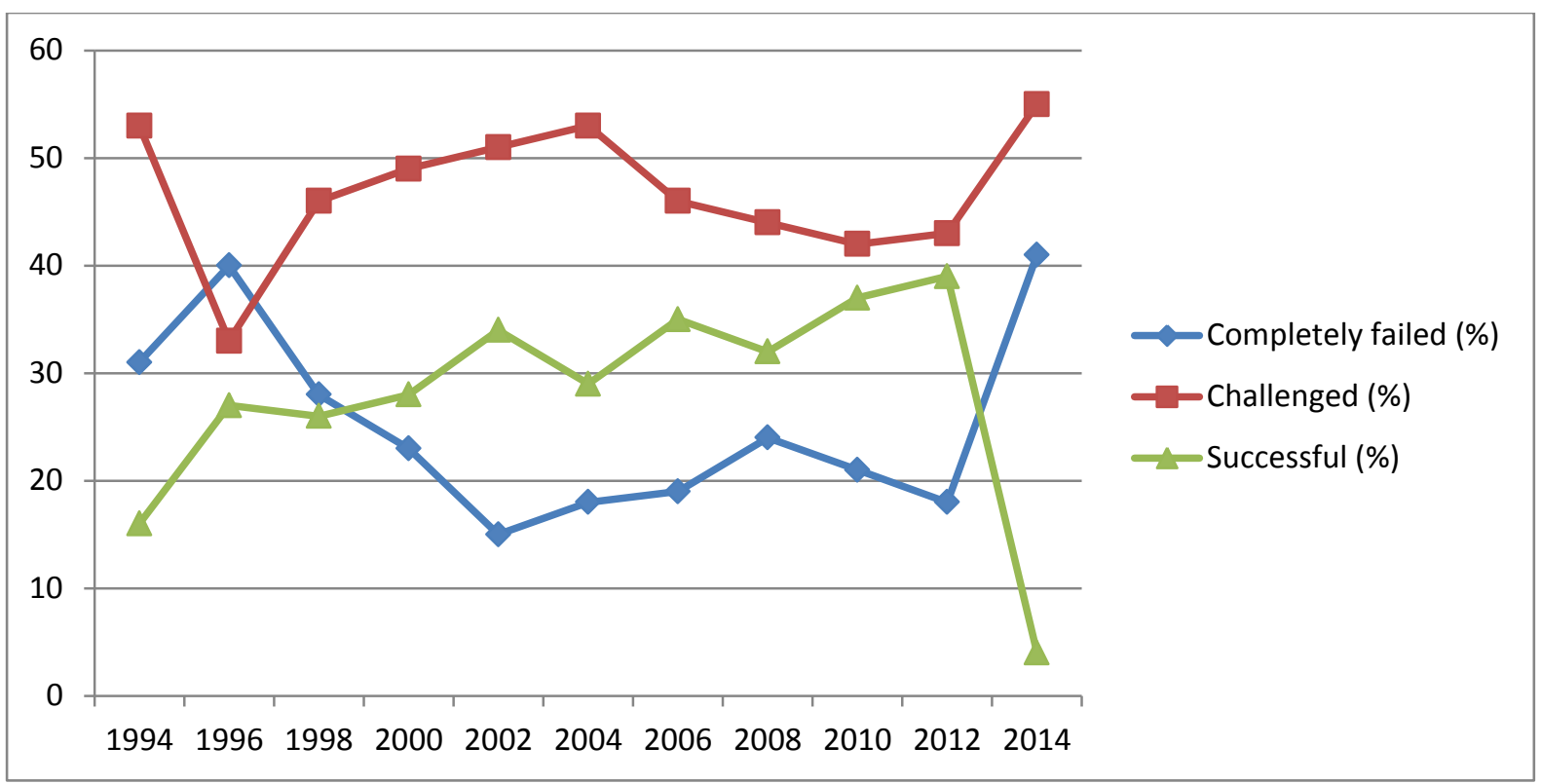

Figure 1: Project performance trend (Standish Group)

Source: Chaos reports compiled by author

As illustrated, the trend of success of SD projects is lower and declining compared to projects that are challenged which is higher and increasing. Most of the SD projects are challenged and hence do not deliver on the expected time or budget goals and do not meet their objectives (PMI's Pulse report, 2013; KPMG report, 2013). Besides, the trend for completely failed SD projects appears to be increasing at a faster rate than the one for SD successful projects. If this alarming trend of SD projects failure is not reversed, organizations will continue to lose a lot of funds through failed SD projects.

Similarly, the project management national survey 2013 from New Zealand indicates that, although there has been a significant increase in project activity in the past two years across all sectors of the economy, only one-third deliver the desired outcome (KPMG report, 2013, p.4). Worryingly, this suggests a staggering wastage of resources. Further, the project management survey data for 2012 demonstrates a significant decrease in project success rates with only $29 \%$ of respondents indicating consistently delivered projects on time, only $33 \%$ consistently delivered on budget, and only $35 \%$ of projects consistently delivered on scope 
compared to previous success rates of $36 \%, 48 \%$ and $58 \%$ respectively for 2010 (KPMG report, 2013, p.19). Thus, project activity is on the increase but so is the project failure rate.

The KPMG report (2013, p.11) further highlights that, despite some practices correlating to project success, project managers are seen slow at adopting them. Specifically, $90 \%$ of the organisations that consistently delivered projects successfully had 'always' or 'often' used a project management approach (KPMG report, p.12). Similarly, the Pulse of the Profession report 2013 indicates that $73 \%$ of the successful projects had conducted training on use of tools and techniques, particularly, methodologies (PMI Pulse report 2013, p.8). Thus, there is some compelling evidence that an appropriate project management methodology approach is essential for project success. Yet, in practice, project managers appear to hardly consider alternative methodologies or fit to characteristics, objectives and the environment of a given project, which then seems to be counterproductive to project success.

\subsection{Research background}

A project is a temporary endeavour undertaken to create a unique product, service or result (PMI, 2013, p.3). Specifically, a software development project is a complex undertaking within the boundaries of time; budget and staff resources intended to generate a new or enhanced computer code that adds significant business value to a new or existing business process (Wysocki, 2006, p. 5). Software development in its narrow sense refers to the activity of computer programming, which is the process of writing and maintaining some source code. In a broader sense, it also includes all that is involved between the conception of the desired software through to the final manifestation of the software, either in a planned or unstructured process.

Project management methodologies seek to provide and lay the high-level framework of the project as well as providing a framework for macro-level matters (Charvat, 2003). They are often organized in phases from initiating to closing the project. According to Charvat (2003), Software Development Methodologies (SDMs) provide the detail that covers software structural design, development and testing but does not cover some project management issues such as financial justification of the project or sales. Although there are many software development methodologies on the market and their number keeps increasing, generally, in the software development methodology ecosystem, two major project management methodologies are distinguished. These are the traditional or heavyweight methodologies (e.g. PRINCE2 and PMI) and the agile or 'lightweight' methodologies. Examples of 
traditional SDM's include: waterfall, Structured Systems Analysis and Design Method (SSADM), Information Engineering, Rational Unified Process (RUP) and OPEN. Agile methodologies include Extreme Programming (XP), Scrum, Crystal, Dynamic System Development Method (DSDM), Adaptive Software Development (ASD) and Lean Software Development (LD) among others. The list of SDMs may vary in the literature depending on different viewpoints. As pointed out by Iivari and Huisman (2007), the usage of SDMs remains a versatile concept. One reason is the ambiguity related to the term methodology. It can be used to cover the totality of system development methodologies, process models, specific methods and specific techniques in an organization. The second source of ambiguity relates to the difficulty in defining and measuring SDM usage. SDM use can be distinguished as implicit and explicit. Explicit refers to consulting the method (documentation) while implicit may be an unconscious process in which method knowledge is tangled with practical experience (Iivari \& Huisman, 2007, p.38). Therefore, in this study the question is not about the details of specific methods and techniques (technical nuts and bolts), but rather contrasts more general approaches and process models. This is because software developers tend to apply a methodology by following the goals, fundamental concepts, guiding principles, and philosophies of the systems development process of a specific systems development methodology (Iivari \& Huisman, 2007, p.38).

Project management methodologies can be used for many different types of projects including software development, and can be used in conjunction with different software development methodologies. Prince2 and PMI are project management methodologies and not SDM's as such, although they may be used to manage and control software projects. However, agile methodologies by contrast are primarily aimed only at software development. According to Dalcher and Brodie (2007), it is important to ensure that the project management and software development methodologies are compatible to achieve project success. Since SDMs must be well aligned with project management methodologies to achieve project success (Sheffield \& Lemetayer, 2013), project management methodology is used here as a broad term or proxy covering methodology in this study. The terms project management approach/process design and methodology are sometimes used in some parts of the thesis to refer to the collection of managerial activities, processes, practices, methods, techniques, and tools employed to achieve project success (PMI, 2013, pg. 48). SDM is an important aspect of project management methodology/process design and the two should be 
configured together to match to achieve project success (Charvat, 2003, p. 3; Cockburn, 2007, p. 149).

SDMs can be mapped onto related project management methodologies so that information can flow between them. According to Sheffield and Lemetayer (2013, p.460), software development practices must align with the management methodologies and processes employed in complex project teams at different hierarchical levels. It is therefore important to ensure that the project management and software development methodologies are compatible, suggesting that project management methodologies have a close relationship with SDMs (Dalcher \& Brodie, 2007).

Figure 2 illustrates the relationship between project management hierarchies, associated methodologies and project environment.

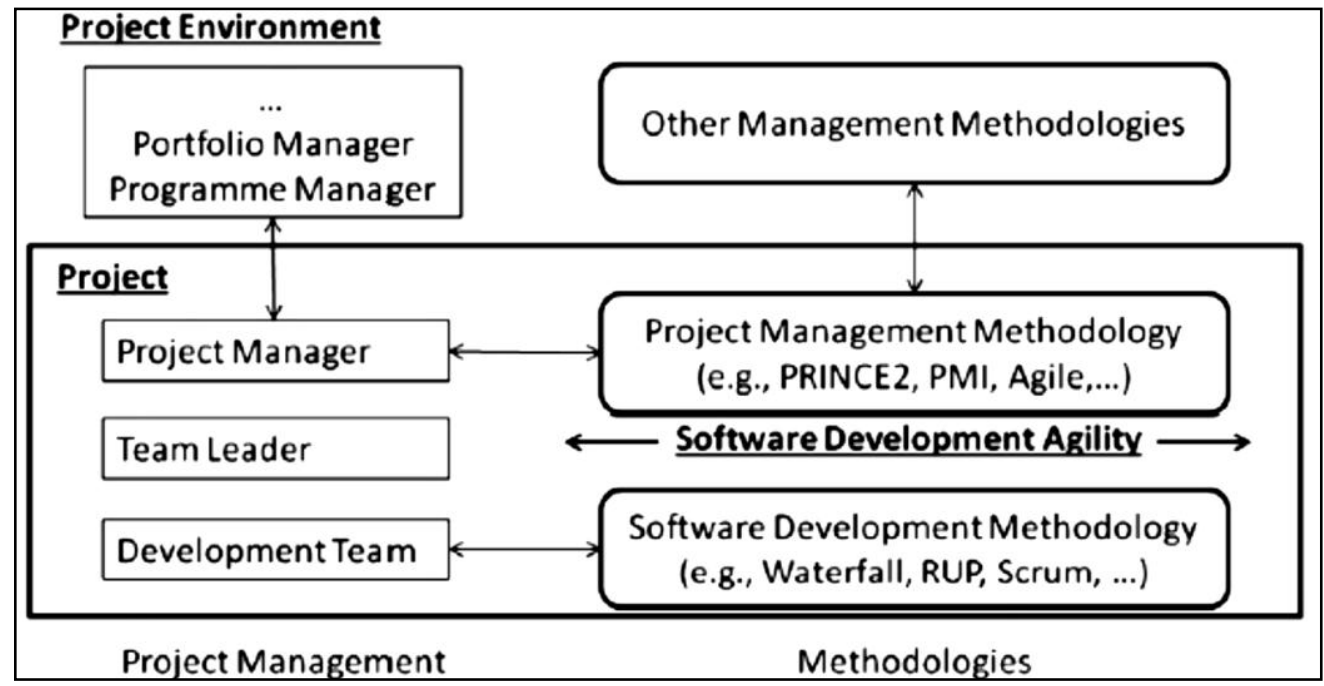

Figure 2: project management hierarchy and associated methodologies (Sheffield \& Lemetayer 2013, Fig.1, p.2)

From the above figure, project management methodologies which include PRINCE2, PMI and agile must fit with the software development methodologies used for a software project. For instance, if a traditional plan-based methodology (PRINCE2, PMI) is selected for a given project then Waterfall is likely to be used, unlike when an Agile approach is chosen, Scrum would be used for alignment. However, most project managers tend to select the methodologies that they are professionally affiliated to, have the most experience with or the ones they feel more comfortable with (Charvat, 2003). When an inappropriate project methodology is used, the outcome of the project becomes uncertain. 


\subsubsection{Communities of project management methodology practices}

Project management is composed of different vendor communities of methodology practices, each with a particular set of principles, philosophy and guidelines. Some practices are formally developed while others are more adhoc with members who share certain methodological commitments. These vendor communities of methodology practice can also be broadly categorised as traditional plan-based and agile. Traditional plan-based approaches encompass PRINCE2 (OGC, 2009) and PMI (PMBOK, 2013) each with a set of contract driven methodologies that seek adherence to a pre-established plan, presumed certainty, stability and ease of planning and controlling of existing processes. On the other side, there is Agile (Agile alliance, 2001) with highly flexible methodologies that seek to embrace the changes and uncertainty frequently involved in software development projects. While various project management methodologies with different underlying values and principles are available on the market, each may present itself as a credible candidate for selection and subsequent adaptation for software development projects depending on the variations noted above. The next sub-sections describe these three major communities of methodology practice i.e. PRINCE2, PMI and Agile.

\subsubsection{PRINCE2}

PRINCE2 refers to 'Projects In Controlled Environments' and is a generic structured method that prescribes principles, processes, and themes (OGC, 2009). It is a structured project management method endorsed by the UK government as the project management standard for public projects and hence it is the leading standard for project management in the UK. The '2' means it is the second version of PRINCE. This methodology encompasses the management, control and organization of projects. The use of the phrase "controlled environments" clearly demonstrates that this method falls into the category of traditional approaches developed to promote compliance to standards in stable or predictable environments.

PRINCE2 can be described as a net of interlinking elements: principles, themes, processes, activities and recommended actions. The focus of this methodology is the popular 'PRINCE2 Model' of processes and activities that describe human activities through a hierarchy of process forward flows, decision points, and feedback loops. Over 500 discrete elements and their interconnections are prescribed. PRINCE2 is based on seven principles (continued business justification, learn from experience, defined roles and responsibilities, manage by 
stages, manage by exception, focus on products and tailored to suit the project environment), seven themes (business case, organization, quality, plans, risk, change and progress) and seven processes. The PRINCE2 model (OGC, 2009) also defines another 40 separate activities and organizes these into seven processes: Starting up a project (SU), Initiating a Project (IP), Directing a Project (DP), Controlling a Stage (CS), Managing stage Boundaries (SB), Managing Product delivery (MP) and Closing a Project (CP).

Figure 3 shows the PRINCE2 process model. The arrows represent flows of information.

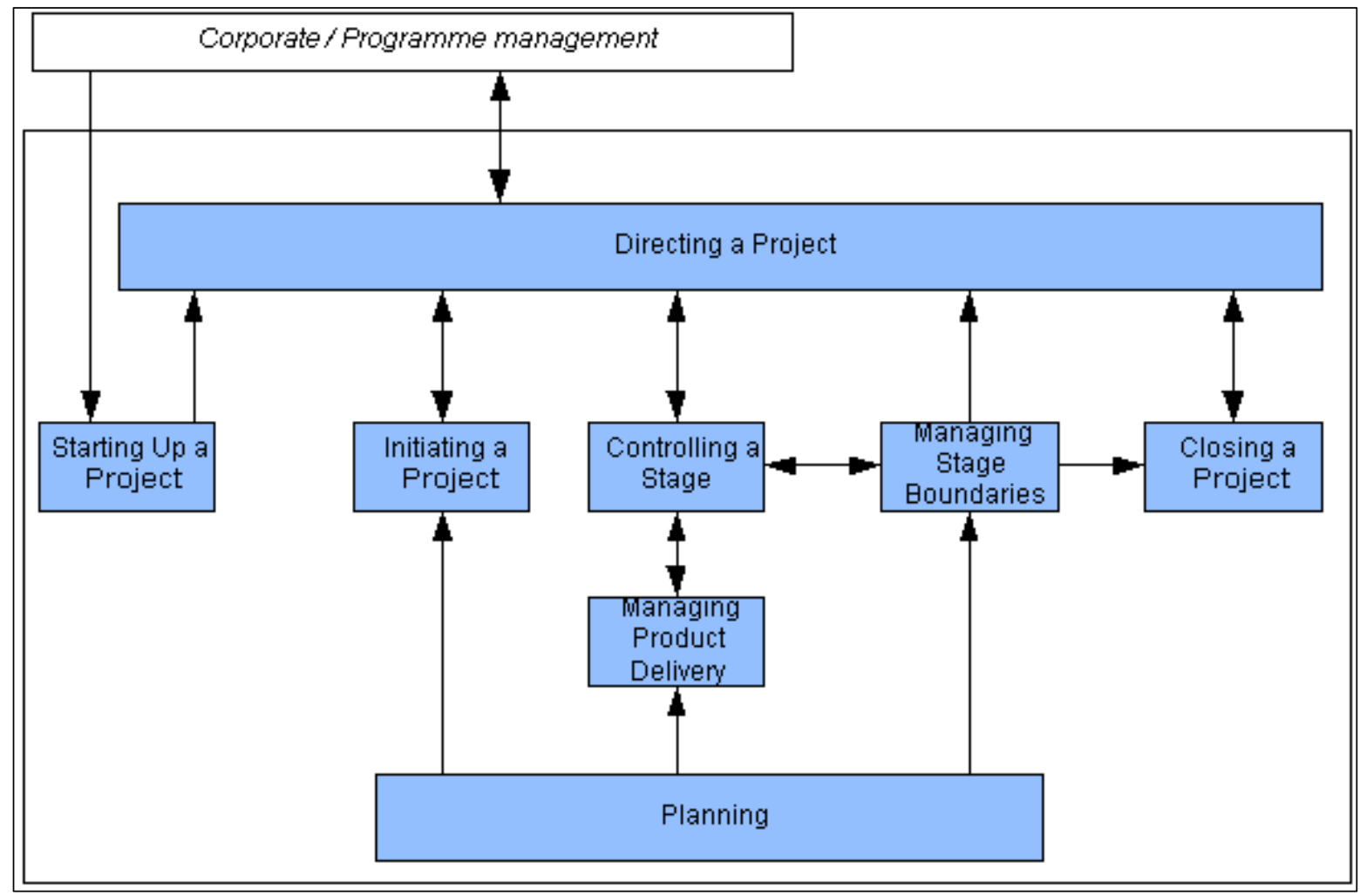

Figure 3:PRINCE2 model (OGC, 2009)

Thus, PRINCE2 provides a managerial approach that is based on the product (OGC, 2009, p.64) and highly structured process plans (OGC, 2009, p.61) that are interspersed with formal decision points (OGC, 2009, p.91). PRINCE2 is also used to refer to the training and accreditation of authorized practitioners of the methodology who must complete accredited qualifications to obtain certification. The main advantage of PRINCE2 as a structured approach to project management is that it provides a clearly-defined framework for managing projects. PRINCE2 describes procedures to coordinate people and activities in a project, how to design and supervise the project, and what to do if the project has to be adjusted if it doesn't develop as planned. Similarly, each process is specified with its key inputs and 
outputs and with specific goals and activities to be carried out, which gives an automatic control of any deviations from the plan (OGC, 2009).

Additionally, since PRINCE2 is divided into manageable stages, the method enables an efficient control of resources. With focus on close monitoring, the project can be carried out in a controlled and organized way. PRINCE2 is also a structured method that is widely recognized and understood since it has been in existence for a while. PRINCE2 like PMI also provides a common language for all participants in the project. The various management roles and responsibilities involved in a project are fully described and can be adapted to suit the complexity of the project and skills of the organization. On the other hand, PRINCE2 is sometimes considered inappropriate for small projects or where requirements are expected to change, due to the work required in creating and maintaining documents, logs and lists. However, the OGC claims that the methodology is scalable and can be tailored to suit the specific requirements and constraints of the project and the environment.

\subsubsection{The Project Management Institute (PMI)}

PMI is vendor community of practitioners and organizations with standards that describe good practices, globally recognized credentials that certify project management expertise, and resources for professional development and networking. Like PRINCE2, PMI falls into the structured traditional plan-driven category of process-based project management. Whereas PRINCE2 is more prescriptive, focussing on compliance and control, the PMI based on the PMBOK guide is descriptive, including a broader treatment of processes, tools, techniques and good practices (Sheffield \& Lemetayer, 2010). Nine project management knowledge areas are described: project integration management, scope management, time management, cost management, quality management, human resources management, communications management, risk management, and procurement management. Each of these nine areas includes processes, activities, tools and techniques. In total, there are 42 project management processes, categorized in five different groups as initiating, planning, executing, monitoring and controlling, and closing. PMI (PMBOK) is a structured methodology that is mostly recognized and understood globally since it has been in existence since 1969. The PMBOK (2013) bureaucratically prescribes all the various processes, skills, tools and techniques that have a significant impact on project success. An efficient tight control of resources is presumed through strict adherence to planning, controlling and monitoring. PMI (PMBOK) like PRINCE2 also provides and promotes a common language within the project 
management profession for using project management concepts. Figure 4 shows these five key project management processes. The darker dotted lines represent relationships between process groups while the lighter dotted lines are external to the process groups.

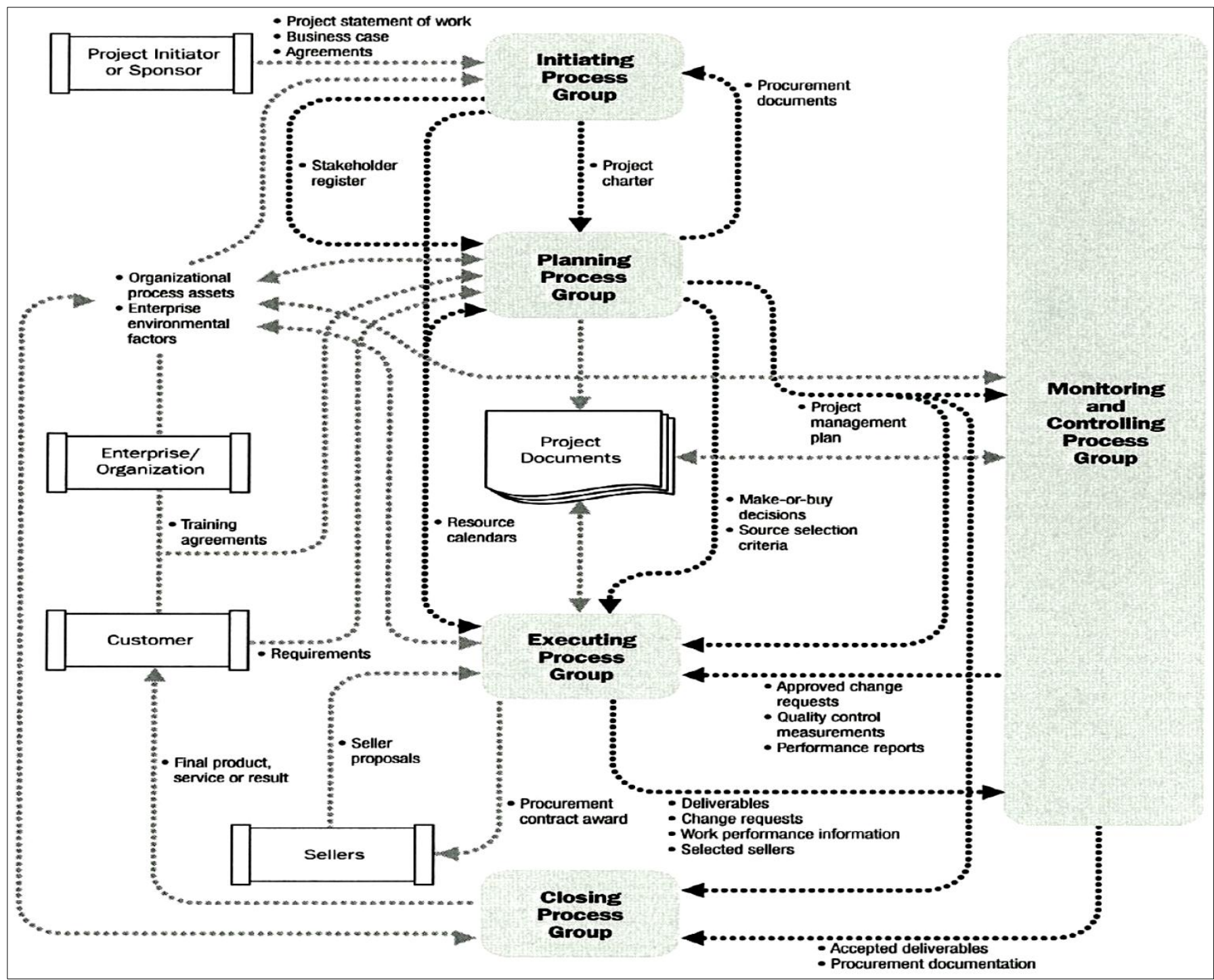

Figure 4: PMI model (PMBOK Guide, 2013, p. 53, Fig. 3-3)

\subsubsection{Agile}

The Agile alliance community was founded in 2001 by proponents of diverse methodologies from around the world who shared similar underlying beliefs and values. Agile is neither prescriptive (like PRINCE2) nor descriptive (like PMI's PMBOK guide), both of which are based on a set of structured processes or tools. Instead it is appreciative, based on the values or philosophy that underlies a variety of methods such as XP, Crystal and Scrum (Sheffield \& Lemetayer, 2010). The developers of most of these methods collaboratively wrote the Agile 
manifesto and agreed to use the word "Agile" as an umbrella term for several iterative and incremental methods (Agile Alliance, 2001). The agile manifesto was developed by initial signatories of the Agile Alliance (2001) for software development (Figure 5).

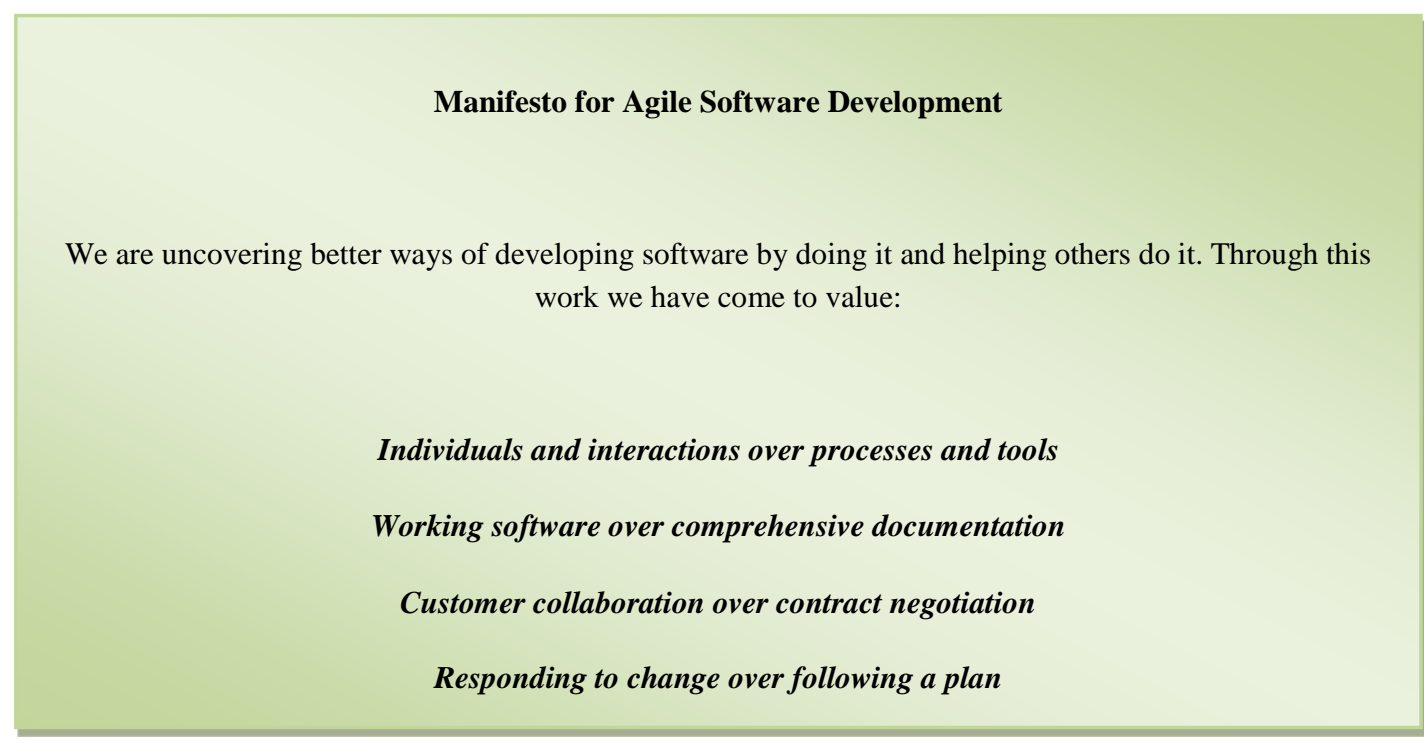

Figure 5: The Agile Manifesto (Agile Alliance, 2001)

In short, the Agile Manifesto contrasts traditional methodologies which are characterized by the items on the right (process and tools, comprehensive documentation, contract negotiation and following a plan) while agile methodologies are characterized by the items on the left (individuals and interactions, working software, customer collaboration and responding to change) (Sheffield, Lemetayer \& Ahimbisibwe, 2011). The values in the Agile Manifesto are not mutually exclusive; the left and right hand items can actually reinforce each other. The right items are not considered unimportant but simply less important than the items on the left (Sheffield et al., 2011). The agile manifesto is supported by 12 principles which define the basic philosophy of Agility (Leffingwell, 2007). Agile methodologies have numerous advantages including that they can: adapt very well to change and dynamism (Charvat, 2003), are people-oriented and value-driven, rather than process-oriented and plan-driven (Leffingwell, 2007), mitigate risks by demonstrating values and functionalities up front in the development process (Perrin, 2008), provide a faster time to market, improve productivity (by reducing the amount of documentation) (Charvat, 2003), fail early/quickly and painlessly, if a project is not doable (Leffingwell, 2007). On the other hand, the main risks stemming from an agile approach are: limited scalability, use of too simple a design which may cause 
expensive rework, high personnel turnover which means a loss of knowledge and lack of people skilled in agile methods (Boehm \& Turner, 2003).

\subsubsection{Contrasting two major vendor communities of methodology practice}

Table 1 contrasts the two major communities of project management methodology practice. These respective principles and procedures can be used as guidance for selecting and adapting a methodology that can help to achieve project success.

Table 1: Two major communities of project management methodology practice: Traditional and Agile

\begin{tabular}{|c|c|c|c|c|c|}
\hline $\begin{array}{c}\text { Methodology } \\
\text { practice }\end{array}$ & $\begin{array}{c}\text { Estimated } \\
\text { membership }\end{array}$ & $\begin{array}{c}\text { Key } \\
\text { attribute }\end{array}$ & Principles & Certifications & Guiding Document \\
\hline $\begin{array}{l}\text { PRINCE2 } \\
\text { (Traditional } \\
\text { approach) }\end{array}$ & $>250.000$ & Prescriptive & $\begin{array}{l}\text { Seven } \\
\text { principles } \\
\text { defined in the } \\
\text { PRINCE2 } \\
\text { manual }\end{array}$ & $\begin{array}{l}\text { Two exams and no experience } \\
\text { required }\end{array}$ & $\begin{array}{l}\text { Managing Successful Projects with } \\
\text { PRINCE2 (2009), Directing } \\
\text { Successful Projects with PRINCE2 } \\
(2009)\end{array}$ \\
\hline $\begin{array}{l}\text { PMI } \\
\text { (Traditional } \\
\text { approach) }\end{array}$ & $>700.000$ & Descriptive & $\begin{array}{l}\text { PMI (PMBOK } \\
\text { guide) }\end{array}$ & $\begin{array}{l}\text { Project Management Professional } \\
\text { certification and experience of } 3 \text { to } 5 \\
\text { years of project management }\end{array}$ & $\begin{array}{l}\text { A Guide to the Project Management } \\
\text { Body of Knowledge (PMBOK } \\
\text { Guide) 5th edition (2013) }\end{array}$ \\
\hline Agile & $\begin{array}{l}>6.000 \\
\text { signatories }\end{array}$ & Appreciative & $\begin{array}{l}\text { Defined by the } \\
\text { Agile } \\
\text { Manifesto }\end{array}$ & $\begin{array}{l}\text { Skills are acquired by practice on } \\
\text { agile projects not by training alone }\end{array}$ & $\begin{array}{l}\text { Agile manifesto (2001), Declaration } \\
\text { of Interdependence (2005), Personal } \\
\text { statements of signatories }\end{array}$ \\
\hline
\end{tabular}

According to Shenhar (2001), 'one size does not fit all'. Instead, project characteristics define the extent to which a particular project management methodology may be suitably applied. Wysocki (2009) identifies the key project characteristics to include: levels of project risk, project complexity, project size, market stability, and business value and technology type used. The level of developers' and users' experience has also been identified as another potentially significant situational factor (Jun et al., 2011). Other researchers subsumed the complexity of the situation facing the users or developers within a broader single factor: project uncertainty (Nidumolu, 1995; Ratbe et al., 2000). Project uncertainty has also been regarded as a function of project size (time and cost), complexity, degree of structure (goal clarity and existence and definition of general model of the process), user task comprehension (problem understanding and application system understanding) and developer task comprehension (previous experience with similar systems as well as previous experience in the user application area). For instance, if the system has a high level of uncertainty, is operating in a dynamic environment, and has high developer competences, an agile approach could be used depending on users' system experience. However, if the system has low uncertainty, functions in a stable environment where plans and controls can be applied for managing specifications/goals that are clear and stable, the appropriate development methodology is more likely to be a traditional plan-based approach regardless of the user's 
experience with the system. These and other candidate CSFs are argued to adjust the best-fit project management methodology. Therefore, it is anticipated that the degree of alignment, or fit, of candidate critical success factors (CSFs) like project characteristics, project environment, and project management methodology combine to affect project success.

\subsection{Relevance of methodologies in software project management}

In software development, methodologies are strategically important to be able to counter the requirements of an often dynamic environment and rapidly changing market (Charvat, 2003; Cockburn, 2007). In fact, irrespective of the size of the project, an appropriate methodology that matches the project characteristics and the project environment should provide a consistent framework through the life cycle of the project and improve the likelihood of better performance of the team (Chow \& Cao, 2008). An appropriate methodology is also argued to enhance the efficiency, productivity and the quality of software development (Misra et al, 2009). Moreover, the adoption of an appropriate methodology delineates a common vocabulary, structures, common formats, processes and a strategy for managing the project (Sheffield \& Lemetayer, 2013). When team members are substituted during the development process, the methodology implemented informs them about how previous work was carried out on the project in addition to defining responsibilities and duties (Lemetayer, 2010). As a result, this substantially reduces conflict and confusion and increases the likelihood of project success. Although methodologies are not a panacea to all IT project problems, without the selection of an appropriate methodology, the risk of failure may be increased. In short, there is strong evidence that methodologies can increase project productivity and quality while reducing time, budget and effort. However, these may well be contingent on project and client organizational characteristics.

\subsection{Focus on software development projects}

Software development projects were chosen in this study because of their unique characteristics. Most of the research on project management has focused on identifying CSFs for projects in industries like engineering, manufacturing and construction rather than focusing specifically on software development projects. Yet, managing software development is idiosyncratic due to the complexity, conformity, costs, flexibility and invisibility of the software itself (Nasir \& Sahibuddin, 2011). Moreover, software projects have unique characteristics like code management (e.g. version control, backup, confidentiality, copyrights, etc.) and issues related to testing such as methodology, tester characteristics, time, 
budget, releases, etc. Unlike other engineering industries, software development projects involve complicated work of revision control which makes it possible to revert to a previous version, a critical capability for allowing programmers to track each other's edits, correct mistakes and defend against vandalism and spam. In addition, the volume of data, speed of response and accuracy of expected results make the software projects relatively critical and complex (Sudhakar, 2012). Reliability, confidentiality, accountability, reportability and completeness are also crucial for software systems. Moreover, software development involves many stakeholders (e.g. client customer senior business managers, customer senior IT managers, customer IT staff, customer IT users, supplier senior managers, supplier account managers, and supplier IT staff, team members, system architects, testers and subcontractors etc.) and each has his or her own priorities and interests that may impact on project success (Lacity \& Willcocks, 2000). Given that the combination of these characteristics could vary greatly across projects, it suggests that the importance of particular CSFs will also be affected and the impact of CSFs on project success criteria may be moderated by key characteristics of software development projects (Wysocki, 2009). Such effects may in part be the reason why there is variation in the CSFs identified by different studies in the literature to date. Furthermore, since studies have not considered this breadth of CSFs simultaneously, it is possible that the interrelationships with omitted CSFs have affected their findings.

\subsection{Focus on outsourced projects and vendor perspective}

Unlike prior research which has largely focused on in-house software development projects, this study takes a vendor perspective, "rather than the client perspective that is mainly employed in the literature" (Jun et al., 2011, p.923). Most of the previous CSFs studies focus on in-house development projects, where developers and users are members of the same organization. However, over the last decade, firms have shown an increasing tendency towards outsourcing their IS activities (Jun et al., 2011). Although interest in the client perspective on the CSFs related to software projects is increasing, the vendor perspective has received less attention (Taylor, 2007). Taylor's (2007) study suggests that compared with the usual in-house development projects, outsourcing may give rise to additional insights or different CSFs from the perspectives of both the client and the vendor. The main difference in outsourcing environments is that the client and vendor share the responsibilities for managing outsourced IS projects. Some research evidence suggests that the two sides may have different perceptions of CSFs, management mechanisms, and project success, because of 
their different goals and structures (Jun et al., 2011). Similarly, Sabherwal's (2003) case study revealed that the vendor and the client had different perspectives on the coordination of outsourced software development projects, while in a study based on semi-structured interviews, Taylor (2007) found that the vendors involved in outsourced projects had different perspectives on the risk and some CSFs than the clients.

Since vendors play a significant part and absorb considerable risk (Jun et al., 2011), an integrated framework is needed for managing software development from a vendor perspective. Equally, the contingency relationships found in prior research need to be theorized further to determine how they may also apply to the study of the outsourced software projects from a vendor perspective (Jun et al., 2011). Thus, this study develops and validates an integrative contingency fit framework to describe the effects of CSFs and their interaction on project success from the vendor's perspective. This study is expected to advance our understanding of the CSFs of outsourced software development projects and to provide system vendors with new understanding that may be helpful for the effective management of outsourced software development projects.

\subsection{Purpose and Research Objectives}

The purpose of this study is to identify CSFs, develop and test an integrative contingency fit model for contrasting perspectives of traditional plan-based and agile methodologies specifically for outsourced software development projects from a vendor's perspective. Thus, the research is guided by the following research objectives:

1. To find out the CSFs for outsourced software development projects from a vendor's perspective.

2. To examine the differences in CSFs for traditional plan-driven and agile methodologies towards project success from a vendor's perspective.

In so doing, this study contributes to a body of knowledge which seeks to understand why software development projects succeed or fail, and how project success might be improved. 


\subsection{Research design}

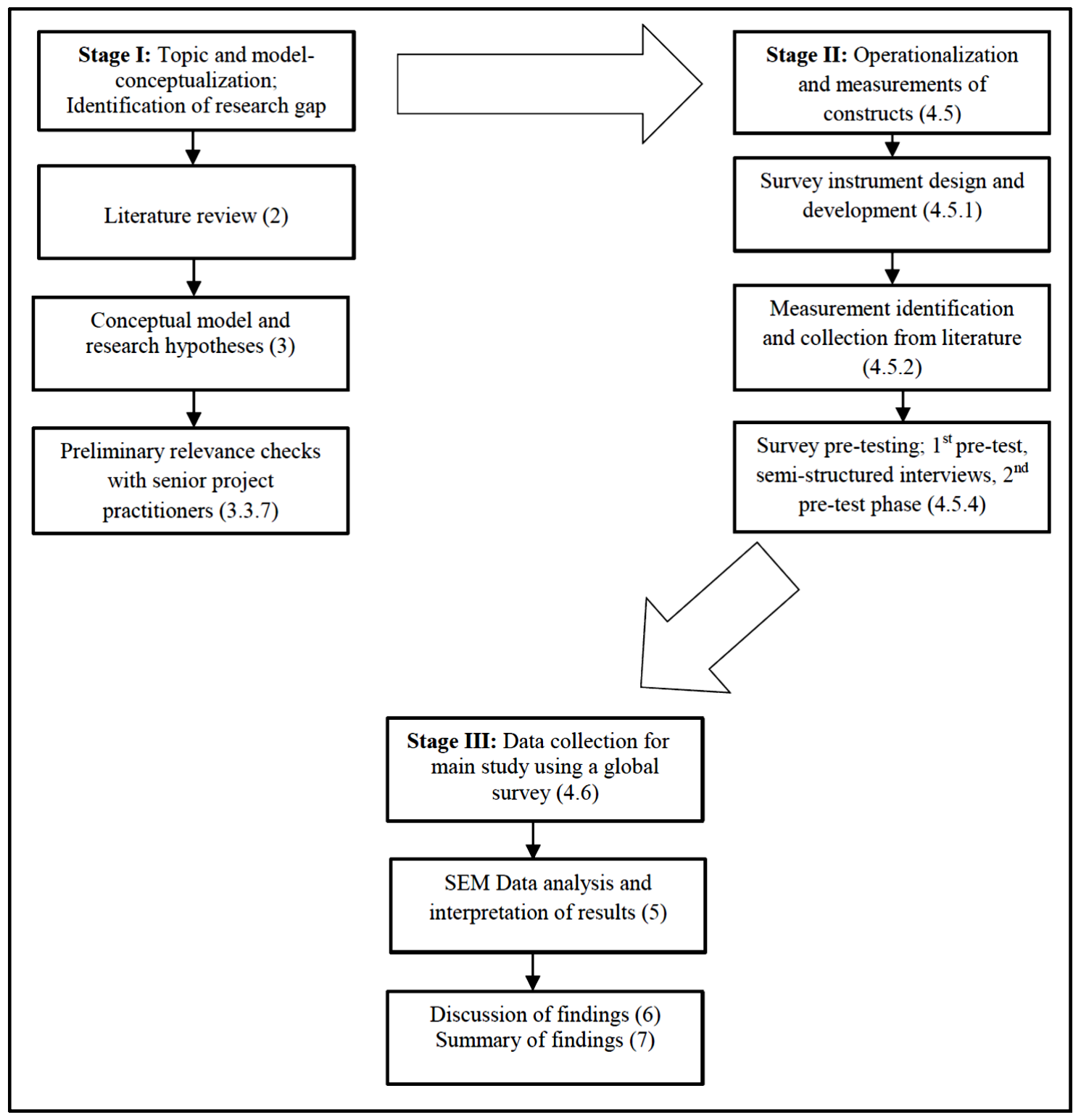

Figure 6: Research design overview

The research design of this study comprised of three major stages. In the first stage, a comprehensive literature review was conducted to identify the research gaps and inform the conceptualization of the research model. The literature review focused on theoretical perspectives on outsourcing and outsourcing relationships, traditional and agile software development methodologies, the CSFs research approach and contingency theory (chapter 2). Based on this, the conceptual research model was developed (chapter 3). In order to ensure that the research outcomes of this are valuable to practitioners, some preliminary checks were conducted among nine senior vendor project managers. 
The second stage aimed to operationalize the research model, identify construct measurements and to thoroughly develop a survey questionnaire instrument. Subsequently, the usability of the survey questionnaire was pretested through two rounds consisting of senior local project managers in Wellington, New Zealand (chapter 4). The third stage consisted of data collection of 984 using a global survey. The data was analysed with SEM to test the hypotheses, results were interpreted (chapter 5), discussed (chapter 6) and a summary was provided (chapter 7).

\subsection{Thesis outline}

This thesis is structured in seven chapters as follows: Chapter one,-introduction, discusses the motivation of this study, describes the research problem and explains why this is a topic of interest. The purpose and research objectives, design and the outline of the thesis are also presented. The second chapter; literature review, examines the research that has been undertaken in the field. Theoretical perspectives on Information Technology (IT) outsourcing and types of outsourcing relationships are examined; traditional plan-based and agile software development methodologies are compared and contrasted. Research on CSFs in software project management is examined. The role of contingency theory and 'fit' in software development are also examined. The aim of this chapter is to highlight research gaps and develop a research conceptual model. Chapter three; conceptual model and research hypotheses, based on literature review, a research conceptual model is expounded further and the hypotheses associated with the research are formulated. Chapter four; methodology, covers the research philosophy, study population, the sampling procedure, measurements, reliability and validity, data collection methods and analysis procedures. Then Chapter five; data analysis and results, presents and interprets the descriptive statistics and SEM analyses from the main survey. Chapter six; discussion, analyses findings in relation to the previous relevant literature and the research gaps identified. Finally, Chapter seven concludes the thesis with a summary of the key research findings, contributions of the research, limitations, delimitations and recommendations for future research. 


\section{CHAPTER TWO: LITERATURE REVIEW}

\subsection{Introduction}

This chapter reviews the literature relevant to this study. In order to develop a strong understanding of the accumulated literature in this field, numerous research streams have been examined.

- Theoretical perspectives on IT outsourcing

- Approaches, types and quality of IT outsourcing relationships

- Traditional and agile software development methodologies

- The concept of project success

- The CSFs research approach

- Contingency theory

- Research gaps

\subsection{Theoretical perspectives on IT outsourcing}

Outsourcing is now emerging as a prevalent practice aimed at market-testing the procurement needs with some research suggesting it can improve project success and organizational performance (Lacity \& Willcocks, 1998, 2000; Gottschalk \& Solli-Saether, 2005). Although the precise definitions of IT outsourcing appear to differ in the literature (Hancox \& Hackney, 2000), there is general agreement that it is when IT functions are carried out by third parties or vendors (Gottschalk \& Solli-Saether, 2005). A number of theories have been put forward in the literature that attempt to justify the unprecedented rate of IT outsourcing (Gottschalk \& Solli-Saether, 2005). Core competencies theory suggests that activities should be performed either in house or by suppliers depending on what is core or noncore (Hancox \& Hackney, 2000). According to the resource-based theory of the firm, outsourcing is a strategic decision, which can be used to fill gaps in the firm's resources and capabilities (Grover, Teng \& Cheon, 1998).

This is supported by the transactional cost theory (Williamson, 1979) that argues transaction costs arise because complete contracting is often impossible and incomplete contracts give rise to subsequent renegotiations when the balance of power between the transacting parties shifts. Thus, outsourced contracts appear cheaper and easier to design and enforce. The proponents of contractual theory such as Luo (2002) argue that an outsourced contract 
provides a legally bound, institutional framework in which each party's rights, duties, and responsibilities are codified, and the goals, policies and strategies underlying the arrangement are specified. Every outsourcing contract has the purpose of facilitating exchange and preventing opportunistic behaviour. Neo classical economic theory posits that firms outsource to attain cost advantages from assumed economies of scale and scope possessed by vendors (Ang \& Straub, 1998). Lambe, Spekman and Hunt (2002) reveal that according to partnership and alliance theory, partnerships can reduce the risk of inadequate contractual provision, which may be comforting for clients about to outsource a complex and high-cost activity such as IT. Relational exchange theory, which is based on relational norms, suggests that the key to determining how efficiently contract governance is carried out lies in the relational norms between the parties (Artz \& Brush, 2000).

Each theory provides a new lens through which IT outsourcing relationships can be viewed with recommendations for actions that can contribute to managing successful IT outsourcing relationships in a given context. These theories can be compared in terms of what they recommend to be successful in IT outsourcing relationships, and identify critical issues in the complex process.

\subsubsection{Approaches, types and quality of IT outsourcing relationships}

Literature shows that some of the first approaches of a systematization of IT outsourcing relationships exist (e.g. Nam et al. 1996; Lacity \& Willcocks, 2000; Kern et al., 2002; Franceschini et al., 2003; Kishore et al. 2003; Gottschalk \& Solli-Saether, 2006 etc.).

Table 2 summarizes key frameworks on IT outsourcing relationships. 
Table 2: Key frameworks on IT outsourcing relationships

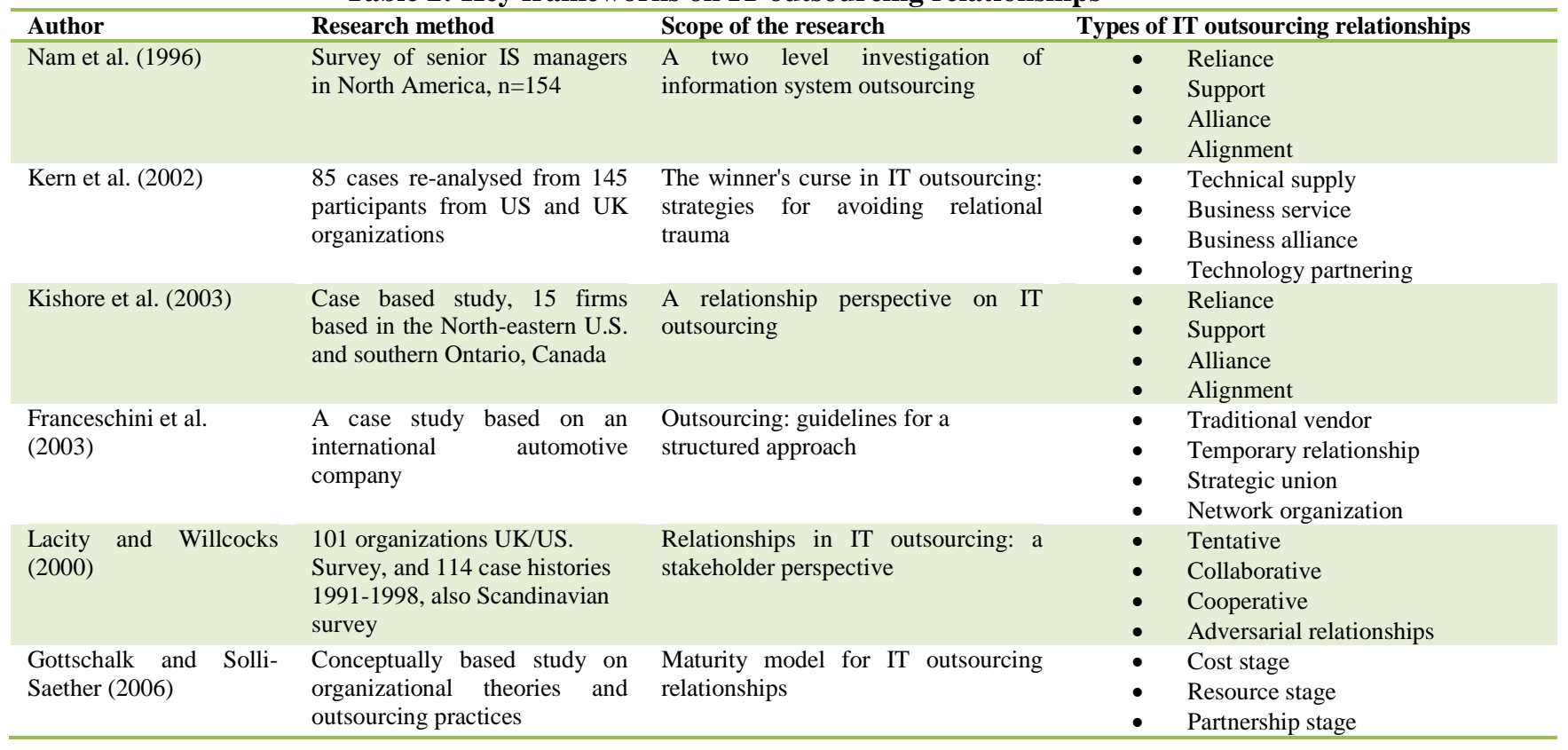

Nam et al. (1996) proposed a two-dimensional framework for describing outsourcing relationships along the dimensions "extent of substitution by vendors" and "strategic impact of IS applications." The framework classifies outsourcing relationships into four types: support, alignment, reliance, and alliance (Figure 7).

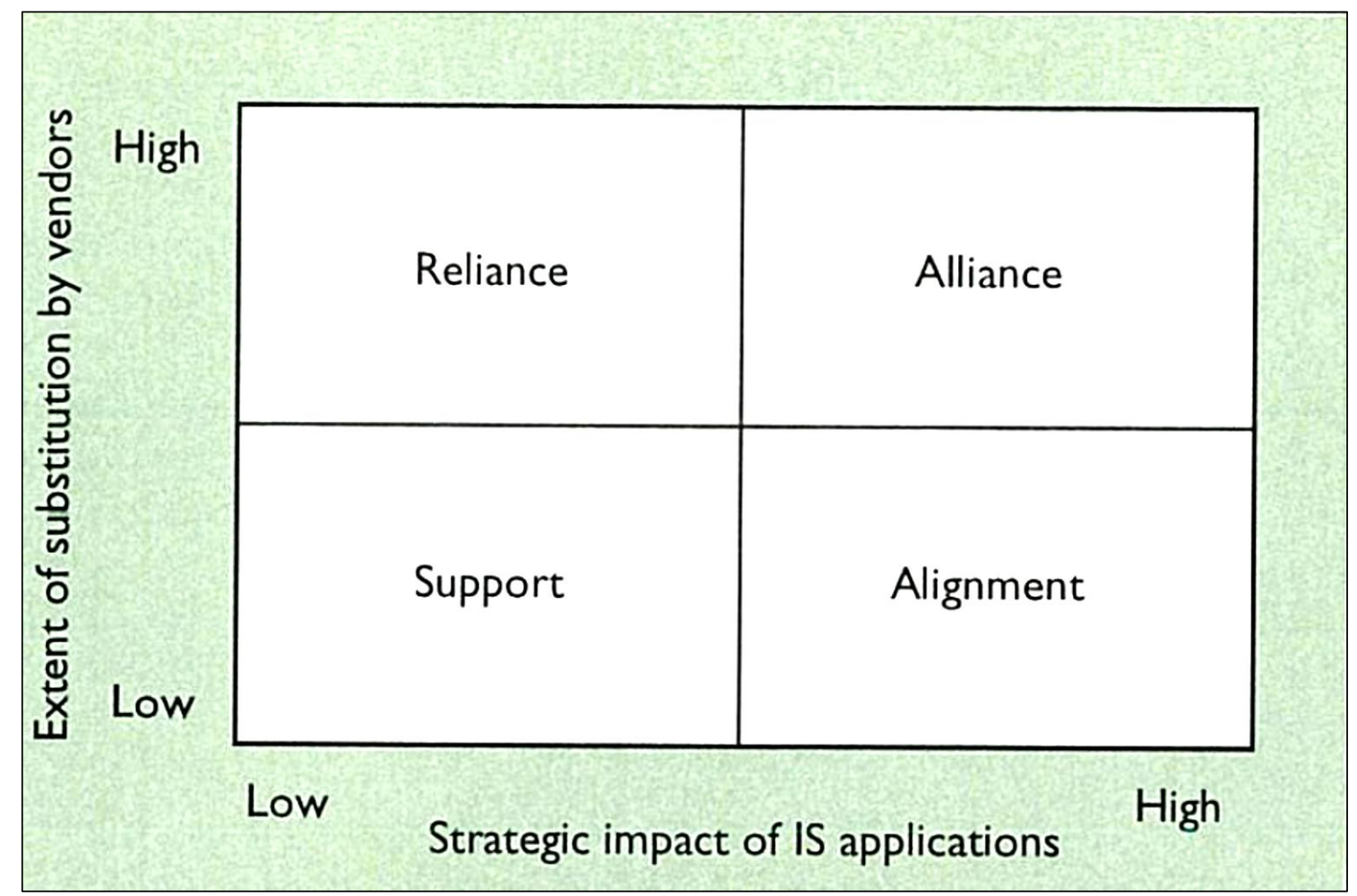

Figure 7: Four types of outsourcing relationships (source: Nam et al, 1996, p.38) 
The support cell corresponds to the traditional IS vendors' service and the simplest type of outsourcing relationships. Vendors are usually restricted to non-core IS activities when the size of the contract is small e.g. contract programming and installation of a software. The duration of such outsourcing is usually short, and it is relatively easy to find alternative vendors. The reliance cell corresponds to IS outsourced functions that are mostly non-core activities, and cost reduction is one of the major motivations. However, the length of contract is longer than in the support cell, because outsourcing in this cell requires more commitments from vendors and clients. Thus, if the contract is small for an IS non-core activity and lasting for a relatively short duration, support and reliance relationships are highly likely.

The alignment cell shows that even though vendors are not significantly involved with client firms' IS operations; vendors' impact lasts longer than in the support cell, e.g., IS consulting or technical supervision for IS planning and design, and system conversion. In the alliance cell, outside vendors not only substitute for in-house IS operations but are also completely responsible for highly strategic IS activities, e.g., IS planning and design for new product development or systems that help penetrate a new market. This type of outsourcing evolves to being based on mutual relationships. Term of contract is usually longer than with other types of relationships. Highest commitments from vendors and clients are required. The implication is that IT vendors/clients are likely to be engaged in any one of these outsourcing relationships for specific software development projects. Thus, if the contract is larger for an IS core activity and lasting for a relatively longer duration, alignment and alliance relationships are highly likely.

Kern et al. (2002) classify four main types of IS outsourcing relationships along two other dimensions: "strategic intent" and "technical capability." The matrix sets up four possible relationships: technical supply, business service, business alliance, and technology partnering (Figure 8). 


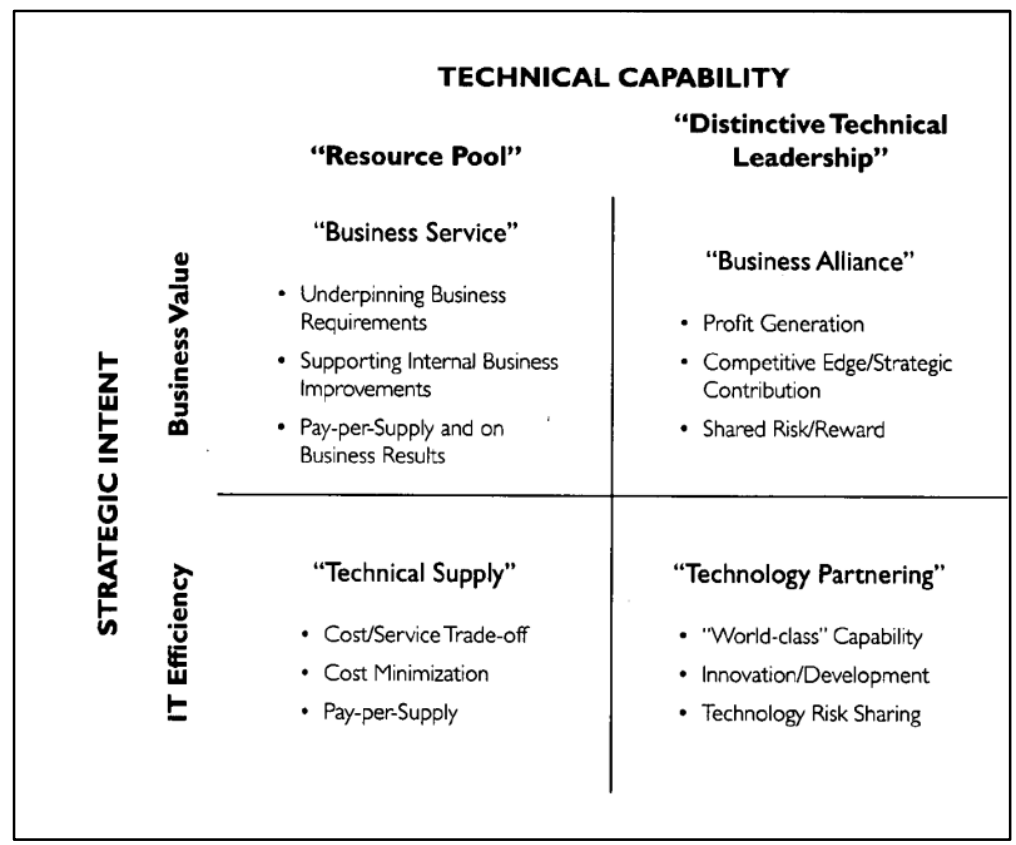

Figure 8: Types of outsourcing relationships (Kern et al. 2002, Figure 4, p.66)

Accordingly, the technical supply relationship is the most common, where the objective is to achieve IT efficiencies by hiring external resources, focusing on cost minimization that renders IT as a variable cost (Kern et al. 2002). Business service, uses the external IT supplier who does not only deliver more IT efficiency for changing business requirements, but is involved in business improvement projects. The third type is technology partnering where suppliers are chosen for best in class capability and pro-active innovations in technological applications. Strategic partnerships (large scale outsourcing arrangements), involve working together and sharing the risks and rewards; with a focus on business expansion. Depending on the circumstances of the software development project, any of these relationships is possible.

As outsourcing relationships are not static, but rather likely to change and evolve over time due to changes in the external and client's internal environment, Kishore et al. (2003) proposed an evolution framework of outsourcing relationships in terms of 'the extent of ownership substitution by IT outsourcing service providers' and 'the strategic impact of outsourced ITS portfolio'. Consistent with Nam et al. (1996), the four resulting types of outsourcing relationships in the FORT framework are support, alignment, reliance, and alliance. Figure 9 represents the movement within the FORT framework of each of the four firms that were examined. 


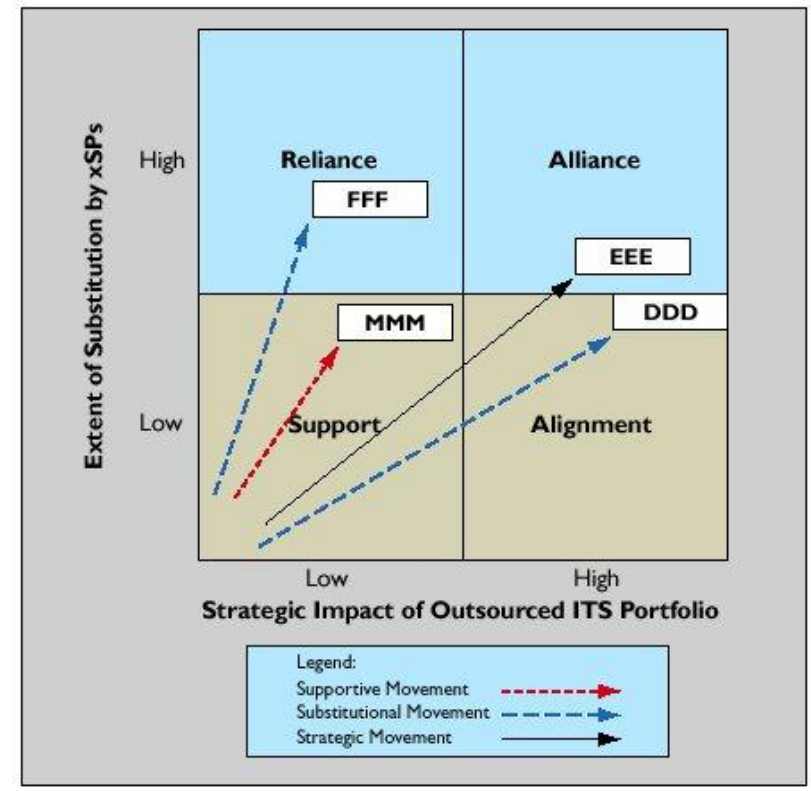

Figure 9: Evolution of outsourcing relationships (Kishore et al., 2003, Figure 2, p.91)

This framework differs from the one of Nam et al. (1996) in that it can be used to depict both static and dynamic aspects of client-provider relationships and then be utilized to examine the movement of organization's changing IT outsourcing relationships over time within or across the four relationship cells. The implication for this is that outsourcing relationships can evolve over time due to changes in the environment.

Further, Franceschini et al. (2003) examined types of relationships between "outsourced" and "outsourcer". To analyse them, they investigated two main characteristics: "specificity" and "complexity". Two levels of evaluation, low and high, were used to define each characteristic. The combination of the two characteristics again gives rise to four types of relationships: (1) traditional vendor; (2) temporary relationship; (3) strategic union; and (4) network organization. A schematic presentation of four types of outsourced-outsourcer relationships by Franceschini et al. (2003) are displayed in Figure 10. 


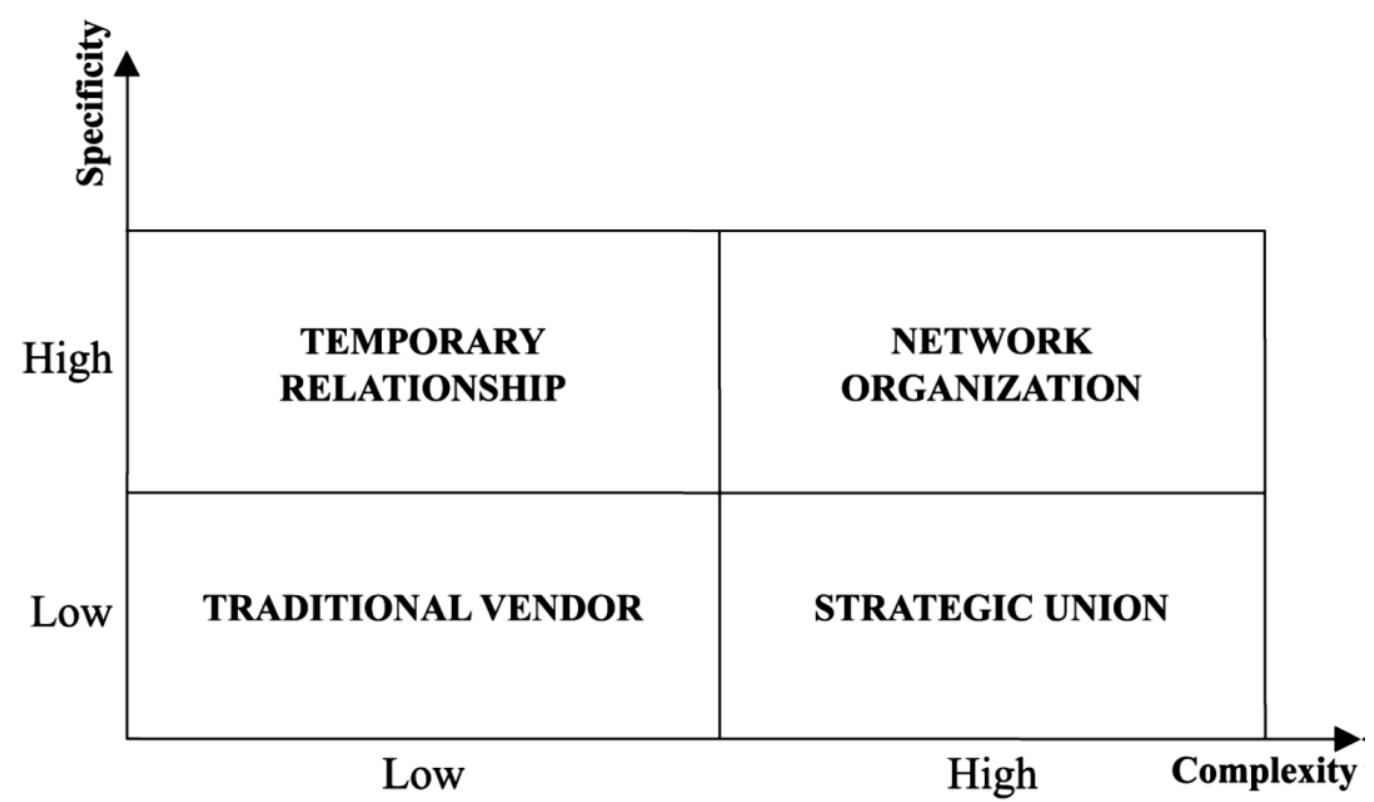

Figure 10: Four types of outsourced-outsourcer relationships (Franceschini et al. 2003, Figure 3, p. 251)

According to Franceschini et al.'s (2003), the traditional vendor relationships is aimed at resolving an immediate problem with a short term focus of minimising costs and the trust is not essential while the temporary relationship targets competences and process improvements. The other two types of relationships are different in that they focus on long term strategies like building partnerships which are based on trust and a price model of winwin. The implication is that different software development projects can pursue any of these outsourcing relationships. This also appears to suggest that when the client is a part-owner or partner of the outsourcing vendor, the interests, vision, and mission of the two organizations are frequently well aligned, while if the outsourcing relationship is simply a one-off contract, they may have very different goals and success criteria for the project.

Consistent with Lacity et al. (1995; 1996) and Lacity and Willocks (1998), Lacity and Willcocks (2000) identified four types of relationships in IT outsourcing as: tentative, collaborative, cooperative and adversarial relationships. Figure 11 illustrates Lacity and Willcocks' (2000) types of relationship in IT outsourcing. 


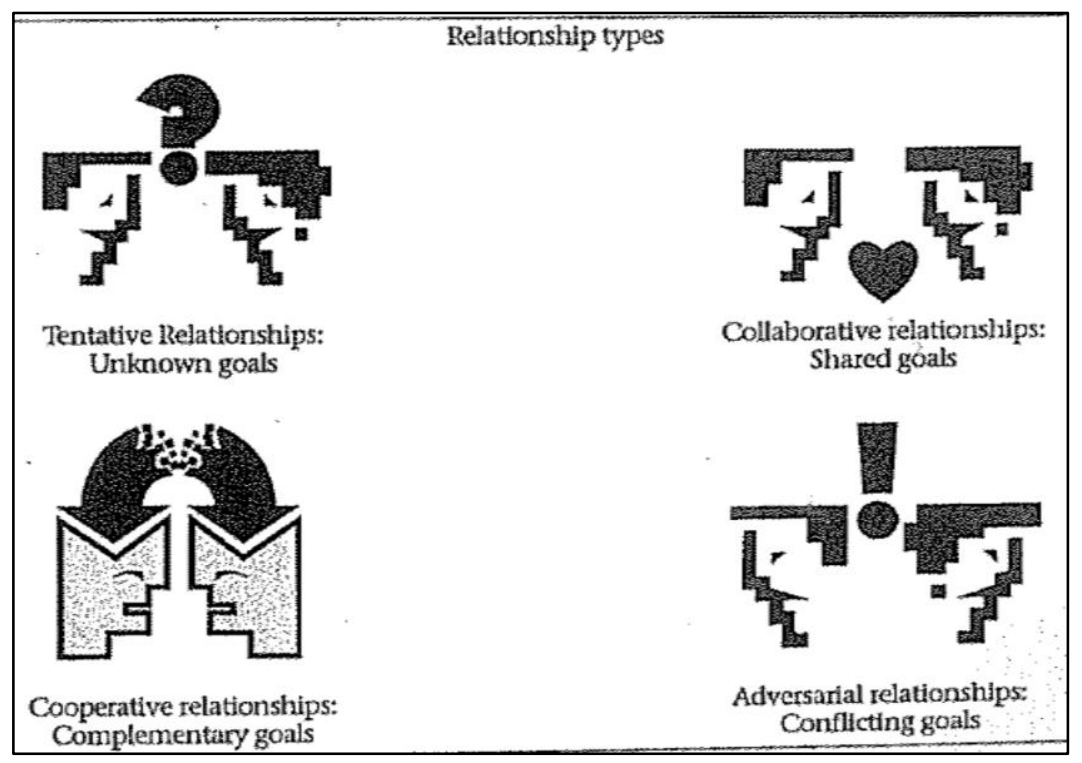

Figure 11: Types of relationship in IT outsourcing (Lacity \& Willcocks, 2000, Figure 18.1, p. 365)

The implication is that tentative relationships are quite common when clients and vendors have no shared history, while collaborative relationships occur when clients' and vendors' goals are shared; cooperative relationships manifest themselves when goals are complementary, and that adversarial relationships occur when stakeholder goals are in conflict (Lacity \& Willcocks, 2000). These types of relationships are potentially likely for any software development project.

Gottschalk and Solli-Saether (2006) propose a three-stage maturity model for the evolution of an IT outsourcing relationship. The model explains the relationships in terms of a specific stage and develops strategies for moving to a higher stage in the future (Figure 12).

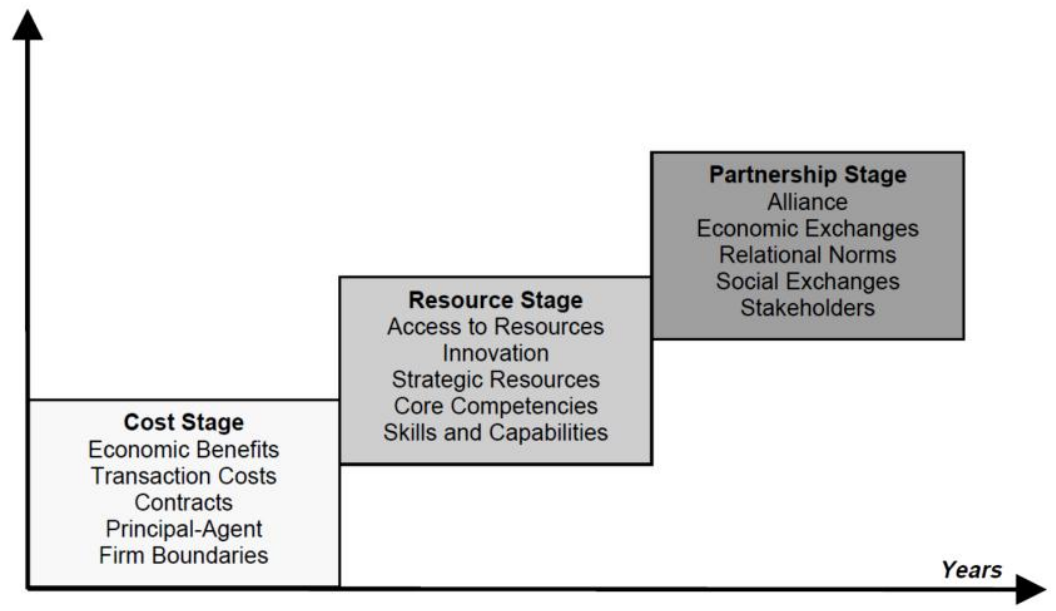

Figure 12: Three stage-maturity model of outsourcing relationships (Gottschalk \& Solli-Saether, 2006, Figure 1, p.205) 
The implication is that as an IT outsourcing relationship matures, the performance measures develop beyond cost minimization and operational efficiency into business productivity and technology innovation, and further into business benefits and achievement of mutual goals for client and vendor (Gottschalk \& Solli-Saether, 2006). The IT outsourcing contract changes focus from specified obligations and service level agreements, to availability of strategic resources, management of key competence and critical projects, to arrangements for profit sharing and personnel exchanges between vendor and client.

Depending on the circumstances, the literature suggests that IT outsourcing relationships can vary considerably. However, the outsourcing market appears to be changing in customer's favour (Lacity \& Willocks, 1998), despite once being dominated by a few players (vendors). As competition increases in the IT outsourcing market, companies have more power to bargain for shorter contracts, more select services, better and more flexible financial packages/ performance based contracts (Lacity \& Willocks, 1998). Also in the customer's favour is a growing experience with IT outsourcing, which allows for negotiating outsourcing deals with greater sophisification.

The review of literature reveals that prior researchers have approached the topic of IT outsourcing relationships from many different points of view. While IT outsourcing relationships have received attention in the literature, few researchers have provided insights into the IT outsourcing relationship between client and vendor and most have produced inconclusive results. Although these contributions help us to understand IT outsourcing relationships in general, our understanding of managing the IT outsourcing relationships remains limited. Until now, there has been no single practical model that fully explains the dynamics of managing IT outsourcing relationships from a vendor perspective. Many studies focus on the client's perspective, yet vendors are the other half of the outsourcing equation and are equally important and influential. Thus, a study that considers outsourcing relationships from vendor perspectives can provide more insights when compared with client perspectives.

\section{Whose perspective and why?}

This study examines the differences between traditional plan-based and agile methodologies based on the perceptions of vendor respondents with regard to their client organizations. In an outsourced context, there are two organizations involved, each with their own perspectives 
which may or may not be congruent. The various perspectives involved include: vendor perceptions of client organization, the clients own perspective on their organization, and the vendor's perspective of their own organization, and presumably also the clients' perspective on the vendor organization. It is also possible that a project team comprising of members of both organizations may have yet a further perspective. Of these, only the vendor perspective of the client organization has been considered.

This is because clients and vendors share the responsibilities for managing outsourced IS projects. Problems arise from the differences between their goals, structures and success, which can cause each side to feel vulnerable to opportunism or shirking of responsibilities by the other (Taylor, 2007). The two sides may therefore pull project management in different directions. The candidate CSFs, along with the difficulties in obtaining quick feedback, meeting frequently, and building interpersonal relationships, make the management of outsourced IS development projects an arduous task. However, such problems especially from the vendor perspective are usually ignored in the IS outsourcing literature, which somehow depicts an overly optimistic view of IS outsourcing (Taylor, 2007).

In outsourcing, both client and vendor may feel vulnerable to opportunistic behavior by the other. For example, the client may be concerned about the vendor providing inadequate effort or developing poor quality software while the vendor may be concerned about the client not providing the needed help in requirements analysis or adding new requirements as the project proceeds. Moreover, the two sides may also have different perceptions of vulnerability and management. The client may be less concerned about the costs the vendor incurs in developing the system, especially in fixed-price contracts. These differences between vendor and client views may be amplified when the relationship quality is poor, e.g., when there are considerable differences in the two organizations' goals and outsourcing is across national boundaries.

Accordingly, in outsourcing the client typically does not have full control over what the vendor-is doing or how the work is done, and some authority is delegated to the vendor to get the work done. The client and the vendor may have differing goals relating to what they hope to achieve from the completion of the work, and hence differing perceptions of the potential CSFs and the risks that might threaten the successful achievement of these goals. For example, a vendor's goals in an outsourcing project are likely to include making a profit, 
while the client may have a goal of reducing overall IT costs. The concern and risk for the client is that differences in goals may lead to the vendor taking actions more favorable to the client's self-interest than to the vendor's interests.

On the other hand, however, the challenge for the vendor is to meet the client's goals for the project, while ensuring that the vendor's own, possibly differing, goals are also met, but this vendor perspective has received little attention. Yet, clearly, if the outsourcing arrangement is to be successful for both parties, while the vendor must accept the transferred risks in the project and agree to the controls imposed by the client to manage the relationship, the vendor must also stand to gain from the relationship, and vendor interests must also be met.

Thus, although some of the candidate CSFs for in-house project outcome can also apply to outsourced projects, the outsourcing situation may give rise to additional and different CSFs both from the client and the vendor perspective. It is not surprising that Taylor (2007) identified some additional risk factors and goals from the vendor viewpoint, while Sabherwal's (2003) highlighted extra different vendor and client coordination concerns.

Apart from few scholars, (e.g., Jun et al., 2011; Taylor, 2007; Sabherwal, 2003) previous CSFs studies focus on in-house development projects; where developers and users are members of the same organization. However, over the current period, most firms outsource their software development activities. Vendors play a significant part in outsourced software development by absorbing considerable risk; however, there is lack of an integrated framework for managing software development from a vendor perspective (Jun et al., 2011). Therefore, there is a need to theorize further the contingency relationships in prior research to determine how they may also apply to the study of the outsourced software projects from a vendor perspective. Though, the challenge that arises from this choice is that there are some candidate CSFs relating to the client organization that vendor staff may not necessarily be best qualified to report on. The next section reviews SDM approaches. Traditional plan-based and agile perspectives of methodologies are then compared and contrasted in more detail.

\subsection{Software development methodology approaches}

Although projects play a critical role in modern business operations, many studies reveal that projects in general and specifically software projects, continue to have unacceptably low success rates (Petit, 2012; Standish Group, 2012; Nasir \& Sahibuddin, 2011). The Chaos report (Standish Group, 2012) suggests that $86 \%$ of all software development projects are 
unsuccessful. One of the most common reasons noted is an inappropriate software development methodology (KPMG, 2013, p.12; Sheffield \& Lemetayer, 2013).

Most of these studies or reports have, though, been critiqued such that the findings cannot be broadly generalised. For instance, globally, the Chaos reports are often used to indicate problems in software development project management (e.g. issues associated with methodology choice decisions). However, the validity of these figures is questioned because these reports have been argued to contain shortcomings (Glass, 2006, p.15). For instance, the Standish Group hasn't explained, how it chooses the organizations it surveys, what survey questions are asked, or how many good responses it receives, thus, leaving many questions about Chaos reports unresolved (Cohn, 2012).

Similarly, Eveleens and Verhoef (2010) in their popular article entitled "The Rise and Fall of the Chaos Report Figures" strongly discredit the Chaos reports based on the following. First, they indicate that these reports are misleading because they are based solely on estimates of cost, time, and functionality. Second, they illustrate that their estimation accuracy measure is one-sided, leading to potentially biased success rates. Third, they demonstrate that their definitions may negatively impact good estimation practice. Fourth, they contend that the resulting figures may be meaningless because they average numbers with an unknown bias, numbers that are introduced by different underlying estimation processes.

The Chaos report (Standish Group, 2012) reveals that projects that use agile are successful three times more often than waterfall (traditional plan-driven methodology projects). They further suggest that agile process is the universal remedy for software development project failure. Figure 13 shows the specific results reported by Chaos report (Standish Group, 2012) that agile methodology projects are successful three times (42\%) more often than traditional plan-driven (waterfall) methodology projects (14\%). 


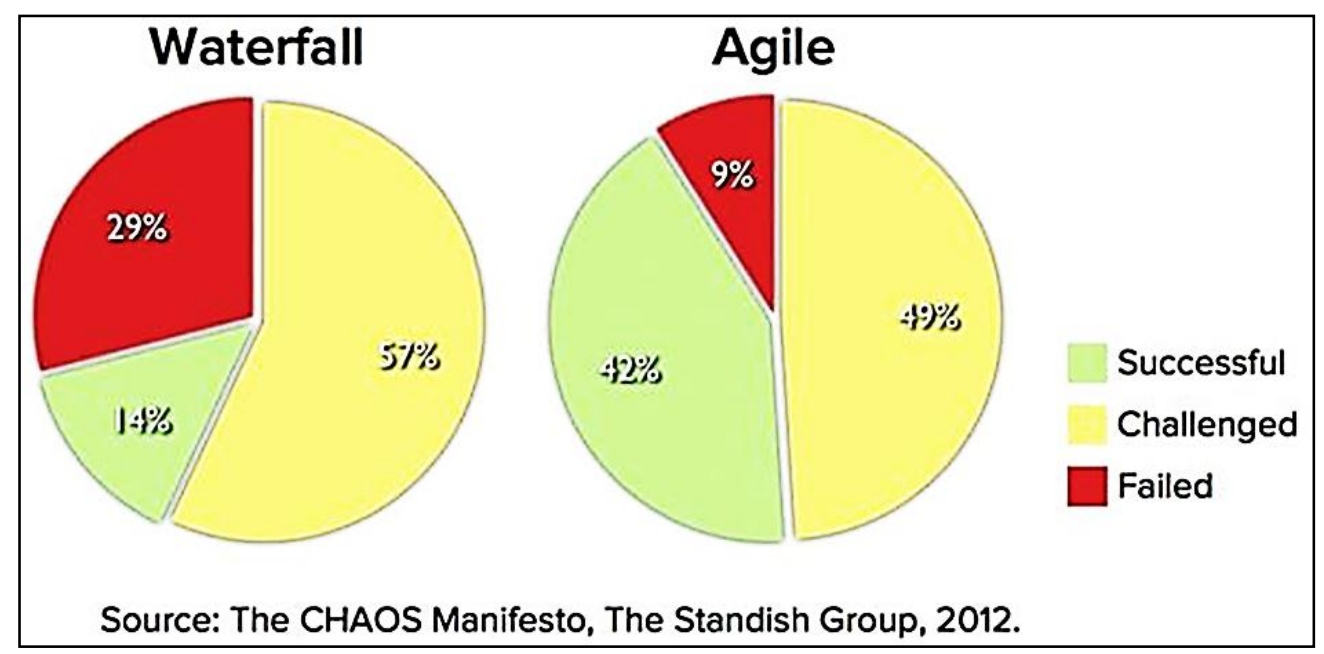

Figure 13: Success rates of Agile and waterfall or traditional projects

Overall, debate remains about what might be the best approach for managing software projects. This debate mainly focuses on whether to conduct software project management using traditional plan-driven methodologies, which views projects as sequential collection of processes, or to adopt agile techniques, which are more suitable in managing the demands of a volatile development environment with a social process perspective of projects (Bajwa et al., 2012). Some authors have also used the concept of "methodology wars" to demonstrate the often unfriendly dispute between advocates of agile and traditional plan driven software development (Singh et al., 2012, p.33). The next section compares and contrasts software development methodologies in more detail.

\subsubsection{Comparison of software development methodologies}

Generally, research on SDM segments the selection of software development methodologies into two broad categories, the traditional formal approaches and more open agile approaches (Ramesh et al., 2012, p.324). The traditional methods are essentially plan-driven approaches that follow the philosophies of the PMBOK Guide (2013) and PRINCE2 manual (OGC, 2009), while agile methodologies are less planned and assume many IT projects take place in volatile environments, requiring projects to adapt quickly to changes (Singh et al., 2012).

\subsubsection{Traditional plan-based software development methodologies}

The traditional approaches rely on what has been described as a linear or incremental lifecycle (Wysocki, 2009). These methods are plan-driven and characterized by a design approach to development (Ramesh et al., 2012). In this kind of project the requirements are clearly specified, little change is expected and uncertainty is low (Cockburn, 2000). Thus, the environment is viewed as predictable and planning tools can be used to optimise the 
management of the project. These methodologies are usually accompanied by a changeresistant focus on compliance-to-plan as their measure of success (Wysocki, 2009). Consequently, they are somewhat prescriptive and heavy on process and documentation (Wysocki, 2009). These methodologies work well until change is required (Chavart, 2003). These changes then do not fit with compliance plan-driven methodology and may be perceived to be a threat to such methodologies, which is why project managers may tend to be change-resistant. When and if change is accepted, the plans need to be updated and previous work may become obsolete. Thus, time may be perceived to be wasted on tasks that do not end up adding value to the project. As a result, traditional plan-based methodologies are referred to as rigorous, predictive or heavy weight.

\section{Linear and Incremental models}

In the linear or sequential life cycle, the project is designed in such a way to be completed in one unique cycle (Wysocki, 2009). Each stage of the project from analysis to support is executed only once. The project moves from one stage to another when the predefined milestones or objectives are achieved. At the end of each stage, the deliverable is not the software itself but the documentation that reflects the milestones of the work undertaken (for example, business requirements or design). The waterfall model (Royce, 1970) is a wellknown example of a linear model. With the Waterfall model, system development is broken down into a number of sequential stages represented by boxes. Only after a stage is finished, is the next stage started and a completed stage is not revisited again.

Figure 14 shows the water fall model of software development which is usually employed when the requirements are clearly specified, little change is expected and where uncertainty is low. 


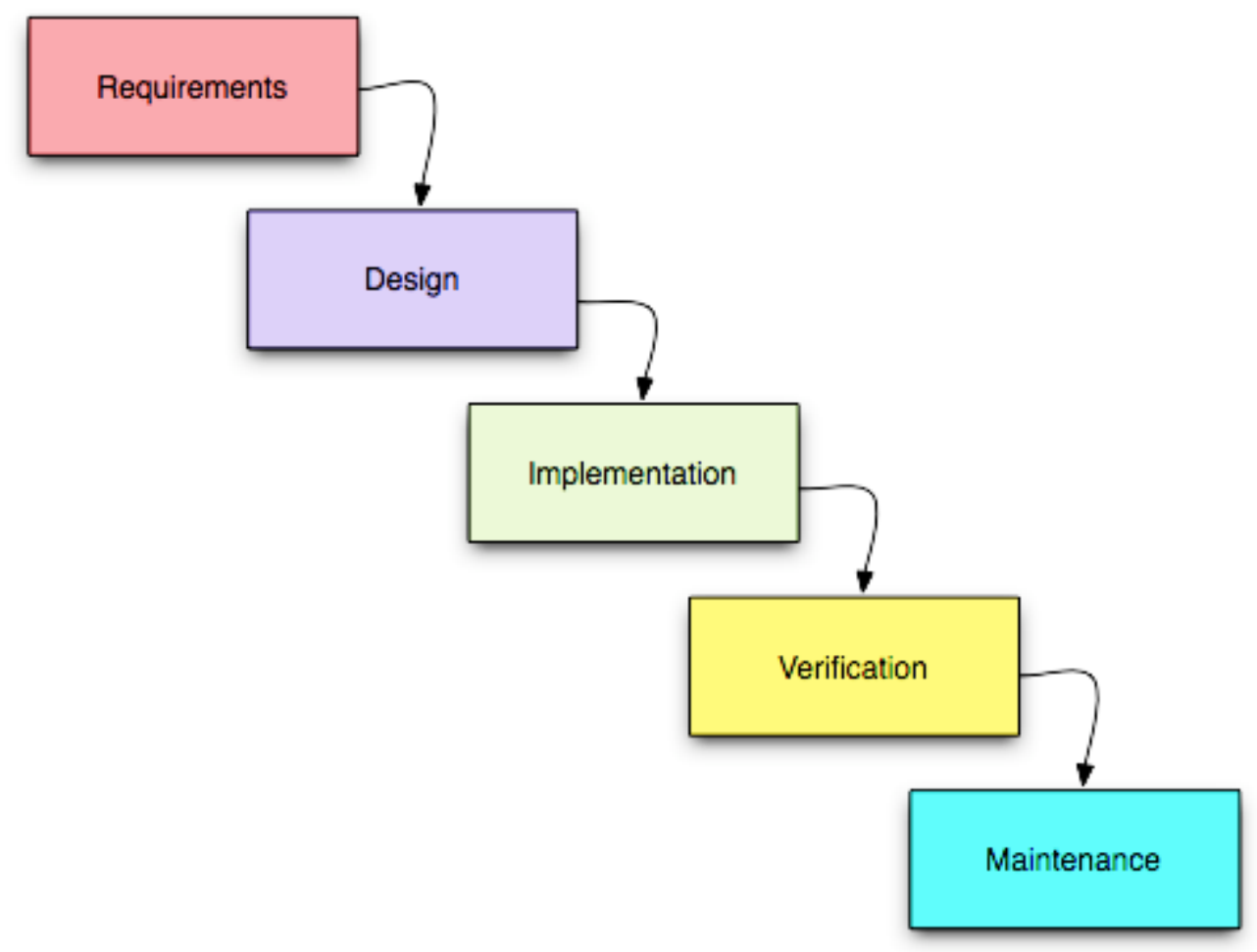

Figure 14: Waterfall model (Royce, 1970)

As illustrated, all stages are planned upfront and there is strict adherence to the set plan. There are no changes expected to occur during software development process. Communication is unidirectional supporting only conformance to plan with no opportunity for innovation. Commitment is demonstrated by meeting the fixed baseline project goals of time, budget and scope.

In this category, there are also traditional-type approaches based on an incremental model. In contrast to the linear model, the development phases (i.e. design, build, and test) may be executed more than once. At each increment, the scope is expanded according to a prespecified plan. This allows phased delivery to the client (Charvat, 2003). Even though this approach allows more flexibility, it still follows a pre-determined plan developed at the beginning of the project, where adherence to that plan is expected.

\subsubsection{Agile Software Development Methodologies}

Agile approaches are based on an iterative or adaptive lifecycle and are designed to accept and embrace change (Chavart, 2003). They may be considered to be value-driven rather than plan-driven and operate using tacit knowledge between team members in place of copies of 
documentation (Wysocki, 2009). In agile methods, the major, upfront, one-time planning task is replaced by an iterative and adaptive series of just-in-time tasks, each of which is executed only when needed (Wysocki, 2009). This provides flexibility and adaptability to the project, enabling it to cope more readily with change requests. At a team level, agility can be explained as the capability of a team to speedily accomplish software development tasks while adapting itself to changing conditions in a rapid manner based on (1) personnel and technology, (2) utilizing software development methodologies, (3) creating and maintaining linkages across communicative and cultural barriers among the project teams (Sheffield \& Lemetayer, 2013). Therefore, in an agile context, both technology and human aspects are important for project success. Conflicts that would arise due to lack of alignment between the set plans and changes are resolved by practices that are tailored to the project teams and its organisational context. However, such methodologies may turn out to cause disaster for larger projects because of lack of plans (Hajjdiab et al, 2012; Mishra \& Mishra, 2011, p. 551). The next section describes iterative and adaptive models as examples of agile software development methodologies.

\section{Iterative and adaptive models}

The iterative life cycle focuses on re-doing the project at each iteration. Therefore, at each iteration there is some learning as a result of feedback, and the next iteration might change or adapt what has been done before, in contrast to an incremental development where increments are planned to fit together and follow each other in a pre-specified order. Thus, an increment does not modify previous work (Charvat, 2003) but iteration may. This is well illustrated by the agile principle of simplicity. This principle states that future features should not or need not be prepared in the current iteration as they are likely to evolve as a natural outcome of the rapid learning experienced on agile projects (Boehm \& Turner, 2004). Iterative and adaptive life cycles have an advantage that arises from a continual testing throughout the project, which has a positive impact on quality (Dyck \& Majchrzak, 2012). Agile methodologies suggest short iterations of less than three months and usually around four weeks (Imreh \& Raisinghani, 2011), where each iteration would cover an entire development life cycle (from the requirement specifications of a specific set of functionalities to the testing and release to the client).

An example is Scrum, an iterative agile software development method for managing software projects or application development. In Scrum, iterations are called sprints and constitute the 
core element (Singh et al., 2012, p.34). The product backlog is an ordered list of requirements that is maintained for a product while the sprint backlog is the list of work the development team must address during the next sprint (Singh et al., 2012, p.34). In this approach, once the scope of the sprint is approved, no additional functionality can be added. In other words, the work done to meet the sprint is fixed, but the product backlog which contains all the features that still need to be implemented is dynamic. The latter is prioritized according to the needs of the customer. Features that deliver the most value will have a higher priority and will be developed in the following sprint. All the features could be reprioritized as client's needs change. Figure 15 shows the scrum process for agile software development in which projects progress via a series of iterations called sprints. Each sprint is typically 2-4 weeks long and sprint planning is essential.

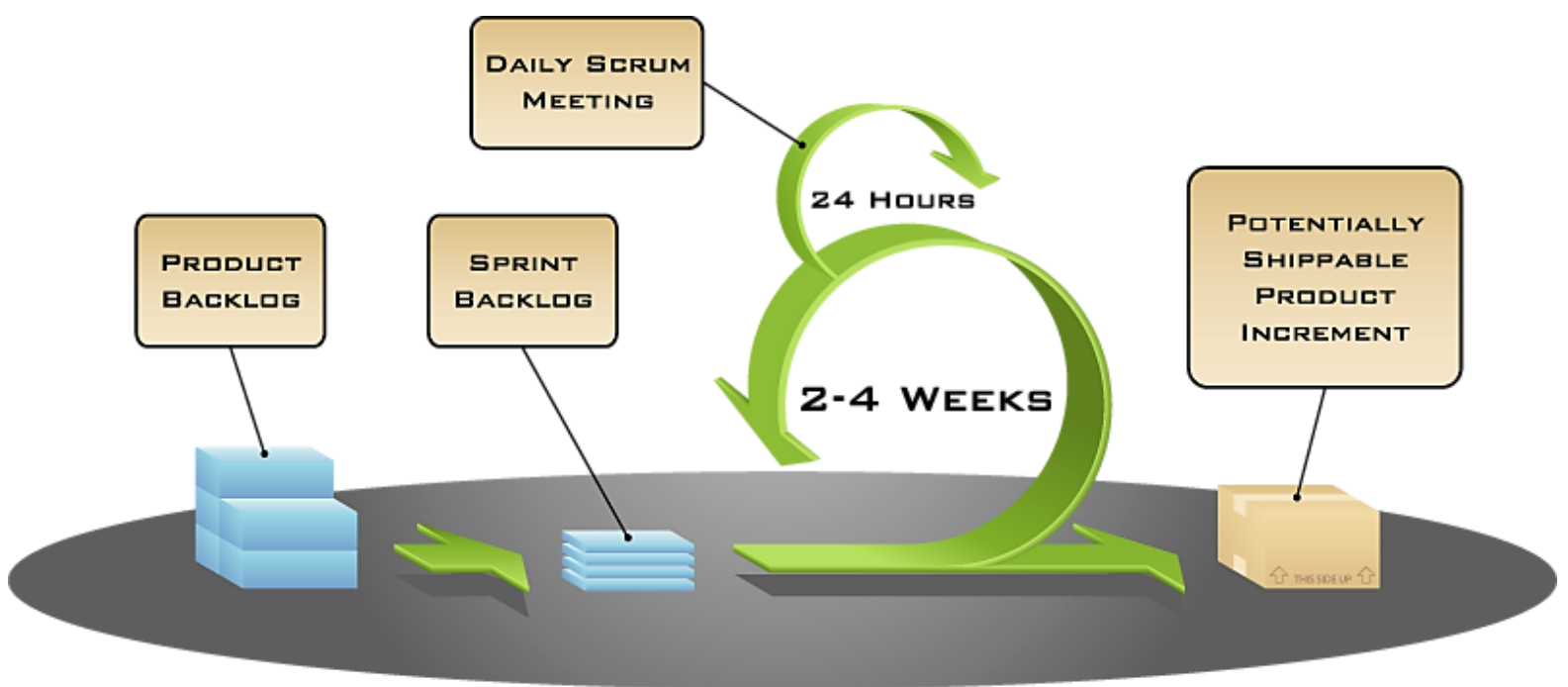

Figure 15: The Scrum process (Cohn, 2012).

As illustrated, the scrum does not require heavy planning and controlling with a lot of documentation. Instead it allows for changes and dynamism and can provide a faster time to market. The process relies on user participation for continuous feedback which enables improvement. Informal communication in form of face-to-face within teams and knowledgeable users is paramount. Teams are self-organizing and collaborative.

The adaptive model is a more extreme version of the iterative model; and is recommended when there is a very high degree of uncertainty and complexity and very little is known about the project (Wysocki, 2009). Learning and discovery are major elements of each cycle of adaptive models that set them apart from iterative models. Thus, each cycle seeks to address 
task completion for newly defined functions, and the discovery of new features and requirements. Unlike the iterative model, where the scope is known, but all the functionalities are not, the adaptive model envisages that the scope of the project will change during development. Therefore, each cycle proceeds on an implicit limited understanding of the solution and attempts to converge pragmatically to an acceptable solution. The requirements are obtained and altered through a feedback loop as the system develops (Wysocki, 2009).

Table 3 contrasts the key aspects of the two approaches (Traditional plan-based and Agile) described in the previous sections.

Table 3 Traditional plan based vs. Agile software development methodology

\begin{tabular}{|c|c|c|}
\hline Project parameter & Traditional software development & Agile software development \\
\hline Development team & $\begin{array}{l}\text { Plan oriented, adequate skills, access to } \\
\text { external knowledge, pre-structured teams }\end{array}$ & $\begin{array}{l}\text { Agile, knowledgeable, co-located and collaborative, self- } \\
\text { organizing teams }\end{array}$ \\
\hline Customers & $\begin{array}{l}\text { Minimal commitment, not co-located and } \\
\text { not empowered }\end{array}$ & $\begin{array}{l}\text { Dedicated, knowledgeable, co-located, collaborative, } \\
\text { representative and empowered }\end{array}$ \\
\hline Requirements & Known early, largely stable & Largely emergent, rapid change \\
\hline Architecture & Designed for current requirements & Designed for current and foreseeable requirement \\
\hline Size & Larger teams & Smaller teams \\
\hline Refactoring & Expensive & Inexpensive \\
\hline Primary objective & High assurance & Rapid value \\
\hline $\begin{array}{l}\text { Fundamental } \\
\text { assumption }\end{array}$ & $\begin{array}{l}\text { Systems are fully specifiable, predictable } \\
\text { and built through meticulous and extensive } \\
\text { planning }\end{array}$ & $\begin{array}{l}\text { High quality adaptive software developed based on } \\
\text { principles of continuous design improvement and testing } \\
\text { based on rapid feedback and change }\end{array}$ \\
\hline Management style & Command and control & Leadership and collaboration \\
\hline $\begin{array}{l}\text { Knowledge } \\
\text { management }\end{array}$ & Explicit & Tacit \\
\hline Development model & Linear or incremental (anticipatory) & Evolutionary-delivery model (Iterative or adaptive models) \\
\hline Communication & Formal & Informal \\
\hline $\begin{array}{l}\text { Desired organisational } \\
\text { form/ structure }\end{array}$ & $\begin{array}{l}\text { Mechanistic (bureaucratic with high } \\
\text { formalization) aimed at large organisations }\end{array}$ & $\begin{array}{l}\text { Organic (flexible and participative encouraging cooperative } \\
\text { social action) aimed at small and medium size organisations }\end{array}$ \\
\hline Quality control & $\begin{array}{l}\text { Heavy planning and strict control, late, } \\
\text { heavy testing }\end{array}$ & $\begin{array}{l}\text { Continuous control of requirements, design and solutions, } \\
\text { continuous testing }\end{array}$ \\
\hline Organizational culture & Risk averse & Risk taking (innovativeness) \\
\hline Market & Mature, stable & Dynamic/early markets \\
\hline Measure of success & Conformance to plan & Business value delivered \\
\hline
\end{tabular}

Despite rhetoric and debates about the superiority of one project methodology over the other, neither appears to be a perfect fit for all types of software development projects (Shenhar, 2001). Instead, project characteristics and project environment should guide the choice of these methodologies (Wysocki, 2009), and these may also affect candidate CSFs for the project.

While the distinctions in the literature may suggest the existence of clear distinctions between agile and plan-based software development methodologies (SDMs), there is not always an easy and entirely clear, black-and-white categorization possible as some projects and organizations use approaches that combine elements of both - for example, the overall project may be plan-based with an incremental approach, but within those increments, an 
agile approach may be taken to the development of detailed functions and features. Or an organization may use a largely plan-based approach, but adopts some agile principles (for example, avoiding excessive heavy-weight documentation) and so on. Thus, the usage of methodologies appears to exist on a continuum from very traditional plan-based to agile SDMs. Next section provides different perspectives on the meaning of project success.

\subsection{The concept of project success in software project management research}

The literature review reveals that the concept of project success has been defined and measured in a range of different ways (Ika, 2009; Jugdvev \& Muller, 2005). This possibly arises from the fact that success criteria may differ from one project to another due to project characteristics. Ika (2009) argues that, although the concept of project success requires different approaches to its study, the idea of a universal set of project success criteria has always dominated. Pinto and Slevin (1988) had earlier acknowledged three aspects of project success as the implementation process, the perceived value of the project, and client satisfaction with the delivered project outcome. Shenhar et al. (1997) suggest two additional measures: business success and preparing for the future. However, empirical results by Lipovetsky et al. (1997) indicate that the importance of the latter measurement is all but negligible. Sheffield and Lemetayer (2013) adopted a relatively narrow criterion of project success based on the first two of the three levels described by Cooke-Davies (2002). The measures at level one were time, budget, functionality and quality while measures at level two were: addresses a need, product is used, customer is satisfied and team is satisfied. Thus, despite research in project management, there is no single simplistic agreement on the concept of project success that works for all projects. But there is agreement about some perspectives on measuring project success.

There appears to be more agreement within IS research on describing and assessing outsourced software project performance/success around the project's process and product performance (e.g. Nidumolu, 1995, 1996; Rai \& Hindi, 2000; Barki et al., 2001; Wallace et al., 2004a; Wallace et al, 2004b; Jun et al., 2011). Project process performance or project management success describes how well the software development process has been undertaken, measuring the extent to which a project is delivered on schedule/time, and within budget and scope (Jun et al., 2011, p. 925). On-time and on-budget completion refer to the extent to which a software project meets its baseline goals for duration/schedule and cost 
respectively (Jun et al., 2011, p.928; Wallace et al., 2004, p.292). Software project scope refers to the work that needs to be accomplished to deliver a product, service, or result with the specified features and functions.

The second dimension, project product performance, describes the performance of the system actually delivered to the users (Jun et al., 2011, p. 925) and measures the quality of the resulting system. System quality, however, is a multidimensional, and also a multifaceted, concept that potentially changes over the project and product life cycle.

Based on previous studies that have examined software development projects using a vendor perspective (e.g. Jun et al., 2011), measures for assessing the outsourced system quality address whether: (1) the application developed is reliable, (2) the application developed is easy to use, (3) flexibility of the system is good, (4) the system meets the user's intended functional requirements, (5) the users, the project team and top management are satisfied with the system delivered, and (6) the overall quality of the developed application is high. This categorization is aligned with the approach undertaken by Pinto and Prescott (1988) who made a distinction between the implementation process (efficiency) and the perceived 'value' of the project (effectiveness), as well as with recent empirical studies by Zwikael et al. (2014) and Zwikael and Smyrk (2012) who distinguished between project management success (efficiency) and project ownership success (effectiveness). Understanding the range of project success dimensions is important because candidate CSFs may well affect some success measures differently.

However, it remains a challenge on how to determine what degree are staff of an outsourced vendor really qualified to evaluate the product success of something they may have helped to build but are not involved in managing the benefit stream. As noted by Zwikael and Smyrk (2012), a project manager from the vendor organization is a temporary position to which the long-term benefits of the project (product success) in the outsourcing organization may not be easily assessed since in outsourcing projects managers are frequently appointed from outside the funding organization on a short and medium term basis. If the project clients' do not utilize the project output and outcomes to a desired level, the benefits cannot be generated. The product success may take a considerable time to be realized, and this implies that the point of time at which the project is measured is likely to have a significant influence on this variable. 
Table 4 presents a summary of criteria of project success used in previous studies.

Table 4: Summary of the criteria on project success used in previous studies

\begin{tabular}{|c|c|}
\hline Dimension & References \\
\hline Time & $\begin{array}{l}\text { Jun et al. (2011), Lee \& Xia (2010), Misra et al. (2009), Chow \& Cao (2008), Sheffield \& } \\
\text { Lemetayer (2013), Shenhar et al. (2002), Lipovetsky et al. (1997), Zwikael et al. (2014). }\end{array}$ \\
\hline Budget & As above \\
\hline Scope & Chow \& Cao (2008), Zwikael \& Smyrk (2012), Zwikael et al. (2014) \\
\hline Reliability & Jun et al. (2011) Shenhar et al. (2002). \\
\hline Easy to use & Jun et al. (2011), Shenhar et al. (2002), Lipovetsky et al. (1997) \\
\hline Flexibility & Jun et al. (2011), Misra et al. (2009), Shenhar et al. (2002). \\
\hline Functionality & Sheffield \& Lemetayer (2013), Jun et al. (2011), Shenhar et al. (2002), Lipovetsky et al. (1997). \\
\hline User is satisfied & $\begin{array}{l}\text { Sheffield \& Lemetayer (2013), Jun et al. (2011), Misra et al. (2009) Shenhar et al. (2002), } \\
\text { Lipovetsky et al. (1997). }\end{array}$ \\
\hline Team is satisfied & As above \\
\hline Top level management is satisfied & As above \\
\hline Overall quality is good & Sheffield \& Lemetayer (2013), Jun et al. (2011), Chow \& Cao (2008), Lipovetsky et al. (1997) \\
\hline
\end{tabular}

It should be noted that there are other many scholars who have contributed various frameworks for assessing software project success, impact, and benefit measurement and the literature is diverse. For instance, DeLone-McLean's (1992) study proposed many interrelated and interdependent measures of success. Likewise, Gable, Sedera, and Chan (2008) suggested information system success was a multi-dimensional phenomenon based upon individual and organizational perceptions of the impact of system and information quality. The aforementioned scholars go beyond simple interpretations of meeting project specifications and illustrate the difficulty in capturing product success with a single measure.

Zwikael and Smyrk (2012) also proposed a general framework for gauging the project performance of initiatives to enhance organizational value. According to this approach, project performance is judged at three separate levels: project management, project ownership and project investment. These three tests allow distinct judgements to be made about the respective performances of the project manager, the project owner and the investment represented by the original funding decision.

There are also very many important quality and success measurement approaches that cannot be given full coverage in this study. For instance, ISO/IEC 9126 Software engineeringProduct quality which was originally an international standard for the evaluation of software quality but currently has been replaced by ISO/IEC 25010:2011. The fundamental objective of the ISO/IEC 9126 standard is to address some of the well-known human biases that can adversely affect the delivery and perception of a software development project. These biases include changing priorities after the start of a project or not having any clear definitions of "success." By clarifying, then agreeing on the project priorities and subsequently converting 
abstract priorities (compliance) to measurable values (output data can be validated against schema $\mathrm{X}$ with zero intervention), ISO/IEC 9126 tries to develop a common understanding of the project's objectives and goals.

A balanced score card can also be used by providing feedback around both the internal project processes and outcomes in order to continuously improve strategic performance and results. Since there are many different stakeholder groups involved in a typical project, it is understandable that each of these stakeholder groups has different goals and objectives for assessing project outcomes. Current research in this area finds that there is a real lack of agreement on not only what constitutes project success, but on methods for more comprehensive assessment of project outcomes.

\section{Summary on the concept of project success and its implications for this study}

Research has suggested that candidate CSFs impact distinctly different success measures. For example, Zwikael et al. (2014) found that the importance of planning is contingent upon the type of success measures employed, Pinto and Prescott (1990) found that planning factors have stronger impact on 'external' success measures (perceived value of the project and client satisfaction) than on efficiency. For this reason, this thesis analyzes the impact of candidate CSFs on two common success measures separately-process success/ efficiency and product success/ effectiveness (Jun et al., 2011; Wallace et al., 2004). Process success or efficiency measures the extent to which time and cost targets and scope mentioned in the project plan have been met (Zwikael et al. 2014; Jun et al., 2011), whereas product success focuses on the realization of target benefits included in the business case (Zwikael and Smyrk, 2012; Jun et al., 2011).

Table 5 demonstrates the mapping of some previous measures of project success in relation to the current study.

Table 5: Mapping of some previous measures of software development project success 


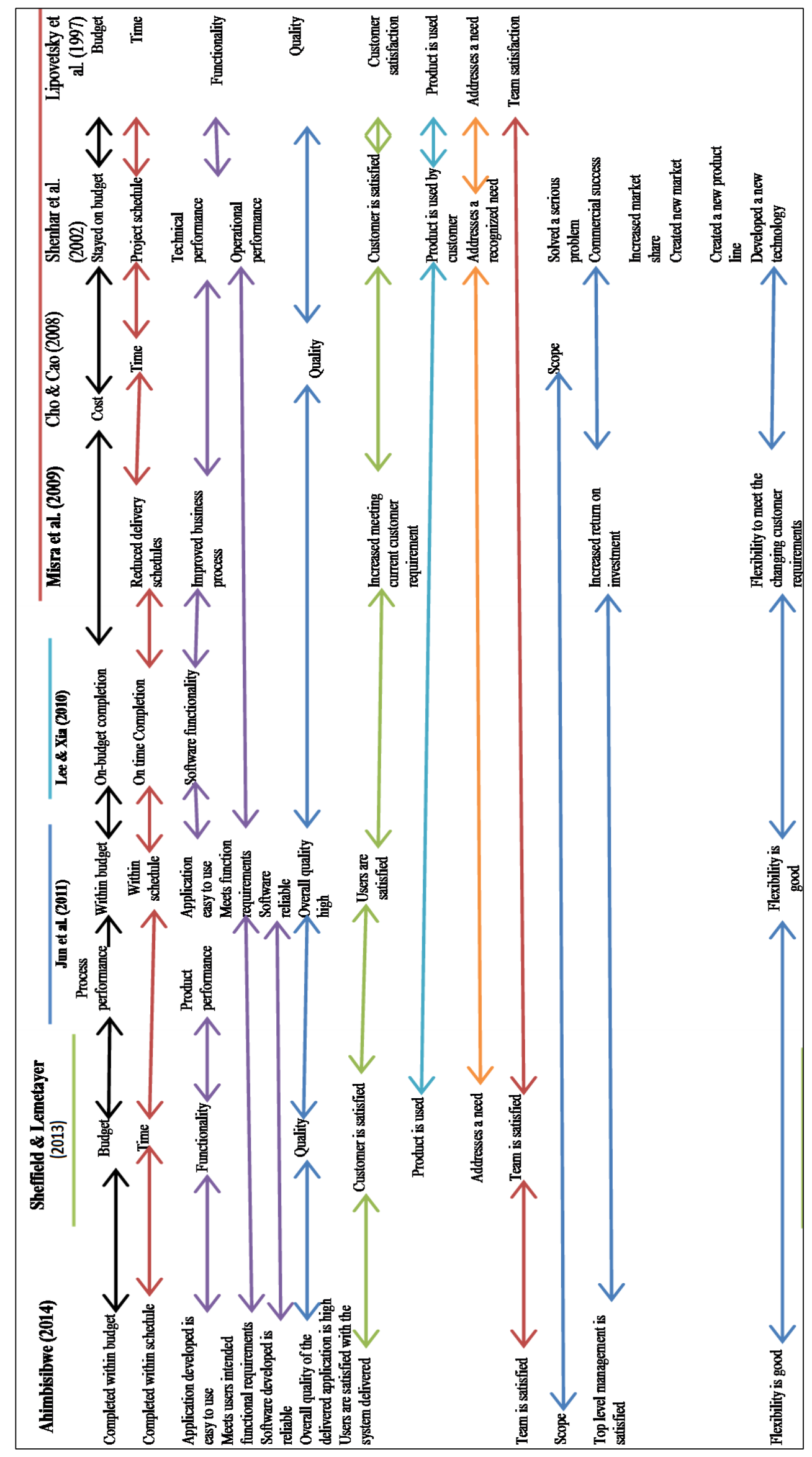




\subsection{CSF research approach in software development projects}

The CSFs research approach for project management seeks to understand why software development projects succeed or fail and how project success might be improved (Yetton et al., 2000). The CSFs research approach can be probably traced to the early 1960s in an effort to distinguish between critical and non-critical information for business decisions with respect to information stored and supplied through Management Information Systems (MIS). This later went on and influenced research in the 1970s whereby the definition of CSFs for information systems design was contingent upon various types of data and often relied upon subjective assessments of top executives.

The seminal work of Slevin and Pinto (1987) was perhaps the first one to extend the CSFs approach into project management. Slevin and Pinto (1987) argued and systematically documented that project managers needed to think in both tactical and strategic terms to achieve successful project management. In this regard, they proposed a list of ten factors that they thought were critical to success of projects and divided those factors into two categories. Strategic factors included planning and goal setting while tactical factors were actions designed to achieve goals. Slevin and Pinto (1987) also proposed that project success was dependent upon an appropriate mix of effort and resources between tactical and strategic categories of success factors. Slevin and Pinto (1987) further suggested that due to different factors associated with strategic and tactical goals, different management strengths might also be more important at different stages of the project, as the project evolves many changes transpire requiring alterations to management approaches.

Pinto and Prescott (1988) built on Slevin and Pinto's (1987) study and reduced the set of candidate CSFs from ten to eight and demonstrated that success factors occurred in different combinations throughout a project's lifecycle. This work began the trend in project management research where measuring project success was in some way related to measuring the presence of success factors. As a result, the examination of attributes associated with successful projects became a major focus of IT project management research over the last 30 years to date.

The project management literature remains unclear about what critical factors work together to make software project successful and moreover, little research has been undertaken on how these candidate factors may interrelate and interact to influence project success. In this vein, Yetton et al. (2000) recommended further studies to investigate the inter-relationships and 
direction of causality between candidate CSFs themselves and project performance. Zwikael and Globerson (2006) examined and recommended some clear procedures of how to move from CSFs to critical success processes. Nonetheless, such studies remain rare. One possible reason for this could be that many candidate critical factors have been proposed. The current candidate CSFs are possibly 'too general and do not contain specific enough know-how to better support project managers' decision-making' (Zwikael \& Globerson, 2006). There is also little evidence indicating whether the practice of IT project management integrates results from studies of candidate CSFs (Sauser et al., 2009).

Moreover, most of the CSFs research effort has focused on the outcomes of software development projects rather than the process of developing software itself. Yet, it is probably efficiency and effectiveness of the whole development process that determines the outcome (Nasir \& Sahibuddin, 2011). Research has often focused on identifying candidate CSFs for project success in various industries like engineering, manufacturing and construction rather than focusing on software development projects, yet managing a software development involves uniqueness due to the complexity, conformity, costs, visibility and softness of the software itself (Nasir \& Sahibuddin, 2011).

Indeed, some scholars seem to agree that there are differences in project management among different project types. For instance, Shenhar et al. (2001) found that candidate CSFs are not universal to all projects and proposed a contingency approach for examining project success. Similarly, a study by Sauser et al. (2009) echoed Shenhar et al.'s (2001) findings as do Howell et al.'s (2010). Hence, candidate CSFs identified in other industries cannot be taken as valid candidate CSFs for software projects (Nasir \& Sahibuddin, 2011), without specific testing.

Another possible reason why CSFs have remained unclear in software development is that there are considerable differences of opinion on what constitutes success for software development and IT projects in general since these projects often involve diverse stakeholders representing many different perspectives.

Most CSF studies have also mixed critical success factors and Key Performance Indicators (KPIs) and yet the two are different. Generally, CSFs are issues that, if addressed appropriately, will substantially increase the likelihood of chances of project success (Nasir \& Sahibuddin, 2011, p.2175). Therefore, CSFs represent those areas that must be given 
special and continual attention to bring about high performance. According to Chow and Cao (2008) CSF's are the few key areas where things must go right for the project to flourish and for the manager's goals to be attained (p.962). They are the limited number of areas in which results, if they are satisfactory, will insure successful competitive performance for the organization and hence, are the few key areas where things must go right for the business to flourish.

\subsubsection{Strength and weaknesses of the CSF approach}

The CSFs approach is vital for establishing the key factors for projects upon which project teams/management can focus their attention and actions to achieve certain common goals by making efforts in ways that the CSFs they have set will be attained. It is highly important that there are some certain points on which project members can base their actions to achieve these goals. Identifying CSFs therefore aids every member on the project to have a common point of reference to know exactly what the most important thing to do is, and this might help them perform their tasks in the right perspective and pull them together into the same overall aims. In general, the CSFs approach helps to identify few factors that provide the critical points which serve as target points for the project to achieve their goals.

Despite the usage of CSFs approach as the common approach in project management research, many researchers have questioned its appropriateness. Firstly, this approach does not address the relationships between these factors and yet interrelationships between candidate CSFs have also been found to be at least as important as the individual factors.

For instance, Yetton et al. (2000) argue that CSFs interact in different ways but CSFs studies have not examined such relationships between candidate CSFs. Also, the CSF approach has been critiqued as an approach for understanding project success, in particular, for not offering a dynamic perspective, and not providing any mechanism for understanding the relationship between factors. Similarly, Fortune and White (2006, p. 54) advanced two main criticisms of the CSF approach. The first is that "the inter-relationships between factors are at least as important as the individual factors but the CSF approach does not provide a mechanism for taking account of these inter-relationships and that "the factor approach tends to view implementation as a static process instead of a dynamic phenomenon...".

Further, the concept of CSFs is difficult to define and can involve vagueness that requires subjective assessments. Third, the usage of the concept of CSFs at both the operational and 
strategic levels has been found to be confusing since workers at lower levels generally have limited capacity of dealing with complexity of strategic issues. From an operational perspective, candidates CSFs usually provide simple assumed unsatisfying accounts that do not accurately represent the complete environment picture.

Sauser et al. (2009) assert that most of the findings coming out of the CSFs approach are not convincing enough to practitioners since there is little evidence to support a positive impact of candidate CSFs on project success. Last but not least, although, the CSFs approach has been dominant in project management research, it is fundamentally constructed on an incorrect hypothesis that projects fail because of similar CSFs.

In conclusion, CSFs research has accumulated mixed findings that are confusing, disconnected and fragmented. For instance, most CSFs studies have not built on previous literature and most especially, most publications by practitioners have only been based on experience with no connection to literature.

\subsubsection{An overview of significant CSFs models in software projects}

In the years since the original works of identifying candidate critical factors for software projects (Slevin \& Pinto 1987; Pinto \& Prescott, 1988; Pinto \& Slevin, 1988), the list of candidate CSFs has expanded significantly in order to allow the inclusion of multiple perspectives of stakeholders' viewpoints and different project types. The most recent studies (e.g., Chow \& Cao, 2008; Misra et al., 2009; Lee \& Xia, 2010; Haijjdiab et al., 2012; Sheffield \& Lemetayer, 2013) empirically tested candidate CSFs of agile software development projects based on previous conceptual theorization as well as anecdotal and practical descriptions. In contrast, other authors such as Mohammad and Al-Shargabi (2011) reviewed literature based on previous research studies (such as Ratbe et al., 2000; Cockburn, 2000; Boehm \& Turner, 2003; Henderson-Sellers \& Serour, 2005) and proposed several frameworks of candidate CSFs also for agile projects. Other frameworks focused on traditional plan-based approaches (e.g., Yetton, 2000; Cooke-Davies, 2002; Baccarini et al., 2004; Humphrey, 2005; Charette, 2005; Standing et al., 2006; Fortune \& White, 2006).

\subsubsection{The search process of identifying candidate CSFs in this study}

Using a vendor perspective (introduced in chapter 1), the method of content analysis (Cavana et al., 2001) was adopted in this study. The identification process of candidate CSFs was based on reading of the titles, abstracts and the entire papers. This is because some of the 
candidate factors described by the authors in the articles were not explicitly clear and required careful reading, understanding and interpretation to produce accurate aggregated findings. The use of this approach is consistent with previous studies such as Fortune and White (2006), Nasir and Sahibuddin (2011) and Sudhakar (2012) who adopted a similar content analysis approach in deriving candidate CSFs for software development project success. While these aforementioned studies also reviewed published research consisting of case studies, surveys and theoretical studies, covering different project sizes in various domains and multiple countries, the major difference is that this current research focussed specifically on contrasting traditional-plan driven and agile approaches to software development projects rather than candidate CSFs across IT projects more broadly. Figure 16 shows a summary of the research process used to search for candidate CSFs.

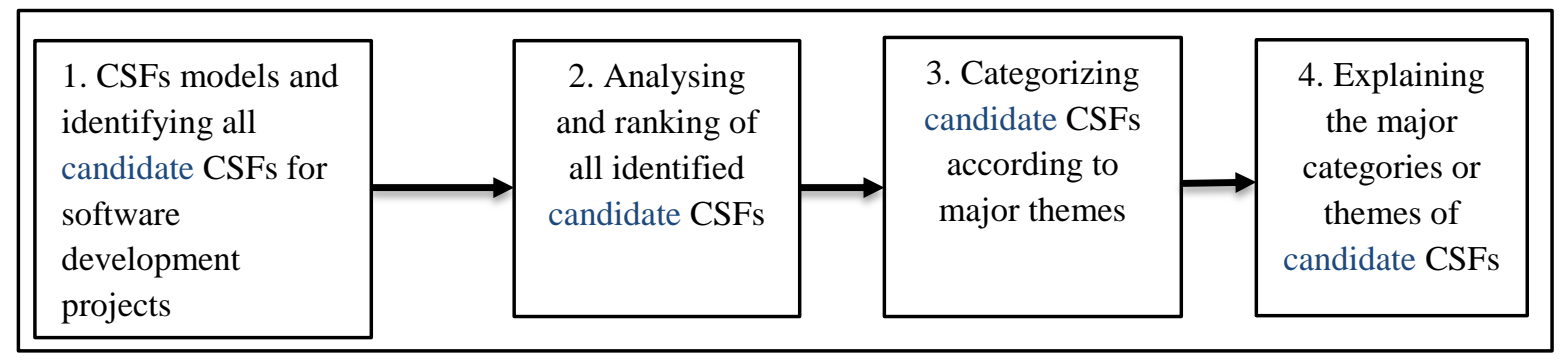

Figure 16: The search process of identifying CSFs

Next, these four stages are explained in detail one by one.

\subsubsection{Step 1-CSFs models and identifying candidate CSFs for software development projects}

One of the earliest published studies on the selection of an appropriate software development methodology was conducted by Burns and Dennis (1985). They compared traditional approaches with iterative prototyping approaches and suggested a contingency approach based on project size and project uncertainty. Burns and Dennis (1985) synthesized literature about prototyping and contrasted prototyping with the more traditional systems life cycle approach to application development. They recognised that some software projects are more suited to one methodology than the other and proposed a contingency approach, based on project size and project uncertainty, to selecting the most appropriate application development methodology for a given project.

In an extension of this research, Ratbe et al. (2000) adopted a contingency approach to examine the fit between project characteristics and application development methodologies. Ratbe et al. (2000) distinguish three contingency variables: project uncertainty, project 
complexity and user's system experience. By focusing on the three major application development methodologies: SDLC, Prototyping at each of its three levels, and End User Development (EUD), they assessed the impact of this contingency approach in terms of user satisfaction and system utilisation. Based on a quantitative analysis of survey responses of 391 (representing a response rate of 28.1\%) members of Association for System Management (ASM), t-tests were conducted. They demonstrated that the level of satisfaction and system utilisation with the application system is higher when there is a match or fit between the methodology utilised and one that has been prescribed for each of the combination of contingency variables. Neither of these studies though included agile methodologies but included the prototyping approach since agile came into existence in 2001. Ratbe et al. (2000) also did not include a full survey of candidate CSFs. Figure 17 displays the research model proposed by Ratbe et al. (2000).

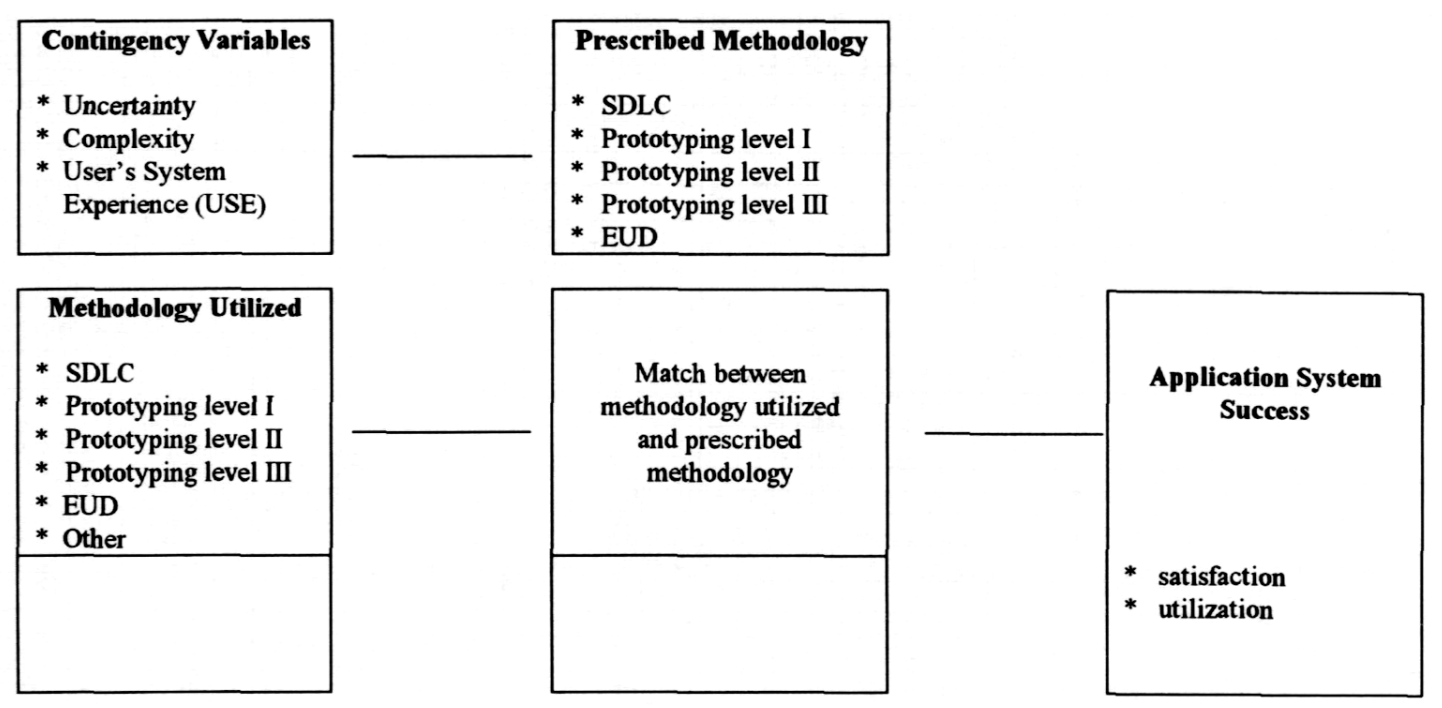

Figure 17: Application system success (Ratbe et al., 2000, Exhibit 2, p.28)

Yetton et al. (2000) extended the CSFs literature by developing a model of success and failure of IS development projects and identifying a robust set of factors and then testing a number of hypotheses concerning their relationship to project performance. The proposed model was based on their view of the relative lack of theory on success and failure at the development stage of IS projects. Yetton et al. (2000) presented hypotheses under four subheadings: project characteristics (size, newness, strategic nature), technical factors (technical risk) and organizational factors (management support, planning, conflict, staff instability, user participation) and their link with project performance (project completion, 
budget variances). Figure 18 illustrates a description of Yetton et al.'s (2000) research model with hypothesized relationships of various CSFs and project performance.

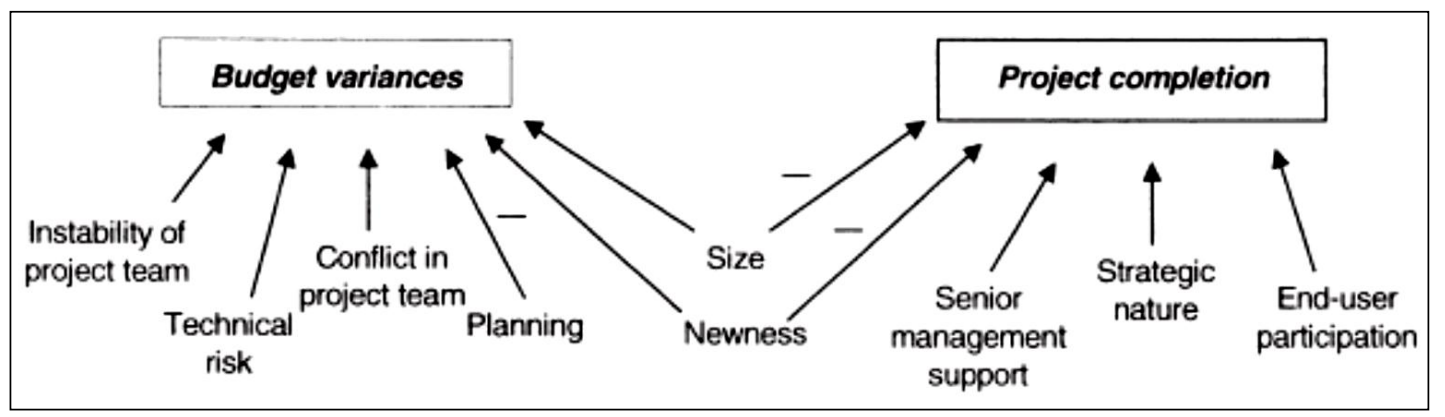

Figure 18: CSFs for project performance (Yetton et al., 2000, Figure 1, p. 269)

A quantitative survey of 28 IS projects in the UK and 72 projects from New Zealand was analysed to test hypotheses concerning performance in terms of both project completion and budget (time-cost) variances (Yetton et al., 2000). Using factor analysis and regression analysis their findings revealed that: the size of the project does not have a direct influence on budget variances. But in comparison with small projects, large project completion is more problematic and, at a minimum, there is a tendency to redefine the project. The greater the newness of the project to the organization, the more likely that completion is problematic and that the project is redefined as the threats and opportunities become apparent. In contrast, the positive relationship between newness and budget variances was not significant. Projects which are strategic to the business are less likely to be redefined than non-strategic projects and also the technical risk of the project did not positively influence budget variances.

Yetton et al.'s (2000) study also found that planning has a strong negative influence on budget variances, i.e. planning reduces budget overruns while budget variances are a positive function of both team conflict and project team instability. User participation increases the likelihood that the project is completed. Strategic value positively influences completion but it does not influence budget variances. Similarly, while planning reduces budget variances it does not increase project completion rates. There was a marginally positive significant relationship between senior management support and project completion implying that higher support from senior management leads to completing the budget on schedule. Table 6 shows a summary of Yetton et al.'s (2000) hypotheses testing findings. 
Table 6: Yetton et al.'s (2000) summary of hypothesis testing results

\begin{tabular}{|l|l|l|}
\hline Independent variables & Budget variances & Project completion \\
\hline Project size & $(+) \mathrm{H} 2 \mathrm{a}$ & $(-) \mathrm{H} 2 \mathrm{~b} \sqrt{ }$ \\
\hline Newness & $(+) \mathrm{H} 3 \mathrm{~b}$ & $(-) \mathrm{H} 3 \mathrm{a} \sqrt{ }$ \\
\hline Strategic nature & No hypothesis stated & $(+) \mathrm{H} 4 \sqrt{ }$ \\
\hline Technical risk & $(+) \mathrm{H} 5$ & No hypothesis stated \\
\hline Senior management support & No hypothesis stated & $(+) \mathrm{H} 6 \sqrt{ }$ \\
\hline Planning & $(-) \mathrm{H} 7 \sqrt{ }$ & No hypothesis stated \\
\hline Conflict project team & $(+) \mathrm{H} 8 \sqrt{ }$ & No hypothesis stated \\
\hline Unstable project team & $(+) \mathrm{H} 9 \sqrt{ }$ & No hypothesis stated \\
\hline User participation & No hypothesis stated & $(+) \mathrm{H} 10 \sqrt{ }$ \\
\hline Note $: \sqrt{ }$ indicates hypotheses that were supported. \\
\hline
\end{tabular}

While Yetton et al.'s (2000) study contributes much to our understanding of managing IS projects, some limitations can be identified in their study. For instance, some relationships were not hypothesized ex ante, and thus, although, the statistical controls might be rigorous, the results might not be as robust as they appear. Although, the authors report a post-hoc power analysis, the overall sample size of 72 is too small and could have resulted in a high Type II error rate. The study did not include a full survey of candidate CSFs, does not specify between traditional plan-based and agile projects, and only examines the direct relationships between candidate CSFs and project success; yet, literature suggests that moderated or mediated paths are more likely.

Another influential work of both traditional and agile methodologies was produced by Boehm and Turner $(2003 ; 2004 ; 2005)$. They built on Cockburn's work (2002) to develop a riskbased approach proposing that methodology selection, engineering and tailoring should be based on an assessment of environmental, agility-oriented and plan-driven risk. They argued that the risk associated with an inappropriate choice of project methodology is reduced by first assessing project factors to ascertain how well the project fits with either the agile or the plan-driven approach. Following their line of thinking, an appropriate project methodology choice can be facilitated by an assessment of five candidate critical factors that include; need for personnel supervision, criticality, project size, culture, and dynamism, which are measured on a scale from pure plan-driven to pure agile.

Figure 19 demonstrates a description of the framework developed by Boehm and Turner (2003) based on key discriminators of agile and plan-driven home grounds. 


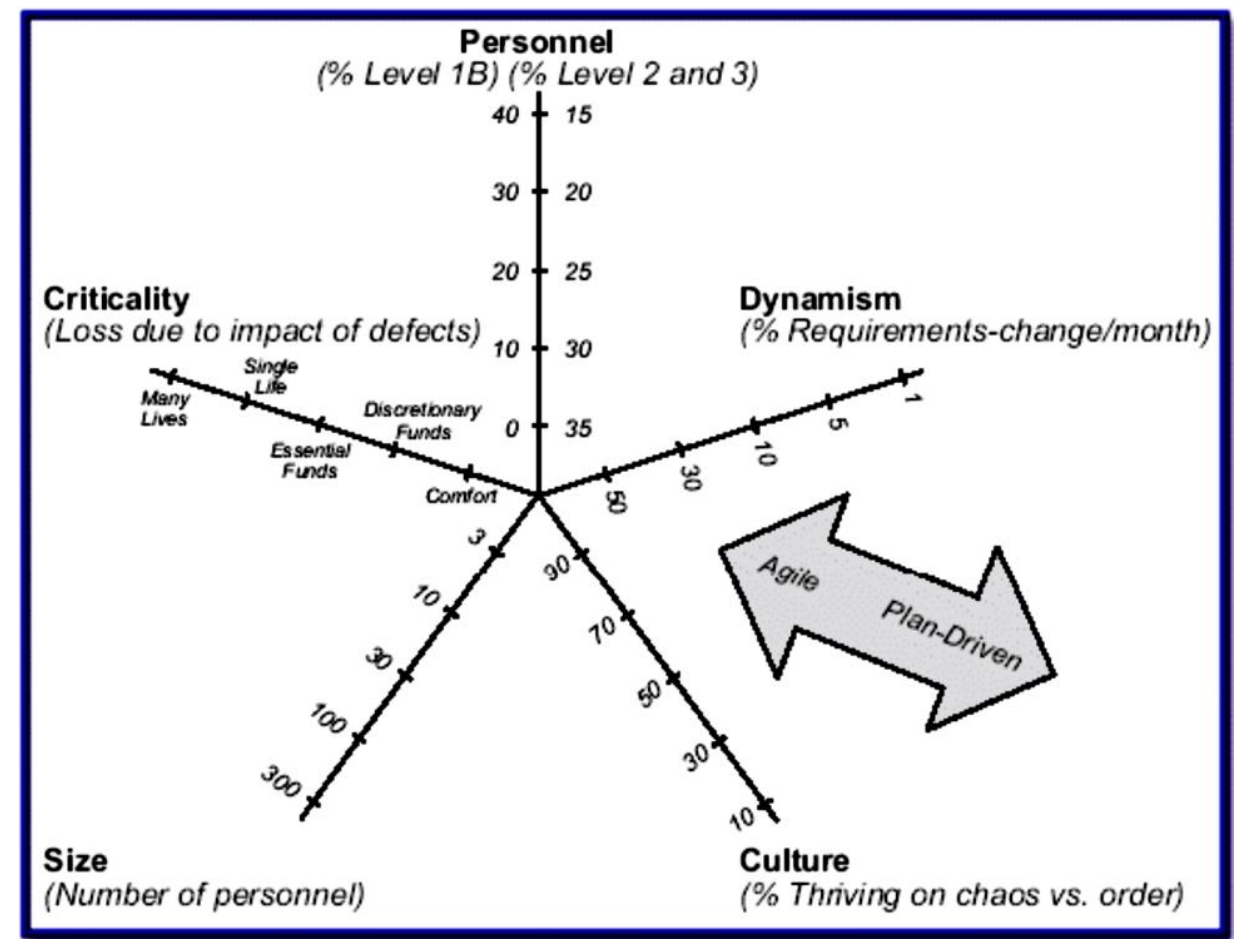

Figure 19: Agile and plan-driven methodologies (Boehm \& Turner, 2003, Figure 2, p.6)

However, Boehm and Turner's (2003) study was conceptual, and the framework does not indicate how candidate CSFs can be linked with project success. Their scales are also not entirely easy to apply in different settings.

Chow and Cao (2008) also proposed a research model for candidate CSFs of agile software projects. In their model, candidate CSFs were categorised as organizational, people, process, technical and project factors. Figure 20 illustrates a description of Chow and Cao's (2008) research model for success factors in agile software projects. 


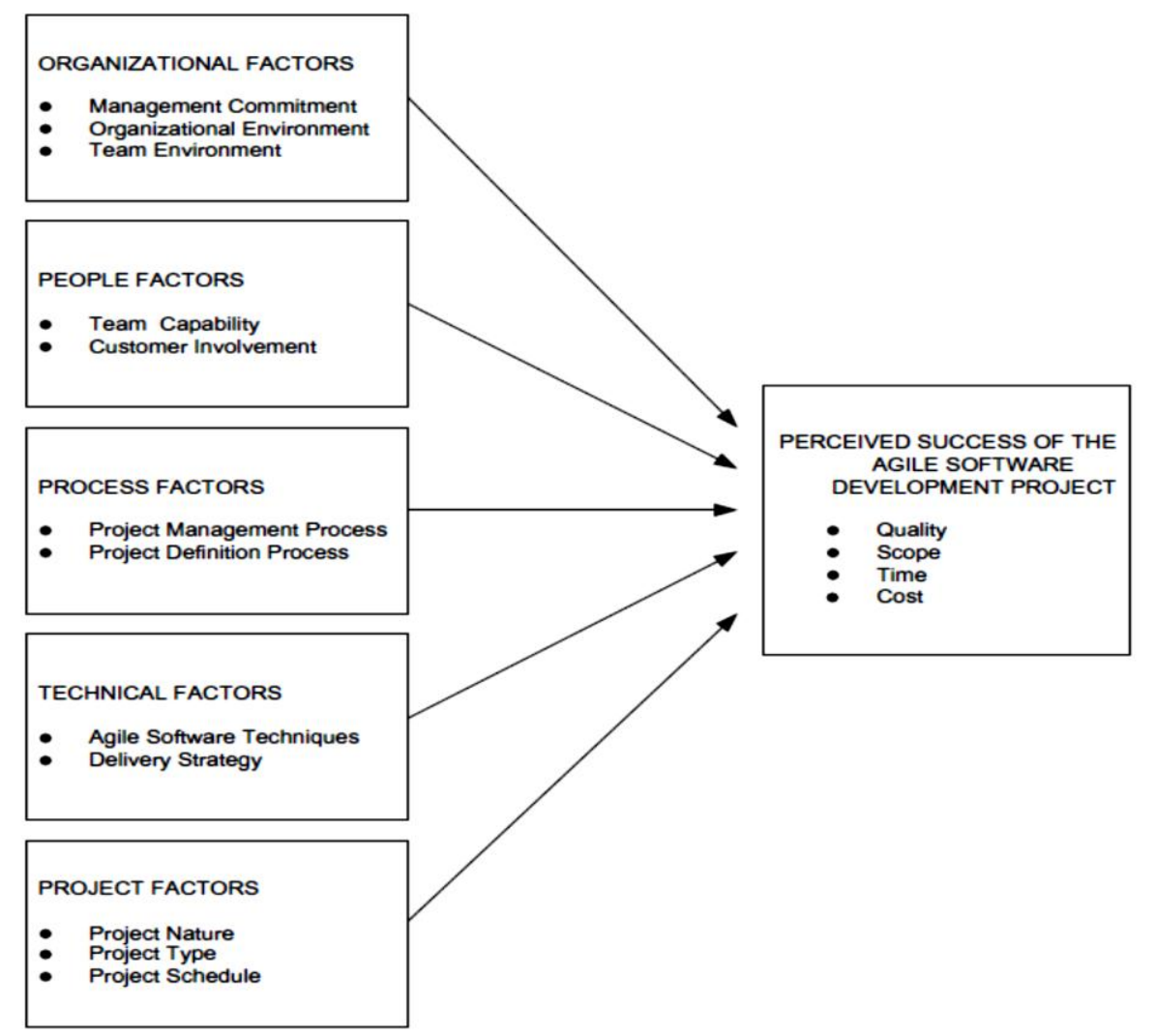

Figure 20: Success factors for agile software projects (Chow \& Cao, 2008, figure 1, p.964)

Chow \& Cao (2008) surveyed 109 agile projects and found that despite the large number of factors suggested by various scholars in literature to be affecting agile software development projects, the actual number of CSFs found to be statistically significant was quite small. This was demonstrated by the fact that out of the 48 hypotheses tested only 10 were supported (Table 7).

Table 7: Chow \& Cao's (2008) summary of hypothesis testing results

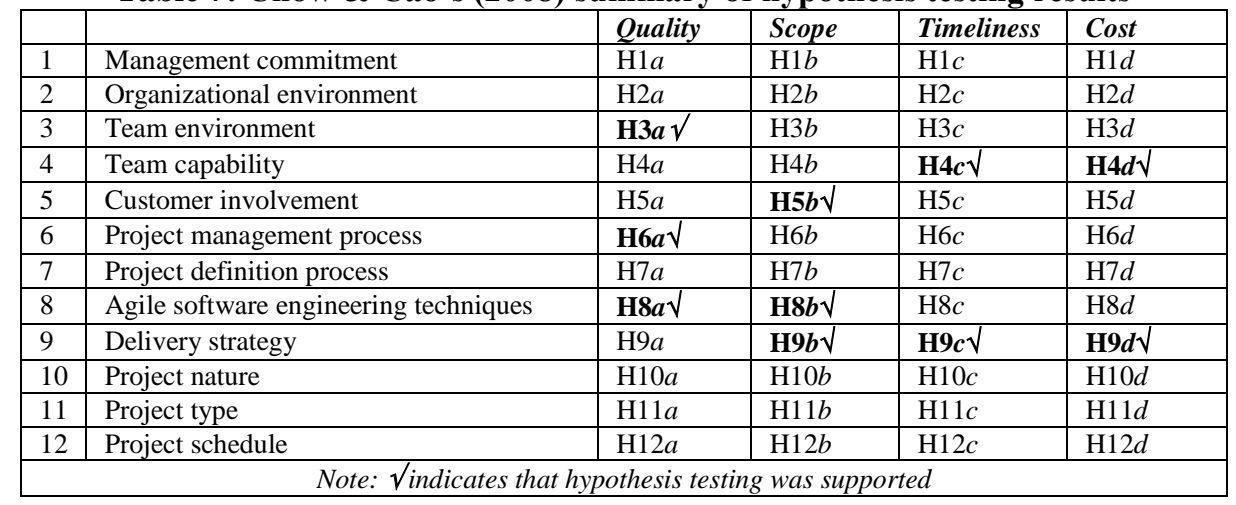

Their regression analysis revealed that the statistically significant CSFs were team environment, customer involvement, project management process, a correct delivery strategy, 
proper practice of agile software engineering techniques and a high calibre team. Surprisingly, their study results did not find evidence for the commonly hypothesised candidate CSFs of agile projects like strong executive support, strong sponsor commitment, availability of physical agile facility or agile appropriate types. However, the study did not include a full survey of candidate CSFs and only examines the direct relationships between candidate CSFs and project success, and is focused on only agile projects. Multiple responses came from similar vendor organisations which limit its generalizability.

Similarly, Misra et al. (2009) reviewed candidate critical factors of adopting agile software development methodologies based on previous anecdotal and practical experience stories. They developed a research framework illustrating the relationships between the agile software development project success and its predictors. In their framework, CSFs were categorised as organisational factors and people factors. Figure 21 illustrates a clear description of the framework developed by Misra et al (2009) for CSFs of adopting agile SDM.

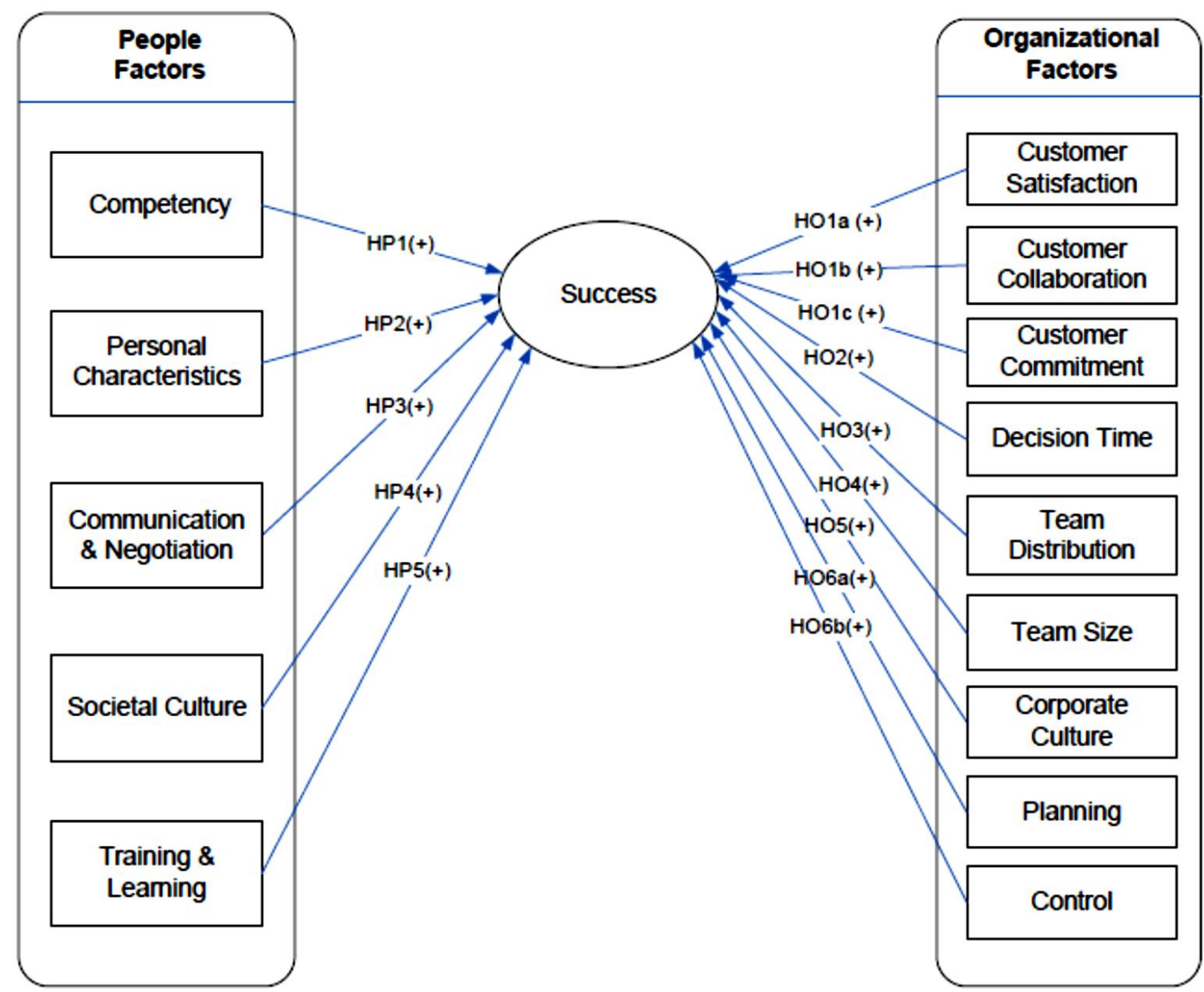

Figure 21: CSFs (Misra et al., 2009, Figure 1, p. 1873) 
Based on a study sample of 174 Agile professionals (vendors) collected by a web-based questionnaire to survey agile software development (ASD) professionals that were widely geographically distributed across continents, Misra et al's (2009) study reported that 9 out of 14 hypotheses were supported. The candidate CSFs that were found to be significant are: customer satisfaction, customer collaboration, customer commitment, decision time, corporate culture, control, personal characteristics, societal culture, and training and learning. However, hypotheses on team distribution (HO3), team size (HO4), informal planning (HO6a), team competence (HPl) and communication and negotiation (HP3) were not supported. This is surprising, because agile methodologies use informal planning and rely on communication in teams for exchanging knowledge. Similarly, based on the current literature one would expect team colocation, team competence and small team size to positively influence ASD projects.

Table 8 summarises hypothesis testing results conducted by Misra et al's (2009).

Table 8: Misra et al's (2009) summary of hypothesis testing results

\begin{tabular}{|l|l|l|}
\hline & \multicolumn{1}{|c|}{ Hypothesis tested } & Findings \\
\hline 1 & HO1a: The greater the satisfaction of the customers in projects, the more likely would be the success of ASD projects. \\
\hline 2 & HO1b: The greater the collaboration of the customers in projects, the more likely would be the success of ASD projects. & Accepted. \\
\hline 3 & HO1c: The greater the commitment of the customers in projects, the more likely would be the success of ASD projects. & Accepted. \\
\hline 4 & HO2: The quicker the decisions are taken in projects, the more likely would be the success of ASD projects. & Accepted. \\
\hline 5 & HO3: The more closely located the project teams are, the more likely would be the success of ASD projects. & Not Accepted. \\
\hline 6 & HO4: The smaller the size of the teams in a project, the more likely would be the success of ASD projects. & Not Accepted. \\
\hline 7 & HO5: The stronger the corporate culture for ASD projects, the more likely would be their success. & Not Accepted. \\
\hline 8 & HO6a: The more informalized the plans are in an agile project, the more likely would be the success of ASD projects. & Accepted. \\
\hline 9 & HO6b: The more qualitative controls the projects have, the more likely would be the success of ASD projects. & Not Accepted. \\
\hline 10 & HP1: The more technically competent the team members are in a project, the more likely would be the success of ASD projects. & Accepted. \\
\hline 11 & HP2: The better the personal characteristics of the team members in a project, the more likely would be the success of AS projects. \\
\hline 12 & HP3: The more the communication and negotiation is between people, the more likely would be the success of ASD projects. & Not Accepted. \\
\hline 13 & HP4: The more favourable the societal culture is for agile projects, the more likely would be the success of ASD projects. & Accepted. \\
\hline 14 & $\begin{array}{l}\text { HP5: The more the environment is for continuously learning, and informal training, the more evident would be the success of ASD } \\
\text { projects. }\end{array}$ & Accepted. \\
\hline
\end{tabular}

Like other studies, Misra et al.'s (2009) exploratory study is not without any limitations. For instance, the list of candidate CSFs in their model remains relatively small compared to candidate CSFs in literature, the study focused on agile projects only, the study does not consider the interrelationships and indirect effects of candidate CSFs on project success.

Lee and Xia (2010) expanded on the work of Chow and Cao (2008) and Misra et al. (2009) and used an integrated research approach that combined both quantitative and qualitative data analyses to empirically examine the relationship between the two dimensions of software development agility (i.e. software team response extensiveness and software team response efficiency). 
Figure 22 illustrates a clear description of Lee and Xia (2010) research model in which software team characteristics were hypothesised to influence software development agility and performance.

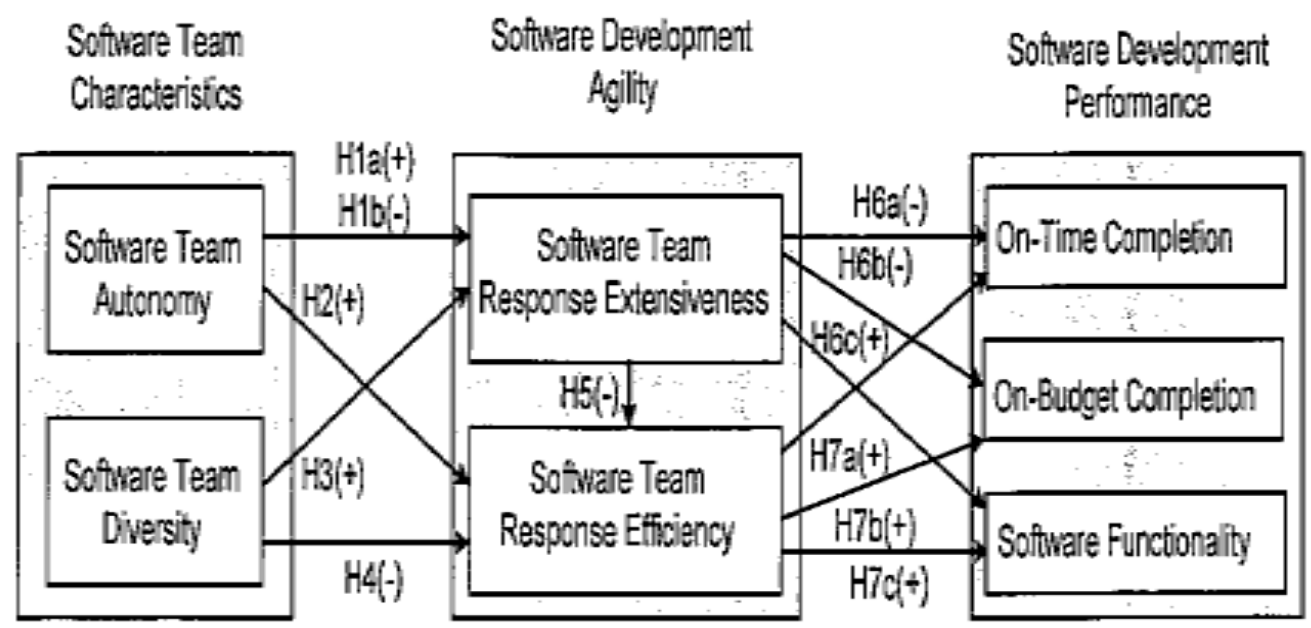

Figure 22: Software development performance (Lee \& Xia, 2010, Figure 1, p. 92)

Using Partial Least Squares (PLS) and a survey of 399 software project managers who were members of the information system specific interest group of the Project Management Institute from North America (vendors), they established statistical evidence for the hypothesised relationships between software team characteristics, software development agility and software development performance. However, hypotheses H4-: Software team diversity negatively affects the efficiency of the team's response to user requirement change, H6a-: Software team response extensive-ness negatively influences on-time completion of software development and $\boldsymbol{H} \boldsymbol{6} \boldsymbol{b}$ - Software team response extensive-ness negatively influences on-budget completion of software development; were not supported in their structural model. Figure 23 presents a summary of research results conducted by Lee and Xia (2010). 


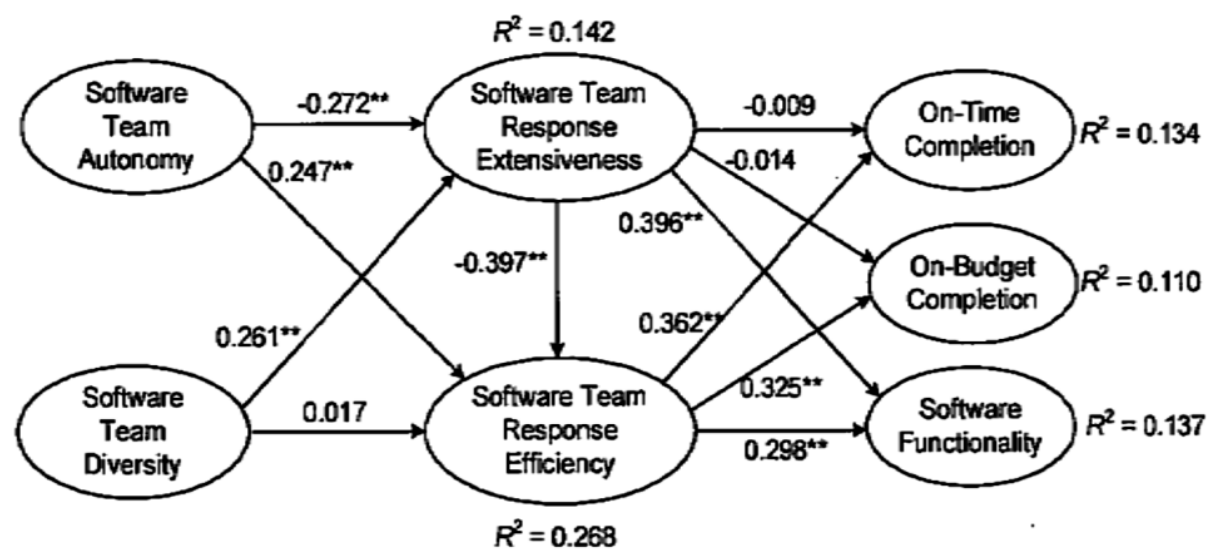

Note: $" p<.05 ; " \mathrm{pt}<.01$

Figure 23: Lee and Xia (2010) PLS results (Figure 2, p.98)

Like previous studies there are some limitations; Lee and Xia (2010) study focuses only on the role of team characteristics in SD performance and hence their model is not specified comprehensively enough to represent most of the candidate CSFs in reality of software development. Second, Lee and Xia (2010) focused their study to only one sample of agile projects.

Using an organisational case based study in China, Wan and Wang (2010) empirically examined the candidate CSFs of agile software development process improvement. In their research model, candidate CSFs were categorised into five categories of: leading, organisation, tools and technology, appropriate import and training and education. Figure 24 presents Wan and Wang (2010) candidate CSF model in agile process improvement.

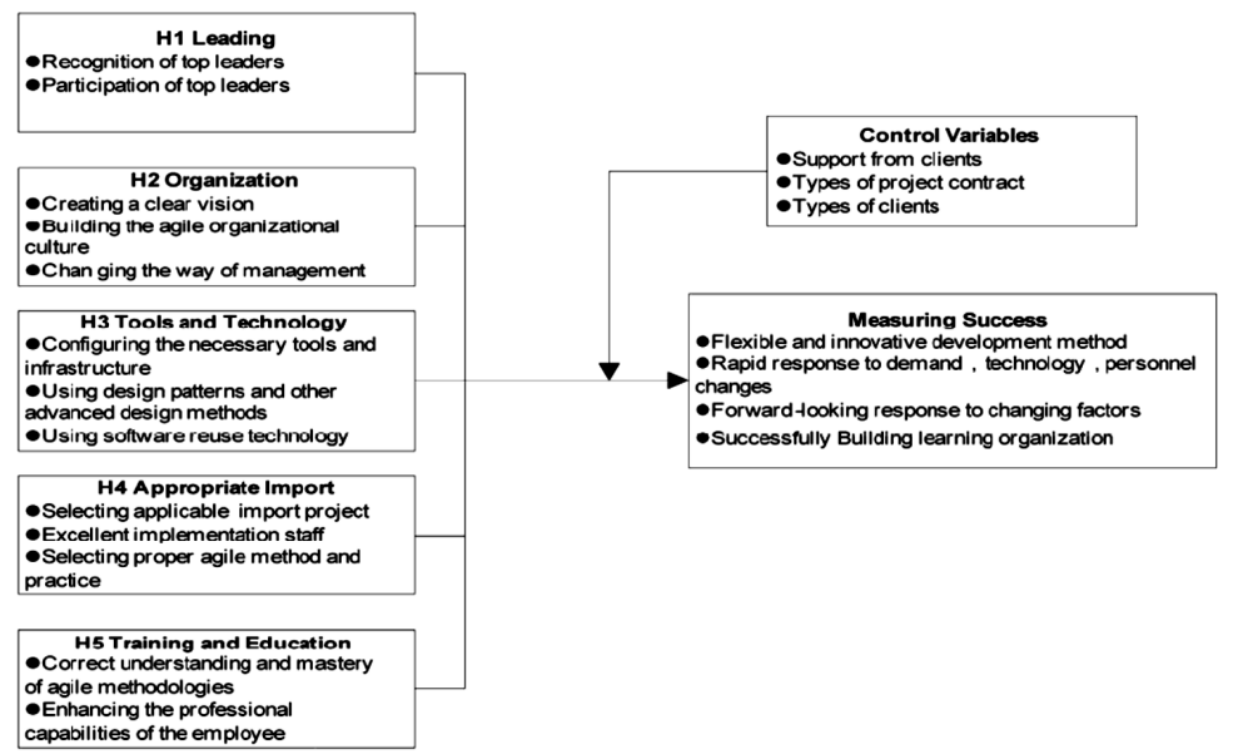

Figure 24: CSFs model for agile process improvement (Wan \& Wang, 2010, Fig 1, p.1134) 
Factor analysis with a principal component analysis was conducted to explore and extract the underlying structure of a set of factors that explained agile software process improvement. Their regression analysis findings suggest that: 1) education and training play a positive role in promoting successful implementation of agile process improvement. 2) Agile methods must be established within an agile culture, which mainly refers to mutual trust and cooperation of the corporate culture. 3) Attention to the design and application of advanced technology do not receive widespread support. All the 5 hypotheses were supported as shown in Table 9.

Table 9: Wan and Wang's (2010) summary of hypothesis testing results

\begin{tabular}{|l|l|}
\hline \multicolumn{1}{|c|}{ Hypothesis tested } & Findings \\
\hline (H1): Support from top leaders plays a positive role in promoting successful implementation of agile process improvement. & Accepted. \\
\hline (H2): Support from organization plays a positive role in promoting successful implementation of agile process improvement. & Accepted. \\
\hline (H3): Use of the tools and technology plays a positive role in promoting successful implementation of agile process improvement. & Accepted. \\
\hline (H4): Suitable import method plays a positive role in promoting successful implementation of agile process improvement. & Accepted. \\
\hline (H5): Education and training play a positive role in promoting successful implementation of agile process improvement. & Accepted. \\
\hline
\end{tabular}

However this study was organisational case based moreover, focusing only on agile projects and hence is difficult to generalise to traditional plan-based projects. It also excludes many candidate CSFs like project uncertainty and complexity which are vital for dealing with the inherent software project risk that influences success of outsourced agile projects. The study only examines agile projects and the overall research sample size also remains significantly small i.e. $n=51$ out of 80 questionnaires that were sent out even though the response rate of 0.6 seems to be relatively high.

Mohammad and Al-Shargabi (2011) studied what they referred to as employee, customer and organisational factors as the candidate CSFs for software development. Consistent with the previous research studies by Henderson-Sellers and Serour (2005), Ratbe et al. (2000) and Cockburn (2000), they proposed a framework for success of software projects that use agile methodology. Their conceptual study concluded that organisational, employee and customer factors were some of the candidate CSFs in agile software development.

Figure 25 depicts Mohammad and Al-Shargabi (2011) framework for candidate CSFs of agile software projects consisting of three main factors categorised as organisational, employee and customer factors. 


\section{Organization Factors}

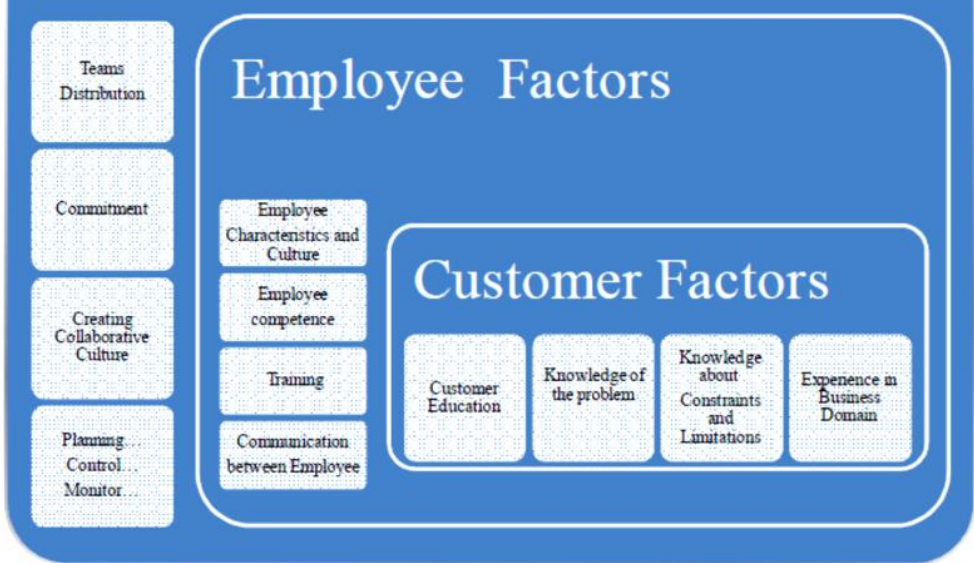

Figure 25: Theoretical Framework for Successful Agile projects (Mohammad \& Al-Shargabi, 2011, Figure 4.1, p.1204)

Although Mohammad and Al-Shargabi (2011) developed a framework for the candidate CSFs of project success, it is only limited to agile projects, conceptual and not empirically validated, and lacks a direct link to project success.

Jun et al. (2011) advanced an integrative model to explore the moderating effects of uncertainty on the relationship between risk management and IS development project performance using a vendor perspective, rather than the client perspective that is mainly employed in the literature. Based on previous research models of Zmud (1980), McFarlan, (1981), Beath (1987), Nidumolu (1995, 1996) Barki et al. (2001), Jiang et al. (2006), Sauer et al. (2007), a quantitative survey based research design was used to collect data from 181 software project managers to test the proposed model.

Figure 26 illustrates Jun et al. (2011) integrative model for moderating effects of uncertainty on the relationship between risk management and IS development project performance using a vendor's perspective. 


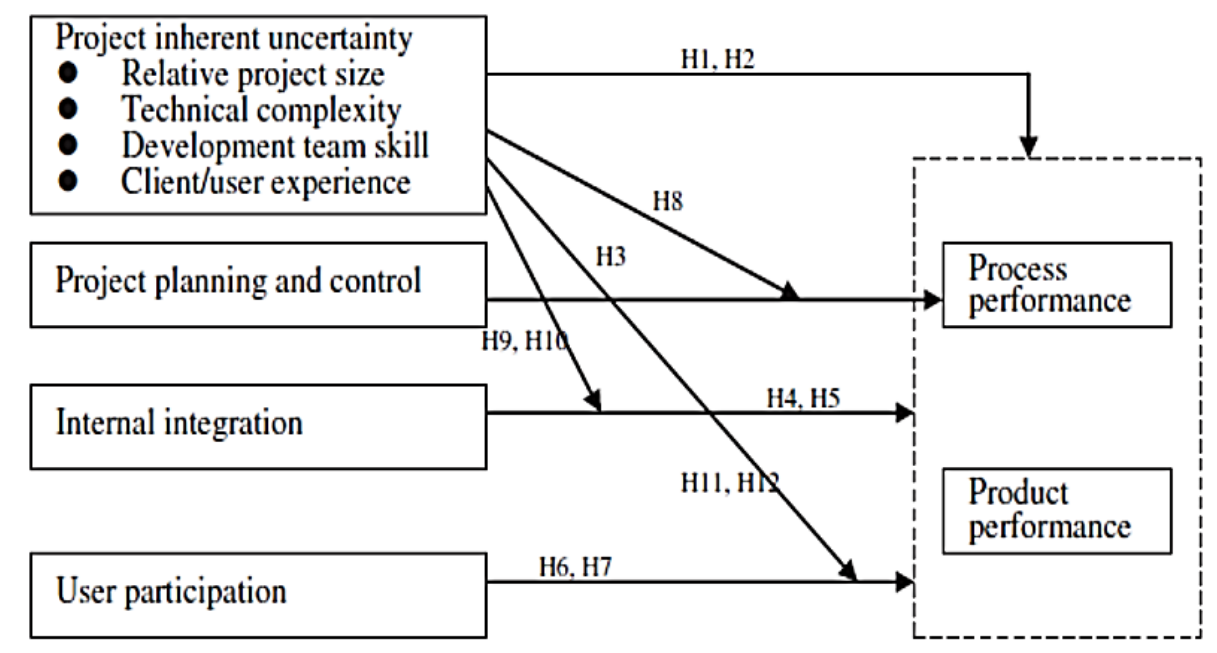

Figure 26: An integrative model for project performance (Jun et al., 2011, Fig. 1, p.926)

Jun et al's (2011) study reported that 9 out of the 12 hypotheses tested were supported. Jun et al (2011) found that project uncertainty, planning and controlling, internal integration and user participation significantly impacted both outsourcing process and product performance. More importantly, the PLS results also reveal that project uncertainty can moderate the effects of project planning and control on process performance and the effects of user participation on product performance.

Their results indicate that project planning and control makes a greater contribution to process performance when there is a low level of inherent uncertainty and that user participation makes a greater contribution to product performance when there is a high level of inherent uncertainty. Table 10 summarises Jun et al. (2011) hypothesis testing results. The strength and sign of standardized path coefficients and t-statistics of all hypothesized paths is also indicated.

Table 10: Jun et al's. (2011) summary of hypothesis testing results

\begin{tabular}{|l|c|c|c|}
\hline \multicolumn{1}{|c|}{ Path } & Path coefficient & $\boldsymbol{t}$-statistics & Supported \\
\hline H1: inherent uncertainty to process performance & -0.324 & $-4.4105 * *$ & Yes \\
\hline H2: inherent uncertainty to product performance & -0.166 & $-2.7179 * *$ & Yes \\
\hline H3: project planning and control process performance & 0.215 & $2.9894 * *$ & Yes \\
\hline H4: internal integration to process performance & 0.242 & $3.6332 * *$ & Yes \\
\hline H5: internal integration to product performance & 0.232 & $3.3797 * *$ & Yes \\
\hline H6: user participation to process performance & 0.011 & 0.2944 & No \\
\hline H7: user participation to product performance & 0.482 & $8.5938^{* *}$ & Yes \\
\hline H8: planning and control-uncertainty to process performance & -0.162 & $-2.2059 *$ & Yes \\
\hline H9: internal integration-uncertainty to process performance & -0.009 & -0.1270 & No \\
\hline H10: internal integration-uncertainty to product performance & 0.041 & 0.8065 & No \\
\hline H12: user participation-uncertainty to product performance & 0.135 & $1.9977 *$ & Yes \\
\hline Note: $* p<0.05 * * p<0.001$ & & & \\
\hline
\end{tabular}

Although this contingency approach (moderation/interaction/fit) of examining outsourced software development project success based on risk is quite robust, its limited by the fact that 
they lumped projects that had used traditional plan-based and agile methodologies together, making it difficult to distinguish between specific candidate CSFs for each methodology; and the study does not typically include a full survey of most candidate CSFs.

In addition to the above-mentioned studies, Sudhakar (2012) conducted a thorough literature review to identify candidate CSFs for software project success. A total of 35 candidate CSFs from seven CSF categories were identified from secondary research of the candidate CSFs for software development projects. The categories that were identified as important as candidate CSFs for software projects were communication, team, technical, organisational and environmental factors.

Figure 27 shows Sudhakar's (2012) proposed research model of candidate CSFs for software project success.

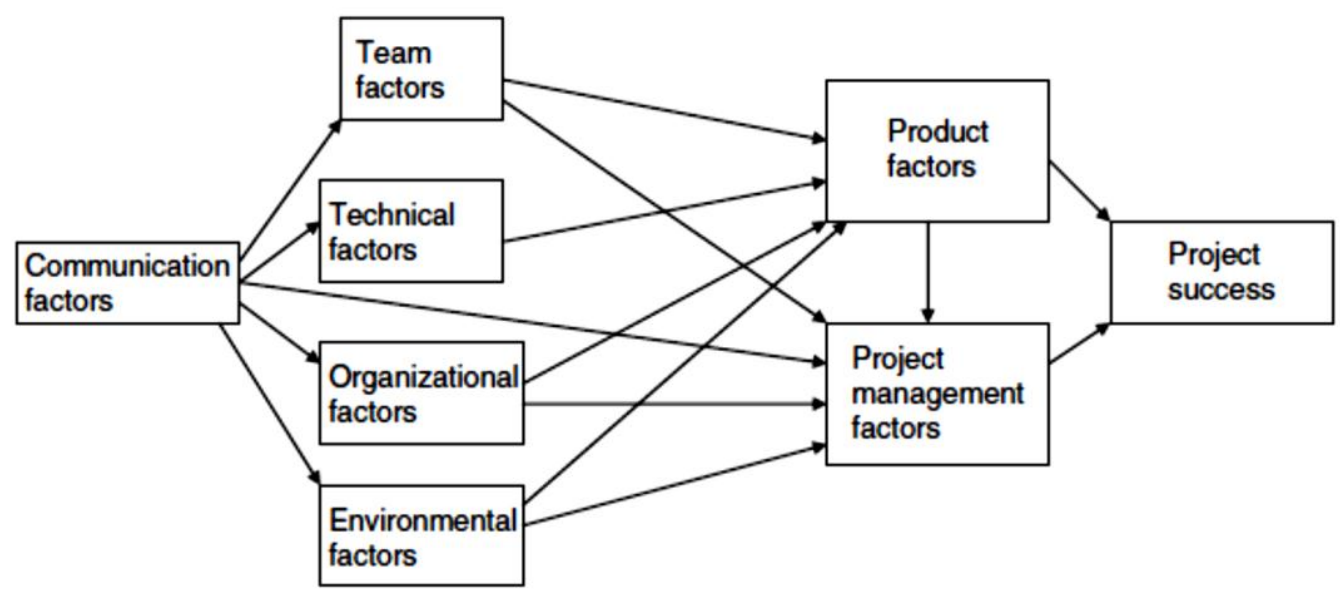

Figure 27: A proposed model of CSFs for software projects (Sudhakar, 2012, Figure 4, p.552)

While this study extensively surveys candidate CSFs for project success, it remains conceptual and the proposed model needs to be empirically tested.

Furthermore, Sheffield and Lemetayer (2013) built on Boehm and Turner, (2003) and some other concepts beyond Boehm and Turner's (2003) study, and explicated what they determined to be the factors associated with software development agility of successful projects. In other words, Sheffield and Lemetayer (2013) study aimed at building a representation of the candidate CSFs in determining software development agility. An online international survey of members of the PRINCE2, PMI and agile (vendor communities of practice) was conducted which yielded 106 mixed responses. 
Their study had initially set two hypotheses for testing that were somewhat broad and not clear which were: HI: Project environment factors influence SD agility and, H2: Project factors influence SD agility. A multiple regression analysis of the survey data found that software development agility was indicated by a project environment factor (organizational culture) and a project factor (empowerment of the project team).

Figure 28 illustrates a clear description of Sheffield and Lemetayer's (2012) work and findings.

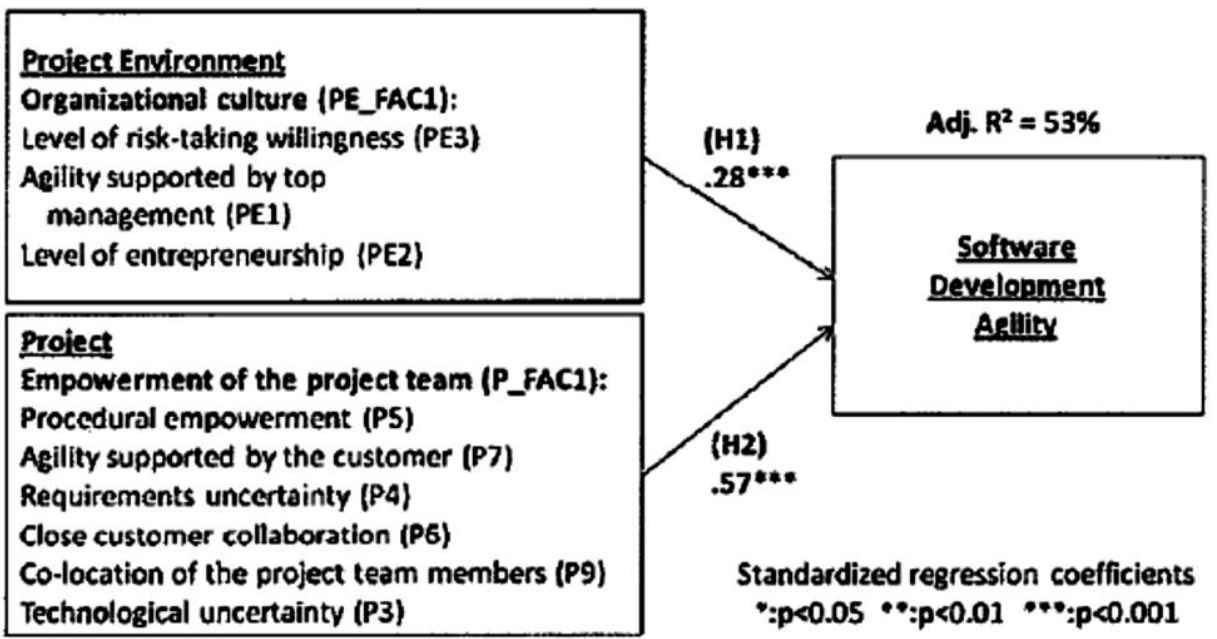

Figure 28: Regression on software development agility (Sheffield \& Lemetayer, 2013, p.468, Fig. 3)

Sheffield and Lemetayer's (2013) research sample size remains relatively small; a larger sample size could have provided more accurate statistical evidence. A large number of constructs were simply measured by a single question in the survey and these findings cannot be easily replicated. The study only focuses on agile projects, and only theorizes direct relationships between candidate CSFs and project success, whereas other literature suggests that moderated or mediated paths may be more likely. The criterion used to select candidate CSFs is also not explained.

Based on the Boehm and Turner (2003) framework for contextual factors of personnel, dynamism, culture, size and criticality, Haijjdiab et al. (2012) extended and examined the adoption of agile methods in a government entity in United Arab Emirates (UAE). Their findings revealed that among the challenges that led to the failure of agile adoption was lack of top level management to fully invest in the project. Their findings also revealed that departure from the plan-driven software development to agile approaches was a significant step that required careful planning and full allocation of resources. Haijjdiab et al. (2012) also 
demonstrated graphically that agile method were more suitable in instances of low criticality, small project size, required few people and change culture.

Figure 29 illustrates a clear description of Haijjdiab et al. (2012) work and findings in relation to Boehm and Turner's (2003) framework.

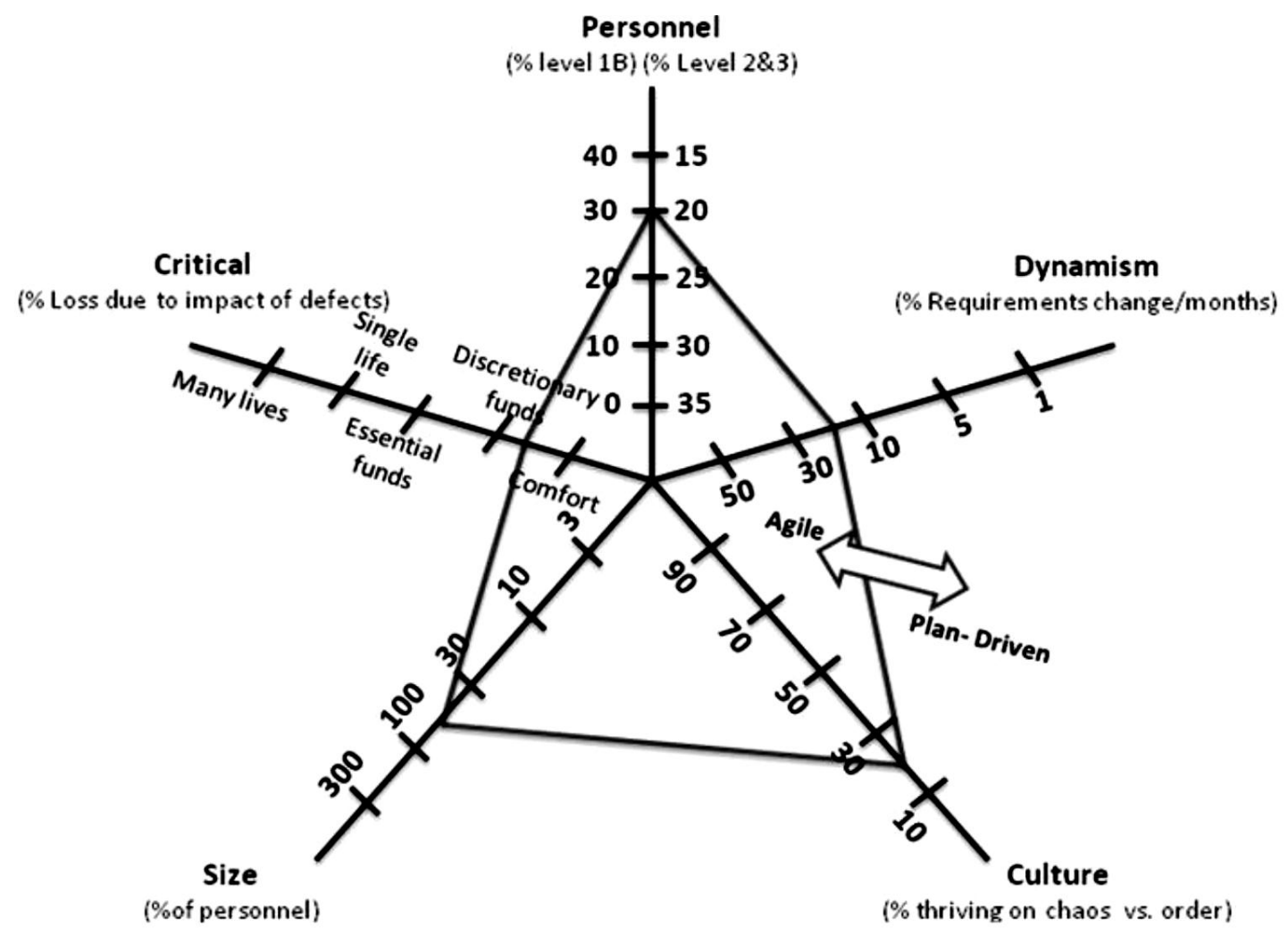

Figure 29: Critical factors (Haijjdiab et al., 2012, Figure 2, p. 238)

However, findings by Haijjdiab et al. (2012) are limited by the fact that they are case specific and cannot be generalised without further validation. Their model also inherited all the weaknesses earlier discussed in Boehm and Turner's (2003) framework.

Table 11 shows a critical analysis of key research models of candidate CSFs that have been discussed using various studies over time since 2000 to date. 
Table 11: Mapping of candidate CSFs from previous models to the current study

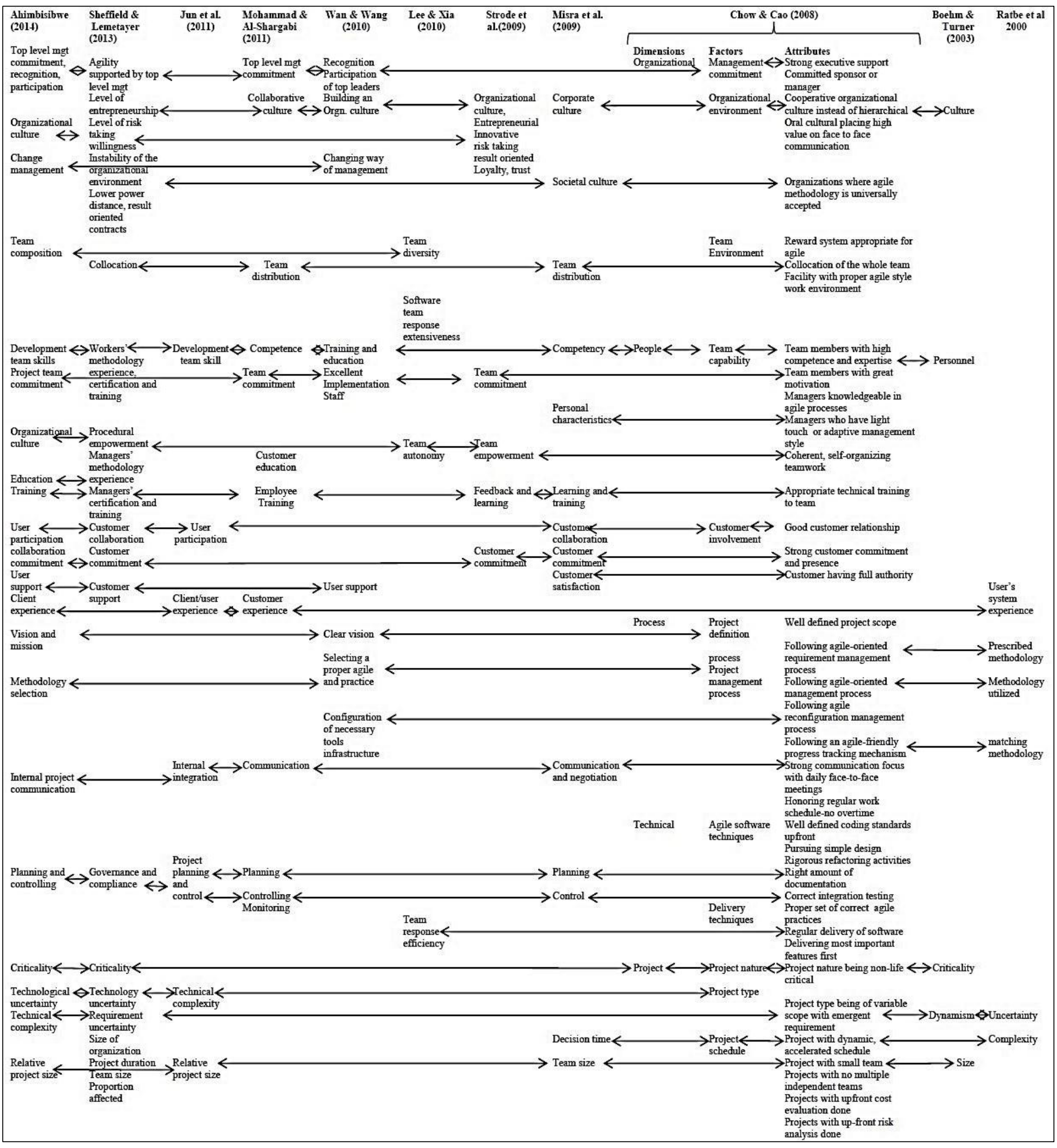

The above table demonstrates the different candidate CSFs for software project success noted across the literature spectrum. However, it appears most scholars have not always built 
on previous studies. In addition the more recent studies are difficult to generalize to traditional plan-driven projects because they focussed primarily on the agile project context. Therefore, it would be valuable to include research on projects that use traditional plan-based methodology to enable a comparative analysis of candidate CSFs for both projects that adopt agile and traditional plan-driven methodologies. This would help guide theorization of contingencies in software development projects across the different methodologies. Second, these studies tended to rely on smaller samples $(n<200$, with validity and reliability tests rarely reported) that are potentially restricted to specific sub-populations (individual countries, successful projects, types of respondents) (Table 12).

Table 12: Empirical studies related to only candidate CSFs for software development methodologies

\begin{tabular}{|c|c|}
\hline Author(s) & Methods \\
\hline Chow \& Cao (2008) & Survey-multiple regression analysis, $n=408$, Agile \\
\hline Cooke-Davies (2002) & Empirical research, survey of 70 organizations in UK -Regression analysis \\
\hline Fortune \& White(2006) & Survey, N=995 PRINCE2 project managers in Israel, used cross tabulation. \\
\hline Hajjidiab et al. (2012) & An industrial case study for scrum adoption in UAE \\
\hline Jun et al (2011) & A quantitative survey, $n=181$ \\
\hline $\begin{array}{l}\text { Karlstrom \& Runeson } \\
\text { (2006) }\end{array}$ & $\begin{array}{l}\text { Case studies on ABB Automation, Ericson Microwave Systems, Vodafone Group global } \\
\text { product development }\end{array}$ \\
\hline Karen et al. (2010) & Survey-ANOVA \& MANOVA, $n=142$ \\
\hline Lee \& Xia (2010) & Multi method-Interviews and survey, factor analysis, $n=399$ project managers, Agile \\
\hline Mills et al (2006) & Case study based on International Market (IM) project, Agile \\
\hline Misra et al (2009) & Survey based, ANOVA, regression, $n=167$, Agile \\
\hline $\begin{array}{l}\text { Mnkandla \& Dwolatzky } \\
\text { (2004) }\end{array}$ & Case study of one software development company in South Africa \\
\hline Mishra \& Mishra (2012) & Case study of supply chain management software project, Agile \\
\hline Oya et al. (2001) & Survey of PMI project managers from USA, $n=117$, Chi-square and T-tests \\
\hline Petit (2012) & Case study based two portfolios in two companies (coded soft \& Fin) \\
\hline Saarinen (1990) & $\begin{array}{l}\text { Multi method-Interviews (number not shown) and survey, } 43 \text { IS projects, ANOVA, Cluster } \\
\text { analysis }\end{array}$ \\
\hline Sauser et al (2008) & Case study on NASA's Mars Climate Orbiter (MCO) \\
\hline $\begin{array}{l}\text { Sheffield \& Lemetayer } \\
\text { (2013) }\end{array}$ & $\begin{array}{l}\text { Multi method using } 9 \text { Interviews and a survey of } n=106 \text { respondents and data analysed using } \\
\text { correlation and regression analysis, Agile }\end{array}$ \\
\hline Shenhar (2001) & $\begin{array}{l}\text { Multi method- } 26 \text { case studies, } 115 \text { interviewed and survey of } 127 \text { projects in Israel, ANOVA, } \\
\text { regression analysis (sample not indicated) }\end{array}$ \\
\hline Shenhar et al. (2002) & $\begin{array}{l}\text { Survey of } 127 \text { projects in Israel based on survey analysed using canonical correlation (sample } \\
\text { size not cited) }\end{array}$ \\
\hline Ratbe et al. (2000) & Survey based and data analysed using t-tests, $n=391$ \\
\hline Tiwana \& Keil (2004) & Survey based $n=720$ MIS directors and data analysed using PLS regression \\
\hline Wan \& Wang (2010) & Survey but case based on Corporation $\mathrm{P}, \mathrm{n}=51$, Factor analysis \\
\hline Yetton et al. (2001) & Survey based on 72 IS project managers from New Zealand and 28 from UK. \\
\hline
\end{tabular}

Most of these studies also do not empirically examine the direct link to project success (see Chow \& Cao, 2008; Misra et al, 2009; Lee \& Xia, 2010 for exceptions). Others studies draw on a risk perspective and as a result may have identified similar candidate CSFs but use different labels. Some constructs used to measure SDM (e.g., in Sheffield \& Lemetayer, 2013) were not based on previous tested instruments- the large number of constructs and the 
need for a brief survey mandated that many constructs be measured by a single question. Their findings cannot be easily replicated. Other researchers can't easily follow every protocol described in their research to be able to arrive on similar results.

The other studies, conducted by Cockburn (2002), Boehm and Turner (2003, 2005), Mohammad and Al-Shargabi (2011) were conceptual and largely anecdotal while others were practitioner based. Yetton et al. (2000) and Jun et al. (2010), meanwhile, proposed models but neither of these studies considers the various development methodologies nor their impact on project success. In short, there has not been any formal large scale study of a comparison of candidate CSFs for both agile and traditional software development methodology projects. Thus, although the research stream seems mature in some sense, it retains a relatively qualitative and descriptive flavour that could limit future measurement and predictive model development.

\subsubsection{Step 2-Analysis and ranking of all identified candidate CSFs from the literature}

Step 1 examined the key candidate CSFs models that have been proposed in recent studies up to 2013. The purpose is to extend and develop a conceptual model based on previous models in software development projects. Other articles that did not have any candidate CSFs models were also reviewed and all candidate CSFs identified were added on the list developed. In the literature search for candidate CSFs, both failure and success factors for software development projects were considered since failures can contribute to the understanding of how to avoid certain serious pitfalls that are critical to the success of a project.

To approach RQ1, a list of CSFs for software development projects was broadly identified from 148 publications. Based on content analysis (Cavana et al., 2001) of this extensive literature search, 37 candidate CSFs were identified that are argued to affect project success. For each candidate CSF identified, the frequency (number of publications) of its occurrence was counted and then expressed as a percentage of the total citation count in the literature survey $(n=148)$.

Next, the identified candidate CSFs were ranked in order of occurrence. The frequencies of candidate CSFs for each software development methodology were also calculated and then plotted (see Figure 30). Studies were coded as relating to agile when this was stated as their focal context $(n=43)$. The remainder $(n=105)$, often those pre-dating 2001, were classified as relating to traditional methodology projects. Following this, broader categories of candidate 
CSFs were developed with their relative rankings of candidate CSFs within agile and traditional studies displayed in Table 13.

Table 13: Candidate CSFs identified across 148 publications

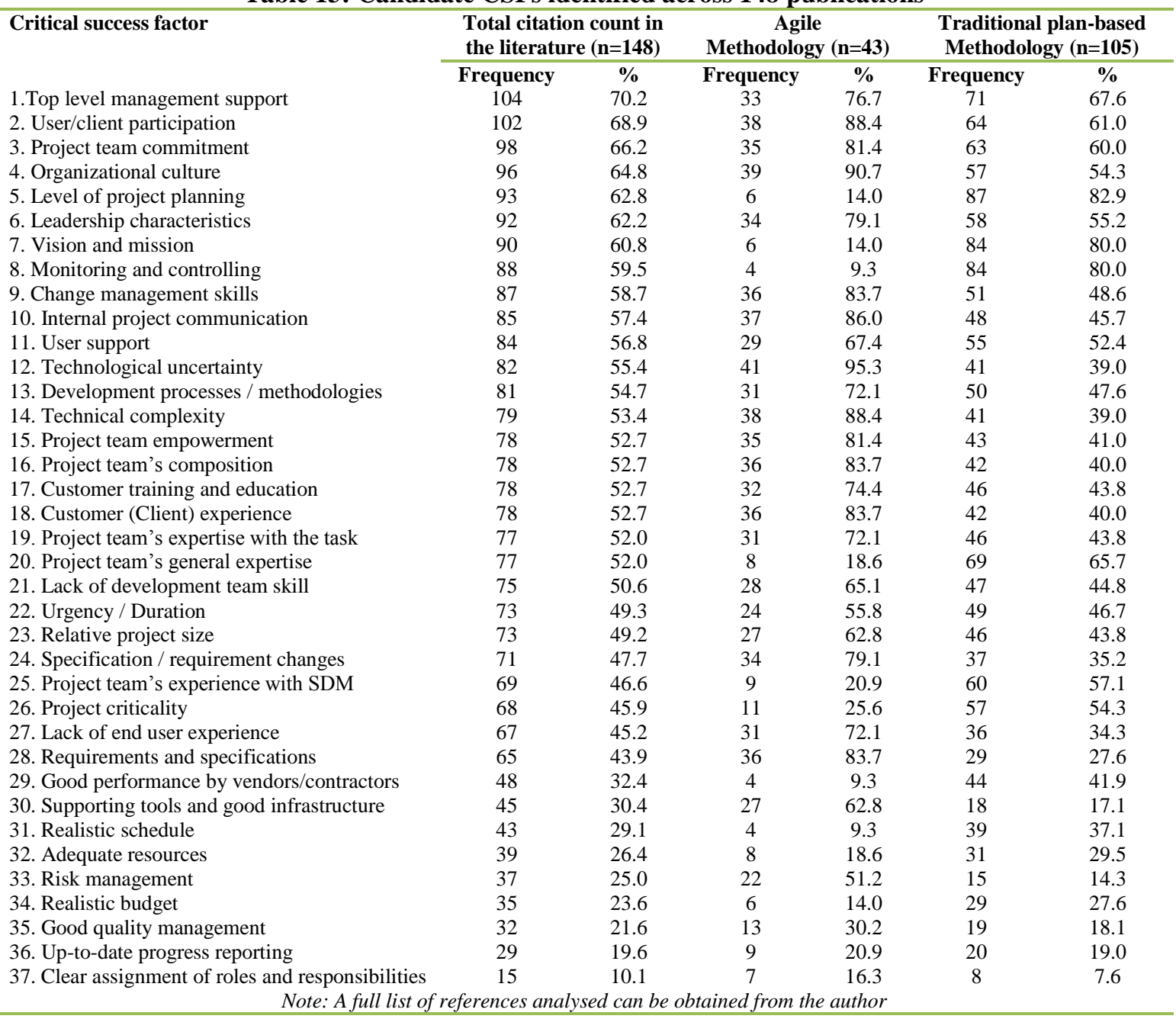

While many studies have been carried out in the last 30 years to establish candidate CSFs for software development projects, there remains only limited agreement on what the candidate CSFs are. Some studies have potentially created confusion by including key performance indicators (success criteria) in their list of candidate CSFs (e.g., Oz \& Sosik, 2000; Schmidt et al., 2001; Sauer \& Cuthbertson, 2003; Baccarini et al., 2004; Charette, 2005; OGC, 2005; Standish Group, 1995; 2001, 2009). As a result, project management literature has not developed a comprehensive theory for assessing candidate CSFs that influence software development project success. 


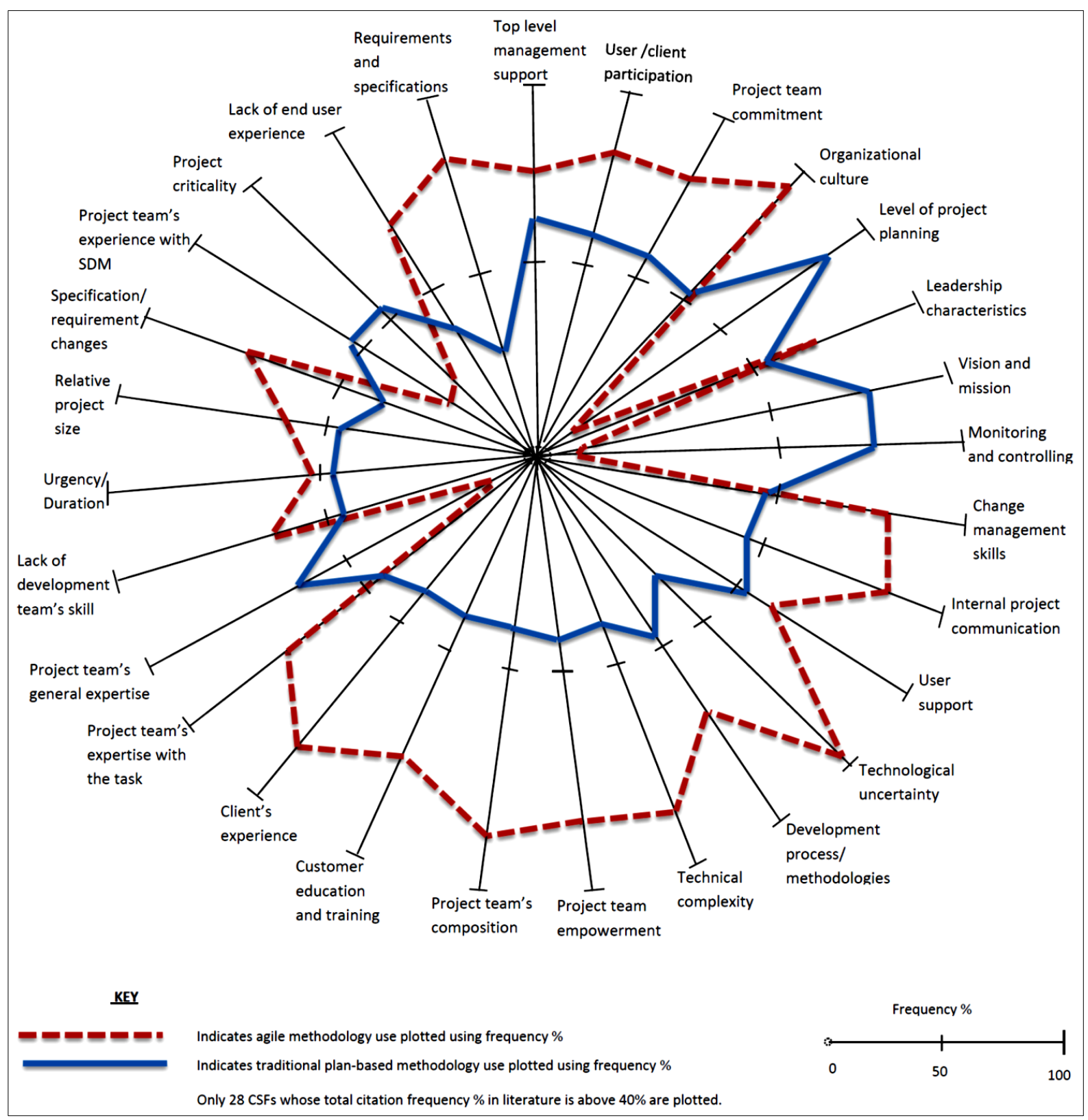

Figure 30: Comparing CSFs frequencies of traditional plan-based and agile methodologies

\subsubsection{Step 3-Categorizing candidate CSFs according to major themes}

Based on the examination, 37 distinct candidate CSFs were identified with the most frequent cited by about $70 \%$ of the publications and 28 candidate CSFs cited in over $40 \%$ of the publications. The more frequently cited candidate CSFs across the 148 publications tend to be factors related to top management, strategic decision making and organizational culture. Characteristics of the project teams (such as commitment, communication, composition and 
empowerment) are also highlighted in over $50 \%$ of the publications, as are some factors associated with the customer/user.

What is also apparent in Table 13 and Figure 30 is that some factors are cited much more frequently in studies relating to an agile context (e.g., technological uncertainty, specification/requirement changes, organizational culture, change management, communication) whereas others appear to be of primary concern for traditional approaches (e.g., project planning, monitoring / controlling, vision and mission, team expertise, project criticality). These data clearly imply that a universal set of candidate CSFs across all software development methodologies is unlikely and that, instead, the importance of candidate CSFs will vary for each methodology. Some candidate CSFs, such as top management support, specification changes may be relatively important for all software development methodologies.

When considering the literature more closely, it was also apparent that many studies have grouped these candidate CSFs within key themes (categories). However, as concluded by Fortune and White (2006), there is again no broad consensus among researchers and practitioners in categorizing candidate CSFs for software projects. This issue remains, with recent scholars (e.g. Chow \& Cao, 2008; Misra et al., 2009; Wan \& Wang, 2010; Mohammad \& Al-Shargabi, 2011; Sudhakar, 2012; Sheffield \& Lemetayer, 2013) suggesting alternative frameworks for categorizing candidate CSFs.

Based on extensive literature review on outsourcing within a software development context from a vendor's perspective, 4 key themes emerged within the candidate CSFs, each with a separate identity from other categories. These are: vendor perceptions of client (VPoC) organizational factors, vendor perceptions of team factors, vendor perceptions of customer factors and vendor perceptions of project factors. This categorization matches and elaborates on that utilized by others. For example, Nasir and Sahibudan's (2011) found that people factors seem to dominate the candidate CSFs. This is not surprising because software projects more rarely fail because of technical reasons, despite the fact that people and process problems may manifest technically. It is also likely the project's technical factors can be improved with proper management of people and processes. People issues may be further elaborated on by considering which 'people' associated with the project are creating the 
'problem' - those in the client organization commissioning the project, those in the development team or the end user or customer.

This categorization also matches Sheffield and Lemetayer's (2013) argument that, in order to achieve project success, the client's top management, the project team and the customer must settle on a software development approach that is aligned with the nature of the project and the environment in which it is embedded (p. 459). In other words, project stakeholders should agree and determine the appropriate software development strategy, i.e. methodology, based on project characteristics and organizational environment (CSFs) to achieve project success.

VPoC organizational factors CSFs, here, include all factors that are affected by top level management, leadership, strategic direction, and client organizational culture. The vendor perception of Team CSFs relate to those employees from the client and vendor who form the development team (communication, commitment, expertise, ...) while, the third category, vendor perception of Customer CSFs, includes factors specific to the customer's (potentially the client organization) use of the software. The fourth and last set of factors covers vendor perception of project situational parameters and changes therein-corresponds to Nasir and Sahibudan's (2011) technical category and Jun et al.'s (2011) project uncertainty factors.

Table 14 shows a list of candidate CSFs for software development projects identified from the literature review with their rankings based on these four major categories.

Note: Candidate CSFs from Table 13 that addressed project success criteria are not ranked here in Table 14. It should also be noted that for parsimony reasons, to avoid redundancy and potential confusion, some candidate CSFs with similar underlying meanings were merged under the most frequently cited factor. The rankings remain based on Table 14 to enable subsequent model building. 
Table 14: Candidate CSF categories and rankings based on methodologies

\begin{tabular}{|c|c|c|c|c|}
\hline \multirow[t]{2}{*}{ Category } & \multirow[t]{2}{*}{ Candidate CSFs } & \multicolumn{3}{|c|}{$\begin{array}{c}\text { Ranking based on number of occurrences in the } \\
\text { considered literature (from Table 15) }\end{array}$} \\
\hline & & Overall & $\begin{array}{c}\text { Agile } \\
\text { methodology }\end{array}$ & $\begin{array}{l}\text { Traditional plan-based } \\
\text { methodology }\end{array}$ \\
\hline \multirow{7}{*}{$\begin{array}{l}P O C \\
\text { organizational } \\
\text { factors }\end{array}$} & Top level management support & 1 & 13 & 4 \\
\hline & Organizational culture & 4 & 2 & 10 \\
\hline & Level of project planning & 5 & 25 & 1 \\
\hline & Leadership & 6 & 12 & 9 \\
\hline & Vision and mission & 7 & 27 & 2 \\
\hline & Monitoring and controlling & 8 & 26 & 3 \\
\hline & Change management skills & 9 & 6 & 13 \\
\hline \multirow{8}{*}{$\begin{array}{l}\text { Vendor } \\
\text { perception of } \\
\text { Team factors }\end{array}$} & Project team commitment & 3 & 10 & 7 \\
\hline & Internal project communication & 10 & 5 & 16 \\
\hline & Project team empowerment & 15 & 11 & 21 \\
\hline & Project team's composition & 16 & 7 & 22 \\
\hline & Project team's expertise with the task & 19 & 16 & 19 \\
\hline & Project team's general expertise & 20 & 24 & 5 \\
\hline & Lack of development team skill & 21 & 19 & 17 \\
\hline & Project team's experience with SDM & 25 & 23 & 8 \\
\hline \multirow{5}{*}{$\begin{array}{l}\text { Vendor } \\
\text { perception of } \\
\text { customer factors }\end{array}$} & User participation & 2 & 3 & 6 \\
\hline & User support & 11 & 18 & 12 \\
\hline & Customer training and education & 17 & 14 & 18 \\
\hline & Customer (Client) experience & 18 & 8 & 23 \\
\hline & Lack of end user experience & 27 & 17 & 27 \\
\hline \multirow{7}{*}{$\begin{array}{l}\text { Vendor } \\
\text { perception of } \\
\text { project factors }\end{array}$} & Technological uncertainty & 12 & 1 & 24 \\
\hline & Development methodologies & 13 & 15 & 14 \\
\hline & Technical complexity & 14 & 4 & 25 \\
\hline & Urgency & 22 & 21 & 15 \\
\hline & Relative project size & 23 & 20 & 20 \\
\hline & Specifications changes & 24 & 9 & 26 \\
\hline & Project criticality & 26 & 22 & 11 \\
\hline
\end{tabular}

The rankings in Table 14 suggest that not only will candidate CSFs vary by methodology applied, but that some candidate CSFs may be closely related (particularly those within categories). For example, the high rankings for vision and mission, project planning, monitoring / controlling and top management support within traditional plan-based approaches is likely to indicate that the combination of these candidate CSFs is essential for project success and that these candidate factors may mutually support each other. In contrast, change management skills and an adaptive organizational culture may be important if the flexible methods of agile approaches are to cope with the technological uncertainty that features more prominently in these projects.

Methodology selection, therefore, should be guided by an assessment of the various CSFs identified and the conditions under which the methods are most likely to succeed. In organizational cultures where top level management support for risk-averse attitudes is high, rigorous planning and controlling will prevail and traditional methodologies are more likely (Sheffield \& Lemetayer, 2013). Where the top level management support is high for flexible 
cultures, changes and adaptations in budgets (costs) and schedules (deadlines) occurring with agile methodologies can be more effectively accommodated.

A key difference between traditional and agile approaches is the way each handles change. The traditional approach attempts to minimize change, while the agile approach embraces it. Thus, as suggested by Vinekar et al. (2006) traditional methodologies should be used when the future can be easily predicted while agile (adaptive and innovative) methods should be adopted under conditions of uncertainty.

Likewise, if a team lacks experience, it is more appropriate to choose a methodology with more structure and more pre-identified processes (i.e. a traditional methodology) to guide the project team members. When both the teams and customers are highly committed, knowledgeable, representative, and empowered, agile methodologies may be suitable and vice versa. With insufficient commitment and limited communication from both the project team and customers, agile projects will suffer and may fail.

The literature also emphasizes that under conditions of high technological uncertainty, relatively large projects and high specification changes, agile methodologies should be used (Charvat, 2003; Boehm \& Turner, 2003). Whereas Jiang and Kline (2000, p.77) argue that complexity requires structure and discipline from traditional methodologies, according to Wysocki (2009, p.312) as technical complexity increases so does the importance of process flexibility and the need to be creative and adaptive.

In summary, the rankings and categorization of candidate CSFs clearly support arguments in the literature for contingent relationships between candidate CSFs with software development methodology choice being a critical factor where 'fit' is essential.

\subsubsection{Step 4-Explaining the major categories or themes of candidate CSFs}

The first theme observed from the literature review is that of VPoC organizational factors. This concept of organisational factors is applied in a broader sense than the normal organizational usage to mean all dimensions that are related to the organizational top level management (leadership) influence, styles and culture. According to Howell et al. (2010), the parent organization is frequently a dominant part of the project's environment, and therefore, it is reasonable to hypothesise both that different projects will face different parent-imposed constraints and that this might yield different optimal project characteristics. 
The second identified theme, vendor perception of team factors, also affects project success. These are candidate CSFs specifically about project teams and are theorized to impact on the success of any software projects. The success or failure of a project greatly depends on the team's communication, team empowerment, expertise and experience, commitment and composition. Following Howell et al.'s (2010) contingency view, the inherent project uncertainty or consequences of unexpected events vary from one project to another and depend heavily on the extent to which they are manageable.

Factors such as authority levels, team size, membership, and geographic distribution are frequently dictated by the parent organisation, and the broader corporate culture is inherited from it. This implies that the team's size, dispersal, and organizational boundaries all affect the team's ability to communicate, and communication, expertise or skill, experience, and authority or empowerment dictate a team's ability to quickly comprehend and respond to the unexpected, improving the chances that an unexpected event will be dealt with without having a major impact on the project.

Team expertise (general or task) is composed of the ability to work with uncertain objectives, ability to work with top management, ability to work effectively as a team, ability to understand human implications of a new system, and ability to carry out tasks effectively. These are interpersonal, team and technical skills that can be considered during the formation of the project team. If insufficient skills are available in the pool of developers, then it may be worthwhile to perform some training in these areas before the commencement of the project.

The third theme, vendor perception of customer factors, includes all the variables that are related to project customers' behaviours and activities of the user in relation to involvement in product development such as customer participation and support, education and training, and experience. It is evident in the IT literature that user participation and support can improve the chances of successful system implementation. According to Jun et al. (2011), customer participation and support in project management can be traced to the theory of "Participative Decision Making (PDM)" and "Planned Organizational Change (POC)" (p.926).

User participation and support are likely to enhance project success. User experience, education and training can also affect project success. Mohammad and Alshargabi (2011) contend that if customer or group of people who are chosen to work with the project team 
have an acceptable level of education or training, they easily explain their requirements and needs in a clear form. It's therefore, highly recommended that those people should have a background or the necessary basic information about Information Technology (IT).

Finally, the literature on software project management identified the vendor perception of project factors or uncertainty, as a key factor influencing project success. In these studies, risk/uncertainty management is not considered to be a separate management process. Instead, it is seen as embedded in the various processes and procedures of the project. A software project's overall level of risk or uncertainty can be obtained by assessing specific risk factors (Boehm \& Turner, 2003; Barki et al., 2001: Howell et al., 2010). Therefore, researchers have identified various risk or uncertainty factors that can threaten the successful completion of a software development project (Jun et al. 2011; Charette, 2005; Baccarini et al., 2004; Sauer \& Cuthbertson, 2003; Schmidt et al., 2001; Jiang \& Klein, 2000). The key management role is to improve the development process by identifying sources of these risk factors and controlling them to produce quality software.

According to Jiang and Klein (2000), controlling can be accomplished by using methods, tools and procedures that bring more structure and formalism to complexity and risks of software development. Taking into consideration project type and the research perspective of this study, technological uncertainty, relative project size, technical complexity, client/user experience, criticality and specification changes are identified as risk/project factors. According to Howell et al. (2010), these project-specific characteristics initially exist in a project rather than emerge during the course of its implementation and can significantly impact the outcome of the IT development effort.

Furthermore, Howell et al. (2010) contend that there is little change in the perceived nature of these characteristics as the project is being completed. For example, they argue that the project doesn't become more or less complex over time, nor does it become smaller or larger in size (p.925). Together, these factors constitute the construct of project factors. Although the concept of software development risks has been examined, it still lacks a general accepted means of assessment.

Literature search on candidate CSFs and the implications for this study

The 4 major categories of CSFs will be used as the basis for further research to develop the conceptual model. However, it should be noted that the distinction made between these 
dimensions is for analytical purposes. Many of these factors are interrelated and often project outcomes or success involves multiple factors that interact in complex ways. Indeed, the theoretical concepts of the VPoC organizational factors, vendor perception of team factors, vendor perception of customer factors and vendor perception of project factors are typically interlinked and in a continuous interplay.

The purpose of conceptual research model is to indicate the theorised relationships between individual candidate CSFs and perceived project success and also demonstrate how these candidate CSFs inter relate or interact amongst themselves to influence project success for various project methodology types. This is further discussed in detail in the next chapter on conceptual model and hypotheses development to further examine the hypothesised relationships between all these individual candidate CSFs and project success for different project methodologies, as well as investigating the interactions or combined factors that influence project success which are situated within the broader context in which software systems development occurs. In practice, all candidate CSFs are interrelated and are mutually interactive. The next section discusses contingency theory, its strength and limitations in software development projects research. The concept of 'fit' and 'fit theory' is also examined.

\subsection{Contingency theory in software development}

Contingency theory generally presents a body of work which argues that not all organisations are the same, and therefore they should be structured and managed differently (Fielder, 1964). Equally, contingency approach, as a concept in management suggests that there is no one universally applicable set of management styles to manage organizations. This is based on the fact that organizations are individually different, face different situations, and require different ways of managing. In project management, contingency theory presents a body of literature that argues that not all projects are the same, and therefore they should not all be structured and managed the same way (Howell et al., p.256).

Contingency theory is used in the current study to relate development methodologies and candidate CSFs; and the "contingency" in this case of research is to do with the "fit" between the candidate CSFs and the method. According to Sauser et al. (2009, p. 666), a contingency approach to project management necessarily investigates the extent of fit or misfit between project characteristics and project management approach. Wysocki (2009) explains that the best development methodology is based on both the project characteristics and the business 
and organizational environment in which the project is conducted. Potentially, in analysing empirical data, the detection of fit/misfit may help better explain project success/failure. More important, understanding the elements of such fit/misfit may provide recommendation for a preferred managerial approach before a project is launched, or for bringing a troubled project back on track.

The study of contingency theory in project management has gradually emerged during the last two decades with specific frameworks for project management that have been influenced by research from disciplines and fields of study like innovation, organisational theory, management, computer science, product management and engineering (Sauser, Reilly \& Shenhar, 2009, p.667). Specifically, Howell et al. (2010, p.256) eloquently discuss how Project Contingency Theory (PCT) has developed from classical organisational contingency theory building upon research from innovation (Shenhar \& Dvir, 2007) and organisational (Van Donk \& Molloy, 2008) perspectives of the project. Classical organisational contingency theory proposes that the effectiveness of an organisation is related to its 'fit' to its environment (Burns \& Stalker, 1961; Lawrence \& Lorsch, 1967). PCT similarly argues that the best approach to managing a project depends on context; that different conditions require different project organisational characteristics and that the effectiveness of the project is related to how well organisation and conditions fit each other (Howell et al., 2010).

This is consistent with the research examining enduring organisations and contingency theory that suggests that organisational effectiveness is dependent upon the organisation's ability to adapt to the environment, and that there is a need for congruence between the environment and structure (Miles \& Snow, 1978; Morgan, 1986). In a similar vein, it has often been suggested that more turbulent environments should be addressed by organic structures because coping with uncertainty is a core problem for complex organisations (Thompson, 1967).

Although there are various forms of contingency theory, most scholars agree and suggest that contingency theories are a class of behavioural theory that contend that there is no one best way of organizing or leading and that an organizational or leadership style that is effective in some situations may not be successful in others. This is consistent with Fielder's (1964) original view of contingency theory. Thus, the optimal course of action is contingent (dependent) upon the internal and external constraints and there is no universal or one best 
way to manage. The philosophical roots of contingency theory are based on four important assumptions which are: (1) there is no one best way of organizing; (2) the design of an organization and its subsystems must 'fit' with the environment; (3) effective organizations not only have a proper 'fit' with the environment but also between its subsystems; and (4) the needs of an organization are better satisfied when it is properly designed and the management style is appropriate both to the tasks undertaken and the nature of the work group. There are also contingency theories that relate to decision making (Vroom \& Yetton, 1973) and these theories suggest that the effectiveness of a decision procedure depends upon a number of aspects of the situation such as the importance and acceptance of the decision. In summary, contingency theory suggests that there is no optimal strategy for all organizations and posits that the most desirable choice of strategy variables varies according to contextual factors, sometimes termed as contingency factors.

Although, contingency theory was not a major focus in the past, it is more relevant today for two reasons. A growing diversity of projects is now reflected in a growing diversity of ways to manage them. Thus, contingency theory seems to be more appropriate for studying project management success due to the temporary nature of IT projects and the existence of a large number of success factors that may provide an optimal performance. However, contingency relationships are often been complex to investigate and demonstrate.

Furthermore, contingency theory with respect to systems rests upon the principle of equifinality (Tossi \& Slocum, 1984, p.15). The concept of equifinality holds that the same outcomes may be achieved even when a system begins from different conditions or situations (Koskinen, 2011, p.59). This also implies that same outcomes may be achieved through the use of different processes. This view also suggests that the project-based company can accomplish its objectives, such as project delivery, with varying inputs and with varying internal activities if the social system is not restrained by the simple cause-and-effect relationships of closed systems (Koskinen, 2011).

\subsubsection{Empirical studies of contingency theory in software development}

In the context of project management, since projects involve many dependent and contingent activities, contingency theory has been adopted as a framework for examining project success. For instance, based on the argument that one size does not fit all projects, Shenhar 
(2001) examined how different types of projects are managed in different ways, and explored the domain of traditional contingency theory in the more modern world of projects. Along similar lines, Sharma and Yetton (2003) also used a meta-analysis to investigate the hypothesized contingency relationship between the level of management support and the degree of interdependency for information system projects. Their findings revealed that management support has a small effect on implementation success when the task interdependency is low and a medium to large effect when interdependency is high. Likewise, by adopting contingency theory, Andres and Zamud (2002) established that coordination strategies for software projects were contingent upon the degree of task interdependency.

Additionally, Mathiassen et al. (2007) advanced an integrative contingency model for software development projects and revealed that the most effective methods for prompting system requirements were contingent upon specific characteristics of both the project and organization. Consistently, based on Minzberg's (1979) five types of organizational structures; (the entrepreneurial organization, the machine organization (bureaucracy), the professional organization, the divisional (diversified) organization and the innovative organization (adhocracy), Van Donk and Molloy (2008) found that each type of a project uses a different organizational structure contingent upon a combination of Minzberg's (1979) organizational design parameters. In their thesis they also argue that projects fall under the category of 'the innovative organization (adhocracy), because companies need to innovate and function on an ad hoc basis to survive in the market place. However, Von Don and Molloy (2008) study relied on only one text of one author i.e. Minzberg (1979), for their major part of analysis and arguments which limits their findings.

Vinekar et al. (2006) used a contingency approach and found that the adoption of agile or traditional approach is contingent upon the product or organizational factors like size, criticality, dynamism, staff competence and culture. Likewise, Sauser et al. (2009) adopted the contingency approach to investigate the extent of fit (success) or misfit (failure) between project characteristics and project management approach. By conducting a comparative analysis of NASA's Mars Climate Orbiter (project) loss, their findings revealed that contingency theory provided the new insights as to why one of the biggest American NASA's projects had failed. Howell et al. (2010) also adopted a contingency approach and extensively reviewed literature on the alternative project management approaches in the context of 
project contingency theory and developed a project contingency framework based on uncertainty and its consequences (Uncertainty-Consequence Framework). However, their orthogonal placement of factors, and suggested use of Cartesian coordinates to compute for the Euclidean distance (space) in their two dimensional framework of uncertainty and its consequences makes it difficult to replicate in different settings.

Thus, the project management literature reveals that typically, most theorists of contingency theory have focused on project/organizational structure as the characteristic under consideration and technological change as the contingency factor (Howell et al., 2010). Similarly, most scholars (e.g. Lawrence \& Lorsch, 1967; Morgan, 1986; Burgeron et al., 2001; Shenhar, 2001; Sauser et al., 2009) have focused on the study of contingency models that share the underlying premise that context and structure must fairly fit together if the organization is to perform well. It has also often been hypothesized that organic organizations cope better with rapid change than mechanistic ones however the mechanistic organizations also outperform in stable environments (Burns \& Stalker, 1961). The mechanistic structures are associated with centralized control, vertical communications, high degrees of formalization and rigid task definitions similar to those of traditional plan-based IT project management. In contrast, the organic structures typically have decentralized control, lateral communications, low degrees of formalization and flexible task definitions, similar features upon which agile methods depend (Ramesh et al., 2012). This suggests that the basic philosophy of traditional plan-based and agile project management practices reflect those analogous extremes.

Despite these intense differences between organic and mechanistic structures some projects may employ some hybrid elements of both models (Austin \& Devin, 2009; Barlow, et al., 2011; Ramesh et al., 2012: Vinekar et al., 2006). There are also some limited projects that may not adopt any of the above models i.e. use no formal methodology at all. However, as described by KPMG report (2013) the probability of success of such projects is very narrowless than $10 \%$ (p.12). Thus, the mechanistic view point of traditional project management and the organic perspective of agile project management have been used as a guiding feature to distinguish between these two approaches in this study. 


\subsubsection{The concept of fit and fit theory}

In this study, 'fit' refers to an alignment between candidate CSFs and project management methodology. It is therefore an acknowledgement for the appropriate choice of the methodology that can aid the successful completion of the project. Although many theorists commonly hypothesise relationships using phrases such as matched with, contingent upon, consistent with, fit, congruence and coalignment, precise guidelines for translating these verbal statements to the analytical level are rarely provided (Venkatraman, 1989). Consequently, Venkatraman (1989) reveals that there is lack of correspondence between the concepts and respective statistical schemes. This lack of correspondence between the concept of fit and its mathematical formulation has potentially led to contradictory and inconsistent results that could have not only caused confusion among scholars but have also weakened the contingency theory itself.

The analysis of 'fit' literature suggests that researchers have often used various approaches to conceptualize fit. Venkatraman (1989) identified six alternative perspectives for conceptualising and measuring fit. The six perspectives are fit as moderation, fit as mediation, fit as matching, fit as gestalts, fit as profile deviation and fit as covariation. Venkatraman's (1989) framework classifies each perspective based on three dimensions: the degree of specificity of the functional form of fit, the number of variables in the equation, and the presence of or absence of a criterion variable.

\subsubsection{Fit as moderation}

One of the underlying assumptions of a great number of contingency models in several areas of research has been based on the idea that there is no one best way to manage an organization which is attributed to seminal works of Burns and Stalker (1961). Following this ground breaking study, many organizational theorists have focused on the study of contingency models that share the underlying premise that context, structure and strategy must somewhat fit together for the organization to perform well. According to the moderation perspective, the fit between the predictor and the moderator variable is the primary determinant of the criterion variable (Venkatraman, 1989, p.424). The researchers usually invoke this perspective when the underlying theory specifies that the impact of the predictor varies across different levels of moderator to affect the relationship with the dependent variable (Venkatraman, 1989). Figure 31 illustrates the alternate path diagram representations of fit as moderation. 


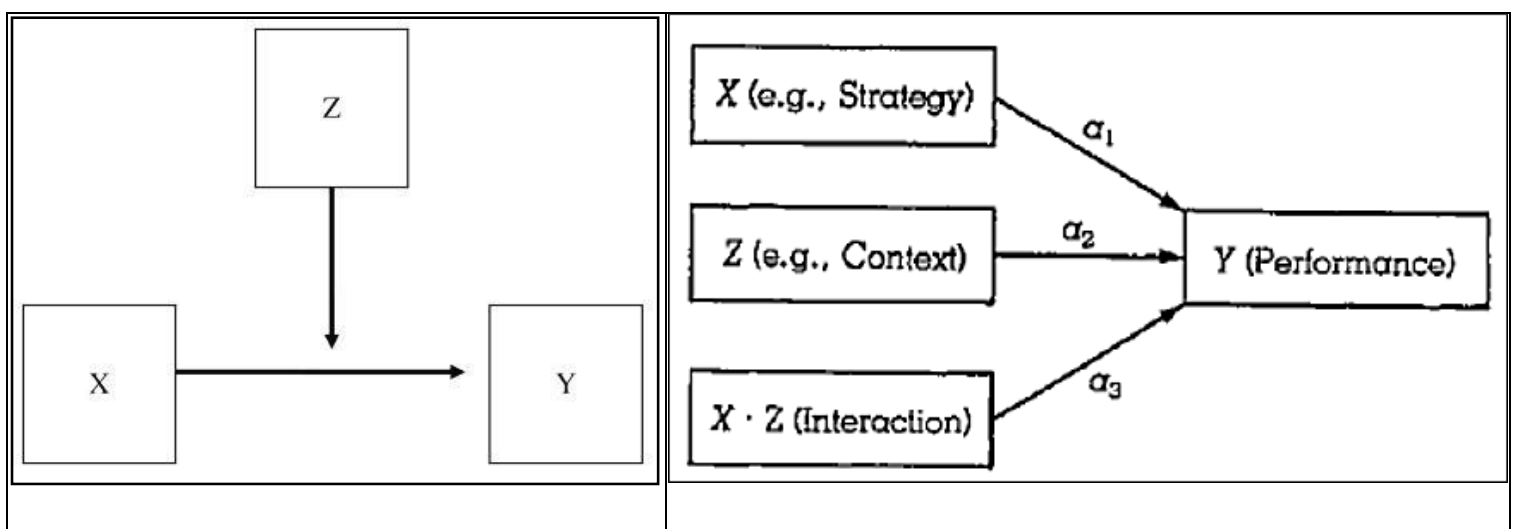

Figure 31: Alternate path diagrams of fit as a moderator (Fairchild \& MacKinnon, 2009, Figure 4, p.90; Venkatraman, 1989, Figure 2, p.425 respectively)

Note. $\mathrm{X}=$ the independent variable, $\mathrm{Y}=$ the dependent variable, $\mathrm{Z}=$ the moderator variable, $\mathrm{XZ}=$ the product of $\mathrm{X}$ and the moderator variable, $\alpha_{1}=$ the effect of $\mathrm{X}$ on $\mathrm{Y}, \alpha_{2}=$ the effect of $\mathrm{Z}$ on $\mathrm{Y}$, and $\alpha_{3}=$ the effect of $\mathrm{XZ}$ on $\mathrm{Y}$. Then the model is: $\mathrm{Y}=\alpha_{0}+\alpha_{1} \mathrm{X}+\alpha_{2} \mathrm{Z}+\alpha_{3} \mathrm{X} * \mathrm{Z}+\mathrm{e}$ (Venkatraman, 1989, p.426, equation 3). The moderation hypothesis is thus supported if the coefficient, $\alpha_{3}$, differs significantly from zero, attesting to the effects of fit between $\mathrm{X}$ and $\mathrm{Z}$ on Y. Previous studies (e.g. Prescott 1986; Goodhue 1995; Chan et al. 1997; Dishaw \& Strong 1999; Parker \& Van Witteloostuijn 2010), adopted moderation approach and computed fit between two variables by capturing interaction effects. Specifically, Chan et al. (1997) applied the moderation approach to investigate IS strategic alignment between business strategic orientation and information systems strategic orientation.

Given the research model developed in chapter 3, fit as moderation seems more appropriate for studying fit due the underlying contingency theoretical assumptions. Fit as mediation seemed to be inappropriate for studying fit due to the underlying contingency theoretical assumptions while fit as gestalt (archetypes or configurations), is only appropriate for a set of theoretical attributes involving multiple variables (Venkatraman, 1989). Fit as profile deviation was not used because it was unclear on how to develop an ideal profile for the fit concept while use of fit as covariation would not easily allow fit to be numerically specified if causal relationships were to be investigated with other variables. Direct measures of assessing fit would shift the responsibility of creating a difference score from the researcher to the respondent which could have severely compromised research reliability and validity.

\subsubsection{Strengths and weaknesses of contingency theory}

Contingency theory has survived over the decades as valid and reliable in explaining of how to achieve effective management because it is grounded in empirical research. Researchers 
who have followed Burns and Stalker (1961), Fiedler (1964) or Lawrence and Lorsch (1967) have validated contingency theory with their own research. Thus, Contingency theory is now widely accepted (Tosi \& Slocum, 1984). The overall strategy of contingency theory is reasonably clear asserting that in order to be most effective, organizational/project structures should be appropriate to the work performed and/or to the environmental conditions facing the organization/project.

Contingency theory has broadened the scope of management understanding from a focus on a single, best type of management to emphasizing the importance of a manager's approach and the demands of different situations. Contingency theory has also been proved to have predictive powers in determining the type of management that is most likely to be effective in particular contexts. Contingency theory does not suggest to expect managers to be equally effective in all situations and therefore organizations/project should consider managers in optimal situations according to their management styles.

Although contingency theory has been an important aspect of management literature for a number of decades and has gained acceptance largely due to weaknesses in the classical theories that advocated for one best way of managing, this theory has been hampered by a number of limitations. These limitations, among others include: contradictory and inconsistent empirical results with low explained variance, ill-defined concepts of fit and performance (Nidumolu, 1995, p.193). This research perspective has also been dominated by deterministic assumptions and often disregarding the equifinality property of contingency theory (Tosi \& Slocum, 1984). Subsequently, some scholars (e.g., Schoonhoven, 1981) have argued that contingency is not a theory at all since in the conventional sense it lacks welldeveloped set of interrelated propositions. Instead, Schoonhoven (1981) argues that it is rather more of an orienting strategy or meta-theory, suggesting ways in which a phenomenon ought to be conceptualized or an approach to the phenomenon ought to be explained (p.350).

Many contingency theorists commonly use phrases such as matched with, contingent upon, consistent with, fit, congruence and coalignment etc., to hypothesize relationships but precise guidelines for translating these verbal statements to the analytical level are rarely provided (Venkatraman, 1989, p.423). Lack of correspondence between these concepts and their statistical schemes or mathematical formulation could possibly explain contradictory and inconsistent results (Venkatraman, 1989). 
Several scholars seem to have been frustrated because contingent relationships have been rarely found to be straight forward and simple. For instance, Pennings' (1975) empirical study of the relevancy of structural contingency model for organizational effectiveness found no support for explaining variation in organisational effectiveness based on simple environmental variables. In a similar fashion, Drazin and Van de Ven (1985) also investigated the alternative forms (selection, interaction and systems approaches) of fit in structural contingency theory and did not get any empirical evidence for a simple interaction model.

Equally, some concerns have also been raised regarding the reductionist orientation dominating contingency research based on bivariate practices and statistics. However, holistic approaches have also been limited by difficulty to establish clear hypotheses (Venkatraman, 1989). Linearity is also often assumed to characterize the relationships between variables yet; organizations are more than a set of linear relationships. Finally, contingency theory has been criticised for remaining relatively narrowly based and failing to accommodate a range of project management approaches (Howell et al., 2010).

Nonetheless contingency theory still appears relevant. Indeed, and according to the criteria suggested by Miles et al. (1978, p.558), a theory of management has three basic components. These are (a) a set of assumptions about human attitudes and behaviours, (b) managerial policies and actions consistent with these assumptions, and (c) expectations about employee performance if these policies and actions are implemented. As noted above, contingency theory meets the above criteria for a theory of management.

\subsection{Research gap and research questions}

A thorough examination of literature reveals that the research gap in the literature is centred on the concept of fit of different candidate CSFs for diverse software development methodologies and the impact of this fit or misfit on project success. What is clear in the literature is that there is a substantial agreement among several authors that the fit between candidate CSFs and project management methodology is likely to positively impact on project success. However, no study undertakes a systematic comparative empirical study focused on both methodologies. Previous software development projects studies have addressed usually one methodology per study and perceived candidate CSFs as a form of 
reasons of success amidst a wide range of project success criteria. No previous study has proposed a comprehensive contingency fit model for comparative analysis of candidate CSFs for both agile and traditional plan-based methodologies. Studies have typically not included a full survey of candidate CSFs and empirical tests; tended to sample only one SDM, most recently mainly Agile (although traditional still seems to be used extensively); tended to examine direct relationships between candidate CSFs and project success (if they include this link) whereas other literature suggests that indirect paths may be more likely. Although contingency theory has been previously argued for software development projects, empirical models have frequently not fully incorporated contingency as fit or fit as moderation (traditional vs agile) when the issues above have been accounted for. This study seeks to fill this research gap.

Thus, the research seeks to answer these questions:

(RQ1) what are the CSFs for outsourced software development projects from a vendor's perspective?

(RQ2) What are the differences in these CSFs for traditional plan-driven and agile methodologies towards project success from a vendor's perspective?

The examination of candidate CSFs for each methodology associated with software project success is likely to provide a better understanding of how candidate CSFs relate to project management methodology and interrelate with one another to influence project success in outsourced projects from a vendor's perspective. In doing so, the study contributes to an area in need of additional research.

\subsection{Chapter summary}

This chapter presented literature review with the purpose of establishing the theoretical foundations underlying this research and identifying research gaps. An overview of theoretical perspectives of IT outsourcing as well as types of relationships were presented. A justification was provided as to why the selected vendor view point of the client organization is the most important, and why the other viewpoints are omitted.

Traditional and agile software development methodologies were compared and contrasted. Next, the concept of project success in software project management research was examined. 
The CSFs research approach, its strengths and weaknesses in software project management were examined. Using a vendor perspective, 37 different candidate CSFs were identified to be related to project success. The frequencies of these candidate CSFs were plotted on a diagram to illustrate how different candidate CSFs can match different methodologies. Commonly occurring candidate CSFs were then grouped into four general categories. These major categories or themes of candidate CSFs were further explained and will be used subsequently to develop the research model.

Contingency theory in software project management and more examination of fit and fit theory from strategic management other than the project management literature was undertaken. The section concluded by discussing the strengths and weaknesses associated with the applicability of contingency theory in project management research.

Lastly, the research gaps were identified from the extant literature. The literature review suggests that there is research gap in the literature that focuses around the concept of fit between candidate CSFs and project management methodology. It is theorised that the aforementioned fit is likely to influence project success. Previous software development projects studies have addressed generally one methodology per study and perceived candidate CSFs as a form of reasons of success amidst a wide range of project success criteria. While contingency theory has been previously argued for software development projects, empirical models have frequently not fully incorporated contingency as fit or fit as moderation (traditional vs agile). 


\section{CHAPTER THREE: CONCEPTUAL MODEL AND RESEARCH HYPOTHESES}

\subsection{Introduction}

The previous chapter presented the literature review. This chapter develops the conceptual model and research hypotheses. Based on the vendor perspective, four categories of CSFs were identified and grouped as 1.VPoc organisational factors, 2. Vendor perception of team factors, 3.Vendor perception of customer factors, and 4. Vendor perception of project factors. Through these 4 categories of candidate CSFs, a conceptual model is presented specifying the theorised relationship with project success.

Subsequently, the variables in the model are discussed and the literature is integrated to allow clear research hypotheses to be formulated, suitable for empirical model validation. The intent is to develop research hypotheses that clearly demonstrate how the different individual candidate CSFs identified from the literature associate with software project success. The strength and direction of each hypothesised path in the domain of theory is explained.

The last section of this chapter reports on the research findings of relevant checks that were conducted with experienced software project managers based in Wellington, New Zealand. Nine experienced software development managers were interviewed about their perceptions in regard to the conceptual model and their feedback is discussed.

\subsection{Conceptual Research Model}

This section develops a conceptual model by using the major categories or themes of the CSFs that were identified from comprehensive literature review. The conceptual research model demonstrates the theorized relationships between the 4 major categories of candidate CSFs and software development project success.

\subsubsection{Development of the conceptual model}

As noted earlier the focus of this study is on the selection of project management methodologies that can be adopted to match candidate CSFs related to project characteristics and the project environment to manage software development projects to success. These candidate CSFs (contingencies) have been named as VPoc organizational factors, vendor perception of team factors, vendor perception of customer factors and vendor perception of project factors. 


\subsubsection{VPoc organizational factors and software development project success}

VPoc organizational factors (from clients' organization) are influences that are external to the project environment itself but are from the broad organization's environment which impact on the way a project can be managed to success (Howell et al., 2010). As noted, the project's environment is often dominated by the parent organisation and hence the management of projects is often influenced by the VPoc organizational factors. VPoc organizational factors include top level management support, organisational culture, project planning and controlling, leadership characteristics, change management and vision and mission. These are constructs that broadly encompass top management (leadership) strategic decisions and the inherited organisational culture. Since software projects exist within the broader organisation; these factors can greatly impact on the management methodologies of such projects.

Therefore, it can be logically hypothesised that different projects face different parent imposed constraints and that this yields different optimal project characteristics (Howell et al., 2010). For instance, traditional plan-based methodologies should be used in organizations that are characterized with mechanistic and bureaucratic structures that emphasize planning and controlling procedures while agile approaches should be used in organisations with organic and flexible structures that support more informal communication and empowerment of the project teams.

Among all VPoc organizational factors, top level management support has been suggested to be the primary candidate CSF for software development projects. This is probably because top level management commitment drives and influences other organisational factors (Jung et al., 2008). Imreh and Raisinghani (2011, p. 464) and Mansor et al. (2011, p.3) also emphasize that no project can finish successfully unless the project manager secures commitment from the senior management. This appears to suggest that for any project's success, there is a necessity for sustained upper management commitment to provide resources, authority and influence. Consistently, Dyck and Majchrzak (2012) found that top management commitment has a positive impact on agile software development success and Jung et al.'s (2008) findings provided support for the hypothesis that top level management commitment was a significant predictor of project performance. It is, thus, clear that a positive relationship between top level management commitment and software development project success is expected. In regard to project management methodology selection, if the top level management of an organization supports an adaptive behaviour, flexible leadership styles and entrepreneurial 
culture, they are not likely to support a rigid traditional approach that requires up-front detailed planning and formal specification, but rather agile methodologies should be adopted.

The literature also suggests that organizational culture; in particular, risk taking attitude has a positive effect on the extent to which agile projects succeed (Misra et al., 2009; Sheffield \& Lemetayer, 2013). Such cultures are characterised by teamwork, flexibility and participation that encourages social interaction (Strode et al., 2009). Thus, software projects in these organizational environments should be managed by agile methodologies rather than the traditional plan-based approaches because agile approaches are designed to manage changes and environmental uncertainty. Agile culture has high tolerance for risk taking and innovative practices.

However, some conflicting results have been reported about the effect of organizational culture on software development project success. While Misra et al. (2009) found that corporate culture influences success of agile projects and Wan and Wang (2010) found that agile methods succeeded when matched with agile corporate culture, in contrast, Chow and Cao (2008) found no statistical support for the effect of organizational culture on the perceived success of agile projects.

Project planning and controlling refer to the extent to which planning and controlling practices are used in a project. Previous research has demonstrated a positive relationship between planning and process performance (Yetton et al., 2000; Jun et al., 2011). Poor planning is likely to be associated with inefficiencies in development and, thus, lead to large budget and time variances. Rigorously tracking and monitoring a project according to a project plan can ensure that the final product is delivered within budget and on schedule. With regard to choosing an appropriate methodology, if there is no or little change expected during the project, the plan does not need to be modified and traditional approaches that plan for every task in advance should work well. Future features should be prepared in the design phase as should interconnections between features. However, since responding to change is one of the key principles of the agile manifesto and change usually occurs faster than a plan can be updated, agile methodologies are argued to be used when planning and controlling are not possible.

Other VPoc organizational factors have also been found to influence software project success e.g. Wan and Wang (2010), Sheffield and Lemetayer (2013) and Strode et al. (2009) found 
that leadership characteristics positively influence agile software development project success. This implies that if the management style has leadership characteristics and is willing to take some significant amount of risks as there is uncertainty, agile approaches should be used. In contrast, if there is a conservative environment where there is command and usually many control procedures in place, traditional approaches should be used. Similarly, Wan and Wang (2010) indicated that change management characteristics, vision and mission significantly and positively impacted software project success. Again, if the project is in a highly volatile environment of change, agile methodologies should be used instead of traditional approaches since agile methodologies embrace change and uncertainty. Overall, the literature consistently proposes that the VPoc organizational factors can have a direct primarily positive influence on outsourced project success (Figure 32).

\subsubsection{Vendor perception of team factors and software development project success}

The Vendor perception of team factors are specifically about issues of project teams and are also theorized to have a primarily positive impact on the success of any software projects. The success of an outsourced software project is argued to depend greatly on the team's communication, team empowerment, expertise and experience, commitment and composition. Although these factors specifically relate to employees or project teams, some factors such as empowerment, team's composition, size and geographic distribution are also frequently influenced by the parent organisation, and the broader corporate culture that is inherited from it (Howell et al., 2010). This implies that the team's empowerment, composition, size, dispersal, and organisational boundaries may all affect the team's ability to communicate or team's commitment. Similarly, a team's communication, commitment, expertise or skill, experience, and empowerment determine a team's ability to quickly comprehend and respond to the risk, thereby improving the chance of project success. Under circumstances where there are small teams that are self-organizing, autonomous, composed of best skilled expertise and experienced people, who are highly collaborative and committed, agile methodologies are seen as highly suitable and the reverse is true for traditional methodologies.

Project team commitment is the willingness by a team to devote energy and loyalty to a project as expressed in three forms: affective, continuance and normative (Meyer \& Allen, 1997). Affective commitment is team's emotional attachment with the project. Continuance commitment refers to the team's recognition of the benefits of continued association with the 
project compared to the perceived cost of leaving the project. Normative commitment refers to the team's feeling of obligation to remain in the project. All these three forms of commitment affect the team members' willingness to remain with a project and their workrelated behaviour. Chow and Cao (2008) found that team members with great motivation positively influenced the perceived success of the agile software development projects. Correspondingly, Wan and Wang (2010) found significant positive relationships between team commitment and agile project success. This suggests that committed project team members more often do not have intentions to quit, which saves the project the costs of recruiting and orienting new members in terms of both time and money. Similarly, costs of supervision are mitigated if the project team members are committed to their project tasks. If the team is highly committed e.g. for working full time, knowledgeable, representative and empowered, then agile approaches should be used and vice versa.

Internal project communication is defined as the practices that increase information exchange and cohesion among development team members. Internal project communication enhances the levels of information sharing and collaboration between the members of the project team which decreases the amount of team conflict and keeps the team stable. Along similar lines, Jun et al. (2011) found that internal project communication had a significant positive effect on both process and product performance. Similarly, Yetton et al. (2000) demonstrated that project team conflict leads to instability in a project team and, thus, result in a project being delayed and exceeding budget. This is because software development is a knowledgeintensive and human-intensive activity that requires collaboration between team members with diverse skills and specialties.

Additionally, effective internal project communication creates a feeling of responsibility and attachment between team members and the project tasks that makes team indebted to the project. As a result, this creates an atmosphere for individual team members to act without much control and coercion. Under such circumstances, what drives a person to work is the emotional attachment to the project as fostered through communication. This is consistent with the findings of Jun et al. (2010), Misra et al. (2009) and Chow \& Cao (2008) who found that those workers with a positive attitude about project tasks carry out certain role behaviours well beyond the basic minimum levels required of them. They, for example, may not take extra breaks and they tend to obey the project rules and regulations even without supervisions. They attend meetings that are not mandatory but are considered important. 
They also keep abreast of changes within the project and elsewhere that affects or are affected by the project and responsibly discuss them with those concerned. With reference to methodology selection, if the organizational culture encourages information sharing freely and collaboration between the members with no large power distance at work place, agile methodologies should be used and vice versa.

Other Vendor perception of team factors have also been found to influence software project success e.g. team capability, competences and skills have also been found to positively influence software project success (Wan \& Wang, 2010; Misra et al. 2009; Chow \& Cao, 2008; Jiang \& Klein, 2000; Boehm \& Turner, 2003; Ratbe et al. 2000; Lindavall et al. 2002; Little, 2005). Team's expertise (general or task) includes the ability to work with uncertain objectives, ability to work with top management, ability to work effectively as a team, ability to understand human implications of a new system, and ability to carry out tasks effectively (technically) (Jiang \& Klein, 2000). These are interpersonal, team or technical skills that can be determined early during the formation of the project team. Although, these skills can be addressed from a number of viewpoints, generally, management can communicate early the basic project parameters and management guidelines to the project team to allow for skill matching. The building of team's skills can also be conducted by project managers throughout the life cycle which enhances project success.

If a team's experience and expertise are low, it is more appropriate to choose a traditional methodology which provides more structure and more pre-identified processes to correctly guide the project team members. Traditional projects need highly skilled and senior staff at the beginning of the project (during the project definition phase), and then junior or lowerskilled staff can do the assigned work by following pre-established plans. On the other hand, if the talents, skills and competences of individuals in teams are high, agile methodologies should be used. This is because agile projects need highly skilled and senior people throughout the entire project in order to continuously adapt to change. Thus, vendor perceptions of team factors are proposed to have a primarily direct positive influence on project success (Figure 32).

\subsubsection{Vendor perception of customer factors and software development project success}

The Vendor perception of customer factors relate to the characteristics of the behaviours and practices of the users' enthusiasm to be involved in activities of product development once they have decided to outsource software development process to an outside vendor. In this 
study, vendor perception of customer factors cover user participation and support, level of customer training and education, and client experience. User participation and support consist of the behaviours and activities of the customer in relation to software development (Jun et al., 2011). The literature reveals that user participation significantly increases the likelihood of the chances of software development projects success. Empirical studies by Chow and Cao (2008), Misra et al. (2009) and Sheffield and Lemetayer (2013) have provided data to support significant and positive relationships between user participation and support and agile software development project success.

In relation to choosing an appropriate methodology, if the level of commitment of the user to participate in requirements definition and provide full time support for the project team is high, agile methodologies should be used and vice versa. This is because unlike traditional methodologies, agile methodologies require full time customer involvement right from the initial specification phase to the end. Although it can be argued that user participation tends to increase budget variance by encouraging suggestions for changes to specification, Yetton et al. (2000) found that user participation also decreases budget variance by managing expectations and quickly resolving potential problems. Similarly, Jun et al. (2011) also demonstrated that resolving potential conflicts early arising from greater user participation plays a vital role in the perceived system satisfaction of software developers and users.

Further, customers who are well experienced in software development projects and have an acceptable level of basic education or training in IT can easily explain their requirements and needs in a clear form. Similarly, customers who have basic knowledge about business domain accurately identify their requirements which save time, costs and contribute to process and product quality (Mohammad \& Alshargabi, 2011). Also, customers who are knowledgeable of the exact problem of the organisation to be solved are likely to help to shorten the development time in producing the product. Equally, customers who have some basic knowledge about constraints in hardware and software world can easily make choices and justify their selection of any specific hardware or software. Previous scholars have shown that end user training, experience and education play a positive role in project success (Jun et al. 2011; Livermore, 2008; Misra et al. 2009; Charvat, 2003; Jiang \& Klein, 2000).

For choosing an appropriate methodology, if the software customers are highly educated, trained or experienced in IT and are willing to participate in the development process and are 
supportive of the new software product, agile methodologies should be used rather than traditional methodologies and vice versa. Simply because, for agile projects, the customer must be able to articulate his needs clearly, otherwise this threatens the whole project. Accordingly, vendor perception of customer factors have a direct and predominantly positive relationship with project success (Figure 32).

\subsubsection{Vendor perception of project factors (moderating variables)}

One of the fundamental assumptions of classical structural contingency theory is the information processing viewpoint of organizations. It is assumed that context and structure must fairly fit together if the organization is to perform well (Burns \& Stalker, 1961; Lawrence \& Lorsch, 1967). It is argued that organisational structures or designs enable information processing capabilities that are appropriate to the level of uncertainty challenging each organisation unit (Jun et al., 2011). Consequently, as the level of uncertainty facing an organisation increases, decision makers must process an increasing amount of information to achieve a given level of performance. This suggests that needs of an organization are better satisfied when it is properly designed and the management style is appropriate both to the tasks undertaken and the nature of the work group. Barki et al. (2001) general contingency hypothesis supported the view that high risk software projects call for high information processing capacity management approaches. Similarly, based on this perspective, Jun et al. (2011) found that project planning and control fitted low information processing capability approaches while, internal integration and user participation presented the high information processing capability approaches to managing software project uncertainty.

Taking into consideration project types and the research perspective of this study, the key vendor perception of project factors identified from literature are: technological uncertainty, technical complexity, relative project size, specification changes, and project criticality. Complexity and uncertainty are frequently regarded as independent (e.g. Ratbe et al., 1999; Shenhar \& Dvir, 2007). However, authors such as Petit (2012) and Hass (2008) consider complexity and uncertainty to be aspects of the same variable. As Howell et al. (2010) argues, the project management issues surrounding complexity centre upon capacity to understand what is going on, and consequently predict the relationship between inputs and outputs. Lack of predictability is identical with uncertainty, and thus complexity becomes a factor in uncertainty (Howell et al., 2010). Use of new or unfamiliar technologies increases project uncertainty (Howell et al., 2010). Nidumolu (1996) and Jun et al. (2011) found the 
use of unfamiliar technologies can lead to software problems that reduce the performance of the software product and delay the project. Some empirical evidence reveals that large project size increases project risk (Sauer et al., 2007, Jun et al., 2011, Turner \& Zolin, 2012). Project criticality is also argued to potentially increase risk, thereby indirectly affecting project success (Boehm \& Turner, 2003; Cockburn, 2007).

Most of these risks are project-specific characteristics that initially exist in a project itself (Howell et al., 2010). These risk management factors with different levels of inherent project uncertainty can influence different contributions of different candidate CSFs to project success (Jun et al., 2011). For instance, project planning and controlling are likely to make a greater contribution to process or product success of traditional plan-driven projects which are characterized with low levels of project risks than for agile projects perceived to have high risks.

Similarly, project communication is likely to make a greater contribution to process or product success of agile methodology projects considered to be with high levels of inherent project uncertainty than traditional plan-based methodology projects with perceived low risk levels. Equally, user participation and support are expected to make a greater contribution to process or product performance for agile methodology projects perceived with high levels of inherent uncertainty than traditional plan-driven methodology projects with low levels. Jun et al (2011) established that project uncertainty had a moderating effect on the relationship between planning and control, internal integration, user participation and project performance. Therefore, vendor perception of project factors moderate the relationship between VPoc organizational factors, vendor perception of team factors, vendor perception of customer factors and vendor perception of project success (Figure 32).

However, suffice to note is that vendor perception of project factors can also negatively affect project success (Jun et al, 2011; Sheffield \& Lemetayer, 2013). Jiang et al., 2002) also demonstrated that uncertainty is negatively associated with project success. Some empirical evidence reveals that project size can also negatively affect project performance (Sauer et al., 2007). Specification changes make it difficult to define complete, unambiguous or consistent requirements (Nidumolu, 1996). As a result this can lead to a software product that cannot meet the client's needs, and decrease process performance (Jun et al, 2011). Thus, generally, 
the level of project inherent uncertainty or risk is also primarily negatively associated with both process and product success (Figure 32).

IT literature suggests that the arguments for specific software development risks on outsourced software project success are largely based on anecdotal evidence or armchair theorizing (Jiang \& Klein, 2000). Empirical evidence on the relationship between various risk factors and project success is rare and often fails to take into account various risk factors that may hinder project success. Yet, not all the various software development risks have the same effects on overall project success. The risks that are more influential than others are also not known. Similarly, risks that have greater impact on the schedule, budget, goals, efficiency, speed, quantity and quality of work performed are hardly assessed. Certainly, if these issues are addressed it can help both IT researchers and practitioners better understand how the risks affect project success. In addition, appropriate procedures can then be adopted to control these risks and achieve the desired results. Figure 32 illustrates the conceptual model. Note: For simplicity and clarity not all interrelationships are illustrated. 


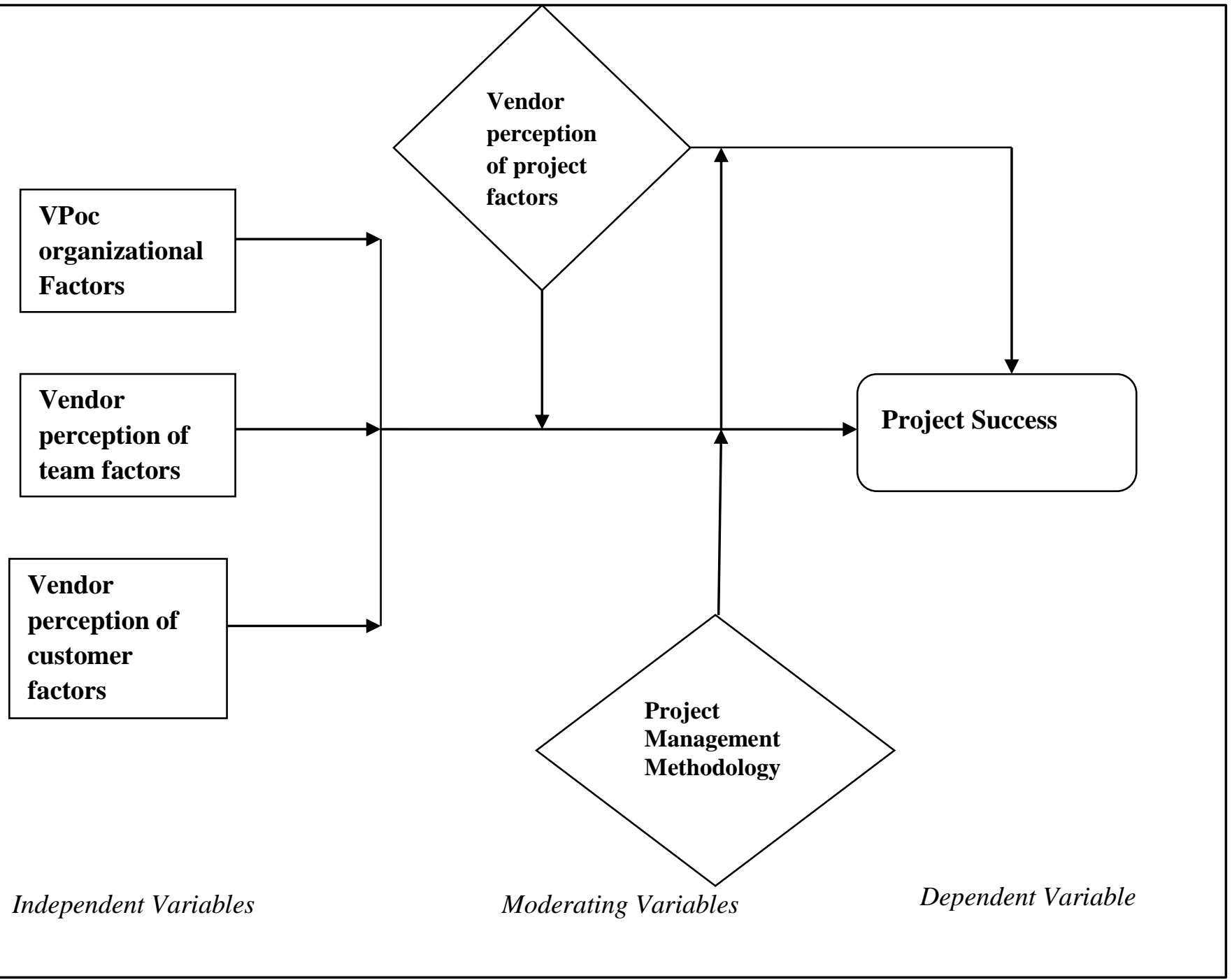

Figure 32: The conceptual model

\subsubsection{Explaining the conceptual model}

As shown in the conceptual model, VPoc client organizational factors, vendor perception of team factors and vendor perception of customer factors are predicted to have a predominantly direct positive influence on project success. In contrast, however, vendor perception of project factors are hypothesised to have a moderating effect on the relation between VPoc client organizational factors, vendor perception of team factors and vendor perception of customer factors with project success. Equally, vendor perception of project factors primarily negatively contribute to project success. Finally, the contingent relationships are incorporated in the model with project management methodology as a moderator. Having presented the 
conceptual model, the next section explains each variable in detail and formulates the research hypotheses.

\subsection{Development of research hypotheses}

This section describes the development of research hypotheses from the literature review that will be used to test the research model. It concludes by summarizing the research hypotheses developed for this study and relates them to studies that have previously tested relationships between similar constructs. Because of different and conflicting results on the impact of each candidate CSF for various methodologies, two competing hypotheses are formulated for each candidate CSF. Whereas the null hypothesis $\left(\mathrm{H}_{0}\right)$ assumes the effect of the candidate CSF on the two major project success measures (i.e., process and product success) for traditional plan-based methodologies, the alternate hypothesis $\left(\mathrm{H}_{1}\right)$ assumes the effect of the candidate CSF on the two major project success measures (process/product) for agile methodologies. Where the contribution or relationship between a candidate CSF and project success measures is assumed to be relatively greater or less important depending on the methodology that is implemented as argued for in the literature is also specified. Research hypotheses are illustrated in Figure 33 and summarized in Table 15 at the end of this chapter.

\subsubsection{Hypotheses for VPoC organizational Factors}

In this study VPoc organizational factors are categorised as top management support, organizational culture, planning and controlling, leadership characteristics, change management, vision and mission. The next section discusses these individual VPoC organizational factors in detail and their postulated relationships with project success.

\subsubsection{Top level management support (TMS) and project success}

Amongst all VPoC organizational factors, TMS has been proposed to be the primary success factor for software development projects. This is probably because TMS drives and influences other VPoC organizational factors. According to Nah et al (2001), top management needs to publicly and explicitly identify the project as a top priority. Senior management must be committed with its own involvement and willingness to allocate and approve valuable resources to the implementation effort. This may involve providing the needed staff for the implementation of the projects and giving them an appropriate amount of time to get the job done (p.291). Jung et al. (2008) found top level management commitment was a significant predictor of project performance. This implies that for any project to succeed there is a necessity for sustained top management support to provide resources, 
authority and influence. However, the purpose of Jung et al.'s (2008) study was slightly different, that is, to explore the relationship between competitive strategy, Total Quality Management (TQM), and Continuous Improvement of International Project Management (CIIPM). Their study also combined both agile and traditional methodology projects so it is difficult to determine the influence of top level management support on different projects methodologies.

In contrast, however, Chow \& Cao (2008) did not find a strong management commitment as a significant CSF that contributes to the successful agile software development projects. However, this study was dominated by multiple responses from similar organizations which could have biased their findings. Wan and Wang (2010) found top management support through both dimensions of participation and recognition significantly contributed to agile software development process improvement in China. However, this study was organizational case-based and cannot be generalized. Similarly, Nah and Delgado's (2006) case-based research of candidate CSFs for ERP planning implementation and upgrade in USA also found top management support and championship positively influenced project success. The only difference was that Nah and Delgado (2006) methodologically adopted a multiple case study approach. Equally, previous researchers such as Imreh and Raisinghani (2011, p. 464), Mansor et al. (2011, p.3), Mishra \& Mishra, 2011) and Dyck and Majchrzak (2012) also theorised a similar relationship between top level management and agile project success but their claims are not empirically validated.

Although there is a unanimous agreement in the project management literature regarding the great effectiveness of top management support, some others underplay its role in projects. Young and Jordan (2008) argue that what begins as support easily turns into interference, which is harmful especially in highly innovative environments. Swink (2011) found top management support did not matter in really new product innovations. Although the relationship of TMS on project success for each methodology is not clear from the literature, the use and success of agile methodologies appears to be a major initiative in most companies that requires more TMS. Based on this discussion it can therefore be hypothesized that:

\section{H1 (T): There is a significant positive relationship between TMS and (i) process and (ii) product success for traditional plan-based methodologies $(T+)$.}

$H 1$ (A): There is a significant positive relationship between TMS and (i) process and (ii) product success for agile methodologies $(A+)$. 
H1 (TA): TMS is more important when agile methodologies are used than when traditional plan-based methodologies are implemented $(A+>T)$.

\subsubsection{Organizational culture and project success}

Organizational culture can determine the choice decisions of software methodology to use, criteria of project team's composition, communication, planning and controlling, or project budgeting, duration and other project parameters (Jung et al., 2008; Chow \& Cao (2008). Some project methodology types are agued to be more suited to certain organizational environments than others (Strode et al., 2009). Sheffield and Lemetayer (2013) argue that agile is not appropriate in bureaucratic and mechanistic organizations because it is not easy to respond to changes and uncertainty.

However, based on the software projects literature some conflicting results have been reported about the effect of organizational culture on project success. For instance, Chow and Cao (2008) did not find a significant relationship between organizational culture (i.e. cooperative and oral culture, where managers are adaptive and teamwork is coherent and selforganizing) and the perceived success of agile projects. In contrast, however, Misra et al. (2009) found that corporate culture influences success of agile projects. Similarly, Wan and Wang (2010) found that agile methods must be established within agile corporate culture to succeed. Equally, Sheffield and Lemetayer (2013) found that organizational culture positively influenced software development agility. Similarly, Strode et al. (2009) found a positive and significant relationship between low formality organizations and the use of agile methodologies.

Consistently, in a study that focused on the intermediate level of values applying the Competing Values Model (CVM) as a theoretical model of organizational culture (OC), Iivari and Huisman's (2007) found a significant positive relationship between hierarchical rational organizations and the deployment of traditional methodologies. However, Iivari and Huisman (2007) did not fully examine OC and neither distinguishes between organizational and national cultures. Yet, this is one of the key studies that primarily focuses on traditional plandriven SDMs and their impact on OC. However, since agile is considered to be a cultural issue and flexible or risk taking cultures can more easily accommodate adventurous methodologies like agile, it can be proposed that:

H2 (T): There is no significant relationship between flexible organizational cultural 
and (i) process and (ii) product success for traditional plan-based methodologies $(\mathrm{T \# )}$.

H2 (A): There is a significant positive relationship between flexible organizational cultural and (i) process and (ii) product success for agile methodologies $(A+)$.

H2 (TA): Flexible organizational culture is more important when agile methodologies are used than when traditional plan-based methodologies are implemented $(A+>T)$.

\subsubsection{Project planning and controlling and project success}

Although professional bodies of knowledge advocate planning as a core process for all projects (OGC, 2009; PMI, 2013); literature is inconsistent regarding the importance of planning for success (Zwikael et al., 2014). Previous research has demonstrated a positive relationship between planning and project success. Pinto and Slevin (1987) found a positive impact between planning and project success. Jun et al. (2011) found that project planning and controlling are positively correlated with process performance. Similarly, Yetton et al. (2000) found that project planning and controlling was negatively related to budget variances and that budget variances are a negative function of planning. This implies that proper project planning is likely to be associated with efficiencies in development and, thus, prevent budget and time variances. Equally, rigorously tracking or monitoring and controlling a project according to a project plan can ensure that the final product is delivered within budget and on schedule (Jun et al. 2011).

Empirical results of Wallace et al. (2004a, b) also confirm the negative relationship between planning \& controlling risks and both process and product project performance. In contrast, however, the management of agile methodology projects may not need formalized planning and controlling since formal plans and controls may become absolute due to continuous changes initiated by high levels of environmental uncertainty (Ceschi et al., 2005; Boehm \& Turner, 2005; Vinekar et al., 2006). Barki et al. (2001) found that formal planning and control are particularly important for project success but did not clarify for which type of software projects. Zwikael et al. (2014) found planning was positively related to project efficiency when risk level was higher and planning was positively related to project effectiveness when risk level was lower. However, surprisingly, Misra et al. (2009) did not find a significant relationship between in-formalized plans and agile methodology projects success. Yet, it argued that plans and controls easily become obsolete for agile methodology projects since 
change usually occurs faster than they can be updated (Ramesh, 2012; Sheffield \& Lemetayer, 2013). Thus, from this discussion it can be hypothesized that:

\section{H3 (T): There is a significant positive relationship between project planning and controlling and (i) process and (ii) product success for traditional plan-based methodologies $(T+)$.}

H3 (A): There is no significant relationship between project planning and controlling and (i) process and (ii) product success for agile methodologies (A\#).

H3 (TA): Project planning and controlling are more important when traditional plan-based methodologies are used than when agile methodologies are implemented $(T+>A)$.

\subsubsection{Vision and mission and project success}

A clear business vision and mission that can steer the strategic direction of the project throughout the project life cycle is critical for success. A vision is the preferred future, a desirable state, and ideal state while the mission is an organization's basic purposes, often in terms of broad outcomes that it is committed to achieving or the major function it carries out. Subsequently, a strategic business plan that outlines proposed strategic and tangible benefits, resources, costs, risks and timeline is derived. This keeps focus on business benefits (Nah \& Delgado, 2006). Project management reflected in organizational missions keeps future strategies in line with the objectively chosen short and long-term goals (Jung et al., 2008). A clear business model provides how the project should operate behind the implementation effort and helps to complete the project on time, budget, scope and quality (Nah \& Delgado, 2006).

Jung et al. (2008) found that clear vision and mission directly impacted on the continuous improvement of international project management. They also found that project plans that aligned the business strategy and IT strategy make project work easier and positively impact on project performance in meeting deadlines, budgets, scope and product quality. Similarly, Nah \& Delgado (2006) found clear business plans and vision that were well aligned with project objectives led to project success. Again, since vision and mission are closely associated with rigorous long term planning and controlling, agile methodology projects might not require emphasis on the vision and mission due to dynamic changes in the software project environment. Therefore, it can be hypothesized that:

H4 (T): There is a significant positive relationship between vision and mission and ( $i)$ process and (ii) product success for traditional plan-based methodologies $(T+)$. 
H4 (A): There is no significant relationship between vision and mission and (i) process and (ii) product success for agile methodologies (A\#).

H4 (TA): Vision and mission are more important when traditional plan-based methodologies are used than when agile methodologies are implemented $(T+>A)$.

\subsubsection{Change management and project success}

Change management is a project management process where changes to a project are formally introduced and approved while in a broader organizational context, it is an approach to transitioning individuals, teams, and organizations to a desired future state. It is critical to recognize change in order to stay competitive. According to Nah et al. (2006), a culture of change should be managed. Formal education and training provided to users or staff can gain an understanding of how the system or software works and how it will impact their work.

Although training can lead to budget overruns for the projects, it is critical to the success of the implementation project as well as the quality of decisions that will be taken based on the software or system. Jung et al. (2008) found that effective change management process positively and significantly impacted on project performance. Similarly, Nah and Delgado (2006) found that change management was essential for successful software implementation throughout the project life cycle. Since agile methodologies deal with dynamic changes and uncertainty management than traditional methodology projects, it can be theorized that:

H5 (T): There is a significant positive relationship between change management and (i) process and (ii) product success for traditional plan-based methodologies $(T+)$.

H5 (A): There is a significant positive relationship between effective change management and $(i)$ process and (ii) product success for agile methodologies $(A+)$.

H5 (TA): Change management skills are more important when agile methodologies are used than when traditional plan-based methodologies are implemented $(A+>T)$.

\subsubsection{Leadership characteristics and project success}

There are numerous definitions but leadership characteristics simply relate to the traits which a person possesses to influence others to accomplish an objective with or without formal authority. Misra et al. (2009) found that the better the personal leadership characteristics of the team members in a project, the more likely would be the success of agile methodology software development projects. Similarly, having right people and team leaders on big teams in terms of leadership experience, is believed to be key to project success. Lindvall et al. ( 2002) suggests that it is not only the experience and competency of the team members that is 
important, but also their personal leadership characteristics such as honesty, collaborative attitude, sense of responsibility, readiness to learn, and work with others are considered equally important for software development project success.

For the successful implementation of agile methodology projects, the client's organizational leaders need to be ambitious and willing to take on initiatives (Strode et al., 2009). The client's organizational leaders must be truthful and do what they said, enjoy taking risks, possess technical expertise in their businesses, be perceptive, and conceptually skilled (Misra et al., 2009). Equally, the client's organizational leaders need to be approachable with good interpersonal skills and be liked in their positions to provide a conducive environment for developing and completing the software on time, budget, scope and quality. Along similar lines, Strode et al. (2009) found that leadership characteristics significantly and positively impact on agile methodology software development. Agile SDM studies appear to emphasize that better leadership characteristics (e.g., innovative and entrepreneurial) positively impact more on agile project success. Thus, it can be postulated that:

H6 (T): There is a significant positive relationship between client's effective leadership characteristics and (i) process and (ii) product success for traditional plan-based methodologies $(T+)$.

H6 (A): There is a significant positive relationship between client's effective leadership characteristics and (i) process and (ii) product success for agile methodologies $(A+)$.

H6 (TA): Effective leadership is more important when agile methodologies are used than when traditional plan-based methodologies are implemented $(A+>T)$.

\subsubsection{Hypotheses for vendor perception of team factors}

The next section discusses individual vendor perception of team factors and their hypothesized relationships with software project success. They include: internal project communication, project team commitment, expertise and experience, and composition.

\subsubsection{Internal project communication and project success}

The construct of internal project communication is defined as the practices that increase information exchanges and cohesion among development team members (Jun et al., 2011, p.926). Although communication can be defined differently, what is clear in the literature is that communication is about exchange of information, data, emotions, feelings, knowledge, plans etc. Thus, internal project communication enhances the levels of information sharing and collaboration between the members of the project team which decreases the amount of 
team conflict and keeps the team stable. According to Jiang and Klein (2000), conflicts and confusion lead to wastage of time and cause variations in project schedules and budgets. This suggests that when there is effective communication to all team members about the overall project objectives conflicts and confusions among the team members are avoided which enables the project team to do the work well and project outcomes can be delivered on time, budget, scope or quality.

However, Misra et al's (2009) did not find a significant relationship between communication and agile methodology project success. This is surprising, because agile methodology projects rely on intense communication within teams for exchanging knowledge. Fast and effective communication is frequently cited as one of the important requirements for agility (Agile, 2001, Sheffield \& Lemetayer, 2013). Jun et al. (2011) found that internal project communication had a significant positive effect on both process and product performance. Similarly, Yetton et al. (2000) found that poor internal communication can escalate project team conflicts leading to instability in a project team which can result in a project being delayed and exceeding budget. This is possibly because software development is a knowledge-intensive and human-intensive activity that requires collaboration and communication between team members with diverse skills and specialties.

Effective project communication creates a feeling of responsibility and attachment between team members and the project tasks that makes one indebted to the project, thereby creating an atmosphere for individual team members to act without much control and coercion (Ahimbisibwe \& Nangoli, 2012, p102). Agile methodologies tend to require more face-toface communication and short meetings within teams and continuous interactions with management than traditional plan-based methodologies do. Thus, it can be hypothesized that:

$H 7(T):$ There is a significant positive relationship between internal project communication and ( $i$ ) process and (ii) product success for traditional plan-based methodologies $(T+)$.

$H 7(A):$ There is a significant positive relationship between internal project communication and $(i)$ process and (ii) product success for agile methodologies $(A+)$.

$H 7$ (TA): Internal project communication is more important when agile methodologies are used than when traditional plan-based methodologies are implemented $(A+>T)$. 


\subsubsection{Project team commitment and project success}

According to the attitudinal and behaviour perspectives on organizational commitment, team commitment is the willingness by a team to devote energy and loyalty to a project as expressed in three forms: affectivity, continuance and normative (Meyer \& Allen, 1997). Chow and Cao (2008) found that team members with great motivation greatly influenced the perceived agile methodology project success. Correspondingly, Strode et al. (2009) found significant relationships between team commitment and agile methodology project success. This implies that committed project team members more often do not have intentions to quit, which saves the project the costs of recruiting and orienting new members in terms of both time and money. Similarly, costs of supervision are mitigated if the project team members are committed to their project tasks. Project teams with a positive attitude about project tasks carry out certain role behaviours well beyond the basic minimum levels required of them (Ahimbisibwe \& Nangoli, 2012). They, for example, may not take extra breaks and they tend to obey the project rules and regulations even without supervisions. They also attend project meetings that are not mandatory but are considered important. They also keep abreast of changes within the project and elsewhere that affects or are affected by the project and responsibly discuss them with those concerned. As a result, time and costs are saved while the quality of the product also significantly improves. However, agile methodology projects success might require a higher level of team commitment than traditional plan-based methodology projects because of changes that must be done under various tighter constraints, based on this discussion it can be hypothesised that:

$H 8(T):$ There is a significant positive relationship between project team commitment and $(i)$ process and (ii) product success for traditional plan-based methodologies $(T+)$.

H8 (A): There is a significant positive relationship between project team commitment and ( $i$ ) process and (ii) product success for agile methodologies $(A+)$.

H8 (TA): Project team commitment is more important when agile methodologies are used than when traditional plan-based methodologies are implemented $(A+>T)$.

\subsubsection{Development team expertise and project success}

Although various labels have been used, previous studies have theorized positive and significant relationship between development team expertise and project success (e.g., Jiang \& Klein, 2000; Ratbe et al. 2000; Lindavall et al. 2002; Boehm \& Turner, 2003; Little, 2005; Chow \& Cao, 2008; Misra et al. 2009; Wan \& Wang, 2010; Jun et al, 2011; Sheffield \& 
Lemetyer, 2013). However, Misra et al. (2009) did not find a significant relationship between technical team competence and agile methodology project success. In contrast, however, Jiang and Klein (2000) found that team's expertise was significantly and positively related to the overall project effectiveness. Similarly, Chow and Cao (2008) found that having a team of high calibre is a significant CSF that contributes to the perceived agile project success.

Team's expertise can also reduce project team conflicts and create project team stability. Yetton et al. (2003) found that lack of development team's expertise can escalate project team's conflicts and create project team's instability and that both are positively correlated with variances in budgets and failure to complete the project on time. Likewise, Nah and Delgado (2006) found that team's skill contributed to ERP implementation success throughout all the stages of the project life cycle. Correspondingly, Nah and Delgado (2006) (2006) also suggest that where team's skills are not adequate to meet the required tasks or project operations, consultants should be called in to help. In the same vein, Wan and Wang (2010) found that enhancing the professional capabilities of an employee was correlated with agile methodology software process improvement. Agile methodologies require more senior and skilled staff to continuously adapt to change (Cockburn \& Highsmith, 2001). If a team lacks expertise, it is more appropriate to choose a traditional plan-based methodology with more structure or plan and pre-identified processes to correctly guide the project team members. Correspondingly, it can be postulated that:

H9 (T): There is a significant positive relationship between development team expertise and (i) process and (ii) product success for traditional plan-based methodologies $(T+)$.

H9 (A): There is a significant positive relationship between development team expertise and (i) process and (ii) product success for agile methodologies $(A+)$.

H9 (TA): Development team expertise is more important when agile methodologies are used than when traditional plan-based methodologies are implemented $(A+>T)$.

\subsubsection{Team's composition and project success}

Project team composition is all about which people constitute the project team and these individuals composing the team have characteristics that create the profile of the team. There is a debate on whether project team composition should be homogeneous or heterogeneous i.e. containing differences. Some homogeneous project teams may perform better due to similarities in experience, skills and thought, while heterogeneous project teams may perform better due to diversity and greater ability to take on multiple roles. What is clear though is 
that the composition of a project team is considered to have a strong influence on team processes and outcomes (Nah \& Delgado, 2006). The importance placed on team design derives from the need to align a team's composition with project goals and resources. Additionally, project teams can vary in size, work in various contexts, and have a variety of team structures and possess several competences. The team size can influence team composition and is also determined by the project task types, goals and processes.

According to Nah and Delgado (2006), the project team should be well balanced, crossfunctional, and have representatives from the internal 1T department as well as consultants. The top managers, vendors, IT department and consultants should be included on the team. This is consistent with Lacity and Willocks (2000, p.362) who demonstrate how customer IT stakeholders and supplier stakeholders are needed on project teams for IT outsourcing success.

Chow and Cao (2008) found that cross functional teams which involved customers contributed to successful agile SDM projects in terms of scope but not time, cost and quality. Similarly, Nah and Delgado (2006) found that software projects with best people on the team, made up of balanced cross-functional teams and working on a full-time basis were successfully implemented. Equally, Nah and Delgado (2006) established that teams composed of business, technical knowledge and consultants that were empowered in decision making had completed projects on time, budget, scope and quality. Agile methodologies require small teams that are autonomous and self-organizing with best skilled expertise and experience than traditional plan-based methodologies (Sheffield \& Lemetayer, 2013). Consistently, it can be postulated that:

$H 10(T):$ There is a significant positive relationship between project team composed of best people and ( $i$ ) process and (ii) product success for traditional plan-based methodologies $(T+)$.

$H 10$ (A): There is a significant positive relationship between project team composed of best people and (i) process and (ii) product success for agile methodologies $(A+)$.

H10 (TA): Project team composition is more important when agile methodologies are used than when traditional plan-based methodologies are implemented $(A+>T)$. 


\subsubsection{Hypotheses for vendor perception of customer factors}

The vendor perception of customer factors are user participation and support and experience. The next section discusses these factors in detail and their conjectured relationships with project success.

\subsubsection{User participation and project success}

The IT literature reveals that user participation significantly increases the likelihood of software development projects success. Empirical studies by Chow and Cao (2008), Misra et al. (2009) and Sheffield and Lemetayer (2012) have provided empirical data to support significant and positive relationship between user participation and software development project success. Although it can be argued that user participation tends to increase budget variance by encouraging suggestions for changes to specification, Yetton et al. (2000) contends that user participation also decreases budget variance by managing expectations and quickly resolving potential problems. Similarly, Jun et al. (2011) argues that resolving the potential conflicts early through greater user participation plays a vital role in the perceived satisfaction of software developers and users, which is a key dimension of project success.

Along similar lines, Jun et al. (2011) found a significant positive relationship between user participation and product performance. However, in Jun et al.'s (2011) study, the direct relationship between user participation and process performance was not supported. Similarly, Yetton et al. (2000) found that user participation tends to increase budget variance by encouraging suggestions for changes to specifications. Likewise, Nidumolu's (1995) findings revealed that increased interaction between users and IS staff does not necessarily lead to a project that converges well (i.e., improved project performance).

However, on the other hand, user participation is necessary for project success and participation in the requirements analysis stage can decrease the risk of there being insufficient requirements. Yetton et al. (2003) found that user participation increases the likelihood that the project is completed on time and not redefined or abandoned but can also increase budget variances. Equally, Chow and Cao (2008) found that having a strong customer involvement contributes to perceived agile methodology project success in terms of scope but not time, cost and quality. Agile methodology projects require onsite and full time user participation from specification phase up to the end; in contrast, however, traditional plan-driven methodology projects do not necessarily require user participation once requirements are clearly specified. Therefore, it can be hypothesized that: 
$H 11(T):$ There is no significant relationship between user participation and (i) process and (ii) product success for traditional plan-based methodologies (T\#).

H11 (A): There is a significant positive relationship between user participation and

(i) process and (ii) product success for agile methodologies $(A+)$.

H11 (TA): User participation is more important when agile methodologies are used than when traditional plan-based methodologies are implemented $(A+>T)$.

\subsubsection{User support and project success}

User support is the willingness and cheerful compliance of the customer to use and adapt to the new software (Jiang \& Klein, 2000). It is critical that the customer is willing to work as part of team members. As a member, the customer is expected to work together with project team to ensure that their needs are met. Thus, the customer will contribute to the requirements, approves the final result and making trade-offs between which features are added, changed or removed from a release. Compared to the traditional methods, in agile customers are more involved from the early beginning of the software development process till the end and it is desired to include customers as part of project team's adaptability. More willingness and involvement from customers can reduce difficulty to understand the process and output by the project teams. Active customer willingness and involvement provides the project team with a clear picture of the whole process and expected output. It helps project team to understand well and clear the scopes, objectives and requirements. Active willingness of customers also helps to verify the end product that cope their needs.

Jiang and Klein (2000) did not find a statistically significant relationship between user support (willingness) and project effectiveness. In contrast, however, Yetton et al. (2003) found that customer support increases the possibility that the project is completed on time and not redefined or abandoned but can also increase budget variances. Equally, Chow and Cao (2008) found that having a strong customer support contributes to the successful agile SDM projects in terms of scope but not time, cost and quality. Likewise, Misra et al. (2009) found a statistically significant and positive relationship between customer collaboration and agile SDM project success. Jun et al. (2011) also found a significant positive relationship between user support and product performance. However, traditional plan-driven methodology projects may not necessarily require user support once specifications are clearly done compared to agile methodology projects. Therefore, it can be theorized that:

\section{H12 (T): There is no significant relationship between user support and (i) process}


and (ii) product success for traditional plan-based methodologies (T\#).

H12 (A): There is a significant positive relationship between user support and (i) process and (ii) product success for agile methodologies $(A+)$.

H12 (TA): User support is more important when agile methodologies are used than when traditional plan-based methodologies are implemented $(A+>T)$.

\subsubsection{User experience and project success}

Jiang and Klein (2000) describe customer experience as a configuration of users who are very familiar with system development tasks and have wealthy experience with the activities to be supported by the future applications (p.6). Equally, Jun et al. (2011) defined users who lacked experience as characterized by not being familiar with the type of application, didn't know what they wanted, didn't have a good understanding of the problems they wanted solved and were not familiar with data processing as a work tool (p. 927). Additionally, Jun et al. (2011) argues that client experience and knowledge or understanding of requirements within a specific application area of the development team make it easy to define complete, unambiguous or consistent requirements, which can lead to a software product that can meet the client's needs, and decreasing process performance.

Equally, Chow and Cao (2008) found that involvement of experienced customers significantly and positively impacted on project scope but not timeliness, cost or quality. Likewise, Jiang and Klein (2000) found that lack of user experience significantly and negatively impacted on the quality of work done but no other success measures. Jun et al. (2011) also found that lack of user experience negatively and significantly affected project performance in terms of both process and product performance. This implies that the customer should have basic experience, training, education and knowledge about business domain (Mohammad \& Alshargabi, 2011). This knowledge makes them realistic to identify their requirements specifically which help to deal easily and achieve both process product performance. Similarly, customer experience and knowledge about constraints and limitations is critical since there is always constraints and limitation for every product. It is critical that the customer or person or group of people who deal with project team members has some basic experience or knowledge about constraints in hardware and software world. This helps producers to justify their selection of any specific hardware or software in regard to the project requirements and achieve project success. However, in comparison to traditional plan-based methodology projects, agile methodology projects require very 
experienced users that must be able to articulate their needs clearly otherwise the project cannot meet its objectives since developers heavily rely on client's feedback to make changes. Therefore, it can be advanced that:

H13 (T): There is no significant relationship between user experience and (i) process and (ii) product success for traditional plan-based methodologies (T\#).

H13 (A): There is a significant positive relationship between user experience and (i) process and (ii) product success for agile methodologies $(A+)$.

H13 (TA): User experience is more important when agile methodologies are used than when traditional plan-based methodologies are implemented $(A+>T)$.

\subsubsection{Hypotheses for vendor perception of project (uncertainty) factors}

Previous scholars (e.g., Jiang \& Klein 2000; Barki et al., 2001; Schmidt et al., 2001; Jun et al, 2011; Howell et al, 2010) have identified various risk or uncertainty factors that can threaten the successful completion of a software development project. However, the distinction between the concepts of risk and uncertainty remains ambiguous in literature (Ward \& Chapman, 2003). Both PMI (2013) and APM (2006) define the concept of risk as an uncertain event which might have positive events (opportunities) or negative effects (threats). PMI (2013) traditionally describes risk as an uncertain event (p. 310), however, it is not specified what "uncertainty" is moreover, uncertainty is not a self-explanatory term. Accordingly one can make a conclusion that risk is uncertainty (PMI, 2013, p.310). However, these two phenomena are not synonymous (Perminova et al., 2007). Petit (2012) asserts that the term risk is limited in scope and refers to events rather than being associated to more general sources of uncertainty. Other scholars (e.g., Cleden, 2009; Perminova et al., 2007, 2008; Ward \& Chapman, 2003) seem to advocate for the use of a broader concept of uncertainty management instead of risk management. Consistently, this study adopts uncertainty management perspective instead of traditional risk management, despite the latter being more established in the project management community. The next section discusses different vendor perception of project or uncertainty factors in detail and their conjectured relationships with project success.

\subsubsection{Technological uncertainty and project success}

Technological uncertainty refers to the ambiguity concerning technologies such as tools and techniques needed to convert requirements to a software system (Nidomolou, 1995, p.195). According to Nidomolou (1995) technological uncertainty entails both technological unpredictability and technological analysability. Technological unpredictability describes the 
extent to which unexpected and novel events occur during the conversion process while technological analysability designates the extent to which the task of converting requirements specifications to software can be undertaken using well-established procedures (p.202). Thus, it arises when doing something that is scientifically or technologically impossible, or of how to achieve it in practice, is not publicly available or deducible by a competent professional working in the field. Accordingly, technology uncertainty might also have other different dimensions such as dynamism and complexity (Boeh \& Turner, 2003; Chow \& Cao, 2008).

Technological uncertainty can influence decisions to abandon, redefine or complete a project. Equally, technological uncertainty could have significant influence on budget (time-cost) overruns, because of the unproven availability, performance, timeliness and functionality of new products and services. Surprisingly, according to Yetton et al. (2003), the technical risks of the project do not influence budget variances. Similarly, Jiang and Klein (2000) found that technological acquisition risks did not significantly impact on the project effectiveness. These findings appear unusual and possibly could be attributed to sampling error and statistical chance effects.

On the other hand however, Jun et al. (2011) found that the use of unfamiliar technologies lead to software problems that reduce the performance of the software product and delay the project. This appears to suggest that the use of new technologies and technological changes that alter system design are key sources of project uncertainty in software development projects. The application of advanced technologies such as distributed data processing or Artificial Intelligence that otherwise push off the boundaries of current technological capabilities; equally, increase the risk of project failure. The organization's experience with technology e.g. the hardware, operating systems, databases, and application languages can be constrained leading to project failure. Unlike traditional plan-based, agile development methodologies were developed to respond to change and uncertainty in requirements and to reduce the cost of change throughout the project (Cockburn \& Highsmith, 2001). New technologies may not go as planned and require a team to adapt as they learn and gradual learning is more suitable for agile methodologies as they use short iterations and constant feedback. Therefore, it can be advanced that:

$H 14(T):$ There is a significant negative relationship between technological uncertainty and (i) process and (ii) product success for traditional plan-based methodologies (T-). 
H14 (A): There is a significant negative relationship between technological uncertainty and (i) process and (ii) product success for agile methodologies (A-).

H14 (TA): Technological uncertainty has a greater negative effect when traditional planbased methodologies are used than when agile methodologies are implemented ( $T$ $>A)$.

\subsubsection{Technical complexity and project success}

In general usage, complexity is used to describe something with many parts in an intricate arrangement and the study of these complex linkages is the main goal of complex systems theory. Specifically, technical complexity simply refers to the degree of predictability about, and control over, the final product permitted by the technology used. Howell et al. (2010) asserts that complexity difficulties essentially focus on ability to predict the relationship between inputs and outputs synonymous with uncertainty (p.260). According to Barki et al. (2001) the technical complexity of a project includes: hardware (computers and networks), software or databases.

Jiang and Klein (2000) found that application complexity did not significantly impact on the project effectiveness in terms of quality of work done, meeting schedules, operation efficiency, and operation speed and meeting budgets. In their study, application complexity was examined in terms of technical complexity (e.g., hardware, software, and database), large number of links to existing systems and large number of links to future systems. However, in contrast, Jun et al. (2011) reported that technical complexity adversely and negatively affected software project performance in terms of both process and product performance. In Jun et al's (2011) study, technical complexity was examined in terms of: projects that involve the use of new technology, projects that have high level of technical complexity and projects that involve the use of technology that has not been used in prior projects. In terms of methodologies, whereas Wysocki (2009, p.312) indicates that as the level of technical complexity increases so does the importance of process flexibility and the need to be creative and adaptive delivered by agile methodologies, Jiang and Kline (2000, p.77) argue that complexity requires structure and discipline that comes from traditional methodologies. Likewise, Highsmith (2010) argues that dealing with complexity requires structure and discipline provided by traditional plan-based methodologies.

Therefore, it can be hypothesized that: 
H15 (T): There is a significant negative relationship between technical complexity and

(i) process and (ii) product success for traditional plan-based methodologies (T-).

H15 (A): There is a significant negative relationship between technical complexity and $(i)$ process and (ii) product success for agile methodologies (A-).

H15 (TA): Technical complexity has a greater negative effect when agile methodologies are used than when traditional plan-based methodologies are implemented $(A->T)$.

\subsubsection{Relative project size and project success}

Relative project size can be defined in terms of number of person-days, the scheduled number of months and the dollar budget allocated to the project (Jun et al., 2011, p. 928). Jiang and Klein (2000) describe relative project size based on the number of people on team, different stakeholders on team, the number of users who use the system and the number of hierarchical levels occupied by users who use the system (p.6). In some circumstance, some scholars have used project team size and relative project size interchangeably (Chow \& Chao, 2008: Misra et al. 2009; Jun et al, 2011; Sheffield \& Lemetayer, 2013).

Boehm and Turner (2003) identified project size as one of the candidate CSFs of software development project success. Empirical evidence reveals that project size in terms of effort and duration can negatively affect project performance (Sauer et al., 2007, p. 81). Large projects are likely to be characterized by high complexity and high levels of task interdependence (Yetton et al., 2003). The increase of project size can complicate project planning and coordination thereby negatively affecting both process and product performance. In large projects, communication increases making coordination impossible, and the process of sharing information gets constrained as the number of people on a project increases.

Surprisingly, Yetton et al.'s (2003) study did not find budget variances as a positive function of project size. Yet, budget variances are expected to increase on complex, interdependent tasks. Instead, Yetton et al.'s (2003) study found that project completion was a negative function of project size. This is possibly because large projects are likely to have project passed times which extend across annual budget cycles. Not only are they subject to potential budget variances, they are also likely to be redefined or abandoned as business demands and external conditions change over time. 
Likewise, Jun et al. (2011) found that the project size was negatively and significantly associated with project performance in terms of both process and product performance. Sauer et al.'s (2007) empirical evidence suggests that large project size negatively affects project performance. Boehm and Turner (2003) argue that it is very difficult to apply agile methodologies to large projects whereas it is more rigid and cumbersome to apply traditional plan-based methodologies to small projects. Therefore, it can be conjectured that:

$H 16(T)$ : There is a significant negative relationship between relative project size and $(i)$ process and (ii) product success for traditional plan-based methodologies ( $T$-).

H16 (A): There is a significant negative relationship between relative project size and $(i)$ process and (ii) product success for agile methodologies (A-).

H16 (TA): Relative project size has a greater negative effect when agile methodologies are used than when traditional plan-based methodologies are implemented $(A->T)$.

\subsubsection{Specification changes and project success}

Requirements analysis is one of the most important stages in the software development process, because it has the greatest impact on future stages. Although the project must be completed within the confined resources; it must meet the requirements of customers in the market which are usually dynamic. Changes in specifications are usually an outward imposed factor on the success of a project. In this regard, at the beginning of many projects, users and analysts do not have a clear sense of what is needed, leading to an ambiguous and incomplete set of requirements. This is especially true for applications that are new or involve unstructured tasks. Incomplete, ambiguous or inconsistent requirements due to frequent changes in them make it difficult to predict performance outcomes, because they often necessitate redoing requirements analysis. Lack of information about requirements therefore makes it difficult to predict the time and effort that the project will consume, thus increasing its performance risk.

Boehm and Turner (2003) suggest that specification changes can negatively affect project performance. Ratbe et al. (2000) found that changes in specifications negatively affect project success. Equally, Nidumolu (1995) found that requirements instability had a negative effect on project performance. Too much user specification changes may have a negative effect on project success and in particular, variations in delivery time, scope and budget (Nidumolu, 1995). However, agile methodology projects are likely to be successful if the requirements change rate is high since they have been specifically developed to address the problems of 
rapid change. Iterative delivery helps reduce uncertainty and leads the project through uncertainty while basing on customer feedback for continuous quality improvements, unlike for traditional approaches that plan for every task in advance and many of them will not be executed if changes occur. In the case of a low specification changes, the traditional methodology and its big, up-front design works best. As there is no or little change expected during the project and the plan does not need to be modified. Future features are prepared in the design and all the pieces are designed to fit well together. Thus, it can be hypothesised that:

$H 17(T):$ There is a significant negative relationship between specification changes and (i) process success and (ii) product success for traditional plan-based methodologies ( $T$-).

H17 (A): There is a significant negative relationship between specification changes and (i) process success and (ii) product success for agile methodologies (A-).

H17 (TA): Specification change has a greater negative effect when traditional plan-based methodologies are used than when agile methodologies are implemented (T->A).

\subsubsection{Project criticality and project success}

Criticality refers to "the effects or consequences of the unexpected events i.e. what can be lost if something unforeseen and unmanageable happens" (Howell et al., 2010, p.260). Little (2005) treated criticality as the major influence of project success arguing that if the project puts lives or business-critical functions at risks, then it should be treated differently than if the only cost of failure is the project investment. Criticality is also related to project size although small and simple IT projects can have considerable monetary or safety consequences; criticality, particularly in financial terms, tends to be comparative to project size for software projects (Howell et al., 2010).

Cockburn (2007) divides criticality into four categories defined in terms of the loss caused by defects in the operational product: (1) Loss of comfort where a defect only has a very minor impact. (2) Loss of discretionary monies where a defect can be fixed pretty easily because of good backup procedures. According to Cockburn (2007), most software projects' criticality is at this level. (3) Loss of essential monies where the company may go bankrupt because of a defect in the system software. (4) Loss of life where defects can be catastrophic, such as in nuclear power stations or flight control systems. Boehm and Turner (2003) added another category to distinguish between the loss of a single life and the loss of several lives. 
When criticality increases, the controls in place should be tighter and the tolerances should decrease hence traditional plan-based methodologies are more appropriate (Cockburn, 2007). However, one can also argue that continuous testing and pair programing used in Agile methodologies may reduce the chances of a defect to occur. Although criticality has often been cited in IT project management literature as a candidate CSF (Imreh \& Raisinghani, 2011; Howell et al., 2010; Lindvall et al., 2002; Little, 2005; Boehm \& Turner, 2003; Dyba \& Dingsor, 2008), its direct link with project success has been rarely examined. However, in one such recent study, Sheffield and Lemetayer (2013) did not find a direct relationship between low project criticality and software development agility. Therefore, in order to investigate this relationship further, it can be proposed that:

$H 18(T):$ There is a significant negative relationship between project criticality and (i) process and (ii) product success for traditional plan-based methodologies (T-).

H18 (A): There is a significant negative relationship between project criticality and (i) process and (ii) product success for agile methodologies (A-).

H18 (TA): Project criticality has a greater negative effect when agile methodologies are used than when traditional plan-based methodologies are implemented $(A->T)$.

\subsubsection{Hypotheses on other interrelationships between candidate CSFs and moderation}

The literature review on the interrelationships between candidate CSFs themselves and how they interact to influence project success remains highly anecdotal. The primary intent of previous studies appears to have been testing and establishing direct relationships between candidate CSFs and project success. Thus, interrelations are often inferred as part of explaining mechanisms for these direct relationships. No previous studies though have included such interrelationships in their models. E.g., neither Misra et al (2009) nor Chow and Chao (2008) hypothesize interrelationships in their studies. However, these significant correlations may be in part due to the combined impact of indirect and interrelationship effects. Thus, given the earlier review of contingency theory, it seems important to allow effects to be estimated in the modelling. Furthermore, given the numerous potential interrelationships and indirect effects, explicitly hypothesising these relationships might divert the focus to these complex interactions or indirect effects. Instead, the aim here is to include such effects so that findings supporting the presence or lack of a direct significant relationship are not due to the omission of indirect/mediating pathways to project success. 


\subsubsection{Moderating effects of project management methodology}

Jun et al. (2011) findings show project planning and controlling have a bigger impact on process or product success of low risk software projects (traditional plan-based methodology) than for projects perceived with high risk (agile methodology projects). Jun et al. (2011) also found internal project communication has a bigger effect on process or product success of high risk (agile methodology) projects than low risk (traditional plan-based methodology projects). Likewise, user participation and support has a greater contribution to process or product performance for high risk projects or agile methodology projects than traditional plan-driven methodology projects perceived to have low risks (Jun et al., 2011). Zwikael et al. (2014) found that in the presence of high risk, increasing the quality of planning improves project efficiency, whereas in the presence of low risk, it improves project effectiveness. According to Sheffield and Lemetayer (2013), if a project has high technological uncertainty, high specification changes, high development team expertise and high user experience, agile methodologies are likely be effective and the reverse is true for traditional plan-based methodologies.

Similarly, according to Boehm and turner (2003), software projects that thrive in a chaotic culture with a high rate of requirements changes (dynamism) are more likely to follow an agile methodology rather a traditional plan-based methodology. Likewise small sized projects that are less critical are likely to be successful if managed with agile methodologies and vice versa (Charvart, 2003). Thus, if the top level management of an organization supports an adaptive behaviour, flexible leadership styles and entrepreneurial culture, they are more likely to support agile methodologies and not traditional plan-based methodologies (Ceschi et al., 2005; Boehm \& Turner, 2005). Agile methodologies are likely to be more successful than traditional methodologies for projects that operate in a culture of teamwork, flexibility and participation with social interaction (Vinekar et al., 2006; Strode et al., 2009). Hence, although the direction of each moderating effect may not be clear from the literature, based on this discussion, it can be hypothesized that: H19: Project management methodology moderates the relationships between candidate CSFs and project success.

\subsubsection{Moderating effects of vendor perception of project (uncertainty) factors}

Empirical research evidence indicates that the influence of different candidate CSFs to project success can be moderated by various risks or vendor perception of project (uncertainty) factors (Jun et al., 2011). Barki et al. (2001) found that project risks moderated 
the relationship between internal integration (e.g. integration of technological and business process knowledge including communication) and project success. Jun et al (2011) found that inherent project uncertainty had a moderating effect on the relationships between planning and control, internal integration, user participation and project performance. By investigating the effectiveness of planning through an analysis of 183 project manager-supervisor dyads, results by Zwikael et al. (2014) show that the level of risk moderates the impact of planning on success, and in different ways for various success measures. Sheffield and Lemetayer (2013) also assert that decisions about the best way of planning and controlling are influenced by the level of risk. Hence, although the direction of the interaction for all candidate CSFs with project uncertainty is not clear from the literature, and may vary across various vendor perception of project (uncertainty) factors and various project success measures, the following hypotheses can be formulated:

H20: Technological uncertainty moderates the relationships between candidate CSFs and project success.

H21: Technical complexity moderates the relationships between candidate CSFs and project success. H22: Relative project size moderates the relationships between candidate CSFs and project success. H23: Specification changes moderate the relationships between candidate CSFs and project success. H24: Project criticality moderates the relationships between candidate CSFs and project success.

\subsubsection{The conceptual research model including the associated hypotheses}

Figure 33 illustrates the research model that was pilot tested and used in this research. The constructs are depicted and the research hypotheses are indicated (H1-H24). 


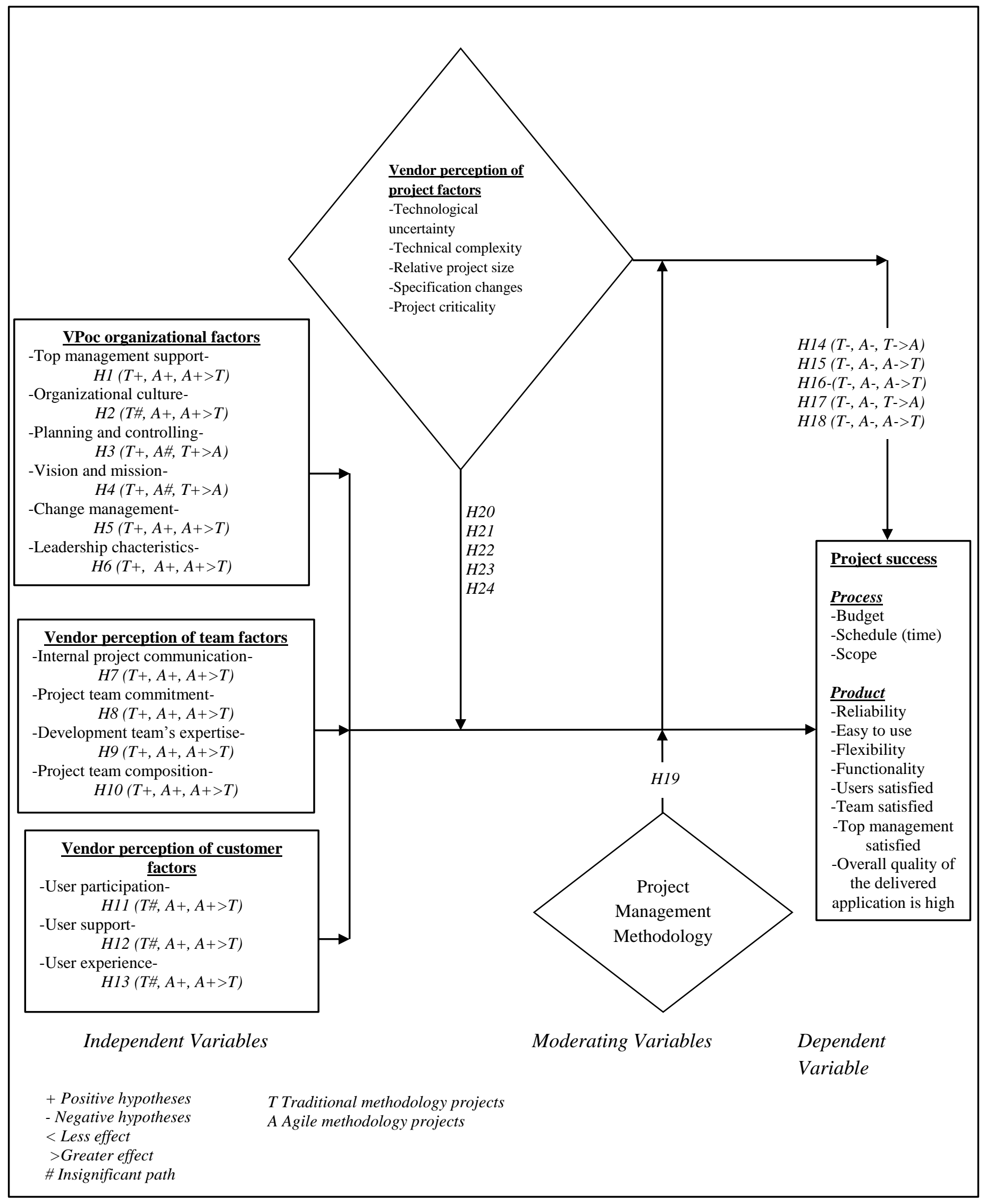

Figure 33: Research model including hypotheses 
Table 15 summarises the research hypotheses of this study and relates them to previous studies that have tested the underlying relationships between similar constructs. 
Table 15: Summary of research hypotheses and supporting references Hypothesis

H1 (T): There is a significant positive relationship between TMS and (i) process and (ii) product success for traditional plan-based methodologies $(T+)$.

HI (A): There is a significant positive relationship between TMS and (i) process and (ii) product success for agile methodologies $(A+)$.

H1 (TA): TMS is more important when agile methodologies are used than when traditional planbased methodologies are implemented $(A+>T)$.

$H 2$ (T): There is no significant relationship between flexible organizational cultural and (i) process and (ii) product success for traditional plan-based methodologies (T\#).

$H 2$ (A): There is a significant positive relationship between flexible organizational cultural and $(i)$ process and (ii) product success for agile methodologies $(A+)$.

$H 2$ (TA): Flexible organizational culture is more important when agile methodologies are used than when traditional plan-based methodologies are implemented $(A+>T)$.

$H 3(T)$ : There is a significant positive relationship between project planning and controlling and ( $i)$ process and (ii) product success for traditional plan-based methodologies $(T+)$.

H3 (A): There is no significant relationship between project planning and controlling and (i) process and (ii) product success for agile methodologies (A\#).

H3 (TA): Project planning and controlling are more important when traditional plan-based methodologies are used than when agile methodologies are implemented $(T+>A)$.

$H 4(T)$ : There is a significant positive relationship between vision and mission and (i) process and (ii) product success for traditional plan-based methodologies $(T+)$.

$H 4$ (A): There is no significant relationship between vision and mission and (i) process and (ii) product success for agile methodologies (A\#).

H4 (TA): Vision and mission are more important when traditional plan-based methodologies are used than when agile methodologies are implemented $(T+>A)$.

$H 5(T)$ : There is a significant positive relationship between change management and (i) process and (ii) product success for traditional plan-based methodologies $(T+)$.

H5 (A): There is a significant positive relationship between effective change management and (i) process and (ii) product success for agile methodologies $(A+)$.

H5 (TA): Change management skills are more important when agile methodologies are used than when traditional plan-based methodologies are implemented $(A+>T)$.

H6 (T): There is a significant positive relationship between client's effective leadership characteristics and (i) process and (ii) product success for traditional plan-based methodologies $(T+)$.

H6 (A): There is a significant positive relationship between client's effective leadership characteristics and (i) process and (ii) product success for agile methodologies $(A+)$.

H6 (TA): Effective leadership is more important when agile methodologies are used than when traditional plan-based methodologies are implemented $(A+>T)$.

$H 7(T):$ There is a significant positive relationship between internal project communication and $(i)$ process and (ii) product success for traditional plan-based methodologies $(T+)$.

$H 7(A)$ : There is a significant positive relationship between internal project communication and $(i)$ process and (ii) product success for agile methodologies $(A+)$.

$H 7(T A):$ Internal project communication is more important when agile methodologies are used than when traditional plan-based methodologies are implemented $(A+>T)$.

$H 8(T)$ : There is a significant positive relationship between project team commitment and ( $i)$ process and (ii) product success for traditional plan-based methodologies $(T+)$.

$H 8(A)$ : There is a significant positive relationship between project team commitment and (i) process and (ii) product success for agile methodologies $(A+)$.

H8 (TA): Project team commitment is more important when agile methodologies are used than when traditional plan-based methodologies are implemented $(A+>T)$.

$H 9(T)$ : There is a significant positive relationship between development team expertise and $(i)$ process and (ii) product success for traditional plan-based methodologies $(T+)$.

$H 9$ (A): There is a significant positive relationship between development team expertise and (i) process and (ii) product success for agile methodologies $(A+)$.

H9 (TA): Development team expertise is more important when agile methodologies are used than when traditional plan-based methodologies are implemented $(A+>T)$.

$H 10(T)$ : There is a significant positive relationship between project team composed of best people and (i) process and (ii) product success for traditional plan-based methodologies $(T+)$.

$H 10$ (A): There is a significant positive relationship between project team composed of best people and $(i)$ process and (ii) product success for agile methodologies $(A+)$.

H1O (TA): Project team composition is more important when agile methodologies are used than when traditional plan-based methodologies are implemented $(A+>T)$.

$H 11(T)$ : There is no significant relationship between user participation and (i) process and (ii) product success for traditional plan-based methodologies (T\#).

Supporting references Nah et al. 2001; Jung et al. 2008; Nah \& Delgado 2006; Imreh \& Raisinghani 2011; Mishra \& Mishra, 2011; Dyck \& Majchrzak, 2012

Strode et al., 2009; Chow \& Cao, 2008; Misra et al. 2009; Wan \& Wang, 2010; Sheffield \& Lemetayer, 2013; Iivari \& Huisman 2007

Jun et al. 2011; Misra et al. 2009; Wallace et al. 2004; Barki et al. 2001; Yetton et al. 2000

Jiang et al. 2008; Nah \& Delgado, 2006; Wan \&Wang, 2010

Nah et al. 2006; Jiang et al. (2008); Wan \& Wang 2010; Nah \& Delgado, 2006

Misra et al. 2009; Lindvall et al. 2002; Strode et al. 2009; Schmidt et al., 2001; OGC, 2005; Baccarini et al., 2004; Humphrey, 2005; Standing et al., 2006

Jun et al., 2011; Misra et al. 2009; Jiang \& Klein 2000; Yetton et al. 2000; Ahimbisibwe \& Nangoli, 2012

Chow \& Cao 2008; Strode et al. 2009; Ahimbisibwe and Nangoli 2012

Jun et al, 2011; Wan \&Wang 2010; Nah \& Delgado, 2006; Misra et al. 2009; Yetton et al.2003;

Jung \& Klein 2000

Nah \& Delgado (2006); Chow\& Cao (2008)

Jun et al. (2011) Chow \& Cao, 2008; Yetton et al., 
H11 (A): There is a significant positive relationship between user participation and (i) process and (ii) product success for agile methodologies $(A+)$.

$H 11$ (TA): User participation is more important when agile methodologies are used than when traditional plan-based methodologies are implemented $(A+>T)$.

H12 (T): There is no significant relationship between user support and (i) process and (ii) product success for traditional plan-based methodologies (T\#).

H12 (A): There is a significant positive relationship between user support and (i) process and (ii) product success for agile methodologies $(A+)$.

H12 (TA): User support is more important when agile methodologies are used than when traditional plan-based methodologies are implemented $(A+>T)$.

$H 13(T):$ There is no significant relationship between user experience and (i) process and (ii) product success for traditional plan-based methodologies (T\#).

$H 13$ (A): There is a significant positive relationship between user experience and (i) process and (ii) product success for agile methodologies $(A+)$.

$H 13$ (TA): User experience is more important when agile methodologies are used than when traditional plan-based methodologies are implemented $(A+>T)$.

$H 14$ (T): There is a significant negative relationship between technological uncertainty and $(i)$ process and (ii) product success for traditional plan-based methodologies ( $T$-).

$H 14$ (A): There is a significant negative relationship between technological uncertainty and (i) process and (ii) product success for agile methodologies (A-).

H14 (TA): Technological uncertainty has a greater negative effect when traditional plan-based methodologies are used than when agile methodologies are implemented $(T->A)$.

$H 15$ (T): There is a significant negative relationship between technical complexity and (i) process and (ii) product success for traditional plan-based methodologies ( $T$-).

$H 15$ (A): There is a significant negative relationship between technical complexity and (i) process and (ii) product success for agile methodologies (A-).

H15 (TA): Technical complexity has a greater negative effect when agile methodologies are used than when traditional plan-based methodologies are implemented $(A->T)$.

$H 16$ (T): There is a significant negative relationship between relative project size and (i) process and (ii) product success for traditional plan-based methodologies (T-).

H16 (A): There is a significant negative relationship between relative project size and (i) process and (ii) product success for agile methodologies $(A-)$.

H16 (TA): Relative project size has a greater negative effect when agile methodologies are used than when traditional plan-based methodologies are implemented $(A->T)$.

$H 17(T)$ : There is a significant negative relationship between specification changes and (i) process success and (ii) product success for traditional plan-based methodologies (T-).

$H 17$ (A): There is a significant negative relationship between specification changes and ( $i$ ) process success and (ii) product success for agile methodologies (A-).

$H 17$ (TA): Specification change has a greater negative effect when traditional plan-based methodologies are used than when agile methodologies are implemented (T->A).

$H 18(T)$ : There is a significant negative relationship between project criticality and (i) process and (ii) product success for traditional plan-based methodologies (T-).

$H 18$ (A): There is a significant negative relationship between project criticality and (i) process and (ii) product success for agile methodologies $(A-)$.

H18 (TA): Project criticality has a greater negative effect when agile methodologies are used than when traditional plan-based methodologies are implemented $(A->T)$.

H19: Project management methodology moderates the relationships between candidate CSFs and project success.

H20: Technological uncertainty moderates the relationships between candidate CSFs and project success.

H21: Technical complexity moderates the relationships between candidate CSFs and project success.

H22: Relative project size moderates the relationships between candidate CSFs and project success.

H23: specification changes moderate the relationships between candidate CSFs and project success.

H24: Project criticality moderates the relationships between candidate CSFs and project success.
2003 ;Misra et al.,2009; Sheffield \&

Lemetayer, 2012;

Nidumolu, 1995

Jiang \& Klein, 2000;

Jun et al.,2011;

Misra et al., 2009;

Yetton et al., 2003

Jiang \& Klein, 2000;

Jun et al., 2011;

Chow \& Cao, 2008

Jun et al., 2011;

Yetton et al., 2003;

Jiang \& Klein 2000;

Nidomolou, 1995

Nidomolou, 1995;

Jun et al. 2011;

Yetton et al. 2003;

Jiang and Klein, 2000

Howell et al., 2010; Sauser

et al., 2009; Shenhar \&

Dvir, 2007; Jiang \& Klein,

2000; Yetton et al., 2003;

Jun et al., 2011 ; Dvir et al., 2007

Jun et al., 2011; Sheffield \&

Lemetayer, 2013; Yetton et

al., 2003; Jiang \& Klein,

2000; Chow \& Chao, 2008;

Misra et al. 2009

Boehm \& Turner, 2003;

Cockburn, 2007; Shefffield

\& Lemetayer, 2013

Barki et al., 2001); Strode

et al., 2009; Jun et al.,

2011; Shefffield \&

Lemetayer, 201

Barki et al., 2001;Jun et al., 2011; Zwikael et al., 2014

Barki et al.,2001; Jun et al., 2011

Barki et al., 2001; Jun et

al.,2011

Barki et al.,2001; Jun et al., 2011

Boehm \& Turner, 2003;

Cockburn, 2007; Shefffield

\& Lemetayer, 2013 
This section introduced the research model to be pilot-tested in this study and stated the hypotheses. The next section reports on a relevant check that was conducted to assess the relevancy of the concept of fit between project management methodologies and candidate CSFs.

\subsubsection{Preliminary relevant checks with software development project practitioners}

In order to ensure that the research outcomes of this are valuable to practitioners, some preliminary checks were conducted during the early stages of this research. These preliminary checks are evaluations by practitioners of the theories, model and frameworks that the academic community either uses or produces. In this approach, the relevancy of the research can be improved and the researcher can ascertain if the conceptual research project is appreciated or would be found useful by the industry.

In this regard, an exploratory investigation was conducted to discover practitioners' views of vendors towards the proposed conceptual framework and the research generally. The next section presents the methodology used to conduct the preliminary relevant checks with senior software development project managers and introduces the profile of research participants. The section concludes by outlining the key evaluations of the conceptual framework.

\subsubsection{Methodology used to discuss the conceptual framework with practitioners}

In order to solicit for participation, project managers from different private and public organizations were contacted through the monthly meetings of project management in Wellington, New Zealand. These groups include: PRINCE2 User Group, PMI and Agile. During these meetings, several contacts were made, requests for meeting appointments initiated and subsequently these appointments were confirmed through e-mail or phone. Overall, 9 senior project managers volunteered to participate and were interviewed for the relevant checks. All the interviews were informal and conducted in Wellington city, New Zealand. The purpose of the interview was to assess how senior project managers perceive the concept of fit of methodologies across the project types of soft development projects. The interviews took place between May and June 2013. All the 9 interviews were conducted face to face and lasted between 30 and 60 minutes. All the interviews were conducted separately, at different venues and times across the city. During these interviews, initially the concept of fit between project management methodologies and project types was introduced and 
subsequently discussed with each project manager. The field notes were taken by the interviewer but discussions were not tape recorded.

Table 16 introduces the research participant's profile. The participants' characteristics (e.g. experience in software development projects, current position, education and the organization) as well as their gender are summarized.

Table 16: Research participants who contributed to the conceptual model discussion

\begin{tabular}{|l|l|}
\hline $\begin{array}{l}\text { Professional } \\
\text { affiliation }\end{array}$ & \multicolumn{1}{c}{ Participant's characteristics } \\
\hline \multirow{4}{*}{ PRINCE2 } & 1: Experienced in software development (38 years), senior projects advisor, bachelors computer science, public organization, male \\
\cline { 2 - 3 } & 2: Experienced in software development (26 years), project manager consulting, MBA, private organisation, female \\
\cline { 2 - 3 } PMI & 3: Experienced in software development (16 years), project manager, masters IT, private organisation, female \\
\cline { 2 - 3 } & 5: Experienced in software development (22 years), senior project manager, bachelor programming, private organisation, male \\
\cline { 2 - 3 } Agile & 6:Experienced in software development (28 years), software integrator manager, bachelors software engineering, public organisation, female \\
\cline { 2 - 2 } & 7: Experienced in software development (24 years), software development manager, bachelors software design, private organisation, female \\
\cline { 2 - 2 } & 9: Experienced in software development (25 years), software tester/ Analyst, bachelors software engineering, private organisation, male \\
\hline
\end{tabular}

\subsubsection{Preliminary research findings from discussing the conceptual framework with practitioners}

Generally, the discussions indicated that the research participants perceived concept of fit as being valuable concept for project management and success. They argued that currently project workers and managers do not have any well-established framework to judge which projects types fit each development methodology.

For instance, Participant 1 argued:

"It would be valuable for us to have a framework that guides us in matching methodologies and project types. Although I have personally used the traditional project management methodology for 38 years, and also applied agile occasionally, am not comfortable with combining both because of value conflict. Having a contingency fit model would be very useful in this regard."

Similarly, Participant 2 said that:

"For my previous SD project that I worked on, it was an organizational policy to use PRINCE2 as a methodology. However, I had to use my experience and combine it with some iterative approaches to adapt to the project environment and project characteristics. Otherwise, I was getting trouble all the time! It was always a wrong methodology selected. If such a contingency fit model existed, it would have been very helpful here." 
Accordingly Participant 3 observed that:

"In many cases instead of the project manager, executives are the people who choose which methodology will be used on the project. So it is messed up! I was forced to abandon agile methodology on the project and replace it by a plan-driven approach for unclear or 'political reasons', yet the project was showing good signs of success. The consequence was a failure to deliver anything. Executives had to recognize their mistake and because of the lack of results, again allowed me to use agile”.

In addition the participants argued that it is always an organizational policy to use a given project methodology irrespective of project environment or characteristics especially in public sector. As a result, project managers are always challenged.

For instance, Participant 4 reasoned that:

"About choosing the right methodology for a given SD project, it's very important for project success. However, in most public organizations that I have worked for, it is always an organizational policy to use PRINCE2.

Participant 6 also revealed that the concept of fit is critical in SD but unfortunately most people are not familiar with it.

"It's absolutely very essential for project success but not sure of which methodology is better. 'It all depends on circumstances'. However, in most projects I have worked for, am the one who proposes the project methodology. Most business owners do not know or understand methodology issues because it is more technical."

Additionally Participant 7 said that:

"Big organisations dictate methodologies. Obviously, project characteristics are important for methodology selection. May be this model can help to explain this."

Participant 8 made another observation that:

'When a wrong project management methodology is implemented, it hinders project success. When a wrong methodology is imposed on a project team, they are more likely to spend most of the time fighting the methodology rather than doing the project work." 
Finally, Participant 9 had this to say:

"Refer to KPMG report (2013); 90\% of SD projects that are successful usually follow some correct development methodology. Methodology is very important but the challenge is how to select them because now we don't have a clear criterion per se".

In summary, all nine experienced senior project managers confirmed that a contingency fit model and a study examining the impact of fit between development methodologies and candidate CSFs would be beneficial for software development project management. Equally, no manager suggested major modifications of the contingency fit model. It was also interesting to note that all project managers welcomed the preliminary relevance checks and they appreciated their involvement in academic research.

\subsection{Chapter summary}

This chapter introduced the conceptual research model and established the research hypotheses outlining the relationships between the constructs. The research model was displayed with the associated hypotheses and the research hypotheses were summarised. The last section of this chapter reported the findings of preliminary relevance checks that were conducted with project managers. Nine senior vendor managerial staff of various projects in Wellington, New Zealand, were interviewed about their perceptions towards the fit concept. Overall, all interviewees confirmed that the fit theory would be valuable for software development projects and organizations applying various related project management approaches. The next chapter outlines the research design proposed for this study. 


\section{CHAPTER FOUR: METHODOLOGY}

\subsection{Introduction}

The previous chapter presented the conceptual research model and established the research hypotheses outlining the relationships between the constructs. This chapter describes the methodology used in addressing the set of objectives and hypotheses. The chapter covers the research paradigms, research design, sampling design, data collection methods, research instruments, operationalization and measurement of variables and the feedback on the pilot study. It concludes by discussing the main phase of data collection and data analysis.

\subsection{Research philosophy}

Research in project management has adopted diverse research methodologies. This study adopts the positivistic philosophical view based on the ontological assumption that reality is external and objective (Cavana et al., 2001, p.8). This research philosophy is deemed appropriate here because the research aims to test hypotheses through an empirical scientific process with measurements to identify and establish the relationships between latent variables and obtain statistically significant findings that can be generalized to a population under study.

\subsection{Research design}

Consistent with positivists' studies, a quantitative approach was chosen and it aimed to collect a large data sample to generate findings that are statistically significant and which could be generalized. A cross-sectional study, which looks at a cross-section of each population at a single point in time, enabled gathering of data from a large number of people, and comparing these data (Cavana et al., 2001, p.121).

Some scholars (e.g. Killen et al., 2012; Soderland, 2004) have documented debate on research design in project management. However, the decision and choice regarding which approach to take greatly depends on personal beliefs and the way the researcher regards human action as opposed to the choice between the diverse alternatives (Cavana et al., 2001). Although the literature reveals that quantitative studies in project and portfolio management are limited, they exhibit diversity in terms of theoretical foundations, significance, strengths, direction and findings (Killen et al., 2012). Those interested in generating an in-depth understanding of the project management phenomena and processes have analyzed small 
sample case studies, while those based on medium to large samples have rather adopted a cross-sectional and quantitative approach (Soderland, 2004).

A review of current literature reveals that most studies in project and portfolio management have been non-empirical in nature, consisting of conceptual reviews (Sanderson, 2012; Killen \& Kjaer, 2012; Nasir \& Sahibuddin, 2011; Imreh \& Raisinghani, 2011) and opinion-type contributions (Bajwa et al., 2012; Singh et al., 2012; Hass, 2008), while a few have been empirical based on case studies (Petit, 2012; Adolph et al., 2012; Mishra \& Mishra, 2012). Among these empirical studies, very few have adopted quantitative approaches (Misra et al., 2009; Chow \& Cao, 2008; Lee \& Xia, 2010; Jun et al., 2011; Keren et al., 2010; Sheffield \& Lemetayer, 2013; Zwikael et al., 2014), while many have adopted a qualitative approach (e.g., Hajjidiab et al., 2012; Sauser et al., 2008), with limited or no triangulation of methods to increase the credibility and validity of the results.

\subsection{Study population}

The study population comprised of all software development projects using the senior project managers (vendors) who are engaged in working on software development projects around the world as the respondents. The unit of analysis was projects while the project managers were the units of inquiry. The software development project methodologies are seen to vary from plan-driven projects to agile projects. Typically, because these software developers are affiliated to various professional bodies of software development or communities of practice (e.g. PRINCE2, PMI and Agile), each with different beliefs and values, these aforementioned communities of practice provide different guidelines for managing software projects and have a significant influence on the selection of the development methodology to be used.

There is no concrete information on the definite number of members for these professional bodies globally. What is clear is that PRINCE2 is used extensively by the UK Government and also widely recognised and sometimes adopted in the private sector, both in the UK and internationally. It is a structured project management method endorsed by the UK government as the project management standard for public projects. Similarly, PMI is also one of the world's largest not-for-profit membership associations for the project management profession, with more than 700,000 members and credential holders in more than 185 countries (PMI, 2013). In contrast, Agile software development is a group of software development methods based on iterative and adaptive development, where requirements and 
solutions evolve through collaboration between self-organizing and cross-functional teams, with more than 80,000 signatories globally (Agile, 2001).

Based on the above, one can say that the documented and recognised current membership for PMI is approximately 700,000 (PMI, 2013), PRINCE2 is about 250,000 (PRINCE2, 2009) while Agile is estimated to have over 80.000 signatories according to Agile website 2013. Essentially, membership to these software professional bodies is acquired based on qualifications, experience and personal choice. These professional bodies undertake certification programs, run conferences, organise local chapters and support research globally. They have branches in Wellington and conduct conferences and discussion sessions where members meet monthly to share knowledge, experiences and challenges in project management (IT). Membership sometimes overlaps, with some members belonging to more than one professional body. The researcher obtained membership to all the three international project management bodies for easier access to all participants for this research and had often attended the local chapters in Wellington for over 2 years before collecting the data.

\subsubsection{Sample size and Sampling technique}

This study adopts stratified sampling, a technique that selects subjects from each stratum (Cavana et al, 2001, p.258). This non-probability sampling technique was used for this study as it was not possible to randomly select practitioners from the populations that were being studied. Consistently, a disproportionate sample of 3,800 senior software development vendors was generated from the population of two major communities of practice i.e. traditional plan-based (PRINCE2, PMI) and Agile. This study required a large sample size to enable SEM analysis as well as splitting of the data set to examine sub group comparisons.

Kline (2005), Cunningham (2010) and Iacobucci (2010), consistent with Anderson and Gerbing (1988), have provided decision criteria for sample sizes in SEM analyses and most importantly, demonstrated that SEM methods of estimation especially those using Maximum Likelihood Estimation (MLE) require large samples. While no definitive guidelines for sample size have been established, these scholars have proposed that an optimal ratio of number of research participants to the number of parameters estimated in confirmatory factor analysis to be at least 20:1 (Kline, 2005). However, testing more complex models that may include mediating and moderating hypotheses require even larger sample sizes (Kline, 2005). 
Initially, this study had about 190 survey questions in the questionnaire for the 28 constructs identified in literature review, which required at least 3,800 research participants. A review of SEM studies in project management indicates that the response is usually approximately less than $20 \%$. It was calculated and established that at least 760 valid responses were appropriate. This number was expected to represent about $20 \%$ response rate with a margin of error of 5\%. This study sample resonates well and is consistent with Krejcie and Morgan's (1970) table of determining the sample size for research studies. Table 17 illustrates the sample size that was derived using disproportionate stratified sampling from the study population made up of two major communities of practice i.e. traditional plan-based (PRINCE2, PMI) and Agile methodologies.

Table 17: Sample size

\begin{tabular}{|l|l|l|}
\hline Community of Practice (Stratum) & Population size & Sample size \\
\hline \multirow{2}{*}{ Traditional plan-based approach } & (PRINCE2) Over 250,000 & \multirow{2}{*}{1,900} \\
\cline { 2 - 3 } & (PMI) Over 700,000 (185 countries) & 1,900 \\
\hline Agile & Over 80,000 & 1 \\
\hline
\end{tabular}

Disproportionate stratified random sampling was deemed more efficient in this study because, for this sample size each important segment of the population is better represented and more valuable and differentiated information can be obtained with respect to each group (Cavana et al., 2002, p. 260).

\subsection{Operationalization and measurements of constructs}

All study variables were operationalized based on previous studies and a review of the existing literature. Variables were measured using the existing measurement items of earlier researchers, with minor modifications where necessary to suit this research. The questions assessed perceptions regarding candidate CSFs in the context of methodology selection practices. The respondents were requested to show the extent of agreement or disagreement with these questions on a seven point Likert scale (1-strongly disagree to 7-strongly agree). A seven point Likert scale was chosen because: (1) It provides many alternatives to select from ( 7 choices) than a 5 point Likert scale, and (2) It also provides a neutral point which helps to avoid forced responses (Alwin, 1997). This is essential in some instances where respondents may not simply have an opinion. Similarly, as most constructs in this study are multidimensional, it was appropriate to use scales with a neutral point in order to increase the response rate and reduce the possibility of low reliability that can be caused by random 
guessing and forced answers (Alwin, 1997). For instance, Alwin's (1997) comparison of various point scales found that the 7- point Likert rating scales transmitted more information, were inherently more precise in their measurement and less vulnerable to systematic measurement errors or shared variance. In addition, the use of a seven point Likert scale is consistent with other previous SEM studies in information system and management research. Although most of the adopted measures (e.g. Jun et al, 2011; Chow \& Cao, 2008, Misra et al., 2009) have been tested elsewhere and used in other studies and found to be valid and reliable; they were further tested for reliability and validity to confirm their applicability in this study.

\subsubsection{Instrument (survey) design and development}

In strategic management and information system research, survey research is a common method of collecting primary quantitative data. This method offers a structured way to ask respondents a variety of questions about their practices, perceptions, behaviours and attitudes. Survey questionnaires are relatively simple to administer and respondents can complete the survey in their own time and at their own pace while maintaining anonymity at the same time (Collis \& Hussey, 2003; Cavana et al., 2001).

In order to ensure that the survey instrument (questionnaire) measured what it was intended to measure, more emphasis and attention was paid to its development. A common procedure for developing a survey instrument was to create a pool of items that have been adopted previously and validated by other studies (Collis \& Hussey, 2003). There was not much need for developing new items tailored to the context of the study.

The following section discusses different measurement items previously used by scholars in the literature in order to identify and gather measures for this study. The section aims at compiling a pool of measurement scales intended to be used in the online survey.

\subsubsection{Measurement items identification and collection from literature}

All the items for the VPoC organizational factors, vendor perception of team factors, vendor perception of customer factors, vendor perception of project factors and project success factors as categorised in the conceptual framework are discussed. 


\subsubsection{Construct 1: VPoC organizational factors}

The next section examines the measurement scales for VPoC organizational factors based on previous studies.

\section{Measurement scales for top level management support}

The search of the existing literature revealed that most of the research involving this dimension was either conceptual (e.g. Mohammad \& Al-Shargabi, 2011; Imreh \& Raisinghani, 2011; Mansor et al. 2011; Mishra \& Mishra, 2011; Dyck \& Majchrzak, 2012), or the scales were inadequately developed and validated in dissimilar contexts. For instance, Sheffield and Lemetayer (2013) included top level management support in their regression model, but only a single item was used to capture the whole dimension making it unsuitable for this study since SEM uses multiple measurement items to measure latent constructs. In contrast, Jiang et al.'s (2008) measures of top management commitment were a combination of many organizational factors such as top management's commitment to quality, vision and mission and short-term and long-term goals which are unsuitable for this study.

Nah and Delgado (2006) was the only empirical study identified from literature that previously assessed only the top level management support dimension with several items. However, the reliability and validity of these items were not reported. Table 18 shows the measurement items previously used to assess top level management by Nah and Delgado (2006) and adopted for this study.

Table 18: Scales used previously to measure top level management support (Nah \& Delgado, 2006)

\begin{tabular}{|l|l|}
\hline \multicolumn{1}{|c|}{ Item wording } \\
\hline 1 & Approval and support from top level management \\
\hline 2 & Top level management publicly and explicitly identified the project as a top priority \\
\hline 3 & Allocate resources \\
\hline 4 & Existence of project champion \\
\hline 5 & High level executive sponsor as champion \\
\hline 6 & Project sponsor commitment \\
\hline
\end{tabular}

\section{Measurement scales for organizational culture}

Strode et al. (2009) was the only study that was identified that had empirically measured organizational culture in the context of software development projects. Although they assessed organizational culture in relation to project success, their study did not indicate reliability and validity of these items. Other studies encountered in the literature were conceptual (e.g. Mishra \& Mishra, 2011; Dyck \& Majchrzak, 2012) or used single items to 
measure organizational culture (e.g. Chow and Cao, 2008; Misra et al. 2009; Sheffield \& Lemetayer, 2013) so they are less suitable for this study. Table 19 shows the measurement items that were previously used by Strode et al. (2009) to assess organizational culture on agile method use and adopted for this study.

Table 19: Scales previously used to measure organizational culture (Strode et al. 2009)

\begin{tabular}{|l|l|}
\hline \multicolumn{2}{|l|}{ Item wording } \\
\hline 1 & The leadership of the organization in which the project was conducted was entrepreneurial, innovative and risk-taking. \\
\hline 2 & The organization culture was flexible and participative, valued teamwork and encouraged social interaction. \\
\hline 3 & The management style was that of leadership and collaboration. \\
\hline 4 & The organization was based on loyalty and mutual trust and commitment. \\
\hline 5 & The organization valued feedback and learning. \\
\hline 6 & The organization enabled empowerment of people and teams. \\
\hline 7 & The organization was result oriented. \\
\hline
\end{tabular}

\section{Measurement scales for planning and controlling}

The measurement scales for planning and controlling were obtained from Jun et al.(2011).

These items had been initially validated for both reliability and validity and were found to perform well. Previous studies such as Barki et al. (2001) have used similar measurement scales. Table 20 summarises the measurement items previously used to assess planning and controlling by Jun et al. (2011) and adopted for this study.

Table 20: Scales previously used to measure project planning and controlling (Jun et al., 2011)

\begin{tabular}{|c|c|c|c|c|c|}
\hline & Item wording & $\begin{array}{l}\text { Factor } \\
\text { loadings }\end{array}$ & $\begin{array}{l}\text { Composite } \\
\text { reliability }\end{array}$ & AVE & $\begin{array}{c}\text { Cronbach } \\
\text { alpha }\end{array}$ \\
\hline 1 & Special attention was paid to project planning. & 0.72 & \multirow{5}{*}{0.90} & \multirow{5}{*}{0.65} & \multirow{5}{*}{0.87} \\
\hline 2 & Project milestones were clearly defined. & 0.79 & & & \\
\hline 3 & Project progress was monitored closely e.g. using PERT or CPM tools. & 0.84 & & & \\
\hline 4 & Periodic formal status reports were used instead of the project plan. & 0.85 & & & \\
\hline 5 & There were strict audits at each milestone. & 0.80 & & & \\
\hline
\end{tabular}

As indicated, all the factor loadings $(\lambda)$ were above 0.7 (Hair et al., 2009), Composite Scale Reliabilities (CSR) were greater than 0.7 (Fornell \& Larcker, 1981; Anderson \& Gerbing, 1988), AVE exceeded 0.5 (Fornell \& Larcker, 1981) and Cronbach's alpha $(\alpha)$ was above 0.7 (Nunnally, 1978).

\section{Measurement scales for change management}

The measurement scales for change management were obtained from $\mathrm{Nah}$ and Delgado (2006). This combination of items was viewed to be appropriate for this study because they cover various phases of implementing change during software development e.g., during planning, implementation and upgrade but their reliability and validity were not reported. 
Other studies encountered in the literature were conceptual (e.g. Mishra \& Mishra, 2011; Dyck \& Majchrzak, 2012) or used single items to measure change management (e.g. Chow and Cao, 2008; Misra et al. 2009; Sheffield \& Lemetayer, 2013) so they are less suitable for this study. Table 21 shows the items previously used to assess change management by Nah and Delgado (2006) and adopted for this study.

Table 21: Scales previously used to measure change management (Nah \& Delgado, 2006)

\begin{tabular}{|l|l|}
\hline \multicolumn{2}{|c|}{ Item wording } \\
\hline 1 & Recognition for the need for change. \\
\hline 2 & Organizational wide change for culture and structure management. \\
\hline 3 & Commitment to change-perseverance and determination. \\
\hline 4 & Business process re-engineering to match the new software. \\
\hline 5 & Analysis of user feedback \\
\hline 6 & User education and training. \\
\hline 7 & User support and involvement. \\
\hline 8 & IT workforce re-skilling. \\
\hline
\end{tabular}

\section{Measurement scales for vision and mission}

The measurement scales for vision and mission were obtained from Wan and Wang (2010) and Nah and Delgado (2006). These were found to be appropriate for this study because they assess vision and mission using a combination of items in the context of software development projects. But the reliability and validity tests for Nah and Delgado' (2006) measures were not reported. In contrast, the measures used by Wan and Wang (2010) were previously validated and showed some reasonable factor loadings $(\lambda>0.6$.) Tables 22 and 23 show the items used to measure vision and missions.

Table 22: Scales previously used to measure Vision and mission (Wan \& Wang, 2006)

\begin{tabular}{|l|l|l|}
\hline \multicolumn{1}{|c|}{ Item wording } & Factor loadings \\
\hline 1 & Creating a clear vision & 0.62 \\
\hline 2 & Building the agile organizational culture & 0.76 \\
\hline 3 & Changing the way of management & 0.72 \\
\hline
\end{tabular}

Table 23: Scales previously used to measure vision and mission (Nah \& Delgado, 2006)

\begin{tabular}{|l|l|}
\hline \multicolumn{2}{|c|}{ Item wording } \\
\hline 1 & The organization had a strategic business plan and a vision. \\
\hline 2 & The organizational had a mission with clearly defined goals. \\
\hline 3 & There was a business case approval for justification for investment in software. \\
\hline
\end{tabular}

The measurement items used by both Wang and Wang (2010) and Nah and Delgado (2000) were therefore subsequently integrated and used together in this study.

\section{Measurement scales for leadership characteristics}

The literature suggests that no previous study has developed measurement items for leadership characteristics and assessed them in the context of software development projects. 
Other studies encountered in the literature used single items to measure leadership characteristics (e,g., Misra et al. 2009; Lindvall et al. 2002; Strode et al. 2009; Schmidt et al., 2001; Baccarini et al., 2004; OGC, 2005; Humphrey, 2005; Standing et al., 2006; Oz and Sosik, 2000) so they are less suitable for this study. Therefore, new items were created by combining all these measures to measure leadership characteristics based on the construct definition as applied in the general management literature. Table 24 shows the combined measurement items developed and used to measure leadership characteristics.

Table 24: Scales previously used to measure leadership characteristics

\begin{tabular}{|l|l|}
\hline & \multicolumn{1}{|c|}{ Item wording } \\
\hline 1 & Drive- Leaders are ambitious and take initiative. \\
\hline 2 & Motivation- Leaders want to lead and are willing to take charge. \\
\hline 3 & Honesty and integrity-Leaders are truthful and do what they say they will do. \\
\hline 4 & Self-confidence-Leaders are assertive and decisive and enjoy taking risks. \\
\hline 5 & Business knowledge-Leaders have technical expertise in their businesses. \\
\hline
\end{tabular}

After considering all the measurement items used by different scholars, the best scales with highest reliability and validity were collected and a pool of them was created to be used to examine the influence of organizational factors on project success in this study. For details of final measures please prefer the full questionnaire in the appendix G.

\subsubsection{Construct 2: Vendor perception of team factors}

According to PMI (2013), project teams include the project manager and the group of individuals who work together in performing the work of the project to achieve objectives. Therefore, the project team includes the project manager, project management staff and other team members who carry out the work but are not necessarily involved in the management of the project (p.35). The dimensions for team factor include: internal project communication, project team commitment, and development team expertise and team composition.

\section{Measurement scales for internal project communication}

The measurement items for internal project communication were adopted from Jun et al. (2011) who had validated them. Their reliability $(\alpha>0.7)$ and validities $(\lambda>0.7$, CSR $>0.7$, AVE $>0.5)$ were very good. Similar measures were previously used by Barki et al. (2001). Table 25 displays the items previously used to measure internal project communication and adopted for this study. 
Table 25: Scales previously used to measure internal project communication (Jun et al., 2011)

\begin{tabular}{|c|c|c|c|c|c|}
\hline \multicolumn{2}{|r|}{ Item wording } & $\begin{array}{l}\text { Factor } \\
\text { loadings }\end{array}$ & $\begin{array}{l}\text { Composite } \\
\text { reliability }\end{array}$ & AVE & $\begin{array}{c}\text { Cronbach } \\
\text { alpha }\end{array}$ \\
\hline 1 & The project team meets frequently & 0.91 & \multirow{4}{*}{0.95} & \multirow{4}{*}{0.83} & \multirow{4}{*}{0.93} \\
\hline 2 & $\begin{array}{l}\text { Project team members are kept informed about major decisions } \\
\text { concerning the project. }\end{array}$ & 0.93 & & & \\
\hline 3 & Every effort is made to keep project team turnover at a minimum. & 0.87 & & & \\
\hline 4 & $\begin{array}{l}\text { Project team members actively participate in the definition of } \\
\text { project goals and schedules. }\end{array}$ & 0.94 & & & \\
\hline
\end{tabular}

\section{Measurement scales for project team commitment}

Although many studies have cited team commitment as a candidate CSF, all previous studies have either used single items to measure team commitment (e.g. Chow \& Cao, 2008; Misra et al. 2009; Sheffield \& Lemetayer, 2013 or others have been conceptual (e.g. Mohammad \& Al-Shargabi, 2011; Imreh \& Raisinghani, 2011; Mansor et al. 2011; Mishra \& Mishra, 2011; Dyck \& Majchrzak, 2012). Single items could not be used because they cannot effectively measure a latent variable for SEM. Instead based on management literature, a set of measurement items were developed from Meyer \& Allen (1991) but no reliability were reported. These measures were also previously used in Ahimbisibwe and Nangoli's (2012) study and showed reasonably good factor loading. The context of their study though was slightly different because they focused on citizenship projects in a less developed country. The items previously used to measure project team commitment are shown in Table 26.

Table 26: Scales previously used to measure team commitment (Meyer \& Allen (1991)

\begin{tabular}{|l|l|}
\hline \multicolumn{1}{|c|}{ Item wording } \\
\hline 1 & No other activities could match the benefits that the software development project activities presented to me. \\
\hline 2 & It would have been very hard for me to abandon the software development project activities even if I wanted to. \\
\hline 3 & My life would have been upset if I decided not to engage in software development project activities. \\
\hline 4 & It would have been too costly for me to quit software development project activities during that project. \\
\hline 5 & Taking part in that software development project was a matter of necessity as much as desire. \\
\hline 6 & Team members proudly accepted any job assignments related to serving on this software development project. \\
\hline 7 & Team member's personal values and those of the software development project were very similar. \\
\hline 8 & Individual team members felt like part of the family of the software development project team. \\
\hline 9 & Team members were emotionally attached to the software development project. \\
\hline 10 & Team members felt a strong sense of belonging to the software development project. \\
\hline 11 & Team members felt they had an obligation to keep performing software development activities. \\
\hline 12 & Team members had a sense of obligation to the client of software development project. \\
\hline 13 & Team members owed a great deal to the software development project. \\
\hline
\end{tabular}

Table 27 shows the measurement items that were originally used by Meyer \& Allen (1991) and adopted by Ahimbisibwe and Nangoli (2012) to assess project team commitment and their respective factor loadings. These measures were reasonably good and were subsequently adopted for this study. 
Table 27: Scales previously used to measure project team commitment (Ahimbisibwe \& Nangoli, 2012)

\begin{tabular}{|l|l|c|}
\hline \multicolumn{1}{|c|}{ Item wording } & Factor loadings \\
\hline 1 & I think no other activities can match the benefits that project activities present to me & 0.67 \\
\hline 2 & It would be very hard for me to abandon project activities even if I wanted to & 0.72 \\
\hline 3 & My life would be upset if I decided not to engage in project activities & 0.70 \\
\hline 4 & It would be too costly for me to quit project activities right now & 0.81 \\
\hline 5 & Taking part in projects is a matter of necessity as much as desire & 0.60 \\
\hline 6 & I would proudly accept any job assignments related to serving community & 0.70 \\
\hline 7 & I find that my personal values and those of projects are very similar & 0.75 \\
\hline 8 & I feel like part of the family of the project teams & 0.60 \\
\hline 9 & I feel emotionally attached to projects & 0.86 \\
\hline 10 & I feel a strong sense of belonging to projects & 0.71 \\
\hline 11 & I feel I have an obligation to keep performing activities & 0.53 \\
\hline 12 & I have a sense of obligation to the recipients of projects & 0.77 \\
\hline 13 & I owe a great deal to projects & 0.76 \\
\hline
\end{tabular}

\section{Measurement scales for team's expertise and experience}

The scales for measuring team's expertise were borrowed from Jun et al. (2011). Table 28 indicates the items previously used to measure team's expertise and experiences in software projects. Jun et al. (2011) used measures that were initially advanced by Barki et al. (2001). The factor loadings were above 0.7 (Hair et al., 2009), composite scale reliabilities were greater than 0.7 (Fornell \& Larcker, 1981; Anderson \& Gerbing, 1988), AVE exceeded 0.5 (Fornell \& Larcker, 1981) and Cronbach'S alpha was also above 0.7 (Nannally, 1978). As a result, these measures were adopted for this study.

Table 28: Scales previously used to measure team's expertise and experience (Jun et al., 2011)

\begin{tabular}{|c|c|c|c|c|c|}
\hline \multicolumn{2}{|r|}{ Item wording } & $\begin{array}{l}\text { Factor } \\
\text { loadings }\end{array}$ & $\begin{array}{l}\text { Composite } \\
\text { reliability }\end{array}$ & AVE & $\begin{array}{c}\text { Cronbach } \\
\text { alpha }\end{array}$ \\
\hline 1 & $\begin{array}{l}\text { Development team's lack of experience with development } \\
\text { platform/environment used in this project }\end{array}$ & 0.79 & \multirow[t]{3}{*}{0.89} & \multirow[t]{3}{*}{0.74} & \multirow[t]{3}{*}{0.82} \\
\hline 2 & $\begin{array}{l}\text { Development team is very unfamiliar with this type of } \\
\text { application }\end{array}$ & 0.90 & & & \\
\hline 3 & $\begin{array}{l}\text { Development team's lack of knowledge of application } \\
\text { domain involved in this project }\end{array}$ & 0.88 & & & \\
\hline
\end{tabular}

\section{Measurement scales for team's composition}

The scales for measuring the team's composition were derived from Nah and Delgado (2006). Similar measures were combined to measure team's composition and empowerment by the same scholars. Table 29 shows the items used to measure team's composition by Nah and Delgado (2006) and used in this study. 
Table 29: Scales previously used to measure team's composition (Nah \& Delgado, 2006)

\begin{tabular}{|l|l|}
\hline \multicolumn{2}{|c|}{ Item wording } \\
\hline 1 & Best people on team \\
\hline 2 & Balanced or cross functional teams \\
\hline 3 & Full time members \\
\hline 4 & Partnerships, trust, risk-sharing and incentives \\
\hline 5 & Empowered decision makers \\
\hline 6 & Business and technical knowledge of team members and consultants \\
\hline
\end{tabular}

All measurement items that had good reliability $(\alpha>0.7)$ and validities $(\lambda>0.7$, CSR $>0.7$, $A V E>.5)$ in measuring team factors were used and for more details of all items used please refer to the questionnaire in appendix $\mathrm{G}$.

\subsubsection{Construct 3: Vendor perception of customer factors}

The third factor, vendor perception of customer factor encompasses all those variables that relate to project customers (clients). According to PMI (2013), a customer or user is any person who has to accept the deliverables or products of the project that he/she is assigned on to act as a representative or liaison to ensure proper coordination, advise on requirements, or validate the acceptability of the products results (p.36). Based on literature review in this study the vendor perception of customer factor include: user participation, user support and user experience. The subsequent section discusses the measurement scales for these dimensions.

\section{Measurement scales for user participation}

The measurement items for user participation were drawn from Jun et al. (2011) that had initially validated them. Their reliability $(\alpha>0.7)$ and validities $(\lambda>.7, \operatorname{CSR}>0.7$, AVE $>0.5)$ were also very good as indicated. Similar measures were also used by Barki et al. (2001). Table 30 shows the items previously used to measure user participation by Jun et al. (2011) and used in this study.

Table 30: Scales previously used to measure user participation (Jun et al., 2011)

\begin{tabular}{|c|l|c|c|c|c|}
\hline \multicolumn{1}{|c|}{ Item wording } & $\begin{array}{c}\text { Factor } \\
\text { loadings }\end{array}$ & $\begin{array}{c}\text { Composite } \\
\text { reliability }\end{array}$ & $\begin{array}{c}\text { AVE } \\
\text { Cronbach } \\
\text { alpha }\end{array}$ \\
\hline 1 & Users actively participated in requirements definition. & 0.86 & & & \\
\cline { 1 - 3 } 2 & $\begin{array}{l}\text { The project team kept users informed concerning project } \\
\text { progress and problems. }\end{array}$ & 0.91 & \multirow{2}{*}{0.91} & 0.72 & 0.87 \\
\hline 3 & Users formally evaluated the work done by the project team. & 0.89 & & \\
\hline 4 & Users formally affirmed the work done by the project team. & 0.73 & & \\
\hline
\end{tabular}




\section{Measurement scales for user support}

The measurement items for user support were drawn from Jiang and Klein (2000) who had initially validated them. Their reliability Cronbach alpha coefficient was also good $(\alpha>0.7)$. Table 31 shows the previous measurement items used to measure user support by Jiang \& Klein (2000) and used in this study.

Table 31: Scales previously used to measure user support (Jiang \& Klein, 2000)

\begin{tabular}{|l|l|l|}
\hline \multicolumn{2}{|c|}{ Item wording } & $\begin{array}{c}\text { Cronbatch } \\
\text { coefficient }\end{array}$ \\
\hline 1 & Users have a negative opinion about the system meeting their needs. & \\
\hline 2 & Users are not enthusiastic about the project. & \\
\hline 3 & Users are an integral part of the development team. & \multirow{2}{*}{0.90} \\
\hline 5 & Users are available to answer the questions. & \\
\hline 6 & Users are ready to accept the changes the system will entail. & \\
\hline 7 & Users quickly respond to development team request. & \\
\hline 8 & Users have positive attitudes regarding the use of computers in their work. & \\
\hline
\end{tabular}

\section{Measurement scales for user experience}

The measurement items for user experience were borrowed from Jun et al. (2011) and had been initially validated. Their reliability $(\alpha>0.7)$ and validities $(\lambda>0.7$, CSR $>0.7$, AVE $>0.5)$ were very good. Table 32 shows the previous measurement items used to measure user experience by Jun et al. (2011).

Table 32: Scales previously used to measure user experience (Jun et al., 2011)

\begin{tabular}{|c|c|c|c|c|c|}
\hline & Item wording & $\begin{array}{l}\text { Factor } \\
\text { loadings }\end{array}$ & $\begin{array}{l}\text { Composite } \\
\text { reliability }\end{array}$ & AVE & $\begin{array}{c}\text { Cronbach } \\
\text { alpha }\end{array}$ \\
\hline 1 & client is not familiar with this type of application & 0.77 & \multirow{4}{*}{0.88} & \multirow{4}{*}{0.64} & \multirow{4}{*}{0.81} \\
\hline 2 & client doesn't know what they want & 0.86 & & & \\
\hline 3 & $\begin{array}{l}\text { client doesn't have a good understanding of the problems } \\
\text { they want solved }\end{array}$ & 0.90 & & & \\
\hline 4 & users are not familiar with data processing as a work tool & 0.67 & & & \\
\hline
\end{tabular}

After carefully assessing all the measurement items used by different scholars, the best scales were collected and a pool of them was created to be used to assess customer factors in this study. Specifically, the selection criteria was that previous items all had the factor loadings above 0.7 (Hair et al., 2009), composite scale reliabilities greater than 0.7 (Fornell \& Larcker, 1981; Anderson \& Gerbing, 1988), AVE exceeding 0.5 (Fornell \& Larcker, 1981) and Cronbach alpha above 0.7 (Nannally, 1978) were preferred and used for this study.

\subsubsection{Construct 4: Vendor perception of project factors}

The fourth factor, vendor perception of project factor covers all those variables that relate to the project's inherent risks. The dimensions for vendor perception of project factor include: 
technological uncertainty, technical complexity, relative project size, specification changes (instability), and project criticality.

\section{Measurement scales for technological uncertainty}

The measurement items for technological uncertainty were adopted from Nidumolu (1995). These measures used by Nidumolu (1995) were previously validated and their factor loadings $(\lambda>.7)$ were also very good. Table 33 shows the previous measurement items used to measure technological uncertainty by Nidumolu (1995).

Table 33: Scales previously used to measure technological uncertainty (Nidumolu, 1995)

\begin{tabular}{|l|l|l|}
\hline \multicolumn{2}{|c|}{ Item wording } & Factor loadings \\
\hline 1 & Lack of a clearly known way to develop software that would meet these requirements & 0.767 \\
\hline 2 & No available knowledge was of great help in developing the software & 0.589 \\
\hline 3 & No established procedures and practices could be relied upon to develop software & 0.782 \\
\hline 4 & Lack of an understandable sequence of steps that could be followed for developing software & 0.814 \\
\hline
\end{tabular}

\section{Measurement scales for technical complexity}

The measurement items for technical complexity were drawn from Jun et al. (2011) who had initially validated them. Their reliability $(\alpha>0.7)$ and validities were also very good $\quad(\lambda>0.7$, CSR>0.7, AVE>0.5). Similar measures were used by Barki et al. (2001) and Wallace et al. (2004). Table 34 shows the previous measurement items used to measure technical complexity by Jun et al. (2011) from Barki et al. (2001) and adopted in this study.

Table 34: Scales previously used to measure technical complexity (Jun et al., 2011)

\begin{tabular}{|c|l|c|c|c|c|}
\hline \multicolumn{2}{|c|}{ Item wording } & $\begin{array}{c}\text { Factor } \\
\text { loadings }\end{array}$ & $\begin{array}{c}\text { Composite } \\
\text { reliability }\end{array}$ & $\begin{array}{c}\text { AVE } \\
\text { Cronbach } \\
\text { alpha }\end{array}$ \\
\hline 1 & Project involves the use of new technology. & 0.86 & 0.90 & 0.75 & 0.83 \\
\hline 2 & Project has high level of technical complexity. & 0.86 & & & \\
\hline 3 & $\begin{array}{l}\text { Project involves the use of technology that has not } \\
\text { been used in prior projects. }\end{array}$ & 0.87 & & \\
\hline
\end{tabular}

\section{Measurement scales for relative project size}

The measurement items for relative project size were drawn from Jun et al. (2011) and Jiang and Klein (2000) who had initially validated them. Jun et al.'s (2011) reliability $(\alpha>0.7)$ and validities $(\lambda>0.7, \mathrm{CSR}>0.7, \mathrm{AVE}>0.5)$ were also very good. Similar measures were used by Barki et al. (1993). Tables 35 and 36 show the previous measurement items used to measure relative project size. 
Table 35: Scales previously used to measure relative project size (Jun et al., 2011)

\begin{tabular}{|c|c|c|c|c|c|}
\hline \multicolumn{2}{|r|}{ Item wording } & $\begin{array}{c}\text { Factor } \\
\text { loadings }\end{array}$ & $\begin{array}{l}\text { Composite } \\
\text { reliability }\end{array}$ & AVE & $\begin{array}{c}\text { Cronbach } \\
\text { alpha }\end{array}$ \\
\hline 1 & $\begin{array}{l}\text { Compared to other information systems projects } \\
\text { developed in your organization, the scheduled number } \\
\text { of person-days for completing this project is much } \\
\text { higher. }\end{array}$ & 0.91 & \multirow[t]{3}{*}{0.95} & \multirow[t]{3}{*}{0.86} & \multirow[t]{3}{*}{0.92} \\
\hline 2 & $\begin{array}{l}\text { Compared to other information systems projects } \\
\text { developed in your organization, the scheduled number } \\
\text { of months for completing this project is much higher. }\end{array}$ & 0.93 & & & \\
\hline 3 & $\begin{array}{l}\text { Compared to other information systems projects } \\
\text { developed in your organization, the dollar budget } \\
\text { allocated to this project is much higher. }\end{array}$ & 0.93 & & & \\
\hline
\end{tabular}

The measures of Jiang and Klein (2000) were combined with Jun et al's (2011) measures. Jiang and Klein's (2000) measures demonstrated good reliability $(\alpha=0.86)$.

Table 36: Scales previously used to measure project size (Jiang \& Klein, 2000)

\begin{tabular}{|c|c|c|}
\hline & Item wording & Cronbatch coefficient \\
\hline 1 & Large number of people on team & \multirow{5}{*}{0.86} \\
\hline 2 & $\begin{array}{l}\text { Large number of different "stakeholders" on team (e.g., IS } \\
\text { staff, users, consultants, suppliers, customers) }\end{array}$ & \\
\hline 3 & Large project size & \\
\hline 4 & Large number of users will be using this system & \\
\hline 5 & $\begin{array}{l}\text { Large number of hierarchical levels occupied by users who } \\
\text { will be using the system (e.g., office clerks, supervisors) }\end{array}$ & \\
\hline
\end{tabular}

\section{Measurement scales for specification changes}

The measurement items for specification changes were adopted from Nidumolu (1995). These measures used by Nidumolu (1995) were previously validated and showed some reasonable factor loadings. However, two measurement items were below the cut off criteria for factor loadings in exploratory factor analysis which is 0.6 (Hair et al., 2009). Table 37 shows the previous measurement items used to measure specification changes by Nidumolu (1995) and used in this study.

Table 37: Scales previously used to measure specification changes (Nidumolu, 1995)

\begin{tabular}{|c|l|c|}
\hline \multicolumn{2}{|c|}{ Item wording } & Factor loadings \\
\hline 1 & Requirements fluctuated quite a bit in earlier phases. & 0.431 \\
\hline 2 & Requirements fluctuated quite a bit in later phases. & 0.695 \\
\hline 3 & Requirements at beginning of project were quite different from those existing at end. & 0.827 \\
\hline 4 & Requirements will fluctuate quite a bit in the future. & 0.474 \\
\hline
\end{tabular}

\section{Measurement scales for project criticality}

The measurement items for project criticality were adopted from Sheffield and Lemetayer (2013). However, the reliability and validity of these measures are not reported. Table 38 shows the previous measurement items used to measure project criticality. 
Table 38: Scales previously used to measure project criticality (Sheffield \& Lemetayer (2013)

\begin{tabular}{|l|l|}
\hline \multicolumn{2}{|c|}{ Item wording } \\
\hline 1 & A defect only has a very minor impact leading to loss of comfort. \\
\hline 2 & A defect can be fixed pretty easily because of good backup procedures \\
\hline 3 & A defect in the system makes the company go bankrupt leading to loss of essential monies. \\
\hline 4 & A defect can be catastrophic, leading to loss of lives such as in nuclear power stations or flight control systems. \\
\hline
\end{tabular}

Again, all measures with factor loadings above 0.7 , composite scale reliabilities greater than 0.7, AVE exceeded 0.5 and Cronbach alpha was also above 0.7 were given first priority in this study. Further details of final items used can be found in the questionnaire attached.

\subsubsection{Construct 5: Project success}

According to PMI (2013), project success refers to completing the project within the constraints of scope, time, cost, quality, resources, and risks approved between the project managers and senior management (p. 35). The following section presents measures of project success that were adopted.

The following were the measures of project success used in Chow and Cao's (2008) study; however, the authors did not report validity and reliability tests.

Table 39: Scales previously used to measure project success (Chow \& Cao, 2008)

\begin{tabular}{|l|l|}
\hline \multicolumn{1}{|c|}{ Item wording } \\
\hline 1 & Quality (delivering good product or project outcome) \\
\hline 2 & Scope (meeting all requirements and objectives) \\
\hline 3 & Time (delivering on time) \\
\hline 4 & Cost (delivering within estimated cost and effort) \\
\hline
\end{tabular}

The following were the measures of project success used by Misra et al. (2009) in identifying some CSFs in adopting agile software development practices. However, this study also did not report validity and reliability tests.

Table 40: Scales previously used to measure project success (Misra et al., 2009)

\begin{tabular}{|l|l|}
\hline \multicolumn{2}{|l|}{ Item wording } \\
\hline 1 & Reduced delivery schedules \\
\hline 2 & Increased return on investment (ROI) \\
\hline 3 & Increased ability to meet with the current customer requirements \\
\hline 4 & $\begin{array}{l}\text { Increased flexibility to meet with the } \\
\text { changing customer requirements }\end{array}$ \\
\hline 5 & Improved business processes \\
\hline
\end{tabular}

The following were the measures of project success used by Jun et al. (2011) and their measurement scales demonstrated worthy reliability and validity $(\lambda>0.7, \mathrm{CSR}>0.7, \mathrm{AVE}>0.5$, $\alpha>0.7)$ and were therefore deemed suitable. 
Table 41: Scales previously used to measure project success (Jun et al., 2011)

\begin{tabular}{|c|c|c|c|c|c|}
\hline \multicolumn{2}{|r|}{ Item wording } & $\begin{array}{c}\text { Factor } \\
\text { loadings }\end{array}$ & $\begin{array}{l}\text { Composite } \\
\text { reliability }\end{array}$ & AVE & $\begin{array}{c}\text { Cronbach } \\
\text { alpha }\end{array}$ \\
\hline \multicolumn{2}{|r|}{ Process performance } & & \multirow[t]{3}{*}{0.94} & \multirow{3}{*}{0.88} & \multirow[t]{3}{*}{0.87} \\
\hline 1 & The project was completed within budget & 0.94 & & & \\
\hline 2 & The project was completed within schedule & 0.94 & & & \\
\hline \multicolumn{2}{|r|}{ Product performance } & & \multirow{7}{*}{0.96} & \multirow{7}{*}{0.79} & \multirow{7}{*}{0.95} \\
\hline 1 & The application developed is reliable & 0.92 & & & \\
\hline 2 & The application developed is easy to use & 0.91 & & & \\
\hline 3 & Flexibility of the system is good & 0.82 & & & \\
\hline 4 & The system meets user's intended functional requirements & 0.87 & & & \\
\hline 5 & Users are satisfied with the system delivered & 0.91 & & & \\
\hline 6 & The overall quality of the developed application is high & 0.91 & & & \\
\hline
\end{tabular}

Sheffield and Lemetayer (2013) used the following measures for project success in exploring factors associated with the software development agility of successful projects.

Table 42: Scales previously used to measure project success (Sheffield \& Lemetayer, 2013)

\begin{tabular}{|l|l|c|}
\hline & \multicolumn{1}{|c|}{ Item wording } & Factor loadings \\
\hline 1 & The project was completed on time or earlier & 0.69 \\
\hline 2 & The project was completed within or below budget & 0.74 \\
\hline 3 & The project met the customer's requirement & 0.85 \\
\hline 4 & The project delivered a good working product & 0.90 \\
\hline 5 & The product addresses a recognized need & 0.81 \\
\hline 6 & The product is used by the customer & 0.83 \\
\hline 7 & The product satisfied the customer & 0.86 \\
\hline 8 & The project team was highly satisfied & 0.74 \\
\hline
\end{tabular}

All the survey items whose reliability and validity was tested previously and found to be reasonably good $(\lambda>0.7, \mathrm{CSR}>0.7, \mathrm{AVE}>0.5, \alpha>0.7)$ were preferred for measuring project success. Please refer to the questionnaire in appendix for details about final items used.

\subsubsection{Initial survey questionnaire design and structure}

Next, the listed items were compiled in a preliminary survey draft consisting of two major sections: A) background information and B) evaluation of software vendors' responses in respect to CSFs for project success.

The first part was intended to determine the demographic characteristics of the respondents as well as the important characteristics of the projects (sample characteristics). There were initially 12 questions in part one which all aimed at capturing respondents' or projects' profiles. These were: experience of the respondents in SD, size of the client firm in which the last project was conducted, type of economic sector industry, position held on the last project, 
project management methodology used (categorical variable), who made the decision of choosing the methodology, development life cycle, total number of project members, estimated project budget (USD), estimated duration in months, country in which the reported project was conducted.

The other part (section B) consisted of the operationalized and itemized components of all candidate CSFs and project success. All these questions aimed at assessing level of response/ perception regarding candidate CSFs and practices. Initially, section B consisted of a total of 178 questions that had been compiled from previous studies in the literature. Therefore, there were a total of 190 questions in the whole preliminary survey questionnaire draft.

A cover letter was also designed to introduce the research participants to the questionnaire and the purpose and goals of the study were stated. In the letter, the research participants were also informed about the data confidentiality and it was acknowledged that Human Ethics Committee (HEC) approval had been gained prior to the study. Equally, the introduction explained the purpose and intended use of survey data and promised anonymity of respondent and company in the reporting. The introduction was also intended to seek the respondents/participants consent before they participated in this research, specifically by agreeing to move on to the next part from the introduction section (represented by qualtrics link or an >> button), the respondents had consented to participate in this study.

\subsubsection{Survey questionnaire pretesting}

The survey questionnaire was pre-tested with the major goal to assess its usability and face validity (Cavana et al., 2001). This included finding out software developers' perceptions of the length of the questionnaire, the wording of the items as well as the questionnaire structure. The pre-test of the questionnaire was administered to a sample of respondents who came from the same target population (Collis \& Hussey, 2003). Importantly, the participants were selected in such a way so as to achieve a wide variety of individual characteristics across the different project management groups in New Zealand.

Due to the questionnaire design described earlier, it was decided to use an online survey system for the pilot and main study for the following reasons. First, as this was a global survey the nature of this study's geographical scope was very big so it would not have been 
easy to travel to all countries in the entire world. Second, this study targeted vndors as the respondents who have easy and continuous access to computers and online facilities. Third, it was intended to receive general useful feedback for the main study regarding the questions and the general structure of the survey. This allowed for potential changes since using online survey applications like Qualtrics.com enables researchers to modify the survey questionnaire during the data collection.

Additionally, online questionnaires are generally similar to mail questionnaires in that they are easy to administer and respondents can complete them at their convenience (Cavana et al. 2001). Another major advantage of online questionnaires is that they can be distributed quickly and inexpensively (Cavana et al. 2001). By asking the respondents to complete the survey questionnaire online, testers could provide feedback on the usability of the online survey tool as well as giving feedback on the "look and feele of the online survey questionnaire (e.g. how long it would take to load the website, visual layout of the online survey, etc.). Qualtrics (qualtrics.com) was used as the software application to collect data for the pilot test and main study. Qualtrics is an industry-leading survey software application and is accessible to all $\mathrm{PhD}$ students at Victoria University of Wellington.

\subsubsection{Ethical considerations}

Human ethics committee approval was obtained from the Victoria University of Wellington before both the semi-structured interviews and the global survey were conducted. Each respondent was asked to sign a research agreement confirming that confidentiality would be maintained and that the reporting will be done in a non-attributable form. Participants in the pre-test had the right to withdraw any time before the beginning of data analysis and the questions that were asked were not sensitive or intended to collect strategic or confidential information relating to themselves, their organisation or specific projects but rather to elicit information on how the selected variables are practiced. The participants were assured that the findings in this report and other publications would only be presented in an aggregated and non-identifiable form. Exploratory semi-structured interviews were neither audio recorded nor transcribed. Data were secured and only accessible by the researcher and his supervisors. An assurance was made that all the materials will be destroyed within a period of three years after the end of this study. This delay allows verification of the data for future publication in academic journals. 


\subsubsection{Survey operationalization}

In order to conduct the pre-test, survey software from qualtrics.com was used and the preliminary survey questionnaire instrument was entered online. The online survey included a text box at the end in order to give respondents the opportunity to comment on the survey questionnaire instrument. Before sending out email invitations, the $\mathrm{PhD}$ supervisory team checked the online survey system. Next, email invitations were sent out to personal contacts of the research participants. For the first pre-test round, theoretically motivated purposive sampling methods were employed in selecting participants in Wellington. As explained, the email invitations stated the purpose of the pre-test and invited the recipients to respond via the qualtrics-survey URL.

\subsubsection{First pre-testing phase}

A total of forty-five senior software vendors from New Zealand volunteered to participate during the first round of pre-testing and all testers completed the online survey questionnaire. Most of them commented on the survey questionnaire instrument as well as the wording of some of the items. Most comments were provided within the embedded text box at the end of the questionnaire but some testers chose to send a separate email or provided personal feedback to the researcher. Similarly, some respondents that initially completed the online survey draft, made some separate detailed notes in cases where items/sections were confusing. Subsequently 15 respondents in Wellington, New Zealand, were requested to participate in the semi-structured interviews to discuss the whole online survey in detail. These interviews aimed at finding out more about the details of the questions/items that the respondents had found unclear or ambiguous, as well as discussing the instructions, structure and length of the questionnaire.

\subsubsection{General feedback from the initial online survey draft}

Overall, the feedback given by the respondents indicated that the structure of the survey questionnaire was reasonable but the length was long. Furthermore, some testers indicated that they had issues with the wording of some items. However, in these cases every participant suggested minor modifications to improve the quality of the online survey questionnaire. The following were some of the relevant comments made by the initial respondents in regard to the general structure, content and length. 
Respondent A stated that: 'the size of the online questionnaire is okay. Questions are comprehensive and clear to understand'.

Similarly, respondent B said that: 'the survey questions are clear in general. Please add a function in qualtrics to put a backward button in the questionnaire in case one wants to move backwards to change something that has already been filled in.'

Respondent $\mathrm{C}$ made almost similar observation that: 'the questionnaire is clear to understand in general but long'.

However, respondent D noted that: 'there are many questions'. Apart from this, this tester did not point out any further issues with the survey questionnaire and provided positive feedback on the survey questionnaire.

Respondent E commented that: 'the size of the questionnaire is slightly longer than I anticipated. The questionnaire should be slightly trimmed if possible to avoid respondents feeling bored and failing to complete it.'

Likewise, respondent $\mathrm{F}$ noted that: 'the size of the questionnaire is really long. The questionnaire should be reduced by at least $25 \%$ to increase responses.'

Next, a meeting was held with the PhD supervisory team and all these comments outlined were thoroughly discussed. Apart from the issue of the length of the online survey draft that raised mixed comments from testers as either being long or not, other suggestions from testers were only minor and did not seem to affect the initial questionnaire draft significantly. It was resolved in this meeting that the researcher should go ahead to conduct all semistructured interviews and also to investigate further the issue of the size of the online survey with the interviewees.

\subsubsection{Semi-structured interviews}

Of the 45 research participants who had completed the online survey, 15 respondents also volunteered to contribute via semi-structured interviews. Subsequently each respondent was interviewed and asked if they found items unclear or ambiguous. The instructions, structure and length of the questionnaire were also discussed. All the semi-structured interviews were conducted after all the 15 participants had first completed the initial online survey draft. This 
exercise was done before the global web-based survey which would be the main method of data collection. The aim of the interviews was to pre-test the international web-based survey. Specifically, the semi-structured interviews contributed to the subsequent main survey and study in the following ways:

- The interaction with software development experts and executives helped to improve the questionnaire, thereby increasing its content validity.

- Interviews helped the researcher to get a deeper insight into the study variables of interest from the vendors' practical project management perspective.

- Through interaction with senior software vendors who are affiliated to diverse professional bodies like PRINCE2, PMI and Agile the researcher was able to identify more firms and the relevant respondents for questionnaires.

- Where appropriate, the responses from the semi-structured interviews were used to complement or discuss the non-significant or significant findings.

\section{Characteristics of the interview participants}

Senior vendors who are members of traditional plan-based project management (PRINCE2, PMI) and agile communities with practical experience in project management Wellington Central, New Zealand, were interviewed. Basically, all the interviewees who participated had high experience within a range of 12-37 years in software development projects. These had a wide knowledge about the subject matter of the study and were very resourceful in providing the required detailed explanations on outsourcing as well. They are members of local chapters who organize monthly meeting sessions in Wellington Central. Through attendance of these meetings a wider social network of project managers was established. Table 43 shows the profile of the research participants who contributed in the semi-structured interviews. The interviewees were drawn from PRINCE2, PMI and Agile. 
Table 43: Profile of research participants who contributed in the semi-structured interviews

\begin{tabular}{|c|c|c|}
\hline $\begin{array}{l}\text { Professional } \\
\text { affiliation }\end{array}$ & Participant & Participant's job description \\
\hline \multirow{5}{*}{ PRINCE2 } & 1 & $\begin{array}{l}\text { Develops software for trading, investing, hedging and funding multiple asset classes, including } \\
\text { interest rates, credit, currencies, equities and commodities, } 28 \text { years' experience. }\end{array}$ \\
\hline & 2 & $\begin{array}{l}\text { Ensures customers receive the best solution, on-schedule and according to strict project } \\
\text { specifications. Consistently monitors project's success, } 12 \text { years' experience. }\end{array}$ \\
\hline & 3 & $\begin{array}{l}\text { Development and delivery of change management programs using PROSCI methodologies, } \\
\text { PRINCE2: Recovery of failing projects, Treasury Business Case Process, } 18 \text { years' experience. }\end{array}$ \\
\hline & 4 & $\begin{array}{l}\text { Manage Online Software Development project activities for multiple projects across all project } \\
\text { phases. Collaborates to determine technical direction and system design, } 25 \text { years' experience. }\end{array}$ \\
\hline & 5 & $\begin{array}{l}\text { Manage multiple teams of engineers, recruits, hires, mentors and coaches software development } \\
\text { managers. Interfaces with internal customers to understand requirements, } 16 \text { years' experience. }\end{array}$ \\
\hline \multirow{5}{*}{ PMI } & 6 & $\begin{array}{l}\text { Monitoring all projects and implementing quality assurance practices. Help develop long-term } \\
\text { development and business technology strategies. Drive improvements in software engineering } \\
\text { practices across engineering teams, } 13 \text { years' experience. }\end{array}$ \\
\hline & 7 & $\begin{array}{l}\text { Integration of the new system SAP with over other } 50 \text { systems, like transactional, human } \\
\text { resources, accounting etc., } 29 \text { years' experience. }\end{array}$ \\
\hline & 8 & $\begin{array}{l}\text { Makes design choices and dictates technical standards, including software coding standards, tools, } \\
\text { and platforms. Analyses design, oversees the software development, } 37 \text { years' experience. }\end{array}$ \\
\hline & 9 & $\begin{array}{l}\text { In charge projects planning, execution, control and completion. Ensures value is added to clients } \\
\text { with best-of-breed solutions to create competitive advantage, } 24 \text { years' experience. }\end{array}$ \\
\hline & 10 & $\begin{array}{l}\text { Provides ongoing project status reporting, strategic planning and asset creation processes. Coaches } \\
\text { and trains project team members on Software processes and best practices. } 33 \text { years' experience. }\end{array}$ \\
\hline \multirow{5}{*}{ Agile } & 11 & $\begin{array}{l}\text { Drives key decisions across projects-trade-offs and risks, recommends to management about } \\
\text { schedules, prioritization and resource allocation, } 36 \text { years' experience. }\end{array}$ \\
\hline & 12 & $\begin{array}{l}\text { Facilitates productivity by ensuring that the team has tools to succeed, removes project roadblocks, } \\
\text { ensures daily sprint and release commitments are being upheld, } 17 \text { years' experience. }\end{array}$ \\
\hline & 13 & $\begin{array}{l}\text { Collaborates with internal and external customers as needed in regards to project deliverables } \\
\text { including managing expectations, presenting and interfacing with sponsors, } 27 \text { years' experience. }\end{array}$ \\
\hline & 14 & $\begin{array}{l}\text { Tailors project management and support processes to meet the needs of individual projects. } \\
\text { Manages the day-to-day activities of projects, } 21 \text { years' experience. }\end{array}$ \\
\hline & 15 & $\begin{array}{l}\text { Facilitates daily stand up meetings, reviews retrospectives, sprints and releases, demos and ensures } \\
\text { practicing the core agile principles of collaboration, prioritization etc., } 29 \text { years' experience. }\end{array}$ \\
\hline
\end{tabular}

\section{Conducting semi-structured interviews}

The purpose of the semi-structured interviews was to assess the content validity of the newly designed questionnaire instrument. Content validity refers to the extent to which the measures include an adequate and representative set of items that tap into the construct (Cavana et al., 2001, p. 213). There are essentially three ways of achieving content validity: using tested measures from the literature, qualitative research and the judgement of a panel of experts. Usually researchers identify specific points that describe a given construct from the literature, interviews or qualitative data of some kind which are then measured in a questionnaire. Similarly, in the current study, to ensure that these specific questions are representative of the constructs in the research model, a full list of questions were referred to practioners and experts for assessment.

To organise these semi-structured interviews, software development project managers (vendors) who are based in Wellington were invited via e-mail or phone to participate in the 
interviews. These semi-structured interviews took about 30 minutes each and were neither audio-recorded nor videotaped. However, field notes were taken during or immediately after the interview. These field notes were also analysed and consolidated in order to identify a list of issues raised during the interviews. To optimise the outcome of the discussions, all these interviews were face-to-face and each interview was conducted separately. The overall targeted number of participants was 15, and a total of 15 interviews were conducted separately in Wellington. As the current research intended to investigate traditional planbased (PRINCE2 \& PMI) and agile projects, 5 participants were selected from each community of methodology practice. A formal arrangement with these 15 respondents was made to meet them at a café, Victoria University or their work place depending on their convenience to ensure confidentiality was preserved.

During each interview, initially the goals of this research were clearly outlined. Next, the survey questionnaire was introduced again and presented to the interviewees, who gave their opinions on this questionnaire based on their practical experience in project management. All the constructs (VPoC organisational factors, vendor perception of team factors, vendor perception of customer factors, vendor perception of project factors and project success) in the research model were again defined and explained carefully to the interviewees. Each of the aforementioned constructs had their respective variables (measures) as specified in the research model; with each variable written down with a corresponding actual survey item (question) wording in the questionnaire. For clarity, accuracy and easy interpretation of these variables during the interviews, a list of theoretical definitions was provided during the interview. It was also brought to the attention of interviewees that the conceptualisation of these constructs was based on the literature review. As the interviews proceeded, the participants were requested to give and explain their comments so that a better understanding of what happens in practice was obtained. Their responses were clearly based on the participants' experience and knowledge.

The interviewees were asked if these questions cover the most essential candidate CSFs for outsourced software project success. Similarly, the interviewees were asked if they know of any other dimensions that can be used as measures or can be used to describe these constructs, with particular emphasis on methodology selection that can help to achieve project success. It is at this stage the interviewees were also asked to identify any 
measurement items that are worded unclearly or that they would describe as being vague. Finally, the participants were asked if they have any further ideas regarding the initial survey questionnaire instrument. Apart from the length of the survey questionnaire which provided mixed comments, all testers did not report any negative issues with the initial online survey draft and they found the structure suitable.

The following sections discuss the feedback received for each section of the initial online survey draft during the interviews. The following comments were also made with regard to each of the survey questionnaire sections.

\section{Interview feedback on the introduction section of the questionnaire}

Generally, the comments that were expected for the introduction part of the questionnaire were largely for improving the outline and presentation of this section rather than the providing specific feedback on the questionnaire items. Interestingly, all the interviewees unanimously agreed that the introduction part of the questionnaire was clear and precise. For instance Participant 3 said that: I found the introduction of the online survey well organized and clear. I liked it! Similarly, Participant 7 said that: 'The clarity of the introduction is okay. 'I'm happy with the content'. Likewise Participant 11 confirmed that: 'I really like the way you have framed your introduction, it sounds good'. All these research participants among others provided support that demonstrates that the introduction was well designed in terms of wording, content and the size.

\section{Interview feedback on section A of the online questionnaire}

As earlier described, this part was intended to determine the demographic characteristics of the respondents as well as the important characteristics of the projects. Most of the interviewees were satisfied with the format and content of section B of the questionnaire. However, participant 14 suggested that: 're-arrange questions $6,7 \& 8$ not as initially done in that current order to allow easy flow of answering and following up by respondents'. This suggestion was taken and changes made to that effect.

\section{Interview feedback on section $B$ of the online questionnaire}

All the research participants consistently agreed that the size/length of section B of the online questionnaire was long. However, apart from this concern, they also agreed based on their different experiences that the variables included in the online questionnaire affect project 
success. They also expressed mixed responses on how they measure project success in practice. The following are some of the comments from the interviews.

For instance, one participant (2) said that: "This section has many questions. However, all factors included affect project success and failure for software development projects either directly or indirectly. I have experience in using PRINCE2-waterfall for over 11 years. Yes, true some of my SD projects have been successful while others failed. The challenge of measuring project success due to stakeholder expectation gap exists”.

Another participant (7) said that: "the questions were many and assumed that one can follow up to the end". However, the research participant agrees that "all factors included in the online questionnaire affect project success and failure for SD projects". The participant also explains a lot about his experience in using PRINCE2 and PMBOK for over 25 years in SD projects mainly in public sector. About measuring project success, he suggests that "it's all about making the customer happy and ensuring that you're on track”.

Another research participant (14) noted that 'the questionnaire is clear to understand in general but very long'. She also agrees that: all factors included in the questionnaire may affect project success and failure for SD projects. She says that: she uses 'expected project benefits to measure project success by making a comparison with the original objectives in the business case'.

Another respondent (3) made this comment: The online survey took me about 26 minutes to complete. This is a long time'. However, the participant also agrees that: all factors included in the questionnaire affect project success and failure for SD projects. When asked about measuring project success in practice, the participant advises that 'project success is all about making business owners happy, evaluating benefit realization and keeping the business owners close to the project'.

Based on these views, it was evident that section B of the questionnaire was long. As a result, the respondents were taking about 26 minutes on average to complete the whole online survey. It was also clear again that the variables and questions included in the questionnaire could impact on project success. However, what remained unclear was which survey questions should be removed and what criteria could be followed. The next section discusses 
the item reduction process. The criteria that were followed to reduce the survey questions in section B of the online survey are also explained.

\subsubsection{Item reduction in section $B$ of the initial online survey draft}

Despite the fact that testers believed that the questions were generally clear and represented what happens in practice, the size of section B made the online questionnaire overly long. In particular, the number of questions for each factor or latent variable in section B was viewed as unnecessarily extensive. Yet, all these questions were based on previous research and literature. Therefore, what was challenging was the best way of reducing these questions without discarding the best ones.

This was not straight forward which questions were better than others for each variable so that they would converge well during the data analysis. If not done carefully, this implied that more suitable questions might be dropped. Yet, at the same time this sample was too small to conduct an exploratory factor analysis and reliability analysis (e.g. Cronbach's alpha).

The total pool of items was discussed in several meetings with my $\mathrm{PhD}$ supervisory team. The primary goal of these discussions was to identify the most appropriate items for each construct before conducting the second pre-testing round. The overall number of items needed to be reduced since 190 items were judged to be too many for the main online survey to achieve a high response. It was finally resolved that only items which have been previously tested for reliability and validity and have demonstrated high psychometric properties should be retained. Therefore, the simple criteria were to consider the previously used survey questions based on respective factor loadings, Cronbach's alpha, composite scale reliabilities and average variance extracted. For the case of questions where these psychometric properties such as factor loadings, Cronbach's alpha, composite scale reliabilities and average variance extracted were not explicitly indicated in previous studies, such questions were discussed further in subsequent interviews and the questions which were suggested to be unclear and not properly stated by participants were also discarded.

Using this criterion, the questions were significantly reduced in section B of the online survey draft from 178 to 125 representing a reduction of $28 \%$. Table 44 depicts a summary of questions that were discarded from the initial online survey draft. A total of $53(28 \%)$ questions were removed. 
Table 44: Summary of questions that were discarded from the initial online survey draft

\begin{tabular}{|l|l|l|}
\hline Section & Initial questions & Final questions \\
\hline A & 12 & 12 \\
\hline B & 178 & 125 \\
\hline Total & 190 & 137 \\
\hline
\end{tabular}

The next section presents the second pre-testing phase that was conducted after reducing the initial online survey draft (final questionnaire in Appendix G).

\subsubsection{Second pre-testing phase}

The survey questionnaire pre-tested in the second round in this study also had an introduction and two major parts. The first part, section A, was intended to determine the demographic characteristics of the respondents as well as the important characteristics of the firm (sample characteristics). The second part, section $\mathrm{B}$, consisted of the operationalized and itemized components of VPoC organizational factors, vendor perception of team factors, vendor perception of customer factors, vendor perception of project factors and project success.

Similarly, the research instrument measured all items in section B on a seven point Likert scale. Further, to ensure validity, the adjusted instrument was discussed with VMS management professors and content experts in the field of project management at Victoria Business School, a major research university in Wellington city, New Zealand. Among these, four professors had worked on software projects for more than four years indicating a wide experience on this area.

The respondents were again requested to read through all the items in the questionnaire and be given an opportunity to indicate whether the question/item was difficult or not clear to them. Similarly, the experts were specifically requested to indicate whether the items in particular sections of the questionnaire adequately measure the respective study constructs, and whether the instrument was appropriate for this kind of study.

\subsubsection{Final feedback from the adjusted online survey instrument}

After significantly reducing the questions in section B by $28 \%$, the second pre-testing round was undertaken. Like the initial draft, the adjusted online survey included a text box at the end in order to give respondents another opportunity to comment on the adjusted survey questionnaire instrument. Next, 200 email invitations were sent out to personal contacts of the researcher, all being project workers working in New Zealand. As with the first pre-test 
round, purposive sampling methods were also employed in selecting participants for the second pre-test. This was consistent with the purpose of this study to collect data from both traditional and agile project to allow group comparisons. Likewise, the email invitations stated the purpose of the study and invited the recipients to follow up the qualtrics-survey URL. After two days, many respondents had completed the survey. The researcher checked the text boxes for comments and found the following.

One participant highly commended the size of the adjusted online survey and said that he was also happy with the clarity and content. 'Very interesting. It made me think of the project as an introspective way. I would be glad to know the study results'. Similarly, another respondent added a comment that: 'Size of the questionnaire is okay. Content and clarity seems pretty good'. Likewise, another respondent commented that: 'The size of the questionnaire is okay. Questions are clear and they cover the whole SD process'.

Another respondent suggested that: 'It would be useful to start the questionnaire with a general enquiry about whether the respondent is involved in software development projects at all (i.e. not all project managers are involved in software development). Also, some questions are not applicable (i.e. whether the company would be bankrupted does not apply to public sector agencies). Otherwise, well done the size of the questionnaire is okay. Questions are comprehensive and clear to understand'.

It was evident that all the comments that were provided after the initial survey drafted was adjusted were all positive and good with none of the testers suggesting major modifications. Therefore, based on these positive comments and the good responses that had come in at that time, it was viewed that the adjusted online questionnaire length was reasonable. Next, another batch of 400 e-mail invitations were sent out but again only limited to New Zealand and Australia. The use of qualtrics.com enabled the researcher to make adjustments to the activated online survey questionnaire. Using this function, the adjustment of the survey questionnaire instrument took place after the pilot study had started. 69 responses were collected using the original survey draft. These responses were all deleted from the system because the wording of some questions was adjusted and they could not be used in subsequent analysis. A total of 255 respondents replied to the adjusted survey questionnaire from New Zealand but the sample size was still relatively small and could not facilitate appropriate SEM analysis. The global data collection exercise followed. 


\subsection{Data collection-main phase}

A global web-based survey was conducted following the guidelines set forth by Dillman (1991). Survey was inexpensive and convenient for senior software vendors who have easy access to a computer. The internet data collection also avoids transcription errors (Tate, 2010) and enables access to a large number of participants all around the world in a very short period of time (Hoehle, 2011). It also ensured anonymity since the respondents did not have to provide identifying information such as email details. However, web-based surveys have been found to suffer from fairly low response rates and the responses can be biased if the respondents are not representative of the population (Cavana et al., 2001, p. 244).

The survey was expected to take about 15 minutes to complete. Questions were to the point, addressed only a single issue at a time and avoided phrases that could elicit social or professional methodological affiliation biased responses. Each construct was measured by at least three questions that were relevant in terms of prior research or established theory. Survey questions were designed to capture the perceptions of senior vendor project managers about software development practices for which they are expected to be the most knowledgeable. However, in using single informants it is also desirable to select the most experienced and knowledgeable person. To reduce the possibility of single-informant bias from exaggeration and self-promotion and to encourage participation, the respondents were informed that results would be completely anonymous.

All the targeted respondents were contacted by email and after one week reminders were sent. The survey remained online for over two months. Additionally, each respondent was asked to fill in the survey for only the last project he/she worked on regardless of whether the project was successful or not. This provided respondents with clear criteria for selecting the required projects and reduced the potential bias of reporting on only successful projects so that even the failed projects were collected in the database for the analysis. The respondents were also contacted progressively to ensure that all the needed data was collected.

\subsubsection{Response rate}

Overall, 3,800 e-mail invitations were sent out to all the intended research participants during data collection of this study. Of these e-mails invitations 1,266 automatic responses were received back confirming that either the owners had changed their original contacts or that the e-mails were no longer valid. 2,080 participants started to complete the survey 
questionnaire. Out of these, only 1,905 respondents completed and submitted the online survey questionnaire. 25 of these responses were identified as skimmers and deleted. 'Skimmer' respondents complete the survey questionnaire in a very short period of time by rating most of the items equally. These responses were identified by comparing the completion time in combination with the responses obtained for the survey questionnaire overall. For example, two respondents completed the entire survey in $4 \mathrm{~min}$. and $12 \mathrm{sec}$ and 3 min. and 16sec respectively and all responses were valued with either a 4 or 5 . But the average duration time of all respondents was approximately about $15 \mathrm{~min}$. In total 1,880 usable responses were received of which 1,367 had clearly specified the project management used. This represents about $49.5 \%$ of the initial valid e-mail invitations sent out. This is far better than the usual response rate of $20 \%$ reported in literature of most SEM studies in project management.

Table 45 summarises the details of the e-mails that were initially sent out as well as the valid responses received. Note: Although the researcher's membership to PRINCE2 was granted, it did not come on time; as a result, most of the members' e-mail contacts which were obtained from the PRINCE2 website were not valid.

Table 45: summary of initially sent out e-mail invitations, the invalid e-mails and final responses

\begin{tabular}{|c|c|c|c|}
\hline Affiliation & Initially sent & Respondents & Non respondents \\
\hline PMI (Traditional) & 1500 & 662 & 838 \\
\hline PRINCE2 (Traditional) & 400 & 41 & 359 \\
\hline Agile & 1900 & 664 & 1236 \\
\hline Others & N/A & 242 & N/A \\
\hline Missing & N/A & 271 & N/A \\
\hline Total & 3800 & 1880 & 2433 \\
\hline \multicolumn{2}{|r|}{ Others include respondents who used a hybrid. Missing means did not specify a methodology used. } \\
\hline
\end{tabular}

Next, the responses which had used a combination (hybrid) of methodologies $(n=242)$ were not considered in the data analysis, because the purpose of this study is to primarily compare the traditional plan-based methodology and agile methodology projects. Therefore, the projects that were indicated in the survey to have used a combination of both project methodologies were excluded from data analysis. Similarly, since this study takes a vendor perspective and only examines outsourced software development projects, all client responses were excluded from data analysis. Likewise, the survey responses which could not be identified by the researcher as either vendors or clients were discarded. This leaves only 984 
valid vendor responses (i.e. total of vendor responses $n=984, n=513$ for traditional plan-based methodology and $n=471$ for agile methodology projects) that were examined in this study.

The following cross tabulation/contingency table (Table 46) summarises the percentages of respondents in traditional plan-based and agile methodology project management data sets respectively that belong to either client or vendor groups.

Table 46: A Cross tabulation for client and vendor groups

\begin{tabular}{|l|c|c|c|}
\hline & $\begin{array}{c}\text { Traditional plan-based } \\
\text { methodology projects }\end{array}$ & $\begin{array}{c}\text { Agile projects } \\
\text { methodology }\end{array}$ & Aggregated \\
\hline Vendors' responses identified & $513(73 \%)$ & $471(71 \%)$ & $984(72 \%)$ \\
\hline Clients' responses identified & $71(10 \%)$ & $106(16 \%)$ & $177(13 \%)$ \\
\hline Total identifiable responses & $584(83 \%)$ & $578(87 \%)$ & $1,162(85 \%)$ \\
\hline Not distinguishable responses & $119(17 \%)$ & $86(13 \%)$ & $205(15 \%)$ \\
\hline Total sample & $703(100 \%)$ & $664(100 \%)$ & $1,367(100 \%)$ \\
\hline
\end{tabular}

The following chart (Figure 34) further depicts the number of respondents in traditional planbased methodology and agile methodology project management data sets that belong to either client or vendor groups.

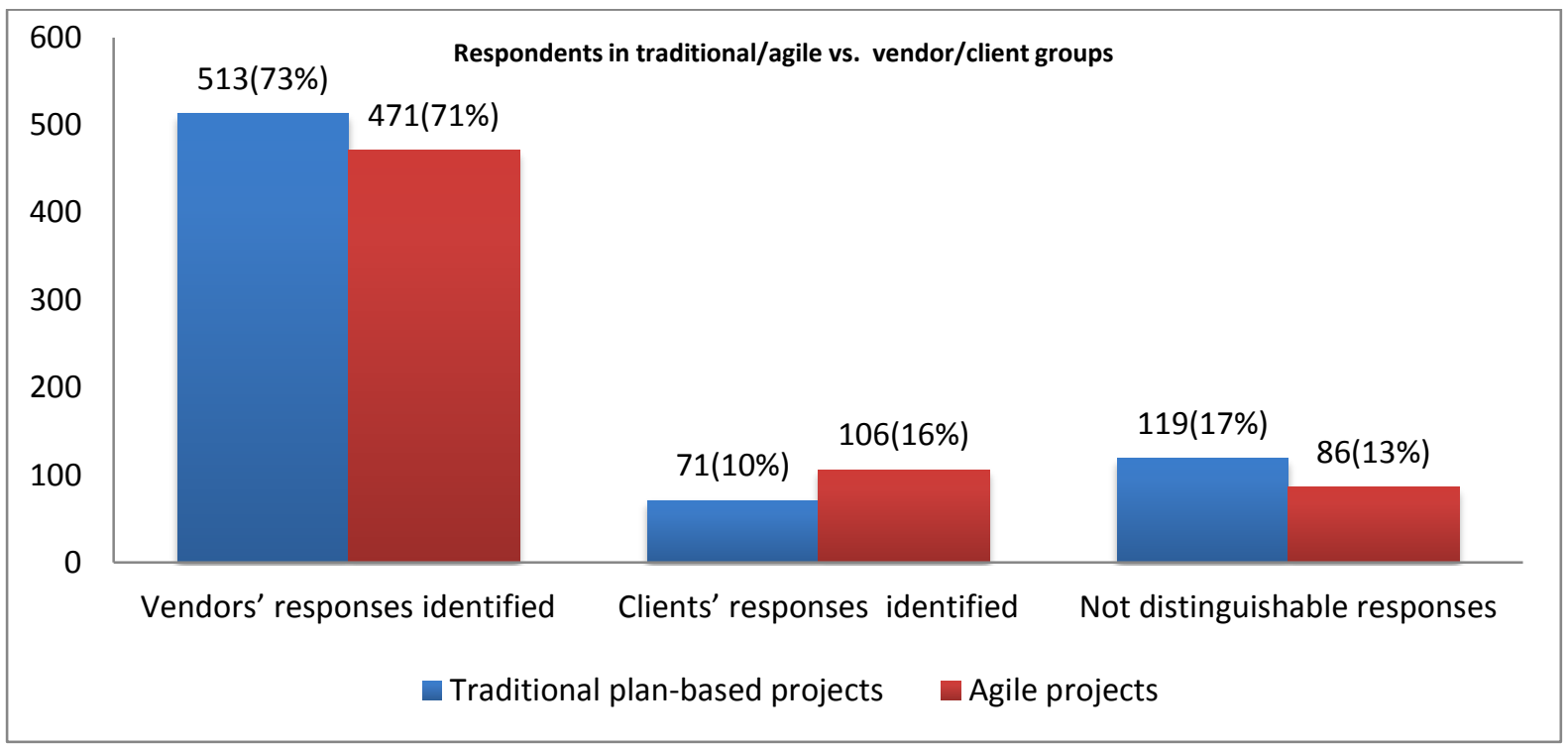

Figure 34: Respondents in traditional/agile methodology vs. vendor and client groups

\subsection{Data Analysis-main study}

The survey data was analysed using SEM. SEM is a multivariate technique in which parameters are estimated by minimizing the discrepancy between the model-implied covariance matrix and the observed covariance matrix was used in tracing structural 
relationships (Joreskog \& Sorbom, 1989). SEM was selected in this study because it (i) tests an overall model rather than individual coefficients (ii) is a confirmatory approach that provides explicit test statistics for establishing convergent and discriminant validity important to management research (iii) allows for error terms (iv) reduces measurement error through the use of multiple indicators and (v) SEM also allows group comparisons (Straub, 1989; Hair et al., 2010). The robustness of SEM using MLE has been demonstrated in prior management/project management/MIS research (Bergeron et al., 2001; Kearns \& Sabherwal, 2007; Jung et al., 2008).

The approach chosen was to separate analysis of the measurement and structural models in a two-step process recommended by Anderson and Gerbing (1988). This is consistent with prior project management research that have used SEM (e.g., Bergeron et al., 2001; Kearns \& Sabherwal, 2007; Jung et al., 2008) and allowed refinement of measures before testing of the structural model. In the first phase, a measurement model was used to measure the fit between the theorized model and observed variables and to establish reliability and validity. In the second phase, results of the measurement model were used to create a structural model in order to measure the strength of the theorized relationships.

Goodness-of-fit was established by multiple indices to negate bias associated with the use of a single index (Joreskog \& Sorbom, 1989; Hair et al., 2009). Chi-square $\left(\chi^{2}\right)$ was used to test the hypothesis if the relationships proposed in the model provide a plausible explanation of those that exist in the data. However, when sample sizes are large as in the present study, a non-significant chi-square is rarely obtained. Because the larger the sample size, the greater the statistical power, and so even smaller differences are reported as indicating statistically significant misfit between the data and the model (Iacobucci, 2010, p.91). Therefore, other additional measures have been developed to assess the fit of the model. In the present study because the $\chi 2$ value is almost always significant in large sample sizes, it was replaced with the ratio of $\chi^{2}$ to the degrees of freedom $\left(\chi^{2} / d f\right)$. Other measures used include; Tucker Lewis Fit Index (TLI), the Comparative Fit Index (CFI), the Root Mean Square Error of Approximation (RMSEA), the Normed Fit Index (NFI), Goodness of Fit (GFI), Adjusted Goodness of Fit (AGFI) and the Parsimony Ratio (PRATIO).

The TLI compares the lack of fit of a target model to the lack of fit of a baseline model, usually the independence model, and is one of the indexes affected least by sample size 
(Joreskog \& Sorbom, 1989). The CFI also has the advantage of reflecting fit at all sample sizes and measures the comparative reduction in noncentrality (Bentler, 1990). The RMSEA avoids issues of sample size by analyzing the discrepancy between the hypothesized model and the observed data, using the optimally chosen parameter estimates and the population covariance matrix (Hu \& Bentler, 1999). The NFI analyzes the discrepancy between the chisquared value of the hypothesized model and the chi-squared value of the null model (Bentler \& Bonnett, 1980). The Parsimony Ratio (PRATIO) is an overall measure of how parsimonious the model is (Byrne, 2001). The GFI is a measure of the relative amount of variance and covariance in the sample covariance matrix that is jointly explained by the population covariance matrix whereas the AGFI adjusts for the number of degrees of freedom in the specified model (Byrne, 2001).

It should be noted that it is it is very difficult with social phenomena to obtain really goodfitting models. There are social factors that can affect the responses collected which impact on the accuracy of model fit indices. Social factors such as self-reports, consistency motif, acquiescence, social desirability, affectivity and transient mood state are proposed to affect fit (Podsakoff, Mackenzie, Lee \& Podsakoff, 2003). These factors can either inflate or deflate observed relationships between constructs, thus leading to both Type I and Type II errors. Method biases are a problem because they are one of the main sources of measurement error (Podsakoff et al., 2003). Measurement error threatens the validity of the conclusions about the relationships between measures and is widely recognized to have both a random and a systematic component. Random error is caused by the accuracy limit of the measuring instrument while systemic error is caused by incorrect calibration of the measuring instrument. Although both types of measurement error are problematic, systematic measurement error is a particularly serious problem because it could provide an alternative explanation for the observed relationships between measures of different constructs that is independent of the one hypothesized (Podsakoff et al., 2003).

Table 47 summarises sources of social phenomena that can lead to measurement error. 
Table 47: Summary of social biases in behavioural research

\begin{tabular}{|c|c|}
\hline Common rater effects & $\begin{array}{l}\text { Any artifactual covariance between the predictor and criterion variable produced by the fact that } \\
\text { the respondent providing the measure of these variables is the same. }\end{array}$ \\
\hline Consistency motif & The propensity for respondents to try to maintain consistency in their responses to questions. \\
\hline $\begin{array}{l}\text { Implicit theories (and illusory } \\
\text { correlations) }\end{array}$ & Respondents' beliefs about the covariation among particular traits, behaviors, and/or outcomes. \\
\hline Social desirability & $\begin{array}{l}\text { The tendency of some people to respond to items more as a result of their social acceptability } \\
\text { than their true feelings. }\end{array}$ \\
\hline Leniency biases & $\begin{array}{l}\text { The propensity for respondents to attribute socially desirable traits, attitudes, and/or behaviors to } \\
\text { someone they know and like than to someone they dislike. }\end{array}$ \\
\hline $\begin{array}{l}\text { Acquiescence biases (yea-saying } \\
\text { and nay-saying) }\end{array}$ & $\begin{array}{l}\text { The propensity for respondents to agree (or disagree) with questionnaire items independent of } \\
\text { their content. }\end{array}$ \\
\hline $\begin{array}{l}\text { Mood state (positive or negative } \\
\text { affectivity; positive or negative } \\
\text { emotionality) }\end{array}$ & $\begin{array}{l}\text { The propensity of respondents to view themselves and the world around them in generally } \\
\text { negative terms (negative affectivity) or the propensity of respondents to view themselves and the } \\
\text { world around them in generally positive terms (positive affectivity). }\end{array}$ \\
\hline Transient mood state & $\begin{array}{l}\text { The impact of relatively recent mood-inducing events to influence the manner in which } \\
\text { respondents view themselves and the world around them. }\end{array}$ \\
\hline Item social desirability & $\begin{array}{l}\text { The fact that items may be written in such a way as to reflect more socially desirable attitudes, } \\
\text { behaviors, or perceptions }\end{array}$ \\
\hline Item demand characteristics & The fact that items may convey hidden cues as to how to respond to them. \\
\hline Item ambiguity & $\begin{array}{l}\text { The fact that items that are ambiguous allow respondents to respond to them systematically using } \\
\text { their own heuristic or respond to them randomly. }\end{array}$ \\
\hline Common scale formats & $\begin{array}{l}\text { Artifactual covariation produced by the use of the same scale format (e.g., Likert scales, } \\
\text { semantic differential scales, "faces" scales) on a questionnaire. }\end{array}$ \\
\hline Common scale anchors & The repeated use of the same anchor points (e.g., extremely, always, never) on a questionnaire. \\
\hline $\begin{array}{l}\text { Positive and negative item } \\
\text { wording }\end{array}$ & $\begin{array}{l}\text { The fact that the use of positively (negatively) worded items may produce artifactual } \\
\text { relationships on the questionnaire. }\end{array}$ \\
\hline Item context effects & $\begin{array}{l}\text { any influence or interpretation that a respondent might ascribe to an item solely because of its } \\
\text { relation to the other items making up an instrument }\end{array}$ \\
\hline Item priming effects & $\begin{array}{l}\text { The fact that the positioning of the predictor (or criterion) variable on the questionnaire can make } \\
\text { that variable more salient to the respondent and imply a causal relationship with other variables. }\end{array}$ \\
\hline Context-induced mood & $\begin{array}{l}\text { When the first question (or set of questions) encountered on the questionnaire induces a mood } \\
\text { for responding to the remainder of the questionnaire. }\end{array}$ \\
\hline Scale length & $\begin{array}{l}\text { The fact that scales have fewer items responses to previous items are more likely to be accessible } \\
\text { in short-term memory and to be recalled when responding to other items. }\end{array}$ \\
\hline $\begin{array}{l}\text { Intermixing (or grouping) of } \\
\text { items or constructs on the } \\
\text { questionnaire }\end{array}$ & $\begin{array}{l}\text { The fact that items from different constructs that are grouped together may decrease intra- } \\
\text { construct correlations and increase inter-construct correlations. }\end{array}$ \\
\hline Measurement context effects & Any artifactual covariation produced from the context in which the measures are obtained. \\
\hline $\begin{array}{l}\text { Predictor and criterion variables } \\
\text { measured at the same point in time }\end{array}$ & $\begin{array}{l}\text { The fact that measures of different constructs measured at the same point in time may produce } \\
\text { artifactual covariance independent of the content of the constructs themselves. }\end{array}$ \\
\hline $\begin{array}{l}\text { Predictor and criterion variables } \\
\text { measured in the same location }\end{array}$ & $\begin{array}{l}\text { The fact that measures of different constructs measured in the same location may produce } \\
\text { artifactual covariance independent of the content of the constructs themselves. }\end{array}$ \\
\hline $\begin{array}{l}\text { Predictor and criterion variables } \\
\text { measured using the same medium }\end{array}$ & $\begin{array}{l}\text { The fact that measures of different constructs measured with the same medium may produce } \\
\text { artifactual covariance independent of the content of the constructs themselves. }\end{array}$ \\
\hline
\end{tabular}

Saurce: Podsakoff et al. (2003)

The underlying dilemma is that the fit statistics are indicative of how well the data fits the data, but not absolute. The decision as to whether a set of data fits the model is a matter for the informed judgement of the analyst, based on theory and the nature of the measurements used to test the sample, rather than a matter of some arbitrary cut-off rule applied to a column of numbers on a computer print-out. 


\subsubsection{Reflective and formative constructs}

There has been an unresolved argument about when reflective and formative constructs should be used in research. Subsequently, many researchers have examined the consequences of mis-specifying constructs as either reflective or formative (e.g., Podsakoff et al., 2006; Diamantopoulos et al., 2008). The debate in modelling literature focuses on topics such as ontology, the assumptions of error measurement, the constraints required to achieve model identification, interpretational confounding, correlation and multicollinearity, the degree to which formative constructs can function as point variables, and whether formative constructs have a place in behavioural models at all or not (Coltman et al., 2008; Tate, 2010).

When using a reflective measurement approach, the indicators are assumed to reflect the variation in the latent variable. Therefore, the direction of causality is assumed to run from the construct to the indicators (Diamantopoulos et al., 2008). Changes in the constructs are expected to impact on all indicators as they are all part of the multi-item scale and, consequently multi-collinearity is desired among reflective measures. In contrast, however, formative measures 'form' the construct and the variation in the measures is assumed to cause variation in the construct rather than the other way round (Diamantopoulos et al., 2008). This means that high correlations among indicators are not desired, since that can result in an unstable construct. Figure 35 illustrates the differences between reflective and formative constructs based on the direction of causality between measures and constructs, and error terms (Diamantopoulos et al., 2008).

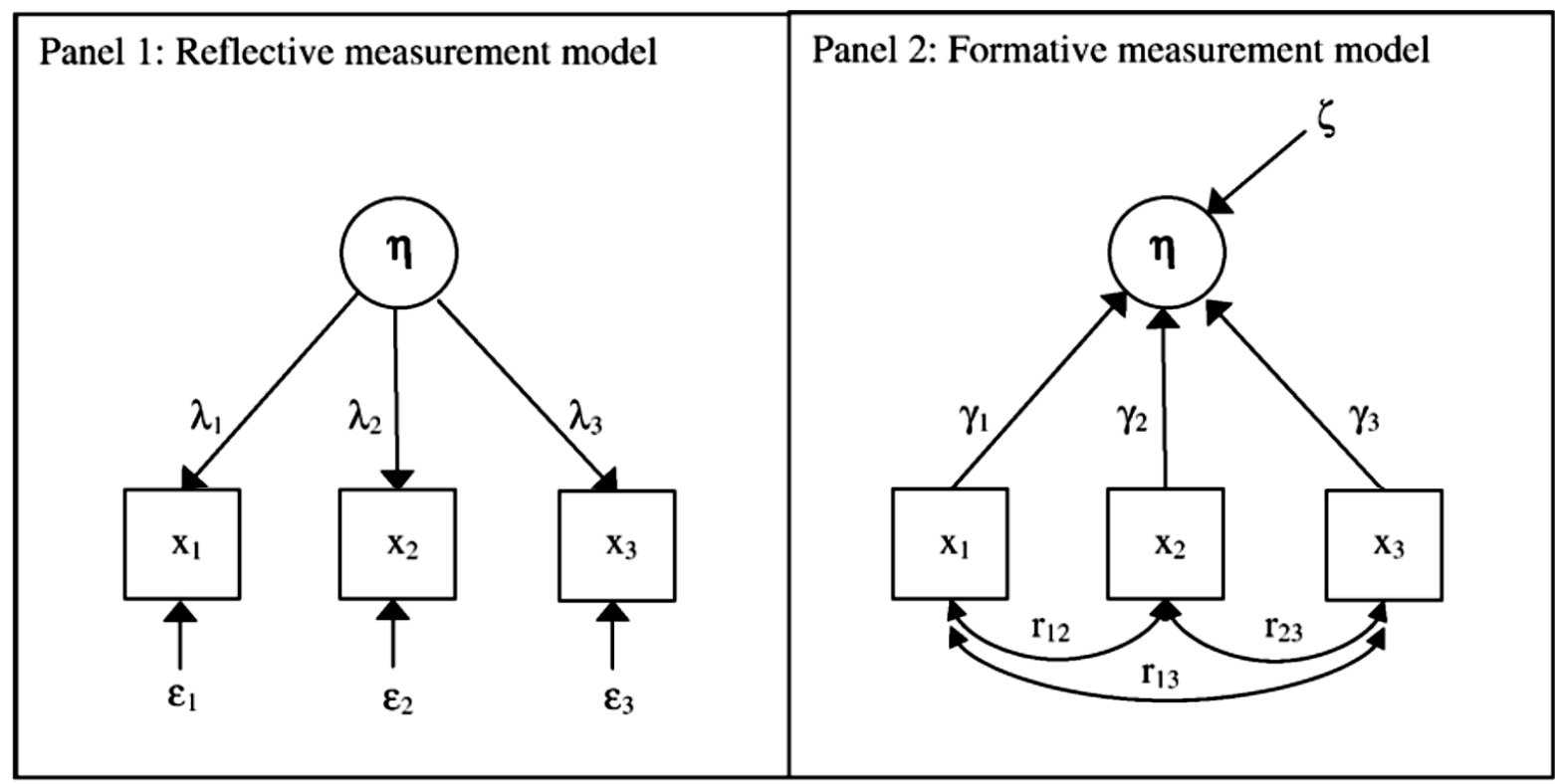

Figure 35: Alternative measurement models (Diamantopoulos et al., 2008, Figure 1, pg. 1205) 
Table 48 summarises differences between reflective and formative constructs.

Table 48: Differences between reflective and formative constructs

\begin{tabular}{|c|c|c|}
\hline Considerations & Reflective construct & Formative construct \\
\hline $\begin{array}{l}\text { 1. Nature of the } \\
\text { construct }\end{array}$ & $\begin{array}{l}\text { Latent construct is existing } \\
\text {-Latent construct exists independent of the } \\
\text { measures used }\end{array}$ & $\begin{array}{l}\text { Latent construct is formed } \\
\text {-Latent constructs is determined as a combination } \\
\text { of its indicators }\end{array}$ \\
\hline $\begin{array}{l}\text { 2. Direction of } \\
\text { causality between } \\
\text { items and latent } \\
\text { construct }\end{array}$ & $\begin{array}{l}\text { Causality from construct to items } \\
\text {-Variation in the construct causes variation in } \\
\text { the item measures } \\
\text { - Variation in item measures does not cause } \\
\text { variation in the construct }\end{array}$ & $\begin{array}{l}\text { Causality from items to construct } \\
\text {-Variation in the construct does not cause } \\
\text { variation in the item measures } \\
\text {-Variation in item measures causes variation in } \\
\text { the construct }\end{array}$ \\
\hline $\begin{array}{l}\text { 3. Characteristics } \\
\text { of items used to } \\
\text { measure the } \\
\text { construct }\end{array}$ & $\begin{array}{l}\text { Items are manifested by the construct } \\
\text { - Items share a common theme } \\
\text {-Items are interchangeable } \\
\text { Adding or dropping an item does not change the } \\
\text { conceptual domain of the construct }\end{array}$ & $\begin{array}{l}\text { Items define the construct } \\
\text {-Items need not share a common theme } \\
\text { - Items are not interchangeable } \\
\text { - Adding or dropping an item may change the } \\
\text { conceptual domain of the construct }\end{array}$ \\
\hline $\begin{array}{l}\text { 4. Item inter- } \\
\text { correlation }\end{array}$ & $\begin{array}{l}\text { Items should have high positive inter- } \\
\text { correlations } \\
\text {-Empirical test: internal consistency and } \\
\text { reliability assessed via Cronbach alpha, average } \\
\text { variance extracted, and factor loadings (e.g., } \\
\text { from common or confirmatory factor analysis) }\end{array}$ & $\begin{array}{l}\text { Items can have any pattern of inter-correlation } \\
\text { but should possess the same directional } \\
\text { relationship } \\
\text {-Empirical test: indicator reliability cannot be } \\
\text { assessed empirically; various preliminary } \\
\text { analyses are useful to check directionality } \\
\text { between items and construct }\end{array}$ \\
\hline $\begin{array}{l}\text { 5. Item } \\
\text { relationships } \\
\text { with construct } \\
\text { antecedents and } \\
\text { consequences }\end{array}$ & $\begin{array}{l}\text { Items have similar sign and significance of } \\
\text { relationships with the antecedents/ } \\
\text { consequences as the construct } \\
\text {-Empirical test: content validity is established } \\
\text { based on theoretical considerations, and } \\
\text { assessed empirically via convergent and } \\
\text { discriminant validity }\end{array}$ & $\begin{array}{l}\text { Items may not have similar significance of } \\
\text { relationships with the antecedents/consequences } \\
\text { as the construct } \\
\text {-Empirical test: nomological validity can be } \\
\text { assessed empirically using a MIMIC model, } \\
\text { and/or structural linkage with another criterion } \\
\text { variable }\end{array}$ \\
\hline $\begin{array}{l}\text { 6. Measurement } \\
\text { error and } \\
\text { collinearity }\end{array}$ & $\begin{array}{l}\text {-Empirical test: common factor analysis can be } \\
\text { used to identify and extract out } \\
\text { measurement error }\end{array}$ & $\begin{array}{l}\text { Error term cannot be identified if the formative } \\
\text { measurement model is estimated in isolation } \\
\text {-Empirical test: vanishing tetrad test can be used } \\
\text { to determine if the formative items behave as } \\
\text { predicted } \\
\text {-Collinearity should be ruled out by standard } \\
\text { diagnostics such as the condition index }\end{array}$ \\
\hline
\end{tabular}

Source: Coltman et al. (2008)

Of these two aforementioned approaches, reflective constructs that are modelled with covariance-based SEM is by far the most widely used and is considered most current and conventional than formative constructs that are based on Partial Least Squares (PLS) (Rouse \& Corbitt, 2008). However, the choice should be based on the psychometric properties of measures (Podsakoff et al., 2006). The reflective measurement model has a long tradition in social sciences and is directly based on classical test theory (Coltman et al., 2008). According to this theory, measures denote effects (or manifestations) of an underlying latent construct. Therefore, causality is from the construct to the measures (Diamantopoulos et al., 2008). Specifically, the latent variable represents the common cause shared by all items reflecting 
the construct, with each item corresponding to a linear function of its underlying construct plus measurement error. All constructs in this study were adopted from previous studies (e.g., Jun et al., 2011; Sheffield \& Lemetayer, 2013) where they were typically assumed as reflective and viewed as underlying factors that give rise to something that is observed. Thus, the reflective model specification was found to be most suitable in this study.

\section{Two tailed and one tailed tests}

There are two alternative ways of computing the statistical significance of a parameter inferred from a data set, in terms of a test statistic: a one-tailed test and a two-tailed test. When using a two-tailed test, regardless of the direction of the relationship you hypothesize, you are testing for the possibility of the relationship in both directions. When using a onetailed test, you are testing for the possibility of the relationship in one direction and completely disregarding the possibility of a relationship in the other direction. One tailed tests are a less conservative option and could lead to false positive support for some hypotheses. The default among statistical packages performing tests is to report two-tailed p-values. Because the most commonly used test statistic distributions (standard normal, Student's t) are symmetric about zero, most one-tailed p-values can be derived from the two-tailed p-values. In this study two tailed tests are used to test hypotheses formulated for each separate groups whereas the one tailed tests are used for hypotheses formulated to make comparisons across the two data sets.

\subsection{Methodological limitations}

The use of a questionnaire where all the data were collected in the same measurement context using a common rater and with common item context could have led to some common methods bias. Future studies should try to obtain measurements of the independent and dependent variables from different sources and at different times.

The study used a cross-sectional research design and the behaviours of the variables over a long time could not be completely analysed; this restricts the applicability of the findings as a longitudinal study may give slightly different results from the ones that were obtained for this study. For cross section data causality can only be inferred not proven. Future studies could extend in a longitudinal fashion which could make possible stronger causal conclusions.

The study uses a non-probability sampling method hence it was not possible to randomly select practitioners from the population being studied. Although stratified sampling was 
undertaken, it was not followed by random selection of respondents from the population as anticipated. Nonetheless this helped to meet the purpose of the study which was to collect data from traditional and agile projects to allow group comparisons. Therefore, the sample might not be entirely random $100 \%$, which could slightly limit to draw inferences or a general conclusion from the findings of this study.

There is no broad consensus among researchers and practitioners in categorising candidate CSFs and the criteria for assessing project success for software development projects. Additionally, some of the candidate CSFs described by previous authors in their articles were not explicitly clear and required subjective but careful reading, understanding and interpretation to determine their best categorization. However, without such categorization, there is a substantial potential for the list of candidate CSFs to grow so large as to be unhelpful and impractical for considering contingencies.

\subsection{Chapter summary}

This chapter described the methodology used in addressing the set of objectives and hypotheses. Specifically, the chapter covered the research paradigms, research design, sampling design, data collection methods, research instruments, operationalization and measurement of variables and feedback from the pilot study. The chapter also discussed the main phase of data collection, response rate as well as the data analysis procedures. Finally, the methodological limitations in this study were highlighted. 


\section{CHAPTER FIVE: DATA ANALYSIS AND FINDINGS}

\subsection{Introduction}

The previous chapter discussed the methodology related to instrument design and data collection used to address the set research questions and hypotheses. This chapter presents the results and findings from the main study undertaken after pilot testing the research instrument. Specifically, the chapter presents the descriptive statistics for the complete dataset and subgroup comparisons. Subsequently, SEM analyses were conducted in a twostep analysis of the measurement and structural models. Group invariance analysis was conducted using SEM to test for any differences between projects that had used traditional plan-based and agile methodologies, by essentially comparing the same model across different samples of respondents. The last section involves testing for interaction effects among candidate CSFs and examining their impact on project success.

The next section presents the descriptive statistics for the main study covering the complete data set ( $\mathrm{n}=984$ ) and subgroup comparisons for both traditional plan-driven methodology data set $(n=513)$ and agile methodology data set $(n=471)$.

\subsection{Descriptive statistics-main study}

This section presents the results from the main study after all pretesting phases were completed. Important to note is that there were three different data sets which were generated for the subsequent analyses. The three categories include: (1) the general or complete data set that included all the data for both projects that used traditional plan-based methodology (i.e. PMI and PRINCE2) and agile methodology (Data set 1, n=984) while data set $2(\mathrm{n}=513)$, only included projects that used the traditional plan- based methodology that had used either PMI or PRINCE2. (Data set 3) included projects which had used only agile methodology $(n=471)$. Next, the sample characteristics are presented.

\subsubsection{Sample characteristics}

For this section, sample characteristics were generated. The frequency distributions cover both individual characteristics of respondents and project characteristics. The aim was to understand various categories of respondents that participated in this study.

\subsubsection{Experience in software development work}

The demographic attributes on experience that respondents had in software development are summarized in a frequency Table 49. 
Table 49: Experience in software development projects

\begin{tabular}{|c|l|c|c|c|}
\hline \# & Classification & $\begin{array}{c}\text { Complete data set, } \\
\mathbf{n = 9 8 4}\end{array}$ & $\begin{array}{c}\text { Traditional plan- } \\
\text { based methodology, } \\
\mathbf{n = 5 1 3}\end{array}$ & $\begin{array}{c}\text { Agile } \\
\text { methodology, } \\
\mathbf{n = 4 7 1}\end{array}$ \\
\hline 1 & Less than 1 year & $21(2.1 \%)$ & $17(3.3 \%)$ & $4(0.8 \%)$ \\
\hline 2 & 1 to less than 2 years & $22(2.2 \%)$ & $18(3.5 \%)$ & $4(0.8 \%)$ \\
\hline 3 & 2 to less than 5 years & $79(8.0 \%)$ & $66(12.9 \%)$ & $13(2.8 \%)$ \\
\hline 4 & 5 to less than 10 years & $214(21.7 \%)$ & $135(26.3 \%)$ & $79(16.8 \%)$ \\
\hline 5 & More than 10 years & $648(65.9 \%)$ & $277(54.0 \%)$ & $371(78.8 \%)$ \\
\hline
\end{tabular}

The responses indicate that the majority of employees had worked on outsourced software development projects for many years, and typically have substantial higher experience and, hence, conversant with the operations and management of software projects. The fact that over $86 \%$ of the respondents have an experience of more than 5 years, they meaningfully answered questions about their client organization based on their experience. This suggests that the data they provided relate to their sufficient experiences in the field. Thus, the data can be used to make valid generalization on outsourced software development projects.

\subsubsection{Position of the respondent}

Table 50 shows the positions held by respondents.

Table 50: Positions held by respondents

\begin{tabular}{|c|l|c|c|c|}
\hline$\#$ & Classification & $\begin{array}{c}\text { Complete data } \\
\text { set, } \mathbf{n = 9 8 4}\end{array}$ & $\begin{array}{c}\text { Traditional plan- } \\
\text { based, } \mathbf{n = 5 1 3}\end{array}$ & $\begin{array}{c}\text { Agile methodology, } \\
\mathbf{n = 4 7 1}\end{array}$ \\
\hline 1 & Project Manager & $830(84.3 \%)$ & $449(87.5 \%)$ & $381(80.9 \%)$ \\
\hline 2 & Team Leader & $100(10.2 \%)$ & $50(9.7 \%)$ & $50(10.6 \%)$ \\
\hline 3 & Developer & $23(2.3 \%)$ & $8(1.6 \%)$ & $15(3.2 \%)$ \\
\hline 4 & Tester & $23(2.3 \%)$ & $2(0.4 \%)$ & $21(4.5 \%)$ \\
\hline 5 & Others & $8(0.8 \%)$ & $4(0.8 \%)$ & $4(0.8 \%)$ \\
\hline
\end{tabular}

With regard to the positions held by the respondents in outsourced software development projects, the majority of respondents were the project managers 830 (84.3\%) and team leaders for all projects 100 (10.2\%). Typically, about 95\% of these respondents occupied senior positions and carried out senior roles on their previous project, hence are very conversant with the operations and management of outsourced software development projects. Thus, these respondents from the vendor organization were able to meaningfully answer questions about their clients' organizations because they are predominantly senior professional staff who are affiliated to communities of methodology practice i.e., PRINCE2, PMI and Agile, and are definitely expected to carry out environment/client organizational analysis for project implementation and planning purposes (PMI, 2013; PRINCE2, 2009; 
Agile, 2001). Typically, these senior staffs are also more engaged with the client organization more often than lower staffs so are expected to know more information about the client organization. The developers and testers had experience of more than five years of working on similar outsourced software development projects and had all previously served in managerial positions. Among other positions indicated were programme manager, project portfolio manager, senior solutions architect, consultant, PMO - manager, product prime, senior applications architect and senior coach in agile methods etc. Again, it is evidently clear that these respondents occupied senior positions and undertook senior roles hence are likely to be familiar with the operations and management of outsourced software projects.

\subsubsection{Client firm size}

Table 51 shows the client firm size in which the last software development was conducted.

Table 51: Client firm size

\begin{tabular}{|l|l|c|c|c|}
\hline$\#$ & Classification & $\begin{array}{c}\text { Complete data } \\
\text { set, n=984 }\end{array}$ & $\begin{array}{c}\text { Traditional plan- } \\
\text { based, n=513 }\end{array}$ & $\begin{array}{c}\text { Agile } \\
\text { methodology, } \\
\text { n=471 }\end{array}$ \\
\hline 1 & $1-10$ & $39(4.0 \%)$ & $15(2.9 \%)$ & $24(5.1 \%)$ \\
\hline 2 & $11-50$ & $66(6.7 \%)$ & $25(4.9 \%)$ & $41(8.7 \%)$ \\
\hline 3 & $51-100$ & $90(9.1 \%)$ & $37(7.2 \%)$ & $53(11.3 \%)$ \\
\hline 4 & $101-500$ & $140(14.2 \%)$ & $66(12.9 \%)$ & $74(15.7 \%)$ \\
\hline 5 & $501-1000$ & $133(13.5 \%)$ & $83(16.2 \%)$ & $50(10.6 \%)$ \\
\hline 6 & $1001-5000$ & $190(19.3 \%)$ & $111(21.6 \%)$ & $79(16.8 \%)$ \\
\hline 7 & More than 5000 & $326(33.1 \%)$ & $176(34.3 \%)$ & $150(31.8 \%)$ \\
\hline
\end{tabular}

The most represented category in firm size was that of organizations which had more than 5,000 employees with 326(33.1\%) and the least had 1 to 10 employees with 39(4.0\%). This typically indicates that there were larger clients' involved, larger projects with larger budgets which had significant importance in determining the reputation of the vendor. This suggests the vendor had a high degree of commitment in regard to these projects hence giving more of their attention and resources to these larger clients. Unlike in some circumstances where a project may be extremely important to small client organizations and the vendors may give more of their attention and resources to larger clients. The data collected shows that there was a large variety of large sized organisations in which software development projects were conducted with a good representation of larger outsourced software development projects that were developed. This data should be suitable for making valid generalization across software development projects in large and medium client organizations. 


\subsubsection{Project budget}

Table 52 shows the various budgets or costs of projects by respondents.

Table 52: Project budget in US Dollars

\begin{tabular}{|c|l|c|c|c|}
\hline$\#$ & Classification & $\begin{array}{c}\text { Complete data set, } \\
\mathbf{n = 9 8 4}\end{array}$ & $\begin{array}{c}\text { Traditional plan- } \\
\text { based, n=513 }\end{array}$ & $\begin{array}{c}\text { Agile methodology, } \\
\mathbf{n = 4 7 1}\end{array}$ \\
\hline 1 & Less than $\$ 100,000$ & $209(21.2 \%)$ & $105(20.5 \%)$ & $104(22.1 \%)$ \\
\hline 2 & $\$ 100,000$ to less than $\$ 1 \mathrm{M}$ & $419(42.6 \%)$ & $188(36.6 \%)$ & $231(49 \%)$ \\
\hline 3 & $\$ 1 \mathrm{M}$ to less than $\$ 10 \mathrm{M}$ & $252(25.6 \%)$ & $161(31.4 \%)$ & $91(19.3 \%)$ \\
\hline 4 & \$10M to less than $\$ 100 \mathrm{M}$ & $70(7.1 \%)$ & $32(6.2 \%)$ & $38(8.1 \%)$ \\
\hline 5 & More than $\$ 100 \mathrm{M}$ & $34(3.5 \%)$ & $27(5.3 \%)$ & $7(1.5 \%)$ \\
\hline
\end{tabular}

In terms of budget, the majority of the software projects representing $419(42.6 \%)$ had project budgets of $\$ 100,000$ to less than $\$ 1 \mathrm{M}$ while the least represented were $34(3.5 \%)$ for projects that had more than $\$ 100 \mathrm{M}$. Hass (2008) considers software development projects of more than $\$ 750,000$ to be large and complex; hence it is evident that about $80 \%$ of all the projects in this study were predominantly large and complex projects.

\subsubsection{Project duration}

Table 53 shows the various amount of time taken to complete different software projects.

Table 53: Project duration

\begin{tabular}{|c|l|c|c|c|}
\hline$\#$ & Classification & $\begin{array}{c}\text { Complete data set, } \\
\mathbf{n = 9 8 4}\end{array}$ & $\begin{array}{c}\text { Traditional plan- } \\
\text { based, n=513 }\end{array}$ & $\begin{array}{c}\text { Agile methodology, } \\
\mathbf{n = 4 7 1}\end{array}$ \\
\hline 1 & Less than 6 months & $187(19.0 \%)$ & $90(20.5 \%)$ & $97(20.6 \%)$ \\
\hline 2 & 6months to less than 12 months & $391(39.7 \%)$ & $197(36.6 \%)$ & $194(41.2 \%)$ \\
\hline 3 & 12 months to less than 24 months & $238(24.2 \%)$ & $150(31.4 \%)$ & $88(18.7 \%)$ \\
\hline 4 & 24 months to less than 36 months & $70(7.1 \%)$ & $48(6.2 \%)$ & $22(4.7 \%)$ \\
\hline 5 & More than 36 months & $98(10.0 \%)$ & $28(5.3 \%)$ & $70(14.9 \%)$ \\
\hline
\end{tabular}

Different project sizes with varying project timeframes or duration are represented, with predominantly more than $80 \%$ of all the projects taking more than more than 6 months to complete. Accordingly, this suggests that most software development projects were large, since software development projects that last for more than 6 months are considered to be large and complex (Hass, 2008).

\subsubsection{Outsourcing project clients by country}

Participants indicated that the outsourcing clients were from over 58 different countries across the globe. The most represented country was the USA (187 or 19\%), followed by Australia (159 or 16.2\%), New Zealand (146 or 14.8\%) and United Kingdom (126 or 12.8\%). This can possibly explain why most of the outsourced client projects were large in terms of size (budgets, duration etc.), the majority of the client organizations were from the United 
States of America, Australia, New Zealand and UK where there are some very big organizations (OECD, 2013). Most of the e-mails for soliciting responses in this study went to these countries because they dominate membership lists for vendor professional bodies (PRINCE2, PMI, and Agile). Table 54 shows the country of the clients where the software was developed.

Table 54: Clients by country

\begin{tabular}{lclc}
\hline \multicolumn{1}{c}{ Country } & Frequency & \multicolumn{1}{c}{ Country } & Frequency \\
\hline Argentina & 5 & Kyrgyzstan & 1 \\
Australia & 159 & Luxembourg & 1 \\
Austria & 2 & Latvia & 1 \\
Belgium & 2 & Liberia & 2 \\
Brazil & 53 & Malawi & 1 \\
Brunei & 1 & Malaysia & 2 \\
Canada & 18 & Netherlands & 5 \\
Chile & 1 & New Zealand & 146 \\
China & 54 & Nigeria & 1 \\
Colombia & 2 & Norway & 3 \\
Congo & 1 & Pakistan & 2 \\
Denmark & 2 & Russia & 5 \\
Dominican Republic & 1 & Saudi Arabia & 5 \\
Egypt & 1 & Singapore & 1 \\
Finland & 3 & South Africa & 2 \\
France & 9 & Spain & 3 \\
Germany & 7 & Sir Lanka & 1 \\
Gibraltar & 1 & Sweden & 7 \\
Greece & 1 & Switzerland & 4 \\
Hong Kong & 1 & Taiwan & 2 \\
Hungary & 1 & Thailand & 2 \\
Iceland & 1 & Turkey & 2 \\
India & 94 & UAE & 3 \\
Indonesia & 2 & UK & 126 \\
Iran & 2 & Ukraine & 1 \\
Ireland & 1 & USA & 187 \\
Israel & 1 & Viet Nam & 1 \\
Italy & 4 & Yemen & 984 \\
\hline Japan & 35 & & \\
Kuwait & 1 & Total & \\
\hline & & & \\
\hline
\end{tabular}

Other countries that dominated the data set according to the responses were from India, China, Brazil and Japan. USA dominated overall but there were also many respondents from European countries but scattered across various countries. The rest of the responses came from across the globe i.e. Asian countries, South and North America, Africa etc. 


\subsubsection{Size of the project teams}

Table 55 below shows the various sizes of projects teams by respondents.

Table 55: Size of the project teams

\begin{tabular}{|c|l|c|c|c|}
\hline$\#$ & Classification & $\begin{array}{c}\text { Complete data set, } \\
\mathbf{n = 9 8 4}\end{array}$ & $\begin{array}{c}\text { Traditional plan- } \\
\text { based, } \mathbf{n = 5 1 3}\end{array}$ & $\begin{array}{c}\text { Agile methodology, } \\
\mathbf{n = 4 7 1}\end{array}$ \\
\hline 1 & $2-5$ & $112(11.4 \%)$ & $42(8.2 \%)$ & $70(14.9 \%)$ \\
\hline 2 & $6-100$ & $750(76.2 \%)$ & $407(79.3 \%)$ & $343(72.8 \%)$ \\
\hline 3 & $101-500$ & $90(9.1 \%)$ & $47(9.2 \%)$ & $43(9.1 \%)$ \\
\hline 4 & $501-1000$ & $8(0.8 \%)$ & $7(1.4 \%)$ & $1(0.2 \%)$ \\
\hline 5 & $1001-5000$ & $4(0.4 \%)$ & $2(0.4 \%)$ & $2(0.4 \%)$ \\
\hline 6 & Above 5000 & $20(2.0 \%)$ & $8(1.6 \%)$ & $12(2.5 \%)$ \\
\hline
\end{tabular}

Majority of project teams ranged between 6-100 with 750 (76.2\%) and smallest size of project teams ranged between 1001-5000 members with only $4(0.4 \%)$ responses. According to Hass (2008) software development projects of less than 5 team members are considered small and less complex while software developments projects of more than 5 team members are considered large teams and complex. Based on this categorization, it is evident that the majority of projects teams were large in this study, which resonates with large client organizations with large budgets for outsourced software development projects. In general, as illustrated a range of various sizes of project teams were well represented in this data, hence can be used to make some valid generalizations.

\subsubsection{Sector}

Table 56 shows the sectors of the clients in this study where the outsourced software was developed.

Table 56: Sectors of the clients

\begin{tabular}{|c|l|c|c|c|}
\hline$\#$ & Classification & $\begin{array}{c}\text { Complete data set, } \\
\mathbf{n = 9 8 4}\end{array}$ & $\begin{array}{c}\text { Traditional plan-based, } \\
\mathbf{n = 5 1 3}\end{array}$ & $\begin{array}{c}\text { Agile methodology, } \\
\mathbf{n = 4 7 1}\end{array}$ \\
\hline 1 & Public sector & $302(30.7 \%)$ & $170(33.1 \%)$ & $132(28.0 \%)$ \\
\hline 2 & Private sector & $643(65.3 \%)$ & $331(64.5 \%)$ & $312(66.2 \%)$ \\
\hline 3 & Others & $39(4.0)$ & $12(2.3 \%)$ & $27(5.7 \%)$ \\
\hline
\end{tabular}

The research participants' results indicate that they had undertaken software developed projects in both private and public sectors which are well represented in this study. This suggests this data can be used to make valid generalizations across all types of sectors. The private sector respondents represent $643(65.3 \%)$ while the public sector had $302(30.7 \%)$. Other sectors were $39(4.0 \%)$ and they included among others, not for profit, NGOs and charities. There are many possible reasons for this sharp difference in responses from private 
and public sectors. First, the public sector outsources most of the software development activities from private sector and second, the private sector is bigger than the public sector and that could have contributed significantly to these differences in responses.

\subsubsection{Industry}

Table 57 shows the type of industry of the participants of this study.

Table 57: Industry of respondents

\begin{tabular}{|c|l|c|c|c|}
\hline$\#$ & Classification & $\begin{array}{c}\text { Complete data } \\
\text { set, } \mathbf{n = 9 8 4}\end{array}$ & $\begin{array}{c}\text { Traditional plan- } \\
\text { based, } \mathbf{n = 5 1 3}\end{array}$ & $\begin{array}{c}\text { Agile methodology, } \\
\mathbf{n = 4 7 1}\end{array}$ \\
\hline 1 & Finance/Insurance & $243(24.7 \%)$ & $138(26.9 \%)$ & $105(22.3 \%)$ \\
\hline 2 & Manufacturing & $109(11.1 \%)$ & $77(15.0 \%)$ & $32(6.8 \%)$ \\
\hline 3 & Marketing/retail & $67(6.8 \%)$ & $18(3.5 \%)$ & $49(10.4 \%)$ \\
\hline 4 & Health & $107(10.9 \%)$ & $49(9.6 \%)$ & $58(12.3 \%)$ \\
\hline 5 & Consulting & $61(6.2 \%)$ & $37(7.2 \%)$ & $24(5.1 \%)$ \\
\hline 6 & Software & $182(18.5 \%)$ & $55(10.7 \%)$ & $127(27.0 \%)$ \\
\hline 7 & Transportation & $42(4.3 \%)$ & $21(4.1 \%)$ & $21(4.5 \%)$ \\
\hline 8 & Utility & $38(3.9 \%)$ & $27(5.3 \%)$ & $11(2.3 \%)$ \\
\hline 9 & Aerospace & $27(2.7 \%)$ & $17(3.3 \%)$ & $10(2.1 \%)$ \\
\hline 10 & Education & $68(6.9 \%)$ & $47(9.2 \%)$ & $21(4.5 \%)$ \\
\hline 11 & Others & $40(4.1 \%)$ & $27(5.3 \%)$ & $13(2.8 \%)$ \\
\hline
\end{tabular}

Other industries reported included security and intelligence, entertainment, social gaming and social security among others. Again, this shows that a large variety of industries were represented in this database hence a good representation of all industries that are involved in software development projects. Thus, this data can be used to make valid generalizations across all industries in which outsourced software development is conducted.

\subsubsection{Project Management Methodology}

Table 58 shows the type of project management methodology that was used in software development by the respondents.

Table 58: Project management methodology used

\begin{tabular}{|c|l|c|c|c|}
\hline \# & Classification & $\begin{array}{c}\text { Complete data } \\
\text { set, } \mathbf{n = 9 8 4}\end{array}$ & $\begin{array}{c}\text { Traditional plan- } \\
\text { based, } \mathbf{n = 5 1 3}\end{array}$ & $\begin{array}{c}\text { Agile methodology, } \\
\mathbf{n = 4 7 1}\end{array}$ \\
\hline 1 & PRINCE2 & $20(2.0 \%)$ & $20(3.9 \%)$ & None \\
\hline 2 & PMI & $493(50.1 \%)$ & $493(96.1 \%)$ & None \\
\hline 3 & Agile & $471(47.9 \%)$ & None & $471(100 \%)$ \\
\hline
\end{tabular}

Furthermore, the data set reveals that 471 (47.9\%) respondents had used agile software development methodology; while 20 (2.0\%) projects used PRINCE2 and 493 (50.1\%) used PMI respectively. Since respondents did not have to choose either traditional plan-based or 
agile methodologies only, yet in this sample they did, it shows that both traditional planbased (i.e. PRINCE2 and PMI) and agile methodologies are both well represented and the data set can serve the purpose of this study which is to make comparisons across these project types.

\subsubsection{Development life cycle}

Table 59 shows the development life cycle on which the software development project was based.

Table 59: Development life cycle used

\begin{tabular}{|c|l|c|c|c|}
\hline$\#$ & Classification & $\begin{array}{c}\text { Complete data } \\
\text { set, n=984 }\end{array}$ & $\begin{array}{c}\text { Traditional plan- } \\
\text { based, n=513 }\end{array}$ & $\begin{array}{c}\text { Agile methodology, } \\
\mathbf{n = 4 7 1}\end{array}$ \\
\hline & Linear or Waterfall & $245(24.9 \%)$ & $236(46.0 \%)$ & $9(1.9 \%)$ \\
\hline 1 & Iterative & $358(36.4 \%)$ & $97(18.9 \%)$ & $261(55.4 \%)$ \\
\hline 2 & Incremental & $167(17.0 \%)$ & $102(19.9 \%)$ & $65(13.8 \%)$ \\
\hline 3 & Adaptive & $194(19.7 \%)$ & $66(12.9 \%)$ & $128(27.2 \%)$ \\
\hline 4 & Others & $20(2.0 \%)$ & $12(2.3 \%)$ & $8(1.7 \%)$ \\
\hline
\end{tabular}

Of the total respondents, those that used linear or waterfall were 245 (24.9\%), Iterative were dominant with $358(36.4 \%)$, incremental were 167 (17.0\%), Adaptive were 194 (19.7\%), while others progressions were $20(2.0 \%)$. Among other life cycles reported were scrum, prototype, Extreme programming, pull-based etc. As indicated, it is evident that both development lifecycles for agile development (iterative and adaptive) and traditional planbased approaches (linear or incremental) were both well represented hence the data can be used to make valid generalisations across all development lifecycles that are used in software development projects.

\subsubsection{Making the decision to use a particular project management methodology}

Table 60 below shows the person or group of people in the client organization who were responsible for making the decision of choosing the project management methodology that was used.

Table 60: Made a decision in the client organization of choosing the project management methodology

\begin{tabular}{|c|l|c|c|c|}
\hline \# & Classification & $\begin{array}{c}\text { Complete data } \\
\text { set, } \mathbf{n = 9 8 4}\end{array}$ & $\begin{array}{c}\text { Traditional plan- } \\
\text { based, n=513 }\end{array}$ & $\begin{array}{c}\text { Agile methodology, } \\
\mathbf{n = 4 7 1}\end{array}$ \\
\hline 1 & Top management & $219(22.3 \%)$ & $113(22.1 \%)$ & $106(22.5)$ \\
\hline 2 & Project team members & $276(28.0 \%)$ & $95(18.5 \%)$ & $181(38.4 \%)$ \\
\hline 3 & Organizational policy & $396(40.2 \%)$ & $258(50.3 \%)$ & $138(29.3 \%)$ \\
\hline 4 & Do not know & $41(4.2 \%)$ & $13(2.5 \%)$ & $28(5.9 \%)$ \\
\hline 5 & Others & $52(5.3 \%)$ & $34(6.6 \%)$ & $18(3.8 \%)$ \\
\hline
\end{tabular}


As illustrated, the decision regarding the selection of the project management methodology was determined by different project stakeholders in the clients' organizations. The projects where the methodology decision was made by top management were 219 (22.3\%), project team members were 276 (28.0\%), organizational policy were 396 (40.2\%), the respondents who did not know how the methodology was selected were 41 (4.2\%) while those projects that had used other approaches were 52 (5.3\%). Other approaches of selecting methodologies reported included among others; the project manager, analysis of the environment, system integrator, based on the vision and the specific project, the portfolio, top managementcustomer and project team member together, top management at recommendation of principal vendor or in some rare instances the customer. Again, this data represents all stakeholders who are responsible for making the decision of choosing the methodology in the clients' organizations that is used in software hence the data can be used to make generalizations across all outsourced software development projects. The results also indicate that most projects use a particular project management methodology because it was an organizational policy without any objective reference to project characteristics or environment. This again provides some supporting evidence that project management methodologies are adopted without necessarily any objective reference to match the project characteristics and environment.

Table 61 shows a summary of the various sample characteristics for the data collected for this study (i.e. the complete sample, traditional plan-based and agile methodologies). 
Table 61: Demographics of the respondents-Group comparisons

\begin{tabular}{|c|c|c|c|c|}
\hline Characteristics & Scale & $\begin{array}{c}\text { Complete data } \\
\text { set (data set 1), } \\
\mathbf{n = 9 8 4}\end{array}$ & \begin{tabular}{|c|}
$\begin{array}{c}\text { Traditional plan- } \\
\text { based (data set 2), } \\
\mathbf{n}=513\end{array}$
\end{tabular} & $\begin{array}{c}\text { Agile methodology } \\
\text { (data set 3), } \\
n=471\end{array}$ \\
\hline \multirow{5}{*}{$\begin{array}{c}\text { Experience of } \\
\text { the vendors }\end{array}$} & Less than 1 year & $21(2.1 \%)$ & $17(3.3 \%)$ & $4(0.8 \%)$ \\
\hline & 1 to less than 2 years & $22(2.2 \%)$ & $18(3.5 \%)$ & $4(0.8 \%)$ \\
\hline & 2 to less than 5 years & $79(8.0 \%)$ & $66(12.9 \%)$ & $13(2.8 \%)$ \\
\hline & 5 to less than 10 years & $214(21.7 \%)$ & $135(26.3 \%)$ & $79(16.8 \%)$ \\
\hline & More than 10 years & $648(65.9 \%)$ & $277(54.0 \%)$ & $371(78.8 \%)$ \\
\hline \multirow{5}{*}{$\begin{array}{l}\text { Positions of the } \\
\text { vendors on the } \\
\text { project }\end{array}$} & Proiect Manaoer & $830(843 \%)$ & $440(875 \%)$ & $381(800 \%)$ \\
\hline & Team Leader & $\frac{050(04.57)}{100(102 \%)}$ & $\frac{4+5(07.07)}{50(07 \%)}$ & $\frac{501(00.970)}{50(10.67)}$ \\
\hline & Developer & $23(2.3 \%)$ & $8(1.6 \%)$ & $15(3.2 \%)$ \\
\hline & Tester & $23(2.3 \%)$ & $2(0.4 \%)$ & $21(4.5 \%)$ \\
\hline & Others & $8(0.8 \%)$ & $4(0.8 \%)$ & $4(0.8 \%)$ \\
\hline & & & & \\
\hline \multirow{7}{*}{$\begin{array}{l}\text { Size of the } \\
\text { client firm }\end{array}$} & $1-10$ & $39(4.0 \%)$ & $15(2.9 \%)$ & $24(5.1 \%)$ \\
\hline & $11-50$ & $66(6.7 \%)$ & $25(4.9 \%)$ & $41(8.7 \%)$ \\
\hline & $51-100$ & $90(9.1 \%)$ & $37(7.2 \%)$ & $53(11.3 \%)$ \\
\hline & $101-500$ & $140(14.2 \%)$ & $66(12.9 \%)$ & $74(15.7 \%)$ \\
\hline & $501-1000$ & $133(13.5 \%)$ & $83(16.2 \%)$ & $50(10.6 \%)$ \\
\hline & $1001-5000$ & $190(19.3 \%)$ & $111(21.6 \%)$ & $79(16.8 \%)$ \\
\hline & More than 5000 & $326(33.1 \%)$ & $176(34.3 \%)$ & $150(31.8 \%)$ \\
\hline \multirow{5}{*}{$\begin{array}{l}\text { Project budget } \\
\text { (USD) }\end{array}$} & Less than $\$ 100,000$ & $209(21.2 \%)$ & $105(20.5 \%)$ & $104(22.1 \%)$ \\
\hline & $\$ 100,000$ to less than $\$ 1 \mathrm{M}$ & $419(42.6 \%)$ & $188(36.6 \%)$ & $231(49 \%)$ \\
\hline & $\$ 1 \mathrm{M}$ to less than $\$ 10 \mathrm{M}$ & $252(25.6 \%)$ & $161(31.4 \%)$ & $91(19.3 \%)$ \\
\hline & $\$ 10 \mathrm{M}$ to less than $\$ 100 \mathrm{M}$ & $70(7.1 \%)$ & $32(6.2 \%)$ & $38(8.1 \%)$ \\
\hline & More than $\$ 100 \mathrm{M}$ & $34(3.5 \%)$ & $27(5.3 \%)$ & $7(1.5 \%)$ \\
\hline \multirow{5}{*}{$\begin{array}{l}\text { Duration in } \\
\text { months }\end{array}$} & Less than 6 months & $187(19.0 \%)$ & $90(20.5 \%)$ & $97(20.6 \%)$ \\
\hline & 6 months to less than 12 months & $391(39.7 \%)$ & $197(36.6 \%)$ & $194(41.2 \%)$ \\
\hline & 12 months to less than 24 months & $238(24.2 \%)$ & $150(31.4 \%)$ & $88(18.7 \%)$ \\
\hline & 24 months to less than 36 months & $70(7.1 \%)$ & $48(6.2 \%)$ & $22(4.7 \%)$ \\
\hline & More than 36 months & $98(10.0 \%)$ & $28(5.3 \%)$ & $70(14.9 \%)$ \\
\hline & & & & \\
\hline \multirow{6}{*}{$\begin{array}{c}\text { Number of } \\
\text { project } \\
\text { members on } \\
\text { each team }\end{array}$} & $2-5$ & $112(11.4 \%)$ & $42(8.2 \%)$ & $70(14.9 \%)$ \\
\hline & $6-100$ & $750(76.2 \%)$ & $407(79.3 \%)$ & $343(72.8 \%)$ \\
\hline & $101-500$ & $90(9.1 \%)$ & $47(9.2 \%)$ & $43(9.1 \%)$ \\
\hline & $501-1000$ & $8(0.8 \%)$ & $7(1.4 \%)$ & $1(0.2 \%)$ \\
\hline & $1001-5000$ & $4(0.4 \%)$ & $2(0.4 \%)$ & $2(0.4 \%)$ \\
\hline & Above 5000 & $20(2.0 \%)$ & $8(1.6 \%)$ & $12(2.5 \%)$ \\
\hline \multirow{3}{*}{$\begin{array}{l}\text { Type of sector } \\
\text { of the clients }\end{array}$} & Public sector & $302(30.7 \%)$ & $170(33.1 \%)$ & $132(28.0 \%)$ \\
\hline & Private sector & $643(65.3 \%)$ & $331(64.5 \%)$ & $312(66.2 \%)$ \\
\hline & Others & $39(4.0)$ & $12(2.3 \%)$ & $27(5.7 \%)$ \\
\hline \multirow[b]{5}{*}{ Industry of the } & Finance/Insurance & $243(24.7 \%)$ & $138(26.9 \%)$ & $105(22.3 \%)$ \\
\hline & Manufacturing & $109(11.1 \%)$ & $77(15.0 \%)$ & $32(6.8 \%)$ \\
\hline & Marketing/retail & $67(6.8 \%)$ & $18(3.5 \%)$ & $49(10.4 \%)$ \\
\hline & Health & $107(10.9 \%)$ & $49(9.6 \%)$ & $58(12.3 \%)$ \\
\hline & Consulting & $61(6.2 \%)$ & $37(7.2 \%)$ & $24(5.1 \%)$ \\
\hline
\end{tabular}




\begin{tabular}{|c|c|c|c|c|}
\hline \multirow[t]{6}{*}{ clients } & Software & $182(18.5 \%)$ & $55(10.7 \%)$ & $127(27.0 \%)$ \\
\hline & Transportation & $42(4.3 \%)$ & $21(4.1 \%)$ & $21(4.5 \%)$ \\
\hline & Utility & $38(3.9 \%)$ & $27(5.3 \%)$ & $11(2.3 \%)$ \\
\hline & Aerospace & $27(2.7 \%)$ & $17(3.3 \%)$ & $10(2.1 \%)$ \\
\hline & Education & $68(6.9 \%)$ & $47(9.2 \%)$ & $21(4.5 \%)$ \\
\hline & Others & $40(4.1 \%)$ & $27(5.3 \%)$ & $13(2.8 \%)$ \\
\hline \multirow{3}{*}{$\begin{array}{l}\text { Project } \\
\text { management } \\
\text { methodology }\end{array}$} & PRINCE2 & $20(2.0 \%)$ & $20(3.9 \%)$ & None \\
\hline & PMI & $493(50.1 \%)$ & $493(96.1 \%)$ & None \\
\hline & Agile & $471(47.9 \%)$ & None & $471(100 \%)$ \\
\hline \multirow{5}{*}{$\begin{array}{l}\text { Development } \\
\text { life cycle } \\
\text { (SDM) }\end{array}$} & Linear or Waterfall & $245(24.9 \%)$ & $236(46.0 \%)$ & $9(1.9 \%)$ \\
\hline & Iterative & $358(36.4 \%)$ & $97(18.9 \%)$ & $261(55.4 \%)$ \\
\hline & Incremental & $167(17.0 \%)$ & $102(19.9 \%)$ & $65(13.8 \%)$ \\
\hline & Adaptive & $194(19.7 \%)$ & $66(12.9 \%)$ & $128(27.2 \%)$ \\
\hline & Others & $20(2.0 \%)$ & $12(2.3 \%)$ & $8(1.7 \%)$ \\
\hline \multirow{5}{*}{$\begin{array}{l}\text { Choosing a } \\
\text { methodology } \\
\text { in the clients } \\
\text { organization }\end{array}$} & Top management & $219(22.3 \%)$ & $113(22.1 \%)$ & $106(22.5)$ \\
\hline & Project team members & $276(28.0 \%)$ & $95(18.5 \%)$ & $181(38.4 \%)$ \\
\hline & Organizational policy & $396(40.2 \%)$ & $258(50.3 \%)$ & $138(29.3 \%)$ \\
\hline & Do not know & $41(4.2 \%)$ & $13(2.5 \%)$ & $28(5.9 \%)$ \\
\hline & Others & $52(5.3 \%)$ & $34(6.6 \%)$ & $18(3.8 \%)$ \\
\hline
\end{tabular}

As displayed the responses came from the projects that had used the two methodologies (traditional plan-based and agile). Non response bias also appears not to be an issue. Non response bias was established using t-test by comparing the average values for each of the constructs for the first quartile completed surveys received versus the last quartile completed surveys, allowing the late surveys to proxy the perceptions of non-respondents (Hair et al., 2010). Mean differences for each of the constructs did not reveal any significant difference between the early and late surveys (2-tailed t-tests, $p<0.05)$. This comparative test depicted the absence of non-response bias in this study.

In order to understand the attributes of the data set, the demographic characteristics of the outsourced software development projects collected in this study as well as the profiles of the respondents were analysed. The sample characteristics indicate the data collected is appropriate for this study. Typically, all respondents were very experienced in outsourced software development and occupied senior positions with senior roles on these projects, and were able to meaningfully answer questions about various aspects in their client organization. In addition, most outsourced software development projects undertaken by respondents were larger from large client organizations with bigger budgets which hold a significant importance to vendor reputation. This suggests the vendors had a high degree of commitment 
in regard to these projects hence giving more of their attention and resources to these larger clients. This is contrary to some circumstances where a project may be critically important to a small client organizations but the vendors gives more of their attention and resources to larger clients, ignoring small projects. All types of economic sectors were represented as well as industries in which outsourced software development projects are undertaken. More importantly, both agile and traditional plan-based methodologies were well represented to allow comparisons between the two groups. Many project stakeholders who make decisions regarding choice of project methodologies in client organizations also participated. A comparative t-test analysis depicted the absence of non-response bias in this study. In short the data collected is representative, valid and reliable to make generalizations in regard to outsourced software development projects across the globe.

Further, in order to summarize the observed data, means and standard deviations were also generated. According to Hair et al. (2009), means represent a summary of the data and standard deviations show how much variation or dispersion from the average exists. A low standard deviation indicates that the data points tend to be very close to the mean; a high standard deviation indicates that the data points are spread out over a large range of values. All survey responses were anchored between strongly disagree (1) and strongly agree (7) so a response of 4.0 would indicate neutrality and responses of or greater than 4.5 would indicate strong agreement. Mean responses for the survey items were in the range of 3.00-6.15 showing that most respondents indicated agreement on the attributes of study variables hence these variables do exist in practice. Because of small standard deviations compared to mean values, it is clear that most data points are close to the means and hence calculated means highly represent the observed data (Byrne, 2001).

Prior to combining both PRINCE2 and PMI as a single traditional plan-based data set in further analyses, t-tests were conducted to establish if any significant differences existed between mean scores for both PRINCE2 and PMI projects. Mean differences for all constructs did not reveal any significant differences between the PRINCE2 and PMI data sets (2-tailed t-tests, $p<0.05)$. Thus PRINCE2 and PMI were combined as traditional plan-based methodologies in all subsequent analyses.

Table 62 shows the mean and standard deviation of the responses given for both groups (traditional and agile data sets). 
Table 62: Descriptive statistics-Group comparisons

\section{Top level management support}

TPLC_1-There was approval and support from top level management.

TPLC_4-Top level management publicly and explicitly identified the project as a top priority.

TPLC_5-The project had a project champion.

TPLC_10-The project champion was very committed to the realization of project benefits.

\begin{tabular}{l|l}
\hline 5.95 & 1.03 \\
\hline 5.55 & 1.26 \\
\hline 5.19 & 1.50 \\
\hline 5.24 & 1.49 \\
\hline 5.19 & 1.50 \\
\hline
\end{tabular}

\begin{tabular}{|l|l|}
\hline 5.85 & 1.14 \\
\hline 5.69 & 1.23 \\
\hline 5.29 & 1.47 \\
\hline 5.32 & 1.46 \\
\hline 5.26 & 1.39 \\
\hline
\end{tabular}

\section{Internal project communication}

IPC_1-The project team met frequently.

IPC_2-Members were kept informed about the major decisions concerning the project

IPC_3-Every effort was made to keep project team turnover at a minimum.

IPC_4-Members actively participated in the definition of project goals and schedules.

IPC_5-The amount of information disseminated by project team leaders was satisfactory.

6.11

5.99

5.53

5.37

5.63

\section{Organizational culture}

OC_2-Flexible and participative, valued teamwork and encouraged social interaction.

OC_3-The management style was that of leadership and collaboration.

OC_4-The organization was based on loyalty and mutual trust and commitment.

OC_5-The organization valued feedback and learning.

OC_6-The organization enabled empowerment of people and teams.

User participation

UP 1 -Users actively participated in requirements definition.

UP_2-The project team kept users informed concerning project progress and problems.

UP_3-Users formally evaluated the work done by the project team.

UP_4-Users formally approved the work done by the project team.

\section{Project team commitment}

PTC_1-No other activities could match the benefits that the project activities presented to me.

PTC_2-Hard for me to abandon the software development project activities.

PTC_9-Team members were emotionally attached to the software development project.

PTC_10 -Team members felt a strong sense of belonging to the software development project

PTC_12-Team members had a sense of obligation to the client of software development project.

PTC_13-Team members owed a great deal to the software development project.

\section{Technical complexity}

TC_1-Project involved the use of new technology.

TC_2-Project had high level of technical complexity.

TC_3-Project involved the use of technology that had not been used in prior projects.

TC_4-Project involved computers, databases and networks that were highly complex.

TC_5-The operating system, procedures, algorithms and programming was complex.

Development team skills

DTS_1-Team lacked experience with the development platform/environment used.

DTS_2-The development team was very unfamiliar with this type of application.

DTS_3-The development team lacked knowledge of application domain involved in this project.

DTS_4-Team generally lacked technically competent and experienced people.

\section{User Experience}

UE_1-The client was not familiar with this type of application.

UE_2-The client didn't know what they wanted.

UE_3 -The client didn't have a good understanding of the problems they want solved.

UE_4-The client was not familiar with data processing as a work tool.

UE_5-The client was not aware of the importance of their roles in the project.

\section{Planning and controlling}

PPC_1-Special attention was paid to project planning

PPC_2-Project milestones were clearly defined.

PPC_3-Project progress was monitored closely e.g. using PERT or CPM tools.

PPC_4-Periodic formal status reports were used instead of the project plan.

PPC_5-There were reviews at each milestone.

\section{Change management}

Q25_1-There was recognition for the need for change.

Q25_2-There was organizational wide change culture and structure.

Q25_3-There was commitment to change.

Q25_4-There was business process re-engineering to match the new software.

Q25_5-Analysis of user feedback was continuously done.

\begin{tabular}{l|l|l|l}
5.14 & 1.36 & 5.29 & 1.35 \\
5.29 & 1.34 & 5.24 & 1.44 \\
\hline 5.24 & 1.34 & 5.18 & 1.44 \\
\hline 5.09 & 1.37 & 5.09 & 1.50 \\
\hline 5.03 & 1.37 & 5.09 & 1.49 \\
\hline
\end{tabular}

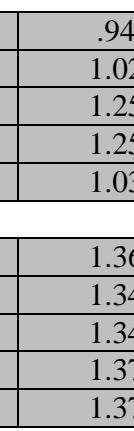

\begin{tabular}{l|l}
\hline 6.15 & 1.04 \\
\hline 5.98 & 1.08 \\
\hline 5.42 & 1.35 \\
\hline 5.42 & 1.35 \\
\hline 5.52 & 1.24 \\
\hline
\end{tabular}

5.57

5.67

5.35

5.58
1.21

1.13

1.34

1.21

\begin{tabular}{|l|l|}
\hline 5.64 & 1.31 \\
\hline 5.68 & 1.32 \\
\hline 5.39 & 1.39 \\
\hline 5.58 & 1.45 \\
\hline
\end{tabular}

4.89

5.14

4.98

5.25

5.40

4.99

5.35

5.60

4.78

5.32

5.17

1.17

1.17

1.27

1.24

1.09

1.27

\begin{tabular}{|l|l|}
\hline 4.88 & 1.26 \\
\hline 5.02 & 1.37 \\
\hline 5.14 & 1.21 \\
\hline 5.34 & 1.18 \\
\hline 5.34 & 1.19 \\
\hline 5.00 & 1.24 \\
\hline
\end{tabular}

1.47

1.27

1.68

1.46

1.38

\begin{tabular}{|l|l|}
\hline 5.54 & 1.27 \\
\hline 5.65 & 1.16 \\
\hline 5.28 & 1.45 \\
\hline 5.41 & 1.29 \\
\hline 5.37 & 1.25 \\
\hline
\end{tabular}

3.45

3.18

3.18
3.17

3.06

1.60

1.60
1.49

1.53

1.56

\begin{tabular}{|l|l}
\hline 3.58 & 1.57 \\
\hline 3.25 & 1.49 \\
\hline 3.36 & 1.52 \\
\hline 3.00 & 1.48 \\
\hline
\end{tabular}

4.04

3.83

3.73

3.63

3.69

\begin{tabular}{|l|}
1.62 \\
\hline 1.66 \\
1.60 \\
1.58 \\
\hline 1.72 \\
\hline
\end{tabular}

\begin{tabular}{|l|l|}
\hline 3.84 & 1.57 \\
\hline 3.84 & 1.58 \\
\hline 3.72 & 1.57 \\
\hline 3.56 & 1.57 \\
\hline 3.69 & 1.64 \\
\hline
\end{tabular}

5.65

5.77

5.25

5.31

\begin{tabular}{|l|l}
\hline & 1.24 \\
1.11 \\
1.46 \\
1.40 \\
1.3
\end{tabular}

1.24
1.11
1.46
1.40
1.31

\begin{tabular}{|l|l|}
\hline 5.19 & 1.31 \\
\hline 5.21 & 1.33 \\
\hline 4.34 & 1.62 \\
\hline 4.85 & 1.47 \\
\hline 5.19 & 1.39 \\
\hline
\end{tabular}

\subsection{4}

4.60

4.60
4.86

4.66

4.96

\begin{tabular}{l}
1.17 \\
1.52 \\
1.40 \\
\hline 1.52 \\
\hline 1.34 \\
\hline
\end{tabular}

\begin{tabular}{|l|l|}
\hline 5.48 & 1.13 \\
\hline 4.64 & 1.40 \\
\hline 4.92 & 1.30 \\
\hline 4.62 & 1.42 \\
\hline 4.78 & 1.39 \\
\hline
\end{tabular}




\section{Leadership characteristics}

Q26_3-The client's organizational leaders were truthful and did what they said.

Q26_4-The client's organizational leaders enjoyed taking risks.

Q26_5-The client's organizational leaders had technical expertise in their businesses.

Q26_6-The client's organizational leaders were perceptive and conceptually skilled.

Q26_7-Leaders were approachable with good interpersonal skills and liked in their positions.

Vision and mission

Q27_1-The organization had a strategic business plan and a vision.

Q27_2-The organization had a mission with clearly defined goals.

Q27_3-There was a business case approval for justification for investment in software.

Q27_4-The project realization benefits were in line with the organization's vision.

Q27_5-The project was part of the strategy to achieve the organizational goals.

Team composition

Q31_1-There were best people available on the team.

Q31_2-The team was cross functional and balanced in its composition.

Q31_3-Team members were full time on the software development project.

Q31_4-The team and incentives supported partnerships, trust and risk-sharing.

Q31_5-The teams were empowered decision makers.

Q31_6-Team comprised of the necessary business and technical knowledge of consultants.

\section{User support}

Q32_1-Users had a positive opinion about the system meeting their needs.

Q32_2-Users were enthusiastic about the project.

Q32_3-Users were an integral part of the development team.

Q32_4-Users were available to answer the questions.

Q32_5-Users were ready to accept the changes the system entailed.

\section{Technological uncertainty}

Q35_1-Lack of a clearly known way to develop software...

Q35_2-No available knowledge was of great help in developing software ....

Q35_3-No established procedures and practices could be relied upon to develop software ...

Q35_4-Lack of an understandable sequence of steps that could be followed for developing...

Relative project size (compared to previous projects)

Q36_1-The scheduled number of person-days for completing this project was much higher

Q36_2-The scheduled number of months for completing this project was much higher.

Q36_3-The dollar budget allocated to this project was much higher.

Q36_4-The number of people on team was much larger.

Q36_6-The overall project size was much larger.

\section{Specification changes}

Q37_1-Requirements fluctuated quite a bit in earlier phases.

Q37_2-Requirements fluctuated quite a bit in later phases.

Q37_3-Requirements identified at beginning of the project were quite different from ...

Q37_4-Requirements may fluctuate quite a bit in the future after the project is completed.

gher

Process project success

PCP_1-The project was completed within budget.

PCP_2-The project was completed within schedule.

PCP_3-The project scope was met.

\section{Product project success}

PCP_4-The software developed is reliable.

PCP_5-The application developed is easy to use.

PCP_6-Flexibility of the system is good.

PCP_7-The system meets users' intended functional requirements.

PCP_8-Users were satisfied with the system delivered.

PCP_9-The project team was satisfied.

PCP_10-Top level management of the client's organization was satisfied.

PCP_11-The overall quality of the delivered application is high.

\begin{tabular}{|l|l|l|l|l|}
\hline 4.17 & 1.48 & 4.04 & 1.42 \\
\hline 4.07 & 1.51 & 3.94 & 1.40 \\
\hline 3.87 & 1.52 & 3.81 & 1.42 \\
\hline 3.63 & 1.50 & 3.35 & 1.36 \\
\hline 3.97 & 1.58 & 3.84 & 1.45 \\
\hline & & & \\
\hline 5.04 & 1.35 & 5.05 & 1.27 \\
\hline 4.34 & 1.53 & 4.54 & 1.38 \\
\hline 3.93 & 1.59 & 4.41 & 1.39 \\
\hline 4.20 & 1.51 & 4.54 & 1.33 \\
\hline \multicolumn{5}{|c|}{} \\
\hline 4.78 & 1.57 & 4.70 & 1.47 \\
\hline 4.44 & 1.65 & 4.54 & 1.56 \\
\hline 5.49 & 1.23 & 5.45 & 1.17 \\
\hline 5.69 & 1.01 & 5.64 & 1.04 \\
\hline 5.54 & 1.06 & 5.54 & 1.02 \\
\hline 5.41 & 1.11 & 5.46 & 1.09 \\
\hline 5.71 & 1.14 & 5.75 & 1.87 \\
\hline 5.43 & 1.13 & 5.49 & 1.05 \\
\hline 5.45 & 1.15 & 5.43 & 1.14 \\
\hline 5.51 & 1.13 & 5.48 & 1.12 \\
\hline 5.53 & 1.08 & 5.49 & 1.12 \\
\hline
\end{tabular}


To establish if any significant differences existed between mean scores for both traditional and agile methodologies, t-tests were conducted. Mean differences for all of the constructs revealed overall there were no significant differences between the agile methodology data set and traditional plan-based methodology data set (2-tailed t-tests, $p<0.05$ ). However, some few measurement items of, e.g., user experience, planning and controlling and technical complexity were slightly different across the two groups $(p<0.05)$. This was somehow expected since the literature review on software development projects (chapter 2) suggests that traditional plan-based and agile methodologies differ across these dimensions.

\subsection{Data screening and preparation for SEM analysis}

It is critical to examine and get to know the nature of the data before conducting any analyses. The common data problems that were checked for are errors, outliers, non-response bias and missing data. Data was checked for any potential errors, outliers, non-response bias; cleaned and negatively-worded scale items were reversed coded. Completed online surveys were further checked for missing values and inconsistencies in responses given by the respondents. Simple frequency runs were made to screen the data so as to identify missing values. The identified missing values were a result of omissions made by respondents and constituted less than $1 \%$ of the data; and, thus, considered too trivial (Little \& Rubin, 2002) and inconsequential to suppress the standard deviation (Field, 2006). The fact that missing values were as a result of omissions which were unrelated to other values or variables, the criteria of data missing completely at random (MCAR) were met (Little \& Rubin, 2002). Though there are a quite number of techniques used to address missing data challenges, such as median imputation, series mean value replacement, pair-wise deletion, list-wise deletion, similar response pattern imputation, expectation maximization and Maximum Likelihood Estimation (MLE), the researcher found MLE method suitable for this study because of its robustness in giving reliable estimates based on large samples (Joreskog \& Sorbom, 1989; Byrne, 2001).

The data screening exercise also aimed at establishing the distribution of data to assess whether the assumptions of parametric data were acceptable (Field, 2006; Hair et al., 2010). Parametric statistics assume that the data has come from a type of probability distribution and makes inferences about the parameters of the distribution. Specific assumptions tested for included normality, homoscedasticity (homogeneity of variance) and linearity of the data. Despite the fact SEM is not constrained by normality assumptions (Byrne, 2001), both 
datasets were initially tested for a normal distribution of the data (Field 2006). To assess the assumption of the normality of the distribution of the data, tests carried out included the drawing of histograms and normal probability plots and the computation of Skewness and Kurtosis statistics for both data sets. The histograms were fairly symmetrical and bell-shaped; for all variables of the projects that had used traditional plan-based and agile methodologies separately, with the normal probability plots following a nearly straight line. This is good evidence that the data meets the assumption of normal distribution which partly underlies the use of MLE in SEM.

Normality was further assessed by calculating the Skewness and Kurtosis ratings in SPSS. Skewness evaluates the asymmetry of a distribution whereas Kurtosis examines the degree to which scores cluster in tails of a distribution (Hair et al. 2009; Field 2006). These authors consider a data set to be normally distributed if the Skewness and kurtosis ratings are within the +2 to -2 range. In the traditional plan-based methodology dataset, the Skewness rating was on average at -0.88 . The kurtosis statistics in the traditional plan-based methodology dataset was on average -1.83 . For the agile methodology dataset, the Skewness statistics had an average of -0.68 . The largest Skewness rating was -0.91 . The Kurtosis statistics averaged 0.51 in the agile methodology dataset. The maximum Kurtosis score was -1.25 for the agile data set. Hence, it seemed reasonable to assume that the data was distributed normally in both datasets.

Next, homoscedasticity and linearity tests were conducted. The assumption of homoscedasticity is that the residuals are approximately equal for all predicted dependent variable scores. Scatter plots were created for both all variables for both traditional and agile data sets and when inspected, it was observed that scatter plots looked like a random array of dots evenly dispersed around zero. Field (2006) observed that if the dots follow a funnelshaped manner (funnels out) or form a curve-linear pattern, then there is likelihood of heteroscedasticity in the data and residual errors are not random. Nevertheless, the results obtained in this study clearly indicated that the data points are evenly dispersed; thus confirming that the assumptions of homoscedasticity and linearity are likely to have been met. The next section discusses the data analysis of the main study using SEM.

\subsection{SEM analysis-main study}

SEM analyses were conducted in a two-step analysis of the measurement and structural models. The two separate phases enabled the refinement of measures before testing of the 
structural model. In the first phase, a measurement model was used to measure the fit between the theorized model and observed variables and to establish reliability and validity. In the second phase, results of the measurement model were used to create a structural model in order to measure the strength of the theorized relationships.

\subsubsection{Measurement model}

Analysis of Moment Structures (AMOS) Version 21, a product of IBM SPSS Statistics was selected for the analysis. AMOS provides for the joint specification of the measurement and structural models as a system of equations that avoids the interpretational confounding of parameters (Joreskog \& Sorbom, 1989). It is also appropriate for non-experimental data (Byrne, 2001) and reports robust goodness-of-fit measures that are more accurate even when data violate the normalcy rule for MLE (Joreskog \& Sorbom, 1989; Byrne, 2001). Based on the larger sample size, the MLE approach was selected as providing more reliable estimates (Bentler \& Bonnett, 1980).

\subsubsection{Testing for unidimensionality of single latent congeneric models}

First, the measurement model was tested for single latent models to evaluate unidimensionality. This is consistent with Joreskog and Sorbom (1989) and Fornell and Larcker (1981) who recommend that an initial step in the analysis of SEM is separately testing a series of one latent models for each latent variable in the model that comprises three or more indicators. It was anticipated that the indicator variables contributing to the overall measurement of the latent variable were unidemensional while observing one of the conditions of unidimensionality for the absence of correlated error terms within the constructs (Cunningham, 2010). The goodness of fit measures were viewed as confirming or disconfirming the unidimensionality of the construct which is a prerequisite for the validity of reporting of internal consistency reliability estimates such as Cronbach coefficients (Cunningham 2010). Although this approach takes a lot of time and it is cumbersome to evaluate each separate single latent model, its main advantages are that it allows measurement problems to be resolved before they form part of a full SEM, ensuring unidimensionality of each latent construct (Cunningham, 2010).

\section{Model specification and Goodness of fit results-single latent models}

Initially, for each construct a single latent model with respective item indicators was specified to evaluate the unidimensionality of the construct using MLE. Using the raw SPSS data file downloaded from qualtrics.com as input, a confirmatory factor analysis was performed to 
verify the reliability and validity of each single latent congeneric model. The model fitting process included assessment of the Goodness of fit Indexes for each initial single congeneric model. All model construct variances were constrained to unity during single factor model analysis to indicate the shared common variance between various items in measuring each construct (Raykov, 2004; Anderson \& Gerbing, 1988). Goodness-of Fit Indexes were found to be unsatisfactory at the beginning for most single congeneric models. Modification Indexes (MIs) were used to indicate that each model fit would improve if some items were added to the analysis. Subsequently, new single congeneric models were run with/without these items until acceptable results were achieved. Each of the constructs retained at least three measurement items.

List of measurements items that were dropped during the analysis of single latent models are shown in Appendix (Table 87).

For each final single latent model output, sample correlations, eigenvalues, standardised residual covariances and MIs were all satisfactory. All the sample correlations were within the acceptable range suggesting that item redundancy was not a problem (Fornell \& Larcker, 1981). Equally, all the eigenvalues suggested that a one factor solution was a reasonable assumption for all single factor models. All the factor loadings were strongly significant and exceeded .60 (Hu \& Bentler, 1999; Hair et al., 2009). Similarly, there were no large standardised residuals exceeding 2 among all the item residual covariances (Rigdon, 1996). This means that the single factor models account for much of the covariations that exist between all these items (Anderson \& Gerbing, 1988). The final MIs also showed that the there was no need for any further change. This was expected given the fact that the scales have been used previously as reflective constructs.

The only exception was for two single latent models i.e. project criticality and project success. The measurement items (indicators) used for the construct of project criticality did not fit the single congeneric model. The model fit indices were all below the acceptable criteria despite several modifications. All the standardised residuals were large (exceeding 2 among all the item residual covariances) indicating that that the single factor model could not account for the covariations that exist between all these items (Anderson \& Gerbing, 1988). This was also reflected in the very low factor loading values suggesting that these items neither contributed relatively nor absolutely to a single project criticality construct. However, 
it should be noted that project criticality is a factor that has been empirically assessed less frequently. Therefore, project criticality was completely dropped from further data analysis since loadings should be higher than 0.60 in CFAs in order to demonstrate convergent validity (Hu \& Bentler, 1999).

For project success, one latent congeneric model comprising of eleven items was initially specified and analysed using MLE to test the unidimensionality of the construct of project success. The data was a poor fit to the model based on the significant chi-square $\left(\chi^{2}\right)=149.73$, $d f=32$ and $p=.001$. While the $R M R=.088, T L I=.76$ and $C F I=.77$ suggested a close fit, other indices of fit $(N F I=.75, P R A T I O=0.68, R M S E A=0.14)$ suggested an unsatisifactory fit of the model. The factor coefficients ranged from a low of 0.64 to a high of 0.88 . Given that these coefficients were of reasonable magnitude, exceeding at least 0.6 , all the items were likely to be retained if the data was a good fit to the model. There were some large standardised residuals exceeding 2 among the item residual covariances. This means that the single project success factor is not able to account for much of the covariation that exists between all these items. The eigenvalues suggested that a two factor solution was more reasonable. Small MIs of 1.36 and 1.56 were shown for linking the error variances e6-e8 and e5-e7 respectively. This implies that that if the covariation between these error terms was freely estimated, the chi-square would decrease by at least the value of the MIs. Based on these MI's and eigenvalues listed in sample correlations, error terms could not be covaried because the model would no longer be unidimensional and a substantive theoretical case would have to be made as to why these error terms should be covaried. Since the theory suggests that project success can be evaluated as process and product success (Jun et al, 2011; Zwikael \& Smyrk 2012; Zwikael et al., 2014) and the eigenvalues also suggest that a two factor solution is a reasonable assumption, a two factor model was re-specified for project success. It was found that a two factor solution was providing a better model fit. Therefore, project success was subsequently divided and specified into process and product project success which fitted the models very well. In further analyses, project success was analysed as two separate components of: (1) process success which assesses the effectiveness of the whole project and (2) product success which measures quality outcomes.

Appendices A and B indicate summary of fitted model results of single latent variables (congeneric models) tested separately and their respective factor loadings. As shown, Appendix A includes the complete data set $(\mathrm{n}=984)$ while Appendix B includes single fitted 
model results for sub-group comparisons i.e. traditional plan-based dataset $(n=513)$ and agile data set $(n=471)$ respectively.

Next, using the raw SPSS data files as input for both traditional plan-based $(n=513)$ and agile methodology data sets $(n=471)$, a Confirmatory Factor Analysis (CFA) was performed to verify the main measurement models. CFA was conducted for both measurement models to assess reliability and construct validity by assessing how well individual items represent their constructs in the main measurement model. Goodness-of-fit indexes for the final measurement models were satisfactory for both data sets i.e. traditional plan-based and agile methodologies. To avoid the bias associated with the use of a single index, multiple indices were used to establish Goodness-of-fit (Joreskog \& Sorbom, 1989; Hair et al., 2009). The measures used were the ratio $\chi 2 / d f$, TLI, CFI, RMSEA, NFI, GFI, AGFI and PRATIO. Although $\chi^{2}$ is recognized as a measure of fit, it is affected by the size of correlations within the model and can produce inaccurate probability values particularly for samples that are outside the 100-200 size range (Joreskog \& Sorbom, 1989; Anderson \& Gerbing, 1988). Because the $\chi^{2}$ value is almost always significant in large sample sizes, it was replaced with the ratio of $\chi^{2}$ to the degrees of freedom $\left(\chi^{2 / d f)}\right.$ with values of less than 3.0 being acceptable (Iacobucci, 2010, p.91).

The TLI compares the lack of fit of a target model to the lack of fit of a baseline model with values close to 0.95 being indicative of good fit for ML based models (Hu \& Bentler, 1999). CFI compares the hypothesized model and the independence model (Iacobucci, 2010). CFI values close to 0.95 indicate a good fit for ML based models (Hu \& Bentler, 1999). However, $\mathrm{Hu}$ and Bentler (1999, p. 214) acknowledge that it is difficult to designate a specific cut off criteria for each fit index because it does not work well equally with various conditions. The NFI specifies the practical fit of the model to the data with acceptable fit of 0.95 or above (Hu \& Bentler, 1999). RMSEA expresses fit per degree of freedom of the model; values of 0.06 are indicative of good fit (Hu \& Bentler, 1999). The PPRATIO is the degrees of freedom of the current model divided by the degrees of freedom of the independence model, with values close to 1 indicating good fit (Byrne, 2001). The GFI is a measure of the relative amount of variance and covariance in the sample covariance matrix that is jointly explained by the population covariance matrix (Byrne, 2001). The AGFI differs from the GFI only in the fact that it adjusts for the number of degrees of freedom in the specified model thereby incorporating a penalty for the inclusion of additional parameters. Both indices range 
between 0 and 1, with values close to 1 being indicative of good fit (Byrne, 2001). All the fit indices suggested an adequate fit of the measurement model as shown in Table 63.

Table 63: Measurement models measures of Goodness-of-fit -Group comparisons

\begin{tabular}{lccc}
\hline Goodness of fit index & $\begin{array}{c}\text { Traditional plan-based } \\
\text { methodology data set, } \mathbf{n = 5 1 3}\end{array}$ & $\begin{array}{c}\text { Agile methodology } \\
\text { data set } \mathbf{n = 4 7 1}\end{array}$ & Heuristics \\
\hline$\chi^{2}$ & 6683.41 & 5984.33 & $\mathrm{p}>0.5$ \\
$\chi^{2 / d f}$ & 2.12 & 2.02 & $<3.00$ \\
TLI & 0.91 & 0.92 & $>0.95$ \\
CFI & 0.89 & 0.89 & $>0.95$ \\
RMSEA & 0.04 & 0.04 & $<0.06$ \\
NFI & 0.88 & 0.89 & $\geq 0.95$ \\
PRATIO & 0.90 & 0.90 & $>0.90$ \\
GFI & 0.93 & 0.94 & $>0.90$ \\
AGFI & 0.90 & 0.91 & $>0.90$ \\
\hline
\end{tabular}

In addition, all factor coefficients were above 0.6 and highly significant $(p<.001)$. Given that these coefficients were of reasonable magnitude, all the measurement items in these final measurement models were retained. Upon inspecting the standardized residuals, there were no large standardized residuals exceeding 2 among the item residual covariances. This confirms that the measurement was able to account for much of the covariation that exists between all these items. The MIs also showed that the there was no need for any further change.

\subsubsection{Reliability}

Reliability is the internal consistency and stability over time of the measuring instrument (Cavana et al., 2001, p.461). Reliability was established by calculating the Cronbach alpha coefficients, Composite Scale Reliabilities (CSR) and Average Variance Extracted (AVE) estimates for the final measurement models. First, the reliability analyses were conducted by calculating the Cronbach's alpha value for each construct and results showed that the Cronbach's alpha measures for all 19 constructs were well above 0.7 indicating internalconsistency reliability (Hair et al., 2009). Although the alpha coefficient is traditionally used it suffers from restrictive assumptions regarding equal importance of all indicators (Raykov, 2004). As a result, Cronbach's $\alpha$ can underestimate or overestimate the composite score reliabilities by assuming that all factor loadings and error variances are constrained to be equal (Raykov, 1998). For this reason, the less-restrictive composite reliability index which is a measure of the overall reliability of a collection of heterogeneous but similar items was 
also calculated (Raykov, 2002). The CSR exceeded the recommended minimum of 0.70 (Fornell \& Larcker, 1981; Hair et al., 2009) for both data sets. Additionally, the AVE which is the amount of variance that is due to the latent construct compared to variance attributed to measurement error was also computed to establish both convergent and discriminant validity (Fornell \& Larcker, 1981). For both reliability and construct validity, the AVE was greater than 0.5 (Fornell \& Larcker, 1981; Anderson \& Gerbing, 1988). This confirms that more than $50 \%$ of the variance in the measurement models is due to hypothesized underlying trait (Fornell \& Larcker, 1981). Table 64 shows reliability and validity scores.

Table 64: Reliability and validity

\begin{tabular}{|c|c|c|c|c|c|c|}
\hline & \multicolumn{3}{|c|}{ Traditional plan-based methodology, $n=513$} & \multicolumn{3}{|c|}{ Agile methodology, $n=471$} \\
\hline Construct & $\begin{array}{l}\text { Composite } \\
\text { scale } \\
\text { Reliability } \\
\text { (CSR) }\end{array}$ & $\begin{array}{c}\text { Cronbatch } \\
\text { alpha } \\
(\alpha) \\
\text { Coefficient }\end{array}$ & $\begin{array}{c}\text { Average } \\
\text { Variance } \\
\text { Extracted } \\
\text { (AVE) }\end{array}$ & $\begin{array}{l}\text { Composite } \\
\text { scale } \\
\text { Reliability } \\
\text { (CSR) }\end{array}$ & $\begin{array}{c}\text { Cronbatch } \\
\text { alpha } \\
(\boldsymbol{\alpha}) \\
\text { Coefficient }\end{array}$ & $\begin{array}{c}\text { Average } \\
\text { Variance } \\
\text { Extracted } \\
\text { (AVE) }\end{array}$ \\
\hline Top management support & 0.87 & 0.89 & 0.58 & 0.88 & 0.89 & 0.62 \\
\hline Organisational culture & 0.94 & 0.95 & 0.77 & 0.96 & 0.97 & 0.80 \\
\hline Planning and controlling & 0.85 & 0.83 & 0.60 & 0.86 & 0.87 & 0.59 \\
\hline Leadership characteristics & 0.88 & 0.89 & 0.64 & 0.90 & 0.93 & 0.71 \\
\hline Change management & 0.84 & 0.85 & 0.58 & 0.85 & 0.85 & 0.56 \\
\hline Vision and mission & 0.89 & 0.92 & 0.67 & 0.88 & 0.90 & 0.67 \\
\hline Int. project communication & 0.84 & 0.85 & 0.55 & 0.87 & 0.88 & 0.63 \\
\hline Project team commitment & 0.88 & 0.89 & 0.66 & 0.88 & 0.89 & 0.65 \\
\hline Dev. team's expertise & 0.91 & 0.92 & 0.72 & 0.89 & 0.90 & 0.64 \\
\hline Team composition & 0.86 & 0.85 & 0.55 & 0.86 & 0.88 & 0.62 \\
\hline User participation & 0.84 & 0.85 & 0.55 & 0.90 & 0.90 & 0.70 \\
\hline User support & 0.86 & 0.87 & 0.61 & 0.93 & 0.95 & 0.63 \\
\hline User experience & 0.90 & 0.90 & 0.70 & 0.93 & 0.94 & 0.72 \\
\hline Technical complexity & 0.87 & 0.89 & 0.70 & 0.88 & 0.87 & 0.75 \\
\hline Technological uncertainty & 0.92 & 0.94 & 0.75 & 0.93 & 0.94 & 0.76 \\
\hline Relative project size & 0.94 & 0.96 & 0.75 & 0.93 & 0.94 & 0.73 \\
\hline Specification changes & 0.85 & 0.86 & 0.62 & 0.87 & 0.88 & 0.53 \\
\hline Process project success & 0.87 & 0.88 & 0.67 & 0.86 & 0.87 & 0.63 \\
\hline Product project success & 0.95 & 0.96 & 0.69 & 0.94 & 0.95 & 0.68 \\
\hline
\end{tabular}

Note: CSR and AVE are not computed by AMOS. The following formulae were used to calculate AVE and CSR: Composite Scale Reliability $=[\operatorname{SUM}(A)]^{2} /\left[(\operatorname{SUM}(A)]^{2}+\operatorname{SUM}(B)\right.$. Where $[S U M(A)]^{2}=$ sum of standardised factor loadings squared and $S U M(B)=$ sum of indicator measurement errors (sum of the variance due to random measurement error for each loading=1-the square of each loading) (Fornell \& Larcker, 1981). AVE $=\left[\left(\operatorname{SUM}\left(A^{2}\right)\right] /\left[\left(\operatorname{SUM}\left(A^{2}\right)\right]+\operatorname{SUM}(B)\right.\right.$. Where $\left[\left(\operatorname{SUM}\left(A^{2}\right)\right]=\right.$ sum of squared standardized loadings and $S U M(B)=$ sum of indicator measurement error) (Fornell \& Larcker, 1981). Cronbach alpha was computed using SPSS V.19 as AMOS does not compute it.

\subsubsection{Validity}

Validity is the extent to which an instrument measures what it is intended to measure (Hair et al., 2009; Iacobucci, 2010). Validity was established by examining content validity, construct validity, convergent validity and discriminant validity.

\section{Content validity}

Content validity is the extent to which a measure represents all facets of a given construct (Cavana et al., 2001, p.213). Therefore content validity establishes the representative sampling of a set of items that measures a concept, and reflects how well the dimensions and elements thereof are delineated. Content validity was achieved through: 1) selection of survey 
items from existing project management theory and the use of tested instruments where available (Byrne, 2001); 2) the use of Victoria University’s School of Management (SoM) Professors for instrument review; and 3) pilot testing by 15 senior and experienced software development project managers in Wellington for instrument refinement.

\section{Construct validity}

Construct validity means that a scale correlates with the theorized construct (Anderson \& Gerbing, 1988). It demonstrates how well the results obtained from the use of the measure fit the theories around which the test is designed (Cavana et al., 2001, p.213). Construct validity was demonstrated by both convergent and discriminant validity (Nanually, 1978).

\section{Convergent validity}

Convergent validity is when the scores obtained by two different instruments measuring the same concept are highly correlated (Cavana et al., 2001, p.213). Convergent validity was established by the high factor loadings $(\lambda \geq 0.6)$ and high levels of significance $(t>2)$ for all the indicator variables (Straub, 1989; Bagozzi \& Yi, 1988; Anderson \& Gerbing, 1988), as shown in Table 65, and by the strong and highly significant correlations between the constructs (Straub, 1989; Bagozzi \& Yi, 1988), as shown in Tables 66 and 67. This provides evidence that the measurement items are significantly related to their construct measures. 
Table 65: Standardised regression weights from the measurement models-Group comparisons

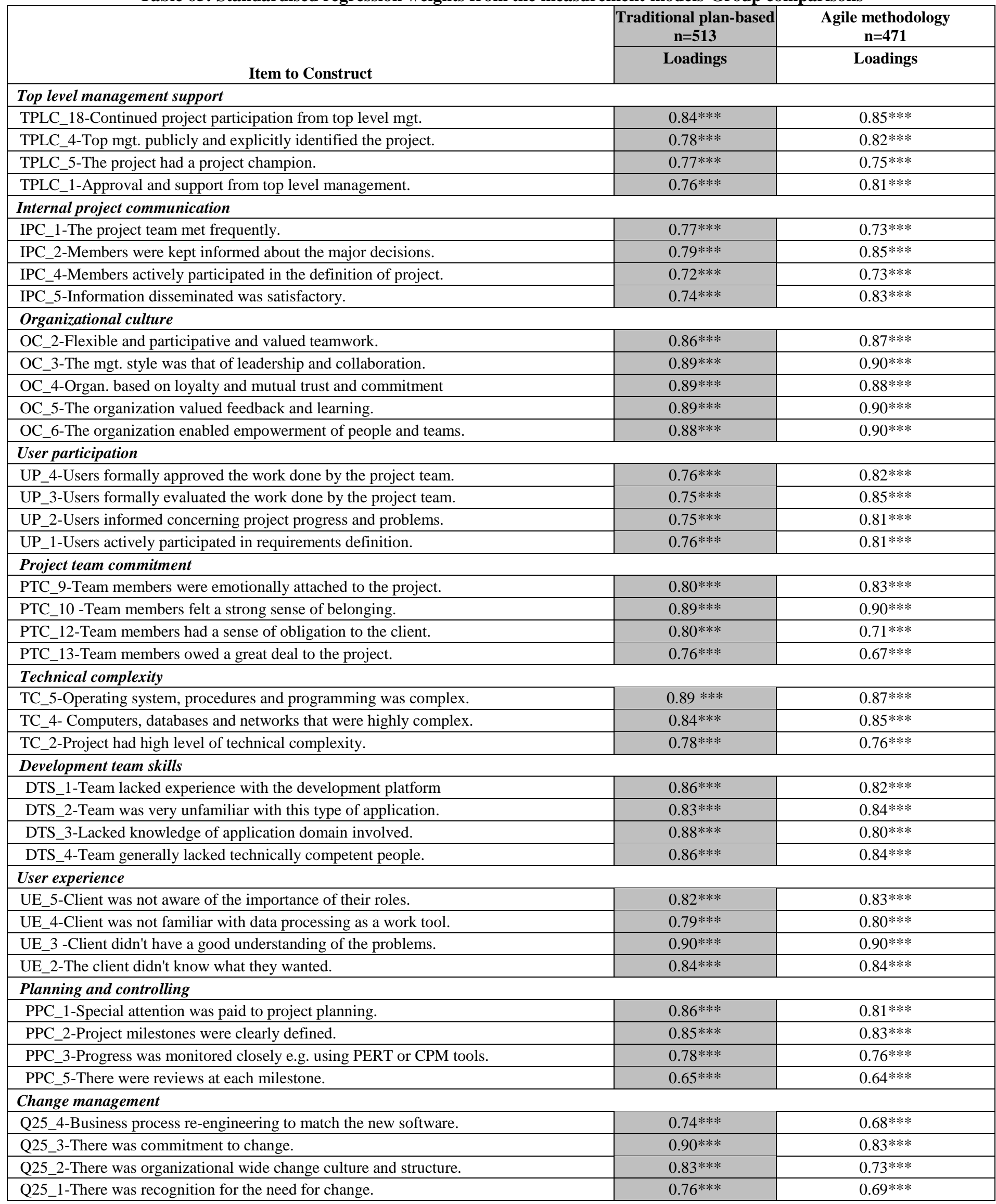




\begin{tabular}{|c|c|}
\hline $\begin{array}{c}\text { Traditional plan-based } \\
\mathbf{n}=513\end{array}$ & $\begin{array}{c}\text { Agile methodology } \\
\mathbf{n}=\mathbf{4 7 1}\end{array}$ \\
\hline Loadings & Loadings \\
\hline
\end{tabular}

\section{Leadership characteristics}

Q26_7-Leaders were approachable with good interpersonal skills

Q26_6-Leaders were perceptive and conceptually skilled.

Q26_5-Leaders had technical expertise in their businesses.

Q26_3-Leaders were truthful and did what they said.

\section{Vision and mission}

Q27_5-The project was part of the strategy.

Q27_4-Realization benefits were in line with the vision.

Q27_2-The organization had a mission with clearly defined goals.

Q27_1-The organization had a strategic business plan and a vision.

\section{Team composition}

Q31_6-Team comprised of necessary technical knowledge and consultants.

Q31_5-The teams were empowered decision makers.

Q31_2-The team was cross functional and balanced.

Q31_1-There were best people available on the team.

User support

Q32_5-Users were ready to accept the changes the system entailed.

Q32_4-Users were available to answer the questions.

Q32_3-Users were an integral part of the development team.

Q32_2-Users were enthusiastic about the project.

Q32_1-Users had a positive opinion about the system.

Specification changes

Q37_4-Requirements may fluctuate quite a bit in the future.

Q37_3-Requirements identified at beginning were quite different.

Q37_2-Requirements fluctuated quite a bit in later phases.

\section{Technological uncertainty}

Q35_1-Lack of a clearly known way to develop software.

Q35_2-No available knowledge was of great help in developing software.

Q35_3-No established procedures and practices could be relied upon.

Q35_4-Lack of an understandable sequence of steps that could be followed.

\section{Relative project size}

Q36_6-The overall project size was much larger.

Q36_4-The number of people on team was much larger.

Q36_3-The dollar budget allocated to this project was much higher.

Q36_2-Months for completing this project were much higher.

Q36_1-Person-days for completing this project were much higher.

\section{Process project success}

PCP_1-The project was completed within budget.

PCP_2-The project was completed within schedule.

PCP_3-The project scope was met.

\begin{tabular}{l|l}
\hline $0.79 * * *$ & $0.84 * * *$ \\
\hline $0.86 * * *$ & $0.90 * * *$ \\
\hline $0.77 * * *$ & $0.78 * * *$ \\
\hline $0.76 * * *$ & $0.82 * * *$
\end{tabular}

\section{Product project success}

PCP_4-The software developed is reliable.

PCP_5-The application developed is easy to use.

PCP_6-Flexibility of the system is good.

PCP_7-The system meets users' intended functional requirements.

PCP_8-Users were satisfied with the system delivered.

PCP_9-The project team was satisfied.

PCP_10-Top level management was satisfied.

PCP_11-The overall quality of the delivered application is high.

\begin{tabular}{l|l}
\hline $0.73 * * *$ & $0.69 * * *$ \\
\hline $0.76 * * *$ & $0.73 * * *$ \\
\hline $0.91 * * *$ & $0.89 * * *$ \\
\hline $0.90 * * *$ & $0.88 * * *$
\end{tabular}

\begin{tabular}{l|l}
$0.81 * * *$ & $0.80 * * *$ \\
\hline $0.72 * * *$ & $0.75 * * *$ \\
\hline $0.78 * * *$ & $0.80 * * *$ \\
\hline $0.70 * * *$ & $0.75 * * *$
\end{tabular}

\begin{tabular}{|l|l|}
\hline $0.78 * * *$ & $0.79 * * *$ \\
\hline $0.72 * * *$ & $0.74 * * *$ \\
\hline $0.66 * * *$ & $0.69 * * *$ \\
\hline $0.78 * * *$ & $0.80 * * *$ \\
\hline $0.81 * * *$ & $0.82 * * *$ \\
\hline
\end{tabular}

\begin{tabular}{|l|l}
\hline $0.77 * * *$ & $0.66 * * *$ \\
\hline $0.86 * * *$ & $0.78 * * *$ \\
\hline $0.81 * * *$ & $0.73 * * *$ \\
\hline
\end{tabular}

\begin{tabular}{|l|l}
\hline $0.82 * * *$ & $0.79 * * *$ \\
\hline $0.84 * * *$ & $0.86 * * *$ \\
\hline $0.89 * * *$ & $0.92 * * *$ \\
\hline $0.90 * * *$ & $0.86 * * *$ \\
\hline
\end{tabular}

*** Significant at $p<0.001$. All regression factor loadings are standardised and greater than 0.6 for convergent validity (Straub, 1989; Bagozzi \& Yi, 1988). 
In summary, all constructs demonstrated good model fit even when subjected to $\mathrm{Hu}$ and Bentler's (1999) restrictive criteria and Rigdon's (1996) criteria. The factor loading of each item in all constructs was above 0.60 and adequate. In addition, all the factor loadings were strongly significant, indicated by their corresponding t-values greater than 2 (Straub, 1989; Bagozzi \& Yi, 1988). The Average Variance Extracted for each of the 19 constructs exceeded the suggested threshold of 0.50 , indicating the variance captured by a construct was larger than the variance due to measurement errors (Fornell \& Larcker, 1981). Hence, the convergent validity of the measurement model was established. 
Table 66: Correlates for projects that used the traditional plan-based methodology, $n=513$

\begin{tabular}{|c|c|c|c|c|c|c|c|c|c|c|c|c|c|c|c|c|c|c|c|}
\hline Construct & TMS & OC & PC & LC & CM & VM & IPC & PTC & TE & TC & UP & US & UE & TCO & TU & RPS & SC & PRC & PRD \\
\hline Top mgt. support(TMS) & 0.58 & 0.26 & 0.24 & 0.19 & 0.17 & 0.24 & 0.24 & 0.18 & 0.01 & 0.19 & 0.23 & 0.14 & 0.05 & 0.05 & 0.03 & 0.01 & 0.01 & 0.08 & 0.12 \\
\hline Organ-culture(OC) & $.54 * * *$ & 0.77 & 0.25 & 0.23 & 0.31 & 0.25 & 0.32 & 0.19 & 0.03 & 0.36 & 0.25 & 0.27 & 0.09 & 0.05 & 0.08 & 0.0 & 0.05 & 0.20 & 0.21 \\
\hline Planning and controlling(PC) & $.49 * * *$ & $.51 * * *$ & 0.60 & 0.11 & 0.23 & 0.23 & 0.45 & 0.20 & 0.02 & 0.30 & 0.29 & 0.28 & 0.09 & 0.08 & 0.13 & 0.00 & 0.11 & 0.23 & 0.23 \\
\hline Leadership characteristics(LC) & $.46^{* * * *}$ & $.49^{* * * *}$ & $.34 * * *$ & 0.64 & 0.22 & 0.24 & 0.11 & 0.08 & 0.01 & 0.22 & 0.25 & 0.36 & 0.22 & 0.02 & 0.04 & 0.00 & 0.12 & 0.22 & 0.22 \\
\hline Change management(CM) & $.44^{* * * *}$ & $.57 * * *$ & $.47 * * *$ & $.48 * * *$ & 0.58 & 0.21 & 0.15 & 0.11 & .00 & 0.26 & 0.15 & 0.24 & 0.06 & 0.06 & 0.03 & 0.00 & 0.03 & 0.17 & 0.21 \\
\hline Vision and mission(VM) & $.49^{* * * *}$ & $.51 * * *$ & $.47 * * *$ & $.49 * * *$ & $.46^{* * * *}$ & 0.67 & 0.12 & 0.09 & 0.04 & 0.22 & 0.17 & 0.19 & 0.13 & 0.05 & 0.06 & 0.00 & 0.04 & 0.12 & 0.15 \\
\hline Int. proj. communication(IPC) & $.49^{* * * *}$ & $.57 * * *$ & $.68^{* * * *}$ & $.33^{* * *}$ & $.39^{* * *}$ & $.35^{* * *}$ & 0.56 & 0.23 & 0.02 & 0.30 & 0.34 & 0.26 & 0.05 & 0.09 & 0.08 & 0.00 & 0.09 & 0.20 & 0.25 \\
\hline Proj. team commitment( PTC) & $.45 * * *$ & $.45 * * *$ & $.45 * * *$ & $.28 * * *$ & $.33 * * *$ & $.31 * * *$ & $.48 * * *$ & 0.66 & .00 & 0.35 & 0.15 & 0.21 & 0.00 & 0.11 & 0.01 & 0.00 & .00 & 0.12 & 0.16 \\
\hline Dev. Team expertise(TE) & -.08 & $-.15^{* * * *}$ & $-.13^{* * *}$ & $-.13^{*}$ & -.02 & $-.20 * * *$ & $-.15^{* *}$ & -.01 & 0.72 & 0.08 & 0.02 & 0.02 & 0.17 & 0.01 & 0.32 & 0.1 & 0.16 & 0.03 & 0.05 \\
\hline Team composition(TC) & $.45^{* * *}$ & $.59 * * *$ & $.54 * * *$ & $.46^{* * *}$ & $.51 * * *$ & $.49^{* * *}$ & $.55^{* * *}$ & $.60^{* * *}$ & $-.28 * * *$ & 0.55 & 0.32 & 0.35 & 0.07 & 0.10 & 0.07 & 0.00 & 0.03 & 0.21 & 0.28 \\
\hline User participation(UP) & $.50 * * *$ & $.49 * * *$ & $.54 * * *$ & $.49 * * *$ & $.39 * * *$ & $.41 * * *$ & $.58 * * *$ & $.40^{* * * *}$ & $-.16^{* *}$ & $.56^{* * * *}$ & 0.55 & 0.34 & 0.10 & 0.04 & 0.07 & 0.00 & 0.06 & 0.19 & 0.24 \\
\hline User support(US) & $.40 * * *$ & $.53 * * *$ & $.52 * * *$ & $.59 * * *$ & $.487 * * *$ & $.44 * * *$ & $.51 * * *$ & $.45^{* * * *}$ & -.15 & $.60 * * * *$ & $.58 * * *$ & 0.61 & 0.19 & 0.02 & 0.07 & 0.00 & 0.15 & 0.31 & 0.28 \\
\hline User experience(UE) & $.25^{* * * *}$ & $-.32^{* * *}$ & $-.31^{* * *}$ & $-.48^{* * *}$ & $-.25^{* * *}$ & $-.36^{* * *}$ & $.21 * * *$ & .05 & $.42^{* * *}$ & $-.27 * * *$ & $-.31^{* * *}$ & $-.43^{* *}$ & 0.70 & 0.04 & 0.23 & 0.4 & 0.35 & 0.09 & 0.09 \\
\hline Technical complexity(TCO) & $.26^{* * *}$ & $.23^{* * *}$ & $.29 * * *$ & $.14^{* *}$ & $.24 * * *$ & $.21 * * *$ & $.30 * * *$ & $.35^{* * *}$ & $.11^{*}$ & $.31^{* * * *}$ & $.21 * * *$ & $.13^{* *}$ & -.01 & 0.70 & 0.01 & 0.04 & 0.01 & 0.01 & 0.06 \\
\hline Technology uncertainty(TU) & $-.19 * * *$ & $-.28 * * *$ & $-.60^{* * *}$ & $-.23 * * *$ & $-.17 * * *$ & $-.25^{* * *}$ & $-.29 * * *$ & -.08 & $.56 * * *$ & $-.26^{* * *}$ & $-.26 * * *$ & $-.3 * * *$ & $.48 * * *$ & .08 & 0.75 & 0.10 & 0.29 & 0.07 & 0.12 \\
\hline Relative project size(RPS) & $.10^{*}$ & -.01 & .07 & -.03 & .04 & .04 & -.03 & .057 & $.26 * * *$ & .03 & -.04 & $-.05 * *$ & $.20 * * *$ & $.21^{* * * *}$ & $.32 * * *$ & 0.75 & 0.13 & 0.05 & 0.03 \\
\hline Specification changes(SC) & $-.12^{*}$ & -.24 & $-.34^{* * * *}$ & $-.36^{* * *}$ & $-.17 * * *$ & $-.19^{* * *}$ & $-.30^{* * *}$ & -.04 & $.39^{* * *}$ & $-.18 * * *$ & $-.24 * * *$ & $-.4 * * *$ & $.59^{* * *}$ & $.10^{* * * *}$ & $.54 * * *$ & $.36^{* * * *}$ & 0.62 & 0.14 & 0.09 \\
\hline Process project success(PRC) & $.12^{*}$ & $.43^{*}$ & $.49 * * *$ & $.46^{* * * *}$ & $.42 * * *$ & $.35 * * *$ & $.46^{* * *}$ & $.34 * * *$ & $-.17 * * *$ & $.47^{* * * *}$ & $.44 * * *$ & $.54 * * *$ & $-.31 * * *$ & $.11^{*}$ & $-.28 * * *$ & $-.21 * * *$ & $-.4 * * *$ & 0.67 & 0.57 \\
\hline Product project success(PRD) & $.37^{*}$ & $.44 *$ & $.49 * * *$ & $.46^{* * * *}$ & $.47 * * *$ & $.38 * * *$ & $.49 * * *$ & $.39^{* * *}$ & $-.22 * * *$ & $.54 * * *$ & $.48 * * *$ & $.52 * * *$ & $-.29 * * *$ & $.23 * * *$ & $-.34 * * *$ & $-.21 * * *$ & $-.2^{* * * *}$ & $.79 * * *$ & 0.69 \\
\hline
\end{tabular}

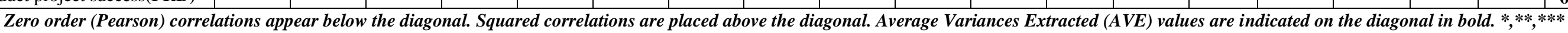
significant at $0.05,0.01,0.001$ respectively. 
Table 67: Correlates for projects that used Agile methodology, $n=471$

\begin{tabular}{|c|c|c|c|c|c|c|c|c|c|c|c|c|c|c|c|c|c|c|c|}
\hline Factor & TMS & $\mathbf{O C}$ & PC & $\mathbf{L C}$ & $\mathbf{C M}$ & VM & IPC & PTC & TE & TC & $\mathbf{U P}$ & US & UE & TCO & TU & RPS & SC & PRC & PRD \\
\hline Top mgt. support(TMS) & 0.62 & 0.33 & 0.17 & 0.21 & 0.27 & 0.22 & 0.26 & 0.11 & 0.06 & 0.19 & 0.16 & 0.16 & 0.05 & 0.09 & 0.03 & 0.00 & 0.01 & 0.05 & 0.09 \\
\hline Organ-culture $(\mathbf{O C})$ & $.56^{* * *}$ & 0.80 & 0.2 & 0.38 & 0.33 & 0.29 & 0.45 & 0.31 & 0.11 & 0.44 & 0.26 & 0.26 & 0.04 & 0.03 & 0.03 & 0.00 & 0.02 & 0.26 & 0.31 \\
\hline Planning and controlling(PC) & $.43^{* * *}$ & $.46^{* * *}$ & 0.59 & 0.19 & 0.32 & 0.25 & 0.30 & 0.12 & 0.02 & 0.28 & 0.26 & 0.17 & 0.06 & 0.05 & 0.02 & 0.00 & 0.07 & 0.18 & 0.18 \\
\hline Leadership characteristics(LC) & $.47_{* * *}$ & $.61^{* * *}$ & $.45 * * *$ & 0.71 & 0.35 & 0.40 & 0.24 & 0.31 & 0.02 & 0.31 & 0.28 & 0.39 & 0.13 & 0.03 & 0.02 & 0.00 & 0.03 & 0.22 & 0.21 \\
\hline Change management $(\mathbf{C M})$ & $.51^{* * *}$ & $.56 * * *$ & $.55 * * *$ & $.59^{* * *}$ & 0.56 & 0.27 & 0.28 & 0.26 & 0.01 & 0.31 & 0.22 & 0.23 & 0.02 & 0.11 & 0.00 & 0.01 & 0.00 & 0.12 & 0.15 \\
\hline Vision and mission(VM) & $.48^{* * * *}$ & $.55 * * *$ & $.49_{* * *}$ & $.63 * * *$ & $.51 * * *$ & 0.67 & 0.15 & 0.19 & 0.01 & 0.27 & 0.20 & 0.24 & 0.04 & 0.10 & 0.03 & 0.00 & 0.01 & 0.10 & 0.17 \\
\hline Int. proj. communication(IPC) & $.50^{* * *}$ & $.66^{* * *}$ & $.54 * * *$ & $.50_{* * *}$ & $.53^{* * *}$ & $.40 * * *$ & 0.63 & 0.35 & 0.08 & 0.43 & 0.30 & 0.28 & 0.02 & 0.06 & 0.04 & 0.01 & 0.02 & 0.26 & 0.32 \\
\hline Proj. team commitment(PTC) & $.32 * * *$ & $.55^{* * *}$ & $.36^{* * *}$ & $.55^{* * *}$ & $.52^{* * *}$ & $.42^{* * *}$ & $.60^{* * *}$ & 0.65 & 0.02 & 0.39 & 0.26 & 0.23 & 0.01 & 0.10 & 0.01 & 0.00 & 0.00 & 0.17 & 0.22 \\
\hline Dev. Team expertise(TE) & $.26^{* * *}$ & $-.34 * * *$ & $-.16^{* * *}$ & -.17 ** & $-.13 *$ & $-.13^{* * *}$ & $-.30_{* * *}^{* *}$ & $-.14 * *$ & 0.64 & 0.11 & 0.00 & 0.03 & 0.23 & 0.00 & 0.29 & 0.05 & 0.09 & 0.07 & 0.09 \\
\hline Team composition(TC) & $.43^{* * *}$ & $.66^{* * *}$ & $.53^{* * *}$ & $.57_{* * *}$ & $.56^{* * *}$ & $.53^{* * *}$ & $.66_{* * *}^{*}$ & $.61^{* * *}$ & $-.35^{* * *}$ & 0.62 & 0.29 & 0.39 & 0.02 & 0.13 & 0.04 & 0.00 & 0.00 & 0.29 & 0.42 \\
\hline User participation(UP) & $.39_{* * * *}$ & $.50^{* * *}$ & $.51^{* * *}$ & $.53^{* * *}$ & $.48^{* * *}$ & $.45^{* * *}$ & $.55^{* * *}$ & $.50_{* * *}^{* *}$ & -.04 & $.53^{* * *}$ & 0.70 & 0.47 & 0.01 & 0.06 & 0.00 & 0.00 & 0.01 & 0.27 & 0.21 \\
\hline User support(US) & $.39^{* * *}$ & $.51_{* * *}^{*}$ & $.43^{* * *}$ & $.61_{* * *}$ & $.49_{* * *}$ & $.50^{* * *}$ & $.54 * * *$ & $.49_{* * *}$ & $-.15^{* *}$ & $.62^{* * *}$ & .68 & 0.63 & 0.05 & 0.05 & 0.03 & 0.00 & 0.00 & 0.23 & 0.31 \\
\hline User experience(UE) & $.22 * * *$ & $-.21^{* * *}$ & $-.24^{* * *}$ & $-.36^{* * *}$ & $-.13 *$ & $-.21^{* * *}$ & $-.17 * *$ & -.08 & $.47_{* * *}$ & $-.16^{*}$ & $-.12 *$ & $-.22^{* * *}$ & 0.72 & 0.00 & 0.22 & 0.06 & 0.34 & 0.04 & 0.02 \\
\hline Technical complexity(TCO) & $.28^{* * * *}$ & $.16^{* * *}$ & $.22 *$ & $.18^{* * *}$ & $.35^{* * * *}$ & $.33^{* * *}$ & $.25^{* * *}$ & $.31^{* * * *}$ & -.03 & $.36^{* * * *}$ & $.24 * * *$ & $.23^{* * *}$ & .03 & 0.75 & 0.00 & 0.05 & 0.03 & 0.00 & 0.02 \\
\hline Technological uncertainty(TU) & $-.17_{* * *}$ & $-.17_{* * *}$ & $-.16^{* * *}$ & $-.14 *$ & -.07 & $-.17 *$ & $-.19_{* * *}$ & -.09 & $.55^{* * *}$ & $-.21_{* * *}^{*}$ & $-.06^{* * *}$ & -.17 & $.47_{* * *}$ & .05 & 0.76 & 0.10 & 0.16 & 0.01 & 0.07 \\
\hline Relative project size(RPS) & .04 & -.07 & -.03 & .07 & .13 & .08 & -.09 & -.02 & $.23^{* * *}$ & .03 & -.02 & .06 & $.25 * * *$ & $.21 * * *$ & .29 & $\mathbf{0 . 7 3}$ & 0.08 & 0.07 & 0.02 \\
\hline Specification changes $(\mathbf{S C})$ & -.07 & $-.14 * * *$ & $-.25^{* * *}$ & $-.17 *$ & .02 & $-.06^{* * *}$ & $-.13^{*}$ & .04 & $.31^{* * *}$ & .03 & -.09 & -.06 & $.58^{* * *}$ & $.18_{* * *}^{*}$ & $.39^{* *}$ & $.30^{* * *}$ & $\mathbf{0 . 5 3}$ & 0.02 & 0.00 \\
\hline Process project success(PRC) & $-.12^{* * *}$ & $.51^{* * *}$ & $.42^{* * *}$ & $.47_{* * * *}$ & $.34 * * *$ & $.32^{* * *}$ & $.50 * * *$ & $.43^{* * *}$ & $-.27^{* * *}$ & $.53^{* * *}$ & $.52^{* * *}$ & $.48^{* * *}$ & $-.19^{* * *}$ & -.01 & $-.11 *$ & $-.25^{* * *}$ & $-.16^{*}$ & 0.63 & 0.54 \\
\hline Product project success(PRD) & $-.19_{* * *}^{*}$ & $.56 * * *$ & $.43^{* * *}$ & $.45^{* * *}$ & $.39_{* * *}$ & $.43^{* * *}$ & $.55 * * *$ & $.45^{* * * *}$ & $-.29_{* * *}$ & $.66^{* * *}$ & $.46^{* * *}$ & $.56^{* * *}$ & $-.15 *$ & $.12^{* * *}$ & $-.25^{* * *}$ & $-.13^{*}$ & $\begin{array}{l}.07 \\
\end{array}$ & $.75^{* * * *}$ & 0.68 \\
\hline
\end{tabular}




\section{Discriminant validity}

The discriminant validity test was performed in order to establish the distinction among all the constructs used in this study (Anderson \& Gerbing, 1988). Discriminant validity was established by three separate tests. First, the Average Variance Extracted (AVE) assesses the amount of variance explained by the construct as compared to the amount ascribed to random measurement error (Fornell \& Lacker, 1981). For all 171 construct pairs, the AVE of each construct exceeded the square of the construct correlations and discriminant validity was established as shown in Tables 66 and 67 for both data sets respectively. As indicated, for the traditional plan based methodology data set, of the 171 construct correlations, 141 were significant at $p<.001,19$ were significant at between $p<0.01$ and $p<0.5$ and only 11 were not significant. For the agile methodology data set, of the 171 construct correlations, 147 were significant at $p<.001,13$ were significant at between $p<0.01$ and $p<0.5$ and only 11 were not significant. No correlation exceeded the prescribed limit of 0.90 suggesting that multicollinearity among latent constructs was not a problem in this study (Kearns \& Sabherwal, 2007, p. 636).

Second, Chi-square $(\chi 2)$ difference tests were run for all possible construct pairs. For each pair, a comparison was made between the $\chi 2$ values for the constrained model and the unconstrained model. The constrained model represents a case in which the covariance and variances for the construct pairs is constrained to unity (Anderson \& Gerbing, 1988). All the $\chi^{2}$ difference were significantly less for the unconstrained model, suggesting that the better model was the one in which the factors were separate but correlated (Anderson \& Gerbing, 1988). Table 68 reports a section of results of $\chi 2$ difference tests conducted on both data sets. Due to space constraints, the whole table of $\chi 2$ difference tests cannot be displayed. 
Table 68: $\chi 2$ difference tests for discriminant validity

\begin{tabular}{|c|c|c|c|c|c|c|}
\hline \multicolumn{4}{|c|}{ Traditional plan-based methodology, $n=513$} & \multicolumn{3}{|c|}{ Agile methodology, $\mathrm{n}=471$} \\
\hline $\begin{array}{l}\text { Construct } \\
\text { pairs }\end{array}$ & $\begin{array}{l}\text { Unconstrained } \\
\text { Model } \chi^{2}\end{array}$ & $\begin{array}{l}\text { Constrained } \\
\text { Model } \chi^{2}\end{array}$ & $\chi^{2}$ Difference* & $\begin{array}{l}\text { Unconstrained } \\
\text { Model } \chi^{2}\end{array}$ & $\begin{array}{l}\text { Constrained } \\
\text { Model } \chi^{2}\end{array}$ & $\chi^{2}$ Difference* \\
\hline TMS/ OC & 144.84 & 263.5 & 118.66 & 148.63 & 266.8 & 118.17 \\
\hline $\mathrm{TMS} / \mathrm{PC}$ & 30.2 & 243.8 & 213.6 & 28.2 & 241.8 & 213.6 \\
\hline TMS /LC & 94.6 & 231.7 & 137.1 & 98.6 & 229.7 & 131.1 \\
\hline TMS /CM & 112.2 & 487.6 & 375.4 & 110.2 & 485.6 & 375.4 \\
\hline TMS /VM & 47.5 & 217.6 & 170.1 & 45.5 & 215.6 & 170.1 \\
\hline TMS /IPC & 76.4 & 245.6 & 169.2 & 74.4 & 243.6 & 169.2 \\
\hline TMS /PTC & 56.3 & 324.6 & 268.3 & 54.3 & 322.6 & 268.3 \\
\hline TMS /TE & 64.4 & 342.8 & 278.4 & 62.4 & 340.8 & 278.4 \\
\hline TMS /TC & 67.7 & 291.2 & 223.5 & 65.7 & 289.2 & 223.5 \\
\hline TMS /UP & 56.9 & 276.3 & 219.4 & 54.9 & 274.3 & 219.4 \\
\hline TMS /US & 43.8 & 454.7 & 410.9 & 41.8 & 452.7 & 410.9 \\
\hline TMS /UE & 61.8 & 521.7 & 459.9 & 59.8 & 519.7 & 459.9 \\
\hline TMS /TCO & 73.3 & 432.2 & 358.9 & 71.3 & 430.2 & 358.9 \\
\hline TMS /TU & 78.5 & 452.8 & 374.3 & 76.5 & 450.8 & 374.3 \\
\hline TMS /RPS & 123.4 & 546.7 & 423.3 & 121.4 & 544.7 & 423.3 \\
\hline TMS /SC & 113.5 & 427.9 & 314.4 & 111.5 & 425.9 & 314.4 \\
\hline TMS /PRC & 126.7 & 246.8 & 120.1 & 124.7 & 244.8 & 120.1 \\
\hline TMS /PRD & 131.4 & 367.6 & 236.2 & 129.4 & 365.6 & 236.2 \\
\hline $\mathrm{OC} / \mathrm{PC}$ & 135.5 & 417.9 & 282.4 & 133.5 & 415.9 & 282.4 \\
\hline $\mathrm{OC} / \mathrm{LC}$ & 123.5 & 437.7 & 314.2 & 121.5 & 435.7 & 314.2 \\
\hline $\mathrm{OC} / \mathrm{CM}$ & 32.7 & 234.6 & 201.9 & 30.7 & 232.6 & 201.9 \\
\hline $\mathrm{OC} / \mathrm{VM}$ & 67.8 & 236.1 & 168.3 & 65.8 & 234.1 & 168.3 \\
\hline $\mathrm{OC} / \mathrm{IPC}$ & 32.5 & 453.9 & 421.4 & 30.5 & 451.9 & 421.4 \\
\hline $\mathrm{OC} / \mathrm{PTC}$ & 35.9 & 457.7 & 421.8 & 33.9 & 455.7 & 421.8 \\
\hline $\mathrm{OC} / \mathrm{TE}$ & 79.7 & 467.8 & 388.1 & 77.7 & 465.8 & 388.1 \\
\hline $\mathrm{OC} / \mathrm{TC}$ & 64.6 & 346.7 & 282.1 & 62.6 & 344.7 & 282.1 \\
\hline Etc. & Etc. & Etc. & Etc. & Etc. & Etc. & Etc. \\
\hline & *Differen & measured at 1 & gree of freedom. & lifferences are si & ant at $p<0.001$ & \\
\hline
\end{tabular}

Finally, a confidence interval of (+/-) 3 standard errors was calculated for each of the construct correlations to determine if the interval contains the value 1 . None of the confidence intervals contains the value 1 indicating that the correlations between these constructs differed significantly from unity and that the probability of perfect correlation was extremely low (Anderson \& Gerbing, 1988).

\section{Common method variance}

Common method variance is the portion of correlation between two variables that results from sharing a common method of measurement (Podsakoff et al, 2003). Because selfreporting, consistency motif, acquiescence, social desirability, affectivity and transient mood state lead to common method variance; it is of concern in survey research when sampling perceptual data (Podsakoff et al., 2003). Common method variance leads to Type 1 and 2 errors whereby researchers may accept or reject the null hypothesis when they should not have done so. Common method variance is a potential problem particularly when all measurements are provided by a single respondent (Kearns \& Sabherwal, 2007). While a popular approach to overcoming "same source bias" problems is to collect responses from two respondents, this approach was not possible to use in this study. Collecting data from separate respondents for same variables would have been very hard to convince the 
respondents and get a higher response rate required for fitting SEM models. Besides, collecting data from two respondents can also introduce some errors of linking up data together for predictor and criterion variables, requiring more time, effort and cost (Podsakoff et al., 2003).

Common method variance was addressed in three ways. Firstly using the strategies to improve the problems of self-reports data during survey design. The questionnaire was designed in such a way to avoid implying that one response is better than the other, paying attention to wording, avoiding socially accepted responses, avoiding vague concepts, keeping questions simple, specific, and concise, avoiding double-barrelled questions, decomposing questions relating to more than one possibility, as well as avoiding complicated syntax.

Second, common method variance was further assessed using Harman's one factor test (Podsakoff et al., 2003). The underlying logic for this test is that if substantial common method bias accounts for the relations among variables, then a factor analysis should yield a single factor or one general factor accounts for most of the variance when all the items are analyzed together. Harman's one-factor test was conducted for all the 97 items, measuring all 19 constructs where they were all entered into an exploratory factor analysis, and the results of the unrotated factor solution were examined. If substantial common method variance is present, either a single factor would emerge or one general factor would account for most of the variance. For both data sets, each single factor had a variance of $23.12 \%$ and 23.42 for both traditional plan-based and agile methodologies respectively which is far less than the cut off criteria of 50\% suggesting that it was not a potential threat in this study (Hair et al., 2009; Podsakoff et al, 2003).

In a third test, a confirmatory factor analysis was conducted and included all the 97 measurement items, the 19 latent constructs, and a $20^{\text {th }}$ latent construct linked to all 97 measurement items. The unstandardized factor loading for a common factor was 0.58 (or 0.336 when squared) for both data sets. Thus, the extracted variance (the measure of common variance between all of the indicator variables) was $33.6 \%$ for both data sets, further indicating that common method variance was not a major problem in this study since this value was less than 0.5 (Hair et al., 2009). This confirms more than $50 \%$ of the variance in the measurement models is due to hypothesized underlying trait rather than the variance due to measurement errors (Fornell \& Larcker, 1981). Table 64 shows reliability and validity 
scores. The common method variance was equal across the two project methodologies because a common method of measurement was used. A similar online survey was used to collect both data sets. The single online survey was structured in the same way and all the questions were constructed in a similar fashion. The way in which questions were asked was also the same as well as the audience (outsourced software development projects) to which they were asked etc. It was merely one of the categorical questions answered by respondents about the project management methodology that was used to distinguish between the two data sets during analysis.

In summary, all these tests were taken to establish the overall discriminant validity of the 19 study constructs suggesting that each construct does represent a separate phenomenon (Anderson \& Gerbing, 1988). Overall reliability and validity measures exceeded the required thresholds and the measurement model was judged acceptable (Hair et al., 2009).

\subsubsection{Structural model}

Results from the final measurement model were used to create the structural model that tested the strength and significance of the theorized relationships. The next section provides the results of the assessment of the structural models using goodness-of-fit indices and briefly explains the results of hypotheses testing.

\subsubsection{Goodness of fit}

Goodness of fit indexes for the initial structural model were unsatisfactory. MIs were used to guide the model fitting where necessary. In most cases the MIs indicated that the overall model fit would improve if some indirect paths were included. These suggested modifications were done one by one and the whole model was run over again each time a structural path was included/excluded. This process went on until the best Goodness of fit indices were obtained.

Table 69 shows goodness-of-fit measures for the final structural models of both traditional plan-based projects and agile methodologies. Based on these values which are satisfactory, the final structural models were deemed acceptable (Bentler \& Bonnett, 1980; Bentler, 1990). 
Table 69: Structural models measures of Goodness-of-fit-Group comparisons

\begin{tabular}{|c|c|c|c|}
\hline Goodness of fit index & $\begin{array}{l}\text { Traditional plan-based } \\
\text { methodology projects, } \\
\text { n=513 }\end{array}$ & $\begin{array}{l}\text { Agile methodology } \\
\text { projects, } n=471\end{array}$ & Heuristics \\
\hline$\chi^{2}$ & 6675.86 & 6626.92 & $\mathrm{p}>0.5$ \\
\hline$\chi^{2 / \mathrm{df}}$ & 2.23 & 2.06 & $<3.00$ \\
\hline TLI & 0.89 & 0.88 & $>.95$ \\
\hline CFI & 0.88 & 0.89 & $>.95$ \\
\hline RMSEA & 0.04 & 0.04 & $<.06$ \\
\hline NFI & 0.90 & 0.91 & $>.95$ \\
\hline PRATIO & 0.91 & 0.93 & $>.90$ \\
\hline GFI & 0.89 & 0.90 & $>.90$ \\
\hline AGFI & 0.89 & 0.89 & $>.90$ \\
\hline
\end{tabular}

\subsubsection{Hypotheses testing results-interpretation of group differences}

Since this thesis largely takes a confirmatory approach rather than an exploratory one, separate competing contingency hypotheses were initially formulated to compare the path differences between the sub-groups. This was based on the general contingency literature that different projects require different project management approaches or methodologies to achieve success. If hypotheses testing findings demonstrate that statistically significant differences between the sub-groups of traditional plan-based and agile methodologies indeed do indeed exist; this implies that the specified model and hypothesized factors in the research model can influence project success differently across the two project types. This conclusion, of course, is highly important for project management since it implies that project practitioners should tailor their methodologies differently for each project characteristics and environment.

\subsubsection{Support for Hypotheses}

Using 513 and 471 observations of both projects that had used traditional plan-based and agile methodologies respectively provided by a global survey of experienced senior software development project managers, survey data supported most of the hypothesised path coefficients shown in Table 70. Some paths did not demonstrate the expected signs. For instance, with the agile data set, the structural path between top level management support and process success was expected to be positive but was negative and significant. Similarly, some structural paths were insignificant. For example in the traditional plan-driven data set, the path between vision and mission and product success was found to not differ significantly from zero. 
Table 70: Hypothesis testing results (only with direct link to project success)-Group comparisons

\begin{tabular}{|c|c|c|c|c|c|c|c|c|c|c|c|}
\hline \multirow{2}{*}{\multicolumn{4}{|c|}{ Hypothesis and the structural path }} & \multicolumn{3}{|c|}{$\begin{array}{c}\begin{array}{c}\text { Traditional plan-based (T) } \\
\text { n=513 }\end{array} \\
\end{array}$} & \multicolumn{3}{|c|}{$\begin{array}{c}\begin{array}{c}\text { Agile methodology data set (A) } \\
\mathrm{n}=471\end{array} \\
\end{array}$} & \multicolumn{2}{|c|}{\begin{tabular}{|c|}
$\begin{array}{c}\text { Comparison hypothesis, TA } \\
\text { (statistical difference test) }\end{array}$ \\
\end{tabular}} \\
\hline & & & & $\begin{array}{c}\begin{array}{c}\text { Hypothesized } \\
\text { relationship }\end{array} \\
\end{array}$ & $\begin{array}{l}\text { Coefficient// } \\
\text { significance }\end{array}$ & Support & $\begin{array}{c}\text { Hypothesized } \\
\text { relationship }\end{array}$ & \begin{tabular}{|l|}
$\begin{array}{l}\text { Coefficient/ } \\
\text { significance }\end{array}$ \\
\end{tabular} & Support & $\begin{array}{c}\begin{array}{c}\text { Hypothesized } \\
\text { relationship }\end{array} \\
\end{array}$ & Support \\
\hline \multirow{2}{*}{$\begin{array}{l}\boldsymbol{H}_{1(i)} \\
\boldsymbol{H}_{1(i i)} \\
\end{array}$} & Top mgt. support & $\rightarrow$ & PS (process) & + & -0.04 & No & + & $-0.16^{* *}$ & $\mathrm{No}^{\mathrm{a}}$ & $\mathrm{A}+>\mathrm{T}$ & $\mathrm{No}^{\mathbf{a}}$ \\
\hline & Top mgt. support & $\rightarrow$ & PS (product) & + & -0.03 & No & + & -0.05 & No & $\mathrm{A}+>\mathrm{T}$ & No \\
\hline \multirow{2}{*}{$\begin{array}{l}\boldsymbol{H}_{2(i)} \\
\boldsymbol{H}_{2(i i)} \\
\end{array}$} & Organizational culture & $\rightarrow$ & PS(process) & \# & 0.02 & Yes & + & $0.06^{*}$ & Yes & $\mathrm{A}+>\mathrm{T}$ & No \\
\hline & Organizational culture & $\rightarrow$ & PS (product) & $\#$ & 0.01 & Yes & + & $0.07^{*}$ & Yes & $\mathrm{A}+>\mathrm{T}$ & No \\
\hline \multirow{2}{*}{$\begin{array}{l}\boldsymbol{H}_{3(i)} \\
\boldsymbol{H}_{3(i i)} \\
\end{array}$} & Planning and controlling & $\rightarrow$ & PS (process) & + & $0.28 * * *$ & Yes & $\#$ & 0.02 & Yes & $\mathrm{T}+>\mathrm{A}$ & Yes \\
\hline & Planning and controlling & $\rightarrow$ & PS (product) & + & 0.05 & No & $\#$ & 0.04 & Yes & $\mathrm{T}+>\mathrm{A}$ & No \\
\hline \multirow{2}{*}{\begin{tabular}{|l}
$H_{4(i)}$ \\
$H_{4(i i)}$ \\
\end{tabular}} & Vision and mission & $\rightarrow$ & PS (process) & + & $0.07 *$ & Yes & $\#$ & -0.02 & Yes & $\mathrm{T}+>\mathrm{A}$ & No \\
\hline & Vision and mission & $\rightarrow$ & PS (product) & + & 0.02 & No & $\#$ & 0.01 & Yes & $\mathrm{T}+>\mathrm{A}$ & No \\
\hline \multirow{2}{*}{$\begin{array}{l}\boldsymbol{H}_{5(i)} \\
\boldsymbol{H}_{5(i i)} \\
\end{array}$} & Change management & $\rightarrow$ & PS (process) & + & $0.06^{*}$ & Yes & + & $0.08 *$ & Yes & $\mathrm{A}+>\mathrm{T}$ & No \\
\hline & Change management & $\rightarrow$ & PS (product) & + & $0.07^{*}$ & Yes & + & $0.21 * * *$ & Yes & $\mathrm{A}+>\mathrm{T}$ & Yes \\
\hline \multirow{2}{*}{$\begin{array}{l}\boldsymbol{H}_{6(i)} \\
\boldsymbol{H}_{6(i i)} \\
\end{array}$} & Leadership characteristics & $\rightarrow$ & PS (process) & + & $0.06^{*}$ & Yes & + & $0.22 * * *$ & Yes & $\mathrm{A}+>\mathrm{T}$ & Yes \\
\hline & Leadership characteristics & $\rightarrow$ & PS (product) & + & 0.05 & No & + & -0.04 & No & $\mathrm{A}+>\mathrm{T}$ & No \\
\hline \multirow{2}{*}{$\begin{array}{l}\boldsymbol{H}_{7(i)} \\
\boldsymbol{H}_{7(i i)}\end{array}$} & Int. proj. communication & $\rightarrow$ & PS (process) & + & $0.08^{*}$ & Yes & + & $0.09 *$ & Yes & $\mathrm{A}+>\mathrm{T}$ & No \\
\hline & Int. proj. communication & $\rightarrow$ & PS (product) & + & $0.06^{*}$ & Yes & + & $0.07 *$ & Yes & $\mathrm{A}+>\mathrm{T}$ & No \\
\hline \multirow{2}{*}{$\begin{array}{l}\boldsymbol{H}_{8(i)} \\
\boldsymbol{H}_{8(i i)} \\
\end{array}$} & Proj. team commitment & $\rightarrow$ & PS (process) & + & $0.08^{*}$ & Yes & + & $0.09 *$ & Yes & $\mathrm{A}+>\mathrm{T}$ & No \\
\hline & Proj. team commitment & $\rightarrow$ & PS (product) & + & $0.07^{*}$ & Yes & + & $0.08^{*}$ & Yes & $\mathrm{A}+>\mathrm{T}$ & No \\
\hline \multirow{2}{*}{$\begin{array}{l}\boldsymbol{H}_{\boldsymbol{9}_{(i)}} \\
\boldsymbol{H}_{\boldsymbol{9}_{(i i)}} \\
\end{array}$} & Devt. team expertise & $\rightarrow$ & PS (process) & + & $0.07 *$ & Yes & + & $0.09 *$ & Yes & $\mathrm{A}+>\mathrm{T}$ & No \\
\hline & Devt. team expertise & $\rightarrow$ & PS (product & + & 0.02 & No & + & $0.06^{*}$ & Yes & $\mathrm{A}+>\mathrm{T}$ & No \\
\hline \multirow{2}{*}{$\begin{array}{l}H_{10(i)} \\
H_{10(i i)}\end{array}$} & Proj. team composition & $\rightarrow$ & PS (process) & + & $0.07 *$ & Yes & + & $0.25 * * *$ & Yes & $\mathrm{A}+>\mathrm{T}$ & Yes \\
\hline & Proj. team composition & $\rightarrow$ & PS (product) & + & $0.08^{*}$ & Yes & + & $0.26 * * *$ & Yes & $\mathrm{A}+>\mathrm{T}$ & Yes \\
\hline \multirow{2}{*}{\begin{tabular}{|l|}
$H_{11(i)}$ \\
$H_{11(i i)}$ \\
\end{tabular}} & User participation & $\rightarrow$ & PS (process) & $\#$ & 0.02 & Yes & + & $0.28 * * *$ & Yes & $\mathrm{A}+>\mathrm{T}$ & Yes \\
\hline & User participation & $\rightarrow$ & PS (product) & $\#$ & 0.05 & Yes & + & $-0.14^{* * *}$ & $\mathrm{No}^{\mathrm{a}}$ & $\mathrm{A}+>\mathrm{T}$ & $\mathrm{No}^{\mathrm{a}}$ \\
\hline \multirow{2}{*}{$\begin{array}{l}H_{12(i)} \\
H_{12(i i)} \\
\end{array}$} & User support & $\rightarrow$ & PS (process) & $\#$ & 0.03 & Yes & + & $0.06^{*}$ & Yes & $\mathrm{A}+>\mathrm{T}$ & No \\
\hline & User support & $\rightarrow$ & PS (product) & $\#$ & 0.02 & Yes & + & $0.18 * * *$ & Yes & $\mathrm{A}+>\mathrm{T}$ & Yes \\
\hline \multirow{2}{*}{$\begin{array}{l}H_{13(i)} \\
H_{13(i i)} \\
\end{array}$} & User experience & $\rightarrow$ & PS (process) & $\#$ & 0.03 & Yes & + & $0.07 *$ & Yes & $\mathrm{A}+>\mathrm{T}$ & No \\
\hline & User experience & $\rightarrow$ & PS (product) & $\#$ & 0.02 & Yes & + & $0.06^{*}$ & Yes & $\mathrm{A}+>\mathrm{T}$ & No \\
\hline \multirow{2}{*}{$\begin{array}{l}H_{14(i)} \\
H_{14(i i)} \\
\end{array}$} & Technological uncertainty & $\rightarrow$ & PS (process) & - & $-0.24 * * *$ & Yes & - & $-0.09 *$ & Yes & $\mathrm{T}->\mathrm{A}$ & Yes \\
\hline & Technological uncertainty & $\rightarrow$ & PS (product) & - & $-0.26 * * *$ & Yes & - & $-0.06^{*}$ & Yes & T->A & Yes \\
\hline \multirow{2}{*}{$\begin{array}{l}H_{15(i)} \\
H_{15(i i)}\end{array}$} & Technical complexity & $\rightarrow$ & PS (process) & - & -0.02 & No & - & $-0.17 * *$ & Yes & $\mathrm{A}->\mathrm{T}$ & $\mathrm{No}^{\mathrm{a}}$ \\
\hline & Technical complexity & $\rightarrow$ & PS (product) & - & $-0.14 * *$ & Yes & - & 0.05 & No & $\mathrm{A}->\mathrm{T}$ & $\mathrm{No}^{\mathrm{a}}$ \\
\hline \multirow{2}{*}{$\begin{array}{l}H_{16(i)} \\
H_{16(i i)}\end{array}$} & Relative project size & $\rightarrow$ & PS (process) & - & $-0.07 *$ & Yes & - & $-0.25 * * *$ & Yes & $\mathrm{A}->\mathrm{T}$ & Yes \\
\hline & Relative project size & $\rightarrow$ & PS (product) & - & -0.05 & No & - & 0.04 & No & $\mathrm{A}->\mathrm{T}$ & No \\
\hline \multirow{2}{*}{$\begin{array}{l}H_{17(i)} \\
H_{17(i i)} \\
\end{array}$} & Specification changes & $\rightarrow$ & PS (process) & - & $-0.14 *$ & Yes & - & $-0.08^{*}$ & Yes & T->A & No \\
\hline & Specification changes & $\rightarrow$ & PS (product) & - & $0.22 * * *$ & $\mathrm{No}^{\mathrm{a}}$ & - & $0.06^{*}$ & $\mathrm{No}^{\mathrm{a}}$ & $\mathrm{T}->\mathrm{A}$ & $\mathrm{No}^{\mathrm{a}}$ \\
\hline \multirow{2}{*}{$\begin{array}{l}H_{18(i)} \\
H_{18(i i)}\end{array}$} & Project criticality & $\rightarrow$ & PS (process) & - & $\mathrm{x}$ & $\mathrm{x}$ & - & $x$ & $\mathrm{x}$ & $\mathrm{A}->\mathrm{T}$ & $\mathrm{x}$ \\
\hline & Project criticality & $\rightarrow$ & PS (product) & - & $\mathrm{x}$ & $\mathrm{x}$ & - & $\mathrm{x}$ & $\mathrm{x}$ & $\mathrm{A}->\mathrm{T}$ & $\mathrm{x}$ \\
\hline
\end{tabular}

When examining the data set of the projects that used traditional plan-based methodology, there is an insignificant association between top level management support and process success $(\beta=-0.04, p>.05)$ and product success $(\beta=-.03, p>.05)$ respectively, not supporting both $H 1(T)(i)$ and $H 1(T)(i i)$ respectively. On the other hand, for the projects that used agile 
methodology, the association between top level management support and process success $(\beta=-.16, p<.05)$ had a significant but negative path coefficient, but is insignificantly associated with product success $(\beta=-.05, p>.05)$, not supporting both $H 1(A)(i)$ and $H 1(A)(i i)$ respectively. Thus, failing to support $H 1(T A)$.

For traditional plan-based methodology data set there are insignificant path association between organizational culture and process success $(\beta=.02, p>.05)$ and product success $(\beta=.01, p>.05)$ respectively, supporting both $H 2(T)(i)$ and $H 2(T)(i i)$ respectively. For the agile methodology data set there are significant path coefficients association between organizational culture and process success $(\beta=.06, p<.05)$ and product success $(\beta=.07$, $p<.05)$ respectively, supporting both $H 2(A)(i)$ and $H 2(A)(i i)$ respectively. However, the contribution of organizational culture is not statistically greater for agile than for traditional plan-based methodologies, failing to support $H 2$ (TA).

As initially hypothesized, for traditional plan-based methodology projects, there is a positive and significant association between planning and controlling and process success $(\beta=.28$, $p<.001)$ and an insignificant path coefficient with product success $(\beta=.05, p>.05)$ respectively, supporting $H 3(T)(i)$ but rejecting $H 3(T)(i i)$ respectively. On the other hand, for the agile methodology projects data set, the association between planning and controlling and process success $(\beta=.02, p>.05)$ and product success $(\beta=.04, p>.05)$ respectively had insignificant positive path coefficients, supporting both $H 3(A)(i)$ and $H 3(A)(i i)$ respectively. Partially supporting $H 3(T A)$.

The direct structural path between vision and mission and process success $(\beta=0.07, p<.05)$ was significant providing support for $H 4(T)(i)$, while the structural path between vision and mission and product success was insignificant $(\beta=.02, p>.05)$, failing to provide support for $H 4(T)(i i)$ in the traditional plan-driven methodology projects model. The agile methodology projects model, had insignificant direct structural paths between vision and mission and process success $(\beta=-.02, p>.05)$ and product success $(\beta=.01, p>.05)$ respectively, thus, providing support for $H 4(A)(i)$ and $H 4(A)(i i)$ respectively. The contribution of vision and mission is not statistically greater for agile than for traditional plan-based methodologies, failing to support $H 4(T A)$.

For traditional plan-based methodology projects data set, there are significant positive path associations between change management and process success $(\beta=.06, p<.05)$ and product 
success $(\beta=.07, p<.05)$ respectively, providing support for both $H 5(T)(i)$ and $H 5 b(T)(i i)$ respectively. On the hand, there were significant positive relationships between change management and process success $(\beta=.08, p<.05)$ and product success $(\beta=.21, p>.001)$ respectively for agile methodology projects, supporting both $H 5(A)(i)$ and $H 5(A)(i i)$ respectively, partially supporting $H 5$ (TA).

The construct of leadership characteristics had a direct positive and significant association with process success $(\beta=.06, p<.05)$ and an insignificant association with product success $(\beta=.05, p>.05)$ respectively, supporting $H 6(T)(i)$ but rejecting $H 6(T)(i i)$ respectively. For the agile methodology projects, leadership characteristics had a direct positive and significant association with process success $(\beta=.22, p<.001)$ and an insignificant association with product success $(\beta=-.04, p>.05)$ respectively, supporting $H 6(A)(i)$ but not $H 6(A)(i i)$ respectively. Partially supporting $H 6(T A)$.

Further, the structural paths between internal project communication and process success $(\beta=.08, p<.05)$ and product success $(\beta=.06, p<.05)$ respectively had significant positive associations for the traditional plan-driven methodology projects, providing support for both $H 7(T)(i)$ and $H 7(T)(i i)$ respectively. Likewise, the structural paths between internal project communication and process success $(\beta=.09, p<.05)$ and product success $(\beta=.07, p<.05)$ respectively had significant positive associations for the agile methodology data set, supporting both $H 7(A)(i)$ and $H 7(A)(i i)$ respectively. Nonetheless, the contribution of internal project communication is not statistically greater for agile than for traditional plan-based methodologies, failing to support $H 7$ (TA).

There are significant positive relationships between project team commitment and process success $(\beta=.08, p<.05)$ and product success $(\beta=.07, p<.05)$ respectively for traditional plandriven methodology projects, providing support for both $H 8(T)(i)$ and $H 8(T)(i i)$ respectively. Similarly, there are significant positive relationships between project team commitment and process success $(\beta=.09, p<.05)$ and product success $(\beta=.08, p<.05)$ respectively for agile methodology projects, supporting both $H 8(A)(i)$ and $H 8(A)(i i)$ respectively. Nevertheless, the contribution of project team commitment is not statistically greater for agile than for traditional plan-based methodologies, failing to support $H 8(T A)$.

There is a significant positive relationships between development team expertise and process success $(\beta=.07, p<.05)$ but insignificant association with product success $(\beta=.02, p>.05)$ 
respectively for traditional plan-driven methodology projects, supporting $H 9(T)(i)$ but not $H 9(T)(i i)$ respectively. There are significant positive relationships between team expertise and process success $(\beta=.09, p<.05)$ and project success $(\beta=.06, p<.05)$ respectively for agile methodology projects, providing support for both $H 9(A)(i)$ and $H 9(A)(i i)$ respectively. However, the contribution of development team expertise is not statistically greater for agile than for traditional plan-based methodologies, failing to support $H 9$ (TA).

The traditional plan-driven methodology projects data set suggests that there are significant positive relationships between team composition and process success $(\beta=.07, p<.05)$ and product success $(\beta=.08, p<.05)$ respectively, supporting both $H 10(T)(i)$ and $H 10(T)(i i)$ respectively. The agile methodology projects data set suggests that there are significant positive relationships between team composition and process success $(\beta=.25, p<.001)$ and product success $(\beta=.26, p<.001)$ respectively, supporting both $H 1 O(A)(i)$ and $H 1 O(A)(i i)$ respectively. Thus, providing support for $H 10$ (TA).

Additionally, the traditional plan-driven methodology projects data set indicates that there are no significant positive relationships between user participation and process success $(\beta=.02$, $p>.05)$ and product success $(\beta=.05, p>.05)$ respectively, supporting both $H 11(T)(i)$ and $H 11(T)(i i)$ respectively. In contrast, however, the agile methodology projects data set suggests that there are significant positive relationships between user participation and process success $(\beta=.28, p<.001)$ and a negative association with product success $(\beta=-.14$, $p<.001)$ respectively, supporting $H 11(A)(i)$ but not $H 11(A)(i i)$ respectively. Thus, partially supporting $H 11$ (TA).

The traditional plan-driven methodology projects model indicates that there are no significant positive relationships between user support and process success $(\beta=.03, p>.05)$ and product success $(\beta=.02, p>.05)$ respectively, supporting both $H 12(T)(i)$ and $H 12(T)(i i)$ respectively. In contrast, however, the agile methodology projects data set suggests there is a significant positive relationship between user support and process success $(\beta=.06, p>.05)$ and product success $(\beta=.18, p<.001)$ respectively, providing support for both $H 12(A)(i)$ and H12(A)(ii) respectively. Partially supporting H12 (TA).

There are no significant relationship between user experience and process success $(\beta=.03$, $p>.05)$ and product success $(\beta=.02, p>.05)$ respectively for traditional plan-driven methodology projects, providing support for both $H 13(T)(i)$ and $H 13(T)(i i)$ respectively. For 
agile methodology projects, there are significant positive relationships between user experience and process success $(\beta=.07, p<.05)$ and product success $(\beta=.06, p<.05)$ respectively, supporting both $H 13(A)(i)$ and $H 13(A)(i i)$ respectively. However, the contribution of user experience is not statistically greater for agile than for traditional planbased methodologies, failing to support $H 13$ (TA).

Technological uncertainty had a negative significant direct association with process success $(\beta=-.24, p<.001)$ and product success $(\beta=-.26, p<.001)$ respectively for traditional plandriven methodology projects, supporting both $H 14(T)(i)$ and $H 14(T)(i i)$ respectively. On the other hand, for the agile methodology projects model, technological uncertainty had a direct negative and significant association with process success $(\beta=-.09, p<.05)$ and product success $(\beta=-.06, p<.05)$ respectively, supporting $H 14(A)(i)$ and $H 14(A)(i i)$ respectively. Thus, supporting H14 (TA).

Technical complexity had an insignificant direct association with process success $(\beta=-.02$, $p>.05)$ and a negative significant association with product success $(\beta=-.14, p<.01)$ respectively for traditional plan-driven methodology projects, not supporting $H 15(T)(i)$ but providing support for $H 15(T)(i)$ respectively. For the agile methodology projects model, technical complexity had a direct negative and significant association with process success $(\beta=-.17, p<.01)$ and an insignificant association with product success $(\beta=.05, p>.05)$ respectively, supporting $H 15(A)(i)$ but rejecting $H 15(T)(i i)$ respectively. Partially supporting $H 15$ (TA).

The traditional plan-driven methodology projects data set suggests that there is a significant negative relationship between relative project size and process success $(\beta=-.07, p<.05)$ but insignificant association with product success $(\beta=-.05, p>.05)$ respectively, providing support for $H 16(T)(i)$ but not $H 16(T)(i i)$ respectively. Equally, the agile methodology projects data set suggests that there is a strong and significant negative relationships between relative project size and process success $(\beta=-.25, p<.001)$ but an insignificant association with product success $(\beta=.04, p>.05)$ respectively, supporting $H 16(A)(i)$ but not $H 16(A)(i i)$ respectively. Partially supporting H16 (TA).

Finally, the traditional plan-driven methodology projects model suggests there is a negative significant association between specification changes and process success $(\beta=-.14, p<.05)$ and a significant positive association with product success $(\beta=.22, p<.001)$ respectively, 
supporting $H 17(T)(i)$ but not $H 17(T)(i i)$ respectively. For the agile methodology projects model, specification changes had a negative significant association with process success $(\beta=-$ $.08, p<.05)$ but a positive significant association with product success $(\beta=.06, p>.05)$ respectively, providing support for $H 17(A)(i i)$ but not $H 17(A)(i i)$ respectively. Partially supporting $H 17$ (TA).

\section{Indirect paths}

There were other interesting findings that came out of multivariate data analysis as well as during the process of collecting the data. Though these findings were outside the scope of study objectives, it is imperative that some of these findings are briefly mentioned and discussed since they are part of the research model. However, these unanticipated findings should be interpreted with caution since they may not be exclusively based on theory and there could be some likelihood that they are based on statistical chance.

Although the survey data does not support some hypothesized direct structural relationships with both process and product successes, many CSFs have indirect effects on project success for both project types. Though not initially hypothesized, some indirect structural paths between CSFs themselves in both models were tested and found to be significant. This indicates that CSFs also interrelate amongst themselves to impact on project success as summarized in Table 71. 
Table 71: Indirect structural paths findings

\begin{tabular}{|c|c|c|c|c|}
\hline \multicolumn{3}{|c|}{ Structural path } & \multirow{2}{*}{$\begin{array}{c}\begin{array}{c}\text { Traditional plan-based } \\
\text { methodology }(\mathbf{T}), \mathbf{n}=\mathbf{5 1 3}\end{array} \\
0.24 * * *\end{array}$} & \multirow{2}{*}{$\begin{array}{c}\begin{array}{c}\text { Agile methodology } \\
\text { data set }(\mathbf{A}), \mathbf{n}=\mathbf{4 7 1}\end{array} \\
0.15^{* *}\end{array}$} \\
\hline Top mgt. support & $\rightarrow$ & Int. proj. communication & & \\
\hline Top mgt. support & $\rightarrow$ & Proj. team commitment & $0.16^{* *}$ & $0.14^{*}$ \\
\hline Int. proj. communication & $\rightarrow$ & Proj. team commitment & $0.18 * *$ & $0.32 * * *$ \\
\hline Vision and mission & $\rightarrow$ & Organizational culture & $0.10^{*}$ & 0.06 \\
\hline Team composition & $\rightarrow$ & Organizational culture & $0.29 * * *$ & $0.29 * * *$ \\
\hline Top mgt. support & $\rightarrow$ & Organizational culture & $0.19 * * *$ & $0.24 * * *$ \\
\hline Change mgt. & $\rightarrow$ & Organizational culture & $0.26 * * *$ & $0.13 *$ \\
\hline Leadership characteristics & $\rightarrow$ & Organizational culture & 0.07 & $0.28 * * *$ \\
\hline User experience & $\rightarrow$ & Organizational culture & -0.04 & $0.13 * *$ \\
\hline Devt. team expertise & $\rightarrow$ & Organizational culture & -0.03 & $-0.18 * *$ \\
\hline User participation & $\rightarrow$ & Organizational culture & 0.07 & $0.09 *$ \\
\hline Organizational culture & $\rightarrow$ & Int. proj. communication & $0.28 * * *$ & $0.37 * * *$ \\
\hline Team composition & $\rightarrow$ & Int. proj. communication & $0.19 * *$ & $0.39 * * *$ \\
\hline Vision and mission & $\rightarrow$ & Int. proj. communication & -0.06 & $-0.14 *$ \\
\hline Relative project size & $\rightarrow$ & Int. proj. communication & -0.02 & $-0.09 *$ \\
\hline User participation & $\rightarrow$ & Int. proj. communication & $0.29 * * *$ & $0.17 * *$ \\
\hline Change mgt & $\rightarrow$ & Int. proj. communication & -0.08 & 0.07 \\
\hline Proj. team composition & $\rightarrow$ & Proj. team commitment & $0.46 * * *$ & $0.24 * *$ \\
\hline Leadership characteristics & $\rightarrow$ & Proj. team commitment & -0.05 & $0.30 * * *$ \\
\hline User participation & $\rightarrow$ & Proj. team commitment & -0.02 & 0.10 \\
\hline Specification changes & $\rightarrow$ & Proj. team commitment & $0.18^{*}$ & $0.13^{*}$ \\
\hline Technical complexity & $\rightarrow$ & Proj. team commitment & $0.12 *$ & $0.10 *$ \\
\hline Team composition & $\rightarrow$ & Planning and controlling & 0.06 & $0.18 *$ \\
\hline Vision and mission & $\rightarrow$ & Planning and controlling & $0.19 * * *$ & $0.24 * * *$ \\
\hline Change magt & $\rightarrow$ & Planning and controlling & $0.18 * *$ & $0.33 * * *$ \\
\hline User participation & $\rightarrow$ & Planning and controlling & 0.06 & $0.16^{* *}$ \\
\hline Specification changes & $\rightarrow$ & Planning and controlling & $-0.13 * *$ & $-0.24 * * *$ \\
\hline Int. proj. communication & $\rightarrow$ & Planning and controlling & $0.42 * * *$ & $0.27 * * *$ \\
\hline Organizational culture & $\rightarrow$ & Planning and controlling & -0.09 & $-0.19 * *$ \\
\hline Proj. team commitment & $\rightarrow$ & Planning and controlling & 0.11 & $-0.14 *$ \\
\hline PS (process) & $\rightarrow$ & PS (product) & $0.59 * * *$ & $0.58 * * *$ \\
\hline$* S i$ & icant & at $p<0.05, * *$ significant at & $n d * * *$ significant at $p<0.0$ & \\
\hline
\end{tabular}

For instance, as indicated there is a significant positive relationship between top management support and internal project communication $(\beta=.24, p<.001)$ and project team commitment $(\beta=.16, p<.01)$ respectively for traditional plan-driven methodology projects. Similarly, there are significant positive relationships between top management support and internal project communication $(\beta=.15, p<.01)$ and a negative association with project team commitment $(\beta=-.14, p<.05)$ respectively for agile methodology projects. There is a significant positive relationship between internal project communication and project team commitment $(\beta=.18$, $p<.01, \beta=.32, p<.001)$ for both traditional plan-driven and agile methodology projects respectively. While there is a positive significant association between vision and mission and organizational culture $(\beta=.10, p<.05)$ for traditional plan-driven methodology projects, there 
is an insignificant association between vision and mission and organizational culture $(\beta=.06$, $p>.05$ ) for agile methodology projects.

\subsubsection{Total variance explained by the final structural models}

The overall predictive power and validity of both final structural models was indicated by the high $\mathrm{R}^{2}$ values of the endogenous latent variables (Joreskog \& Sorbom, 1989; Anderson \& Gerbing, 1988). Both the traditional plan-driven and agile methodology projects' structural models fitted the data well and were very successful at explaining a large variance in both data sets. More than $58 \%$ of variance was explained in organizational culture in traditional plan-based methodology projects while more than $64 \%$ of the variance in organizational culture was explained in the agile methodology projects data set. Further, whereas more than $49 \%$ of the variance in internal project communication was explained in the traditional planbased methodology projects, $61 \%$ of the variance in internal project communication was explained in the data set collected from projects that used an agile methodology. Project team commitment explained more than $46 \%$ of the variation in the traditional plan-based methodology projects; it also explained more than $53 \%$ of the variation in the agile methodology project's model. Additionally, while planning and controlling explained more than $62 \%$ of the variation in the traditional plan-based methodology projects, it also explained more than $53 \%$ of the variation in the agile methodology projects data set. Lastly, more than $46 \%$ and $53 \%$ of variance in process project success for both traditional plan-based and agile methodology projects respectively was accounted for by the research models. Similarly, more than $68 \%$ and $69 \%$ of the variance in product project success for both traditional plan-based and agile methodology projects respectively was accounted for by the research models. Table 72 displays the $\mathrm{R}^{2}$ values (total variances) explained by the two respective final structural models and the respective endogenous variables.

Table 72: $\mathbf{R}^{2}$ values (variance explained)-Group comparisons

\begin{tabular}{|l|c|c|}
\hline Endogenous variable & $\begin{array}{c}\text { Traditional plan based } \\
\text { methodology, } \mathbf{n = 5 1 3}\end{array}$ & $\begin{array}{c}\text { Agile methodology projects, } \\
\mathbf{n = 4 7 1}\end{array}$ \\
\hline Organizational culture & .58 & .64 \\
\hline Internal project communication & .49 & .61 \\
\hline Project team commitment & .46 & .53 \\
\hline Planning and controlling & .62 & .53 \\
\hline PS process & .46 & .53 \\
\hline PS product & .68 & .69 \\
\hline
\end{tabular}




\section{Why a multi-level analysis has not been used?}

It should be noted that although the constructs being used in the thesis are at various levels organizational, project and team, a multi-level analysis has not been used. While multilevel analysis can be used whenever the data is grouped (or nested) in more than one category; there was no substantive interest in multi-level analysis, particularly in this study. Like in many situations the research questions guide the study and subsequently influence the research methodology, configuration and data analysis. Based on the research questions of this study and the purpose of this study there was no substantive interest in adopting multilevel analysis. Instead, since one of the primary objectives of the study was to test for differences across project methodologies implemented and moderation, group invariance analysis was used. Group invariance analysis is a SEM based framework for testing any differences between similar models estimated for different groups of respondents (Hair et al. 2010, p.744). This helped to answer the research questions unlike if multi-level analysis was used.

\subsection{Group invariance testing (group comparisons) using SEM}

Group invariance analysis is a SEM framework for testing any number or types of differences between similar models estimated for different groups of respondents (Hair et al. 2010, p.744). The general objective is to see if there are any significant differences between individual group models by comparing the same model across different samples of respondents. Although very specific tests of differences can be performed for unique research questions, a general framework has emerged for comparing the measurement models and then the structural models across the groups. Byrne $(2001,2009)$ categorizes invariance tests as tests of measurement and structural invariance. Measurement invariance is concerned with tests of relationships between indicators and their latent variables (e.g. equivalent factor loadings) while structural invariance concerns aspects between the latent constructs only (e.g. structural coefficients). This is consistent with the distinction made by Anderson and Gerbing (1988) that measurement invariance models assess the invariances between the construct and its measurement items e.g., factor loadings, item intercepts and error variances while the structural invariance models assess equality of the structural path coefficients between the latent variables.

As a minimum requirement, the relationship between latent variables and their indicators should be identical across the groups if any meaningful group comparisons are to be made 
(Cunningham, 2010). While different researchers in multi-group invariance literature appear to use different terminologies to represent essentially the same concepts, there is a general consensus that the process for establishing multi-group invariance/equivalence progresses in a hierarchical manner in which an initial omnibus test of equivalence of covariances between groups is conducted in order to determine whether any further invariance tests are warranted.

\subsubsection{General procedure of invariance testing in SEM}

The current general procedure in SEM capable of testing for invariance simultaneously across groups derives from the seminal works of Joreskog (1971). Accordingly, Joreskog (1971) recommended that all tests of invariance begin with a global test of the equality of covariance structures across groups. In other words, one tests the hypothesis $\left(\mathrm{H}_{0}\right)$ that $\sum_{1}=\sum_{2}=\ldots=\sum_{\mathrm{G}}$, where $\sum$ is the population variance-covariance matrix, and G number of groups. Rejection of the null hypothesis implies the non-equivalence of the groups and, thus, for the subsequent testing of increasingly restrictive hypotheses in order to identify the source of non-invariance. In contrast, if $\mathrm{H}_{0}$ cannot be rejected, the groups are considered to be equivalent and thus test for invariance are unjustified; group data should be pooled together and all the subsequent investigative work should be based on a single group analysis (Byrne, 2001).

In the Joreskog (1971) tradition, tests of hypotheses related to group invariance typically begin with the scrutiny of the measurement for the pattern of factor loadings for each observed measure that is tested for its equivalence across each group. Once it is known which measures are group invariant, these parameters are constrained equal while subsequent tests of the structural parameters are conducted (Byrne, 2009). As each new set of parameters is tested, those known to be group invariant are constrained equal. Thus, the idea of invariance testing is to progressively test increasingly constrained models that are nested in previous estimated less constrained models.

The fundamental measure of difference used is the $\chi^{2}$ difference (Joreskog, 1971). This measure allows for an overall comparison between two model specifications (i.e. one with parameter constrained and one without). Because the models are nested within one another, the difference in chi-square $(\Delta \chi 2)$ between the nested models is also distributed as a chisquare distribution with degrees of freedom equal to the difference in degrees of freedom $(\Delta d f)$ of the models. If the $\Delta \chi^{2}$ test is not significant, the more constrained models in which more parameter are set to equality is acceptable as the more parsimonious model compared to the less restricted model nested model (Cunningham, 2010). The basic logic is that if a set of 
constraints is applied and the model fit (as measured by $\chi^{2}$ ) does not show a significant increase (meaning worse fit) from a less constrained model, then the constraints can be accepted (Byrne, 2001, 2009).

General practice is to start with the most unconstrained model (i.e., a separate and unique CFA model is estimated for each group). Then between groups constraints are added to reflect specific measurement model comparisons. A between-group constraint estimates a single parameter for the relationship rather than the estimating unique parameter for each group (Hair et al. 2010). Thus, it represents the hypothesis that the relationship being tested is invariant across the groups. If imposing these constraints does not significantly increase model fit, then it can be accepted and assume invariance for that relationship in the measurement model.

Recent research has converged to identify a systematic framework for evaluating all of these aspects in a progressively more rigorous set of comparisons that addresses the most elemental aspects in the earlier stages (e.g. Byrne, 2001, 2009; Hair et al., 2010; Milfont \& Fischer, 2010; Cunningham, 2010). The following six hierarchical stages describe what appears to be a systematic framework for multi-group invariance testing in SEM in terms of model issues addressed as well as the nature of constraints used. The framework is subdivided into measurement and structural invariance.

\subsubsection{Tests of measurement invariance}

Models that test relationships between measured variables and latent variables are measurement invariance tests (Milfont \& Fischer, 2010).

Table 73 shows a summary of the hierarchy and descriptions of the six stage process of measurement invariance testing. 
Table 73: Hierarchy and descriptions of the six stage process of measurement invariance testing

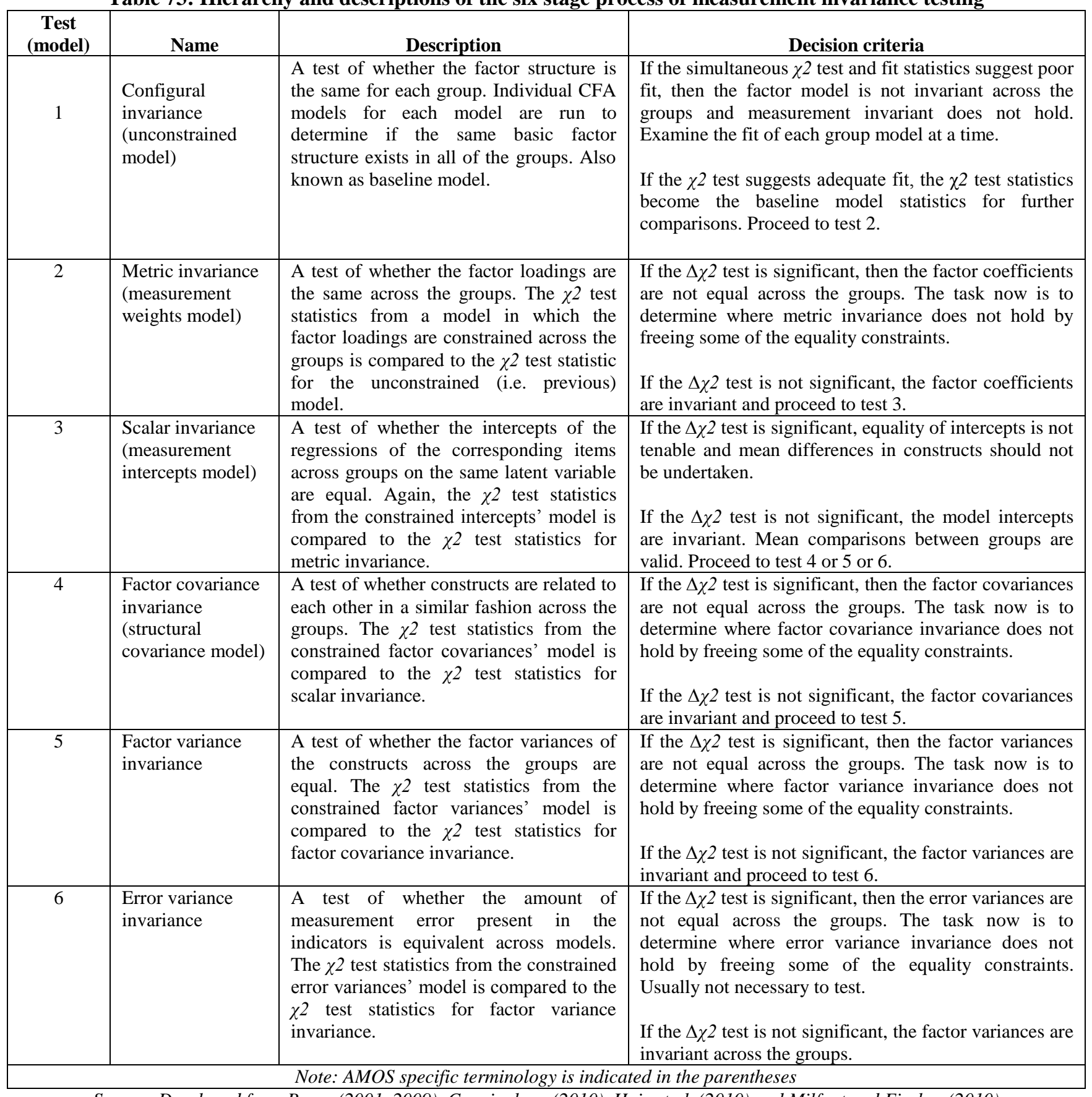

Source: Developed from Byrne (2001, 2009), Cunningham (2010), Hair et al. (2010) and Milfont and Fischer (2010)

5.5.2.1. Configural invariance (Model 1): This is the first stage that confirms that same basic factor structure exists in all of the groups, indicating that respondents from different groups conceptualize the constructs in the same way (Milfont \& Fischer, 2010). Configural invariance is tested by running individual CFAs in each group. Researchers confirm that each group CFA model has the same number of constructs and items associated with each 
construct. It must also be shown that each model meets the appropriate levels of model fit and construct validity (Hair et al. 2010).

A combined baseline model called Multi-group Confirmatory Factor Analysis (MCFA) or Totally Free $(T F)$ model representing both models must be ran to serve as a standard for subsequent tests (Byrne, 2001). The $\chi^{2}$ for the entire group models are added together and measures such as TLI, CFI, RMSEA and others are calculated for the entire set. In this way, comparisons can be made on model fit measures (e.g. the $\Delta \chi^{2}$ ) across the multi-group models with differing sets of constraints (Hair et al., 2010). This model is tested by constraining the factorial structure to be the same across the groups.

5.5.2.2. Metric invariance (Model 2): This provides the empirical comparison of factor loadings between MCFA models and involves the equivalence of factor loadings (Hair et al., 2010). Metric invariance establishes the equivalence of the relationships between latent constructs and their indicators. Constraints are set so that factor loadings are equal across the groups. This model tests if different groups respond to the items in the same way; that is, if the strengths of the relations between specific scale items and their underlying construct are the same across the groups (Milfont \& Fischer, 2010). While the loadings are equal for each indicator across groups, each measured variable has its own unique loading estimate. The $\Delta \chi 2$ is computed between this model and the baseline model with degrees of freedom equalling the number of constrained loading estimates across the groups (Hair et al., 2010).

Research seems to suggest that at least partial metric invariance must be established before proceeding to the next step (e.g. Byrne, 2009; Hair et al., 2010; Milfont \& Fischer, 2010). Partial invariance is a less conservative standard involving at least multiple estimates per construct to be equivalent across groups (Hair et al., 2010). A general consensus has developed that if two parameters per construct (e.g. loadings, intercepts or even error variances) are found to be invariant, then partial invariance is found and the process can proceed to the next stage.

5.5.2.3. Scalar invariance (Model 3): The third stage tests for the equality of the intercepts of the measured variables (i.e. means) on the constructs (Hair et al., 2010). Scalar or intercept invariance is required to compare latent means (Milfont \& Fischer, 2010). Establishing scalar invariance indicates that the observed scores are related to the latent scores on the latent construct, i.e. individuals who have the same score on the latent variable would obtain the 
same score on the observed variable regardless of their group. This model is tested by constraining the intercepts of items to be the same across group (Byrne, 2009).

5.5.2.4. Factor covariance invariance (Model 4): In the fourth stage the model is tested by constraining all the factor covariances to be the same across groups. It tests if constructs are related to each other in a similar fashion across the groups (Hair et al., 2010). The stability of the factor relationships across the groups is assessed and the model implies that all latent variables have same relationships in all groups (Milfont \& Fischer, 2010).

5.5.2.5. Factor variance invariance (Model 5): This model assesses the equality of variances of the constructs across the groups. It indicates that the range of scores on a latent factor do not vary across groups (Milfont \& Fischer, 2010). If factor variances and covariances are equivalent across the groups, the latent construct correlations are also equal (Hair et al., 2010). This model is tested by constraining all factor variances to be the same across the groups (Byrne, 2001).

5.5.2.6. Error variance invariance (Model 6): This model tests for the amount of measurement error present in the indicators and the extent to which it is equivalent across models. All error variances are constrained to be equal across the groups (Hair et al., 2010).

In order to test for the significant differences between the traditional and agile methodology projects models, group models were specified based on the previous six stage process discussed and then progressively estimated. First and foremost, when working with analysis of covariance structures that involve multi-group invariance analysis the data related to each group must be made clearly known to the SEM programme (AMOS). This was accomplished by using the raw data files that reside in SPSS version 19 as input files for AMOS Graphics (version 21); where both the name of each group and the location of its data file were communicated to the programme prior to any analyses involving multi-groups invariance. This procedure was executed in AMOS Graphics via the Manage Group dialog box, which in turn, was made available by pulling down the Model-Fit menu and selecting the "Manage Groups" option (Byrne, 2009). By clicking on the "New" in the Manage Groups dialog box, each click yielded the name "Group", along with an assigned number. Subsequently, "Group number 1" was named Traditional plan-based methodology projects while "Group number 2" was named Agile methodology projects. This name change was invoked by simply typing 
over the former name. Once the group names were established, each respective data file for both traditional plan-based and agile methodology projects were identified and attached (Cunningham, 2010).

The rest of the specification of multi-group analysis was majorly guided by several basic default rules. One such default is that all groups in the analysis had an identical path diagram structure (Byrne, 2001). A model structure was only drawn for one group (agile methodology projects) and the other group (traditional plan-driven methodology projects) had the same structure by default. Having specified the name associated with each group together with the related data file, both model parameters were estimated simultaneously across the two groups. The fit statistics of this simultaneously estimated model provided the baseline values against which all subsequently specified models were compared (Hair et al., 2010).

In other words, this multi-group analysis yielded only one set of fit statistics for overall model fit. Given that the $\chi^{2}$ statistics are summative, the overall $\chi^{2}$ value for the multi-group model was equal to the sum of $\chi^{2}$ values obtained when the baseline model is tested separately for each group of projects (with no group constraints imposed). Table 74 shows the summary of model fit statistics for each model and the corresponding $\chi^{2}$ difference test for each model comparisons.

In the first stage of configural invariance, two separate models for traditional plan-based and agile methodology projects both exhibit acceptable levels of models, as does the combined MCFA model $(\chi 2=19601.53, d f=5818, \chi 2 / d f=3.37, p>.05, R M S E A=.04, C F I=.89$. It can be observed that the MCFA $\chi^{2}$ is equal to the sum of the two project management methodologies group models (traditional plan-based and agile) while the other fit measures signify acceptable fit across the two groups thus, indicating configural invariance.

As the configural invariance was supported, the next step was testing for metric invariance and involved constraining each loading to be equal across the groups. It can be observed that $\Delta \chi 2$ is only 163.085 with 61 degrees of freedom, which indicates a non-significant difference ( $p>.05)$. The 61 degrees of freedom represent the 61 free factor loadings that were constrained to be equal to the other group. Note that one parameter was already constrained to 1.00 to set the scale on each construct, thus leaving 61 free parameters across the measured variables (i.e. 80 observed variables minus 19 parameter constraints). Thus, the two models exhibit full metric invariance. 
The next step tested for scalar invariance. The $\Delta \chi 2$ is 400.666 with 80 degrees of freedom. This difference is statistically significant $(p<0.05)$, indicating that full scalar variance is not supported. Since full invariance could not be achieved, at least partial invariance was conducted to be able to continue to the next stage. Modification indices for the scalar invariance model were examined to identify the constraints of item intercepts that could be freed to most reduce the $\Delta \chi^{2}$ difference. As a result, most items (per construct) were identified to retain their constraints in a test of partial scalar invariance. A model constraining each of these parameters to be equal to one another in each group produced a $\chi^{2}$ difference of only $896.865(d f=05)$ from the metric invariance model, which is insignificant $(p>.05)$. Thus, partial scalar invariance was supported. Thus, comparisons between construct means are possible.

The next stage tested for factor covariance invariance, and the $\Delta \chi^{2}$ of 711.99 with 190 degrees of freedom is insignificant $(p>.05)$. From these results there is supporting evidence for invariance of factor covariances among matching constructs. The next test for factor variances shows goodness of fit statistics for the variance constraint $(\Delta \chi 2=711.987, d f=190)$, indicating that the factor variances are almost identical between the two models. The final measurement invariance test was for the equivalence of the error terms of the indicators. As expected this test had a significant $\chi^{2}$ difference $(\Delta \chi 2=288.013, d f=80, p<.05)$. If this had been supported, then equal reliabilities (e.g. AVE, CSR and $\alpha$-coefficients) would have been found for all constructs in each group in this study. Thus, the error variances are different across the groups.

Thus, the measurement invariance testing process demonstrated that the 19 latent constructs and their respective observed variables used in the traditional plan-based and agile methodology projects models in this study met the criteria for configural, full metric, partial scalar, covariance and variance invariance. As a result, any form of group comparisons can be made without concern that the differences are due to differing measurement properties between the groups. 
Table 74: A summary of Goodness-of-fit statistics from measurement invariance tests

\begin{tabular}{|c|c|c|c|c|c|c|c|c|c|c|c|}
\hline \multirow{3}{*}{$\begin{array}{l}\text { Model Tested } \\
\text { Separately tested groups models }\end{array}$} & \multicolumn{6}{|c|}{ Model fit measures } & \multirow{3}{*}{ Comparison models } & \multicolumn{3}{|c|}{ Model differences } & \multirow[b]{2}{*}{ Decision } \\
\hline & $\chi^{2}$ & $d f$ & $\chi^{2 / d f}$ & $\begin{array}{l}\text { significance } \\
\text { (p-value) }\end{array}$ & RMSEA & CFI & & $\Delta \chi^{2}$ & $\Delta d f$ & significance (p-value) & \\
\hline & & & & & & & & & & & \\
\hline 1. Traditional plan-based $(\mathrm{n}=513)$ & 9478.28 & 2909 & 3.26 & $p>.05$ & .04 & .88 & Not required & $\mathrm{Na}$ & $\mathrm{Na}$ & $\mathrm{Na}$ & Accepted \\
\hline 2.Agile projects $(\mathrm{n}=471)$ & 10123.20 & 2909 & 3.48 & $p>.05$ & .04 & .89 & Not required & $\mathrm{Na}$ & $\mathrm{Na}$ & $\mathrm{Na}$ & Accepted \\
\hline $\begin{array}{l}\text { Model } 1 \\
\text { Configural invariance (unconstrained/baseline model) }\end{array}$ & 19601.53 & 5818 & 3.37 & $p>.05$ & .04 & .89 & Not required & $\mathrm{Na}$ & $\mathrm{Na}$ & $\mathrm{Na}$ & Accepted \\
\hline $\begin{array}{l}\text { Model } 2 \\
\text { Metric invariance (factor loadings constrained equal) }\end{array}$ & 19764.62 & 5879 & 3.36 & $p>.05$ & .042 & .89 & Model 1 vs. model 2 & 163.085 & 61 & $p>.05$ & Accepted \\
\hline $\begin{array}{l}\text { Model } 3 \\
\text { Scalar invariance (intercepts constrained equal) }\end{array}$ & 20165.29 & 5959 & 3.40 & $p>.05$ & .052 & .86 & Model 2 vs. model 3 & 400.666 & 80 & p. $<05$ & Rejected \\
\hline $\begin{array}{l}\text { Model 3a } \\
\text { Partial scalar invariance (some constraints freed) }\end{array}$ & 20661.48 & 5884 & 3.51 & $p>.05$ & .043 & .87 & Model 2 vs. model 3a & 896.865 & 05 & $p>.05$ & Accepted \\
\hline $\begin{array}{l}\text { Model } 4 \\
\text { Factor covariance invariance (covariances constrained equal) }\end{array}$ & 20877.27 & 6149 & 3.40 & $p>.05$ & .043 & .89 & Model 3 vs. model 4 & 711.987 & 190 & $p>.05$ & Accepted \\
\hline Model 5 & & & & & & & Model 4 vs. model 5 & & & & \\
\hline Factor variance invariance (equality constraints of variances) & 20877.27 & 6149 & 3.40 & $p>.05$ & .043 & .88 & & 711.987 & 190 & $p>.05$ & Accepted \\
\hline $\begin{array}{l}\text { Model } 6 \\
\text { Error variance invariance (equality constraints of residuals) }\end{array}$ & 21165.29 & 6229 & 3.40 & $P<.05$ & .043 & .86 & Model 5 vs. model 6 & 288.013 & 80 & p. $<05$ & Rejected \\
\hline Error varlance invanlance (equamty constraints or resiauals) & 21105.29 & 0229 & ${ }^{3.40} \mathrm{Na}=$ & Not applicable & .043 & .80 & & 288.015 & 80 & p. $\times 3$ & Kejected \\
\hline
\end{tabular}




\subsubsection{Tests of structural invariance}

According to Hair et al. (2010) structural model comparisons can provide a test for a number of research hypotheses, but the most common use is the test of moderation. Thus, the process of testing moderation is an extension of multi-group analysis for testing measurement invariance where some form of measurement invariance must be established before examining any differences in structural model parameters.

With the measurement invariances established, this was sufficient to now test for structural invariance (moderation) on the relationships between the 17 latent constructs (i.e. candidate CSFs in the hypothesized research model) and project success (i.e. process and product). At this point, any differences in structural path coefficients cannot be attributed to measurement error but rather to the inherent idiosyncratic structural differences that exist within the two groups. The structural model estimates were then assessed for moderation by a comparison of nested models, much like in measurement invariance testing described earlier. Following the same steps used to specify the two CFA group models to test for measurement invariance; two-group structural models were set up, specified and estimated (Frazier et al., p.120). Next, a combined baseline model (TF structural model) was estimated using an identical structural model in both groups simultaneously. Then nested group model were estimated, the only difference being that the structural parameters were constrained to be equal in both groups (Hair et al., 2010, p.757). The fit statistics and estimates are shown in Table 75.

Table 75: Nested group model comparisons testing for structural invariance

\begin{tabular}{|c|c|c|c|c|c|c|c|c|c|c|c|}
\hline \multirow[b]{2}{*}{ Model } & \multirow[b]{2}{*}{ NPAR } & \multicolumn{7}{|c|}{ Model fit statistics } & \multicolumn{3}{|c|}{ Model difference } \\
\hline & & $\chi^{2}$ & $d f$ & $p$ & $\chi^{2 / d f}$ & TLI & CFI & RMSEA & $\Delta \chi^{2}$ & $\Delta d f$ & $p$ \\
\hline Unconstrained Group model (TF) & 774 & 19784.843 & 5866 & $p>.05$ & 3.37 & .87 & .89 & .042 & - & - & - \\
\hline Measurement weights & 713 & 19945.966 & 5927 & $p>.05$ & 3.36 & .87 & .88 & .042 & 161.123 & 61 & $p>.05$ \\
\hline Measurement intercepts & 633 & 20446.115 & 6007 & $p>.05$ & 3.40 & .86 & .87 & .042 & 661.273 & 141 & $p>.05$ \\
\hline Structural weights & 565 & 20679.614 & 6075 & $p<.001$ & 3.40 & .84 & .86 & .045 & 894.771 & 209 & $P<.05$ \\
\hline Structural covariances/variances & 473 & 20955.498 & 6167 & $p>.05$ & 3.39 & .88 & .89 & .042 & 1170.655 & 301 & $p>.05$ \\
\hline Structural residuals & 467 & 20986.974 & 6173 & $p<.001$ & 3.40 & .85 & .85 & .042 & 1202.131 & 307 & $P<.05$ \\
\hline
\end{tabular}

Both the unconstrained model and constrained structural group models demonstrate reasonably and close acceptable fit indices (TLI, CFI and RMSEA etc.) indicating their overall adequacy. The overall chi-square difference between the unconstrained group model and the structural weights model $(\Delta \chi 2)$ is 894.771 with 209 degrees of freedom. This is significant $(p<.05)$, indicating that constraining the structural path estimates to be equal between groups produces worse fit. 
Since the comparison of the differences between models with a chi-square difference test $\left(\Delta \chi^{2}\right)$ indicates the model fit decreases significantly (i.e. an increase in chi-square) when the estimates are constrained to be equal, the fully constrained structural group model is not supported (Hair et al., 2010). Therefore, the unconstrained (TF) model in which the structural relationships are freely estimated in both groups is fully supported. A statistically significant $\chi 2$ difference between the unconstrained and constrained structural weights models indicates that the structural path coefficients are significantly different across the two groups (Hair et al., 2010, p.757). In other words, the model fit is significantly better when separate structural path coefficients are estimated freely indicating that moderation does indeed exist. These results statistically suggest that project management methodology moderates the relationships between some CSFs and project success.

\subsubsection{Testing for specific moderating effects of project management methodology between candidate CSFs and project success}

The next task was to identify the structural path coefficients that are significantly different across the two groups. To achieve this, some constraints of equality for structural path coefficients were imposed across the two groups each one at a time. A sequence of models that involved constraining each of the remaining structural path parameters to be equal to one another in each group were then estimated until non-significant $\Delta \chi^{2}$ values were produced for the final models. The final results from this hierarchical model testing are shown in Table 76, indicating the respective significance levels of model $\chi^{2}$ differences $\left(\Delta \chi^{2}\right)$ when each path is constrained equal across groups.

When examining the $\chi^{2}$ model differences between the unconstrained and constrained group models, top level management support, planning and controlling, leadership characteristics, team composition, user participation, technological uncertainty, technical complexity and relative project size were found to significantly differ across the two project methodologies, based on their structural paths linked to process success (in this case as the endogenous variable). In contrast, however, when examining the structural paths between the 17 latent constructs with product success and reading the $\chi^{2}$ model differences between the unconstrained and constrained group models; change management, team composition, user participation, user support, technological uncertainty, technical complexity and specification changes were found to significantly differ across the two project methodologies. Thus, these findings statistically indicate that there are significant differences in terms of these structural 
path coefficients of each candidate CSF on process/product project success across the two different project types. Thus as predicted, some relationships hold for one group but not the other group indicating that the two group models differ significantly (Dawson, 2014). In short, the results demonstrate a clear case of moderation (for categorical/non-metric/discrete variable) where the nature of the relationships between candidate CSFs and project success change based on a third variable (Hair et al., 2010), in this case project management methodology. These findings support hypothesis $19\left(H_{19}\right)$.

Table 76: $\chi 2$ difference tests for moderating effects of project management methodology

\begin{tabular}{|c|c|c|c|}
\hline \multicolumn{3}{|c|}{ Structural path constrained equal across groups } & \multirow{2}{*}{$\begin{array}{c}\text { Model } \\
\chi^{2} \text { Difference } \\
\text { Sig. }(p<0.01)\end{array}$} \\
\hline H1(i): Top mgt. support & $\rightarrow$ & PS(process) & \\
\hline H1(ii): Top mgt. support & $\rightarrow$ & PS(product) & Not sig. $(p>0.05)$ \\
\hline H2(i): Organizational culture & $\rightarrow$ & PS(process) & Not sig. $(p>0.05)$ \\
\hline H2(ii): Organizational culture & $\rightarrow$ & PS(product) & Not sig. $(p>0.05)$ \\
\hline H3(i): Planning and controlling & $\rightarrow$ & PS(process) & Sig. $(p<0.001)$ \\
\hline H3(ii): Planning and controlling & $\rightarrow$ & PS(product) & Not sig. $(p>0.05)$ \\
\hline H4(i): Vision and mission & $\rightarrow$ & PS(process) & Not sig. $(p>0.05)$ \\
\hline H4(ii): Vision and mission & $\rightarrow$ & PS(product) & Not sig. $(p>0.05)$ \\
\hline H5(i): Change mgt. & $\rightarrow$ & PS(process) & Not sig. $(p>0.05)$ \\
\hline H5(ii): Change mgt. & $\rightarrow$ & PS(product) & Sig. $(p<0.05)$ \\
\hline H6(i): Leadership characteristics & $\rightarrow$ & PS(process) & Sig. $(p<0.001)$ \\
\hline H6(ii): Leadership characteristics & $\rightarrow$ & PS(product) & Not sig. $(p>0.05)$ \\
\hline H7 (i): Int. proj. communication & $\rightarrow$ & PS(process) & Not sig. $(p>0.05)$ \\
\hline H7 (ii): Int. proj. communication & $\rightarrow$ & PS(product) & Not sig. $(p>0.05)$ \\
\hline H8 (i): Proj. team commitment & $\rightarrow$ & PS(process) & Not sig. $(p>0.05)$ \\
\hline H8 (ii): Proj. team commitment & $\rightarrow$ & PS(product) & Not sig. $(p>0.05)$ \\
\hline H9 (i): Devt. team expertise & $\rightarrow$ & PS(process) & Not sig. $(p>0.05)$ \\
\hline H9 (ii): Devt. team expertise & $\rightarrow$ & PS (product & Not sig. $(p>0.05)$ \\
\hline H10 (i): Proj. team composition & $\rightarrow$ & PS(process) & Sig. $(p<0.001)$ \\
\hline H10 (ii): Proj. team composition & $\rightarrow$ & PS (product) & Sig. $(p<0.001)$ \\
\hline H11(i): User participation & $\rightarrow$ & PS (process) & Sig. $(p<0.001)$ \\
\hline H11(ii): User participation & $\rightarrow$ & PS(product) & Sig. $(p<0.05)$ \\
\hline H12(i): User support & $\rightarrow$ & PS(process) & Not sig. $(p>0.05)$ \\
\hline H12(ii): User support & $\rightarrow$ & PS(product) & Sig. $(p<0.01)$ \\
\hline H13(i): User experience & $\rightarrow$ & PS(process) & Not sig. $(p>0.05)$ \\
\hline H13(ii): User experience & $\rightarrow$ & PS(product) & Not sig. $(p>0.05)$ \\
\hline H14(i): Technological uncertainty & $\rightarrow$ & PS(process) & Sig. $(p<0.001)$ \\
\hline H14(ii): Technological uncertainty & $\rightarrow$ & PS(product) & Sig. $(p<0.001)$ \\
\hline H15(i): Technical complexity & $\rightarrow$ & PS(process) & Sig. $(p<0.01)$ \\
\hline H15(ii): Technical complexity & $\rightarrow$ & PS(product) & Sig. $(p<0.01)$ \\
\hline H16(i): Relative project size & $\rightarrow$ & PS(process) & Sig. $(p<0.001)$ \\
\hline H16(ii): Relative project size & $\rightarrow$ & PS (product) & Not sig. $(p>0.05)$ \\
\hline H17(i): Specification changes & $\rightarrow$ & PS(process) & Not sig. $(p>0.05)$ \\
\hline H17(ii): Specification changes & $\rightarrow$ & PS(product) & Sig. $(p<0.05)$ \\
\hline $\begin{array}{r}\text { Goodness of fit indices for the } u \\
d f=5866, \chi^{2} / d f=3.37, T L I=.87,\end{array}$ & 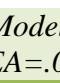 & las baseline $n$ & $\begin{array}{l}\text { parisons were } \chi 2=19784.84, \\
\text { lan-based } n=513 \text {, Agile } n=471\end{array}$ \\
\hline
\end{tabular}

According to Aiken and West (1991), Jose (2013a; 2013b) and Dawson (2014), a relationship between two variables is moderated if (i) the regression lines have different gradients (must not be parallel), and (ii) the effect of one of the variables on the dependent variable differs depending on the level of the other predictor variable (that is, the magnitude of an effect should be significantly greater or less for one group than for another). Jose (2013a; 2013b) 
and Dawson (2014) in line with Aiken and West (1991), further argue that the moderating effects of variables can be appropriately confirmed and interpreted based on the slopes of the plotted moderation graphs. Next, the moderating effects were demonstrated using Dawson's (2014) Stats tool software programme of binary moderators by generating graphs.

From Figure 36, it is evident that the effect of the shown candidate CSFs (i.e. top level management support, planning and controlling, leadership characteristics, team composition, user participation, technological uncertainty, technical complexity and relative project size) on process success differ significantly, depending on the project management methodology used; besides, the graphs have different slopes and hence, are not parallel (Dawson (2014). This truly means that the effect of these candidate CSFs on process success differs as a function of project management methodology (that is, traditional plan-based and agile). Thus, the magnitude of these candidate CSFs on process project success is influenced by the type of project management methodology. Aiken and West (1991) and Jose (2013a) observed that as long as the magnitude of an effect is greater at one level of a variable than at another, it means a significant moderation has occurred. These findings significantly support hypothesis (H19).

To demonstrate further the validity of the conclusion made above (H19) moderation graphs were generated to show how project management methodology moderates the relationships between candidate CSFs and product success. The results are also shown together in Figure 36.

It is clearly illustrated that the effects of some candidate CSFs (i.e. change management, team composition, user participation, user support, technological uncertainty, technical complexity and specification changes) to product success differ significantly depending on the type of the project management methodology, as supported by the graphs which have different gradients. Consistent with the observations of Aiken and West (1991), Jose (2013a) and Dawson (2014), since the magnitude of an effect is different it is sufficient to conclude that moderation has occurred. These findings further support $H 19$. 


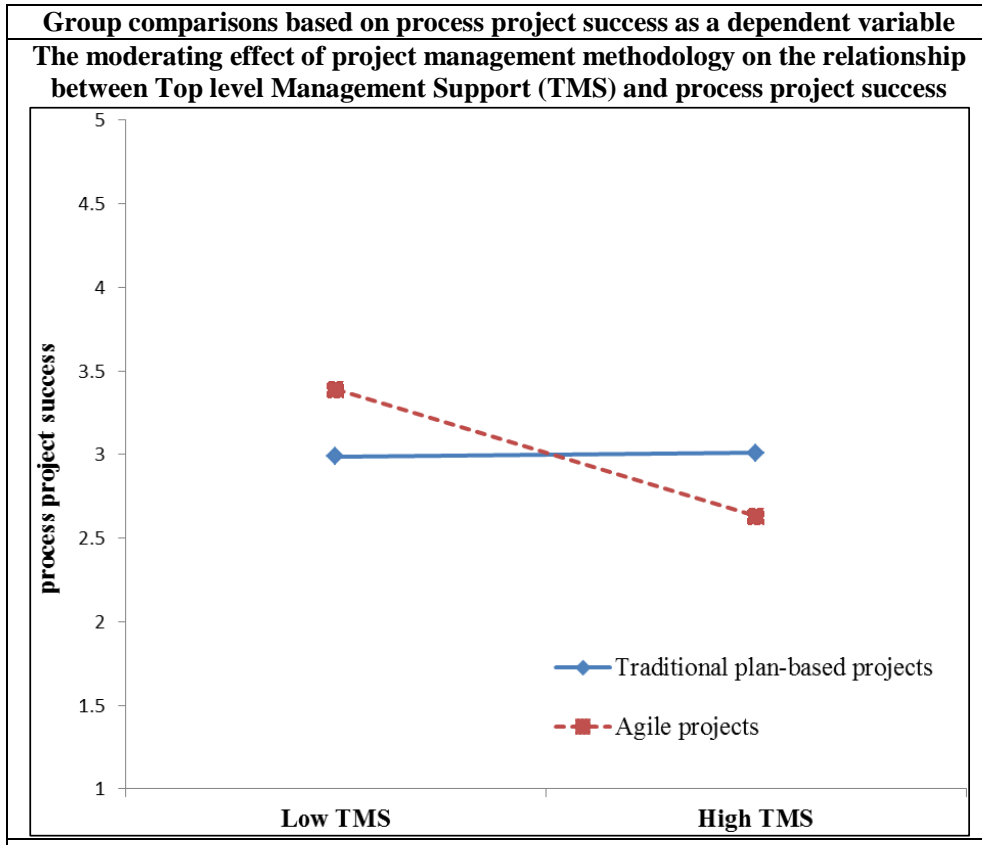

The moderating effect of project management methodology on the relationship between planning and controlling and process project success

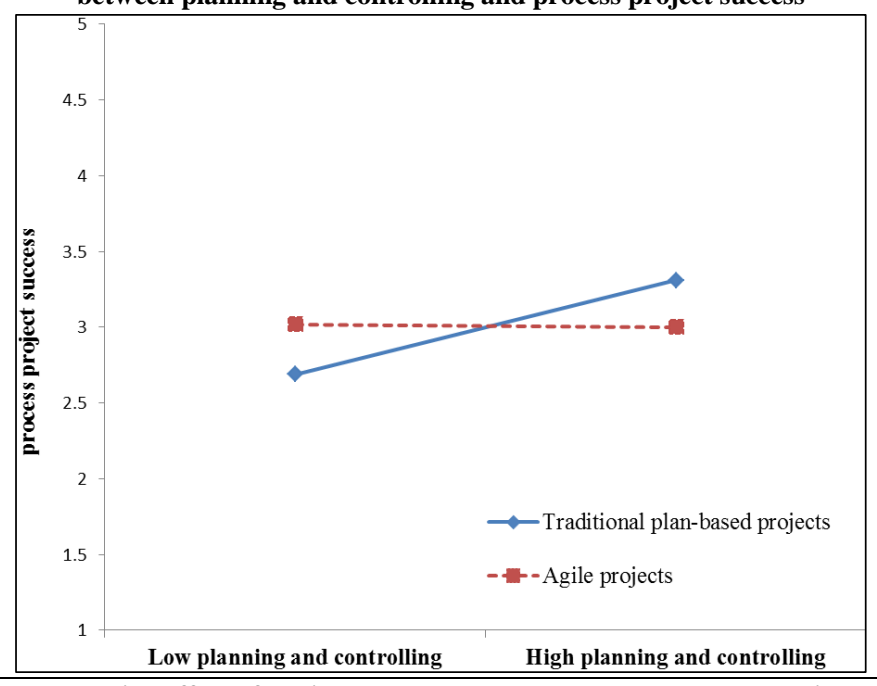

The moderating effect of project management methodology on the relationship between leadership characteristics and process project success

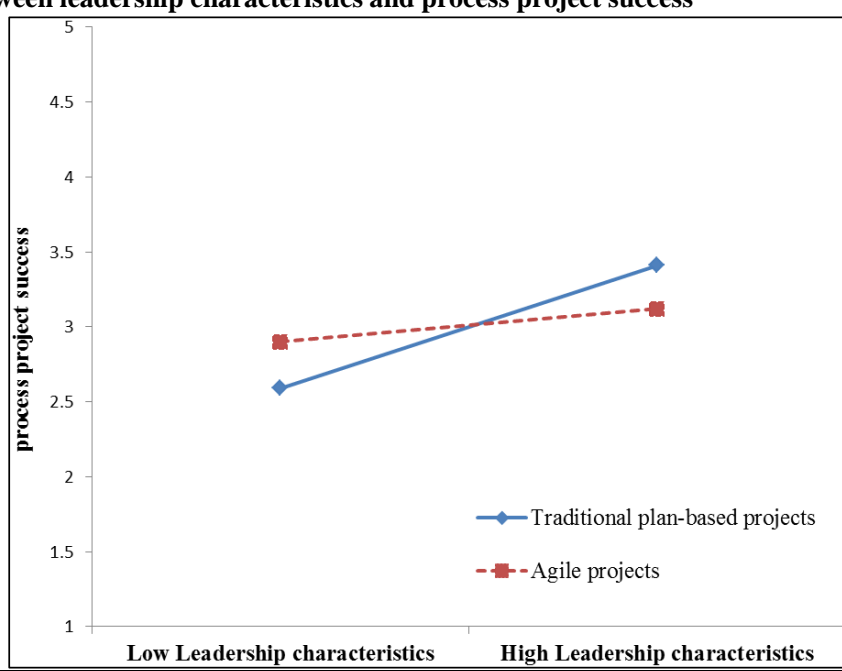

Group comparisons based on product project success as a dependent variable The moderating effect of project management methodology on the relationship between change management and product project success

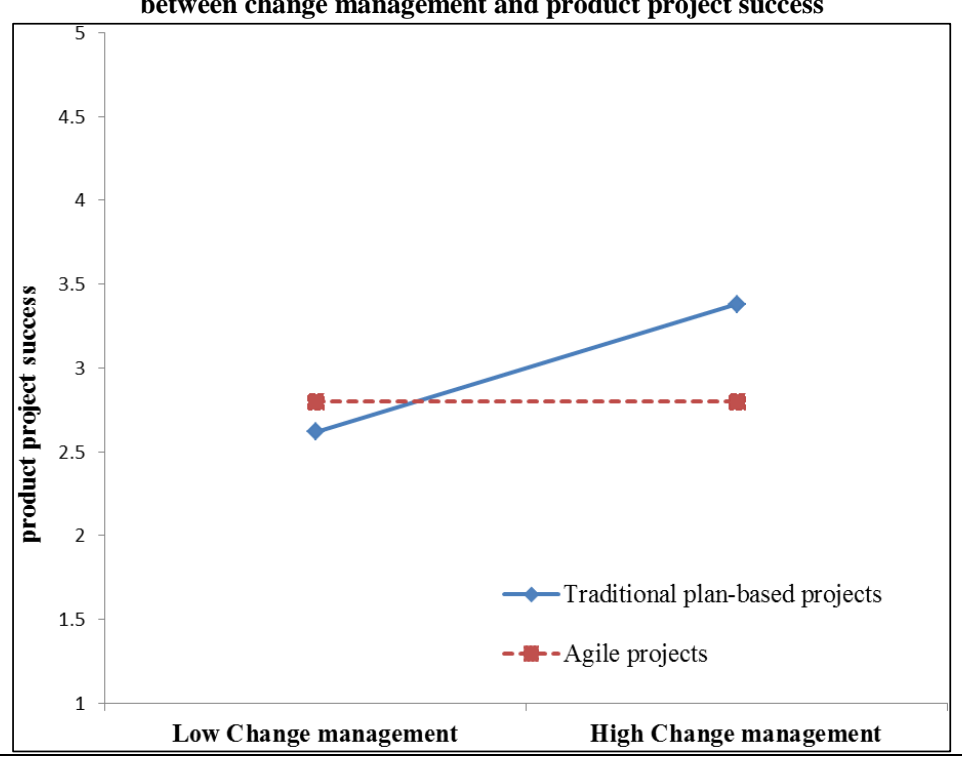

The moderating effect of project management methodology on the relationship between project team composition and product project success

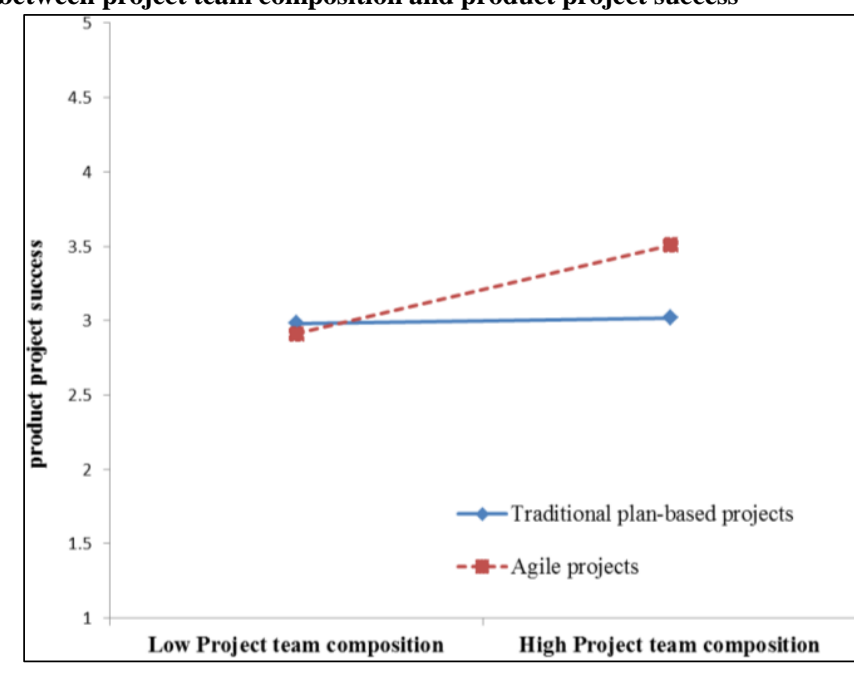

The moderating effect of project management methodology on the relationship between user participation and product project success

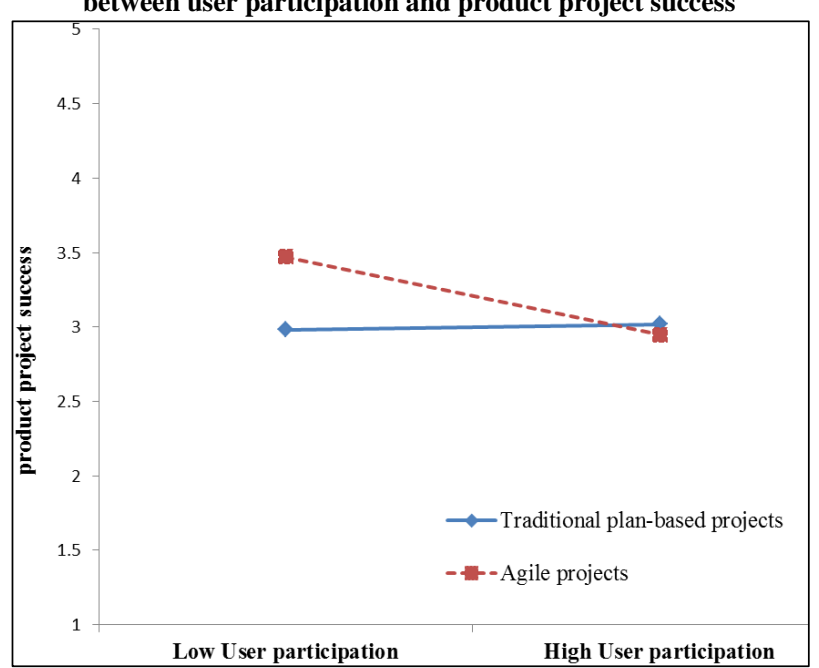




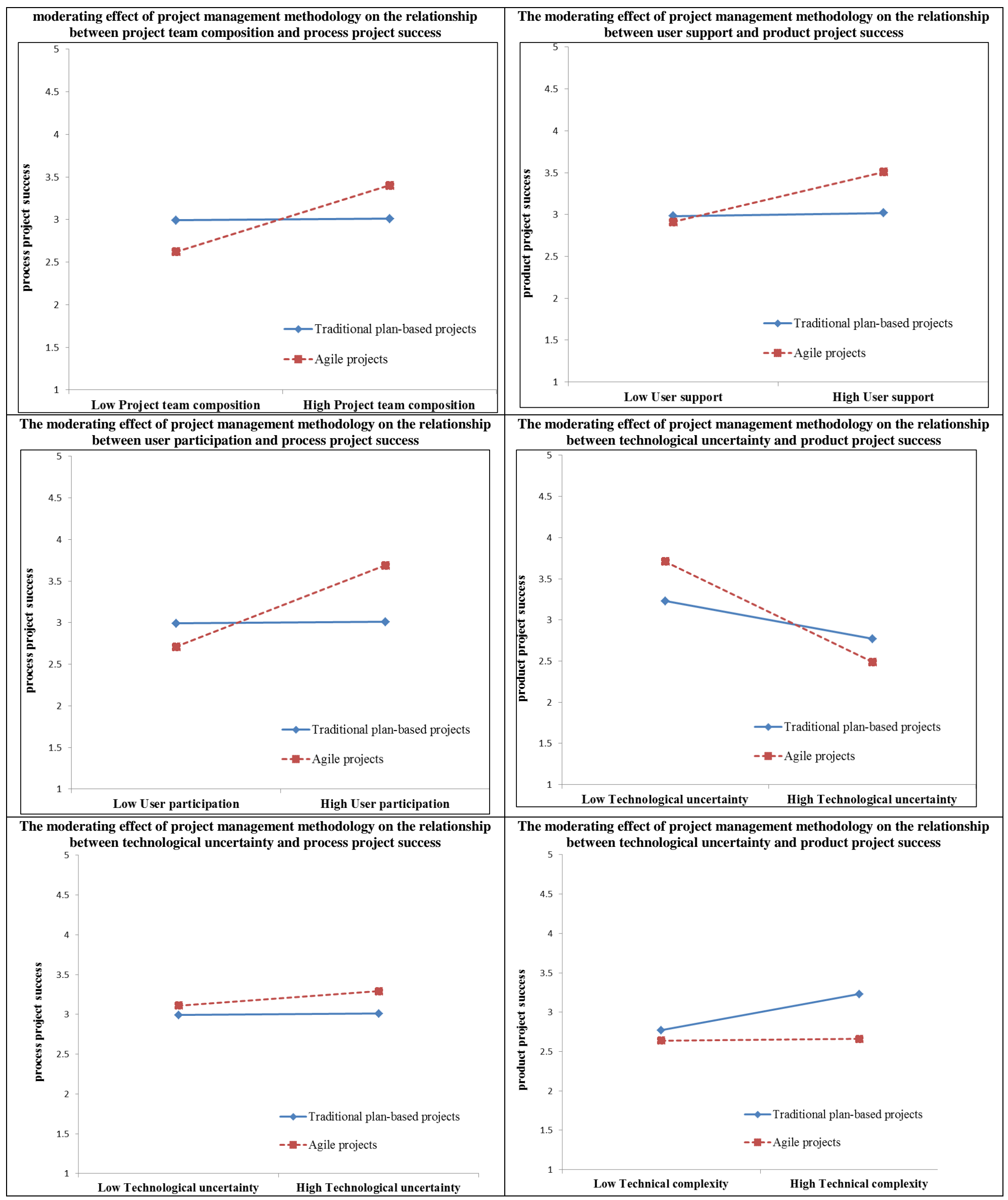




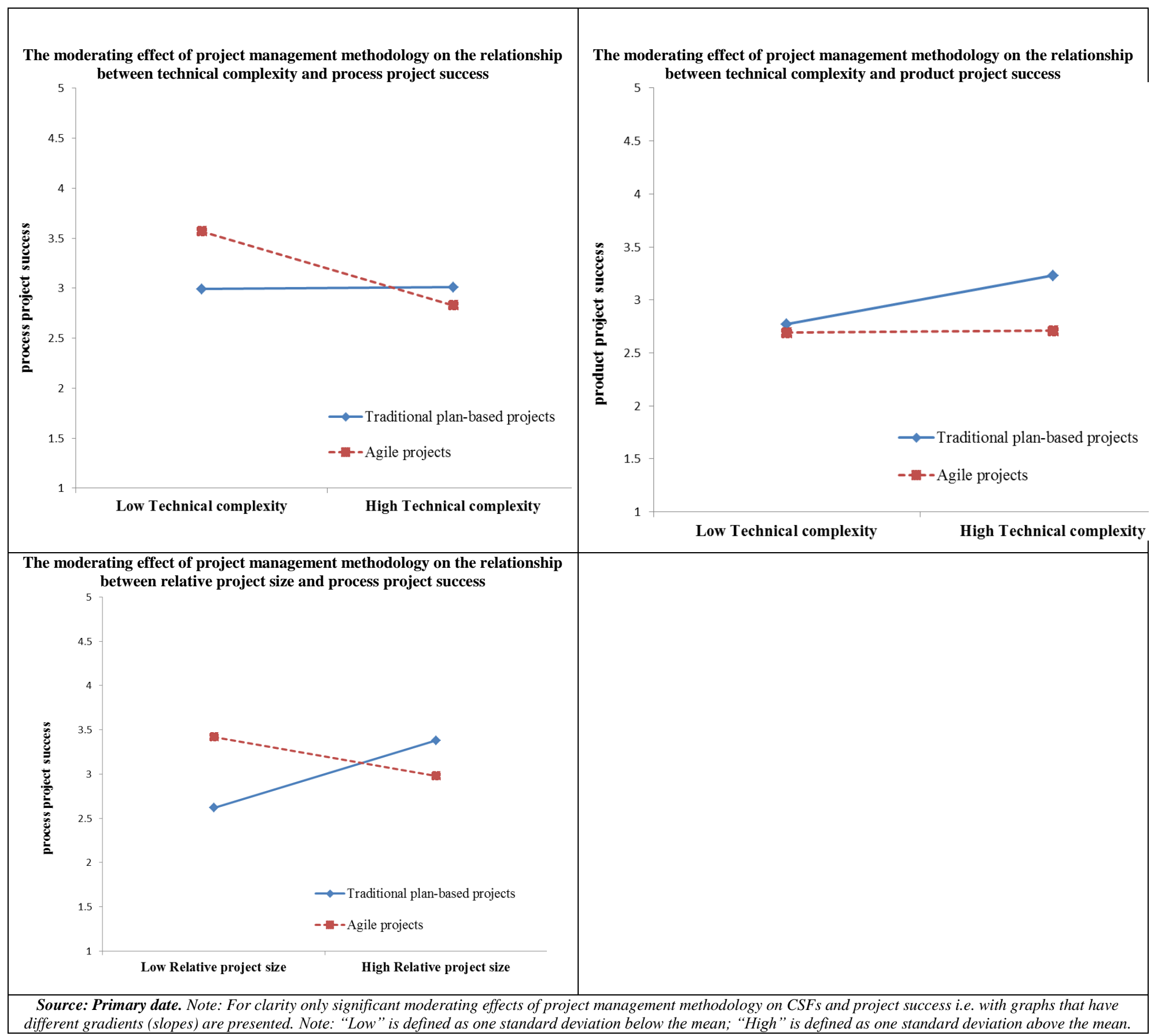

Figure 36: Significant moderating effects of project management methodology

\subsection{Testing interaction effects}

There is a general consensus that tests of discrete/categorical/non-metric moderator variable effects can be performed in SEM by utilizing the moderator to divide the sample into groups and then performing a chi-square of the significance difference between designated structural groups using multi-group invariance (e.g. Byrne, 2001, 2009; Hair et al., 2010; Milfont \& Fischer, 2010; Cunningham, 2010). However, tests of continuous (metric) moderator variable effects are performed by specifying interaction effects within the SEM context (Sauer \& 
Dick, 1993). In this case, the effect of a moderating variable is typically characterized statistically as an interaction (Dawson, 2014). Sauer and Dick (1993) observe that when variables are measured as continuous it is preferred to model moderated variable effects as multiplicative interactions to retain the full information contained in continuous variables. A similar approach is also recommended by Hair et al. (2010) and Dawson (2014). Precisely, Sauer and Dick (1993) contend that modelled interactions are favoured over multi-group analysis because: (1). Some information can be easily lost resulting from transformation of a continuous variable into a qualitative variable. (2). A median split into groups may create groups which do not exit at least for the present sample, raising up theoretical questions about the particular dividing points used. (3). Observed relationships can sometimes be very sensitive to cut off points used to form groups, especially when there is no natural cut off point. Thus, when moderators are continuous, it is better to model their effect using interaction terms in SEM in a manner similar to that used in multiple regression analysis (Sauer \& Dick, 1993; Hair et al., 2010). SEM has demonstrated to be much better than regression analysis because it compounds measurement error when estimating and fitting models with interaction terms (Frazier et al., p.120). Thus, SEM is preferable when you have multiple measures for each of the constructs (Frazier et al., p.115). Figure 37 demonstrates a schematic representation of a Structural Equation Model used to test for moderation for latent variables with many measurement indicators.

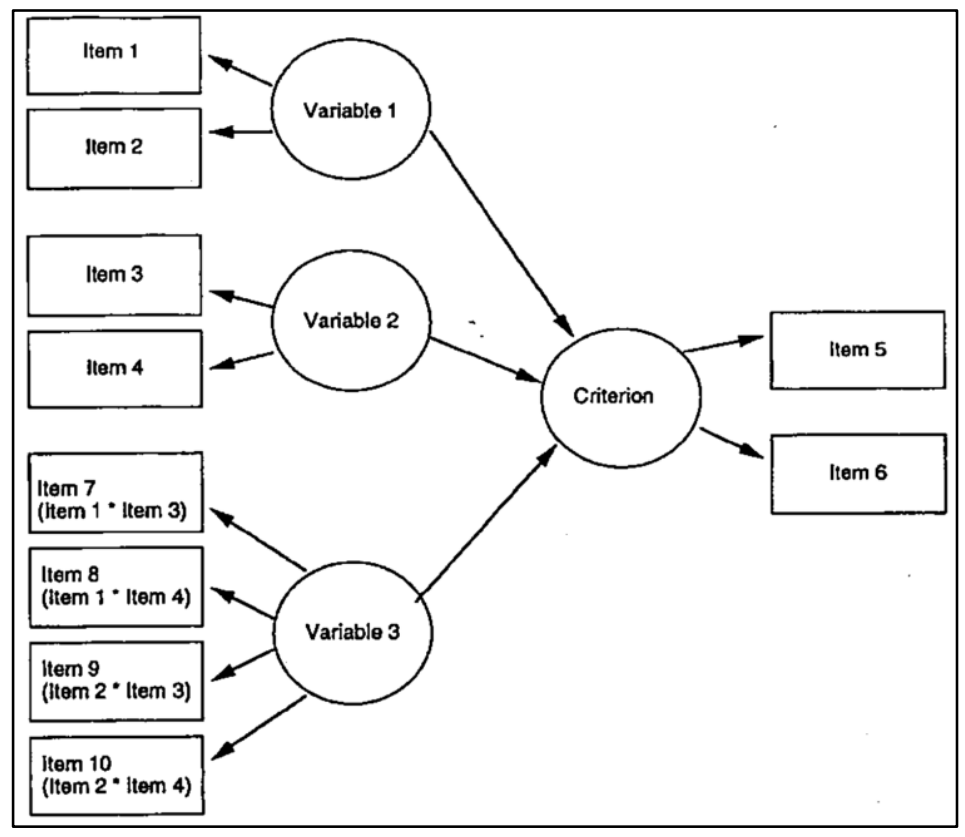

Figure 37: A schematic representation of a SEM used to test for moderation for latent variables with many measurement indicators (Source: Kline \& Dunn, 2000, Figure 1, p.128) 
As illustrated, interaction effects of at least two original exogenous variables and two observed variables associated with each can be modelled using SEM to test for moderation for latent variables with many measurement indicators. A third or interaction latent exogenous variable can be constructed from the cross products of the original observed variables. This interaction variable can then have four indicators. The two original and one interaction exogenous latent variables are then predicted to be related to an endogenous variable that also has at least two indicators.

Since one of the models is assumed to be nested in the other, two AMOS runs are necessary, one in which the formed interaction variables are present as indicators, the other in which they are absent (Sauer \& Dick, 1993, p.638; Jorekog \& Sorbon, 1989). The difference in chisquare values may then be computed and the test using the chi-square difference proceeds as with the case for discrete moderator variables previously described (Hair et al, 2010). Structural parameters can then be compared across models to test for the significance of the difference between the goodness of fit chi-square values for the two models (Frazier et al., p.120). If model 1 (without interaction terms) fits the data better than model 2 (with interaction effects), then there is a significant interaction effect (Sauer \& Dick, 1993). This is because lower (non-significant) chi-square values indicate better fit.

However, according to Kline and Dunn (2000), the problem of dealing with interaction terms in SEM has been a source of aggravation for users of SEM programs (e.g., AMOS, EQS, LISREL, COSAN). The problem occurs if an interaction variable is created by crossmultiplying raw scores of two original variables to create a third variable. Typically, the interactions are usually highly correlated with the raw score terms resulting in multicollinearity problems, and the resulting matrix of covariances or correlations among the variables becomes singular (i.e. there is a linear dependency in the variables). Subsequently, SEM program using MLE does not analyze data where the matrix is singular as it is not positive definite. The measurement error also appears to be exacerbated when product terms are created (Kline \& Dunn, 2000).

The literature suggests that this interaction problem has been around for a while and is not readily tractable (Kline \& Dunn, 2000). Several approaches have been suggested including a technique by Kenny and Judd (1984) that involves constraining the estimation of a number of parameters. However, most techniques appear to be very cumbersome and easily prone to 
errors because of the convenient variables created to estimate the covariances between the observed variables. In addition, the model fit is degraded due to non-normality introduced by specifying many product indicators. Scholars have noted these problems and tried to provide more simplified approaches to estimating interactions and quadratic effects.

For instance, Kline and Dunn (2000) propose the use of mean centering approach whereas Dawson (2004) suggests z-standardization to overcome the problem of interaction terms in SEM. While Mean centering involves the transformation of variables from raw scores to deviation scores scaling by subtracting the variable mean from all observations (Kline \& Dunn 2000), standardizing involves creating of z-scores (Dawson, 2014). Either mean centering or $z$-standardization can alleviate the problem of collinearity among the predictor variables that result from the excessive collinearity among the main effects and their interaction terms when products of variables are simply formed (Dawson, 2014, p.12).

The second characteristic of these approaches is that they help in interpretability of the estimates since standardized parameters are used which are more practically useful. Both methods provide identical findings, with some minor advantages to each. Thus, the choice between mean centering and z-standardizing is more a matter of personal choice (Dawson, 2014, p.12). Nonetheless, $z$-standardization is more easily accomplished with a simple command in SPSS.

Consistently, in this study z-standardization was used whereby the construct indicators of interaction terms were created from multiplicative terms that were initially transformed/ standardized to allow the matrix submitted for SEM analysis to be positive definitive. The interaction terms, were then created from the standardized (z-scores) variables.

Specifically, this procedure was initially executed in a two-step process in the SPSS program by first going to the main menu and selecting "analyze" followed by selecting "descriptive statistics" and then clicking on "descriptives". This prompted an empty variable box in which all the target observed variables for exogenous variables were chosen. The box for "save values as standardized values" was then ticked. These standardized values for each construct indicators were then computed in SPSS and saved as z-scores.

The second step involved going to the main menu again but this time selecting "transform" and then "compute variable" in which new observed variables (cross products) were formed 
under the "new expression" using the $z$-scores. These multiplicative terms were then used in SEM analysis for creating construct indicators of the respective interaction terms.

Next, two SEM models were estimated for each continuous moderator (i.e., project factors), one in which the formed interaction variables are present as indicators, the other in which they were absent (Sauer \& Dick, 1993). Again, an identical structural path diagram was used to estimate the baseline models while in the second model interaction terms were added (Hair et al., 2010). The two SEM models (model 1 without interaction terms and model 2 with interaction terms) were tested hierarchically and the chi-square difference between these models $\left(\Delta \chi^{2}\right)$ together with their respective degrees of freedom were computed using the respective model fit statistics to determine whether interaction effects were significant or not.

\subsubsection{Testing the interaction effects of technological uncertainty and candidate CSFs on project success}

According to Aiken and West (1991), Jose (2013a) and Dawson (2014) two variables interact if a particular combination of variables leads to results that would not be anticipated on the basis of the main effects of those variables. Following the steps provided above, SEM analyses were conducted which generated the results explained below.

In model 1, all 17 model predictor constructs (i.e. top level management support, organizational culture, internal project communication, user participation, project team commitment, technical complexity, development team skills, user experience, planning and controlling, change management, leadership characteristics, vision and mission, team composition, user support, technological uncertainty, relative project size and specification changes) were used to run a single SEM analysis simultaneously. The overall model fit was closely adequate as indicated by the fit statistics $\left(\chi^{2}=12785.54, d f=2935, \chi^{2} / d f=4.35, p>.05\right.$, $T L I=.88, C F I=.89$, RMSEA $=.04)$.

In model 2, all the predictor constructs of model 1 together with the interaction effects of technological uncertainty were entered, specified and estimated simultaneously in a SEM. The model output fit statistics were $\chi^{2}=34486.947, \chi^{2}=5927, \chi^{2} / d f=5.819, p<.05, C F I=.843$, $R M S E A=.05$, Thus, both models show reasonably acceptable fit indices indicating their overall fit. The chi-square difference between models $(\Delta \chi 2)$ is 21701.404 with 2992 degrees of freedom. This is significant $(p<.05)$, indicating that model 1 (without interaction terms) fits the data better than model 2 (with interaction effects) and thus, there is a significant 
interaction effect (Sauer \& Dick, 1993; Frazier et al., 2004). Table 77 illustrates the summary of results from testing the interaction effects of technological uncertainty and candidate CSFs on project success. Where the coefficient of the interaction term is significant, there is an indication of the statistical significance that the relationship between that predictor construct and process/ product success differs significantly according to technological uncertainty.

While the results indicate that technological uncertainty interacts with user support to positively and significantly influence process success; the results also suggest that technological uncertainty interacts positively and significantly with project team commitment and project team composition, but interacts negatively with planning and controlling, and relative project size to affect product success. A close examination of the correlations matrix between the original variables and the created interaction terms confirms that all correlations were low. In addition, the standardized regression weights show that the interaction term is not highly correlated with the criterion variable, indicating that multi-collinearity is not a problem in these two nested models (Sauer \& Dick, 1993). Thus, these findings statistically confirm that the relationships between those candidate CSFs and process/product success (marked with asterisks*) significantly differ according to technological uncertainty. 
Table 77: Hierarchical SEM analysis of interaction terms of technological uncertainty

\begin{tabular}{|c|c|c|c|c|}
\hline \multirow[b]{2}{*}{ Model constructs } & \multicolumn{2}{|c|}{$\begin{array}{l}\text { Process success as the dependent } \\
\text { variable }(n=984)\end{array}$} & \multicolumn{2}{|c|}{$\begin{array}{c}\text { Product success as the dependent } \\
\text { variable }(n=984)\end{array}$} \\
\hline & $\begin{array}{c}\text { SEM } 1 \text { (without } \\
\text { interaction } \\
\text { variables) }\end{array}$ & $\begin{array}{c}\text { SEM } 2 \text { (with } \\
\text { interaction } \\
\text { variables) }\end{array}$ & $\begin{array}{c}\text { SEM } 1 \text { (without } \\
\text { interaction } \\
\text { variables) }\end{array}$ & $\begin{array}{c}\text { SEM 2(with } \\
\text { interaction } \\
\text { variables) }\end{array}$ \\
\hline Top level management support & $-.093^{*}$ & $-.087^{*}$ & -.024 & -.024 \\
\hline Organizational culture & $.102 *$ & $.099 *$ & -.007 & .005 \\
\hline Internal project communication & $.105^{*}$ & $.113 *$ & $.104 * *$ & $.096^{* *}$ \\
\hline User participation & $.087 *$ & .079 & -.026 & -.024 \\
\hline Project team commitment & .042 & .046 & .009 & .013 \\
\hline Dev. Team expertise & -.047 & -.050 & -.041 & -.027 \\
\hline User experience & .049 & .034 & .049 & .038 \\
\hline Planning and controlling & $.112 * *$ & $.098 *$ & -.023 & -.024 \\
\hline Change management & .054 & .067 & .032 & .014 \\
\hline Leadership characteristics & $.151 * * *$ & $.149 * * *$ & .039 & .040 \\
\hline Vision and mission & -.016 & -.020 & .045 & .044 \\
\hline Project team composition & $.124 *$ & .144 & $.106^{*}$ & $.088 *$ \\
\hline User support & $.158 *$ & .129 & .076 & $.069 *$ \\
\hline Technological uncertainty & $.075^{*}$ & .084 & $-.156 * * *$ & $-.356 * * *$ \\
\hline Relative project size & $-.200 * * *$ & $-.201 * * *$ & -.014 & $-.022 *$ \\
\hline Specification changes & $-.085^{*}$ & -.077 & $.080 * *$ & .090 \\
\hline Technical complexity & $-.075^{*}$ & -.085 & $.079 *$ & $-.077 * * *$ \\
\hline Technological uncertainty*Top level management support & & $.019 n s$ & & $-.019 n s$ \\
\hline Technological uncertainty* Organizational culture & & $-.024 n s$ & & $-.045 n s$ \\
\hline Technological uncertainty*Internal project communication & & $-.015 n s$ & & $-.007 n s$ \\
\hline Technological uncertainty*User participation & & $.013 n s$ & & $-.006 n s$ \\
\hline Technological uncertainty*Project team commitment & & $.051 n s$ & & $.041 *$ \\
\hline Technological uncertainty $*$ Dev. Team expertise & & $-.016 n s$ & & $.011 n s$ \\
\hline Technological uncertainty*User experience & & $.020 n s$ & & $-.043 n s$ \\
\hline Technological uncertainty*Planning and controlling & & $-.001 n s$ & & $-.031^{*}$ \\
\hline Technological uncertainty*Change management & & $-.023 n s$ & & $-.009 n s$ \\
\hline Technological uncertainty*Leadership characteristics & & $-.025 n s$ & & $.047 n s$ \\
\hline Technological uncertainty*Vision and mission & & $-.046 n s$ & & $.024 n s$ \\
\hline Technological uncertainty*Team composition & & $-.016 n s$ & & $.212 * *$ \\
\hline Technological uncertainty*User support & & $.129 * * *$ & & $-.006 n s$ \\
\hline Technological uncertainty*Technical complexity & & $-.011 n s$ & & $-.021 n s$ \\
\hline Technological uncertainty*Relative project size & & $.013 n s$ & & $-.050 * *$ \\
\hline Technological uncertainty*Specification changes & & $.019 n s$ & & $.058 n s$ \\
\hline Note: Standardized regression coefficients are reported. $n$ & tion not significan & *****' signific & $0.05,0.01$ and 0 . & el respective \\
\hline
\end{tabular}

Based on Dawson's (2014) guidelines, moderation graphs were generated using Stats tool software programme-an Excel version program for two way interactions, to show how technological uncertainty significantly interacts with some candidate CSFs to influence project success. The results are summarised in Figure 38. It is evident that the interaction effects of technological uncertainty are significant, as supported by the moderation graphs which have different gradients. This essentially means that the effects of these candidate CSFs on project success differ as a function of technological uncertainty levels. Consistent with the observations of Aiken et al. (1991), Jose (2013a) and Dawson (2014), since the magnitude of effects are greater at one level of a variable than at another it is a sufficient evidence to conclude that significant interactions have occurred. These findings support hypothesis $20(H 20)$. 


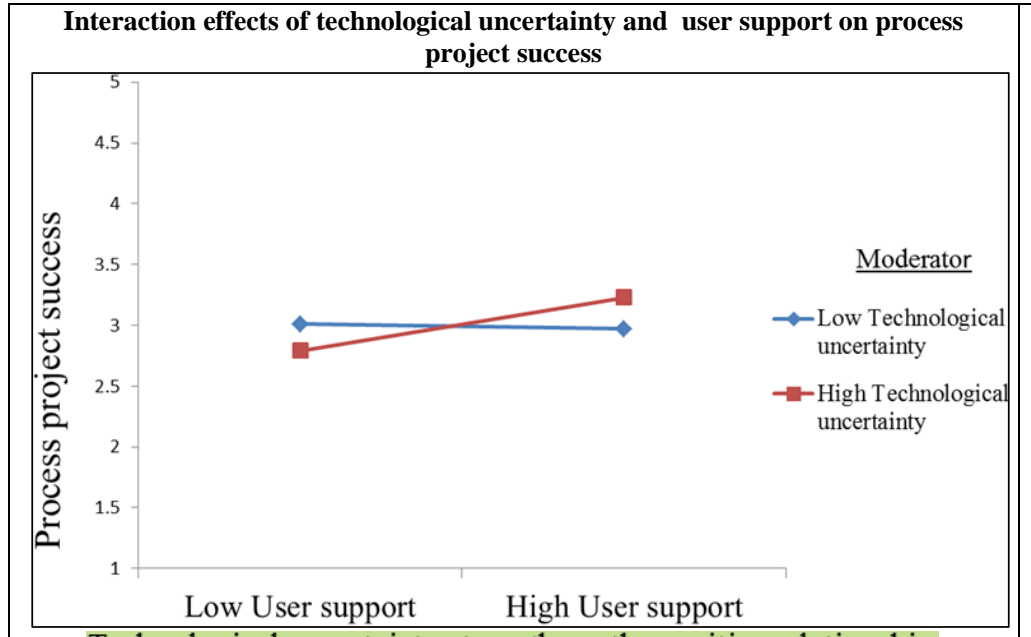

Technological uncertainty strengthens the positive relationship between User support and Process project success.

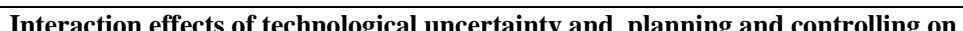
product project success

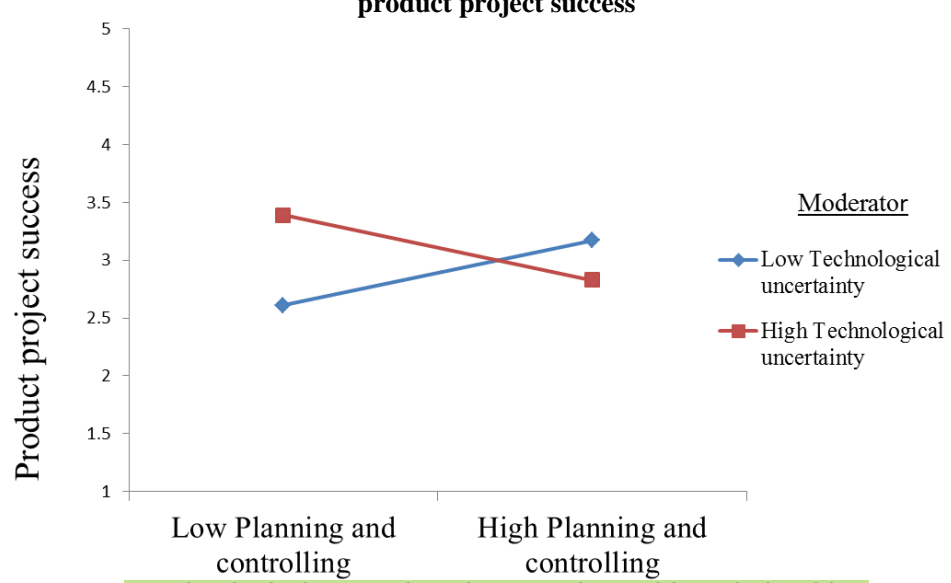

Technological uncertainty dampens the positive relationship between Planning and controlling and Product project success.

Interaction effects of technological uncertainty and relative project size on product project success

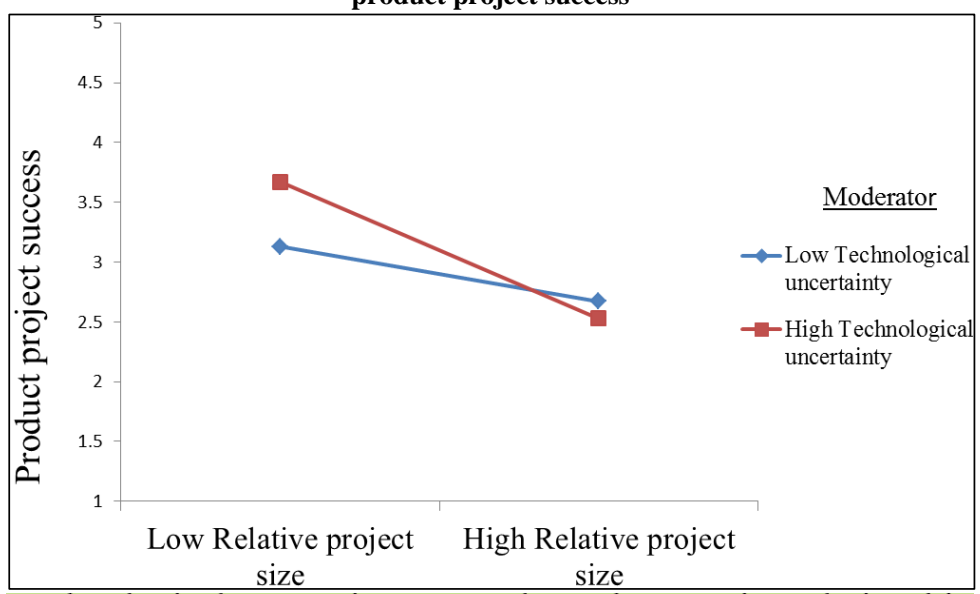

Technological uncertainty strengthens the negative relationship

between Relative project size and Product project success.

Source: Primary data. Note: "Low" is defined as one standard deviation below the mean; "High" is defined as one standard deviation above the mean. For clarity only significant interaction effects i.e. with graphs that have different gradients (slopes) are presented.

Figure 38 : Interaction effects of technological uncertainty and candidate CSFs on project success
Interaction effects of technological uncertainty and project team commitment on product project success

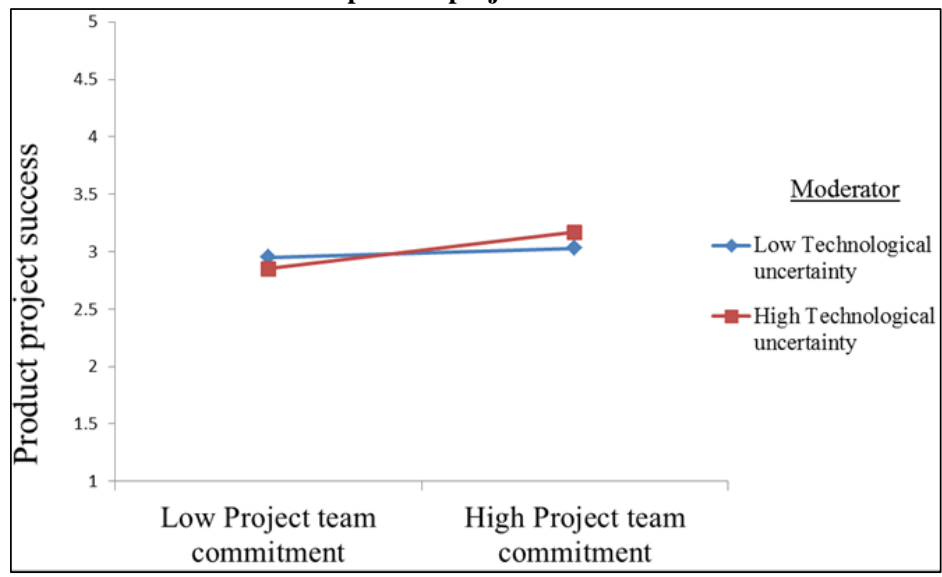

Technological uncertain ty strengthen s the positive relationship between Project team commitment and Product project success.

Interaction effects of technological uncertainty and team composition on product project success

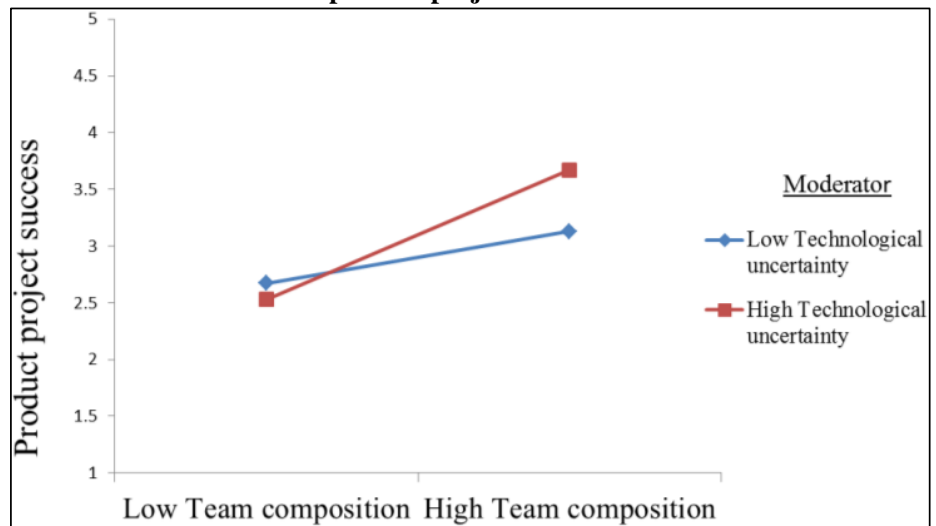

Low Team composition High Team composition

Technological uncertainty strengthens the positive relationship between Team composition and Product project success. 


\subsubsection{Testing the interaction effects of technical complexity and candidate CSFs on project success}

This section tests the interaction effects of technical complexity and candidate CSFs on project success. In model 1, all 17 model predictor constructs were entered and analyzed simultaneously. The overall model fit was adequate as indicated by fit statistics $(\chi 2=12785.54, d f=2935, \chi 2 / d f=4.35, p>.05, T L I=.87, C F I=.88, R M S E A=.050)$.

In model 2, all the predictor constructs of model 1 together with the interaction effects of technical complexity were entered, specified and analyzed simultaneously in a SEM. The model fit statistics were $\chi^{2}=29630.79, d f=5927, \chi 2 / d f=4.99, p<.05, T L I=.871, C F I=.85$, RMSEA $=.054$, Thus, both models $(1 \& 2)$ have some acceptable fit indices indicating their overall acceptability. The chi-square difference between these models $\left(\Delta \chi^{2}\right)$ is 16845.253 with 2992 degrees of freedom. This is significant $(p<.05$ ), indicating that model 1 (without interaction terms) fits the data better than model 2 (with interaction effects) and thus, there is a significant interaction effect (Hair et al., 2010). Table 78 shows summarized results from testing interaction effects of technical complexity and candidate CSFs on project success.

As illustrated, technical complexity interacts positively with user participation and user support, but negatively with project team commitment, development team expertise, change management, leadership characteristics and technological uncertainty to significantly influence process success. The results further indicate that technical complexity interacts with planning and controlling, relative project size, specification change to negatively affect product success.

Together, these findings statistically confirm that technical complexity interacts with those candidate CSFs (interaction terms with asterisks*) to influence process/product project success. 
Table 78: Hierarchical SEM analysis of interaction terms of technical complexity

\begin{tabular}{|c|c|c|c|c|}
\hline \multirow[b]{2}{*}{ Model constructs } & \multicolumn{2}{|c|}{$\begin{array}{c}\text { Process success as the dependent } \\
\text { variable }(n=984)\end{array}$} & \multicolumn{2}{|c|}{$\begin{array}{l}\text { Product success as the dependent } \\
\text { variable }(n=984)\end{array}$} \\
\hline & $\begin{array}{l}\text { SEM } 1 \text { (without } \\
\text { interaction } \\
\text { variables) }\end{array}$ & $\begin{array}{c}\text { SEM } 2 \text { (with } \\
\text { interaction } \\
\text { variables) }\end{array}$ & $\begin{array}{c}\text { SEM } 1 \text { (without } \\
\text { interaction } \\
\text { variables) }\end{array}$ & $\begin{array}{c}\text { SEM } 2 \text { (with } \\
\text { interaction } \\
\text { variables) }\end{array}$ \\
\hline Top level management support & $-.093 *$ & $-.094 *$ & -.024 & -.038 \\
\hline Organizational culture & $.102 *$ & $.106^{*}$ & -.007 & .009 \\
\hline Internal project communication & $.105^{*}$ & $.115 * *$ & $.104 * *$ & .110 \\
\hline User participation & $.087^{*}$ & .103 & -.026 & -.025 \\
\hline Project team commitment & .042 & .043 & .009 & .005 \\
\hline Dev. Team expertise & -.047 & -.040 & -.041 & -.041 \\
\hline User experience & .049 & .044 & .049 & .033 \\
\hline Planning and controlling & $.112 * *$ & $.121 *$ & -.023 & -.012 \\
\hline Change management & .054 & .034 & .032 & .019 \\
\hline Leadership characteristics & $.151 * * *$ & .134 & .039 & .025 \\
\hline Vision and mission & -.016 & .003 & .045 & .050 \\
\hline Project team composition & $.124 *$ & $.110^{*}$ & $.106^{*}$ & .104 \\
\hline User support & $.158^{*}$ & $.170 * * *$ & .076 & $.076^{*}$ \\
\hline Technological uncertainty & $.075^{*}$ & $.083^{*}$ & $-.156 * * *$ & $-.139 * * *$ \\
\hline Relative project size & $-.200 * * *$ & -.182 & -.014 & -.017 \\
\hline Specification changes & $-.085^{*}$ & $-.080 *$ & $.080 * *$ & $.079 *$ \\
\hline Technical complexity & $-.075^{*}$ & -.101 & $.079 *$ & $.082 * * *$ \\
\hline Technical complexity *Top level management support & & $.007 n s$ & & $.009 n s$ \\
\hline Technical complexity * Organizational culture & & $.068 n s$ & & $.050 n s$ \\
\hline Technical complexity *Internal project communication & & $.002 n s$ & & $-.022 n s$ \\
\hline Technical complexity $*$ User participation & & $.102 * * *$ & & $.031 n s$ \\
\hline Technical complexity *Project team commitment & & $-.017 *$ & & $.013 n s$ \\
\hline Technical complexity *Dev. Team expertise & & $-.095^{*}$ & & $.036 n s$ \\
\hline Technical complexity $*$ User experience & & $-.004 n s$ & & $.017 n s$ \\
\hline Technical complexity *Planning and controlling & & $-.004 n s$ & & $-.020 *$ \\
\hline Technical complexity ${ }^{*}$ Change management & & $-.078 *$ & & $-.033 n s$ \\
\hline Technical complexity *Leadership characteristics & & $-.076^{*}$ & & $-.049 n s$ \\
\hline Technical complexity $*$ Vision and mission & & $.012 n s$ & & $.011 n s$ \\
\hline Technical complexity $*$ Team composition & & $-.011 n s$ & & $-.013 n s$ \\
\hline Technical complexity *User support & & $.105^{*}$ & & $-.043 n s$ \\
\hline Technical complexity *Technological uncertainty & & $-.099 *$ & & $-.015 n s$ \\
\hline Technical complexity $*$ Relative project size & & $-.007 n s$ & & $-.074 *$ \\
\hline Technical complexity $*$ Specification changes & & $-.045 n s$ & & $-.116^{* * *}$ \\
\hline Note: Standardized regression coefficients are repor & $\begin{array}{l}s=\text { interaction not } \\
\text { respectively. }\end{array}$ & icant; ${ }^{*} * * * *$ & ificant at $0.05,0.0$ & d 0.001 level \\
\hline
\end{tabular}

Following Dawson's (2014) guidelines, moderation graphs were generated to show the significant interaction effects between technical complexity and some candidate CSFs on project success. The results are shown in Figure 39. 


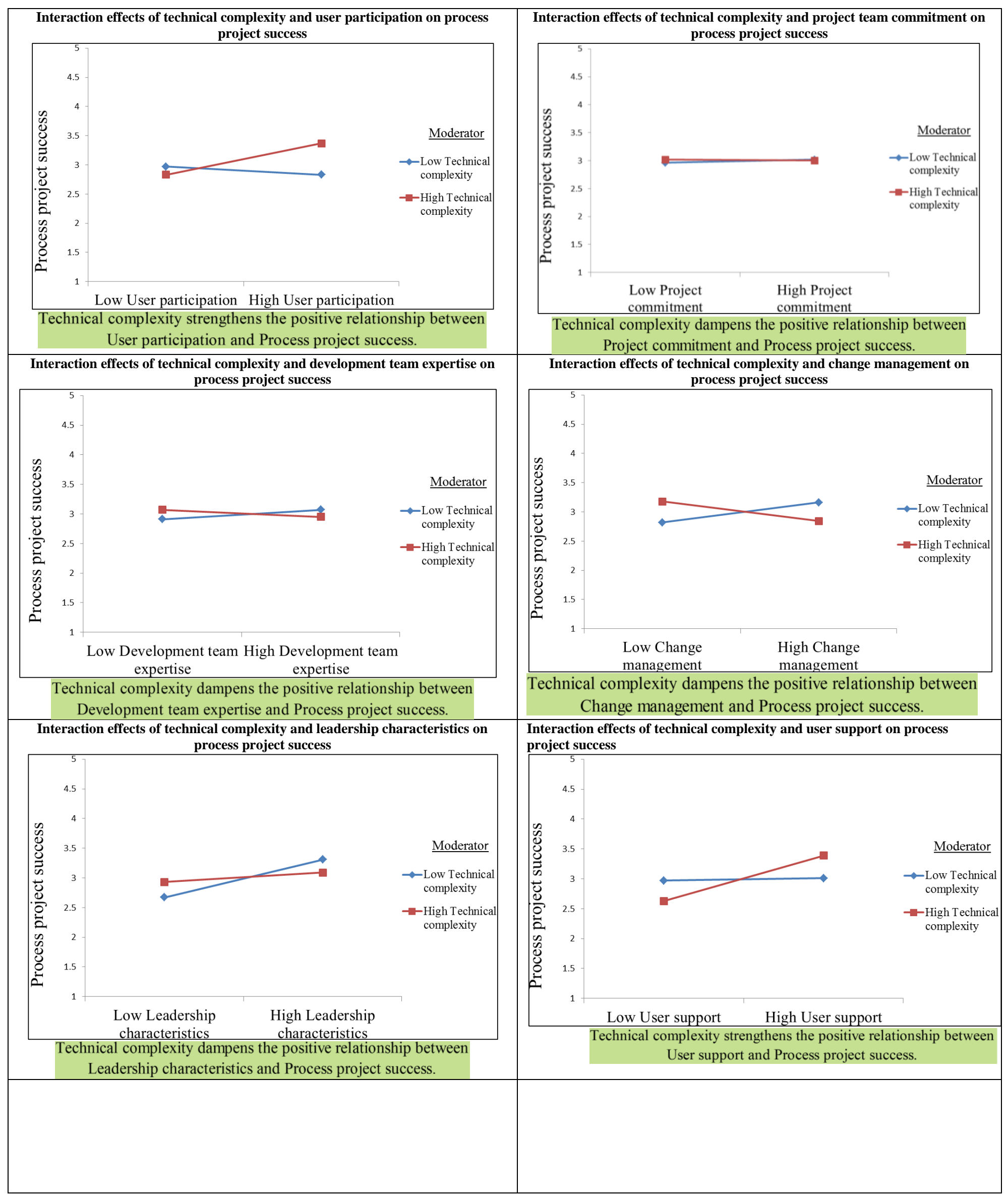




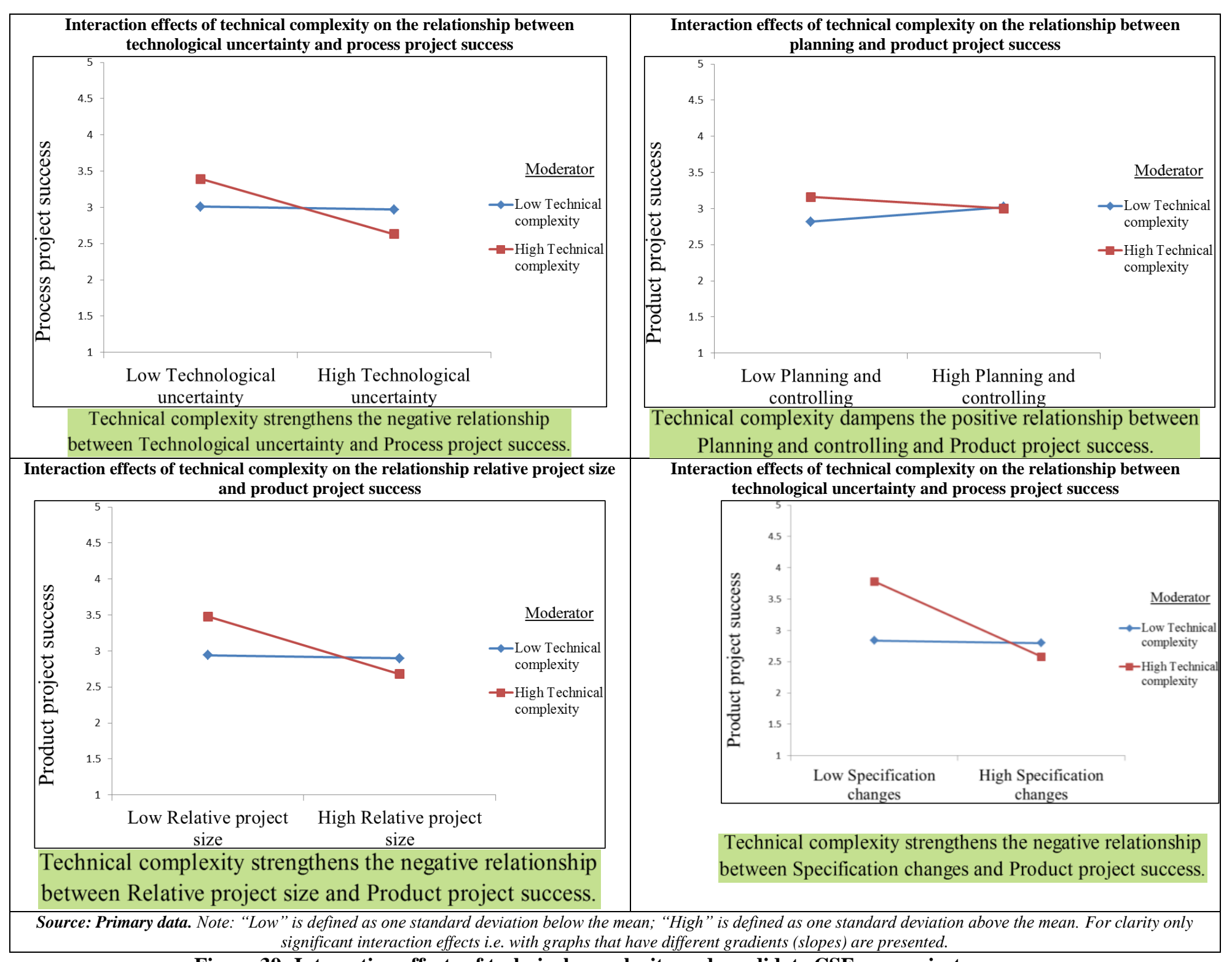

Figure 39: Interaction effects of technical complexity and candidate CSFs on project success

The effects of the shown candidate CSFs on project success differ, depending on the level of technical complexity; besides, the moderation graphs have different slopes and hence, are not parallel (Dawson, 2014). This implies that the effect of these CSFs on project success differs as a function of technical complexity (that is, high and low levels). Thus, the magnitude of these candidate CSFs on project success is influenced by level of technical complexity. This is consistent with Aiken and West (1991) and Dawson (2014) who observed that as long as the magnitude of an effect is greater at one level of a variable than at another, it means a significant interaction effect has occurred. These findings support (H21). 


\subsubsection{Testing the interaction effects of relative project size and candidate CSFs on project success}

This section tests the interaction effects of relative project size and candidate CSFs on project success. Again, in model 1, all 17 model predictor constructs were entered and analyzed simultaneously. The overall model fit was adequate as indicated by fit statistics $\left(\chi 2=12785.54, d f=2935, \chi^{2} / d f=4.36, p>.05, T L I=.871, C F I=.880, R M S E A=.050\right)$.

In model 2, all the predictor constructs of model 1 together with the interaction effects of relative project size were entered and estimated simultaneously in a SEM. The model fit statistics were $\chi^{2}=28622.167, d f=5927, \chi 2 / d f=4.829, \quad p<.05, \quad C F I=.782, \quad$ RMSEA $=.053$. Thus, both models show acceptable fit indices indicating their overall fit. The chi-square difference between models $\left(\Delta \chi^{2}\right)$ is 1583.624 with 2992 degrees of freedom. This is significant $(p<.05)$, indicating that model 1 (without interaction terms) fits the data better than model 2 (with interaction effects) and thus, there is a significant interaction effect. Table 79 illustrates the summarized results from testing relative project size as a moderator.

The results indicate that of all the candidate CSFs, relative project size only interacts with user support to positively influence process success. In addition, the results also suggest that relative project size interacts with top level management support, change management, vision and mission, technical complexity, specification changes to negatively influence product success. The findings statistically suggest that relative project size interacts with those candidate CSFs (with asterisks*) to influence project success. 
Table 79: Hierarchical SEM analysis of interaction terms of relative project size

\begin{tabular}{|c|c|c|c|c|}
\hline & \multicolumn{2}{|c|}{$\begin{array}{l}\text { Process success as the dependent } \\
\text { variable }(n=984)\end{array}$} & \multicolumn{2}{|c|}{$\begin{array}{l}\text { Product success as the dependent variable } \\
\qquad(\mathrm{n}=984)\end{array}$} \\
\hline Model constructs & $\begin{array}{c}\text { SEM } 1 \text { (without } \\
\text { interaction } \\
\text { variables) }\end{array}$ & $\begin{array}{c}\text { SEM } 2 \text { (with } \\
\text { interaction } \\
\text { variables) }\end{array}$ & $\begin{array}{c}\text { SEM } 1 \text { (without } \\
\text { interaction } \\
\text { variables) }\end{array}$ & $\begin{array}{c}\text { SEM } 2 \text { (with } \\
\text { interaction } \\
\text { variables) }\end{array}$ \\
\hline Top level management support & $-.093 *$ & $-.083^{*}$ & -.024 & -.023 \\
\hline Organizational culture & $.102 *$ & $.093 *$ & -.007 & -.008 \\
\hline Internal project communication & $.105^{*}$ & $.121 *$ & $.104 * *$ & $.076^{*}$ \\
\hline User participation & $.087 *$ & .080 & -.026 & -.023 \\
\hline Project team commitment & .042 & .033 & .009 & .027 \\
\hline Dev. Team expertise & -.047 & -.066 & -.041 & -.051 \\
\hline User experience & .049 & .046 & .049 & .033 \\
\hline Planning and controlling & $.112 * *$ & $.109 *$ & -.023 & .002 \\
\hline Change management & .054 & .049 & .032 & .026 \\
\hline Leadership characteristics & $.151 * * *$ & $.149 * * *$ & .039 & .049 \\
\hline Vision and mission & -.016 & -.018 & .045 & $.056^{*}$ \\
\hline Project team composition & $.124 *$ & $.127 *$ & $.106^{*}$ & .077 \\
\hline User support & $.158^{*}$ & $.151 * * *$ & .076 & $.095^{*}$ \\
\hline Technological uncertainty & $.075^{*}$ & .078 & $-.156 * * *$ & -.143 \\
\hline Relative project size & $-.200 * * *$ & -.207 & -.014 & -.027 \\
\hline Specification changes & $-.085^{*}$ & $-.086^{*}$ & $.080 * *$ & .109 \\
\hline Technical complexity & $-.075^{*}$ & $-.068 *$ & $.079 *$ & $.079 * * *$ \\
\hline Relative project size*Top level management support & & $.049 n s$ & & $-.063 * * *$ \\
\hline Relative project size* Organizational culture & & $.031 n s$ & & $-.010 n s$ \\
\hline Relative project size*Internal project communication & & $-.008 n s$ & & $.012 n s$ \\
\hline Relative project size*User participation & & $.009 n s$ & & $.071 n s$ \\
\hline Relative project size*Project team commitment & & $.072 n s$ & & $.043 n s$ \\
\hline Relative project size*Dev. Team expertise & & $.018 n s$ & & $-.024 n s$ \\
\hline Relative project size*User experience & & $-.008 n s$ & & $-.062 n s$ \\
\hline Relative project size*Planning and controlling & & $-.022 n s$ & & $-.009 n s$ \\
\hline Relative project size* Change management & & $.018 n s$ & & $-.080 *$ \\
\hline Relative project size* Leadership characteristics & & $-.033 n s$ & & $-.023 n s$ \\
\hline Relative project size*Vision and mission & & $-.015 n s$ & & $-.116 * * *$ \\
\hline Relative project size*Team composition & & $.010 n s$ & & $-.011 n s$ \\
\hline Relative project size*Technical complexity & & $-.016 n s$ & & $-.030 *$ \\
\hline Relative project size*User support & & $.024 *$ & & $.013 n s$ \\
\hline Relative project size*Technological uncertainty & & $.042 n s$ & & $-.028 n s$ \\
\hline Relative project size $*$ Specification changes & & $-.042 n s$ & & $-.093 *$ \\
\hline
\end{tabular}

Figure 40 illustrates how the effects of candidate CSFs to project success differ depending on the level of relative project size, as supported by the graphs which have different gradients. This implies that the effect of those candidate CSFs on project success differs as a function of relative project size levels. Consistent with the observations of Aiken et al. (1991), Jose (2013a) and Dawson (2014), since the magnitude differs it is sufficient evidence to conclude that significant interaction has occurred. These findings support hypothesis 22 (H22). 


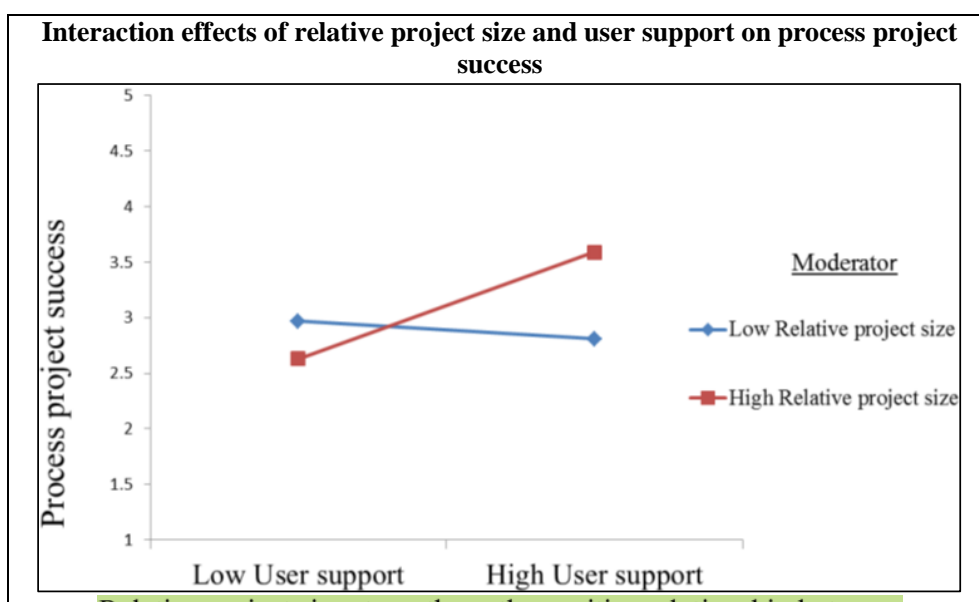

Relative project size strengthens the positive relationship between User support and Process project success.

Interaction effects of relative project size and change management on product project success

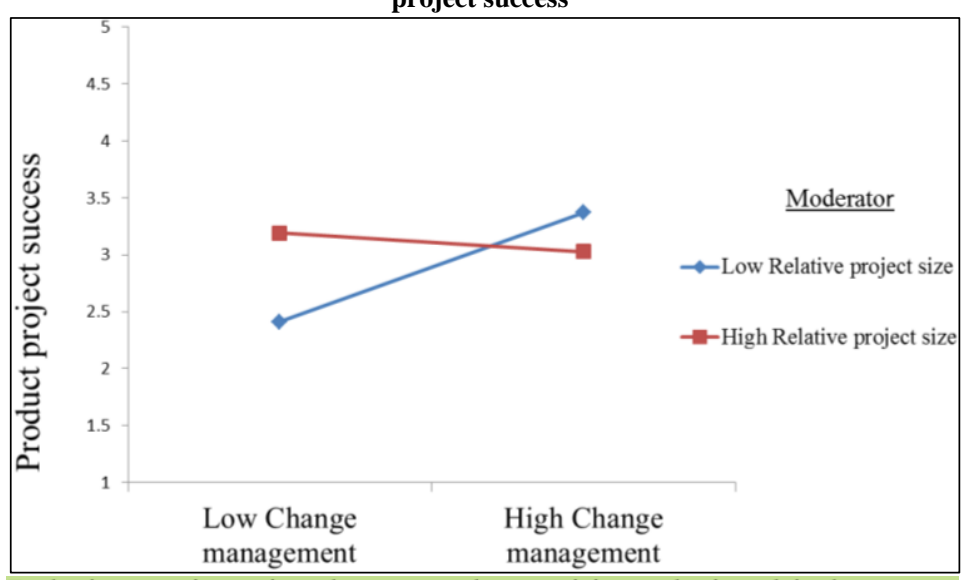

Relative project size dampens the positive relationship between Change management and Product project success.

Interaction effects of relative project size and technical complexity on product project success

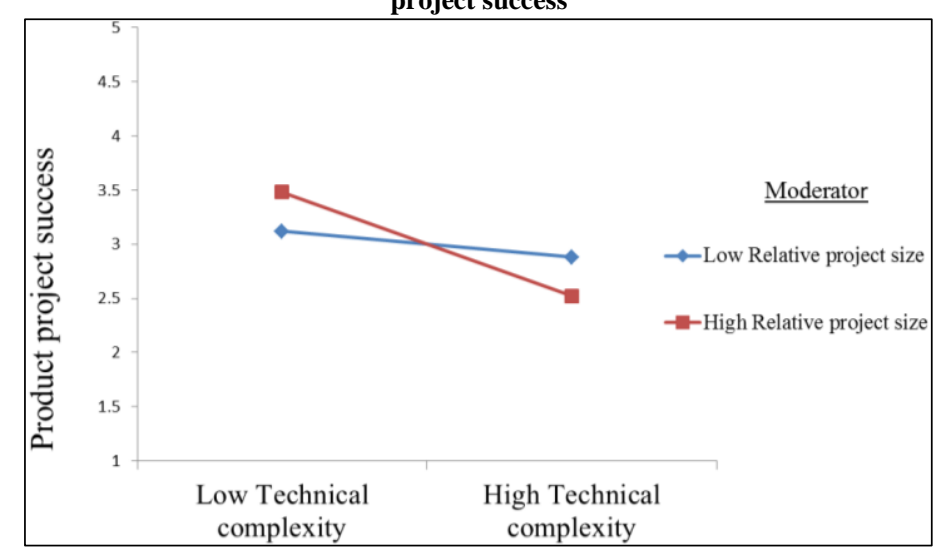

Relative project size strengthens the negative relationship between Technical complexity and Product project success.
Interaction effects of relative project size and top level management support (TMS) on product project success

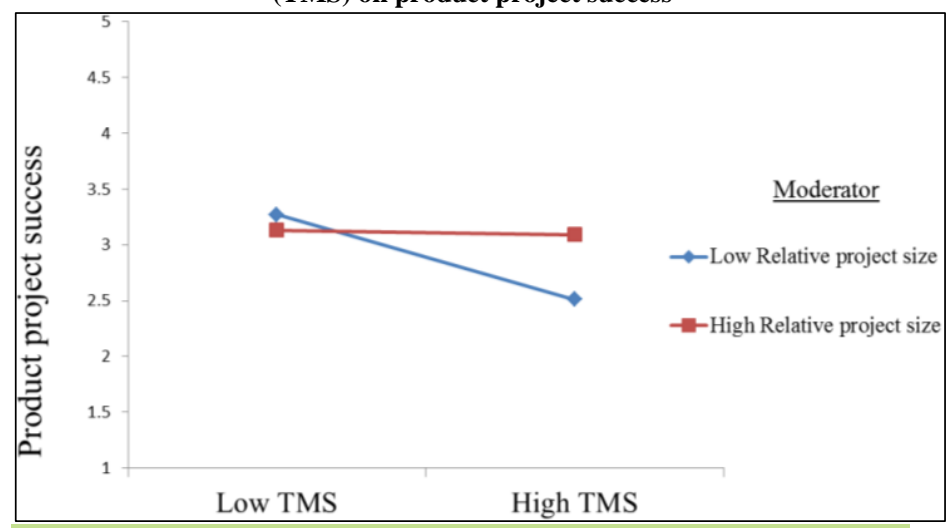

Relative project size dampens the negative relationship between TMS and Product project success.

Interaction effects of relative project size and vision and mission on product project success

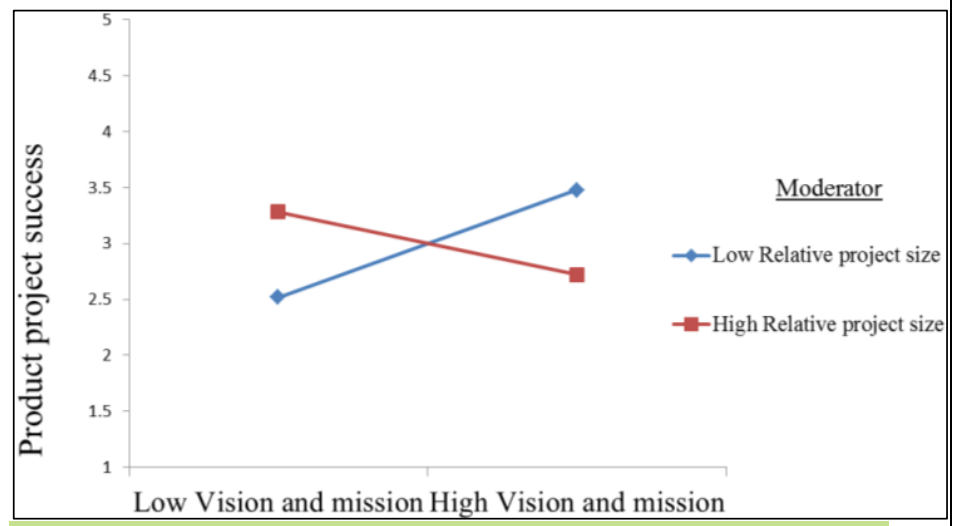

Relative project size dampens the positive relationship between Vision and mission and Product project success.

Interaction effects of relative project size and specification changes on

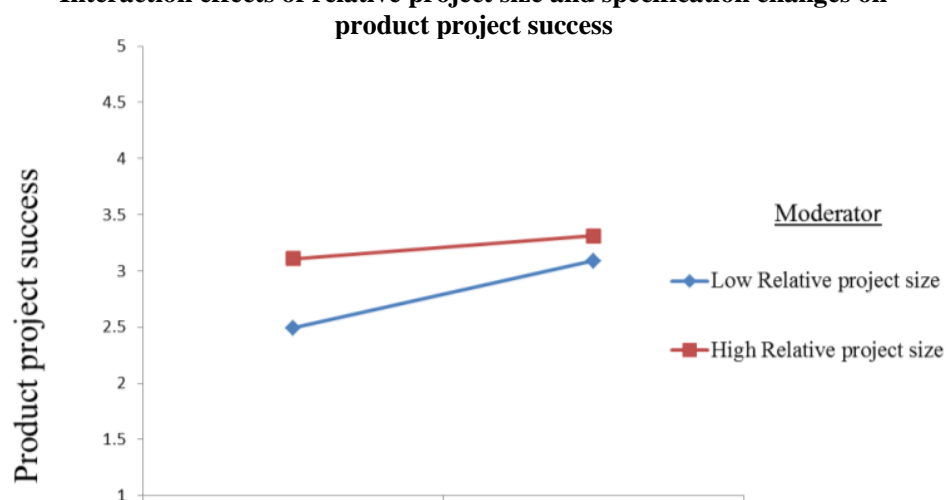

Low Specification High Specification changes changes

Relative project size dampens the positive relationship between Specification changes and Product project success.

Source: Primary data. Note: "Low" is defined as one standard deviation below the mean; "High" is defined as one standard deviation above the mean. For clarity only significant interaction effects i.e. with graphs that have different gradients (slopes) are presented.

\section{Figure 40: Interaction effects of relative project size and candidate CSFs on project success}




\subsubsection{Testing the interaction effects of specification changes and candidate CSFs on project success}

This section tests the moderating effects of specification changes on the relationships between all the candidate CSFs and project success in the model. Again, in model 1, all 17 model predictor variables were entered and analyzed simultaneously. The overall model fit was adequate as indicated by the fit statistics $(\chi 2=12785.543, d f=2935, \chi 2 / d f=4.356, p>.05$, $\mathrm{TLI}=.871, \mathrm{CFI}=.880, \mathrm{RMSEA}=.050)$.

In model 2, all the predictor constructs of model 1 together with the interaction effects of specification were specified and estimated simultaneously in a SEM. The model fit statistics were $\chi^{2}=28784.526, d f=5927, \chi^{2 / d f}=4.857, p<.05, C F I=.777, R M S E A=.053$. Thus, both models show acceptable fit indices indicating their overall fit. The chi-square difference between models $\left(\Delta \chi^{2}\right)$ is 15998.983 with 2992 degrees of freedom. This is significant ( $\mathrm{p}<.05$ ), indicating that model 1 (without interaction terms) fits the data better than model 2 (with interaction effects) and thus, there is a significant interaction effect.

Table 80 depicts the summary results from testing specification changes as a moderator. The results indicate that specification change only negatively interacts with technical complexity but positively interacts with user support to influence process success. In addition, specification changes interacts with top level management support, user participation, project team composition to positively influence product success as well as interacting with planning and controlling, technical complexity, relative project size to negatively influence product success. These findings statistically support that specification changes interacts with those candidate CSFs (interaction effects marked with asterisks*) to influence process/product success. 
Table 80: Hierarchical SEM analysis of interaction terms of specification changes

\begin{tabular}{|c|c|c|c|c|}
\hline \multirow[b]{2}{*}{ Model constructs } & \multicolumn{2}{|c|}{$\begin{array}{l}\text { Process success as the dependent } \\
\text { variable }(n=984)\end{array}$} & \multicolumn{2}{|c|}{$\begin{array}{l}\text { Product success as the dependent } \\
\text { variable }(n=984)\end{array}$} \\
\hline & $\begin{array}{c}\text { SEM } 1 \text { (without } \\
\text { interaction } \\
\text { variables) }\end{array}$ & $\begin{array}{c}\text { SEM } 2 \text { (with } \\
\text { interaction } \\
\text { variables) }\end{array}$ & $\begin{array}{c}\text { SEM } 1 \text { (without } \\
\text { interaction } \\
\text { variables) }\end{array}$ & $\begin{array}{l}\text { SEM } 2 \text { (with } \\
\text { interaction } \\
\text { variables) }\end{array}$ \\
\hline Top level management support & $-.093^{*}$ & $-.098 *$ & -.024 & -.023 \\
\hline Organizational culture & $.102 *$ & $.110 *$ & -.007 & -.009 \\
\hline Internal project communication & $.105 *$ & $.114 *$ & $.104 * *$ & $.107 *$ \\
\hline User participation & $.087 *$ & $.102 *$ & -.026 & -.043 \\
\hline Dev. Team expertise & -.047 & -.053 & -.041 & -.029 \\
\hline User experience & .049 & .055 & .049 & .045 \\
\hline Planning and controlling & $.112 * *$ & $.118^{*}$ & -.023 & .009 \\
\hline Change management & .054 & .042 & .032 & .001 \\
\hline Leadership characteristics & $.151 * * *$ & $.159 * * *$ & .039 & .037 \\
\hline Vision and mission & -.016 & -.020 & .045 & .046 \\
\hline Project team composition & $.124 *$ & .104 & $.106^{*}$ & $.113^{*}$ \\
\hline User support & $.158 *$ & $.150 *$ & .076 & .071 \\
\hline Relative project size & $-.200 * * *$ & $-.202 * * *$ & -.014 & -.009 \\
\hline Specification changes & $-.085^{*}$ & $-.079 *$ & $.080 * *$ & $.097 *$ \\
\hline Technical complexity & $-.075^{*}$ & $-.082 * *$ & $.079 *$ & $.071 *$ \\
\hline Specification changes*Top level management support & & $-.016 n s$ & & $.038 *$ \\
\hline Specification changes* Organizational culture & & $-.036 n s$ & & $.064 n s$ \\
\hline Specification changes*Internal project communication & & $.014 n s$ & & $-.014 n s$ \\
\hline Specification changes*User participation & & $-.001 n s$ & & $.045^{*}$ \\
\hline Specification changes $*$ Project team commitment & & $-.002 n s$ & & $.010 n s$ \\
\hline Specification changes*Dev. Team skills & & $.041 n s$ & & $.045 n s$ \\
\hline Specification changes*User experience & & $-.032 n s$ & & $-.054 n s$ \\
\hline Specification changes*Planning and controlling & & $.037 n s$ & & $-.117 * * *$ \\
\hline Specification changes*Change management & & $-.043 n s$ & & $-.023 n s$ \\
\hline Specification changes*Leadership characteristics & & $-.001 n s$ & & $-.019 n s$ \\
\hline Specification changes $*$ Vision and mission & & $-.004 n s$ & & $-.022 n s$ \\
\hline Specification changes*Team composition & & $-.002 n s$ & & $.049 *$ \\
\hline Specification changes*Technical complexity & & $-.066^{*}$ & & $-.098 * * *$ \\
\hline Specification changes*User support & & $.106 *$ & & $-.017 n s$ \\
\hline Note: Standardized regression coefficients are reporte & $\begin{array}{l}\text { gnificant in the int } \\
\text { respectively. }\end{array}$ & ion model; * & significant at 0.0 & and 0.001 leve \\
\hline
\end{tabular}

Next, based on Dawson's (2014) guidelines, moderation graphs were generated to show the significant interaction effects of specification changes and candidate CSFs on project success. The results are summarised in Figure 41. 


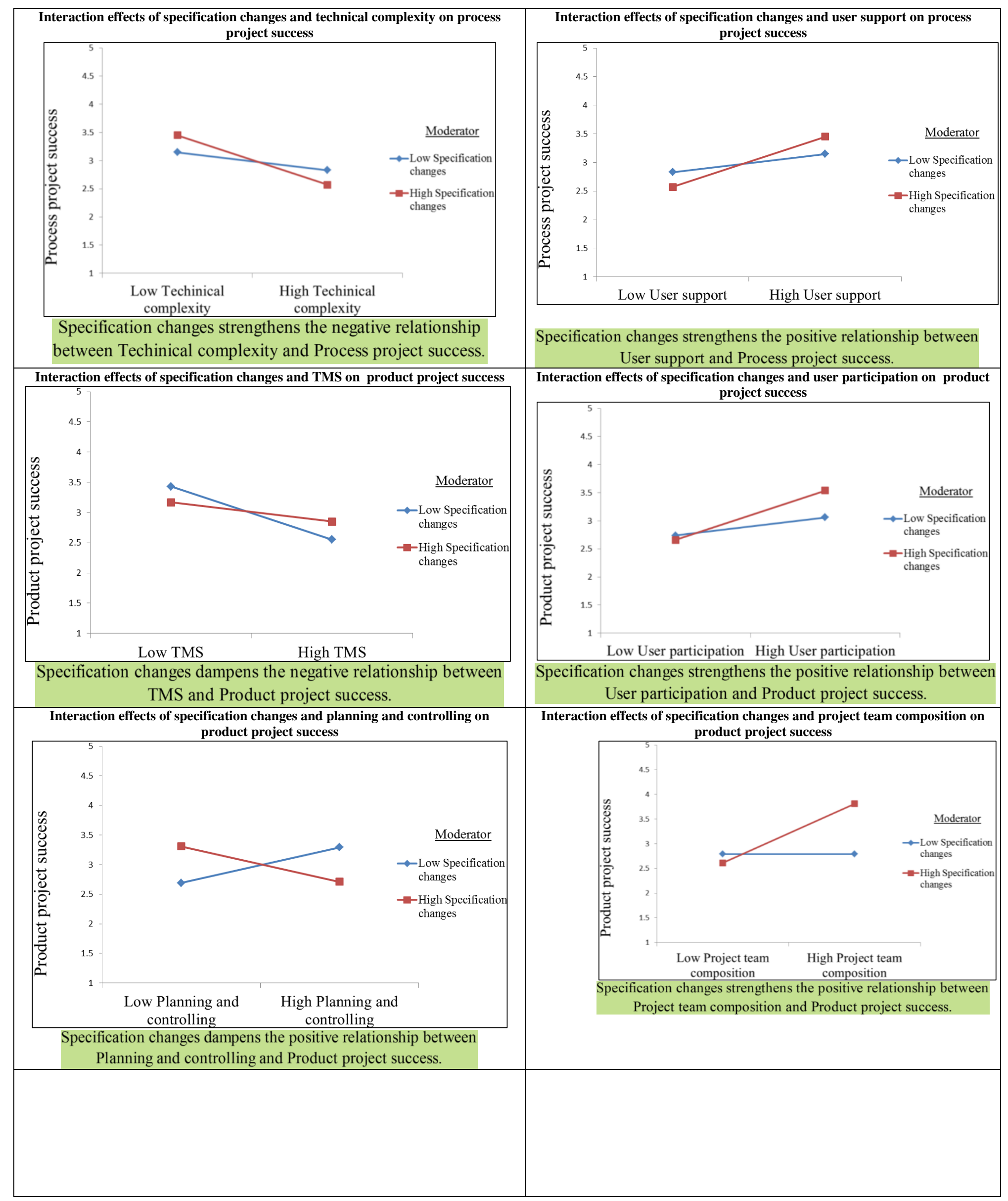




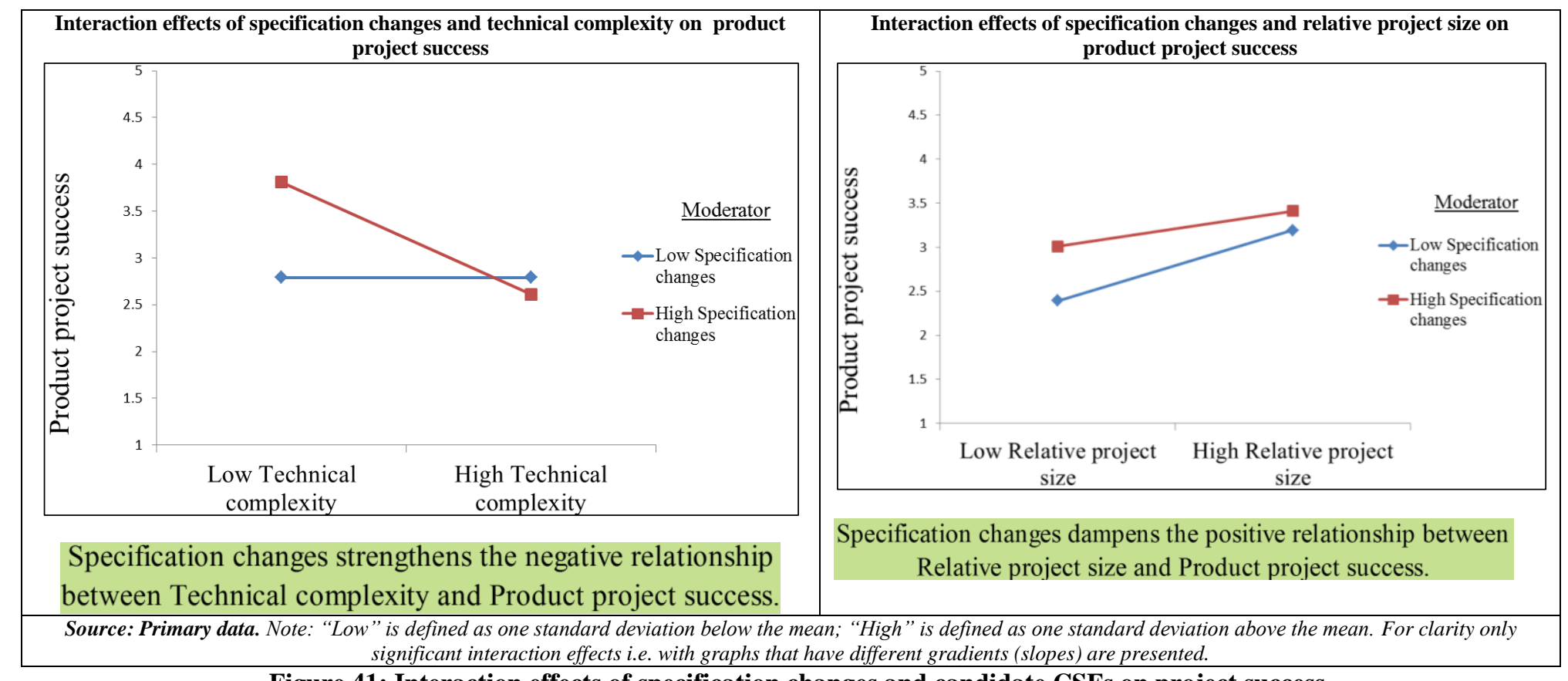

Figure 41: Interaction effects of specification changes and candidate CSFs on project success

It is clear that the effects of CSFs on project success differ, depending on the level of specification changes; since, the graphs have different slopes and hence, are not parallel (Aiken \& West, 1991; Dawson, 2014). Thus, the magnitude of these candidate CSFs on project success is significantly influenced by level of specification changes. These findings support hypothesis (H23).

\subsection{Chapter summary}

This chapter presented the results and findings from the main study undertaken after pilot testing the research instrument. Specifically, the chapter presents the descriptive statistics for the complete data set and sub-group comparisons. Subsequently, SEM analyses were conducted in a two-step analysis of the measurement and structural models. The analysis of the measurement model demonstrated good reliability and validity for both single factor models and combined measurement models. The reflective items loaded very cleanly onto the intended constructs and all tested highly reliable. When all the items were tested together in the measurement model, they were all strong and significant at $p<.001$. The analysis of the measurement model demonstrated that the scales used during the main study advanced the findings of the pilot study. All construct measures indicated strong and significant item loading weights supporting one factor solution for each factor.

The only exception was for project criticality which did not fit the single congeneric model as indicated by the model fit indices which were all below the acceptable criteria despite several modifications. The factor loading values were also very low suggesting that both items 
neither contributed relatively nor absolutely to the project criticality construct. Therefore, project criticality was completely dropped from further data analysis since at least 2 factor loadings per construct should be higher than 0.60 in CFAs. Additionally, a single factor model for project success indicated that a two factor solution was the best fit and project success was subsequently divided into process and product success. These two factor models fitted the data well and were subsequently used separately in all the analysis instead of using a global measure of project success.

The structural model assessment demonstrated an overall good fit of the research models to both sample data sets. All the variances explained were constantly high for both structural models of traditional plan-based and agile methodology projects. This indicates high predictive validity for the research models and confirms that both models capture a large variation of candidate CSFs impacting on project success.

Table 81 shows hypotheses (with direct link to project success) that were supported. 
Table 81: Summary of supported hypotheses

\begin{tabular}{|c|c|c|c|c|c|c|c|c|c|}
\hline \multirow{2}{*}{\multicolumn{4}{|c|}{ Hypothesis and the structural path }} & \multicolumn{2}{|c|}{\begin{tabular}{|c|}
$\begin{array}{c}\text { Traditional plan-based } \\
\text { methodology }(\mathrm{T}) \\
\mathrm{n}=513\end{array}$ \\
\end{tabular}} & \multicolumn{2}{|c|}{$\begin{array}{c}\begin{array}{c}\text { Agile methodology } \\
\text { data set (A) } \\
\mathbf{n}=471\end{array} \\
\end{array}$} & \multicolumn{2}{|c|}{$\begin{array}{c}\text { Comparison hypothesis, } \\
\text { TA } \\
\text { (statistical difference test) } \\
\end{array}$} \\
\hline & & & & \begin{tabular}{|c|}
$\begin{array}{c}\text { Hypothesized } \\
\text { relationship }\end{array}$ \\
\end{tabular} & Support & \begin{tabular}{|c|}
$\begin{array}{c}\text { Hypothesized } \\
\text { relationship }\end{array}$ \\
\end{tabular} & Support & \begin{tabular}{|c|}
$\begin{array}{c}\text { Hypothesized } \\
\text { relationship }\end{array}$ \\
\end{tabular} & Support \\
\hline \multirow{2}{*}{$\begin{array}{l}H_{1(i)} \\
H_{1(i i)}\end{array}$} & Top mgt. support & $\rightarrow$ & PS (process) & + & No & + & $\mathrm{No}^{\mathrm{a}}$ & $\mathrm{A}+>\mathrm{T}$ & $\mathrm{No}^{\mathrm{a}}$ \\
\hline & Top mgt. support & $\rightarrow$ & PS (product) & + & No & + & No & $\mathrm{A}+>\mathrm{T}$ & No \\
\hline \multirow{2}{*}{$\begin{array}{l}\boldsymbol{H}_{2(i)} \\
\boldsymbol{H}_{2(i i)}\end{array}$} & Organizational culture & $\rightarrow$ & PS(process) & \# & Yes & + & Yes & $\mathrm{A}+>\mathrm{T}$ & No \\
\hline & Organizational culture & $\rightarrow$ & PS (product) & \# & Yes & + & Yes & $\mathrm{A}+>\mathrm{T}$ & No \\
\hline \multirow{2}{*}{$\begin{array}{l}\boldsymbol{H}_{3(i)} \\
\boldsymbol{H}_{3(i i)}\end{array}$} & Planning and controlling & $\rightarrow$ & PS (process) & + & Yes & \# & Yes & $\mathrm{T}+>\mathrm{A}$ & Yes \\
\hline & Planning and controlling & $\rightarrow$ & PS (product) & + & No & $\#$ & Yes & $\mathrm{T}+>\mathrm{A}$ & No \\
\hline \multirow{2}{*}{$\begin{array}{l}H_{4(i)} \\
H_{4(i i)} \\
\end{array}$} & Vision and mission & $\rightarrow$ & PS (process) & + & Yes & \# & Yes & $\mathrm{T}+>\mathrm{A}$ & No \\
\hline & Vision and mission & $\rightarrow$ & PS (product) & + & No & $\#$ & Yes & $\mathrm{T}+>\mathrm{A}$ & No \\
\hline \multirow{2}{*}{$\begin{array}{l}H_{5(i)} \\
H_{5(i i)} \\
\end{array}$} & Change management & $\rightarrow$ & PS (process) & + & Yes & + & Yes & $\mathrm{A}+>\mathrm{T}$ & No \\
\hline & Change management & $\rightarrow$ & PS (product) & + & Yes & + & Yes & $\mathrm{A}+>\mathrm{T}$ & Yes \\
\hline \multirow{2}{*}{$\begin{array}{l}H_{6(i)} \\
H_{6(i i)}\end{array}$} & Leadership characteristics & $\rightarrow$ & PS (process) & + & Yes & + & Yes & $\mathrm{A}+>\mathrm{T}$ & Yes \\
\hline & Leadership characteristics & $\rightarrow$ & PS (product) & + & No & + & No & $\mathrm{A}+>\mathrm{T}$ & No \\
\hline \multirow{2}{*}{$\begin{array}{l}\boldsymbol{H}_{7(i)} \\
\boldsymbol{H}_{7(i i)}\end{array}$} & Int. proj. communication & $\rightarrow$ & PS (process) & + & Yes & + & Yes & $\mathrm{A}+>\mathrm{T}$ & No \\
\hline & Int. proj. communication & $\rightarrow$ & PS (product) & + & Yes & + & Yes & $\mathrm{A}+>\mathrm{T}$ & No \\
\hline \multirow{2}{*}{$\begin{array}{l}\boldsymbol{H}_{8(i)} \\
\boldsymbol{H}_{8(i i)} \\
\end{array}$} & Proj. team commitment & $\rightarrow$ & PS (process) & + & Yes & + & Yes & $\mathrm{A}+>\mathrm{T}$ & No \\
\hline & Proj. team commitment & $\rightarrow$ & PS (product) & + & Yes & + & Yes & $\mathrm{A}+>\mathrm{T}$ & No \\
\hline \multirow{2}{*}{$\begin{array}{l}\boldsymbol{H}_{9_{(i)}} \\
\boldsymbol{H}_{9(i i)}\end{array}$} & Devt. team expertise & $\rightarrow$ & PS (process) & + & Yes & + & Yes & $\mathrm{A}+>\mathrm{T}$ & No \\
\hline & Devt. team expertise & $\rightarrow$ & PS (product & + & No & + & Yes & $\mathrm{A}+>\mathrm{T}$ & No \\
\hline \multirow{2}{*}{$\begin{array}{l}H_{10(i)} \\
H_{10(i i)} \\
\end{array}$} & Proj. team composition & $\rightarrow$ & PS (process) & + & Yes & + & Yes & $\mathrm{A}+>\mathrm{T}$ & Yes \\
\hline & Proj. team composition & $\rightarrow$ & PS (product) & + & Yes & + & Yes & $\mathrm{A}+>\mathrm{T}$ & Yes \\
\hline \multirow{2}{*}{$\begin{array}{l}H_{11(i)} \\
H_{11(i i)} \\
\end{array}$} & User participation & $\rightarrow$ & PS (process) & $\#$ & Yes & + & Yes & $\mathrm{A}+>\mathrm{T}$ & Yes \\
\hline & User participation & $\rightarrow$ & PS (product) & $\#$ & Yes & + & $\mathrm{No}^{\mathrm{a}}$ & $\mathrm{A}+>\mathrm{T}$ & $\mathrm{No}^{\mathrm{a}}$ \\
\hline \multirow{2}{*}{$\begin{array}{l}H_{12(i)} \\
H_{12(i i)} \\
\end{array}$} & User support & $\rightarrow$ & PS (process) & $\#$ & Yes & + & Yes & $\mathrm{A}+>\mathrm{T}$ & No \\
\hline & User support & $\rightarrow$ & PS (product) & $\#$ & Yes & + & Yes & $\mathrm{A}+>\mathrm{T}$ & Yes \\
\hline \multirow{2}{*}{$\begin{array}{l}H_{13(i)} \\
H_{13(i i)}\end{array}$} & User experience & $\rightarrow$ & PS (process) & $\#$ & Yes & + & Yes & $\mathrm{A}+>\mathrm{T}$ & No \\
\hline & User experience & $\rightarrow$ & PS (product) & $\#$ & Yes & + & Yes & $\mathrm{A}+>\mathrm{T}$ & No \\
\hline \multirow{2}{*}{$\begin{array}{l}H_{14(i)} \\
H_{14(i i)} \\
\end{array}$} & Technological uncertainty & $\rightarrow$ & PS (process) & - & Yes & - & Yes & $\mathrm{T}->\mathrm{A}$ & Yes \\
\hline & Technological uncertainty & $\rightarrow$ & PS (product) & - & Yes & - & Yes & $\mathrm{T}->\mathrm{A}$ & Yes \\
\hline \multirow{2}{*}{$\begin{array}{l}H_{15(i)} \\
H_{15(i i)}\end{array}$} & \begin{tabular}{|l|} 
Technical complexity \\
\end{tabular} & $\rightarrow$ & PS (process) & - & No & - & Yes & $\mathrm{A}->\mathrm{T}$ & $\mathrm{No}^{\mathrm{a}}$ \\
\hline & Technical complexity & $\rightarrow$ & PS (product) & - & Yes & - & No & $\mathrm{A}->\mathrm{T}$ & $\mathrm{No}^{\mathrm{a}}$ \\
\hline \multirow{2}{*}{$\begin{array}{l}H_{16(i)} \\
H_{16(i i)} \\
\end{array}$} & Relative project size & $\rightarrow$ & PS (process) & - & Yes & - & Yes & $\mathrm{A}->\mathrm{T}$ & Yes \\
\hline & Relative project size & $\rightarrow$ & PS (product) & - & No & - & No & $\mathrm{A}->\mathrm{T}$ & No \\
\hline \multirow{2}{*}{$\begin{array}{l}H_{17(i)} \\
H_{17(i i)} \\
\end{array}$} & Specification changes & $\rightarrow$ & PS (process) & - & Yes & - & Yes & $\mathrm{T}->\mathrm{A}$ & No \\
\hline & Specification changes & $\rightarrow$ & PS (product) & - & $\mathrm{No}^{\mathrm{a}}$ & - & $\mathrm{No}^{\mathrm{a}}$ & $\mathrm{T}->\mathrm{A}$ & $\mathrm{No}^{\mathrm{a}}$ \\
\hline \multirow{2}{*}{$\begin{array}{l}\mathrm{H}_{18(i)} \\
\mathrm{H}_{18(i i)}\end{array}$} & Project criticality & $\rightarrow$ & PS (process) & - & $\mathrm{x}$ & - & $\mathrm{x}$ & $\mathrm{A}->\mathrm{T}$ & $\mathrm{x}$ \\
\hline & Project criticality & $\rightarrow$ & PS (product) & - & $\mathrm{x}$ & - & $\mathrm{x}$ & $\mathrm{A}->\mathrm{T}$ & $\mathrm{x}$ \\
\hline \multicolumn{10}{|c|}{$\begin{array}{l}\text { PS-Project success (process and product), +positive hypothesis, -negative hypothesis, < less effect, >greater effect, } \\
\text { \# insignificant path, } x \text {-construct dropped and excluded from SEM analysis due to poor psychometric measurement properties, } \\
\qquad=\text { significant but not as hypothesised }\end{array}$} \\
\hline
\end{tabular}

Group invariance analysis was conducted using SEM to test for any differences between the two projects management methodologies, by essentially comparing the same model across different samples of respondents. SEM demonstrated to be a more suitable approach due to its ability to model multiple indicators as well as controlling confounding effects. The SEM group comparison framework was subdivided into measurement and structural invariance. The measurement invariance testing process demonstrated that all the 19 latent constructs and the respective observed variables used across the two project types models in this study met 
the criteria for configural invariance, full metric invariance, partial scalar invariance, covariance and variance invariance.

Subsequently, structural invariance group comparisons were conducted without any concern that the differences are due to differing measurement properties (measurement error) between the groups but rather to the inherent idiosyncratic structural differences that exist within the two groups. A statistically significant $\chi 2$ difference between the unconstrained and structural weights models indicated that the path coefficients were different across the two groups; suggesting that project management methodology moderates some relationships between candidate CSFs and project success. The significant moderating effects were assessed, plotted and demonstrated using moderation graphs. The plotted moderation graphs have different slopes and hence, are not parallel. This provides support that the effect of these candidate CSFs on project success differs as a function of project management methodology (that is, traditional plan-based and agile methodology projects). Table 82 summarises path coefficients that were found to significantly differ across the two groups.

Table 82: Summary of path coefficients that differ across the two methodology groups

\begin{tabular}{|c|c|c|c|}
\hline \multicolumn{3}{|c|}{$\begin{array}{l}\text { Structural path } \\
\end{array}$} & \multirow{2}{*}{$\begin{array}{r}\text { Difference } \\
\text { Sig. }\end{array}$} \\
\hline H1(i): Top mgt. support & $\rightarrow$ & PS(process) & \\
\hline H1(ii): Top mgt. support & $\rightarrow$ & PS(product) & $\#$ \\
\hline H2(i): Organizational culture & $\rightarrow$ & PS(process) & \# \\
\hline H2(ii): Organizational culture & $\rightarrow$ & PS(product) & \# \\
\hline H3(i): Planning and controlling & $\rightarrow$ & PS(process) & Sig. \\
\hline H3(ii): Planning and controlling & $\rightarrow$ & PS(product) & \# \\
\hline H4(i): Vision and mission & $\rightarrow$ & PS(process) & \# \\
\hline H4(ii): Vision and mission & $\rightarrow$ & PS(product) & \# \\
\hline H5(i): Change mgt. & $\rightarrow$ & PS(process) & $\#$ \\
\hline H5(ii): Change mgt. & $\rightarrow$ & PS(product) & Sig. \\
\hline H6(i): Leadership characteristics & $\rightarrow$ & PS(process) & Sig. \\
\hline H6(ii): Leadership characteristics & $\rightarrow$ & PS(product) & $\#$ \\
\hline H7 (i): Int. proj. communication & $\rightarrow$ & PS(process) & \# \\
\hline H7 (ii): Int. proj. communication & $\rightarrow$ & PS(product) & \# \\
\hline H8 (i): Proj. team commitment & $\rightarrow$ & PS(process) & \# \\
\hline H8 (ii): Proj. team commitment & $\rightarrow$ & PS(product) & \# \\
\hline H9 (i): Devt. team expertise & $\rightarrow$ & PS(process) & \# \\
\hline H9 (ii): Devt. team expertise & $\rightarrow$ & PS (product & \# \\
\hline H10 (i): Proj. team composition & $\rightarrow$ & PS(process) & Sig. \\
\hline H10 (ii): Proj. team composition & $\rightarrow$ & PS (product) & Sig. \\
\hline H11(i): User participation & $\rightarrow$ & PS (process) & Sig. \\
\hline H11(ii): User participation & $\rightarrow$ & PS(product) & Sig. \\
\hline H12(i): User support & $\rightarrow$ & PS(process) & $\#$ \\
\hline H12(ii): User support & $\rightarrow$ & PS(product) & Sig. \\
\hline H13(i): User experience & $\rightarrow$ & PS(process) & $\#$ \\
\hline H13(ii): User experience & $\rightarrow$ & PS(product) & \# \\
\hline H14(i): Technological uncertainty & $\rightarrow$ & PS(process) & Sig. \\
\hline H14(ii): Technological uncertainty & $\rightarrow$ & PS(product) & Sig. \\
\hline H15(i): Technical complexity & $\rightarrow$ & PS(process) & Sig. \\
\hline H15(ii): Technical complexity & $\rightarrow$ & PS(product) & Sig. \\
\hline H16(i): Relative project size & $\rightarrow$ & PS(process) & Sig. \\
\hline H16(ii): Relative project size & $\rightarrow$ & PS (product) & $\#$ \\
\hline H17(i): Specification changes & $\rightarrow$ & PS(process) & \# \\
\hline H17(ii): Specification changes & $\rightarrow$ & PS(product) & Sig. \\
\hline
\end{tabular}


The last section involved testing for interaction effects among candidate CSFs and examining their impact on project success. In each case, two SEM models were specified and estimated, one in which the formed interaction variables were present as indicators (interaction effects model), the other in which they were absent (main effects model). An identical structural path diagram was used to estimate the baseline models while in the second model interaction terms were added. The two SEM models (model 1 without interaction terms and model 2 with interaction terms) were tested hierarchically and the chi-square differences $(\Delta \chi 2)$ between these models together with their respective degrees of freedom were computed using the model fit statistics to determine whether interaction effects were significant or not.

Using Dawson's (2014) guidelines, moderation graphs were plotted for two way interactions to show the significant interaction effects of candidate CSFs on project success. The combination of some candidate CSFs confirmed to have significant interactive effects in influencing project success, hence providing support for moderating effects. It was also established, however, that the interactive terms of some candidate CSFs are inconsequential and cannot influence project success in software development. Table 83 summarises the significant interaction effects for continuous moderators (technological uncertainty, technical complexity, relative project and specification changes). 
Table 83: Summarized significant moderating effects and supported moderation hypotheses

\begin{tabular}{|c|c|c|c|c|c|c|c|}
\hline \multirow{2}{*}{$\frac{\text { Construct }}{\text { Top mgt. support }}$} & \multicolumn{2}{|c|}{ Structural path } & \multirow{2}{*}{$\begin{array}{c}\text { Technological } \\
\text { uncertainty } \\
\text { (interaction) } \\
\left(H_{20}\right)\end{array}$} & \multirow{2}{*}{$\begin{array}{c}\text { Technical } \\
\text { Complexity } \\
\text { (interaction) } \\
\left(\mathrm{H}_{21}\right) \\
\# \\
\end{array}$} & \multirow{2}{*}{$\begin{array}{c}\text { Relative } \\
\text { project size } \\
\text { (interaction) } \\
\left(H_{22}\right) \\
\#\end{array}$} & \multirow{2}{*}{$\begin{array}{c}\begin{array}{c}\text { Specification } \\
\text { changes } \\
\text { (interaction) } \\
\left(H_{23}\right)\end{array} \\
\#\end{array}$} & \multirow{2}{*}{$\begin{array}{c}\begin{array}{c}\text { Project } \\
\text { criticality }\end{array} \\
\text { (interaction) } \\
\left(\boldsymbol{H}_{24}\right)\end{array}$} \\
\hline & $\rightarrow$ & PS (process) & & & & & \\
\hline Top mgt. support & $\rightarrow$ & PS (product) & $\#$ & \# & - & + & $\mathrm{x}$ \\
\hline Organizational culture & $\rightarrow$ & PS(process) & $\#$ & $\#$ & $\#$ & $\#$ & $\mathrm{x}$ \\
\hline Organizational culture & $\rightarrow$ & PS (product) & $\#$ & $\#$ & $\#$ & $\#$ & $\mathrm{x}$ \\
\hline Planning and controlling & $\rightarrow$ & PS (process) & \# & \# & \# & \# & $\mathrm{X}$ \\
\hline Planning and controlling & $\rightarrow$ & PS (product) & - & - & \# & - & $\mathrm{X}$ \\
\hline Vision and mission & $\rightarrow$ & PS (process) & $\#$ & $\#$ & $\#$ & \# & $\mathrm{x}$ \\
\hline Vision and mission & $\rightarrow$ & PS (product) & $\#$ & $\#$ & - & \# & $\mathrm{X}$ \\
\hline Change management & $\rightarrow$ & PS (process) & $\#$ & - & $\#$ & $\#$ & $\mathrm{x}$ \\
\hline Change management & $\rightarrow$ & PS (product) & $\#$ & $\#$ & - & $\#$ & $\mathrm{x}$ \\
\hline Leadership characteristics & $\rightarrow$ & PS (process) & $\#$ & - & $\#$ & $\#$ & $\mathrm{x}$ \\
\hline Leadership characteristics & $\rightarrow$ & PS (product) & $\#$ & \# & $\#$ & \# & $\mathrm{x}$ \\
\hline Int. proj. communication & $\rightarrow$ & PS (process) & $\#$ & \# & $\#$ & \# & $\mathrm{x}$ \\
\hline Int. proj. communication & $\rightarrow$ & PS (product) & $\#$ & \# & $\#$ & \# & $\mathrm{x}$ \\
\hline Proj. team commitment & $\rightarrow$ & PS (process) & $\#$ & - & $\#$ & \# & $\mathrm{x}$ \\
\hline Proj. team commitment & $\rightarrow$ & PS (product) & + & \# & $\#$ & $\#$ & $\mathrm{x}$ \\
\hline Devt. team expertise & $\rightarrow$ & PS (process) & $\#$ & - & $\#$ & $\#$ & $\mathrm{x}$ \\
\hline Devt. team expertise & $\rightarrow$ & PS (product & $\#$ & \# & $\#$ & $\#$ & $\mathrm{x}$ \\
\hline Proj. team composition & $\rightarrow$ & PS (process) & $\#$ & \# & $\#$ & $\#$ & $\mathrm{x}$ \\
\hline Proj. team composition & $\rightarrow$ & PS (product) & + & \# & $\#$ & + & $\mathrm{X}$ \\
\hline User participation & $\rightarrow$ & PS (process) & $\#$ & + & \# & $\#$ & $\mathrm{X}$ \\
\hline User participation & $\rightarrow$ & PS (product) & $\#$ & $\#$ & $\#$ & + & $\mathrm{X}$ \\
\hline User support & $\rightarrow$ & PS (process) & + & + & + & + & $\mathrm{X}$ \\
\hline User support & $\rightarrow$ & PS (product) & $\#$ & $\#$ & $\#$ & $\#$ & $\mathrm{x}$ \\
\hline User experience & $\rightarrow$ & PS (process) & $\#$ & \# & $\#$ & \# & $\mathrm{x}$ \\
\hline User experience & $\rightarrow$ & PS (product) & $\#$ & $\#$ & $\#$ & $\#$ & $\mathrm{x}$ \\
\hline Technological uncertainty & $\rightarrow$ & PS (process) & $\mathrm{Na}$ & - & \# & \# & $\mathrm{x}$ \\
\hline Technological uncertainty & $\rightarrow$ & PS (product) & $\mathrm{Na}$ & $\#$ & $\#$ & $\#$ & $\mathrm{x}$ \\
\hline Technical complexity & $\rightarrow$ & PS (process) & $\#$ & $\mathrm{Na}$ & $\#$ & - & $\mathrm{x}$ \\
\hline Technical complexity & $\rightarrow$ & PS (product) & $\#$ & $\mathrm{Na}$ & - & - & $\mathrm{x}$ \\
\hline Relative project size & $\rightarrow$ & PS (process) & $\#$ & $\#$ & $\mathrm{Na}$ & $\#$ & $\mathrm{x}$ \\
\hline Relative project size & $\rightarrow$ & PS (product) & - & - & $\mathrm{Na}$ & - & $\mathrm{x}$ \\
\hline Specification changes & $\rightarrow$ & PS (process) & $\#$ & $\#$ & $\#$ & $\mathrm{Na}$ & $\mathrm{x}$ \\
\hline Specification changes & $\rightarrow$ & PS (product) & \# & - & - & $\mathrm{Na}$ & $\mathrm{x}$ \\
\hline
\end{tabular}




\section{CHAPTER SIX: DISCUSSION OF FINDINGS}

\subsection{Introduction}

The previous chapter presented the results and findings from the main study. This chapter presents the discussion of findings. It provides a detailed explanation of the statistical analyses conducted in the previous chapter and discusses how the results obtained relate to both the theoretical foundations and empirical findings in the existing literature. The following section discusses and compares hypotheses testing for the two SEM models for both projects that used traditional plan-based and agile methodologies.

\subsection{Hypotheses testing results-Group comparisons}

This study builds on project contingency theory which argues that not all projects have the same characteristics (Howell et al., 2010), and therefore, they should be managed differently. As earlier noted, one of the many factors proposed as to why software projects fail is the inappropriate choice of a project management methodology (Sauser et al., 2009; Murad \& Cavana, 2012).

\subsubsection{H1: Top level management support (TMS) $\rightarrow$ project success}

Overall, H1 was not supported for both data sets. The results from testing for significant differences of coefficients across the two data sets indicate that the importance of TMS on project success is not greater for projects managed with an agile methodology than for projects that used traditional plan-based methodologies.

When using the data set from projects that used the traditional plan-based methodology, there were no significant relationships between TMS and both process and product project success, thereby failing to provide support for both $H 1(i)(T)$ and $H 1(i i)(T)$ respectively. These results were not as expected. This may be partly because it was the vendor reporting perceptions of the client, and reflects a different construct to an employee reporting their own perceptions of their own organization.

TMS has often been reported in empirical studies as a candidate CSF for software development projects. For instance, in a case based study, Kandelousi et al (2011) found that TMS impacted on projects managed with a traditional plan-based methodology and in a survey conducted by White and Fortune (2006) to determine the current practice in project management, support of TMS ranked third out of 23 CSFs listed. The research done by Young and Jordan (2008) also concluded that TMS had a positive impact on traditional 
project success. Jung et al. (2008) findings provided support for the hypothesis that TMS was a significant predictor of project performance. However, these aforementioned studies focused on "in-house" development projects where developers and users were members of the same organization, rather than the vendor's perspective employed in this study.

Nonetheless, TMS was found to indirectly and positively influence process success through planning and controlling. This means that from a vendor perspective, the TMS only contributes towards setting project plans and controls which if adhered to can improve project success.

For the projects that adopted an agile methodology, $H 1(i)(A)$ and $H 1(i i)(A)$ which theorised a significant positive relationships between TMS and both process and product project success respectively were also not supported. Instead, the results suggest that for agile methodology projects, there is a significant negative association between TMS and process project success, but an insignificant association between TMS and product project success. Although these findings seem surprising, they are not new. Chow and Cao's (2008) study of 906 agile software projects did not find TMS as a significant CSF that contributes to the success of projects managed with an agile methodology. However, Chow and Cao's (2008) study did not distinguish between client and vendor perspectives. On the other hand, however, these findings are inconsistent with other studies (e.g. Wan \& Wang, 2010; Sheffield \& Lemetayer, 2013 Imreh \& Raisinghani, 2011). However, none of these studies focused on the narrow vendor's perspective used in this study.

Nonetheless, the results in both models indicate positive and significant associations of TMS with internal project communication, project team commitment and organizational culture. This seems to raise the possibility that TMS has indirect influence on project success, based on the perceptions of vendor respondents with regard to their client organizations.

Further, although TMS is generally viewed as a positive factor, some researchers argue that what begins as support can easily turn into interference, which is considered counterproductive and detrimental especially in highly innovative environments (Young \& Jordan, 2008). This is also consistent with Swink (2011) who found that the presence of TMS may not matter in really new product innovations. This could imply that in circumstances where agility is coupled with uncertainty and changes, new software development project success depends more on other aspects such as the presence of deep technological expertise. 
It is also highly likely that top management directives and controls often accompany TMS (Swink, 2011). Such directives and controls are potentially counterproductive to agile practices, as revealed by perceptions of vendor respondents with regard to their client organizations.

Additionally, a previous empirical study by Schmidt and Calantone (1998) demonstrated that some managers are prone to greater levels of optimism, psychological commitment and resource support for really new innovative product development projects even when performance forecasts are poor. Perhaps, this finding that TMS is ineffective in projects that were managed with a traditional plan-based methodology and seemingly detrimental in projects that were managed with an agile methodology reflects over optimism. If top level managers find it difficult to do something that prevents an activity from continuing, especially to stop giving money on innovative software projects, then ceteris paribus they are likely to support a greater percentage of project failures (Schmidt \& Calantone, 1998). This would also account for the finding that TMS is insignificant with both process and product project success in what are typically lower innovative contexts (projects managed with a traditional plan-based methodology, but negatively and significantly associated with process project success for highly technological innovations (projects managed with an agile methodology). In terms of comparing vendor and client perspectives for the relationship between TMS and project success for projects managed with an agile methodology, the vendor perspective appears different from previous "in-house" studies that focused on client perspective, which have indicated a positive relationship with project success.

\subsubsection{H2: Organizational culture $\rightarrow$ project success}

$\mathrm{H} 2$ was partially supported. $H 2(i)(T)$ and $H 2(i i)(T)$ which hypothesize no significant relationships between flexible organizational culture and process and product project success respectively for projects that used a traditional plan-driven methodology were supported in line with previous studies. For example, Iivari and Huisman's (2007) study found a significant positive relationship between hierarchical rational organizations and the deployment of traditional methodologies. This would correspond to a flexible organizational culture not being aligned for traditional plan-driven methodologies. This finding is not different with previous "in-house" studies which based on the perceptions of client respondents with regard to their client organizations. 
There are significant relationships between flexible organizational culture and process and product project success for projects that used an agile methodology, supporting both $H 2(i)(A)$ and $H 2(i i)(A)$ respectively. These findings support Misra et al. (2009) who found that agile corporate culture influences success of projects managed with an agile methodology. Similarly, Wan and Wang (2010) found that agile methods need to be established within agile corporate culture to succeed. Sheffield and Lemetayer (2013) found that flexible organizational culture positively influenced software development agility. Strode et al. (2009) found a positive and significant relationship between low formality organizations and the use of agile methodologies. This suggests that agile SDMs are more suited to flexible organizational culture. The vendor perspective appears not to be different from previous "inhouse" studies based on client perception, which have indicated a positive relationship organizational culture and project success for projects managed with an agile methodology. The model comparison results from testing for significant differences of coefficients across the two data sets indicate that the importance of organizational culture is not statistically greater for agile than for traditional plan-based methodologies.

\subsubsection{H3: Project planning and controlling $\rightarrow$ project success}

H3 was partially supported. The model comparison results from testing for significant differences of coefficients across the two data sets indicate that the importance of planning and controlling is statistically greater for plan-based than agile methodologies on only process success but not product success.

There is a significant positive relationship between project planning and controlling and process project success for projects that were managed using a traditional plan-driven methodology supporting $H 3(i)(T)$. These findings are in line with the views expressed by research participants during the pilot study. For instance, research participant 14 who uses traditional plan-driven methodologies said: "It is essential that a detailed plan is in place, this highlights conflicts, resource availability etc. Also gives the project team a structure to work within. If there is no detailed project plan, then the project is doomed to fail from the start”. Similarly, research participant 10 (traditional plan-based project management) said: "Planning and controlling are very important for software project success. Successful projects I know spent more than $60 \%$ of the time on planning and controlling. This helps to set up tolerance levels and meet goals". Thus, for outsourced projects managed with a traditional plan-driven methodology, this strongly suggests that planning and controlling are 
essential for setting milestones and taking corrective actions where the outsourced SD project is not going on well as expected.

These findings concur with previous research which has demonstrated a positive relationship between project planning and controlling and process performance. For instance, based on the perceptions of vendor respondents with regard to their client organizations, Jun et al. (2011) found that project planning and controlling are positively correlated with process performance at low levels of uncertainty. Similarly, Yetton et al. (2000) found that project planning and controlling were negatively related to budget variances and that budget variances are a negative function of planning, based on a client perspective. This implies that effective project planning and controlling for traditional plan-based methods is likely to be associated with efficiencies in development and, thus, prevent budget, scope and time variances. These results also mirror empirical results of Wallace et al. (2004), which confirmed a negative relationship between planning and controlling risks and both process and product project performance. Thus, the vendor perspective appears not to be different from previous "in-house" studies that focused on client perspectives in regard to the relationship between planning and controlling and process success for projects managed with a traditional plan-based methodology.

However, the structural path between project planning and controlling and product project success for traditional plan-driven methodology projects was insignificant, not supporting $H 3(i i)(T)$. This is consistent with Jun et al. (2011) who did not find a statistically significant relationship between planning and controlling and product success, based on the perceptions of vendors in regard to their client organization. This finding indicates that better quality of project plans and controls does not necessarily lead to better quality of outsourced software development project products. This might be because better long-range project plans may be followed by poor planning at the project or team level and mistakes in terms of the assignment of personnel, requirement specification, less customer participation or feedback etc. Thus, a better software project master plan should not be considered as an end-goal in itself; instead, it should be complemented with top managers' participation in IT resource allocation, customer interaction, proper assignment of personnel and requirement specification. These findings are consistent with recent Kearns and Sabherwal's (2008) empirical survey of 274 CIO's indicating that most outsourced software development projects 
practice intensive planning, controlling and alignment but continue to experience failed software quality products and IT investments.

For the projects that used an agile methodology, no significant relationships between project planning and controlling and process and product project success were found, supporting both $H 3(i)(A)$ and $H 3(i i)(A)$ respectively. This is consistent with the pilot study, for example a research participant 9 who uses agile methodologies said: "For agile projects, detailed planning up front is useless and dangerously misleading. Making estimates based on reallyrough order of magnitude estimates and comparisons to prior experience are useful for securing funding and gaining go-ahead, but are likely to change repeatedly as requirements are clarified through iterative processes. There are far too many unknowns in agile projects to be able to create a well-defined plan up-front. The up-front planning should be at the level needed to make the next decision. Detailed planning should be reserved for the near-term work, where there is a chance of being realistic and accurate”.

This clearly suggests that the management of projects using agile methodology does not need formalized planning and controlling since formal plans and controls may become absolute sooner than later due to continuous changes initiated by high levels of environmental uncertainty. By nature, this demonstrates that agile approaches are more risk oriented and cope with uncertainty, unlike traditional plan-based approaches. These findings are consistent with Misra et al. (2009), Jun et al. (2011) and Barki et al. (2001) that when dealing with high project uncertainty and change, planning and controlling are insignificant predictors of process and product success. In terms of vendor and client perspectives, the findings suggest no significant difference from previous "in-house" studies that focused on client perspective.

\subsubsection{H4: Vision and mission $\rightarrow$ project success}

Overall, H4 was partially supported. For projects that used a traditional plan-driven methodology, $H 4(i)(T)$ which hypothesized a significant positive relationships between vision and mission and process success was supported. These research findings are in line with previous "in-house" studies that focused on the client perceptions of their organization. For instance, Jung et al. (2008) found that clear vision and mission directly impacted on project performance of international projects. Similarly, Nah and Delgado's (2006) “in-house” study found that clear business plan and vision that were well aligned with project objectives led to successful software implementation and upgrade. 
However, the relationship between vision and mission and product success was not supported H4(ii)(T). However, previous studies by Jung et al. (2008) and Nah and Delgado (2006) focused on developers and users as members of the same organization, rather than the vendor's perspective used in this study. This could also possibly arise from the fact that the delivery of outputs by vendors can support the realization of outcomes, but does not guarantee the achievement of the desired or anticipated benefits by the clients (Zwikael \& Smyrk, 2012). A project manager (from the vendor organization) is a transient position to which the attachment of long-term accountabilities in the outsourcing organization may be most inappropriate since in outsourcing projects managers are frequently appointed from outside the funding organization on a short and medium term contracts (Zwikael \& Smyrk, 2012). If the project clients' do not utilize the project output and outcomes to a desired level, the benefits cannot be generated.

The direct structural paths between vision and mission and process and product project success were not significant in the model for the projects that used an agile methodology, thus, supporting $H 4(i)(A)$ and $H 4(i i)(A)$ respectively. This confirms that projects which use an agile methodology rely heavily on continuous market analysis rather the vision or mission of the organization in which they operate. They cope with uncertainty and continuous change as a result more innovative (adventurous) and adaptive practices that are highly flexible and entrepreneurial are needed with less up-front detailed planning and formal specifications. This matches vision and mission being usually operationalized through strategic plans which are not consistent with agile projects practices. In reference to vendor and client perceptions, this finding suggests a significant difference from previous "in-house" studies based on client perspective (e.g., Wan \& Wang, 2006; Nah \& Delgado, 2006) in comparison with the current vendor perceptions based on the client organization. This suggests that vision and mission vary considerably between vendors and clients and hence could be a source of tension in the outsourced project management arena. The model comparison results from testing for significant differences of coefficients across the two data sets indicate that the importance of vision and mission is not statistically greater for traditional plan-based than for agile methodologies.

\subsubsection{H5: Change management $\rightarrow$ project success}

H5 was partially supported. For projects that were managed with a traditional plan-based methodology, there are significant positive relationships between change management and process and product success, supporting both $H 5(i)(T)$ and $H 5(i i)(T)$. These findings are 
consistent with Nah \& Delgado (2006) and Jung et al. (2008) who found that change management is essential for traditional software project success, based on the client perceptions of their own organizations. Change management is needed most especially for projects managed with a traditional plan-based methodology where there is likelihood of resistance to change. Even though training tends to be one of the areas to be cut in the case of budget overruns, it is critical to the success of the implementation of outsourced project change as well as the quality of decisions that will be taken based on the system.

For projects that adopted an agile methodology, there are significant positive relationships between effective change management and process and product project success, supporting both $H 5(i)(A)$ and $H 5(i i)(A)$ respectively. The results suggest that agile methodologies require change management skills to deal with changes and uncertainty. The test results for significant differences of coefficients across the two data sets indicate that the importance of change management is statistically greater for agile than for traditional methodologies on product but not process success.

\subsubsection{H6: Leadership characteristics $\rightarrow$ project success}

H6 was partially supported. The model results from testing for significant differences of coefficients across the two data sets indicate that the importance of leadership characteristics is statistically greater for agile than for plan-based methodologies on only process but not product success. For the data set of the projects that used a traditional plan-based methodology, there is a significant and positive association between leadership characteristics and process success, providing support for $H 6(i)(T)$. But there is no significant relationship between leadership characteristics and product success, rejecting $H 6(i i)(T)$. The significant results between leadership characteristics and process success are consistent with Lindvall et al.'s (2002) empirical findings that it is not only the experience and competency of the team members that are important, but also their leadership characteristics such as honesty, collaborative attitude, sense of responsibility, readiness to learn, and work with others that are considered equally important for software development project success. Therefore, based on the perceptions of vendor respondents with regard to their client organizations, having project members with effective leadership on big teams is critical to traditional project success. This is consistent with previous "in-house" studies that focused on client perceptions of their own organization. 
For the projects that used an agile methodology, there is a significant and positive association between leadership characteristics and process project success, providing support for $H 6(i)(A)$. But there is no significant relationship between leadership characteristics and product project success, rejecting $H 6(i i)(A)$. The significant results between leadership characteristics and process success are consistent with Misra et al. (2009) and Strode et al. (2009). Misra et al. (2009) established that for the successful implementation of projects with an agile methodology to be completed on time, budget and scope, the project leaders need to possess some leadership characteristics. For instance, leaders who are ambitious, willing to take on initiatives, truthful and do what they say, enjoy taking risks, possess technical expertise in their businesses, are perceptive, as well as being conceptually skilled significantly contributed towards successful implementation of agile projects.

Surprisingly, based on the perceptions of vendor respondents with regard to their client organizations; these results seem to suggest that leadership characteristics significantly contribute only towards process success but not product success, irrespective of project methodologies i.e. traditional plan-based or agile. One possible explanation is that regardless of the project type, a project leader is required to handle people's emotions, to motivate people when they get disappointed, to take the teams together during the difficult times, and finally to make sure that they have concentrated on the goals (schedule, budget and scope) of the project.

\subsubsection{H7: Internal project communication $\rightarrow$ project success}

H7 was partially supported. The direct structural path between internal project communication and both process and product success were supported by the data collected from projects that used a traditional plan-based methodology, hence supporting $H 7(i)(T)$ and $H 7(i i)(T)$ respectively. Based on the perceptions of vendor respondents with regard to their client organizations, these results were as expected, simply because if software definition and development occurs across organizational boundaries as in outsourced software development, even more communications work is possibly needed to define and evolve a shared system vision and development strategy. This corresponds with previous "in-house" studies by Yetton et al. (2000) and Jiang and Klein (2000) who found lack of effective internal communication can lead to project team conflict that creates instability in a project team and, thus, result in a project being delayed and over budget. 
For the projects that adopted an agile methodology, there is are significant associations between internal project communication and process and product project success, providing support for both $H 7(i)(A)$ and $H 7(i i)(A)$. This is in line with Jun et al. (2011), Boehm and Turner (2003), Chavart (2003), Cockburn (2000, 2007) and Wysocki (2009) that agile projects success needs fast and effective communication for fast decision making, adapting with the dynamicity of projects, and fast knowledge transfer between team members in a project. Internal project communication and collaboration between the members of the project team has shown to decrease the amount of team conflict and keep the team stable. Based on a vendor perspective, Jun et al. (2011) found that lack of internal communication between team members or instability in a project team negatively affects process and product success. Results from testing for significant differences of coefficients across the two data sets indicate that the importance of internal project communication is not statistically greater for agile than for traditional plan-based methodologies.

\subsubsection{H8: Project team commitment $\rightarrow$ project success}

H8 was partially supported. For the projects that used a traditional plan-based methodology, there are significant positive associations between project team commitment and process and product success, supporting both $H 8(i)(T)$ and $H 8(i i)(T)$. The results were as expected; committed team project members have a feeling of responsibility and are attached to the project tasks that make them feel indebted to the project. This creates an atmosphere for individual team members to act without much control and coercion and costs of supervision are mitigated if the project team members are committed to their project tasks. These findings support previous studies (Meyer \& Allen, 1997; Ahimbisibwe \& Nangoli, 2012).

For projects that used an agile methodology, there are also significant associations between project team commitment and process and product success, providing support for both $H 8(i)(A)$ and $H 8(i i)(A)$. This is consistent with Chow and Cao (2008) who found committed team members positively influenced the perceived success of the software development projects that use agile methodology. Wan and Wang (2010) found significant positive relationships between team commitment and agile project success. This suggests that committed project team members more often do not have intentions to quit, which saves the project the costs of recruiting and orienting new members in terms of both time and money. With reference to the focus on vendor perspective, this finding is not different from previous "in-house" studies which based on the perceptions of client respondents with regard to their client organizations. Results from testing for significant differences of coefficients across the 
two data sets indicate that the importance of project team commitment is not statistically greater for agile than for traditional plan-based methodologies.

\subsubsection{H9: Development team's expertise $\rightarrow$ project success}

H9 was partially supported. For projects that used a traditional plan-based methodology, there are significant associations between development team's expertise and process and product success, providing support for both $H 9(i)(T)$ and $H 9(i i)(T)$. This is consistent with Jiang and Klein (2000) who found team's development expertise was significantly and positively related to the overall project effectiveness. Yetton et al. (2003) found that development team's expertise reduces project team's conflicts and creates project team's stability enabling the project to be completed on time. Likewise, Nah and Delgado (2006) found that team's skill contributed to ERP implementation success.

For the projects that used an agile methodology, there are also significant association between development team's expertise and process and product success, supporting both $H 9(i)(A)$ and H9(ii)(A). These findings are consistent with previous agile software success studies. For instance, Wan and Wang (2010) found competency/expertise dimension positively influenced agile project improvement. Similarly, Boehm and Turner's (2003) suggested a principle of using fewer and better people for agile projects. If project teams are composed of the people who have the corresponding required skills, the project is likely to be completed on time, budget, scope and quality.

Equally, from the discussions in Lindvall et al. (2002), it can be inferred that having at least $25 \%$ technically competent people on the agile projects is important. These findings based on a vendor perspective are not different from previous "in-house" studies which focused on the perceptions of client respondents with regard to their client organizations. Results from testing for significant differences of coefficients across the two data sets indicate the importance of development team expertise is not statistically greater for agile than for traditional plan-based methodologies.

\subsubsection{H10: Project team's composition $\rightarrow$ project success}

H10 was supported. The importance of project team composition is statistically greater for agile than for plan-based methodologies on both process and product success. For the projects that used a traditional plan-based methodology, the beta coefficients for the direct structural paths between project team's composition and process and product success were both significant. Thus, providing support for both $H 10(i)(T)$ and $H 10(i i)(T)$. The findings support 
that project teams composed of best various people with both business and technical knowledge in traditional plan-based methodology projects is essential for both process and product project success. These findings are in line with a previous study by Jung et al. (2008). Likewise, Nah and Delgado (2006) found that outsourced software development projects require balanced cross functional teams. The results in this study are also consistent with Lacity and Willocks (2000, p.362) who found that strategic project stakeholders should be included on project teams for IT outsourcing. In case of multi-sourcing, Lacity and Willocks (2000) found that even the number and required knowledge of these stakeholders increases.

For the projects that adopted an agile methodology, the beta coefficients for the direct structural paths between project team's composition and process and product project success were both found to be statistically significant. Thus, providing support for both $H 1 O(i)(A)$ and H1O(ii)(A). The results suggest the agile project team should be well balanced, crossfunctional, and have representatives from the internal organization as well as vendors. The top managers, IT and other departments and consultants should be included on the team as well. The best people in the organization should make part of the implementation team in order to foster innovation and creativity that are important for agile project success and the functional team members involved in the project should preferably be on a full time basis. These findings are not different from previous agile success studies which focused on the perceptions of client respondents with regard to their client organizations. E.g. Chow and Cao (2008) found that well composed teams contributed to successful agile software development projects.

\subsubsection{H11: User participation $\rightarrow$ project success}

Hypothesis 11 was partially supported. The importance of user participation is statistically greater for agile than for traditional plan-based on process but not product success. For the projects that used a traditional plan-based methodology, there are insignificant relationships between user participation and both process and product success, supporting both $H 11(i)(T)$ and $H 11(i i)(T)$. These findings suggest traditional plan-driven projects do not necessarily require substantial user participation during software development. There is no or little change expected during the traditional project execution and therefore, the plan and specification requirements are not expected to change once made. There is no much need for continuous involvement of the user during project implementation. 
For the projects that used an agile methodology, there is a significant positive relationships between user participation and process but a significant negative effect on product success, supporting H11(i)(A) but not H11(ii)(A). This is consistent with empirical studies by Chow and Cao (2008), Misra et al. (2009) and Sheffield and Lemetayer (2013) that have provided data to support significant and positive relationship between user participation and agile software development project success. Yetton et al. (2000) also found that user participation decreases budget variance by managing expectations and quickly resolving potential problems. Similarly, Jun et al. (2011) found that resolving potential conflicts early arising from greater user participation plays a vital role in the perceived system satisfaction of software developers and users. Therefore, user participation is an effective way to know and fulfil the needs of the agile users. It may also create user commitment to the agile projects.

This finding based on the current vendor perspective is not different from previous studies that focused on perceptions of client respondents with regard to their client organizations suggesting that a process of continuous and dynamic evaluation and debate between informed clients and vendors provides the best chance for information system optimisation. It can reduce friction and help to align the different viewpoints of the clients and vendors. Furthermore, user participation through intensive communication can improve the knowledge of management on IT projects-issues and the knowledge of IT-people on the specific management issues as agile project development and implementation progresses.

However, the negative effect between user participation and product success indicates that user participation negatively affect product success. The results possibly suggest that although user participation is necessary for process success, and participation in the requirements analysis stage can decrease the risk of there being insufficient requirements, too much user participation may have a negative effect on product success. Due to the novelty of the technology involved in outsourced software development projects and the corresponding uncertainty about requirements, clients/users can continually change their requirements, which can lead to confusion and conflict and the poor product being delivered. This concurs with Nidumolu's (1995) findings based on the perceptions of client respondents with regard to their client organizations that found increased interaction between users (clients) and IS staff (vendors) does not necessarily lead to a software project that converges well (i.e., improved product performance). Therefore, managers need to be aware of the potential tradeoffs between too much, and extremely limited user participation in requirements analysis. 


\subsubsection{H12: User support $\rightarrow$ project success}

Hypothesis 12 was partially supported. The importance of user support on project success is statistically greater for agile than plan-based methodologies on product success but not process success.

For the projects that adopted a traditional plan-based methodology, there is no significant relationship between user support and process and product success. Thus, supporting both $H 12(i)(T)$ and H12(i)(T) respectively. These findings also support that traditional plan-driven methodology projects do not necessarily require much user support during software development. There is no need for continuous support of the user during project implementation. Changes are not expected and all is planned for every task in advance. The results possibly suggest that for traditional methodology projects, user support is enforced through controlled culture using prescribed rules and regulations rather than seeking the discretionary support from users. Thus, there is compulsory compliance expected from all users.

For projects that used an agile methodology there is a significant and positive relationship between user support and process and product success supporting both H12(ii)(A) and $H 12(i)(A)$. These findings support that user attitudes need to be supportive and positive for agile projects to achieve success. Users and developers need to pay attention to each other using cautious interpersonal approaches to ensure project success. These findings are consistent with other previous scholars; Yetton et al.'s (2003) study found that user support increases project success. Chow and Cao (2008) also found that a strong user support contributed to the success of agile software development projects. Likewise, Misra et al. (2009) found a statistically significant and positive relationship between user support and software development project success. With regard to this vendor perspective used, this finding is not different from previous "in-house" studies which based on the perceptions of client respondents with regard to their client organizations.

\subsubsection{H13: User experience $\rightarrow$ project success}

Hypothesis 13 was partially supported. As initially hypothesized, for the projects that adopted the traditional plan-based methodology, there are insignificant relationships between user experience and both process and product success, supporting both $H 13(i)(T)$ and H13(ii)(T) respectively. This supports that projects managed with the traditional plan-based methodology do not necessarily require very experienced users that must be able to articulate 
their needs clearly. The results suggest that user experience for traditional projects does not necessarily contribute towards meeting objectives since developers do not rely on client's feedback to develop the software.

On the other hand, for the projects that adopted an agile methodology the beta coefficients between user experience and both process and product project success were supported, supporting both $H 13(i)(A)$ and $H 13(i i)(A)$ respectively. These findings are in line with previous studies; for instance, Chow and Cao (2008) found that involvement of experienced users significantly and positively impacted on project scope but not timeliness, cost or quality. Users who have basic experience about business domain help to identify software development project requirements explicitly.

Similarly, users who have an acceptable level of education or training enable them to explain their requirements and needs in a clear form. Clear knowledge of the problem can shorten the development time in producing the product. In regard to client and vendor perspectives, this finding is not different from previous "in-house" studies which based on the perceptions of client respondents with regard to their client organizations. The overall importance of user experience on project success is not statistically greater for agile than for plan-based methodologies.

\subsubsection{H14: Technological uncertainty $\rightarrow$ project success}

H14 was supported. Technological uncertainty has a less negative effect for agile than for plan-based methodologies for both process and product success. For projects that used a traditional plan-based methodology, the beta coefficients between technological uncertainty and process and product success were statistically significant, supporting both $H 14(i)(T)$ and $H 14(i i)(T)$. This suggests that the use of unfamiliar technologies can lead to software problems that reduce process and product success of the software development project.

Similarly, for projects that used the agile methodology, the beta coefficients between technological uncertainty and process and product success were statistically significant and negative, both supporting $H 14(i)(A)$ and $H 14(i i)(A)$. This is consistent with Jun et al. (2011) who found that the use of unfamiliar technologies leads to software problems that reduce the performance of the software product. In terms of vendor and client perceptions, results suggest no significant variation from previous "in-house" studies of the client perception on their client organization. 


\subsubsection{H15: Technical complexity $\rightarrow$ project success}

Overall, H15 was partially supported. Technical complexity has a greater negative effect on agile than on plan-based methodologies on process but not product success. For projects that used a traditional plan-based methodology, the beta coefficient between technical complexity and process success was statistically insignificant while the beta coefficient between technical complexity and product success was significantly negative. Thereby, not supporting $H 15(i)(T)$ but providing support for $H 15(i i)(T)$. This is in line with Jun et al. (2011) who found that technical complexity adversely and negatively affected software project performance in terms of both process and product performance from the vendor perceptive.

On the other hand, for projects that used an agile methodology, the beta coefficient between technical complexity and process success was negative and statistically significant as initially anticipated, providing support for $H 15(i)(A)$. However, the beta coefficient between technical complexity and product success was insignificant, not supporting H15(ii)(A). This clearly indicates that, whereas technical complexity has no direct influence on product success, it is negatively associated with process success.

The findings in this study suggest that technically complex agile projects involve extensive tasks and activities related to requirements' specifications, physical design and code implementation. These responsibilities increase the difficulty of coordination efforts, which in turn cause the implementation process to deteriorate leading to a project being completed behind the schedule and over budget. In relation to the vendor perspective, the variation in the findings compared to previous "in-house" studies which concentrated on the perceptions of client respondents with regard to their client organizations indicates that the vendors involved in outsourced projects have different perspectives on the risk (technical complexity).

\subsubsection{H16: Relative project size $\rightarrow$ project success}

H16 was partially confirmed. The negative influence of project size is statistically greater for agile than plan-based methodologies on process but not product success. For the projects that used a traditional plan-based methodology, the beta coefficient between relative project size and process success was statistically significant and negative, supporting $H 16(i)(T)$. These findings resonate with Yetton et al.'s (2003) study that larger projects are likely to be characterized by high complexity and high levels of task interdependence which increase project failure. The findings are also in line with other empirical evidence that large project size increases project risk (e.g., Sauer et al., 2007, Jun et al., 2011, Turner \& Zolin, 2012). 
However, the beta coefficient between relative project size and product success was statistically insignificant, rejecting $H 16(i i)(T)$.

For projects that used an agile methodology, the beta coefficient between relative project size and process success was statistically significant and negative as originally anticipated, supporting $H 16(i)(A)$. However, the beta coefficient between relative project size and product success was statistically insignificant, not supporting H16(ii)(A). According to these findings, as the size of projects increases, the chances of project success tend to diminish with agile methodologies. The results suggest that agile methodologies work best on small projects as measured in terms of the number people (fewer than 10) and the budget.

These results are in line with Sauer et al.'s (2007) empirical evidence that project size in terms of effort and duration can negatively affect project performance (p.81). This seems to suggest that the increase in project size of agile projects complicates coordination and requires planning and controlling which is counterproductive to agile practices thereby negatively affecting agile process success. In large projects, the size of communication paths becomes too many making coordination impossible for agile methodologies. The process of sharing information gets constrained as the number of people on a project increases. In terms of vendor and client perceptions, there appears to be no significant variation from previous "in-house" studies of the client perception on their client organization.

\subsubsection{H17: Specification changes $\rightarrow$ project success}

Overall H17 was partially supported. The negative effect of specification changes is not statistically greater for traditional plan-based than for agile methodologies for both product and process success. For traditional plan-based methodology projects, the beta coefficient between specification changes and process success was significant and negative supporting $H 17(i)(T)$. These findings are in line with Ratbe et al. (2000) who found that changes in specifications negatively affect project success. Shenhar (2001) also found that specification changes had a negative effect on project performance. This is possibly because for traditional methodology projects any changes in requirement specifications are likely be followed by increases in the budget and schedules.

In contrast, the results indicate that specification changes improve product success of traditional plan-based methodology projects. The beta coefficient between specification changes and product success was significant but positive, not supporting H17(ii)(T). The results suggest that specification analysis of user requirement is necessary for project success 
and can decrease the risk of there being insufficient requirements. However, too much specification may have a negative effect on product success. When clients/users continually change their requirements, it can lead to conflicts and a poor product being delivered late and over budget. Therefore, managers need to be aware of the potential trade-offs between too much, and extremely limited specification changes.

On the other hand, for agile methodology projects, both the beta coefficients between specification changes and process and product success were significant and negative, supporting both $H 17(i)(A)$ and $H 17(i i)(A)$ respectively. This is consistent with Boehm and Turner (2003) that specification changes negatively affect project performance. Equally, Nidumolu (1995) found that requirements instability had a negative effect on project performance. Too much user specification changes may have a negative effect on project success and in particular, variations in delivery time, scope and budget (Nidumolu, 1995). In terms of vendor and client perceptions, these results based on a vendor perspective are not different from previous "in-house" studies that focused on the client perception of their client organization.

\subsection{Discussing indirect effects or interrelationships}

This section briefly discusses some findings for indirect effects or inter-relationships between candidate CSFs themselves other than the hypothesized direct relationships with project success. Some candidate CSFs were found to interrelate in various ways to influence project success. Although some candidate CSFs may not have a direct relationship with project success, they appear to predominantly influence other candidate CSFs which in turn also influence project success. Thus, such indirect relationship might be equally important as direct ones in explaining candidate CSFs for SD projects and can help to provide insights as to why previous studies identified these as candidate CSFs.

There are significant positive relationships between top management support and internal project communication and project team commitment for both projects that used traditional plan-driven and agile methodologies. These findings indicate that top management support helps the project team to communicate and gain support from the organisation regardless of the project methodology used. As a result, this makes the task of the project team easier and reduces the amount of negotiation that needs to be done. An effective communication strategy and infrastructure put in place by top management as well as the project structure would motivate the project team. 
There is a significant positive relationship between internal project communication and project team commitment for both traditional plan-driven and agile methodology projects respectively. These findings suggest that project communication is needed, especially in the early stages, to establish understanding, trust and commitment among members. Expectations at every level need to be communicated and the associated benefits to project members should be clearly stipulated. Management of communication, career development and growth like education, salary, promotion and other expectations related to psychological contracts are critical for project team commitment and project success. This also shows that if project employees are told in advance about the scope, objectives, activities and updates, and where required change will occur, they will be committed to ensure that the project is successful.

Finally, while there is a positive significant association between vision and mission and organizational culture for projects that used a traditional plan driven, there is an insignificant association between vision and mission and organizational culture for projects that used an agile methodology. This suggests that for projects that for projects which are managed using the traditional plan-based methodology, vision and mission influences organizational cultures in which they operate.

\subsection{H19: Moderating effects of project management methodology}

Overall $H 19$ was primarily supported. The direct effects of top level management support, planning and controlling, leadership characteristics, team composition, user participation, technological uncertainty, technical complexity and relative project size on process success were found to differ significantly, depending on the type of the project management methodology. Equally, the direct influence of change management, team composition, user participation, user support, technological uncertainty, technical complexity and specification changes) on product success differs significantly depending on the type of the project management methodology used, as supported by the moderation graphs which have different gradients. Thus, the magnitude of these candidate CSFs on project success is influenced by the type of project management methodology used, providing support for H19: Project management methodology moderates the relationships between candidate CSFs and project success.

These results were expected and are consistent with previous studies that have demonstrated that different candidate CSFs have a statistically significant different impact on project success of different project types. For instance, Jun et al. (2011) found that project planning 
and controlling contributed more towards projects success for low information processing capability approaches (i.e., traditional plan-driven methodology) while internal integration (communication and information exchange), user participation and user support were found to make a greater contribution to project performance for high information processing capability approaches (i.e., agile methodology) than traditional plan-driven methodologies with low levels of risks. Consistently, these findings reflect Barki et al.'s (2001) study. Thus, typically, the relationships between the aforementioned candidate CSFs and project success vary depending on the type of project management methodology used (traditional plan-based vs. agile).

\subsection{H20-24: Moderating effects of vendor perception of project (uncertainty) factors}

As initially hypothesized, the findings indicate that technological uncertainty interacts with user support to positively and significantly influence process success. These results indicate that the magnitude effect of user support on process success depends on technological uncertainty; hence indicating a moderation effect. A significant interaction effect of technological uncertainty and user support signifies that combining the two will enhance or boost further the process performance of software development projects, and register higher performance than what one of the variables would have single-handedly registered. Therefore, hypothesis 20 (H2O), which examines interactive effects between technological uncertainty and candidate CSFs on project success for software development projects, is partly supported. This finding provides an insight that in presence of high technological uncertainty, user support is crucial for process success.

In a related case, the results also suggest that technological uncertainty interacts positively and significantly with project team commitment and project team composition to influence product success. Significant interaction effects between technological uncertainty with either project team commitment or project team composition indicates that neither of the two individual variables has much effect on product success, unless they are combined.

This implies that the magnitude effects of either project team commitment or project team composition on product success is influenced by the levels of technological uncertainty. This signifies that either of the two must co-exist if better product successis to be realized in software development projects. This study has, therefore, shown that the interplay of technological uncertainty with either project team commitment and project team composition 
is major in influencing software product success; and this provides support for hypothesis 20 (H20).

It was discovered, however, that the interaction effects of technological uncertainty with either planning and controlling, or relative project size negatively affect product success. This insight implies that under conditions of high technological uncertainty, setting plans and controls is counterproductive to product success. Equally, this insight suggests that relative project size in the presence of technological uncertainty appears to hinder product success. This is true, large sized projects that require use of novel technology have increased chances of product failure.

Technical complexity interacts with user participation, project team commitment, development team skills, change management, leadership characteristics, user support, and technological uncertainty to negatively and significantly influence process success. The results appear to suggest that technically complex projects increase the potential project risk and are likely to be highly vulnerable to failure, despite the presence and balance of these candidate CSFs. These findings are consistent with previous studies, for instance, based on a vendor perspective, Jun et al (2011) found that uncertainty risks like technical complexity had a negative moderating effect on the relationship between user participation and project performance.

Similarly, the results further indicate that technical complexity interacts with planning and controlling, relative project size, specification change to negatively affect product success. Thus, providing support for H21: technical complexity moderates the relationships between candidate CSFs and project success. These findings are also consistent with the conclusions of Zwikael et al. (2014) who argued that the level of risk moderates the impact of candidate CSFs on success, and in different ways for various success measures.

The results further indicate that relative project size interacts with user support to positively and significantly influence process success. A significant interaction effect between relative project size and user support indicates that neither of the two individual variables has much effect on process success, unless they are combined. This implies that the magnitude effect of user support on process success is influenced by the levels of relative project size. This insight signifies that the two must co-exist if better process performance is to be realized in software development projects. 
In addition, the results also suggest that relative project size interacts with top level management support, change management, vision and mission, technical complexity, specification changes to negatively and significantly influence product success. Thereby supporting, H22: Relative project size moderates the relationships between candidate CSFs and project success. The results imply that when relative project size interacts with each of the above candidate CSFs (top level management support, change management, vision and mission, technical complexity, specification changes); their impact diminishes product success. This insight possibly suggests that as the size of the software project tends to increase, there is always negative counter effect from these candidate CSFs on product success.

The results also indicate that specification change negatively interact with technical complexity but positively interacts with user support to influence process success. This insight suggests that specification changes for technically complex projects produces a combined effect which hinders process success; whereas a significant and positive interaction effect between specification changes and user support indicates that neither of the two individual variables has much effect on process success, unless they are combined. This implies that the magnitude effect of user support on process success is influenced by the levels of specification changes.

In addition, specification changes interacts with top level management support, user participation, project team composition to positively and significantly influence product success as well as interacting with planning and controlling, technical complexity, relative project size to negatively and significantly influence product success. Hence providing empirical support for H23: specification changes moderate the relationships between candidate CSFs and project success.

This insight implies that the combined use of specification changes and top level management support, user participation, project team composition always enhances product success. This insight further signifies that an appropriate mix or blend of these candidate CSFs is a source of product success in software development projects, if they are properly managed. In contrast, however, the interaction or combined effect of specification changes with planning and controlling, technical complexity, relative project size significantly diminishes product success. 
Surprisingly, some interaction effects of specification changes were not significant as expected, for instance, the interaction effects with project planning and controlling and change management on process success were found insignificant. This signifies that the combinations (interactive terms) of specification changes with either project planning or controlling and change management is inconsequential to process success levels of software development projects. This insight possibly suggests when user requirements are changing continuously, formal plans and controls easily become obsolete. It is also more difficult for vendors to implement formal plan and control mechanisms, and change management as in outsourced projects where the developers and users belong to different organizations. In contrast, however, in in-house projects it may be more convenient and effective for project managers to use standards, plans and formal mutual adjustment through a hierarchical structure.

Similarly, the interaction effect between user participation and specification changes on process success was found to be insignificant, but significant on product success. This suggests that involvement of users who are experienced and are familiar with the type of application, know what they want or have a good understanding of the problems they want solved significantly improves product success. However, as earlier noted, there is a need to balance between too much, and extremely limited user participation.

This finding is consistent with the views of Jun et al. (2011) and Yetton et al. (2000) that user participation tends to increase budget variance by encouraging suggestions for changes to specifications. The finding also mirrors the empirical finding of Nidumolu (1995) that increased interaction between users and IS staff does not necessarily lead to a project that converges well (i.e., improved process performance).

User participation is necessary for project success and participation in the requirements analysis stage can decrease the risk of there being insufficient requirements. However, too much user participation may have a negative effect on project success especially on delivery time.

Given the novelty of the technology involved and the corresponding uncertainty about requirements, clients/users can continually change their requirements, which can lead to conflict and the product being delivered late and over budget. Therefore, managers need to be 
aware of the potential trade-offs between too much, and extremely limited user participation. Thus, technological uncertainty, technical complexity, and relative project size and specification changes primarily moderate the relationships between candidate CSFs and project success.

\subsection{Chapter summary}

This chapter discussed the findings of the current study and provided answers to all research hypotheses. The research findings presented in the previous chapter were interpreted and discussed using logical justification, previously published literature and the responses obtained through the pilot study. For each candidate CSF the set hypotheses were discussed in relation to both process and product success. The current study supports that CSFs have a critical role in determining the software development methodology that fits and hence project success. The role of candidate CSFs in light of a selected methodology that fits for project success has often been neglected in the project success literature, especially where developers and users are not members of the same organization and the current study provides evidences that more attention is needed on this aspect.

The moderating influences of project management methodology and vendor perception of project (uncertainty) factors have been discussed. The findings of this study provide a better understanding of which project methodology should be adopted based on specific software project methodologies in a given project context. Practitioners could pursue different strategies for each project methodology they adopt to achieve project success. Researchers should not be frightened by these findings since most of the project management theories are tested without reference to specific project types or methodologies. The next chapter presents conclusions, contributions, recommendations, limitations and areas for further research. 


\section{CHAPTER SEVEN: CONCLUSIONS}

\subsection{Introduction}

The previous chapter discussed the findings from the main study. This chapter presents conclusions, contributions, delimitations, limitations and areas for further research. This is the last chapter of this thesis and concludes the dissertation.

The study examined the differences between the CSFs for traditional plan-based and agile methodologies for outsourced software development projects from a vendor's perspective. The gist of the study was to identify CSFs, develop and test an integrative contingency fit model for contrasting perspectives of traditional plan-based and agile methodologies specifically for outsourced software development projects from a vendor's perspective. This study was motivated by the alarming failure rate of software development projects that has become a habitual phenomenon in the industry. In order to address the above challenge, the researcher set and addressed the following two study objectives; which include:

1) To find out the CSFs for outsourced software development projects from a vendor's perspective.

2) To examine the differences in CSFs for traditional plan-driven and agile methodologies towards project success from a vendor's perspective.

This study involved the formulation and the testing of several contingency hypotheses which were developed from the objectives, and supported from the extant literature.

\subsection{Conclusion}

On the basis of the study findings and discussions in the previous chapters, and guided by the formulated hypotheses, the following conclusions were drawn:

A total of 37 candidate CSFs for outsourced software development projects were identified from 148 publications, and then categorised into four major categories (Tables $13 \& 14$, Figure 30). The identified candidate CSFs were categorized into top level management support, user participation, project team commitment, organizational culture, project planning and control, leadership characteristics, vision and mission, monitoring and controlling, change management skills, internal project communication and technological uncertainty among others. 
The three major sets of candidate CSFs identified for software projects from a vendor's perspective are VPoC organizational factors, vendor perception of team factors, and vendor perception of customer factors and their influence on project success is agreed to be moderated by the vendor perception of project factors and project management methodology. A contingency fit model was developed which augments this by highlighting the necessity to match project management methodology to these candidate CSFs.

Differences between the candidate CSFs for traditional plan-based and agile methodologies for outsourced software development projects from a vendor's perspective were established. The results indicate that some candidate CSFs contribute differently towards various project success measures when traditional plan-based methodologies are implemented than when agile methodologies are used and vice versa. This explains why SD projects succeed or fail under different contexts, and provides insights on how project success might be improved using a distinction of suitable management methodologies.

Table 84 depicts hypothesis testing results (those with a direct link to project success, $\mathrm{HI}$ H18). 
Table 84: Hypothesis testing results (only with direct link to project success)-Group comparisons

\begin{tabular}{|c|c|c|c|c|c|c|c|c|c|c|}
\hline \multirow{2}{*}{$\begin{array}{l}\text { Candidate } \\
\text { CSF } \\
\text { category }\end{array}$} & & & & & \multicolumn{2}{|c|}{$\begin{array}{c}\text { Traditional plan-based } \\
\text { methodology }(T), n=513\end{array}$} & \multicolumn{2}{|c|}{$\begin{array}{c}\text { Agile methodology (A) } \\
\mathrm{n}=471\end{array}$} & \multicolumn{2}{|c|}{$\begin{array}{l}\text { Comparison hypothesis, TA } \\
\text { (statistical difference test) }\end{array}$} \\
\hline & \multicolumn{4}{|c|}{ Hypothesis and the structural path } & \begin{tabular}{|c|} 
Hypothesized \\
relationship
\end{tabular} & Support & $\begin{array}{c}\text { Hypothesized } \\
\text { relationship }\end{array}$ & Support & $\begin{array}{l}\text { Hypothesized } \\
\text { relationship }\end{array}$ & Support \\
\hline \multirow{12}{*}{$\begin{array}{c}\text { VPoC } \\
\text { organisational } \\
\text { factors }\end{array}$} & \multirow{2}{*}{$\begin{array}{l}\boldsymbol{H}_{1(i)} \\
\boldsymbol{H}_{1(i i)} \\
\end{array}$} & Top mgt. support & $\rightarrow$ & PS (process) & + & No & + & $\mathrm{No}^{\mathrm{a}}$ & $\mathrm{A}+>\mathrm{T}$ & $\mathrm{No}^{\mathrm{a}}$ \\
\hline & & Top mgt. support & $\rightarrow$ & PS (product) & + & No & + & No & $\mathrm{A}+>\mathrm{T}$ & No \\
\hline & \multirow{2}{*}{$\begin{array}{l}\boldsymbol{H}_{2(i)} \\
\boldsymbol{H}_{2(i i)}\end{array}$} & Organizational culture & $\rightarrow$ & PS(process) & $\#$ & Yes & + & Yes & $\mathrm{A}+>\mathrm{T}$ & No \\
\hline & & Organizational culture & $\rightarrow$ & PS (product) & $\#$ & Yes & + & Yes & $\mathrm{A}+>\mathrm{T}$ & No \\
\hline & \multirow{2}{*}{$\begin{array}{l}\boldsymbol{H}_{3(i)} \\
\boldsymbol{H}_{3(i i)}\end{array}$} & Planning and controlling & $\rightarrow$ & PS (process) & + & Yes & \# & Yes & $\mathrm{T}+>\mathrm{A}$ & Yes \\
\hline & & Planning and controlling & $\rightarrow$ & PS (product) & + & No & \# & Yes & $\mathrm{T}+>\mathrm{A}$ & No \\
\hline & \multirow{2}{*}{$\begin{array}{l}\boldsymbol{H}_{4(i)} \\
\boldsymbol{H}_{4(i i)}\end{array}$} & Vision and mission & $\rightarrow$ & PS (process) & + & Yes & $\#$ & Yes & $\mathrm{T}+>\mathrm{A}$ & No \\
\hline & & Vision and mission & $\rightarrow$ & PS (product) & + & No & $\#$ & Yes & $\mathrm{T}+>\mathrm{A}$ & No \\
\hline & \multirow{2}{*}{$\begin{array}{l}H_{5(i)} \\
H_{5(i i)}\end{array}$} & Change management & $\rightarrow$ & PS (process) & + & Yes & + & Yes & $\mathrm{A}+>\mathrm{T}$ & No \\
\hline & & Change management & $\rightarrow$ & PS (product) & + & Yes & + & Yes & $\mathrm{A}+>\mathrm{T}$ & Yes \\
\hline & \multirow{2}{*}{$\begin{array}{l}H_{6(i)} \\
H_{6(i i)}\end{array}$} & Leadership characteristics & $\rightarrow$ & PS (process) & + & Yes & + & Yes & $\mathrm{A}+>\mathrm{T}$ & Yes \\
\hline & & Leadership characteristics & $\rightarrow$ & PS (product) & + & No & + & No & $\mathrm{A}+>\mathrm{T}$ & No \\
\hline \multirow{8}{*}{$\begin{array}{c}\text { Vendor } \\
\text { perception of } \\
\text { team } \\
\text { factors }\end{array}$} & \multirow{2}{*}{$\begin{array}{l}\boldsymbol{H}_{7(i)} \\
\boldsymbol{H}_{7(i i)} \\
\end{array}$} & Int. proj. communication & $\rightarrow$ & PS (process) & + & Yes & + & Yes & $\mathrm{A}+>\mathrm{T}$ & No \\
\hline & & Int. proj. communication & $\rightarrow$ & PS (product) & + & Yes & + & Yes & $\mathrm{A}+>\mathrm{T}$ & No \\
\hline & \multirow{2}{*}{$\begin{array}{l}\boldsymbol{H}_{8(i)} \\
\boldsymbol{H}_{8(i i)} \\
\end{array}$} & Proj. team commitment & $\rightarrow$ & PS (process) & + & Yes & + & Yes & $\mathrm{A}+>\mathrm{T}$ & No \\
\hline & & Proj. team commitment & $\rightarrow$ & PS (product) & + & Yes & + & Yes & $\mathrm{A}+>\mathrm{T}$ & No \\
\hline & \multirow{2}{*}{$\begin{array}{l}\boldsymbol{H}_{9_{(i)}} \\
\boldsymbol{H}_{9_{(i i)}}\end{array}$} & Devt. team expertise & $\rightarrow$ & PS (process) & + & Yes & + & Yes & $\mathrm{A}+>\mathrm{T}$ & No \\
\hline & & Devt. team expertise & $\rightarrow$ & PS (product & + & No & + & Yes & $\mathrm{A}+>\mathrm{T}$ & No \\
\hline & \multirow{2}{*}{$\begin{array}{l}H_{10(i)} \\
H_{10(i i)}\end{array}$} & Proj. team composition & $\rightarrow$ & PS (process) & + & Yes & + & Yes & $\mathrm{A}+>\mathrm{T}$ & Yes \\
\hline & & Proj. team composition & $\rightarrow$ & PS (product) & + & Yes & + & Yes & $\mathrm{A}+>\mathrm{T}$ & Yes \\
\hline \multirow{6}{*}{$\begin{array}{l}\text { Vendor } \\
\text { perception of } \\
\text { customer } \\
\text { factors }\end{array}$} & \multirow{2}{*}{$\begin{array}{l}H_{11(i)} \\
H_{11(i i)}\end{array}$} & User participation & $\rightarrow$ & PS (process) & $\#$ & Yes & + & Yes & $\mathrm{A}+>\mathrm{T}$ & Yes \\
\hline & & User participation & $\rightarrow$ & PS (product) & $\#$ & Yes & + & $\mathrm{No}^{\mathrm{a}}$ & $\mathrm{A}+>\mathrm{T}$ & $\mathrm{No}^{a}$ \\
\hline & \multirow{2}{*}{$\begin{array}{l}H_{12(i)} \\
H_{12(i i)}\end{array}$} & User support & $\rightarrow$ & PS (process) & $\#$ & Yes & + & Yes & $\mathrm{A}+>\mathrm{T}$ & No \\
\hline & & User support & $\rightarrow$ & PS (product) & $\#$ & Yes & + & Yes & $\mathrm{A}+>\mathrm{T}$ & Yes \\
\hline & \multirow{2}{*}{$\begin{array}{l}H_{13(i)} \\
H_{13(i i)}\end{array}$} & User experience & $\rightarrow$ & PS (process) & $\#$ & Yes & + & Yes & $A+>T$ & No \\
\hline & & User experience & $\rightarrow$ & PS (product) & $\#$ & Yes & + & Yes & $\mathrm{A}+>\mathrm{T}$ & No \\
\hline \multirow{10}{*}{$\begin{array}{l}\text { Vendor } \\
\text { perception of } \\
\text { project } \\
\text { factors }\end{array}$} & \multirow{2}{*}{$\begin{array}{l}H_{14(i)} \\
H_{14(i i)}\end{array}$} & Technological uncertainty & $\rightarrow$ & PS (process) & - & Yes & - & Yes & $\mathrm{T}->\mathrm{A}$ & Yes \\
\hline & & Technological uncertainty & $\rightarrow$ & PS (product) & - & Yes & - & Yes & $\mathrm{T}->\mathrm{A}$ & Yes \\
\hline & \multirow{2}{*}{$\begin{array}{l}H_{15(i)} \\
H_{15(i i)}\end{array}$} & Technical complexity & $\rightarrow$ & PS (process) & - & No & - & Yes & $\mathrm{A}->\mathrm{T}$ & $\mathrm{No}^{\mathrm{a}}$ \\
\hline & & Technical complexity & $\rightarrow$ & PS (product) & - & Yes & - & No & $\mathrm{A}->\mathrm{T}$ & $\mathrm{No}^{\mathrm{a}}$ \\
\hline & \multirow{2}{*}{$\begin{array}{l}H_{16(i)} \\
H_{16(i i)}\end{array}$} & Relative project size & $\rightarrow$ & PS (process) & - & Yes & - & Yes & $\mathrm{A}->\mathrm{T}$ & Yes \\
\hline & & Relative project size & $\rightarrow$ & PS (product) & - & No & - & No & $A->T$ & No \\
\hline & $\mathrm{H}_{17(i)}$ & Specification changes & $\rightarrow$ & PS (process) & - & Yes & - & Yes & $\mathrm{T}->\mathrm{A}$ & No \\
\hline & $H_{17(i i)}$ & Specification changes & $\rightarrow$ & PS (product) & - & $\mathrm{No}^{\mathrm{a}}$ & - & $\mathrm{No}^{\mathbf{a}}$ & $\mathrm{T}->\mathrm{A}$ & $\mathrm{No}^{\mathrm{a}}$ \\
\hline & $\mathrm{H}_{18(i)}$ & Project criticality & $\rightarrow$ & PS (process) & - & $\mathrm{x}$ & - & $\mathrm{x}$ & $\mathrm{A}->\mathrm{T}$ & $\mathrm{x}$ \\
\hline & $\mathrm{H}_{18(i i)}$ & Project criticality & $\rightarrow$ & PS (product) & - & $\mathrm{x}$ & - & $\mathrm{x}$ & $\mathrm{A}->\mathrm{T}$ & $\mathrm{x}$ \\
\hline
\end{tabular}

Figure 42 illustrates standardised coefficients (from Table 70) of CSFs for each project management methodology based on various measures of project success (process and product). As demonstrated some CSFs for traditional plan-based and agile methodologies differ significantly depending on the success measures. 


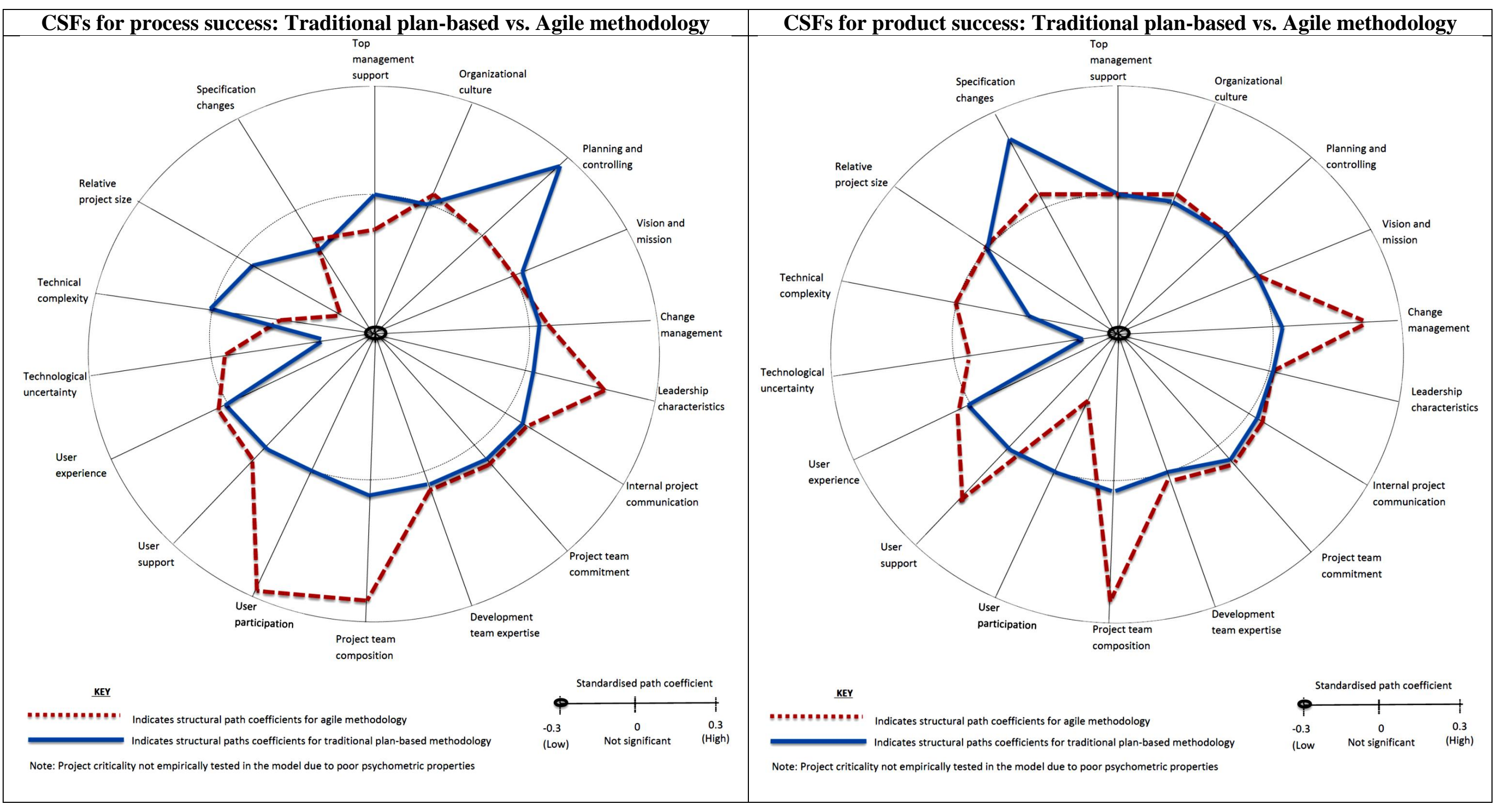

Figure 42: Summary of CSFs for project success (standardised path coefficients) 
Unlike previous research contributions, this study examined indirect links between all of the most frequently cited candidate CSFs and project success. This is important because previous studies have not examined all these factors in a single model simultaneously. Although some candidate CSFs were found not to be directly associated with project success, these often had indirect relationships that influence project success. Such omissions to capture indirect effects could also explain variations of candidate CSFs highlighted in previous research. Table 85 shows the indirect structural paths findings.

Table 85: Indirect structural paths findings

\begin{tabular}{|c|c|c|c|c|}
\hline \multicolumn{3}{|c|}{ Structural path } & \multirow{2}{*}{\begin{tabular}{|c|}
$\begin{array}{c}\text { Traditional plan-based } \\
\text { methodology projects }(\mathbf{T}) \mathbf{n}=\mathbf{5 1 3}\end{array}$ \\
$\sqrt{ }$ \\
\end{tabular}} & \multirow{2}{*}{$\begin{array}{c}\begin{array}{l}\text { Agile methodology } \\
\text { projects (A) } n=471\end{array} \\
\sqrt{ }\end{array}$} \\
\hline Top mgt. support & $\rightarrow$ & Int. proj. communication & & \\
\hline Top mgt. support & $\rightarrow$ & Proj. team commitment & $\sqrt{ }$ & $\sqrt{ }$ \\
\hline Int. proj. communication & $\rightarrow$ & Proj. team commitment & $\sqrt{ }$ & $\sqrt{ }$ \\
\hline Vision and mission & $\rightarrow$ & Organizational culture & $\sqrt{ }$ & $\#$ \\
\hline Team composition & $\rightarrow$ & Organizational culture & $\sqrt{ }$ & $\sqrt{ }$ \\
\hline Top mgt. support & $\rightarrow$ & Organizational culture & $\sqrt{ }$ & $\sqrt{ }$ \\
\hline Change mgt. & $\rightarrow$ & Organizational culture & $\sqrt{ }$ & $\sqrt{ }$ \\
\hline Leadership characteristics & $\rightarrow$ & Organizational culture & \# & $\sqrt{ }$ \\
\hline \begin{tabular}{|l|l|} 
User experience \\
\end{tabular} & $\rightarrow$ & Organizational culture & $\#$ & $\sqrt{ }$ \\
\hline Devt. team expertise & $\rightarrow$ & Organizational culture & $\#$ & $\sqrt{ }$ \\
\hline User participation & $\rightarrow$ & Organizational culture & $\#$ & $\sqrt{ }$ \\
\hline Organizational culture & $\rightarrow$ & Int. proj. communication & $\sqrt{ }$ & $\sqrt{ }$ \\
\hline Team composition & $\rightarrow$ & Int. proj. communication & $\sqrt{ }$ & $\sqrt{ }$ \\
\hline Vision and mission & $\rightarrow$ & Int. proj. communication & $\#$ & $\sqrt{ }$ \\
\hline Relative project size & $\rightarrow$ & Int. proj. communication & $\#$ & $\sqrt{ }$ \\
\hline User participation & $\rightarrow$ & Int. proj. communication & $\sqrt{ }$ & $\sqrt{ }$ \\
\hline Change mgt & $\rightarrow$ & Int. proj. communication & $\#$ & $\#$ \\
\hline Proj. team composition & $\rightarrow$ & Proj. team commitment & $\sqrt{ }$ & $\sqrt{ }$ \\
\hline Leadership characteristics & $\rightarrow$ & Proj. team commitment & $\#$ & $\sqrt{ }$ \\
\hline \begin{tabular}{|l|} 
User participation \\
\end{tabular} & $\rightarrow$ & Proj. team commitment & $\#$ & $\#$ \\
\hline Specification changes & $\rightarrow$ & Proj. team commitment & $\sqrt{ }$ & $\sqrt{ }$ \\
\hline Technical complexity & $\rightarrow$ & Proj. team commitment & $\sqrt{ }$ & $\sqrt{ }$ \\
\hline Team composition & $\rightarrow$ & Planning and controlling & $\#$ & $\sqrt{ }$ \\
\hline Vision and mission & $\rightarrow$ & Planning and controlling & $\sqrt{ }$ & $\sqrt{ }$ \\
\hline Change magt & $\rightarrow$ & Planning and controlling & $\sqrt{ }$ & $\sqrt{ }$ \\
\hline User participation & $\rightarrow$ & Planning and controlling & $\#$ & $\sqrt{ }$ \\
\hline Specification changes & $\rightarrow$ & Planning and controlling & $\sqrt{ }$ & $\sqrt{ }$ \\
\hline Int. proj. communication & $\rightarrow$ & Planning and controlling & $\sqrt{ }$ & $\sqrt{ }$ \\
\hline Organizational culture & $\rightarrow$ & Planning and controlling & $\#$ & $\sqrt{ }$ \\
\hline Proj. team commitment & $\rightarrow$ & Planning and controlling & $\#$ & $\sqrt{ }$ \\
\hline PS (process) & $\rightarrow$ & PS (product) & $\sqrt{ }$ & $\sqrt{ }$ \\
\hline
\end{tabular}

The study conducted SEM group comparisons (invariance analysis) and tested for significant differences of standardised beta coefficients across the two data samples to establish if the candidate CSFs were significantly different for each project type, that is, whether one project 
methodology does not fit all projects. The structural path coefficients between top level management support, planning and controlling, leadership characteristics, team composition, user participation, technological uncertainty, technical complexity and relative project size and process success were found to differ significantly, depending on the type of the project management methodology.

Equally, the structural path coefficients between change management, team composition, user participation, user support, technological uncertainty, technical complexity and specification changes and product success differed significantly depending on the type of the project management methodology used, as supported by the moderation graphs which have different gradients. Thus, these candidate CSFs are significantly moderated by project management methodology. In combination, these suggest that the project methodology should be adopted based on specific software project types in a given project context.

Some of the hypotheses tested how vendor perceptions of project factors interact with candidate CSFs to influence project success. For the interactions effects, the research found that technological uncertainty interacts with user support to positively and significantly influence process success; the results also suggest that technological uncertainty interacts positively and significantly with project team commitment and project team composition, but interacts negatively with planning and controlling, and relative project size to affect product success. The implication is that more user support, best members in teams and project team commitment are needed for process success when technological uncertainty is high, while planning and controlling should not be used when there is high technological uncertainty.

Technical complexity interacts positively with user participation and user support, but negatively with project team commitment, development team expertise, change management, leadership characteristics and technological uncertainty to significantly influence process success. The results further indicate that technical complexity interacts with planning and controlling, relative project size, specification change to negatively affect product success. This suggests that project complexity is likely to increase depending on the relative size of projects and the level of technological uncertainty.

The results further indicate that relative project size interacts with user support to positively and significantly influence process success. In addition, relative project size interacts with top level management support, change management, vision and mission, technical complexity, specification changes to negatively and significantly influence product success. 
The results also indicate that specification change negatively interacts with technical complexity but positively interacts with user support to influence process success. In addition, specification changes interacts with top level management support, user participation, project team composition and user support to positively and significantly influence product success as well as interacting with planning and controlling, technical complexity, relative project size to negatively and significantly influence product success. This perhaps suggests plans and controls should not be relied on when user requirements are changing very fast. Together, these findings provided support for contingency as fit in this study. Table 86 shows significant interaction effects.

Table 86: Significant interaction effects $(n=984)$

\begin{tabular}{|c|c|c|c|c|c|c|c|}
\hline Construct & Stru & ctural path & $\begin{array}{c}\text { Technological } \\
\text { uncertainty } \\
\text { (interaction) } \\
\left(H_{20}\right)\end{array}$ & $\begin{array}{c}\text { Technical } \\
\text { Complexity } \\
\text { (interaction) } \\
\left(H_{21}\right)\end{array}$ & $\begin{array}{c}\text { Relative } \\
\text { project size } \\
\text { (interaction) } \\
\left(H_{22}\right)\end{array}$ & $\begin{array}{c}\text { Specification } \\
\text { changes } \\
\text { (interaction) } \\
\left(H_{23}\right)\end{array}$ & $\begin{array}{c}\text { Project } \\
\text { criticality } \\
\text { (interaction) } \\
\left(\boldsymbol{H}_{24}\right)\end{array}$ \\
\hline Top mgt. support & $\rightarrow$ & PS (process) & $\#$ & $\#$ & $\#$ & $\#$ & $\mathrm{x}$ \\
\hline Top mgt. support & $\rightarrow$ & PS (product) & $\#$ & $\#$ & - & + & $\mathrm{x}$ \\
\hline Organizational culture & $\rightarrow$ & PS(process) & $\#$ & $\#$ & $\#$ & $\#$ & $\mathrm{x}$ \\
\hline Organizational culture & $\rightarrow$ & PS (product) & $\#$ & $\#$ & $\#$ & $\#$ & $\mathrm{x}$ \\
\hline Planning and controlling & $\rightarrow$ & PS (process) & $\#$ & $\#$ & $\#$ & $\#$ & $\mathrm{x}$ \\
\hline Planning and controlling & $\rightarrow$ & PS (product) & - & - & $\#$ & - & $\mathrm{x}$ \\
\hline Vision and mission & $\rightarrow$ & PS (process) & $\#$ & $\#$ & $\#$ & $\#$ & $\mathrm{x}$ \\
\hline Vision and mission & $\rightarrow$ & PS (product) & $\#$ & $\#$ & - & $\#$ & $\mathrm{x}$ \\
\hline Change management & $\rightarrow$ & PS (process) & $\#$ & - & $\#$ & $\#$ & $\mathrm{x}$ \\
\hline Change management & $\rightarrow$ & PS (product) & $\#$ & $\#$ & - & $\#$ & $\mathrm{x}$ \\
\hline Leadership characteristics & $\rightarrow$ & PS (process) & $\#$ & - & $\#$ & $\#$ & $\mathrm{x}$ \\
\hline Leadership characteristics & $\rightarrow$ & PS (product) & $\#$ & $\#$ & $\#$ & $\#$ & $\mathrm{x}$ \\
\hline Int. proj. communication & $\rightarrow$ & PS (process) & \# & $\#$ & $\#$ & $\#$ & $\mathrm{x}$ \\
\hline Int. proj. communication & $\rightarrow$ & PS (product) & $\#$ & $\#$ & $\#$ & $\#$ & $\mathrm{x}$ \\
\hline Proj. team commitment & $\rightarrow$ & PS (process) & $\#$ & - & $\#$ & $\#$ & $\mathrm{x}$ \\
\hline Proj. team commitment & $\rightarrow$ & PS (product) & + & $\#$ & $\#$ & $\#$ & $\mathrm{x}$ \\
\hline Devt. team expertise & $\rightarrow$ & PS (process) & $\#$ & - & $\#$ & $\#$ & $\mathrm{x}$ \\
\hline Devt. team expertise & $\rightarrow$ & PS (product & $\#$ & $\#$ & $\#$ & $\#$ & $\mathrm{x}$ \\
\hline Proj. team composition & $\rightarrow$ & PS (process) & $\#$ & $\#$ & $\#$ & $\#$ & $\mathrm{x}$ \\
\hline Proj. team composition & $\rightarrow$ & PS (product) & + & $\#$ & $\#$ & + & $\mathrm{x}$ \\
\hline User participation & $\rightarrow$ & PS (process) & \# & + & $\#$ & $\#$ & $\mathrm{x}$ \\
\hline User participation & $\rightarrow$ & PS (product) & $\#$ & $\#$ & $\#$ & + & $\mathrm{x}$ \\
\hline User support & $\rightarrow$ & PS (process) & + & + & + & + & $\mathrm{x}$ \\
\hline User support & $\rightarrow$ & PS (product) & \# & $\#$ & \# & $\#$ & $\mathrm{x}$ \\
\hline User experience & $\rightarrow$ & PS (process) & $\#$ & $\#$ & $\#$ & $\#$ & $\mathrm{x}$ \\
\hline User experience & $\rightarrow$ & PS (product) & $\#$ & $\#$ & $\#$ & $\#$ & $\mathrm{x}$ \\
\hline Technological uncertainty & $\rightarrow$ & PS (process) & $\mathrm{Na}$ & - & $\#$ & $\#$ & $\mathrm{x}$ \\
\hline Technological uncertainty & $\rightarrow$ & PS (product) & $\mathrm{Na}$ & $\#$ & $\#$ & $\#$ & $\mathrm{x}$ \\
\hline Technical complexity & $\rightarrow$ & PS (process) & $\#$ & $\mathrm{Na}$ & $\#$ & - & $\mathrm{x}$ \\
\hline Technical complexity & $\rightarrow$ & PS (product) & $\#$ & $\mathrm{Na}$ & - & - & $\mathrm{x}$ \\
\hline Relative project size & $\rightarrow$ & PS (process) & $\#$ & $\#$ & $\mathrm{Na}$ & $\#$ & $\mathrm{x}$ \\
\hline Relative project size & $\rightarrow$ & PS (product) & - & - & $\mathrm{Na}$ & - & $\mathrm{x}$ \\
\hline Specification changes & $\rightarrow$ & PS (process) & $\#$ & $\#$ & $\#$ & $\mathrm{Na}$ & $\mathrm{x}$ \\
\hline Specification changes & $\rightarrow$ & PS (product) & $\#$ & - & - & $\mathrm{Na}$ & $\mathrm{x}$ \\
\hline
\end{tabular}

\subsection{Contribution of the Research}

The current research has contributions for both project management theory and practice. Next both theoretical and practical contributions are discussed. 


\subsubsection{Theoretical Contributions}

This research contributes to contingency theory within project management which essentially argues that projects should be managed and structured differently. It seeks to understand and explain why SD projects succeed or fail under different contexts, and how project success might be improved using a variation of suitable management methodologies.

The strongest contribution is to understanding the differences between agile and plan-based project management, and also to understanding what are the most significant CSFs in different project contexts. The study examined the differences between the CSFs for traditional plan-based and agile methodologies for outsourced software development projects from a vendor's perspective. Previous software development projects studies have addressed only one methodology per study and perceived candidate CSFs as form of reasons of success amidst a wide range of project success criteria.

The extensive analysis of indirect and interaction effects is also a contribution. Previous studies have generally assumed a direct link between all candidate CSFs and project success. Empirical contingency models have frequently not fully incorporated contingency as fit or fit as moderation. This study incorporates vendor perception of project factors as moderating variables on the relationships between $V P o C$ organizational factors, vendor perception of team factors, vendor perception of customer factors (as independent variables) and project success (dependent variable), that is, incorporating fully contingency as fit as well as a comparative analysis of the two project management methodologies. The combination of several candidate CSFs confirmed significant interactive effects in influencing project success and these findings provide new insights towards understanding of managing software development projects.

The other contribution of this research occurs through the synthesis and integration of a large body of previously diverse literature, applying contingency theory, and conducting a large confirmatory study. The study systematically identifies, ranks and examines 37 candidate CSFs for software projects from 148 journal publications and holistically categorises them as VPoC organizational factors, vendor perception of team factors, vendor perception of customer factors and vendor perception of project factors.

The candidate CSFs matching each methodology were established and compared. This study builds on Boehm and Turner's (2003) five candidate CSFs for software development project methodology selection i.e. personnel, dynamism, culture, size and criticality by extending 
from these 5 to 28 candidate CSFs upon which methodology selection is best determined by an assessment of these 28 candidate CSFs which are measured on a continuum (scale) from high to low. These candidate CSFs were developed from the project management literature contrasting key features of agile and traditional plan-based methodologies, which determine the profile of a given software development project and the selection of an appropriate methodology. A conceptual model was also developed to augment how an appropriate choice of a project methodology associated with these 28 candidate CSFs is best evaluated by first ascertaining how well the software development project fits with either the traditional planbased or agile methodology, typically by superimposing it on a radar plot.

Additionally, several contingency hypotheses (a total of over 60) were formulated based on theory (chapter 3) and subsequently empirically validated using a global survey which generated a large sample of 1,880 (984 usable) responses from senior project managers around the globe. This permitted the complete data sample to be split up into two subgroups (traditional plan-based and agile methodology projects) which enabled comparative analyses across the two project types. The robustness of covariance-based SEM using Maximum likelihood method of estimation in data analysis enabled the simultaneously analysis of all these hypotheses (chapter 5). Most of the originally set hypotheses were supported.

This is the first research to develop and test fully an integrative contingency fit model on outsourced software development project success, contrasting traditional plan-driven and agile methodologies from a vendor's perspective. It is a significant and an initial step for quantitative data collection and enables detailed empirical analysis of comparative studies, thus is likely to improve clarity in debate. Through more robust empirical findings, it should help to clear up some contradictions that limit theory development for candidate CSFs of software development projects. In this regard, this study makes an important contribution and hopefully serves as a useful stepping stone for future project management studies.

Although the study appropriates some measures developed for another context from studies based on an in-house perspective, this study contributes to construct specification and measurement. Some scales were not initially developed specifically to represent the vendor perspective adopted in this study. Nonetheless, the measures were pre-tested to confirm if they suited the current context of the study and during the pre-testing phase the respondents did not raise a concern regarding the possibility of influencing the perspectives of staff members on their own organization. Thus, this study developed and validated a single survey 
questionnaire instrument to assess candidate CSFs for project success, contrasting plan-based and agile methodologies. The existing project management literature suggested that there was limited coherency on the best fit between candidate CSFs and project management methodologies, and on how to assess the impact of this fit on project success.

Borrowing more detailed theorization of fit from strategic management studies and MIS; this research developed a more extensive and comprehensive measurement instrument for software development projects. This comprehensive measurement instrument for software development projects hopefully will be useful for researchers in the future studies to examine the concept of fit further.

The approach of assessing fit involved developing a questionnaire which was first pilot tested in three rounds using senior project managers as respondents before undertaking the main global study. The measurement scales generally demonstrated good construct validity and high reliability with high predictive validity of the research model which is supported by theory. This progressive procedure was found to be a reliable method in developing an extensive and comprehensive measurement instrument for software development projects. This approach hopefully will be used by researchers aiming to assess candidate CSFs for various methodologies and the concept of fit in future project management studies.

\subsubsection{Practical Contributions}

The most valuable contribution for practitioners from this research is the more comprehensive evidence that different project types in various project contexts require different management methodologies i.e. traditional plan-based and agile methodologies. The empirical data gathered in two collection rounds generally supported these hypotheses. The entire research model was tested in the two methodology-specific contexts. This research design led to actionable recommendations for practitioners. For instance, traditional project management methodology requires planning and controlling for process success but seems less influenced by user participation and support.

In contrast, agile methodology projects require user participation but not planning and controlling to achieve project success. The composition of project teams and leadership characteristics were found to be more important for agile methodology projects than for traditional methodologies for both process and product success. This suggests that software practitioners should carefully consider the project types, project contexts (CSFs) and project methodologies when managing software development projects. The findings demonstrate that 
project managers and, more generally, top management and organizations should adopt a more project-specific approach to project management and software development based on CSFs. This is likely to improve the current project success rates.

Second, it appears that many software development practitioners are affiliated, committed and loyal to different communities as indicated by responses from different development communities. However, to obtain better desired end results, software development practitioners have to ensure that their allegiance to a particular community of project management methodology practice does not influence them to select an inappropriate software development methodology.

Third, the current study suggests that at least the three parties who are potentially involved in an outsourced software development environment (the client, the vendor, and the team which may consist of representatives from both client and vendor) need to be fully engaged and agree on software development methodology consistent with the project characteristics and environment to achieve project success. The CSFs identified and evaluated by this research may assist the parties to reflect on software development agility, and to negotiate change for traditional plan-based projects to achieve higher rates of project success.

Although the scale was not initially developed particularly to represent the vendor perspective adopted in this study and some operationalization was taken from studies based on an in-house perspective, this research provides a validated and reliable survey questionnaire instrument. The survey questionnaire was cross-validated in two stages and tested with regards to project specific data. During the pre-test phase, the respondents did not raise a concern regarding the possibility of influencing the perspectives of staff members on their own organization and confirmed that the measures used suited the current context of the study. Generally, the higher the project score is on some variables the more appropriate an agile or traditional plan-based methodology is. For instance, leadership characteristics, project team composition and user participation were found to be more important towards achieving process success when agile methodologies are used than when traditional planbased are implemented. Equally, project team composition, user participation and user support contributed more towards product success when agile methodologies were used than when traditional plan-based methodologies were implemented.

In contrast, planning and controlling were found to be more important for process success when traditional plan-based methodologies were implemented than when agile 
methodologies were used. Specification changes were found to be more important for product success when traditional plan-based methodologies were implemented than when agile methodologies were used. Practitioners who need to understand more fully which of the specific methodologies that needs to be adopted for specific project types in different contexts could use the survey tool to explore additional project management methodology/project type combinations. In other circumstances, when these factors do not lead to a particular clear choice of methodology, practitioners could consider adopting a mixed methodology selected to best fit their needs. The use of a hybrid methodology that combines the features of plan-driven and agile methodologies may be needed in some cases.

For agile projects, top management may want to draw a note of caution regarding their close involvement in high technologically innovative development efforts i.e. agile methodology projects. The results of this study suggest that top managers' involvement could do more harm than good. These findings appear to suggest that top management should be mindful in when, why and how, they are providing support and involvement. They should not overstep and implement their own agenda; otherwise, this might distress the project and project manager. Since TMS is usually in the form of providing sufficient resources to the team in ensuring project success and communicating with project team in the case of authority and responsibility, TMS is therefore, particularly important in the times of crisis or when unexpected situations arise.

The study contributes a prescriptive model serving as a tool kit to assist stakeholders involved in outsourced software development project to choose between the two methodologies in light of the candidate CSFs. The framework clearly shows that an agile methodology is preferred over traditional methods when a given candidate CSF is high/low and vice versa. For instance, if the system has low uncertainty, functions in a stable environment where plans and controls can be applied for managing specifications/goals that are clear and stable, the appropriate development methodology appears to be traditional plan-based approach regardless of the user's experience with the system. If the system has a high level of uncertainty, operating in a dynamic environment with high user participation and user support, an agile approach seems preferable depending on users' system experience.

Lastly, the project manager is not authorized in many cases to change the organizational project management methodology. Nor, can they change the type of the project in hand. The major practical contribution then is to understand the CSFs one should focus on, given the 
type of project management methodology in use. However, the focus on individual project manager's decision-making is not a major part of the thesis and frequently might not be relevant to the selection of methodology.

\subsection{Delimitations of the Research}

There are so many categories and types of software projects that no single framework can properly cover all of them simultaneously (e.g. nature of the project: open source, development of proprietary software, custom software, project's contracting scheme: inhouse, outsourced, offshored, mixed). Thus, the scope of the framework/study was narrowed down to only outsourced software development projects to improve its value added and create a more useful outcome academically and practically. Otherwise, the study would likely have been too broad to allow for meaningful relationships to be established.

The software development methodologies are broadly categorised as traditional plan-based and agile. However, while SDMs tend to match one of these more fully than others, there might still be various methodologies in each category. For instance, even though agile methodologies (e.g. XP, Scrum, Crystal, DSDM, ASD and LD among others) share the same underlying agile philosophies, they are not exactly the same and do not use identical methods and techniques. Equally, traditional software development methodologies (like waterfall, SSADM, IE and RUP) follow a structured approach in software development but may use slightly different methods and techniques. While contrasting these broader categories was necessary here, it is possible that this may have affected the research study's ability to distinguish all of the key contingencies.

\subsection{Limitations of the Research}

Although this study makes a significant contribution to both practice and research, there are latent limitations worth noting.

This study empirically analyses the relationships between candidate CSFs and project success from a vendor perspective. This categorization was helpful to provide criteria for identifying the candidate CSFs. However, it was limited to views of suppliers or outsourced firms that develop code rather than the usual clients or beneficiaries of the software made. Other stakeholders' views are also not included. The implication is that this constrains the possible interpretations of the data, since the views of clients might yield different results. It would be better to simultaneously examine client and vendor perspective (Sabherwal, 2003; Taylor, 2007). 
There are many different types of IT outsourcing relationships which have different implications for the parties. For instance, when the client is a partner of the outsourcing vendor, the interests, vision, and mission of the two organizations are frequently well aligned, while if the outsourcing relationship is simply a one-off contract, they may have very different goals and success criteria for the project. Although the data set was restricted to vendors and there tended to be larger outsourced projects relying on senior project managers as respondents to comment on aspects of the client organization, this is more suitable if the client and vendor organizations were well aligned. In an outsourced software development contract, where the organizations have no relationship either prior or beyond the immediate project, it may not be as reliable to expect that the staffs of one organization to offer detailed insights into the management, culture, priorities, vision, project success criteria, etc., of the other organization.

The issue of requiring all respondents, whether they were co-located or not with the client staff or in the same client organization, to comment on internal aspects of their client organization (not their own employer) is also another limitation. While co-located, agile teams may well have meaningful insights into the inner workings of their client organization, geographically dispersed outsourced plan-based teams may in fact have relatively limited understanding of the internal aspects of their client organization. Thus, some respondents may not be that familiar about internal aspects of their client organization. There are some questions relating to the client organization that vendor staff may not necessarily be best qualified to report on. The extent to which a vendor is qualified to evaluate the product success of something may have helped to build but are not involved in managing the benefit stream is restricted. These constrain the possible interpretations of the data, although the respondents did not raise such concerns while commenting on the client as part of their survey response process.

The factors internal to the vendor organization carrying out the project were neither included in the model nor their impact on project success examined, despite factors such as top management support, culture etc., of the vendor organization conducting the project might be argued to be important to project success. Additionally, the culture, degree of commitment, etc. can be very different between an outsourcing vendor and a client with regard to a project (for example, a project may be extremely important to a small client organization, while the outsourcer gives more of their attention and resources to larger clients). Nonetheless, more than $80 \%$ of all the outsourced software development projects in this study were 
predominantly large. This typically indicates that there were larger clients involved, larger projects with larger budgets which likely had significant importance in determining the reputation of the vendor. This suggests the vendor had a high degree of commitment in regard to these projects hence giving more of their attention and resources to these larger clients.

Some of the measurements were drawn from in-house/same organizational studies, and the measurement scales were not initially developed to represent the vendor perspective adopted in this study. This could have an effect on the effect of conflating what are likely to be the differing perspectives, and missing the opportunity to identify genuine and interesting differences between vendor and client viewpoints. Nonetheless, the respondents did not raise this concern while commenting on the survey during the pre-testing phase.

The variable "project management methodologies" aims at representing the "project management methodology being implemented" and this is the question asked in the questionnaire itself. However, one might argue that what may moderate the impact of candidate CSFs on project success is not the methodology used, but the type of project or the environment under which it operates. Accordingly, these two things can be taken to be different and hence one might not make a judgment on the "type of project" only based on the methodology used to manage it. However, the data was collected regarding project management methodology rather than project type.

It should be noted that product success may take a considerable time to be realized. The point of time at which the project was measured is likely to have a significant influence on this dependent variable.

The study of candidate CSFs for project success was limited to factors that are currently documented in the software project management literature. However, there might be other candidate CSFs which are not frequently discussed that might also significantly influence outsourced software project success. Extending the current questionnaire would make the survey too long and, for modelling purposes and parsimony reasons not all candidate CSFs could be incorporated in one model. Some candidate CSFs were combined or represented through their constituent factors (for instance, planning was combined with controlling and monitoring, urgency was combined with complexity, and team's general expertise was also combined with team's expertise with the task). The model fitting process, using fit indices like AGFI, PGFI, PRATIO, PNFI, also punishes complexity in preference of parsimony. 
The construct of project criticality did not fit the single congeneric model as indicated by the model fit indices which were all below the acceptable criteria despite several modifications. The factor loading values were also very low suggesting that both items neither contributed relatively nor absolutely to the project criticality construct. Therefore, project criticality was completely dropped from further data analysis and its impact on project success could not be assessed. However, project criticality is still a potentially important contingency but one for which enhanced measurement approaches and design are needed. Further, some relationships were not hypothesized ex ante because of lack of theoretical evidence, and thus, there is a possibility that during the confirmatory model building, identification of some significant relationships could have been influenced by statistical chance.

Finally, there are general several potential sources of error when executing survey research. Such errors include measurement error, sampling error, internal validity error, and statistical conclusion error (Straub 1989). This research was cognizant of these errors and attempted to mitigate them by using commonly accepted methods such as selection of survey items from existing management/IS theory, use of tested instruments where available; use of SoM professors (experts) for instrument review, pilot testing by using 15 (semi-structured interviews) software workers in Wellington for initial instrument refinement. Pre-tests and a pilot study of 400 local software developers were undertaken to develop the survey instrument thoroughly. In addition, robust statistical techniques including Confirmatory Factor Analysis (CFA) with covariance based SEM (MLE) were used in data analysis to assess the validity and reliability of the survey instrument.

\subsection{Directions for Future Research}

The findings here provide opportunities and directions for studies that can be carried out in future in the area of software project management.

Some of the candidate CSFs did not directly contribute significantly to project success as expected in the database here despite the fact that theoretical evidence suggests these candidate CSFs are important for outsourced software project success. Future studies could proceed to further investigate why some of these candidate CSFs did not directly associate with project success as initially hypothesized based on existing literature.

More research is needed to examine whether the contingency relationships found here also apply from a client perspective and to analyse the differences between vendor and client perspectives. Future researchers are called upon to include other project stakeholders like the 
customers, government, local population, tax payers among others. This would help to understand what works when. Future studies could also incorporate other categories and types of software projects to see if there any significant differences across the spectrum. Future studies could also examine other interaction effects that were not tested in this study.

Future research should examine candidate CSFs for a range of various specific methodologies in each category. For instance, for agile methodologies (such as XP, Scrum, Crystal, DSDM, ASD, Crystal, and LD among others) could be investigated to identify the CSFs for each. Similarly, traditional software development methodologies (e.g. waterfall, SSADM, IE, RUP) that follow a structured approach in software development could each be studied to confirm these findings. Case study research could also be used to test the applicability and usefulness of these candidate CSFs frameworks in practice. This is likely to narrow the existing gap between the research in candidate CSFs and what practitioners do or perceive to be candidate CSFs.

In this study, the necessity to limit the size of the questionnaire did not allow for the full inclusion of all previously used CSFs measures (e.g., three commitment components separately i.e. continuance, normative and affectivity). Instead, such measures were combined. It will be useful for future research, therefore, to examine the independent effects of each of the commitment components on project employees' behaviour for both project methodologies.

\subsection{Chapter Summary}

This last chapter of the dissertation concluded and provided some of the most important aspects of the research findings. The contributions of the research were then discussed, both with regard to the theory value of the research and the practitioner value. Next, the delimitations and limitations of the research were outlined. Finally, directions for future research were identified and discussed. It appears that the bivariate inclusion of numerous potential candidate CSFs leads to a larger set of broadly based CSFs with direct effects. These direct effects could have potentially been the reason for previous studies labelling some factors as candidate CSFs. 


\section{REFERENCES}

Adolph, S., Kruchten, P., \& Hall, W. (2012). Reconciling Perspectives: A Grounded Theory of how People Manage the Process of Software Development. The Journal of Systems and Software, 85, 1269-1286.

Aiken, L. S., \& West, S. G. (1991). Multiple Regression: Testing and Interpreting Interactions. Newbury Park, London, Sage.

Agile Alliance. (2001). Manifesto for Agile Software Development. [Online]. Available at: http://agilemanifesto.org/

Ahimbisibwe, A., Cavana, R.Y., \& Daellenbach, U. (2015). A Contingency Fit Model of Critical Success Factors for Software Development Projects, Journal of Enterprise Information Management, 28 (1): 7-33.

Ahimbisibwe, A., \& Nangoli, S. (2012). Project Communication, Individual Commitment, Social Networks and Perceived Project Performance. Journal of African Business, 13(2), 101-114.

Alwin, D. (1997). Feeling Thermometers Versus 7-Point Scales: Which are better? Sociological Methods \& Research, 3(25), 318-340.

Anderson, J. C., \& Gerbing, D. W. (1988). Structural Equation Modeling in Practice: A Review and Recommended Two-Step Approach. Psychological Bulletin, 103(3), 411-423.

Andres, H., \& Zmud, R. (2002). A Contingency Approach to Software Project Coordination. Journal of Management Information Systems, 18(3), 41-70.

Ang, S., \& Straub, D.W. (1998). Production and Transaction Economics and IS Outsourcing: A Study of the US Banking Industry. MIS Quarterly, 22 (4), 535-552.

APM. (2006). APM Body of Knowledge (5th ed.). Buckinghamshire: Association for Project Management.

Austin, R.D., \& Devin, L. (2009). Weighing the Benefits and Costs of Flexibility in Making Software: Towards a Contingency Theory of the Determinants of Development Process Design. Information System Research, 20 (3), 462-477.

Bagozzi, R.P., \& Yi,Y. (1988). On the Evaluation of Structural Equation Models. Journal of the Academy of Marketing Science, 16(1), 74-94

Bajwa, J., Singh, K., \& Sharma, N. (2012). Analysing the Enablers and Challenges for Successful Methodological Transition. Proceedings of International Conference on recent Advances and Future Trends in Information Technology.

Barki, H., Rivard, S., \& Talbot, J. (2001). An Integrative Contingency Model of Software Project Risk Management. Journal of Management Information Systems, 17(4), 3769.

Baccarini, D., Salm, G., \& Love, P.E.D. (2004). Management of Risks in Information Technology Projects. Ind. Manag. Data Syst., 104(4), 286-295.

Barlow, B.J., Giboney, J.S., Keith, M.J., et al. (2011). Over view and Guidance on Agile Development in Large Organizations. Communication of the Association for Information Systems, 29(2), 25-44.

Bentler, P. M. (1990). Comparative Fit Indexes in Structural Models. Psychology Bulletin, 107(2), 238-246.

Bentler, P. M., \& Bonnett, D. G. (1980). Significance Tests and Goodness of Fit in the 
Analysis of Covariance Structures, Psychol. Bull., 88, 588-626.

Bergeron, F., Raymond, R., \& Rivard, S. (2001). Fit in Strategic Information Technology Management Research: An Empirical Comparison. The International Journal of Management Science, 29, 125-142.

Boehm, B., \& Turner, R. (2003). Using Risk to Balance Agile and Plan-Driven Methods. IEEE Computer Society, 36(6), 57-66.

Boehm, B., \& Turner, R. (2004). Balancing Agility and Discipline: A Guide for the Perplexed. Addison-Wesley.

Boehm, B., \& Turner, R. (2005). Management Challenges to Implementing Agile Processes in Traditional Development Organizations. IEEE software, 22(5), 30-39.

Burns, R. N., \& Dennis, A. R. (1985). Selecting the Appropriate Application Development Methodology. ACM SIGMIS Database, 17(1), 19-23.

Burns, T., \& Stalker, G. M. (1961). The Management of Innovation. London: Tavistock.

Byrne, B.N. (2001). Structural Equation Modeling with AMOS: Basic Concepts, Applications and Programming. Rahwah, NJ Lawrence Erlbaum.

Byrne, B.N. (2009). Testing for Multigroup Invariance Using AMOS Graphics: A Road Less Travelled. Structural Equation Modelling: A Multidiplinary Journal, 11(2), 272-300.

Cavana, R.Y., Delahaye, B.L., \& Sekaran, U. (2001). Applied Business Research: Qualitative and Quantitative Methods. Brisbane: John Willey \& Sons.

Ceschi, M., Sillitti, A., Succi, G., \& De Panfilis, S. (2005). Project Management in PlanBased and Agile Companies. IEEE software, 22(3), 21-27.

Chan, Y.E., Huff, S., Barclay, D. W., \& Copeland, D. G. (1997). Business Strategic Orientation, Information Systems Strategic Orientation and Strategic alignment. Information System Research, 8(2), 125-150.

Charette, R.N. (2005). Why Software Fails. IEEE Spectrum, 42(9), 42-49.

Charvat, J. (2003). Project Management Methodologies: Selecting, Implementing and Supporting Methodologies and Processes for Projects. New York: John Wiley \& Sons, Inc.

Chow, T., \& Cao, D. (2008). A Survey of Critical Success Factors in Agile Software Projects. The Journal of Systems and Software, 81(6), 961-971.

Cleden, D. (2009). Managing Project Uncertainty. Gower, Farnham (UK).

Cockburn, A. (2000). Selecting a Project's Methodology. IEEE software, 17(4), 64-71.

Cockburn, A. (2007). Agile Software Development: The Cooperative Game. Upper Saddle River, NJ: Addison-Wesley.

Cockburn, A. (2002). Agile Software Development. Upper Saddle, NJ: Addison-Wesley.

Cockburn, A., \& Highsmith, J. (2001). Agile Software Development: The People Factor. Computer, 34(11), 131-133.

Cohn, M. (2012, Feb 13). Succeeding with Agile [online forum comment]. Retrieved on November 2, 2012 from http://www.mountaingoatsoftware.com/blog/agile-succeedsthree-times-more-often-than-waterfall.

Collis, J., \& Hussey, R. (2003). Business Research : A Practical Guide for Undergraduate and Postgraduate Students. New York: Palgrave Macmillan.

Coltman, T, Devinney, T.M, Midgley, D.F \& Veniak, S. (2008). Formative versus Reflective 
Measurement Models: Two Applications of Formative Measurement. Journal of Business Research, 61(12), 1250-1262.

Cooke-Davies, T. (2002). The "Real" Success Factors on Projects. International Journal of Project Management, 20(3), 185-190.

Cunningham, E. (2010). A Practical Guide to Structural Equation Modelling Using AMOS.

New Zealand Social Statistics Network (NZSSN) Summer Programme Course Notes.

Dalcher, D., \& Brodie, L. (2007). Successful IT Projects. London: Thomson Learning.

Dawson, J. F. (2014). Moderation in Management Research: What, Why, When and

How. Journal of Business and Psychology, 29, 1-19.

http://www.jeremydawson.co.uk/slopes.htm

DeLone, W., \& McLean, E. (1992). Information Systems Success: The Quest for the

Dependent Variable. Information Systems Research, 3(1), 60-95.

Dillman, D. A. (1991). The Design and Administration of Mail Surveys. Annual review of Sociology, 17, 225-248.

Dishaw, M. T. \& D. M. Strong (1999). Extending the Technology Acceptance Model with Task-Technology Fit Constructs. Information and Management, 36(1), 9-21.

Dyck, S., \& Majchrzak, T.A. (2012). Identifying Common Characteristics in Fundamental, Intergrated, and Agile Software Development Methodologies. IEEE Computer Society. Proceedings of the $45^{\text {th }}$ Hawaii International Conference on Systems Sciences.

Drazin, R., \& Van de Ven, A.H. (1985). Alternative Forms of Fit in Contingency Theory. Administrative Science Quarterly, 30 (4), 514-539.

Dybå, T., \& Dingsøyr, T. (2008). Empirical Studies of Agile Software Development: A Systematic Review. Information and Software Technology, 50(9-10): 833-859.

Eveleens, J. L., \& Verhoef, C. (2010). The Rise and Fall of the Chaos Report Figures. IEEE Software, 30-36.

Fairchild, A. J., \& MacKinnon D.P. (2009). A General Joint Analysis Model for Testing Mediation and Moderation. Preventive Sciences, 10, 87-99.

Fiedler, F. E. (1964). A Contingency Model of Leadership Effectiveness. Advances in Experimental Social Psychology, (1), 149-190. New York: Academic Press.

Field, A. (2006). Discovering Statistics using SPSS (2nd Ed.). Sage, London.

Fornell C. \& Larcker, D. F. (1981). Evaluating Structural Equation Models with Unobservable Variables and Measurement Error. Journal of Marketing Research, 18(1), 39-50.

Fortune J, White D (2006). Framing of Project Critical Success Factors by a Systems Model. International Journal of Project Management, 24(1), 53-65.

Franceschini, F., Galetto, M., Pignatelli. A., \& . Varetto, M. (2003). Outsourcing: Guidelines for a Structured Approach. Benchmarking: An International Journal, 10(3), 246-260.

Frazier, P.A., Tix, A.P., \& Barron, K. E. (2004). Testing Moderator and Mediator Effects in Counseling Psychology Research. Journal of Counseling Psychology, 51(1), 115-134.

Gable, G., Sedera, D., \& Chan, T. (2008, Jul). Re-conceptualizing Information System Success: The IS-Impact Measurement Model. Journal of the Association for Information Systems, 9(7), 377-408. 
Glass, R. (2006). The Standish report: Does it Really Describe a Software Crisis.

Communications of the ACM, 49(8), 15-16.

Goodhue, D. L. (1995). Understanding user evaluations of information systems. Management Science 41(12), 1827-1844.

Gottschalk, P., \& Solli-Saether, H. (2005). Critical Success Factors from IT Outsourcing Theories: An Empirical Study. Industrial Management \& Data Systems, 105(6), 685702.

Gottschalk, P., \& Solli-Saether, H. (2006). Maturity Model for IT Outsourcing Relationships. Industrial Management \& Data Systems, 106(2), 200 - 212.

Grover, V., Teng, T.C., \& Cheon, M.J. (1998). Towards a Theoretically-Based Contingency Model of Information Systems Outsourcing. In Willcocks, L.P. and Lacity, M.C. (Eds.), Strategic Sourcing of Information Systems: Perspectives and Practices (pp. 79-101). Chichester, UK: John Wiley \& Sons.

Hajjdiab, H., Taleb, A., \& Ali, J. (2012). An Industrial Case Study for Scrum Adoption. Journal of Software, 70(1), 237-242.

Hancox, M., \& Hackney, R. (2000). IT Outsourcing: Frameworks for Conceptualizing Practice and Perception. Information Systems Journal, 10(3), 217-237.

Hair, J.F., Black, W.C., Babin, B.J, \& Anderson, R.E (2009). Multivariate Data Analysis (7 $^{\text {th }}$ Ed.). Englewood Cliffs, NJ: Prentice Hall.

Hair, J.F., Black, W.C., Babin, B.J, \& Anderson, R.E (2010). Multivariate Data Analysis (8 ${ }^{\text {th }}$ Ed.). Englewood Cliffs, NJ: Prentice Hall.

Hass, K.,B. (2008). Introducing the New Project Complexity Model. Management Concepts, 22-31.[Online]. Available at: http://www.projecttimes.com/articles/introducing-thenew-project-complexity-model-part-i.html.

Henderson-Sellers, B., \& Serour, M.K. (2005). Creating a Dual-Agility Method: The Value of Method Engineering. Journal of Database Management, 16(4), 1-23.

Highsmith, J. (2010). Agile Project Management: Creating Innovative Products (2nd ed.). Upper Saddle River, NJ: Addison-Wesley.

Hoehle, H. (2011). Consumer Intentions to Use Electronic Banking Channels: The Role of Task-Channel Fit. Unpublished Doctoral thesis. Victoria University of Wellington.

Howell, D., Windahl, C., \& Seidel, R. (2010). A Project Contingency Framework Based on Uncertainty and its Consequences. International Journal of Project Management, 28 (3), 256-264.

Hu, L., \& Bentler, P.M. (1999). Cut off Criteria for Fit Indices in Covariance Structure Analysis: Conventional Criteria versus New Alternatives. Structural Equation Modelling, 6(1), 1-55.

Humphrey, W.S. (2005). Why Big Software Projects Fail: The 12 Key Questions. CrossTalk, The Journal of Defense Software Engineering, 18(3), 25-29.

Iacobucci, D. (2010). Structural Equations Modelling: Fit Indices, Sample Size, and Advanced Topics. Journal of Consumer Psychology, 20, 90-98.

Iivari, J., \& Huisman, M. (2007). The Relationship between Organizational Culture and the Deployment of Systems Development Methodologies. MIS Quaterly, 31(1), 35-58.

Jiang, J., Klein, G. (2000). Software Development Risks to Project Effectiveness. Journal of Systems and Software, 52(1), 30-41. 
Ika, L. (2009). Project Success as a Topic in Project Management Journals. Project Management Journal, 40 (4), 6-19.

Imreh, R., \& Raisinghani, M. (2011). Impact of Agile Software Development on Quality within Information Technology Organisations. Journal of Emerging Trends in Computing and Information Science, 10(10), 460-475.

Jiang.J., \& Klein, G. (2000). Software Development Risks to Project Effectiveness. Journal of Systems and Software, 52, 3-10.

Jiang, J.J., Klein, G., \& Chen, H.G. (2006). The Effects of User Partnering and User Nonsupport on Project Performance. Journal of the Association for Information Systems 7 (2), 68-90.

Joreskog, K. G., \& Sorbom, D. (1989). LISREL 7: A Guide to the Program and Applications, 2nd ed. Chicago, IL: SPSS.

Joreskog, K. G. (1971). Simultaneous Factor Analysis in Several Populations. Psychometrica,36, 409-426.

Jose, P. E. (2013a). Doing Statistical Mediation and Moderation. New York: NY: Guilford Press.

Jose, P. E. (2013b). Moderation/Mediation Help Centre (Ver. 3.0). Victoria University of Wellington, Wellington, New Zealand, School of Psychology. Retrieved [July $12^{\text {th }}$ 2014] at: http://pavlov.psyc.vuw.ac.nz/paul-jose/helpcentre/

Jugdev, K., \& Muller, R. (2005). A Retrospective Look at our Evolving Understanding of Project Success. Project Management Journal, 36(4), 19-31.

Jun, L., Qiuzhen,W., \& Qingguo, M. (2011). The Effects of Project Uncertainty and Risk Management on IS Development project Performance: A Vendor Perspective. International Journal of Project Management, 29, 923-933.

Jung, Y.J., Wang, J.W., Wu, S. (2008). Competitive Strategy, TQM Practice, and Continuous Improvement of International Project Management: A Contingency Study. International Journal of Quality and Reliability Management, 26(2), 161-183.

Kandelousi, N., Ooi. J., \& Abdollahi, A. (2011). Key Success Factors for Managing Projects. World Academy of Science, Engineering and Technology, 5(11), 1291- 1296.

Karen, E. P., Catherine B., \& Jing Q. (2010). Do Project Managers Practice what they Preach, and Does it Matter to Project Success? International Journal of Project Management, 28(7), 650-662.

Karlstrom, D., \& Runeson, P. (2005). Combining Agile Methods with Stage-gate Project Management. IEEE Software, 22 (3), 43-49.

Kern, T., Willcocks, L.P., \& van Heck, E. (2002). The Winner's Curse in IT Outsourcing: Strategies for Avoiding Relational Trauma, California Management Review, 44(2), 47-69.

Kearns, G.S., \& Sabherwal. R. (2007). Antecedents and Consequences of Information Systems Planning Intergration. IEEE Transactions on Engineering Management, 54(4), 628-643.

Kenny, D., \& Judd, C.M.(1984). Estimating the Non-Linear and Interactive Terms of Latent Variables. Psychological Bulletin, 96, 201-210.

Killen, C. P., Jugdev, K., Drouin, N., \& Petit,Y.(2012). Advancing Project and Portifolio 
Management Research: Applying Strategic Management Theories. International Journal of Project Management, 30(5), 525-538.

Killen, C.P., \& Kjaer, C. (2012). Understanding Project Interdependences: The role of Visual Representation, Culture and Process. International Journal of Project Management,30, 554-566.

Kishore, R., Rao, H., Nam, K., Rajagopalan, S., and Chaudhury, A. (2003). A Relationship Perspective on IT Outsourcing. Communications of the ACM, 46(12), 87-92.

Kline, T.J.B., \& Dunn B. (2000). Analysis of Interaction Terms in Structural Equation Models: A Non-Technical Demonstration Using Deviation Score Approach. Canadian Journal of Behavioural Science, 32(2), 127-132.

Koskinen, K. U. (2011). Project-Based Companies as Learning Organisations: Systems Theory Perspective. International Journal Project Organisation and Management, 3(1), 91-106.

KPMG. (2013). Project Management Survey Report 2013. Wellington, New Zealand. KPMG: [Online]. Available at:

http://www.kpmg.com/NZ/en/IssuesAndInsights/ArticlesPublications/Documents/KP MG-Project-Management-Survey-2013.pdf

Krejcie, P., \& Morgan, D. W. (1970). Determining Sample Size for Research Activities. Educational and Psychological Measurement, 30(3), 607-610.

Lambe, C.J., Spekman, R.E., \& Hunt, S.D. (2002). Alliance Competence, Resources, and Alliance Success: Conceptualization, Measurement and Initial Test. Journal of the Academy of Marketing Science, 30(2), 141-158.

Lacity, M., \& Willcocks, L. (1998). An Empirical Investigation of Information Technology Sourcing Practices: Lessons from Experience. MIS Quarterly, 22(3), 363-408.

Lacity, M., Willcocks, L., \& Feeny, D. (1995). IT Outsourcing: Maximize Flexibility and Control. Harvard Business Review, 73(3), 84-93.

Lacity, M., Willcocks, L., \& Feeny, D. (1996). The Value of Selective IT Sourcing. Sloan Management Review, 37(3), 13-25.

Lacity, M., \& Willcocks, L. (2000). Relationships in IT Outsourcing: A Stakeholder Perspective, Cutter Consortium, 2 (3).

Lawrence, P.R., \& Lorsch, J.W. (1967). Differentiation and Integration in Complex Organizations. Administrative Science Quarterly 12(1), 1-47.

Lee, G., \& Xia, W. (2010). Toward Agile: An Integrated Analysis of Quantitative and Qualitative Field Data on Software Development Agility. MIS Quarterly, 34(1), 87114.

Leffingwell, D. (2007). Scaling Software Agility: Best Practices for Large Enterprises. Upper Saddle River, NJ: Addison Wesley.

Lemetayer, J.(2010). Identifying the Critical Factors in Software Development Methodology Fit. Master's thesis, Victoria University, New Zealand.

Lindvall, M., Basili, V., Boehm, B., Costa, P., et al. (2002). Empirical Findings in Agile Methods.. Proceedings of the Second XP Universe and First Agile Universe Conference on Extreme Programming and Agile Methods - XP/Agile Universe,81-92. London. UK.

Lipovetsky, S., Tishler, A., Dvir, D., \& Shenhar, A. (1997). The Relative Importance of 
Project Success Dimensions. $R \& D$ Management, 27(2), 97-106.

Little, R. J. A. \& Rubin, D.B. (2002). Statistical analysis with missing data. NewYork, John wileg \& Sues.

Little, T. (2005). Context-Adaptive Agility: Managing Complexity and Uncertainty. IEEE Software 22(3), 28-35.

Livermore, J.A. (2008). Factors that Significantly Impact the Implementation of an Agile Software Development Methodology. Journal of Software 3 (4), 31-36.

Lonsdale, C. \& Cox, A. (2000). The Historical Development of Outsourcing: The Latest Fad?”. Industrial Management \& Data Systems, 100(9), 444-50.

Luo, Y. (2002). Contract, Cooperation and Performance in International Joint Ventures. Strategic Management Journal, 23(10), 903-19.

Mansor, Z., Yahya, S., \& Arshad, N.H. (2011). Towards the Development Success Determinants Charter for Agile Development Methodology. International Journal of Information Technology and Engineering, 2(1), 1-7.

Mathiassen, L., Tuunanen, T., Saarinen, T., \& Rossi, M. (2007). A Contingency Model for Requirements Development. Journal of the Association for Information Systems, 8(11), 569-597.

Meyer, J. P., \& Allen, N. J. (1991). A Three-Component Conceptualization of Organizational Commitment. Human Resource Management Review 1(1): 61-89.

Meyer, J. P., \& Allen, N. J. (1997). Commitment in the Workplace: Theory, research, and application. Thousand Oaks, CA: Sage.

Miles, R. E., \& Snow, C. C. (1978). Organizational strategy, structure, and process. San Francisco:McGraw-Hill.

Miles, R. E., Snow, C. C., Meyer, A. D., \& Coleman, H. J., Jr. (1978). Organizational Strategy, Structure, and Process. Academy of Management Review, 3 (3), 546-562.

Milfont, T.L., \& Fischer, R. (2010). Testing Invariance Across Groups: Applications in Cross Cultural Research. International Journal of Psychological Research, 3(1), 111121.

Milis, K., \& Mercken, R. (2002). Success Factors Regarding the Implementation of ICT Investment Projects. Int. J. Production Economics 80(1), 105-117.

Mills, D., Sherrel, L., Boydstun, J., \& Wei, G. (2006). Experiences using Agile Software Development for a Marketing Simulation. IEEE Southeast Conference Proceedings, 285-290.

Minzberg, H. (1979). The Structuring of Organizations. Prentice-Hall.

Mishra, D., \& Mishra, A. (2011). Complex Software Project Development: Agile Methods Adoption. Journal of Software Maintenance and Evolution, 23, 549-564.

Misra, S. C., Kumar, V., \& Kumar, U. (2009). Identifying some Important Success Factors in Adopting Agile Software Development Practices. Journal of Systems and Software, 82 (11), 1869-1890.

Mnkandra, E., \& Dwolazky, B., (2004). Balancing the Human and the Engineering factors in Software Development. $7^{\text {th }}$ AFRICON Conference in Africa Proceedings, 2, $1207-$ 1210. Johannesburg. South Africa.

Mohammad, A.L., \& Al-Shargabi, B. (2011). Agile Software Methodologies: Employee, 
Customer and Organization Factors. International Conference on Technology and Business Management, March 28-30.

Morgan, G. (1986). Images of organisations. Sage. Newbury Park, CA.

Mountain Goat Software (2005). Retrieved on 21 September, 2012 from: http://www.mountaingoatsoftware.com/scrum/overview

Murad, R, S. A., \& Cavana, R.Y. (2012). Applying the Viable System Model to ICT Project Management. International Journal of Applied Systemic Studies, 4(3), 186-205.

Nah, F.F., \& Delgado, S. (2006). Critical Success Factors for Enterprise Resource Planning Implementation and Upgrade. The Journal of Computer Information Systems, 46(5), 99-113.

Nam, K., Rajagopalan, S., Rao, H.R. \& Chaudhury, A. (1996). A Two-level Investigation of Information Systems Outsourcing. Commun. ACM 39(7), 36-44.

Nunnally, J. C. (1978). Psychometric Theory, 2nd ed. New York: McGraw-Hill.

Nasir, M. H., \& Sahibuddin, S. (2011). Critical Success Factors for Software Projects: A Comparative study. Scientific Research and Essays, 6(10), 2174-2186.

Nidumolu, S.R. (1995). The Effect of Coordination and Uncertainty on Software Project Performance: Residual Performance Risk as an Intervening Variable. Information System Research, 6(3), 191-219.

Nidumolu, S.R. (1996). A Comparison of the Structural Contingency and Risk-Based Perspectives on Coordination in Software-Development Projects. Journal of Management Information System, 13(2), 77-113.

Office of Government Commerce (OGC). (2005). Common Causes of Project Failure. OGC London.

Office of Government Commerce (OGC). (2009). Managing Successful Projects with PRINCE2. London: TSO.

Organizations for Economic Co-operation and Development (OECD). (2013). OECD Secretariat, Paris, France. [Online]. Available at: http://stats.oecd.org/

Oz E, \& Sosik, J.J. (2000). Why Information Systems Projects are Abandoned: A Leadership and Communication Theory and Exploratory Study. J. Comput. Inform. Syst. 41(1), 66-77.

Parker, S. C. \& Van Witteloostuijn, A. (2010). A General Framework for Estimating Multidimensional Contingency Fit. Organization Science, 21(2), 540-553.

Pennings, J.M. (1975). The Relevance of the Structural-Contingency Model for Organizational Effectiveness. Administrative Science Quarterly, 20(3), 393-410.

Perminova, O., Gustafsson, M., \& Wikström, K. (2008). Defining Uncertainty in Projects: A New Perspective. International Journal of Project Management 26, 73-79.

Perrin, R. (2008). Real-world Project Management: Beyond Conventional Wisdom, Best Practices, and Project Methodologies. Wiley.

Petit, Y. (2012). Project Portfolios in Dynamic Environments: Organising for Uncertainty. International Journal of Project Management, 30, 539-553.

Pinto, J., \& Prescott, J. (1988). Variations in Critical Success Factors over the Stages in the Project Life Cycle. Journal of Management, 14(1), 5-18.

Pinto, J. K., \& Slevin, D. P. (1988). Critical Success Factors Across the Project Life Cycle. Project Management Journal, 19(3), 67-75. 
PMI. (2013). A Guide to the Project Management Body of Knowledge (PMBOK Guide) (5th ed.). Project Management Institute.

PMI. (2013). Pulse of the Profession Report 2013. Project Management Institute.Inc.

Podsakoff, P. M., Mackenzie, S. B., Lee, J., \& Podsakoff, N. P. (2003). Common Methods

Biases in Behavioural Research: A Critical Review of the Literature and

Recommended Remedies. Journal of applied Psychology, 88(5), 879-903.

Prescott, J. E. (1986). Environments as Moderators of the Relationship between Strategy and Performance. Academy of Management Journal, 29(2), 329-346.

Rai, A., \& Hindi, A.H. (2000). The Effects of Development Process Modeling and Task Uncertainty on Development Quality Performance. Information \& Management, 37(6), 335-346.

Ramesh, B., Mohan, K., \& Cao, L. (2012). Ambidexiterity in Agile Distributed Development: An Empirical Investigation. Information System Research, 23(2), 323339.

Ratbe, D., King, W. R., \& Kim, Y.G. (2000). The Fit between Project Characteristics and Application Development Methodologies: A Contingency Approach. Journal of Computer Information Systems, 40(2), 26-33.

Raykov, T. (2004). Estimation of Maximal Reliability: A Note on a Covariance Structure Modelling Approach. British Journal of Mathematical \& Statistical Psychology, 57, 21-27.

Raykov, T. (1997). Estimation of Composite Reliability for Congeneric Measures. Applied Psychological Measurement, 21, 173-184.

Raykov, T. (1998). Coefficient alpha and Composite Reliability with Interrelated NonHomogeneous Items. Applied Psychological Measurement, 22, 375-385.

Rice, M.P., Colarelli O’Connor, G., \& Pierantozzi, R., (2008). Implementing a Learning Plan to Counter Project Uncertainty. MIT Sloan Manage Review. 49(2), 54-62.

Rigdon, E.E. (1996). CFI versus RMSEA: a Comparison of Two Fit Indices for Structural Equation Modelling. Structural Equation Modelling 3(1), 369-79.

Royce, W. (1970). Managing the Development of Large Software Systems. Proceedings of IEEE WESCON 26 (August): 1-9. [online]. Available at: http://www.cs.umd.edu/class/spring2003/cmsc838p/Process/waterfall.pdf

Sabherwal, R. (2003). The Evolution of Coordination in Outsourced Software Development Projects: A Comparison of Client and Vendor perspectives. Information and Organization 13(3), 153-202.

Sanderson, J. (2012). Risk, Uncertainty and Governance in Mega Projects: A Critical Discussion of Alternative Explanations. International Journal of Project Management, 30, 432-443.

Sauser, B. J., Reilly, R. R., \& Shenhar, A. J. (2009). Why Projects Fail? How Contingency Theory Can Provide New Insights - A Comparative Analysis of NASA's Mars Climate Orbiter Loss. International Journal of Project Management, 27(7), 665-679.

Sauer, C. \& Cuthbertson, C. (2003). The state of IT Project Management in UK 2002-2003. Computer Weekly, 15 April.

Sauer, C., Gemino, A., \& Reich, B.H. (2007). The Impact of Size and Volatility on IT Project 
Performance: Studying the Factors Influencing Project Risk. Communications of the ACM 50(11), 79-84.

Saur, P.L., \& Dick, A. (1993). Using Moderating Variables in Structural Equation Models. Advances in Consumer Research, 20, 637-640.

Schmidt, R., Lyytinen, K., Keil, M., \& Cule, P. (2001). Identifying Software Project Risks: An International Delphi Study. Journal of Management Information Systems, 17(4), 5-36.

Schmidt, J.B., \& Calantone, R.J . 1998). Are Really New Product Development Projects Harder to Shut Down? Journal of Product Innovation Management, 15 (2), 111-123.

Schoonhoven, B.C. (1981). Problems with Contingency Theory: Testing Assumptions Hidden within the Language of Contingency Theory. Administrative Science Quarterly, 26 (3), 349-377.

Sharma, R., \& Yetton, P. (2003, Dec). The Contingent Effects of Management Support and Task Interdependence on Successful Information Systems Implementation. MIS Quarterly, 27(4), 533-555.

Sheffield, J., \& Lemétayer, J. (2013). Factors associated with the Software Development Agility of Successful projects. International Journal of Project Management, 31(3), 459-472.

Sheffield, J., \& Lemétayer, J. (2010). Measuring the Critical Success Factors in Project Management Methodology Fit. PMI Global Congress Proceedings. Melbourne, Australia.

Sheffield, J., Lemétayer, J. \& Ahimbisibwe, A. (2011). What Works When? Exploring Contingency in Software Development Methodology. Australian and New Zealand Academy of Management (ANZAM) Conference, 7-9 December, Wellington New Zealand.

Shenhar, A. J. (2001). One Size Does Not Fit All Projects: Exploring Classical Contingency Domains. Management Science, 47(3), 394-414.

Shenhar, A. J., Tishler, A., Dvir, D., Lipovetsky, S., \& Lechler, T. (2002). Refining the Search for Project Success Factors: A Multivariate, Typological Approach. $R \& D$ Management, 32(2), 111-126.

Shenhar, A., \& Dvir, D. (2007). Reinventing Project Management: The Diamond Approach to Successful Growth and Innovation. Boston, MA: Harvard Business School Press.

Shenhar, A. (2008). Unleashing the Power of Project Management. Industrial Management, 50(1), 14-18.

Singh, A., Singh, K., \& Sharma, N. (2012). Managing Knowledge in Agile Software Development. International Conference on Recent Advances and Future Trends in Information Technology (iRAFIT2012) Proceedings published in International Journal of Computer Applications (IJCA).

Slevin, D., \& Pinto, J. (1987). Balancing Strategy and Tactics in Project Implementation. Sloan Management Review, 29(1), 33-41.

Soderlund J. (2004). Building Theories of Project Management: Past Research, Questions for the Future. Journal of Project Management, 22, 183-191.

Standing C, Guilfoyle G, Lin C, Love PED (2006). The Attribution of Success and Failure in IT Projects. Ind. Manag. Data Syst., 106(8), 1148-1165. 
Standish Group (2012). CHAOS 2012 report Agile Projects Successful 3X More than Non-Agile Projects. [Online]. Available at: http://www.sdsconsulting.com/blog/agile-projects-successful-3x-more-non-agile-projects.

Standish Group International, Inc, (2001). Extreme Chaos. Technical Report.

Standish Group (1995). Chaos Report. Boston, MA. [Online]. Available at: http://www.projectsmart.co.uk/docs/chaos-report.pdf.

Standish Group (2009, April 23). Latest Study Shows Rise in Project Failures. [Online]. Available at: http://kinzz.com/resources/articles/91-project-failures-rise-study-shows Straub, D.W. (1989). Validating instruments in MIS research, MIS Q., 13(2), 147-169.

Strode, D. E., Huff, S. H., \& Tretiakov, A. (2009). The Impact of Organizational Culture on Agile Method Use. 42nd Hawaii International Conference on System Sciences (pp. 19). Hawaii: HICSS.

Sudhakar, P. (2012). A Model of Critical Success Factors for Software Development. Journal of Entreprise Information Management, 25(6), 537-558.

Swink, M. (2000). Technological Innovativeness as a Moderator of New Product Design Intergration and Top Management Support. Journal Product Innovation Management, 17, 2008-220.

Tate, M. (2010). Reflections on Perceived Online Service Quality: Structure, Antecedents, Ontology, Theory and Measurement. Unpublished doctoral thesis. Victoria University of Wellington.

Taylor, H. (2007). Outsourced IT projects from the Vendor Perspective: Different Goals, Different Risks. Journal of Global Information Management, 15(2), 1-27.

Thompson, J. D. (1967). Organizations in Action. New York: McGraw-Hill.

Tiwana, A., \& Keil, M. (2004). The One-Minute Risk Assessment Tool. Communications of the ACM, 47(11), 73-77.

Tosi, H., \& Slocum, J. (1984). Contingency Theory: Some Suggested Directions. Journal of Management, 10(1), 9-26.

Van Donk, D. P., \& Molloy, E. (2008). From Organising as Projects to Projects as Organizations. International Journal of Project Management, 26 (2), 129-137.

Venkatraman, N. (1989).The Concept of Fit in Strategy Research: Toward a Verbal and Statistical Correspondence. Academy of Management Review, 14(3), 423-444.

Venkatraman N., \& Prescott, J. E. (1990). Environment-Strategy Coalignment: An Empirical Test of its Performance Implications. Strategic Management Journal, 11(1), 1-23.

Vinekar, V., Slinkman, C. W., \& Nerur, S. (2006). Can Agile and Traditional Systems Development Approaches Coexist? An Ambidextrous View. Information Systems Management, 23(3), 31-42.

Vroom, V.H. \& Yetton, P.W. (1973). Leadership and decision-making. Pittsburgh: University of Pittsburgh Press.

Wallace, L., Keil, M., \& Rai, A. (2004a). Understanding Software Project Risk: A Cluster Analysis. Information \& Management, 42: 115-125.

Wallace, L., Keil, M., \& Rai, A. (2004b). How Software Project Risk Affects Project Performance: An Investigation of the Dimensions of Risk and Exploratory Model. Decision Sciences 35(2), 289-321.

Wan, J., \& Wang, R.(2010). Empirical Research on Critical Success Factors of Agile 
Software Process Improvement. Software Engineering and Applications, 3, 11311140.

Ward, S., \& Chapman, C.B., (2003). Transforming Project Risk Management into Project Uncertainty Management. International Journal of Project Management, 21, 97-105.

Williamson, O.E. (1979). Transaction-Cost Economics: The Governance of Contractual Relations. The Journal of Law and Economics, 22(2): 233-61.

Wysocki, R.K. (2009). Effective Project Management: Traditional, Agile, Extreme. Wiley, Indianapolis, (IN).

Wysocki, R. K. (2006). Effective Software Project Management. Indianapolis, IN: Wiley.

Yetton, P., Martin, A., Sharma, R., \& Johnston, K. (2000). A Model of Information Systems Development Project Performance. Information Systems Journal, 10(4), 263-289.

Young, R., \& Jordan, E. (2008). Top Management Support: Mantra or Necessity? International Journal of Project Management, 26(7), 713-725.

Zwikael, O., Pathak, R. D., Singh, G., \& Ahmed, S. (2014). The Moderating Effect of Risk on the Relationship between Planning and Success. International Journal of Project Management, 32(3), 435-441.

Zwikael, O., \& Smyrk, J. (2012). A General Framework for Gauging the Performance of Initiatives to Enhance Organizational Value. British Journal of Management, 23, S6S22.

Zwikael, O., \& Globerson, S. (2006). From Critical Success Factors to Critical Success Processes. International Journal of Production Research, 44(17), 3433 - 3449. 


\section{Appendix A: Details of SEM analysis}

Table 18: Single latent variables tested separately and factor loadings-complete data set $\mathbf{n = 9 8 4}$

Top level management support

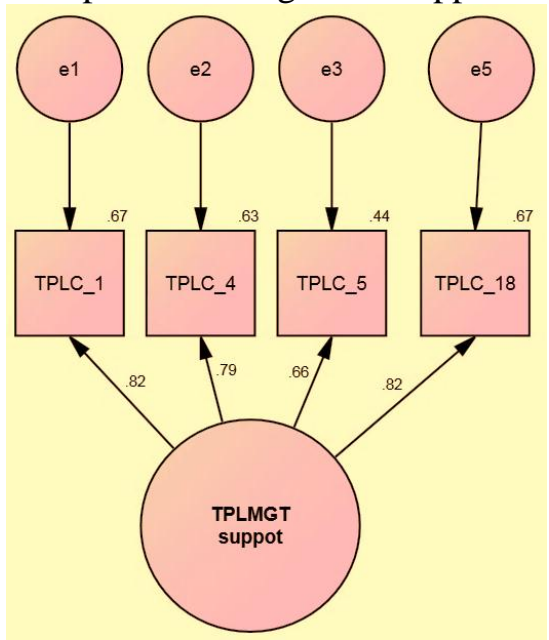

User participation

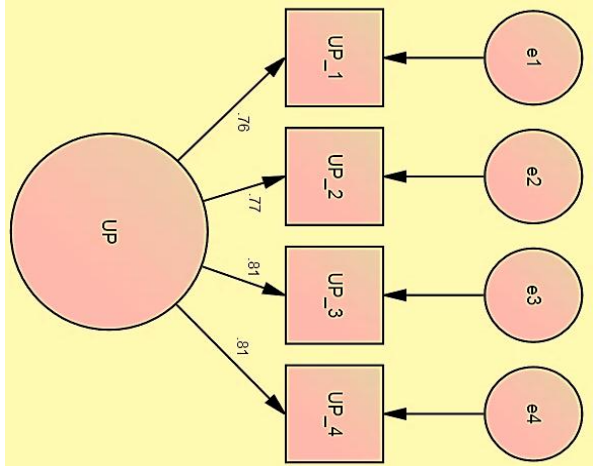

Development team expertise

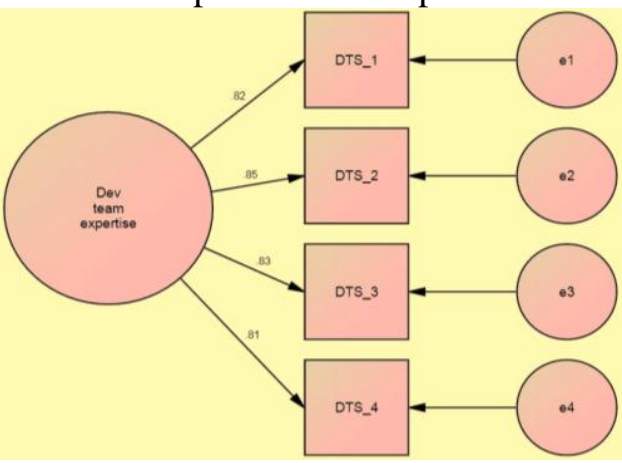

Change management

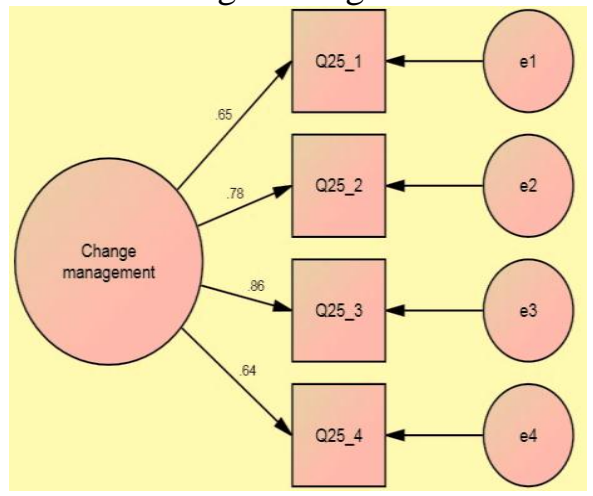

Internal projectcommunication

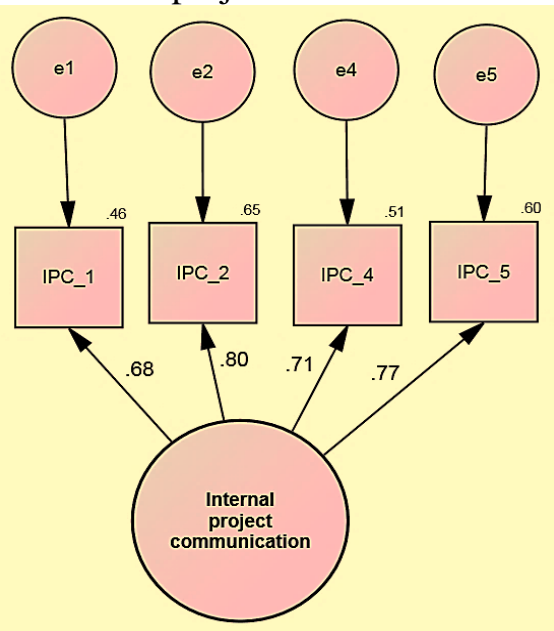

Project team commitment

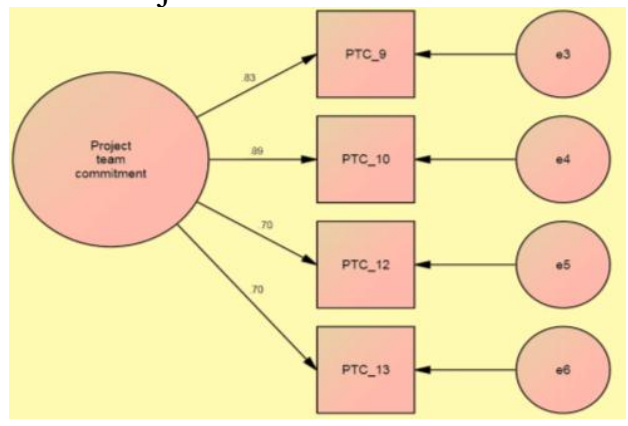

User experience

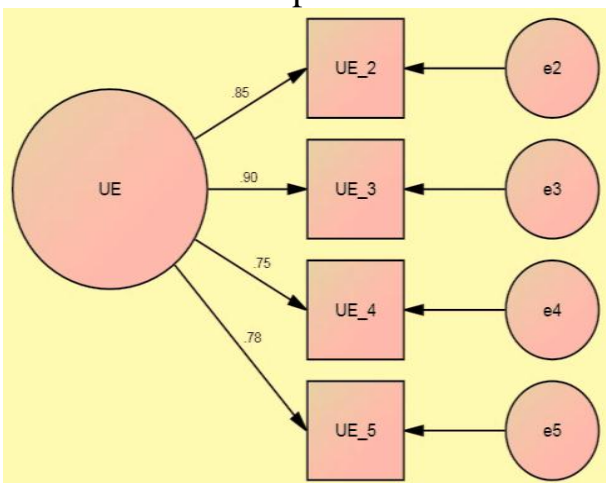

Leadership characteristics

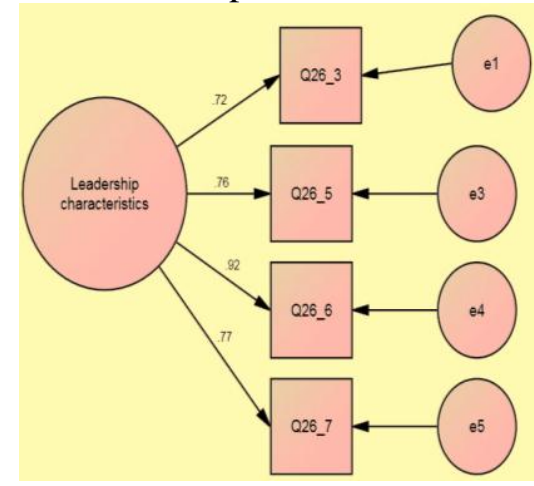

Organisational culture

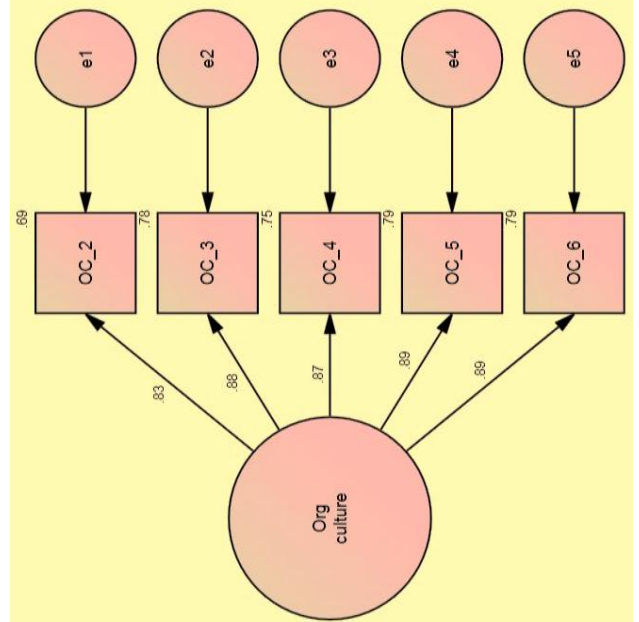

Technical complexity

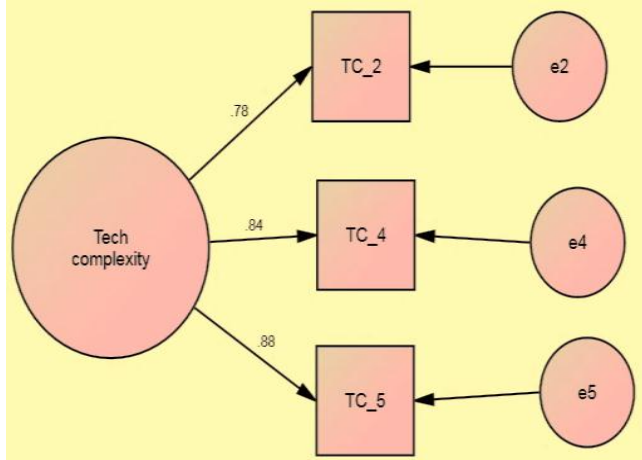

Planning and controlling

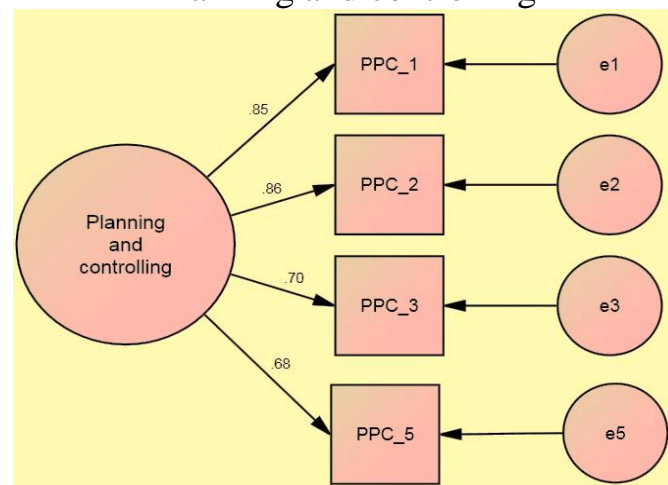

Vision and mission

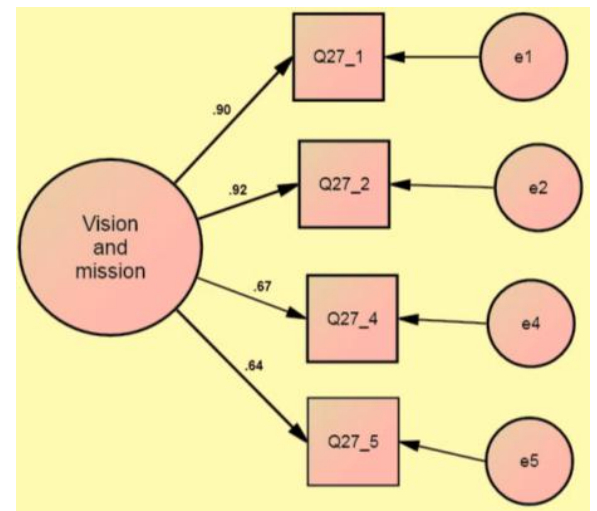



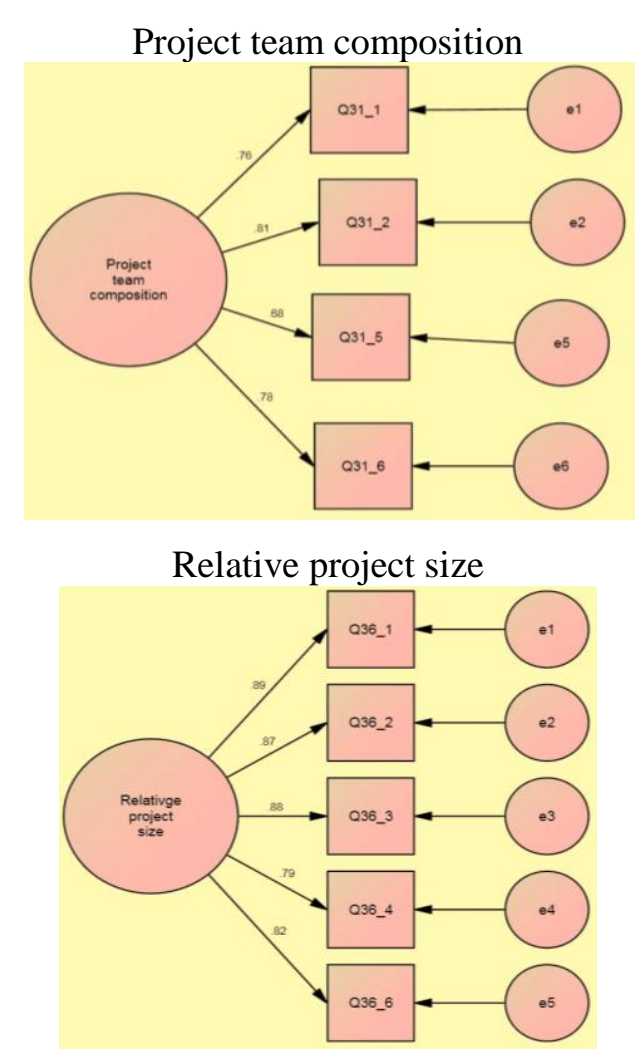

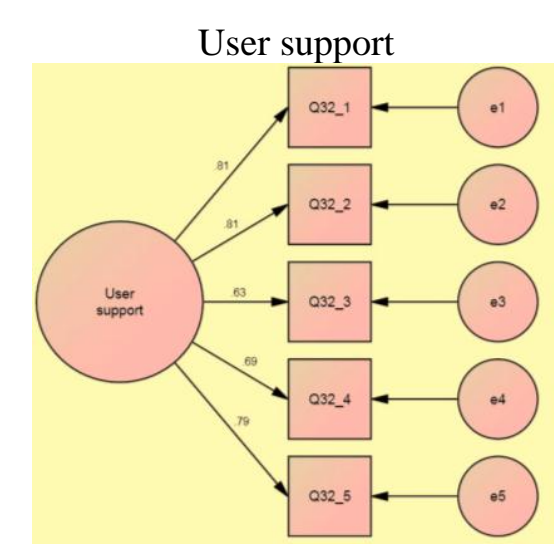

Specification changes

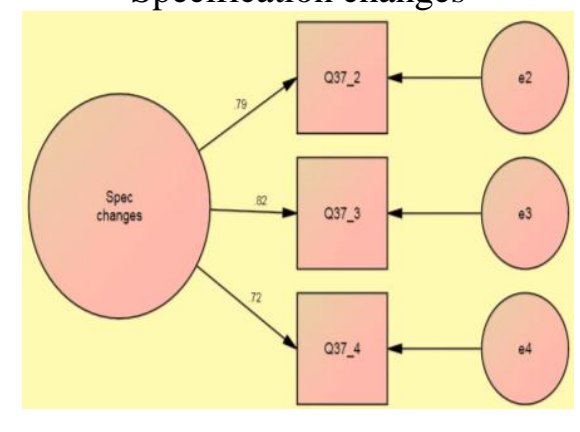

Product project success

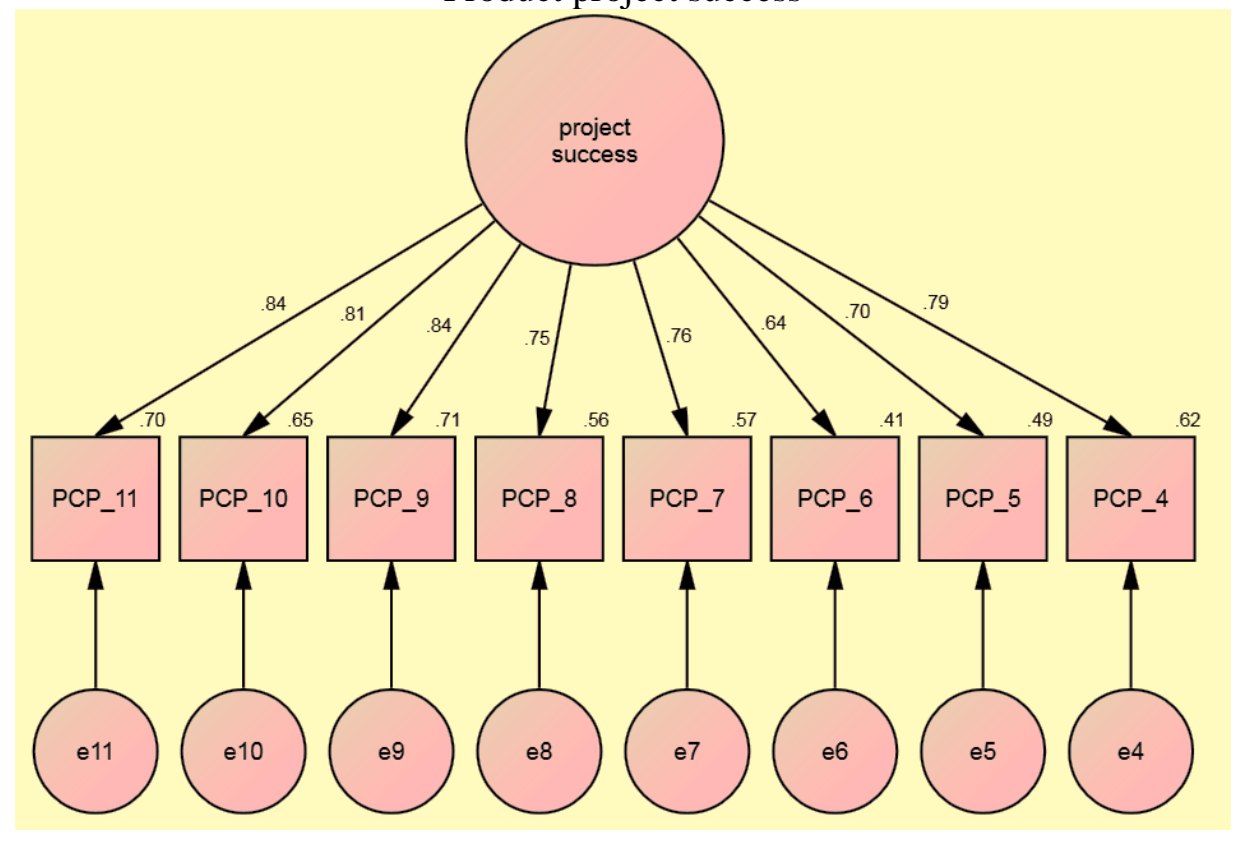

Tecnological uncertainty

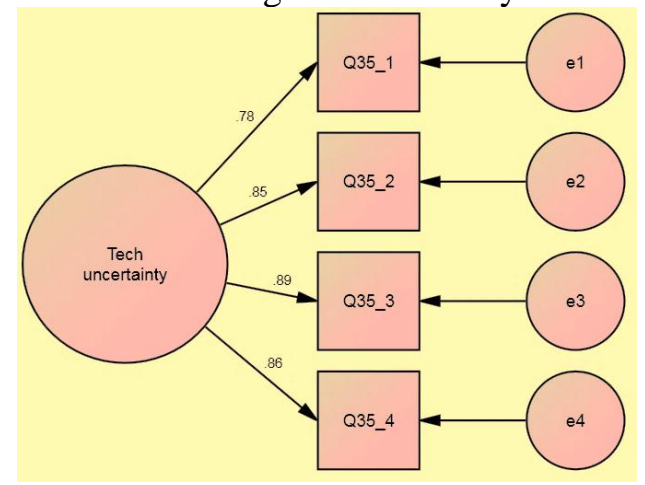

Project process success

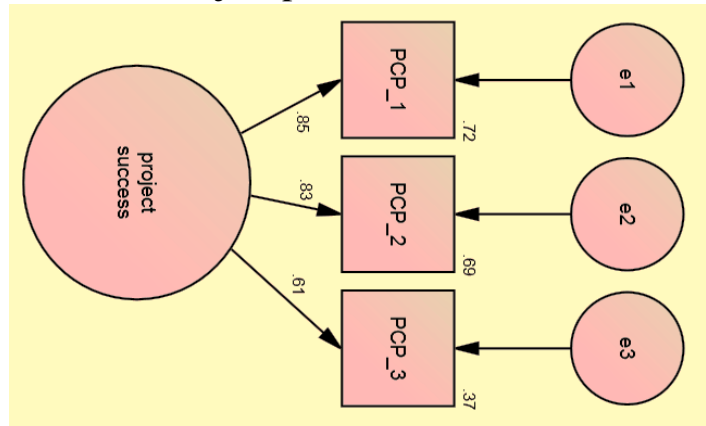




\section{Appendix B: Single latent variables-Group comparisons}

Traditional plan based data set $\mathbf{n}=\mathbf{5 1 3}$

Top level management

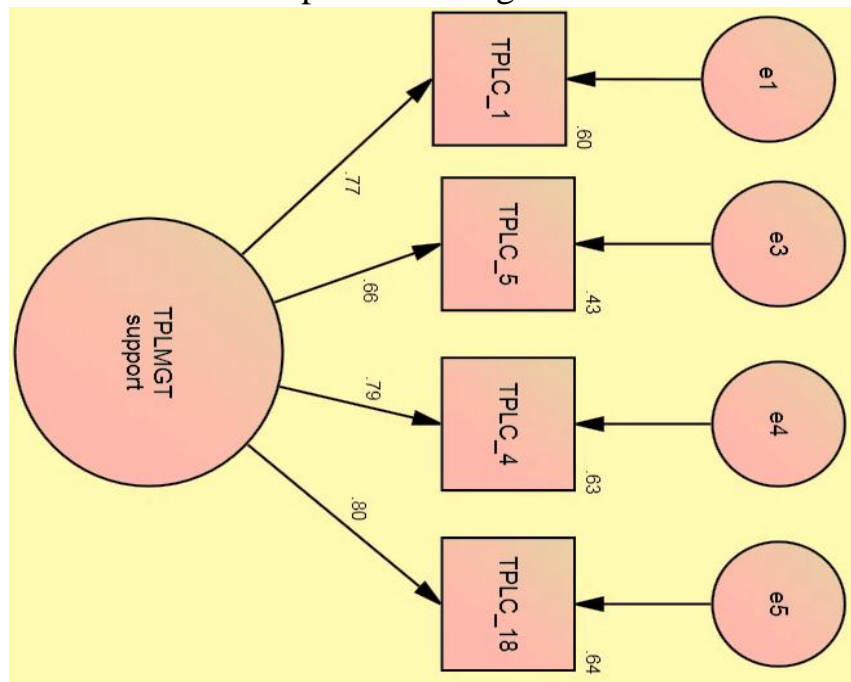

Internal project communication

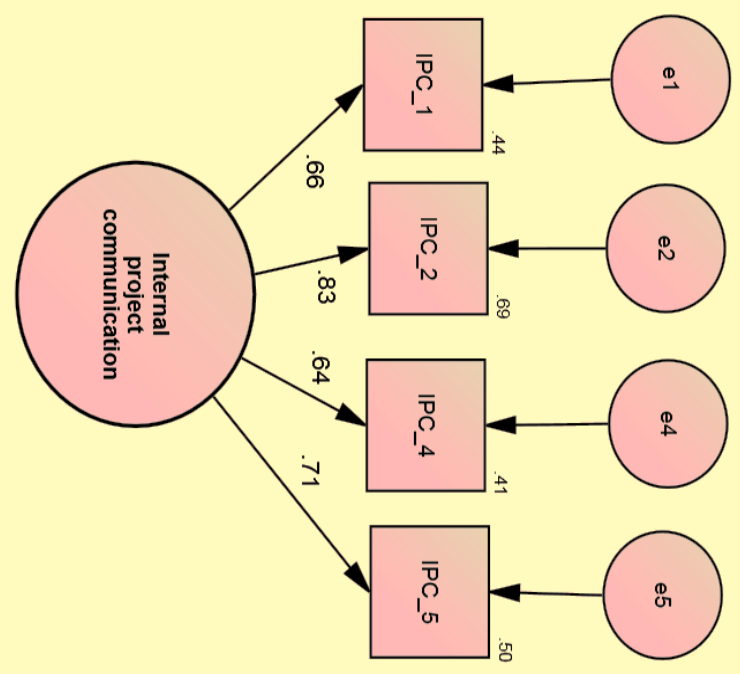

Organisational culture

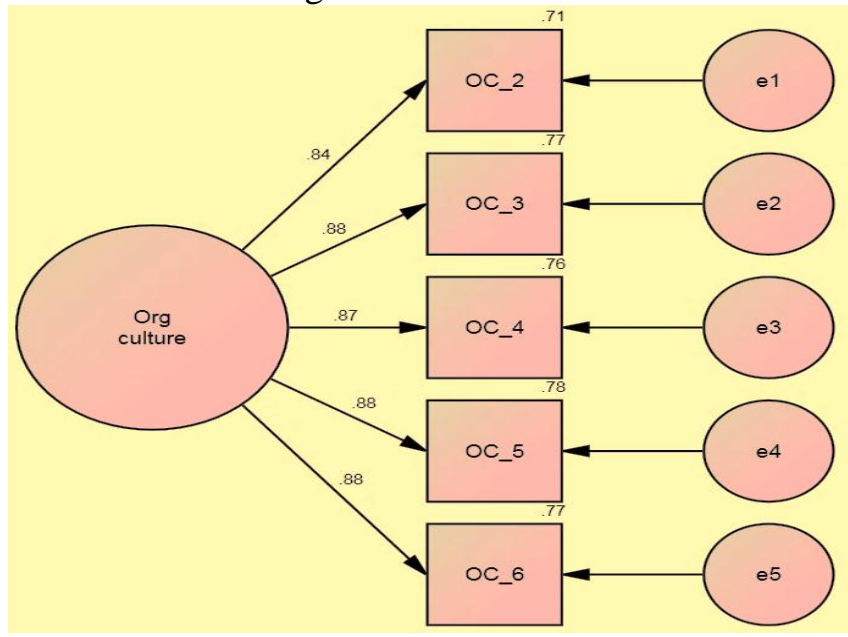

Agile projects data set $n=471$

Top level management

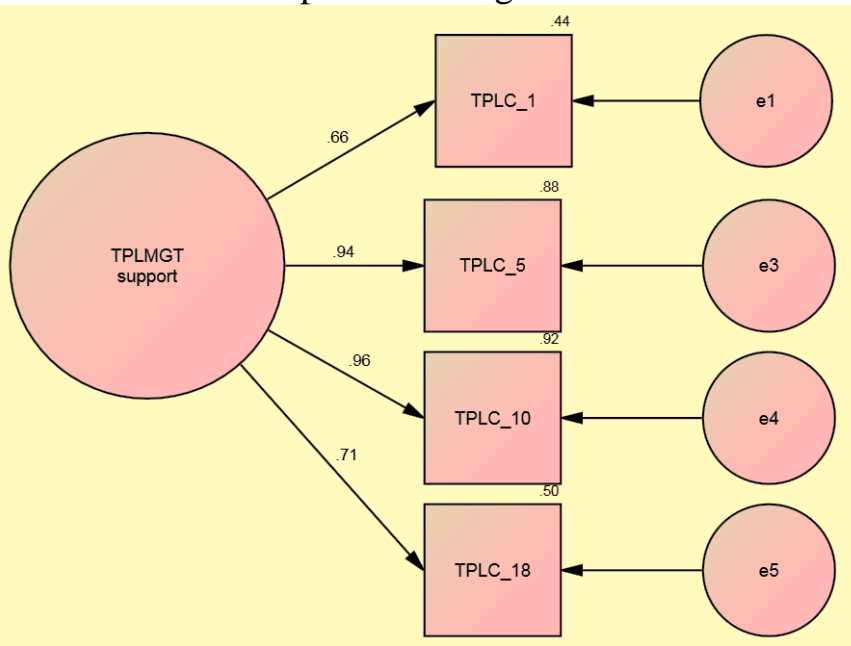

Internal project communication

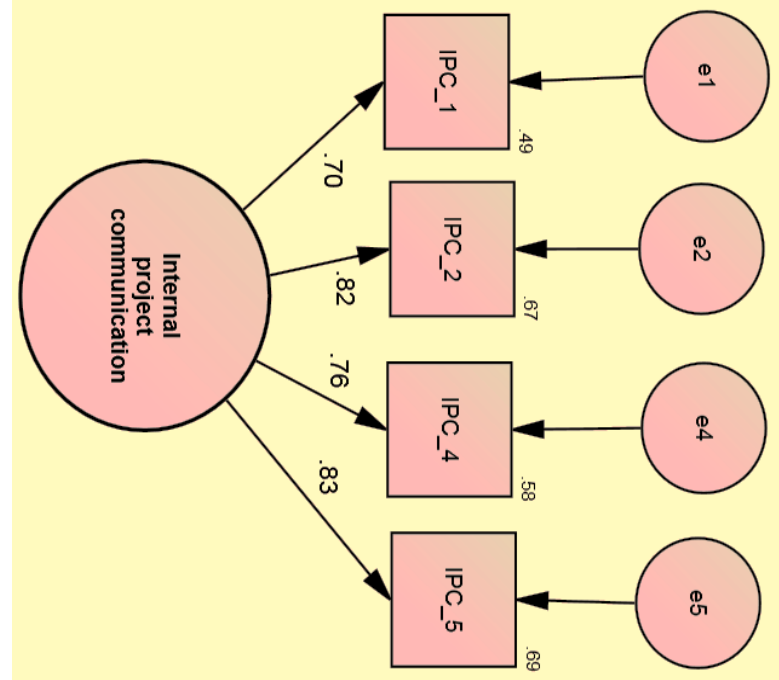

Organisational culture

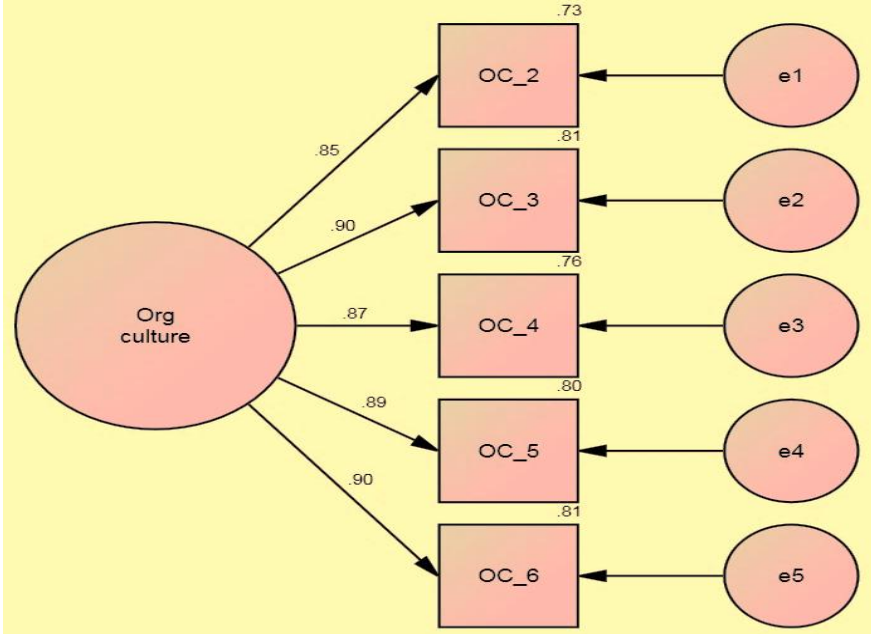




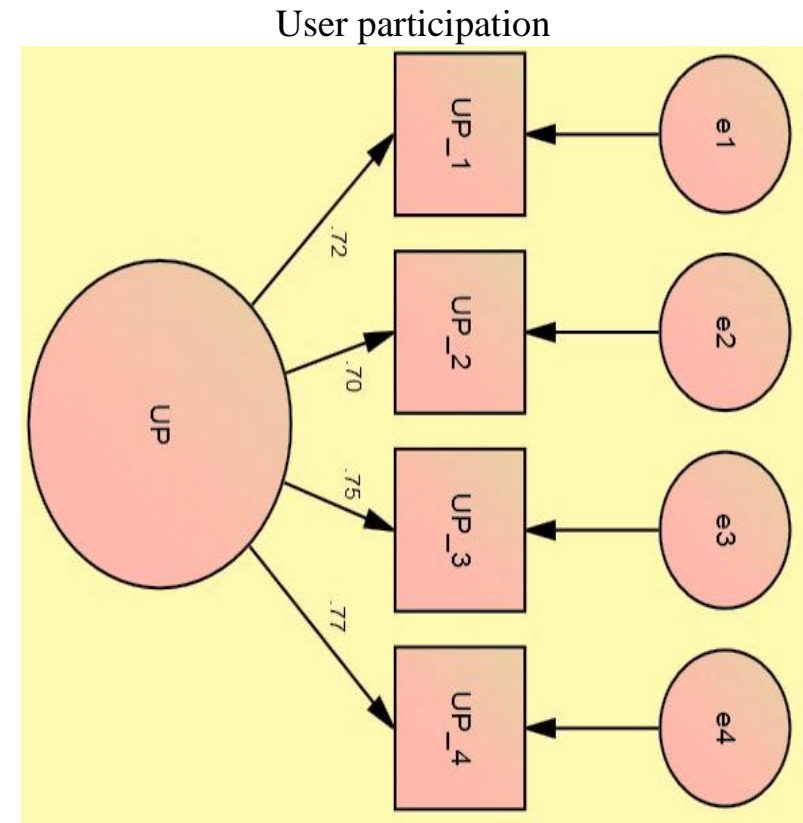

Project team commitment

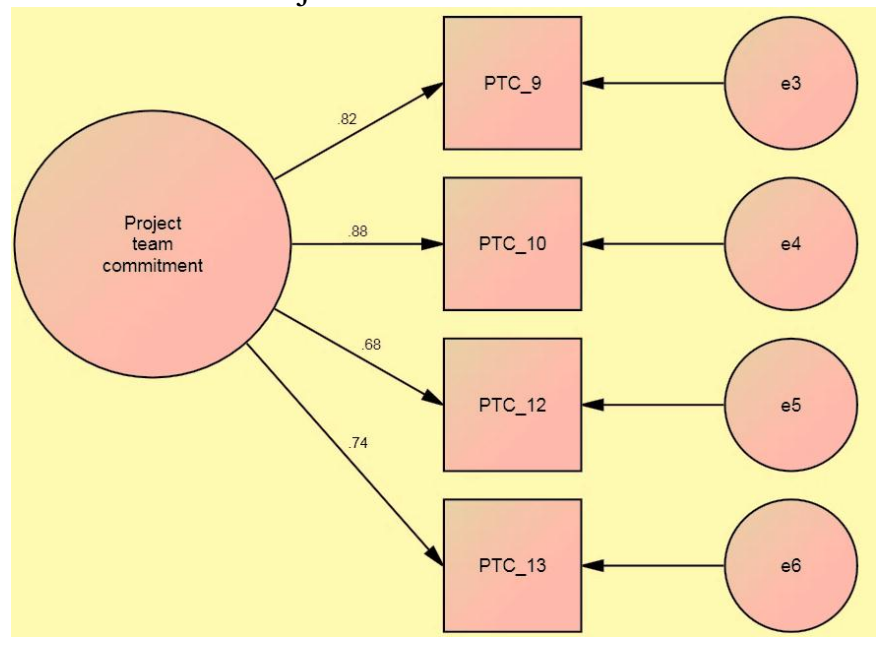

Technical complexity

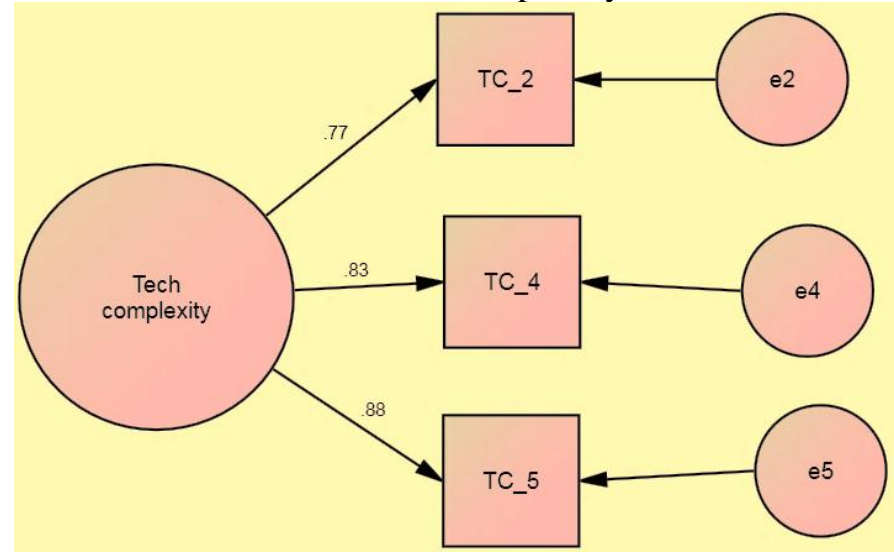

User participation

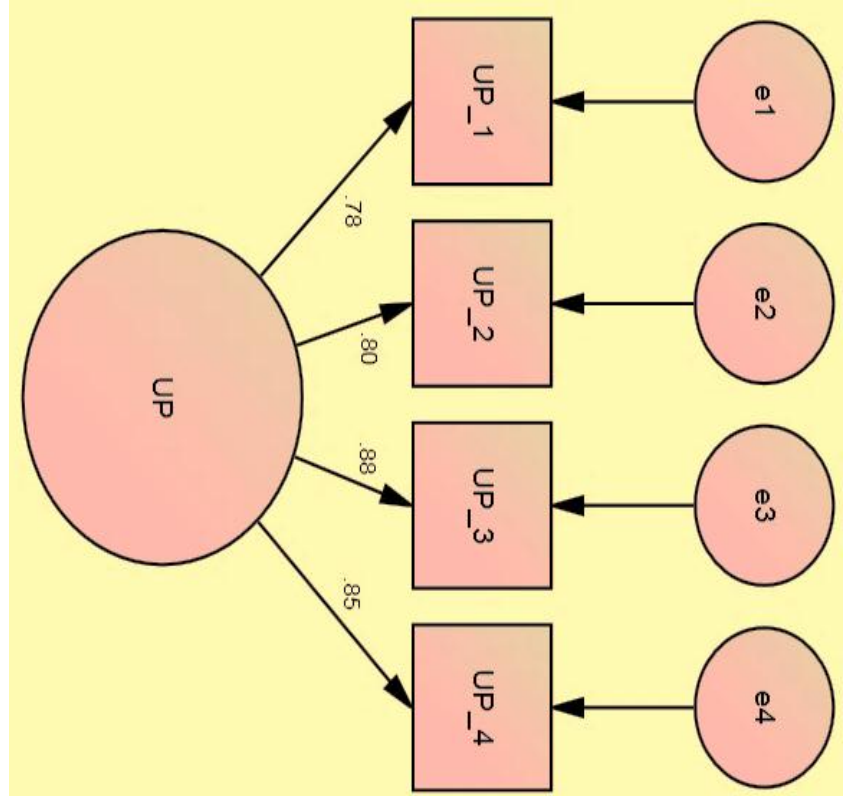

Project team commitment

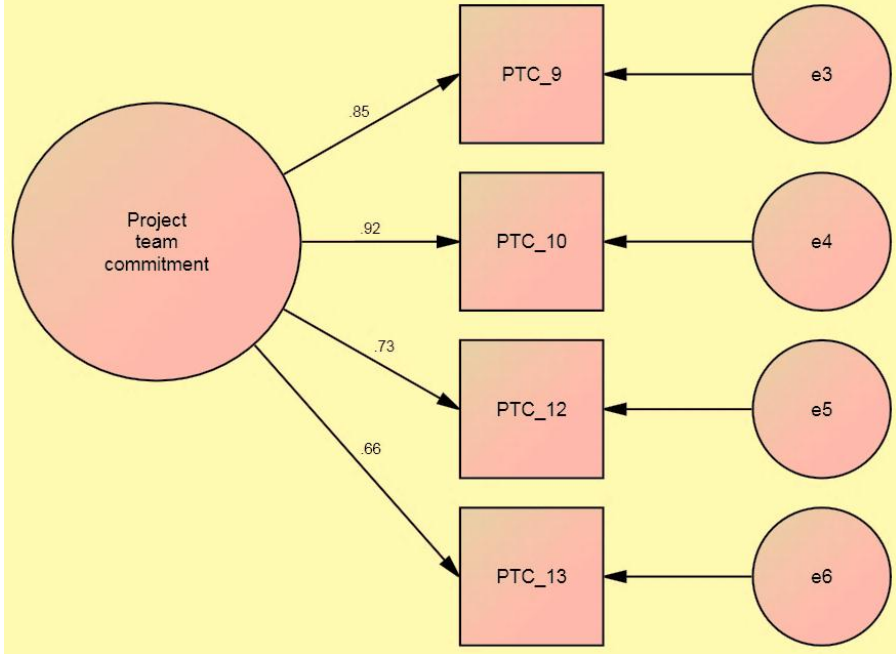

Techinical complexity

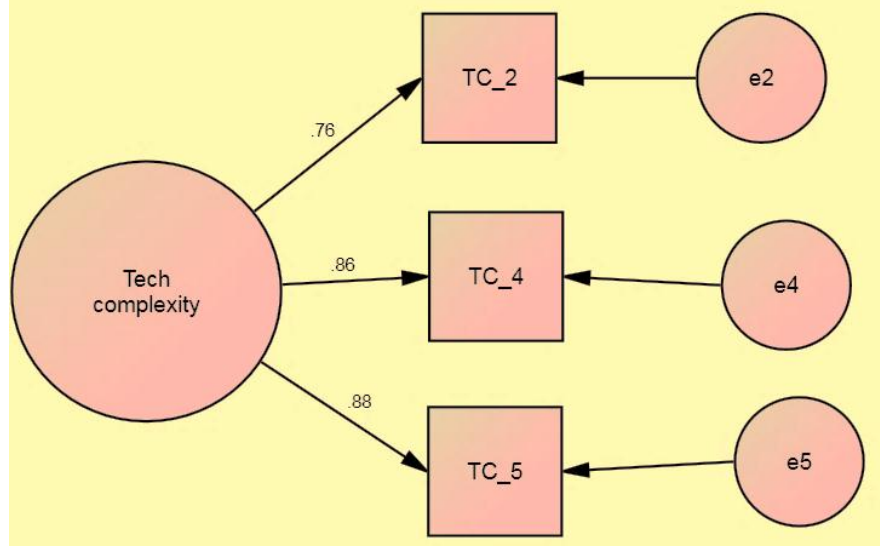



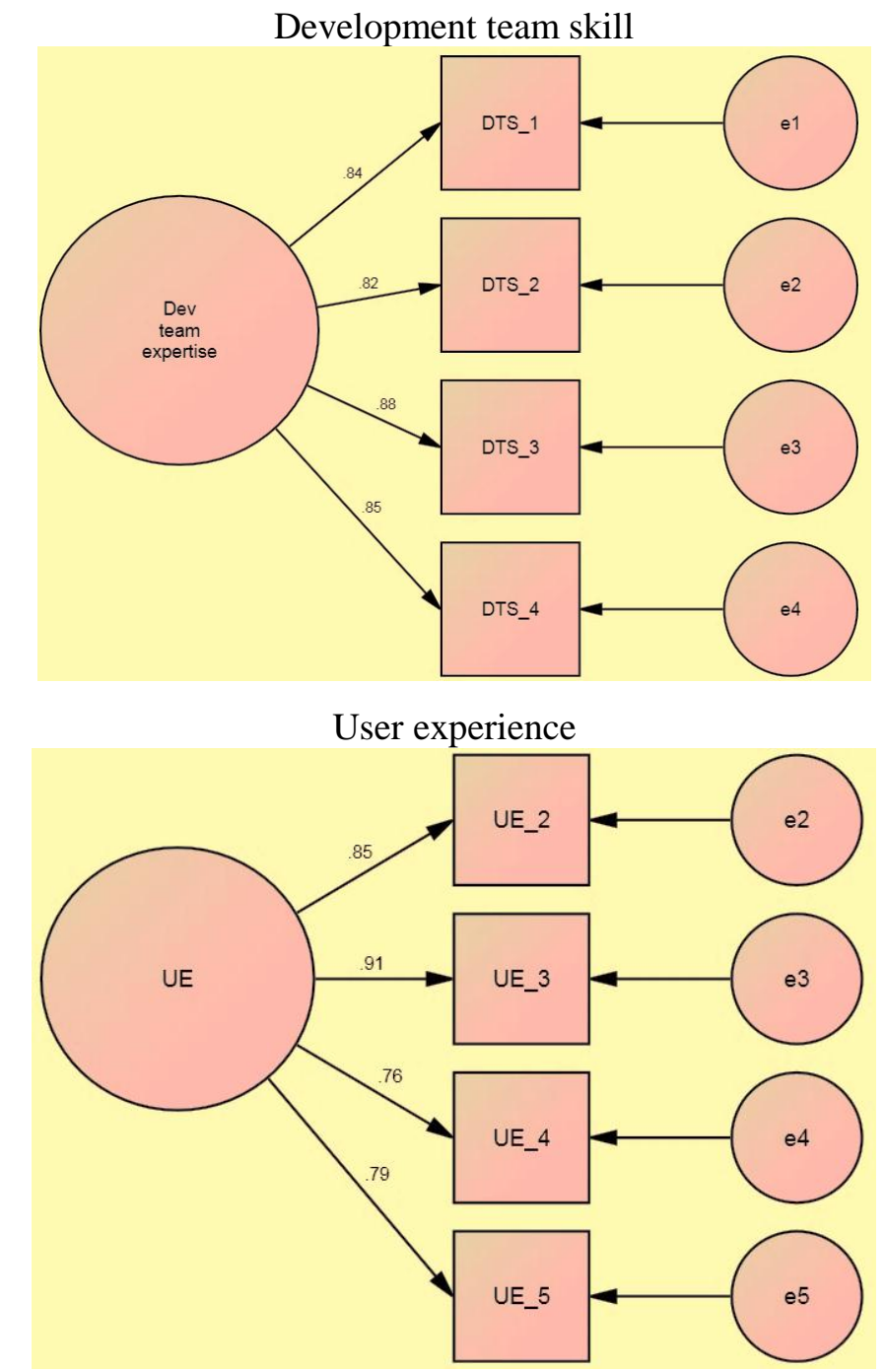

Planning and controlling

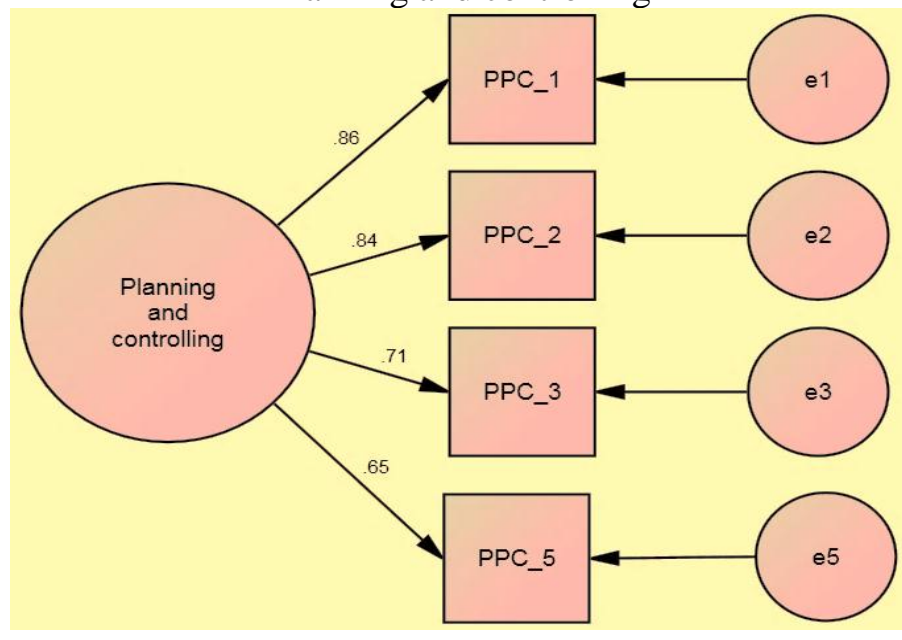

Development team skill

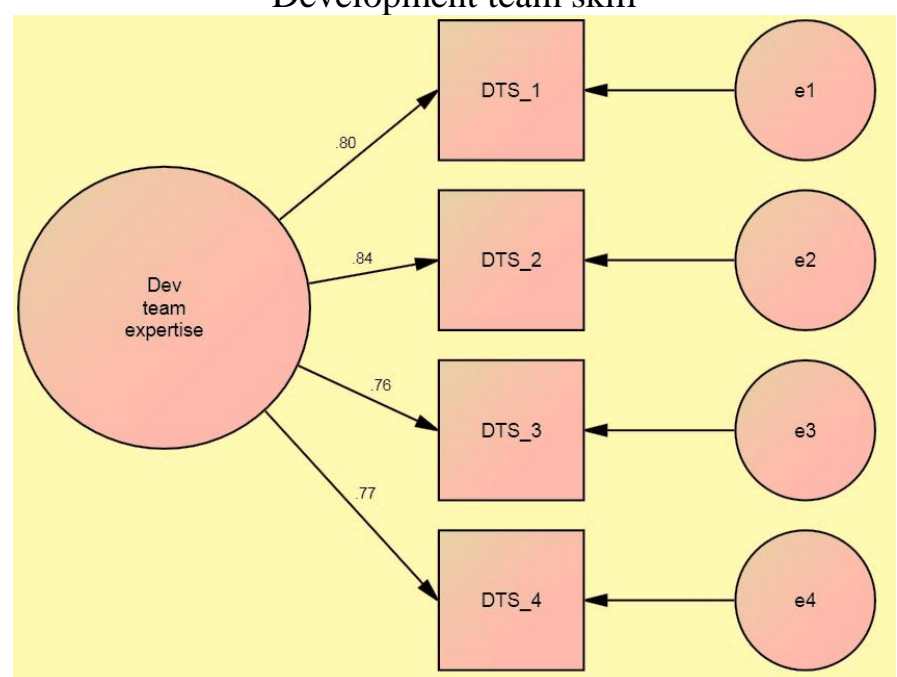

User experience

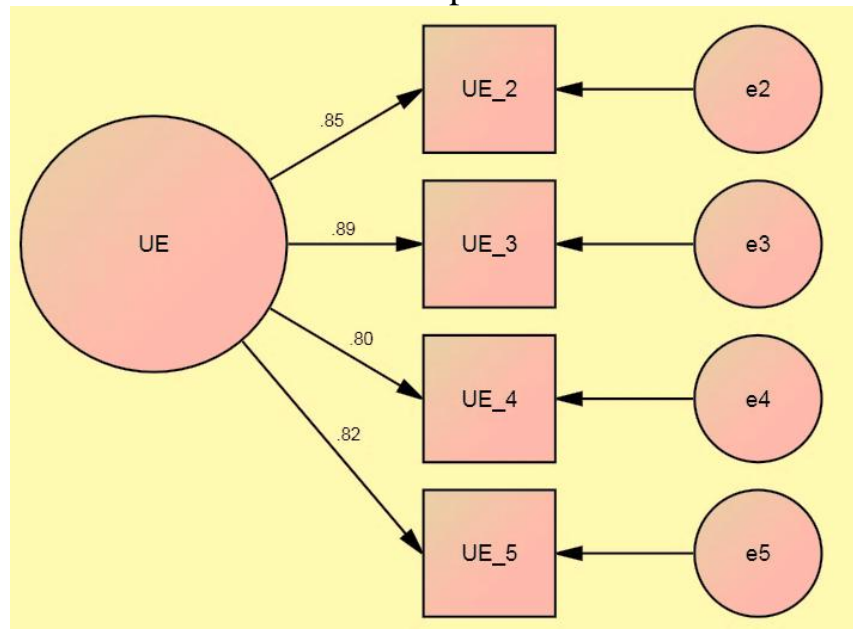

Planning and controlling

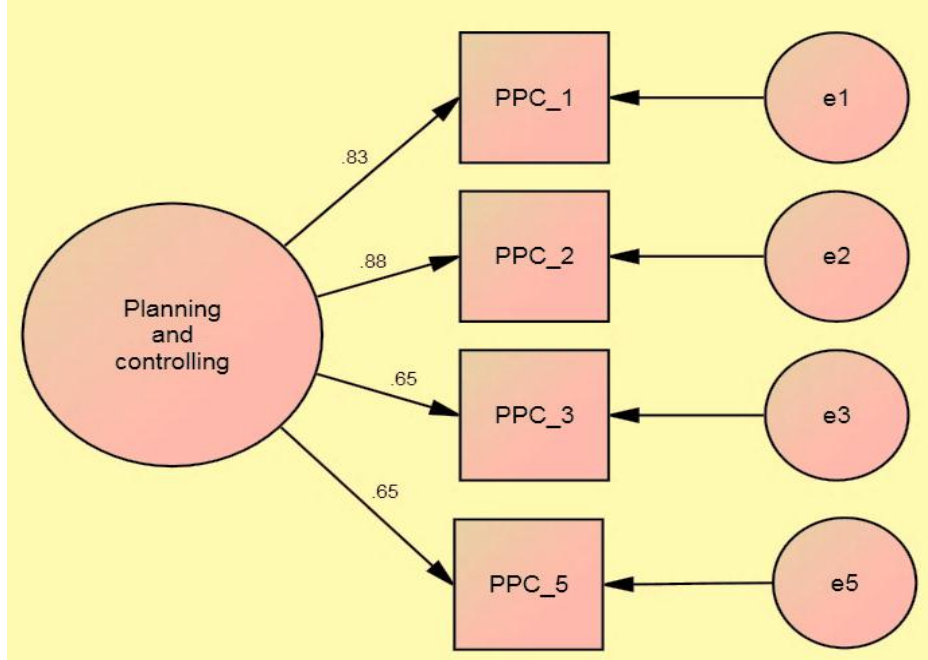


Change management

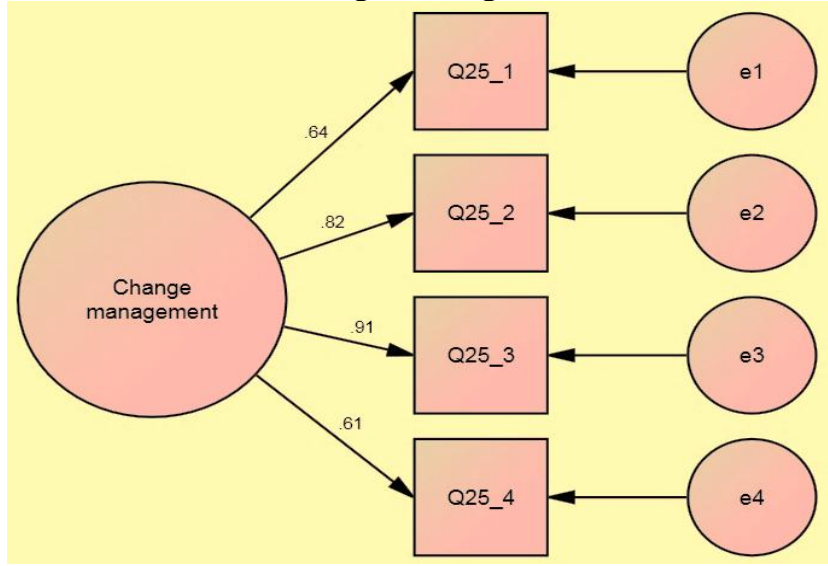

Leadership characteristics

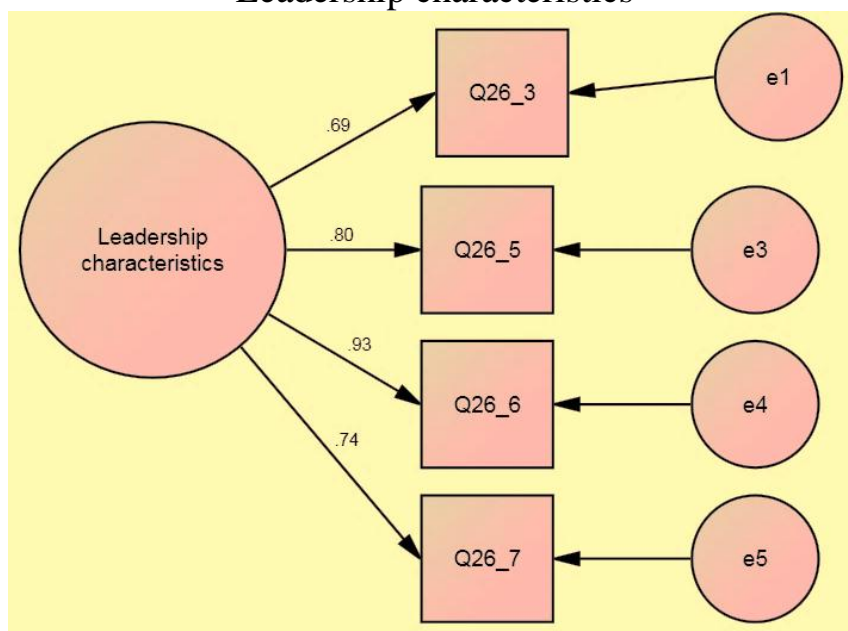

Vision and mission

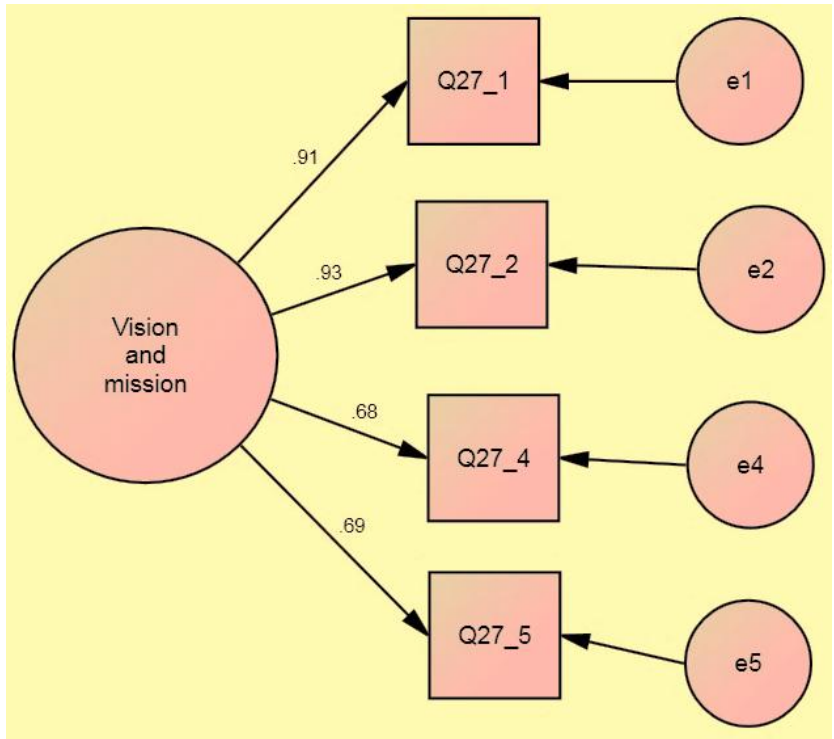

Change management

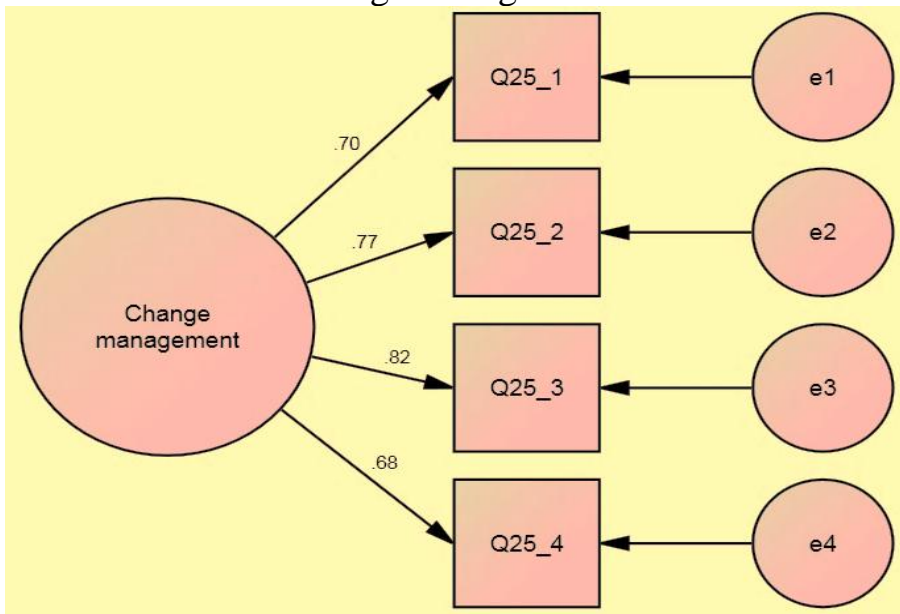

Leadership characteristics

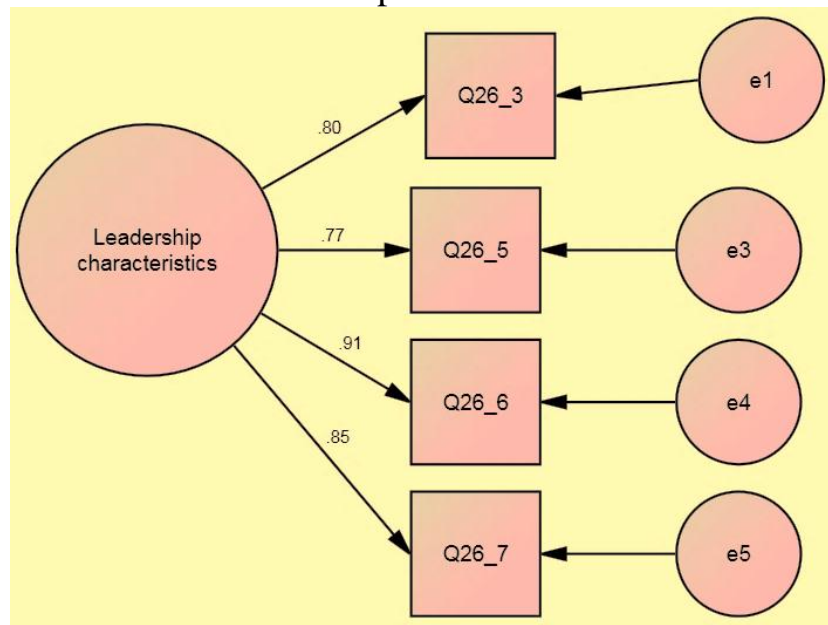

Vision and mission

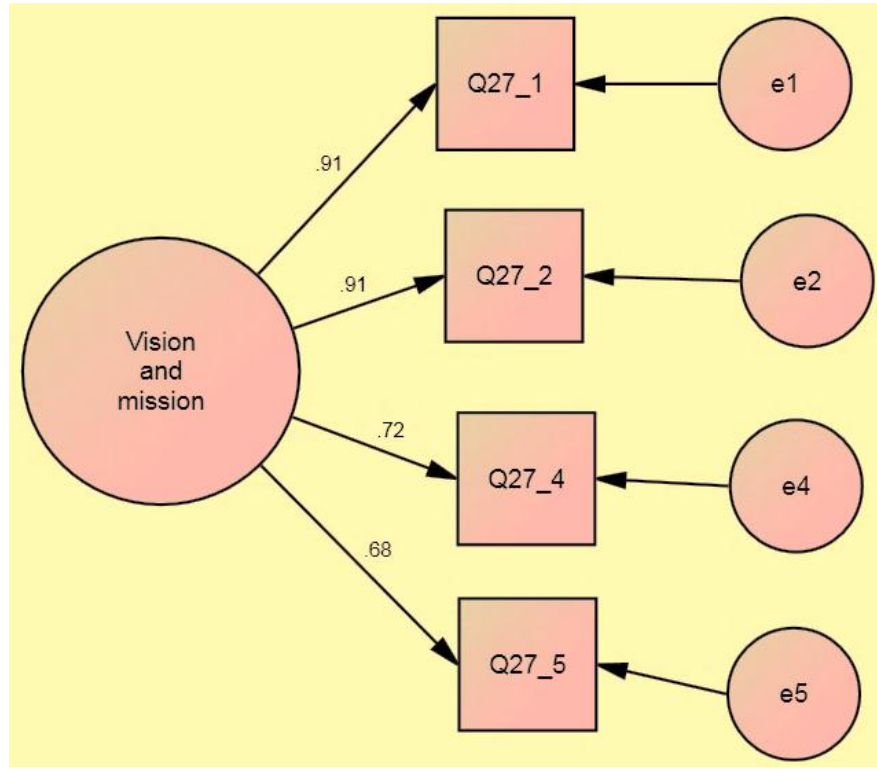



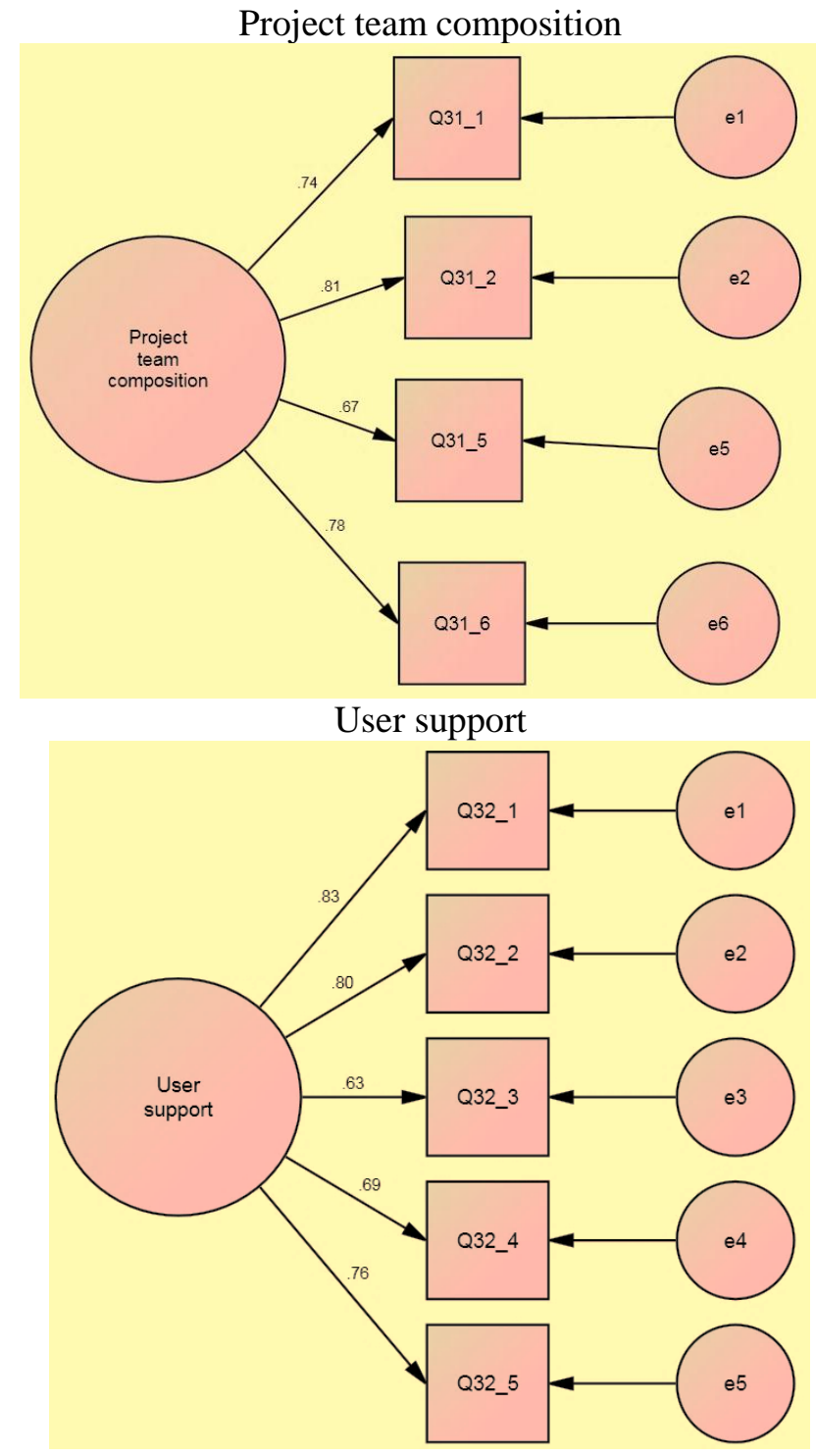

Technological uncertainty

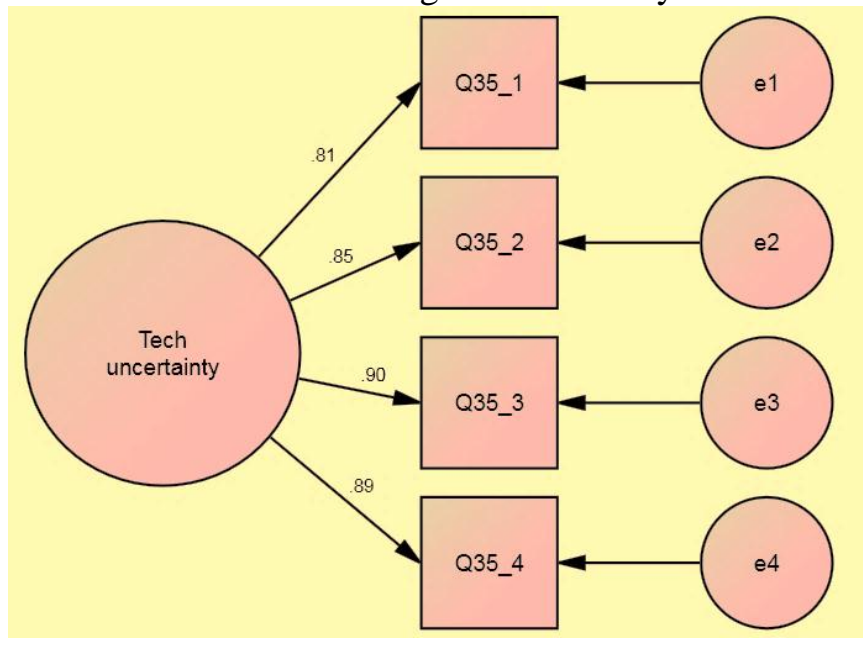

Project team composition

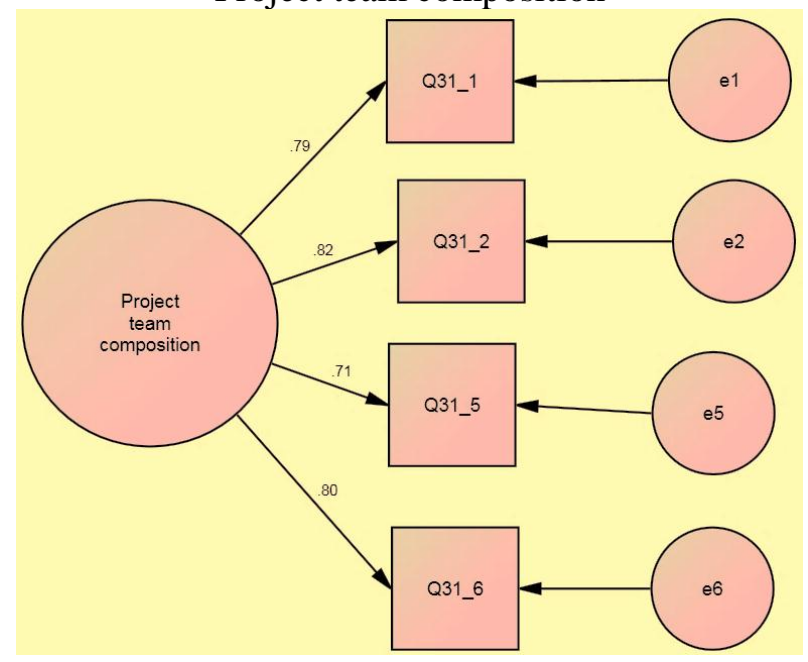

User support

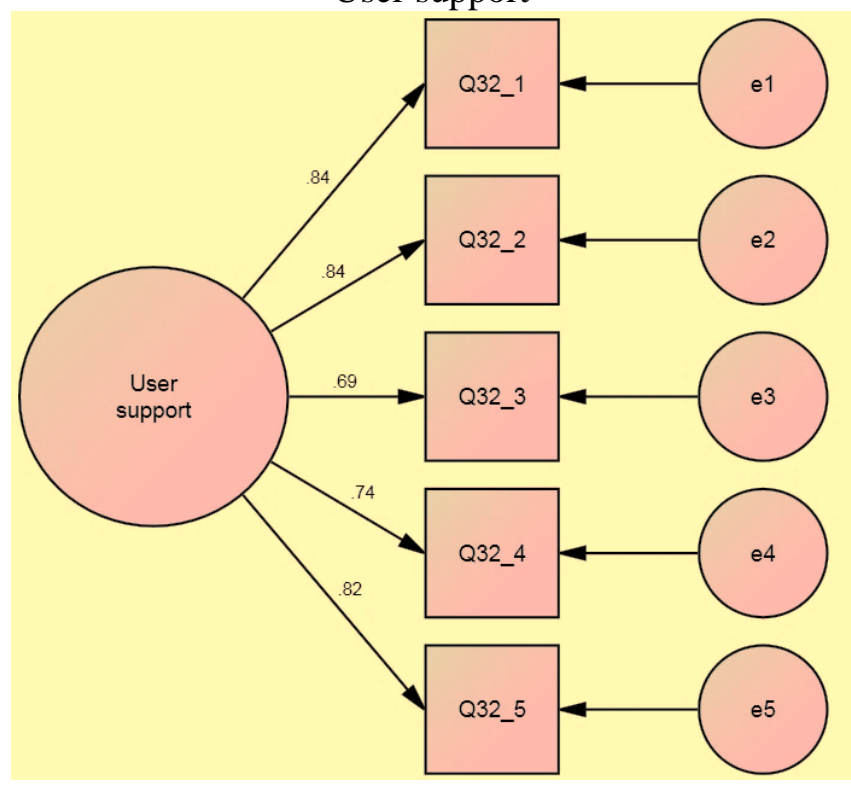

Technological uncertainty

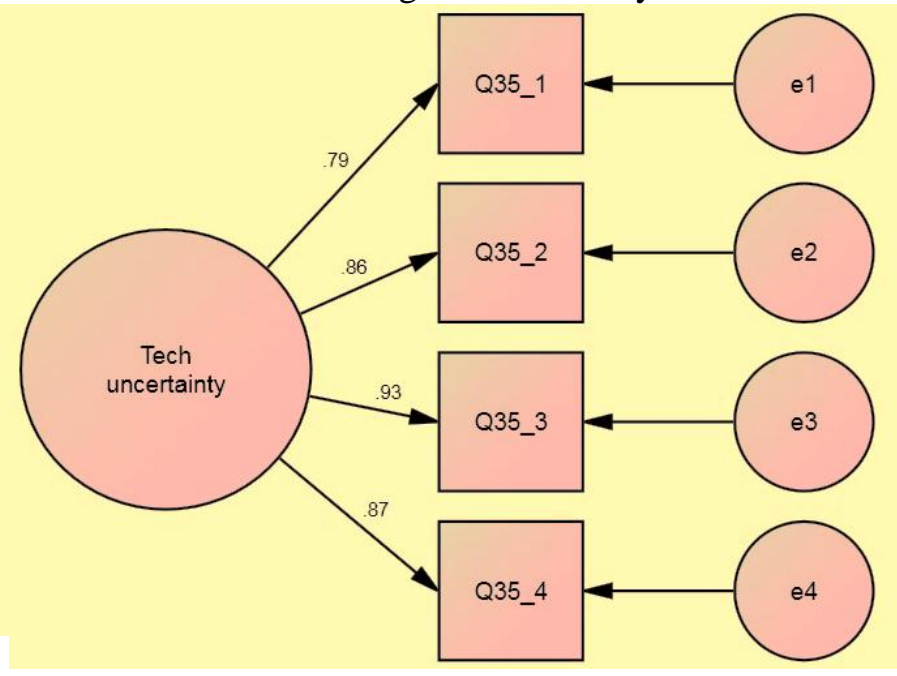




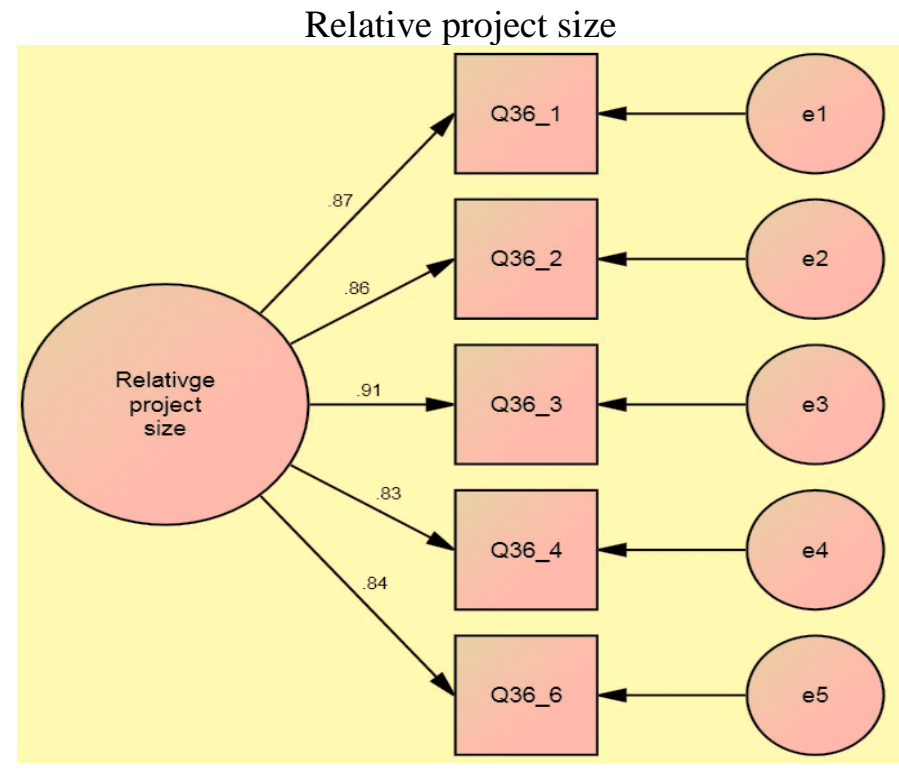

Specification changes

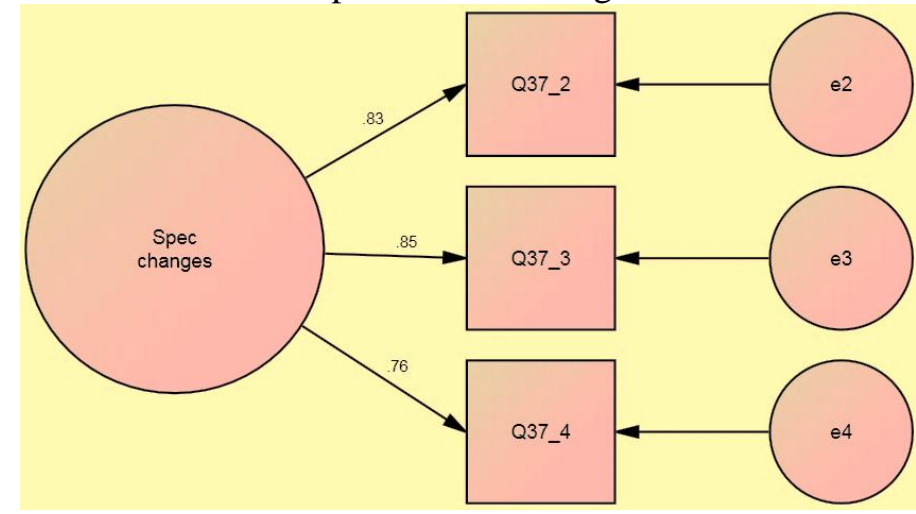

Process project success

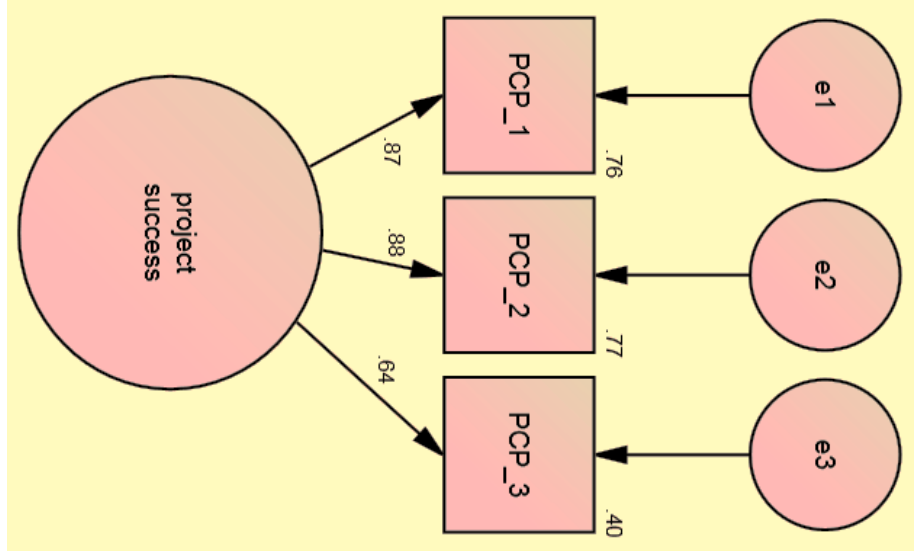

Relative project size

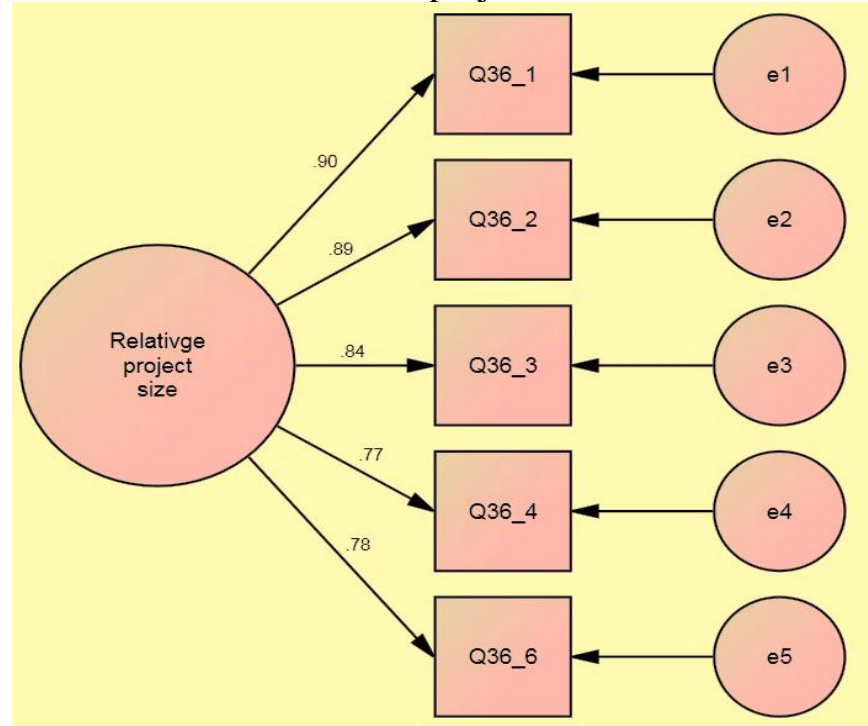

Specification changes

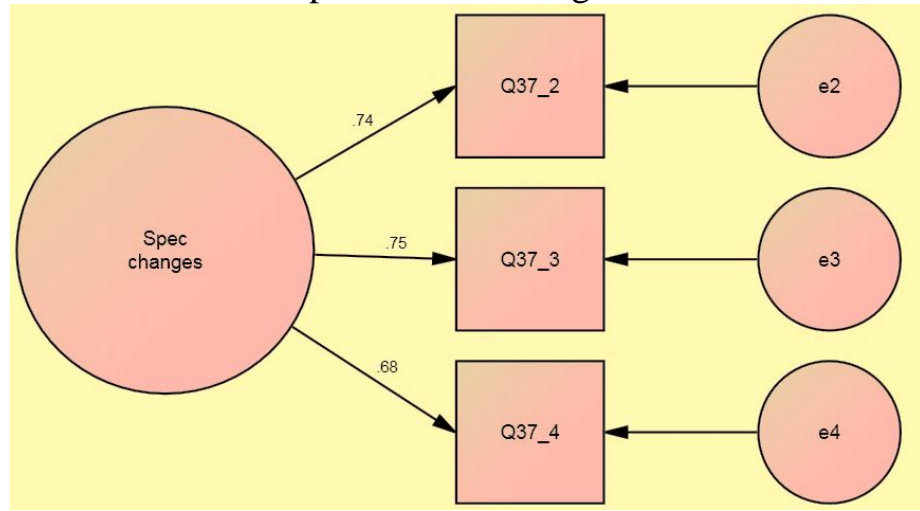

Process project success

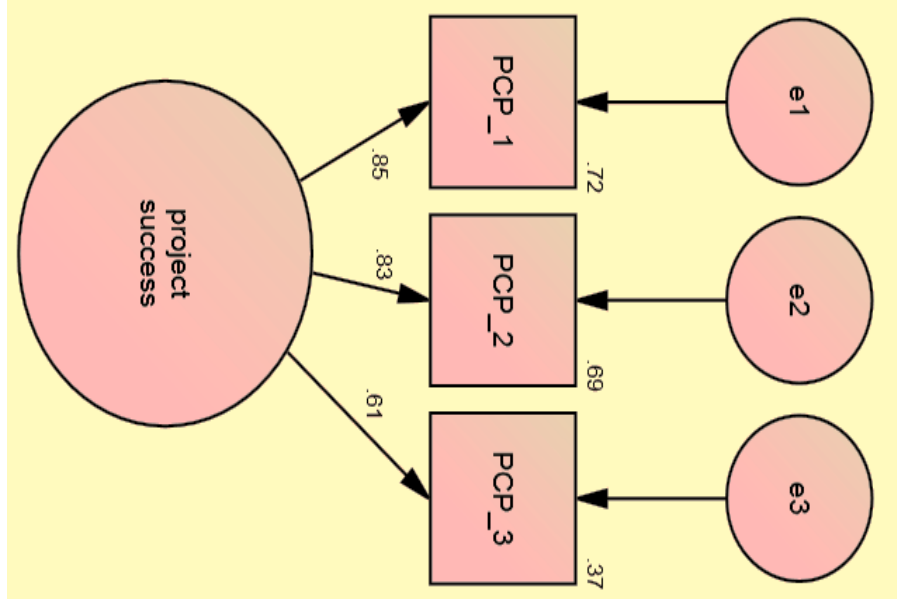



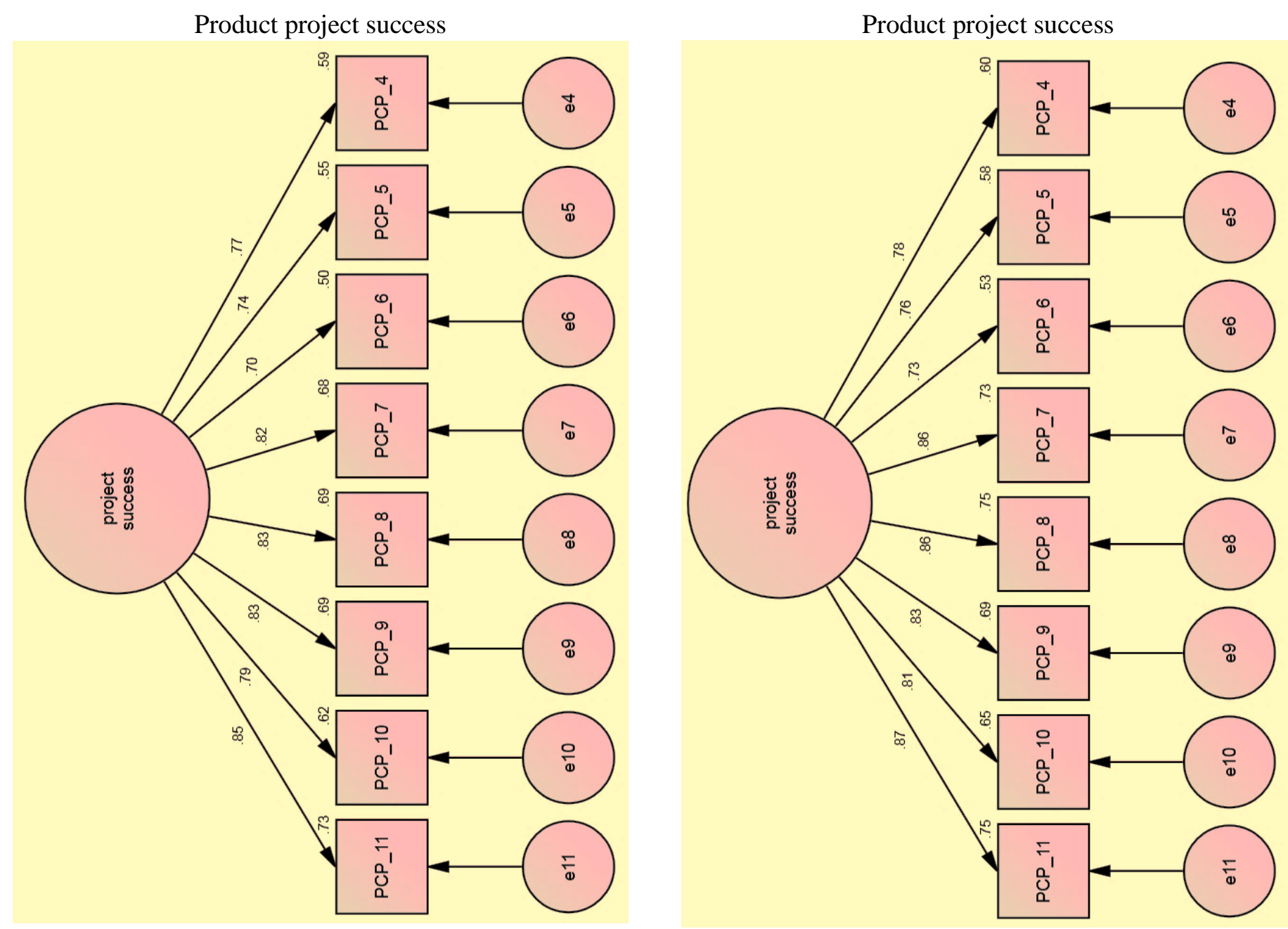


\title{
Appendix C: Information sheet given to interview participants
}

\author{
VICTORIA UNIVERSITY OF WELLINGTON \\ Te Whare Wänanga o te Ûpoko o te Ika a Māui

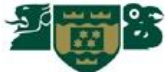 \\ VICTORIA \\ MANAGEMENT SCHOOL \\ Te Kura Whakahaere
}

\section{Research project: Critical Success Factors for Outsourced Software Development Project Success: A comparison of Traditional plan-based and Agile Methodology}

I am a PhD student at Victoria University of Wellington in New Zealand and am conducting research on management of software development projects. This research is being conducted as part of the requirements for the completion of my $\mathrm{PhD}$ degree. Many outsourced software development projects still fail to deliver on time or on budget, or fail to deliver value to the client. One reason for this is suggested to be the choice of an inappropriate project management methodology. I'm investigating how to choose a methodology that can lead to outsourced project success in light of critical success factors. The aim of this interview is to determine the most important factors in methodology selection that helps to achieve project success.

\section{Terms and conditions}

- Participation is entirely voluntary.

- If you agree, the interview will be about 30 minutes long and scheduled at a time that suits you.

- You have the right to withdraw yourself or any information you have provided from this project, without having to supply a reason for doing so, for four weeks after the interview. In which case, information obtained will be immediately destroyed.

- Participants will be interviewed confidentially. All information gathered in these interviews will be treated confidentially - your name will not be used. The results from the interviews will be reported in an aggregated, non-attributable form.

- Ethical approval from Victoria University of Wellington has been obtained for the proposed research.

- All participants will sign a Research Agreement where they can state how they would like the data collected from them to be handled.

- A copy of the research paper or thesis will be deposited in the Victoria University of Wellington Library. Findings may be presented at conferences or published in academic or professional journals at a later date. Any further use will require your written consent.

- A summary of the results will be available to participants who ask for it.

\section{Contact Information}

Thank you for your time and help to make this study possible. If you have any queries please do not hesitate to contact me or my supervisors.

If you would like further information or a summary of key findings, please contact me or my supervisors.

Researcher: Arthur Ahimbisibwe

Supervisor: A/Prof. Bob Cavana

Supervisor: A/Prof. Urs Daellenbach $\underline{\text { arthur.ahimbisibwe@vuw.ac.nz }}$

bob.cavana@vuw.ac.nz

urs.daellenbach@vuw.ac.nz

Thank you in advance for your participation and time.

Arthur Ahimbisibwe 


\section{Appendix D: Research agreement signed by interviewees}

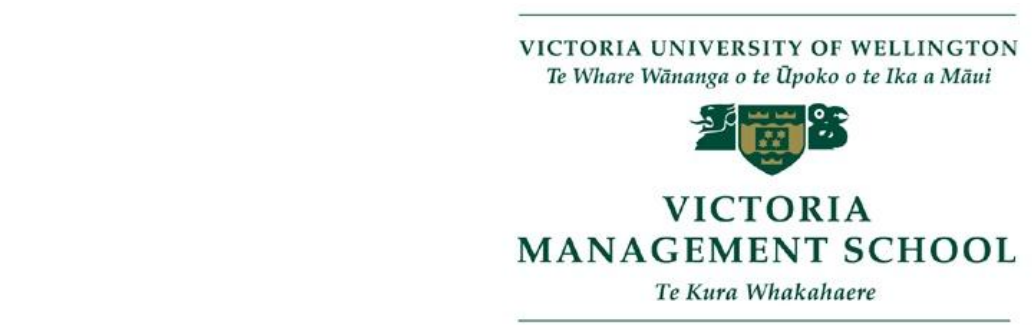

\section{Research project: Critical Success Factors for Outsourced Software Development Project Success: A Comparison of Traditional Plan-Based and Agile Methodologies}

Researcher: Arthur Ahimbisibwe, School of Management, Victoria University of Wellington

\section{Purpose of agreement:}

This agreement is to ensure that you are sufficiently informed about the purpose of the research, and your right to know how data will be collected, analysed and written up.

\section{Consent to participation}

I have been given and have understood an explanation of this research project.

I have had an opportunity to ask any questions and had them answered to my satisfaction.

I understand the data collected will remain confidential and will be reported in an aggregated, nonattributable form.

I understand that I may withdraw myself or any information I have provided from this project (for four weeks after the interview), without having to supply a reason for doing so. In which case, information obtained will be immediately destroyed.

$\square$ I understand that the information obtained will be stored in a locked cabinet or password-protected file. All interview notes and research materials will be destroyed three years after the research is completed.

A copy of the research paper or thesis will be deposited in the Victoria University of Wellington Library. Findings may be presented at conferences or published in academic or professional journals at a later date. Any further use will require my written consent.

$\square$ I agree to participate in this study.

I want to receive a summary of the findings of this study.

I agree to receive an invitation by email to participate in a survey that will be conducted on the same topic.

\section{Participant}

Name:

Email:

Date:

Signature:

\section{Researcher}

Arthur Ahimbisibwe

Victoria University of Wellington

Signature: 


\title{
Appendix E: Interview recording sheet
}

\author{
VICTORIA UNIVERSITY OF WELLINGTON \\ Te Whare Wänanga o te Ûpoko o te Ika a Māui

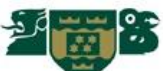 \\ VICTORIA \\ MANAGEMENT SCHOOL \\ Te Kura Whakahaere
}

Interview number:

Participant's tittle-

Job description-

Organization: Anonymous

Sector:

Professional affiliations:

Date:

Time:

Experience in years in software development:

General comments about the questionnaire and possible suggestions for improvement

Based on your practical experiences explain how each of the following factors may lead to project success or failure in outsourced software development projects, from a vendor's perspective.

Top level management support

Organizational culture

Change management

Internal project communication

Project team commitment

User participation and user support

Vision and mission

knowledge and experience

Team composition

Customer training and education

Planning and controlling

Software Development Methodology

Project uncertainty

Technological uncertainty

Project complexity

Project criticality

How do you measure Project success in practice? 


\section{Appendix F: Information sheet sent to survey sample}

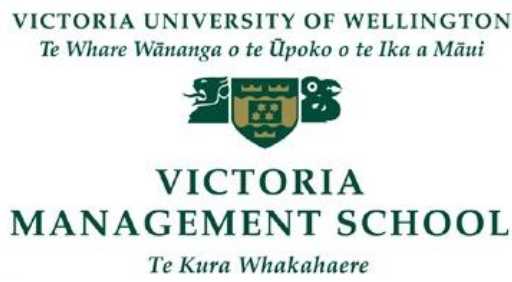

\section{International Survey on Critical Success Factors for outsourced Software Development Projects}

You have been identified as a knowledgeable person in outsourced software development and you're therefore kindly requested to participate in this online survey conducted by researchers at Victoria University of Wellington, New Zealand. To begin, please click this link:

http://vuw.qualtrics.com/SE/?SID=SV_6oqeQxpN9bujgBD

We appreciate your participation in this survey and look forward to receiving your feedback.

If you would like further information about any aspect of this survey, please contact me or any of my supervisors.

Researcher: Arthur Ahimbisibwe

Supervisor: A/Prof. Bob Cavana

Supervisor: A/Prof. Urs Daellenbach arthur.ahimbisibwe@vuw.ac.nz

bob.cavana@vuw.ac.nz

urs.daellenbach@vuw.ac.nz

Thank you for your time,

Kind regards,

Arthur 


\section{Appendix G: Survey questionnaire-Main study}

Qualtrics Survey Software
Introduction
Information to participants
Thank you for accessing this questionnaire. Please kindly read the following
information before you proceed.
My name is Arthur Ahimbisibwe and I am a PhD student at the School of Management,
Victoria University of Wellington. You have been selected as knowledgeable person in the
area of software development and you are therefore kindly invited to participate in this
study. The purpose of this research is to investigate the relationships between project
characteristics, project management approach and project success in the domain of software
development projects. Many IT projects are reported to fail to deliver on time, budget and do
not give value to the client. Please give your responses based on the last software
development project that you have completed, irrespective of whether it was successful or
not. This will aid me in identifying factors that affect project success and failure.

Ethics approval has been obtained for this research from the Victoria University Human Ethics Committee. Your participation is completely voluntary. The questionnaire is anonymous and thus does not collect identifying information such as names from the participants. The completion of this web-based survey should take about fifteen minutes. All the material related to this survey will be kept confidential and only accessible to me and my supervisors A/Profs. Bob Cavana and Urs Daellenbach. During this project, electronic data will be kept under password protection and any printed material will be kept in locked cabinets. All the data will be destroyed two years after the completion of the dissertation. Results of this questionnaire will contribute to my thesis and may appear in publications in academic journals or may be presented at academic conferences. All results from the survey will be reported in an aggregated and non-attributable form.

If you would like further information or a summary of key findings, please contact me or my supervisors.

Researcher: Arthur Ahimbisibwe Supervisor: A/Prof. Bob Cavana

arthur.ahimbisibwe@vuw.ac.nz

Supervisor: A/Prof. Urs Daellenbach

bob.cavana@vuw.ac.nz urs.daellenbach@vuw.ac.nz

Thank you in advance for your participation and time.

Arthur Ahimbisibwe

https://vuw.qualtrics.com/ControlPanel/Ajax.php?action=GetSurveyPrint... 9/02/2014 
Section A: Background information (Please tick in the box of your choice). Please note that all these questions relate to your last completed software development project irrespective of whether it was successful or not.

How long have you been involved in software development projects?
Less than 1 year
1 to less than 2 years
2 to less than 5 years
$\square$ to less than 10 years
More than 10 years

What was the size of the client organization in which your last software development project was conducted?
D $1-10$
$11-50$
$51-100$
101-500
501-1000
1001-5000
More than 5000

In what type of sector was your recent software development project conducted?
$\square$ Public sector
Private sector
Other- please specify

In what industry was your last software development project conducted?
(C) Finance/lnsurance
Manufacturing
Marketing/retail
Health
Consulting
Software
Transportation
Utility

https://vuw.qualtrics.com/ControlPanel/Ajax.php?action=GetSurveyPrint... 9/02/2014 

(6) Aerospace
C) Education
Other - please specify

What was your position on that software development project?
$\square$ Project Manager
$\square$ Team Leader
Developer
$\square$ Tester
$\square$ Other-please specify

What project management methodology was used?
PRINCE2
(5) PMI (PMBOK)
(1) Agile project management
Other - please specify

Who made the decision of choosing the methodology that was used?
Top management
Project team members
(2) It is an organizational policy to use this methodology
Do not know
Other-please specify

On which development life cycle was your last software development project based?

$\square$ Linear or Waterfall
$\square$ Iterative
$\square$ Incremental
$\square$ Adaptive
$\square$ Other-please specify

https://vuw.qualtrics.com/ControlPanel/Ajax.php?action=GetSurveyPrint... 9/02/2014 
What was the estimated total number of people who worked on this project from both the vendor and client organizations?
(1) $2-5$
(1) $6-100$
(C) $101-500$
$501-1000$
$1001-5000$
above 5000

What was the estimated project budget in USA Dollars?
Less than $\$ 100,000$
$\$ 100,000$ to less than $\$ 1 \mathrm{M}$
$\$ 1 \mathrm{M}$ to less than $\$ 10 \mathrm{M}$
$\$ 10 \mathrm{M}$ to less than $\$ 100 \mathrm{M}$
More than $\$ 100 \mathrm{M}$

What was the estimated duration of your last software development project?
Less than 6 months
6 months to less than 12 months
12 months to less than 24 months
24 months to less than 36 months
More than 36 months

In which country was this software development project conducted? (Please indicate the major location for international software development projects)

Section B: Kindly indicate your responses for the following factors based on your last completed software development project irrespective of whether it was successful or unsuccessful. Please, indicate the extent to which you agree or disagree with the following statements by ticking the box of your choice.The word 'organization' refers to the client or customer organization for which the software was developed.

Top level management commitment. A 'project champion' is not a project manager or team leader but a person who provides moral, psychological and physical support to the team and provides them with needed resources and advocates for the project's benefits and advantages to its stakeholders.

\begin{tabular}{|c|c|c|c|c|c|c|c|}
\hline & $\begin{array}{l}\text { Strongly } \\
\text { Disagree }\end{array}$ & Disagree & $\begin{array}{l}\text { Somewhat } \\
\text { Disagree }\end{array}$ & $\begin{array}{l}\text { Neither } \\
\text { Agree nor } \\
\text { Disagree }\end{array}$ & $\begin{array}{l}\text { Somewhat } \\
\text { Agree }\end{array}$ & Agree & $\begin{array}{c}\text { Strongly } \\
\text { Agree }\end{array}$ \\
\hline $\begin{array}{l}\text { There was approval and support } \\
\text { from top level management. }\end{array}$ & (1) & 0 & O & (0) & (1) & (C) & (1) \\
\hline
\end{tabular}

https://vuw.qualtrics.com/ControlPanel/Ajax.php?action=GetSurveyPrint... 9/02/2014 


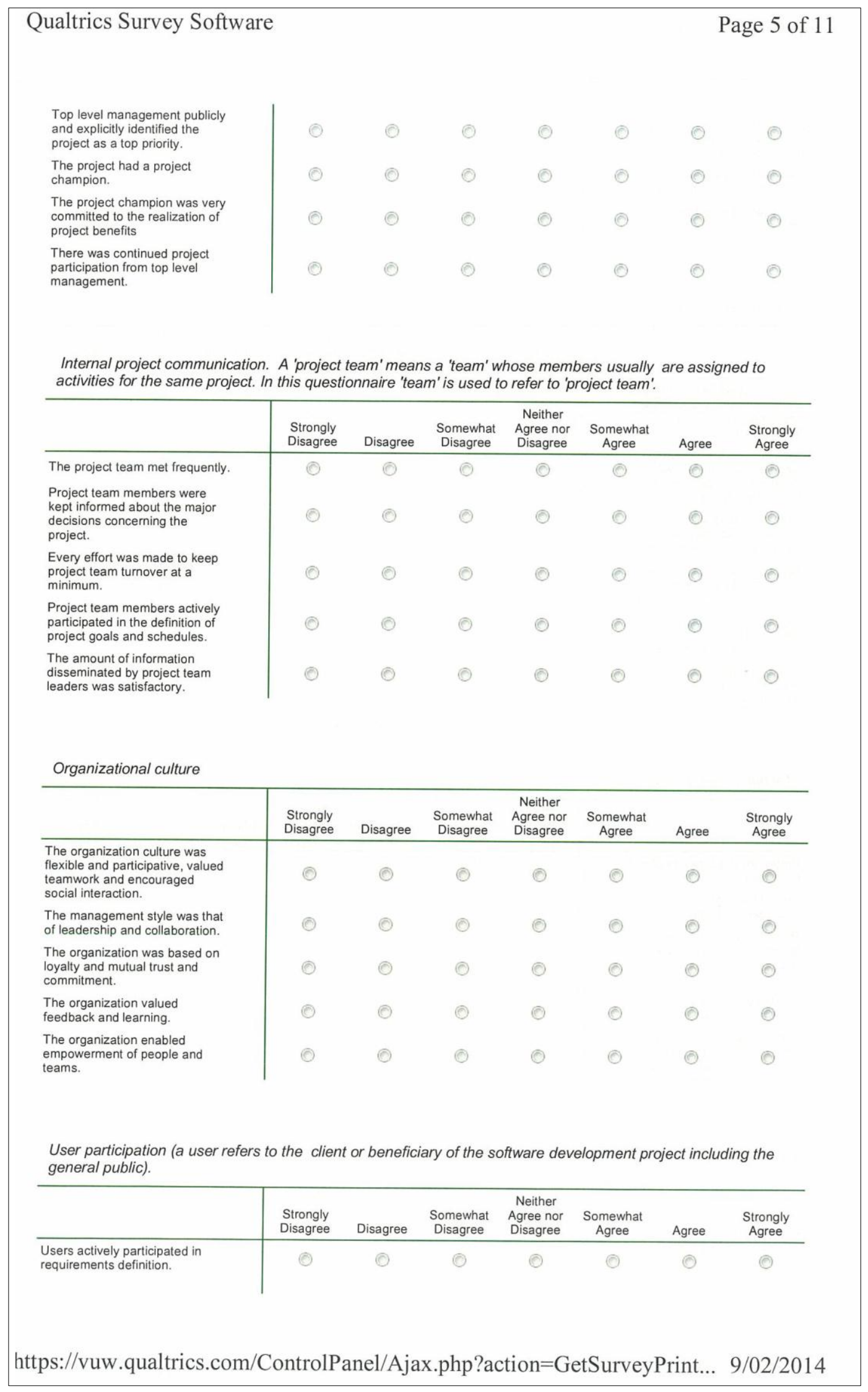




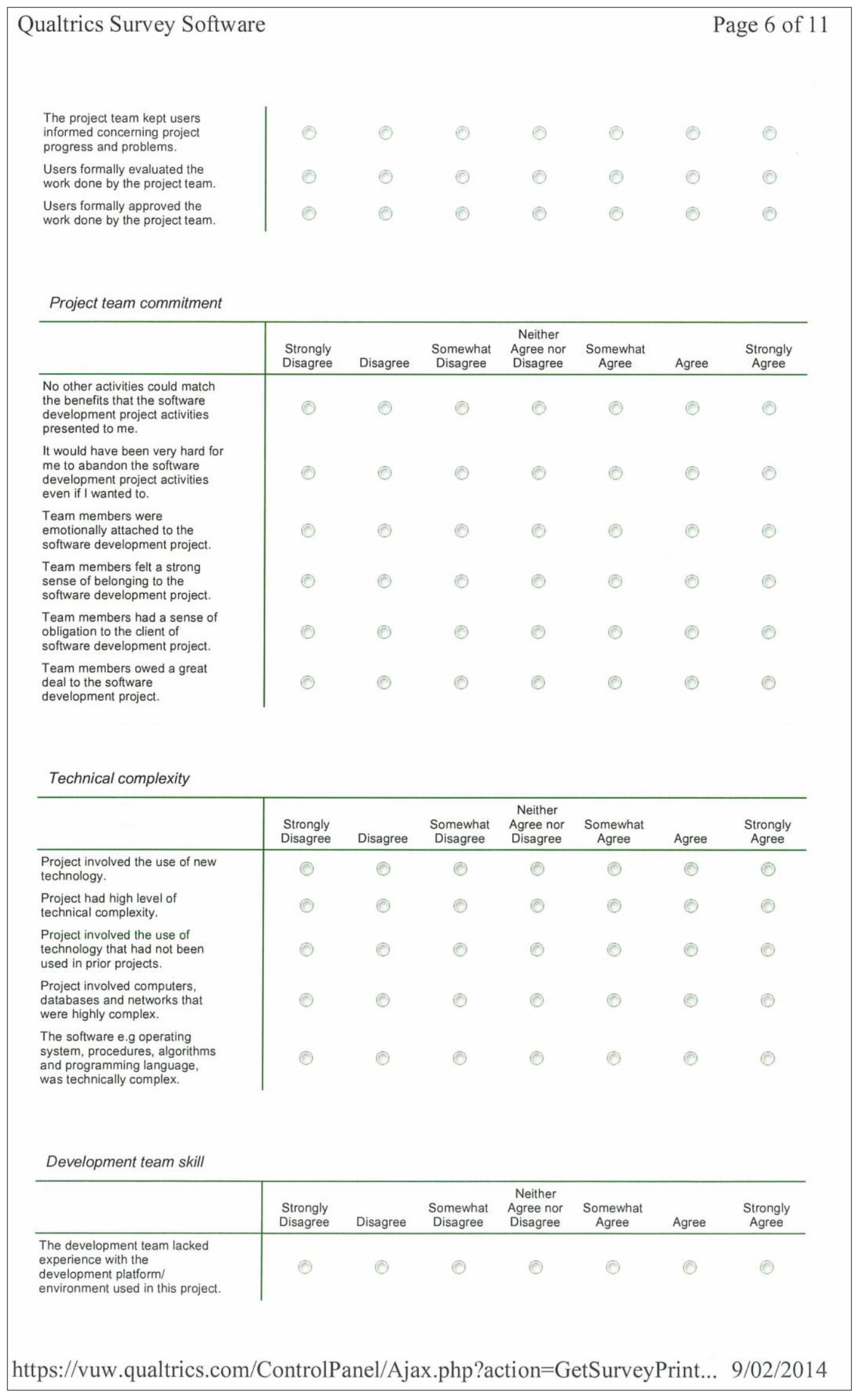


The development team was very unfamiliar with this type of application.

The development team lacked involved in this project.

The development team

generally lacked technically

competent and experienced similar software in the past. knowledge of application domain

\section{$\mid$}

Client experience level

\begin{tabular}{|c|c|c|c|c|c|c|c|}
\hline & $\begin{array}{l}\text { Strongly } \\
\text { Disagree }\end{array}$ & Disagree & $\begin{array}{c}\text { Somewhat } \\
\text { Disagree }\end{array}$ & $\begin{array}{l}\text { Neither } \\
\text { Agree nor } \\
\text { Disagree }\end{array}$ & $\begin{array}{l}\text { Somewhat } \\
\text { Agree }\end{array}$ & Agree & $\begin{array}{c}\text { Strongly } \\
\text { Agree }\end{array}$ \\
\hline $\begin{array}{l}\text { The client was not familiar with } \\
\text { this type of application. }\end{array}$ & (1) & (C) & (1) & (O) & (1) & 0 & (1) \\
\hline $\begin{array}{l}\text { The client didn't know what they } \\
\text { wanted. }\end{array}$ & (0) & (C) & (C) & (1) & (C) & (0) & (0) \\
\hline $\begin{array}{l}\text { The client didn't have a good } \\
\text { understanding of the problems } \\
\text { they want solved. }\end{array}$ & 0 & (C) & ( & (C) & (C) & (C) & (C) \\
\hline $\begin{array}{l}\text { The client was not familiar with } \\
\text { data processing as a work tool. }\end{array}$ & (1) & (C) & (1) & (1) & (1) & (1) & 0 \\
\hline $\begin{array}{l}\text { The client was not aware of the } \\
\text { importance of their roles in } \\
\text { successfully completing the } \\
\text { project. }\end{array}$ & (1) & (C) & (1) & (C) & (C) & (1) & (0) \\
\hline
\end{tabular}

Project planning and controlling

\begin{tabular}{l|ccccccc}
\hline & $\begin{array}{c}\text { Strongly } \\
\text { Disagree }\end{array}$ & Disagree & $\begin{array}{c}\text { Somewhat } \\
\text { Disagree }\end{array}$ & $\begin{array}{c}\text { Neither } \\
\text { Dgree nor } \\
\text { Disagree }\end{array}$ & $\begin{array}{c}\text { Somewhat } \\
\text { Agree }\end{array}$ & $\begin{array}{c}\text { Strongly } \\
\text { Agree }\end{array}$ \\
\hline $\begin{array}{l}\text { Special attention was paid to } \\
\text { project planning. }\end{array}$ & 0 & & & & 0 \\
$\begin{array}{l}\text { Project milestones were clearly } \\
\text { defined. }\end{array}$ & 0 & & & & & \\
\end{tabular}

https://vuw.qualtrics.com/ControlPanel/Ajax.php?action=GetSurveyPrint... 9/02/2014 


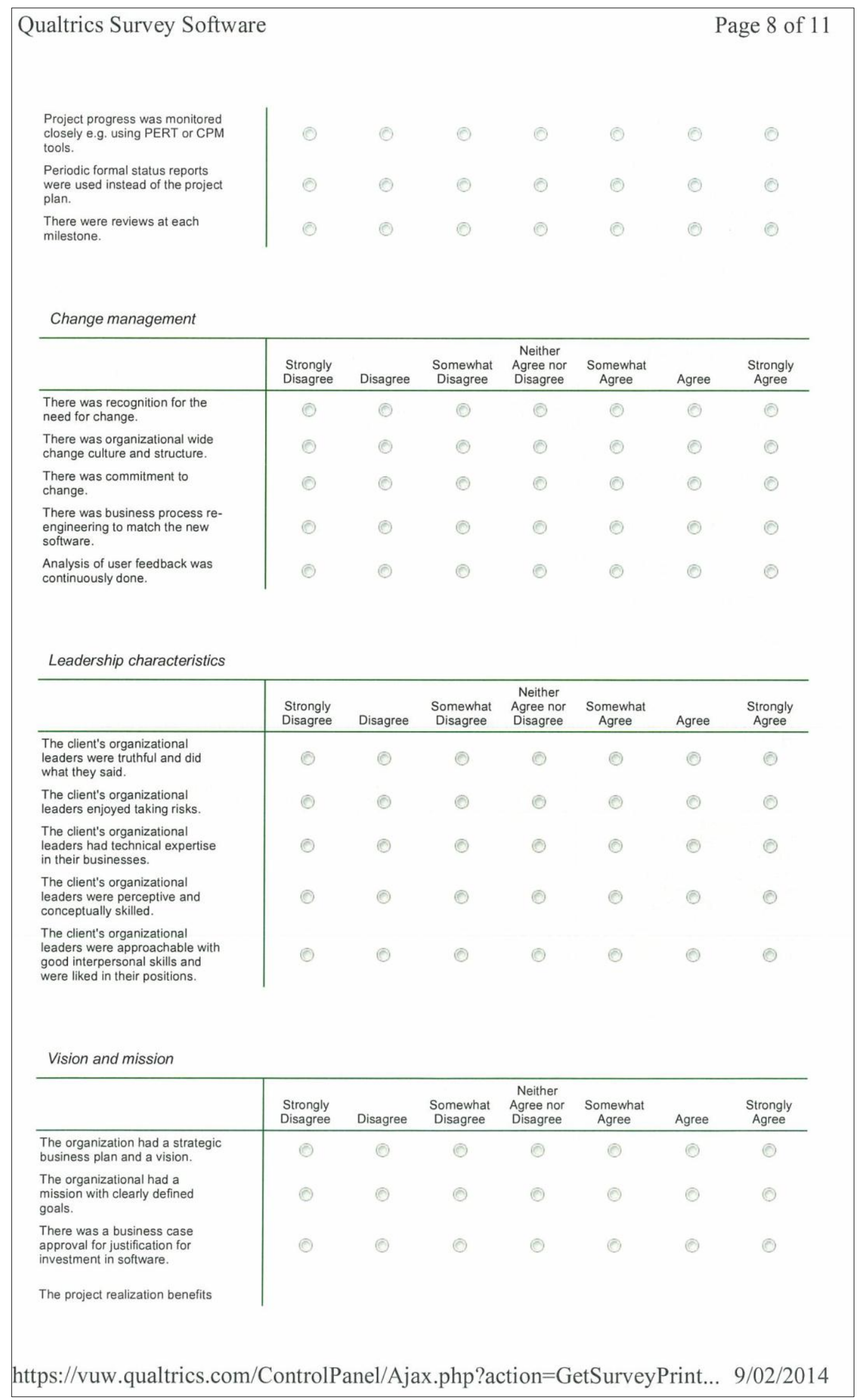




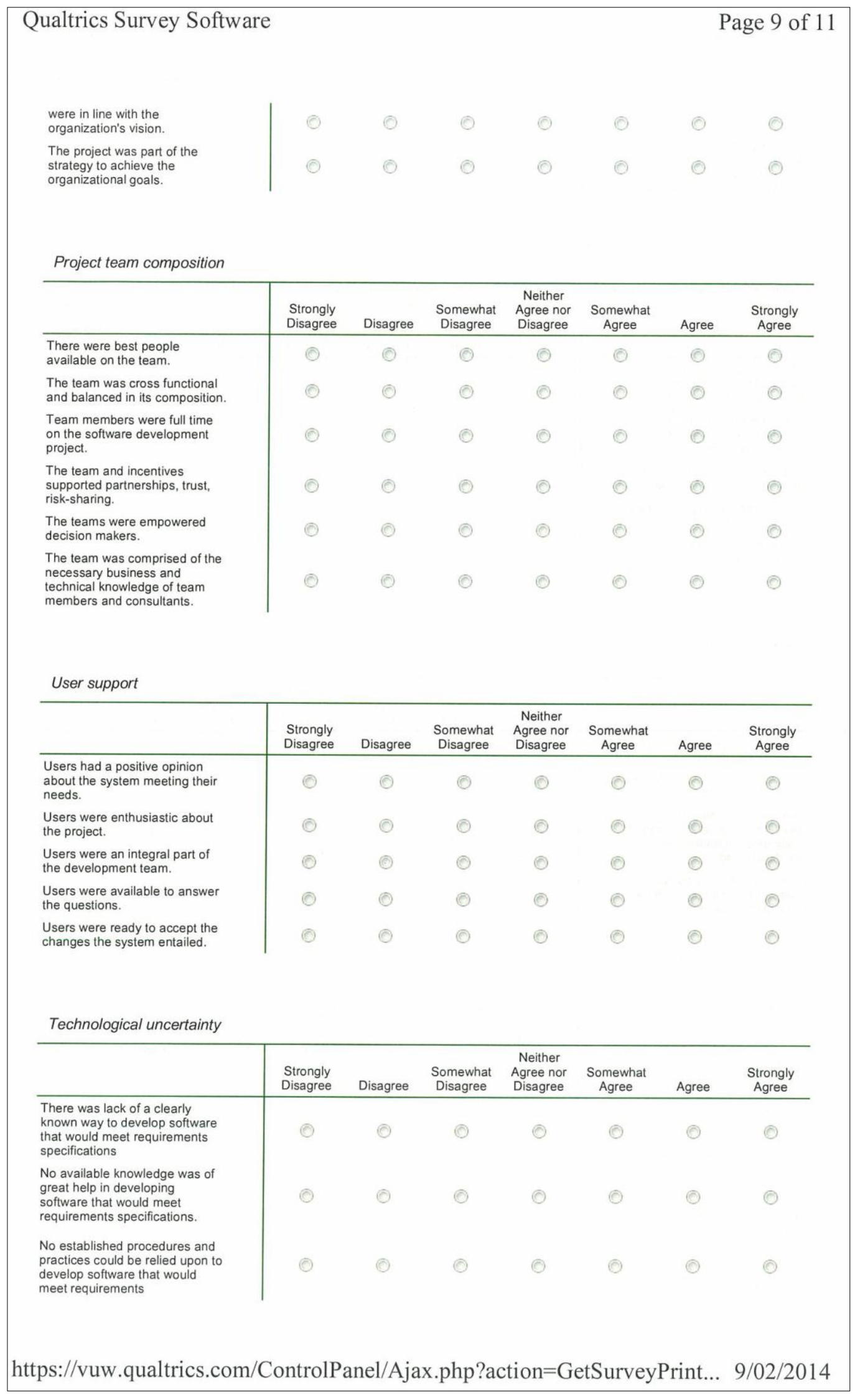




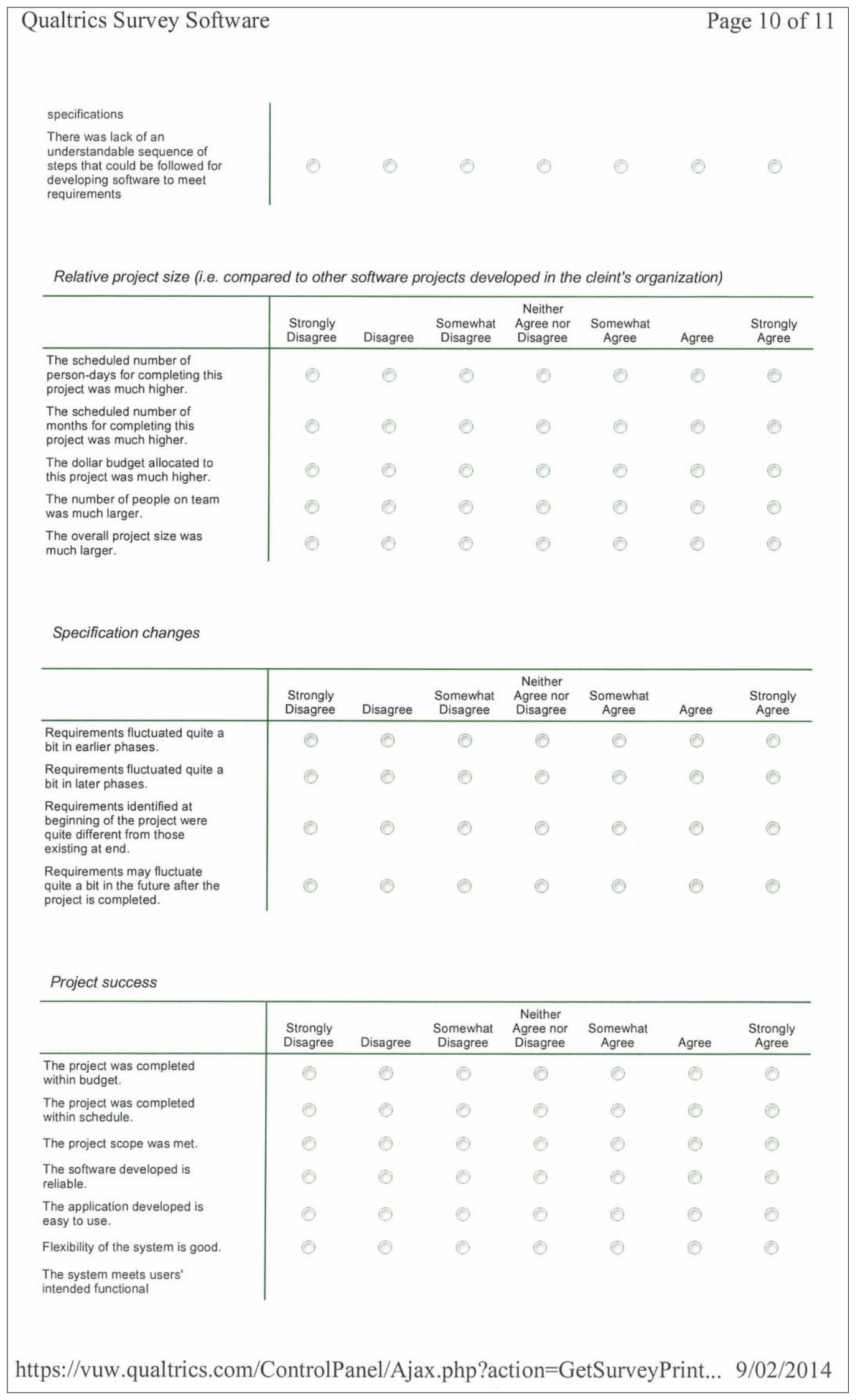


requirements.

Users were satisfied with the system delivered.

The project team was satisfied.

Top level management of the

client's organization was

satisfied.

The overall quality of the

delivered application is high.

\begin{tabular}{|lllllll}
0 & 0 & 0 & 0 & 0 & 0 & 0 \\
0 & 0 & 0 & 0 & 0 & 0 & 0 \\
0 & 0 & 0 & 0 & 0 & 0 & 0 \\
0 & 0 & 0 & 0 & 0 & 0 & 0 \\
0 & 0 & 0 & 0 & 0 & 0 & 0
\end{tabular}

Please kindly write down any general comments that you may have about this questionnaire or the whole study in general.

https://vuw.qualtrics.com/ControlPanel/Ajax.php?action=GetSurveyPrint... 9/02/2014 


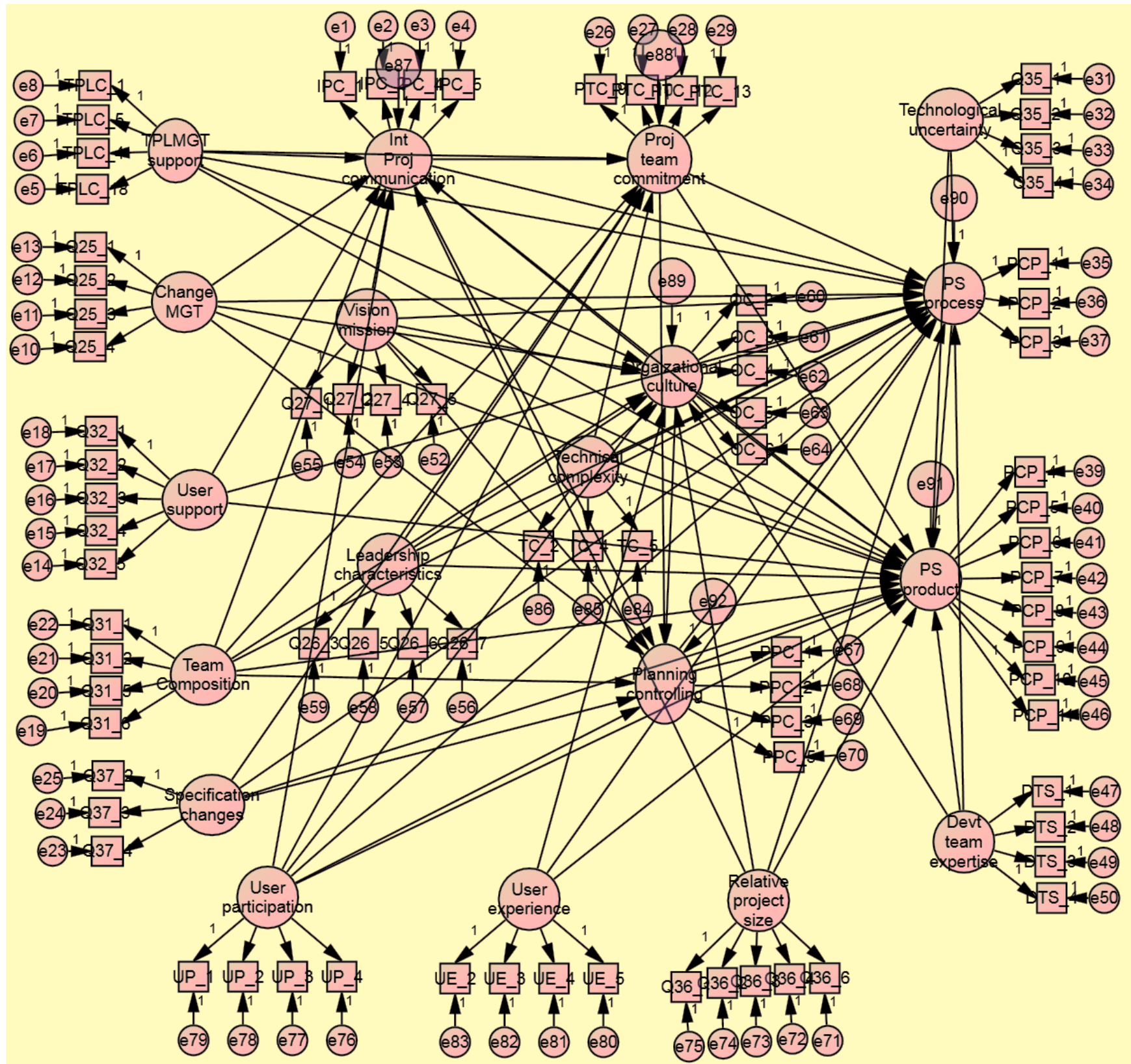

Figure 43: SEM model (main effects model) tested (without covariances for readability and clarity) 
Table 87: Measurement items dropped during SEM analysis

\section{Top management support}

The project champion was part of top level management.

There was an organizational commitment to employing the principles of project management.

The project manager and the project team were given the resources necessary to carry out the strategy for project completion.

The project was well recognized by top level management.

Internal project communication

The language which was used in correspondences was familiar to all team members.

Organizational culture

The leadership of the organization in which the project was conducted was entrepreneurial, innovative and risk-taking.

User participation

Users were cooperative and had a positive attitude towards the project.

\section{Project team commitment}

My life would have been upset if I decided not to engage in software development project activities.

It would have been too costly for me to quit software development project activities during that project.

\section{Technical complexity}

The project involved developing software with many links to the existing system.

User experience

The client was not aware of the importance of their roles in successfully completing the project.

\section{Project criticality}

A defect only could have a very minor impact leading to loss of comfort.

A defect could be fixed pretty easily because of good backup procedures leading to loss of discretionary monies.

A defect in the system could make the company go bankrupt leading to loss of essential monies.

A defect could be catastrophic, leading to loss of lives such as failure in nuclear power stations or flight control system.

Leadership characteristics

The client's organizational leaders were ambitious and took initiatives.

The client's organizational leaders wanted to lead and were willing to take charge.

Vision and mission

There was a business case approval for justification for investment in software.

Project team composition

Team members were full time on the software development project.

The team and incentives supported partnerships, trust, and risk-sharing.

Specification changes

Requirements fluctuated quite a bit in earlier phases. 